\title{
Idaho National Laboratory Directed Research and Development FY-2009
}

March 2010

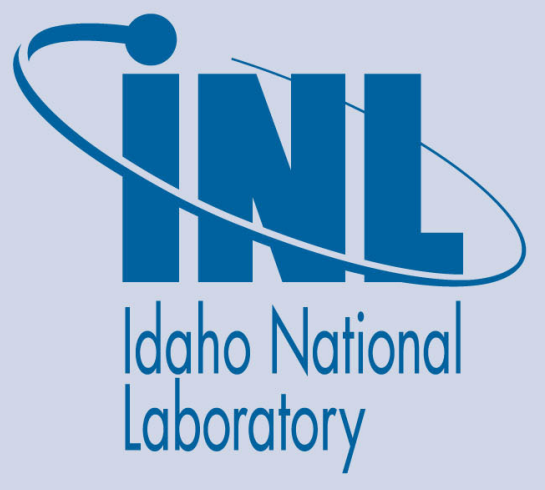

The INL is a U.S. Department of Energy National Laboratory operated by Battelle Energy Alliance 
INL/EXT-09-17391

\title{
Idaho National Laboratory Directed Research and Development FY-2009
}

March 2010

\author{
Idaho National Laboratory \\ Idaho Falls, Idaho 83415
}

http://www.inl.gov

Prepared for the

U.S. Department of Energy

Office of Nuclear Energy

Under DOE Idaho Operations Office

Contract DE-AC07-05ID14517 


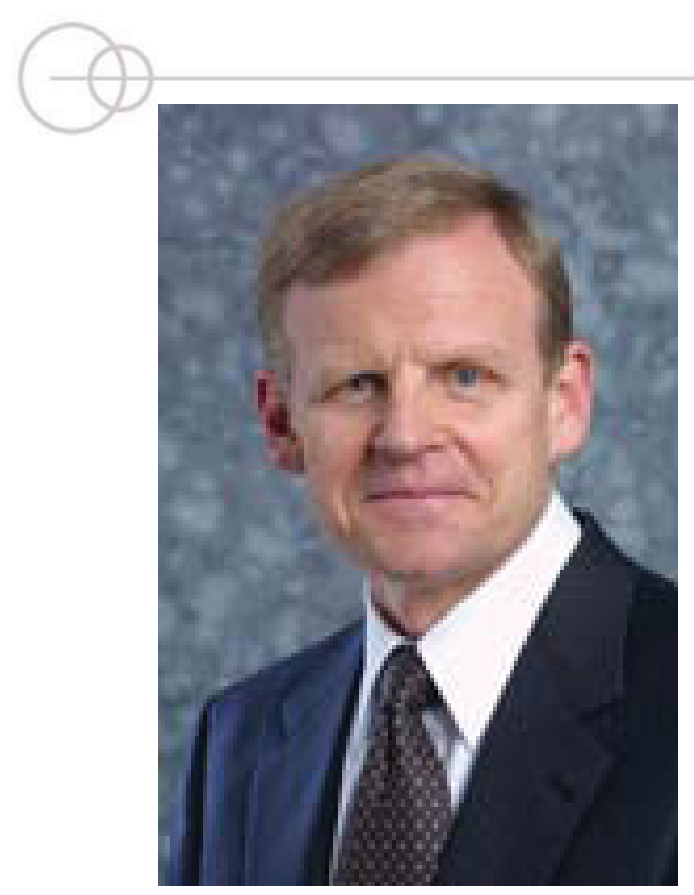

It is my pleasure to present the Idaho National Laboratory's (INL's) fiscal year (FY) 2009 Laboratory-Directed Research and Development (LDRD) Annual Report. This report demonstrates the types of cutting edge research the INL is performing to help ensure the nation's energy security. The research conducted under this program is aligned with our strategic plan, benefits the Department of Energy (DOE) and is in compliance with DOE order 413.2B.

This report summarizes the diverse research and development portfolio with emphasis on the DOE-NE mission, encompassing both advanced nuclear science and technology and underlying technologies.

The INL's LDRD program also serves the nation through research and development that helps sustain homeland security with emphasis on cyber and infrastructure control systems, communications and wireless systems, and explosives detection and effects mitigation. Additionally, our science base is extended through research that advances alternative fuels to lessen the nation's dependence on petroleumbased fuels.

The INL Strategic Plan presents objectives that will transform the Laboratory during the next decade and the LDRD program is a pivotal component of this transformation. These key objectives are defined in five areas:

- Leading nuclear energy

- Multiprogram excellence

- Modern facilities and capabilities

- Outstanding people

- Excellence in operations and community service.

The LDRD research portfolio supports the INL's Strategic Plan by developing the scientific and engineering resource capabilities and collaborations needed to accomplish these objectives. It is an essential competency strengthening activity, attracting and maintaining world-class researchers, and cultivating the collaborative networks with universities, industry and other laboratories required to fully support DOE and other national priorities.

I am proud of the accomplishments and opportunities that the INL's LDRD provides to the nation, and encourage you to take the time to review these project narratives and reflect on those contributions.

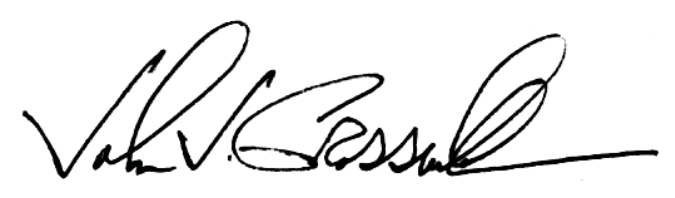

John J. Grossenbacher Laboratory Director 
INL/EXT-09-17391

\title{
Acknowledgments
}

This annual report provides an overview of the Laboratory Directed Research and Development (LDRD) Program at the Idaho National Laboratory (INL). Summarized in this report are 100 technical research projects conducted in fiscal year 2008. The LDRD Program at the INL is managed for the laboratory by Mr. John J. Grossenbacher, Laboratory Director, Dr. Ray Enge, Director, Strategic Planning and Organizational Development, and Mr. John S. Martinell, LDRD Program Manager.

Many people contributed to this report; however, particular thanks go to the principal investigators who conduct the cutting-edge research so important to this laboratory.

\author{
Managing Editor: Dena Tomchak \\ Science Writer: Lisa Plaster \\ Keith Arterburn \\ Mike Wall
}

Text Processing: Mary Bates

Cover Design: Allen Haroldsen

\author{
Prepared for the U.S. Department of Energy \\ Director, Nuclear Energy, Science \& Technology \\ Under DOE Idaho Operations Office \\ Contract Number: DE-AC07-05ID14517
}

\section{Disclaimer}

This report was prepared as an account of work sponsored by an agency of the United States Government. Neither the United States Government nor any agency thereof, makes any warranty, express or implied, or assumes any legal liability or responsibility for the accuracy, completeness, or usefulness of any information, apparatus, product, or process disclosed, or represents that its use would not infringe privately owned rights. Reference herein to any specific commercial product, process, or service by trade name, trademark, manufacturer, or otherwise does not necessarily constitute or imply its endorsement, recommendation or favoring by the United States Government or any agency thereof. The views and opinions of authors expressed herein do not necessarily state or reflect those of the United States Government or any agency thereof. 


\section{Table of Contents}

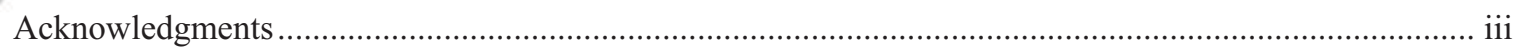

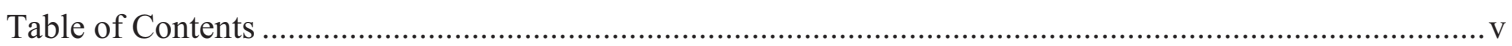

\section{Overview and Highlights}

Highlights

Testing Offers Insight into New Energy-Absorbing Building Materials and Methods for Blast-

Resistant Applications....

Developing Resilient Control System Network Agents for Critical Infrastructure

Addressing the Spectrum of Nuclear-Related NDE Needs .

Developing Numerical Tool to Aid INL Hybrid Energy Systems Testing (HYTEST).............................14

Working Toward a Better Understanding of Radiation Chemistry ….................................................. 16

Next-Generation Modeling for Next-Generation Reactors ........................................................ 18

\section{Nuclear Energy Science and Technology}

Group Actinide Separation from SNF Using a Modified Universal Solvent Extraction Process.................23

Exploration of Electrolyte Complexation and Pulse Deposition for Production of Dense Uranium.............26

A Strategy to Tightly Couple Neutronics and Thermal Hydraulics Models for Next Generation

Reactor Analysis ..... . .28

Integrated Mesoscale Approach for the Simulation of Nuclear-Fuel Behavior......................................31

Process Modeling of Solvent Extraction Separations for Advanced Nuclear Fuel Cycles.........................33

Reactivity of Radiolytically Produced Nitrogen Oxide Radicals toward Aromatic Compounds .................36

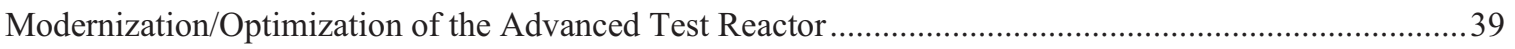

Evaluations of Linear Variable Differential Transformers for ATR In-Pile Instrumentation .....................42

Characterization of a Consolidated Electrochemical Technique for Separation and Recovery of Actinides from Fission Products

Advanced Instrumentation for In-pile Detection of Thermal Conductivity

Human System Simulation and Human Performance Assessment: Testing and Review of Digital

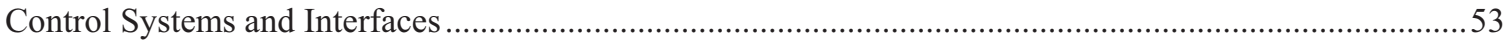

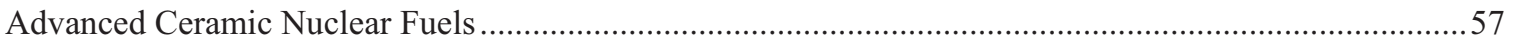

Characterization of Fluidized Beds via Pressure-Fluctuation Analysis..............................................60

Dissolution and Extraction Studies of Fission Products in Room Temperature Ionic Liquids (RTIL) and in Supercritical Fluid $\mathrm{CO}_{2}\left(\mathrm{ScCO}_{2}\right)$ and Determination of Radiolytic Stability.....

High Temperature In-Pile Instrumentation Enhancements

\section{National and Homeland Security}

Multi-Stage Sequential Injection Gas Gun.... 
Enhanced Metal Ion Analysis

Taylor Cylinder Determination of Impact Material Properties.......................................................75

Development and Evaluation of Low Pressure Energy Absorbing Materials and Methods for

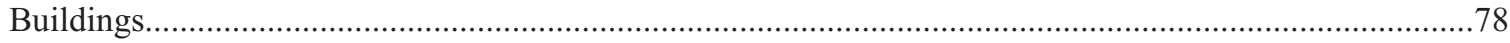

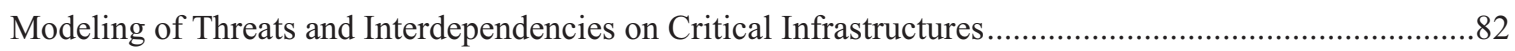

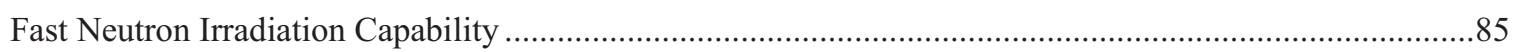

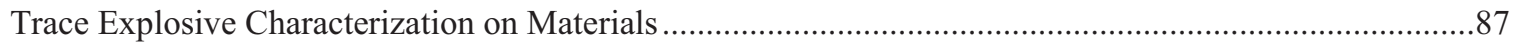

Methodologies for the Design, Analysis, and Validation for Operation of Complex Resilient

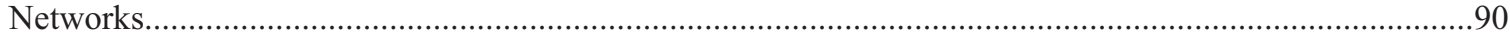

Cognitive Network Engine and Simulation Framework ................................................................96

Application of Dynamic Bayesian Networks to Systems with Ambient Intelligence ...............................97

\section{Science and Technology}

Thermal and Acid Activated In-plant Lignocellulose-degrading System for Eonomically Enhanced

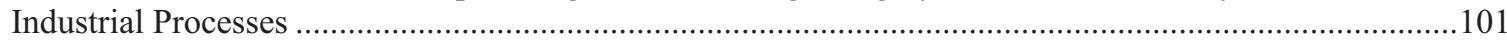

Novel Catalysts for the Reduction of Biomass Pretreatment Severity …...........................................103

Particle-discrete Element Model Simulation of the Coupling between Material

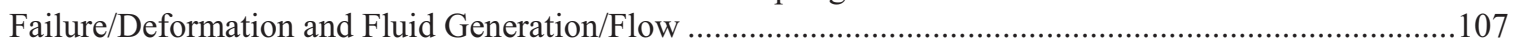

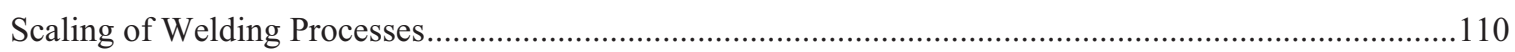

Effect of Glycosylation on the Activity and Stability of Bacterial Enzymes........................................113

A Systems Biology Approach to Understanding Lignocellulose-derived Carbon Metabolism.................115

The Metabolic Engineering of a Gram-positive Thermoacidophile for Lactic Acid ..............................117

Network Interaction in a Thermoacidophile In Response to Different Complex Carbon Sources .............120

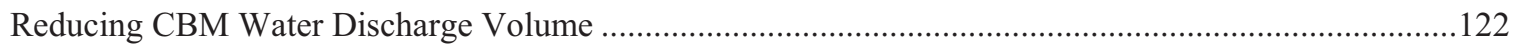

Advanced Adaptive Algorithms in Phased Array Ultrasonics for Material Inspection ............................125

Investigation of Low-Temperature Performance in Membrane Materials and Processes for Gas

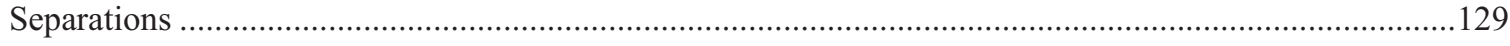

High-Performance Polymer Membranes for High-Temperature Gas Separations .................................132

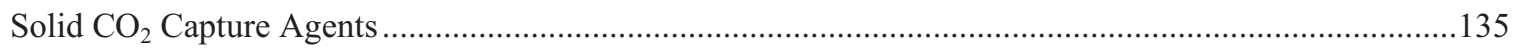

Dynamic Impact Model and Information System to Support Unconventional-Fuels Development ...........137

Near-Field Impacts of In-Situ Oil-Shale Development on Water Quality...........................................139

Advanced Remote Sensing for Energy and Environmental Applications Using Unmanned Aerial

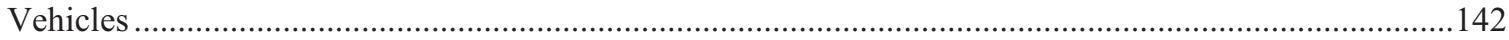

Addressing the Spectrum of Nuclear-Related NDE Needs .............................................................. 145

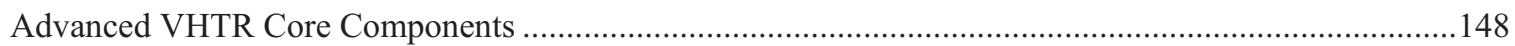


Development of a Sustainable Ecological Engineering Approach to Support Hybrid Energy

System Development in the Western Inland Energy Corridor ....

Development of 3D Multiphase Flow and Reactive Transport Codes and their Applications to

Reactive Flow in Porous Media and Fracture Apertures.....

\section{Advanced Nuclear Energy Initiative}

Multi-Reactor Design and Analysis Platform

A General Framework for Simulating Fully Coupled Mass and Energy Transport .....

System Analysis for Reactor Applications with High Fidelity (SARAH) 166

Acquisition and Improvement of a Modern Lattice Physics Capability 168

Unified Two-phase CFD Modeling of Boiling, Cavitation, and Bubble Collapse. .171

Reactor Physics Sensitivity Analysis, Uncertainty Quantification, and Data Assimilation Capability 176

Fracture Methods for Reactor Fuel Performance Analysis 179

Use of Ice Thermal Storage Systems to Improve LWR Plant Efficiency....

Development of a Next-Generation Production Code for Nuclear Reactor System Analysis and Safety Margin Quantification.....

Multi-physics Simulation Methods for Advanced Reactor Analysis .

\section{Energy Security Initiative}

Chemical Separations and Process Research to Enable Biorefinery Systems

Advanced Predictive Condition Monitoring and Control for Modern Energy Systems:

Gasification-based Processes 198

CFD-Based Simulation Capability of Fischer-Tropsch Reactors and Process Equipment.

Biomass Feedstock Assembly to Gasification Process Computational Interface Development ...............209

Generation and Expulsion of Hydrocarbons from Oil Shale ...........................................................213

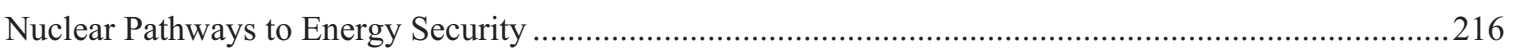

Investigation of Fischer Tropsch catalyst deactivation ............................................................219

Altering Wettability by Chemical Amendments to Improve Gas Production from Tight Sands ..............220

Adaptive Process Modeling Using Parameter Estimation \& Mechanism Sensitivity Analysis .................223

\section{Nuclear Nonproliferation Initiative}

Chemical Signatures of Nuclear Proliferation on Particles ..........................................................2229

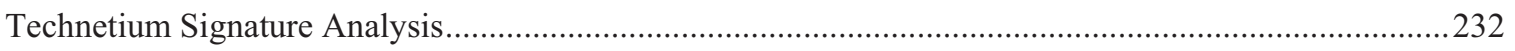

Active Interrogation Die-Away Assay Development Program .........................................................235

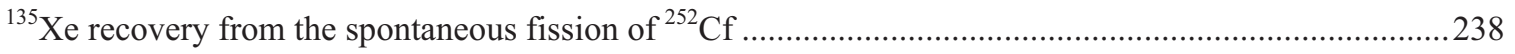


Developing a Next Generation, Risk-Informed Approach for the Physical Protection of Nuclear Facilities ....

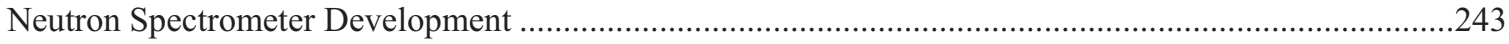

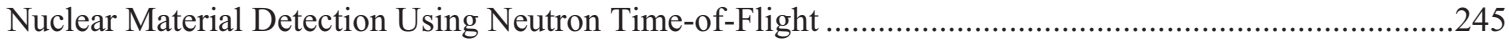

On-line monitoring of Actinide Concentrations for Advanced Aqueous Separations ...................................249

Development of a Safeguards Approach for Pyroprocessing …..................................................................252

Radionuclide Collection-Detection Device for the in situ Remote Monitoring of ${ }^{99} \mathrm{Tc}$ as a

Proliferation Indicator.

\section{Center for Advanced Energy Studies}

Microstructural Evolution During Spark Plasma Sintering of High-Temperature Fuels and Coatings 259

Suitability of Layered Basalt as Targets for Industrial Carbon Dioxide Sequestration 262

Understanding Apomixis: The Basis for a Robust Trait Delivery and Containment Platform for Bioenergy Crops. .265

Enhancement of Separation Methods in Nuclear Fuel Recycling .267

Investigation of Public Discourse Methods in Energy Policy Decision-making ..........................................269

Development of Lignocellulosic Biofuels Production Potential in Idaho. .274

Center for Advanced Modeling and Simulation

Structural and Electronic Properties of Adsorbed Metal Nanoparticles .281

Adaptive Modeling of Geometrically Complex Fuel Rods with a posteriori Error Control .......................284

Uncertainty Quantification for Nuclear Fuels Performance 286

\section{Distinctive Signatures}

\section{INSTRUMENTATION, CONTROL AND INTELLIGENT SYSTEMS DISTINCTIVE}

SIGNATURE

Exploration and Development of Automated Differential Equation-Based System Identification .............293

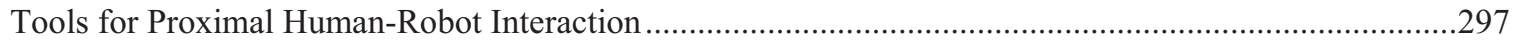

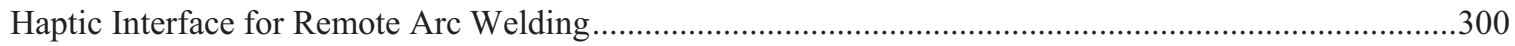

Modeling Interface to Control System Designs...........................................................................................303

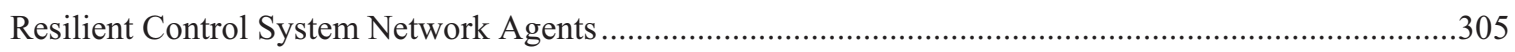

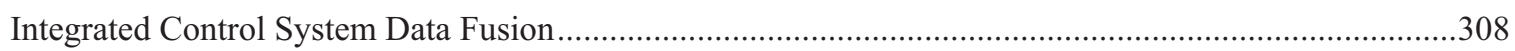

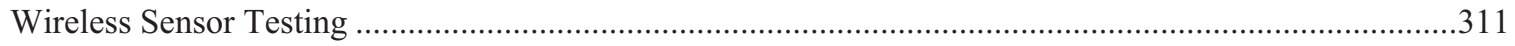

Anomaly Detection, Diagnosis, and Resilient Control in Complex Engineered Systems ............................312

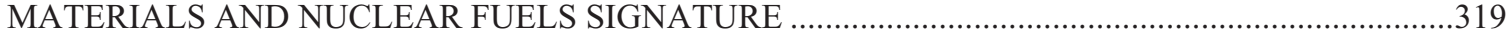


Environmental Effects on Crack Growth in High-Temperature Alloys for Advanced Energy Systems 321

Development of Advanced Burnup Measurement \& Nuclear Forensics ICP-MS Isotopics Analysis Techniques

Development of a Small Sample Volume Mechanical Properties Testing Technique for Irradiated Fuels and Materials

Microstructure and Deformation Physics of Nuclear Materials by Atomistically Informed Mesoscale Simulation 332

SEPARATIONS AND ACTINIDE SCIENDE DISTINCTIVE SIGNATURE

Utility of Unusual Oxidation States of Americium for Separations

Rapid Detection of Plutonium, Neptunium, and Technetium in Water Samples

Evaluation of Covalent Interactions in Actinide Coordination Compounds 343

Measuring Actinide Speciation in High-pH Solutions .... 346

Isotope Ratio Measurement Methods for Direct Analysis of Samples

\section{Appendices}

Appendix A - Authors

Appendix B - Refereed Publications.

Appendix C - Key Terms.

Appendix D — Project Performance.

Appendix E - Relevance to Major DOE Missions

Appendix F — Relevance to Major National Programs. 
PAGE FOR OVERVIEW DIVIDER 


\section{Overview and Highlights}

The FY 2009 Laboratory Directed Research and Development (LDRD) Annual Report is a compendium of the diverse research performed to develop and ensure the INL's technical capabilities can support the future DOE missions and national research priorities. LDRD is essential to the INL - it provides a means for the laboratory to pursue novel scientific and engineering research in areas that are deemed too basic or risky for programmatic investments. This research enhances technical capabilities at the laboratory, providing scientific and engineering staff with opportunities for skill building and partnership development.

Established by Congress in 1991, LDRD proves its benefit each year through new programs, intellectual property, patents, copyrights, publications, national and international awards, and new hires from the universities and industry, which helps refresh the scientific and engineering workforce.

\section{Benefits of LDRD to INL}

The benefits of INL's LDRD research are many as shown in the tables below. Last year, 91 faculty members from various universities contributed to LDRD research, along with 7 post docs and 64 students. Of the total invention disclosures submitted in FY 2009, 7 are attributable to LDRD research. Sixty three refereed journal articles were accepted or published, and 93 invited presentations were attributable to LDRD research conducted in FY 2009.

\begin{tabular}{|l|r|}
\hline \multicolumn{2}{|c|}{ FY09 LDRD Project Performance } \\
\hline National Awards & 0 \\
\hline Chaired Symposia & 19 \\
\hline Invention Disclosures & 7 \\
\hline Patents & 0 \\
\hline Copyrights/Trademarks & 3 \\
\hline Invited Presentations & 93 \\
\hline Refereed Publications & 63 \\
\hline University Personnel & 162 \\
\hline
\end{tabular}

\begin{tabular}{|l|c|}
\hline \multicolumn{2}{|l|}{ FY09 LDRD Program Statistics } \\
\hline LDRD Program Cost & $\$ 24.9 \mathrm{M}$ \\
\hline Number of Projects & 100 \\
\hline Largest Project & $\$ 1 \mathrm{M}$ \\
\hline Smallest Project & $\$ 50 \mathrm{~K}$ \\
\hline
\end{tabular}

\section{The LDRD Process at INL}

The LDRD Program is administered in accordance with requirements set in DOE Order 413.2B, accompanying contractor requirements, and other DOE and federal requirements invoked through the INL contract. The LDRD Program is implemented in accordance with the annual INL LDRD Program Plan, which is approved by the DOE, Nuclear Energy Program Secretarial Office. This plan outlines the method the laboratory uses to develop its research portfolio, including peer and management reviews, and the use of other INL management systems to ensure quality, financial, safety, security and environmental requirements and risks are appropriately handled. The LDRD Program is assessed annually for both output and process efficiency to ensure the investment is providing expected returns on technical capability enhancement.

The call for proposals and project selection process for the INL LDRD program begins typically in April, with preliminary budget allocations, and submittal of the technical requests for preproposals. A call for preproposals is made at this time as well, and the preparation of full proposals follows in June and closes in July. The technical and management review follows this, and the portfolio is submitted for DOE-ID concurrence in early September. Project initiation is in early October.

The technical review process is independent of, and in addition to the management review. These review processes are very stringent and comprehensive, ensuring technical viability and suitable technical risk are encompassed within each project that is selected for funding. Each proposal is reviewed by two or three anonymous technical peers, and the reviews are consolidated into a cohesive commentary of the overall research based on criteria published in the call 
for proposals. A grade is assigned to the technical review and the review comments and grade are released back to the principal investigators and the managers interested in funding the proposals. Management criteria are published in the call for proposals, and

management comments and selection results are available for principal investigator and other interested management as appropriate. The DOE Idaho Operations Office performs a final review and concurs on each project prior to project authorization, and on major scope/budget changes should they occur during the project's implementation.

\section{Report Organization}

This report begins with several research highlights that exemplify the diversity of scientific and engineering research performed at the INL in FY 2009. Progress summaries for all projects are organized into sections reflecting the major areas of research focus at the INL. These sections begin with the DOE-NE Nuclear Science and Technology mission support area, followed by the National and Homeland Security and the Energy and Environmental Science and Technology areas. The major INL initiatives and the INL's Distinctive Signatures areas complete the project summaries. The appendices provide information on project relevance to $\mathrm{DOE}$ missions and major national programs as well as an author index, list of refereed publications and index of key terms. 


\section{Highlights}




\title{
Testing Offers Insight into New Energy-Absorbing Building Materials and Methods for Blast-Resistant Applications
}

\author{
Mark Landon, Ben Langhorst
}

NS161

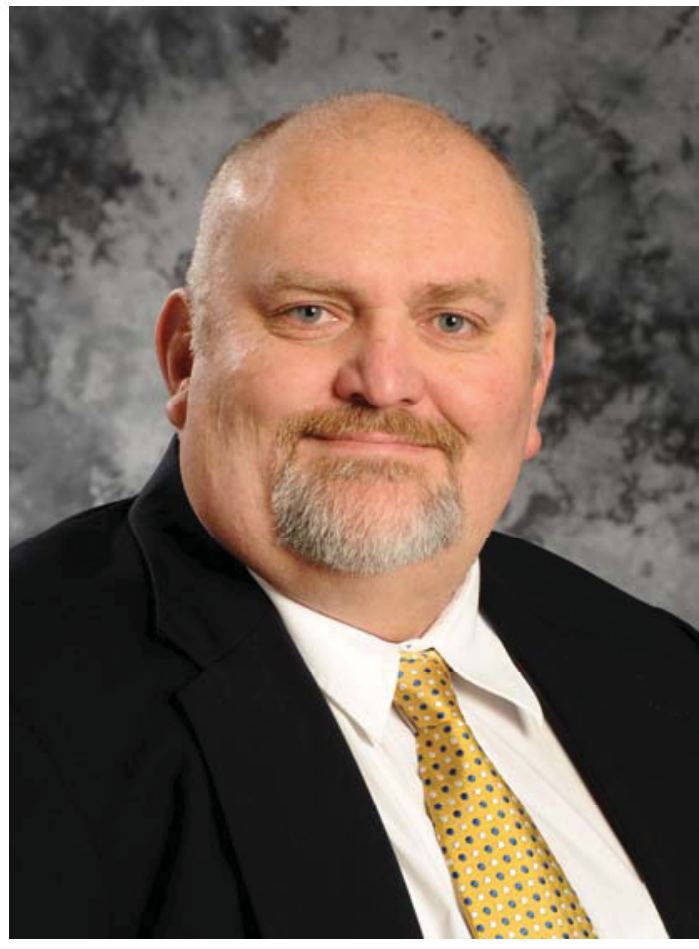

This LDRD research project learned about the possibility of using lighter building materials using specific construction methods to make buildings safer.

The team of INL researchers, including Dr. Mark Landon, Dr. Ben Langhorst, Dr. Henry Chu, Dr. John Weathersby, James Schondel and two summer interns, conducted blast testing on an extensive series of novel energy-dissipating technologies. The team also included three researchers from the University of Florida that investigated cladding-connection systems that could further reduce the amount of blast energy transferred to a building.

"We discovered some very promising building technologies with efficient weights and costs that could be further refined to enhance applicability for full-scale blast testing," Landon said. The report noted that the tests revealed impressive blast dissipative performance. One promising design weighed 3.5 pounds per square foot and cost approximately $\$ 13$ per square foot for a lab-scale sample.

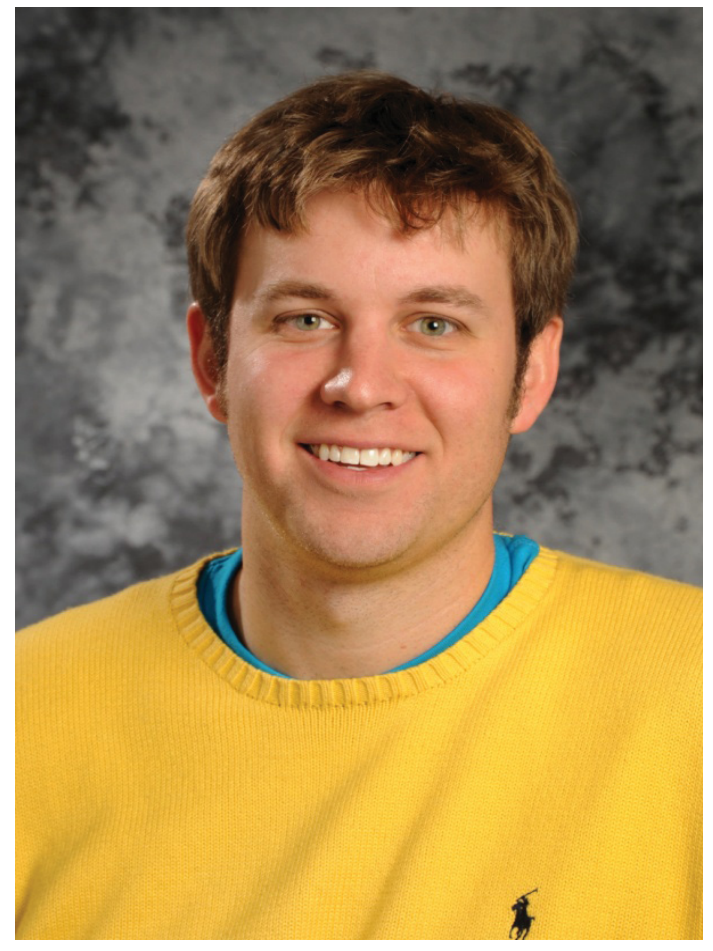

"We tried thirty seven different concepts and many of them performed very well," Langhorst said. "The better-performing technologies would reduce the amount of energy transmitted through the panels to a building by a factor of 5." He also noted that further development is necessary to prepare the top-performers for real-world application.

The research was conducted to discover improved ways of protecting buildings and their contents from blast overpressures. In an urban environment, the detonation of a large explosive device ( $>500 \mathrm{lbs}$ TNT) could expose a few building to large overpressures, and many more building to small overpressures (10-50psi). These pressures can significantly damage windows and facades, causing hazardous debris fields inside and outside of buildings. The application of robust cladding materials and systems could prevent damage to a building's exterior, as well as dissipate the amount of blast energy transferred to the supporting structure.

Material systems were designed to affect two primary parameters of blast loading: peak load and impulse (impulse is analogous to energy) transmitted to a structure by a blast 
wave. Three phases of blast testing were conducted to refine panel design and incrementally improve dissipative performance.

Professor Ted Krauthammer from the University of Florida investigated tunable, energy absorbing frames that can be attached to buildings that permit a controlled amount of energy to be passed from the energy absorbing panel to the building.

This LDRD also included Peridynamics, a fairly new formulation of continuum mechanics based on the integral equation of motion. A particle-based method, it can handle material discontinuities such as cracks, but considers when equation of motion is discretized.

Five technical objectives were achieved, including:

- Developed granular-filled protective panels that utilize a proprietary binding and confining methods and effectively mitigate blast effects on structures

- Developed a hydraulic-filled panel with a proprietary core design and unique hydraulic fill which effectively dissipates blast energy as it crushes

- Conducted twenty two (22) blasts to test a variety of different panel designs collecting high speed transmitted force data and correlated high-speed photography

- Developed a novel technique to measure transmitted force-time histories in specific load ranges

- $\quad$ Filed 5 invention disclosure records, wrote two technical papers to be submitted to refereed journals, and work will be presented at annual SAVIAC (shock and vibration information analysis center) conference in 2010.

Initially, this program included a multifaceted research effort coupled with multi-scale modeling. As the project evolved, the primary emphasis was on experimental development and the construction and blast testing of prototype test articles. tested:

The following classes of technologies were
- Granular material matrices

- Hydraulic dissipative panels

- Honeycomb-core sandwich panels

- Proprietary impulse elongation panels

- Pyramidal truss core sandwich panels

These panels were tested at INL's National Security Test Range using full-scale explosions. Figure 1 shows a frame from high-speed photography of a blast test in progress. The shock wave is moving away from the bomb, which was situated approximately 20 feet off the left edge of the photo.

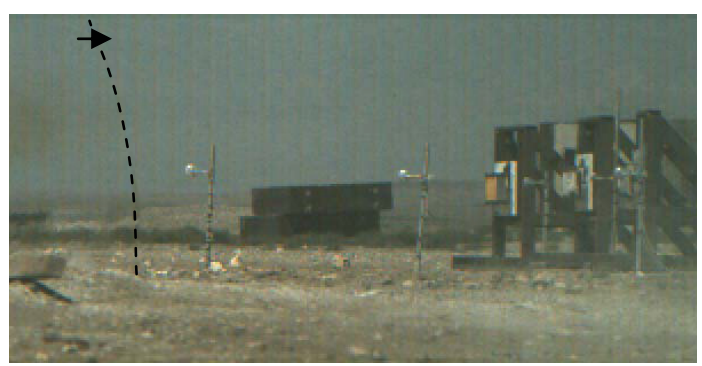

FIGURE 1. High-speed photography of a blast test. The shock wave is barely visible to the right of the dashed line. It is moving to the right at approximately $3000 \mathrm{feet} / \mathrm{sec}$ approaching the test articles (one is yellow).

The lessons learned from the first two rounds of blast testing were incorporated into a new set of panel designs and a new test setup. The revised test setup enabled enhanced measurement capabilities and positioned test articles at higher elevations above the ground to eliminate boundary effects on the blast wave near the ground (Fig. 2). The final phase of testing featured explosions consisting of $175 \mathrm{lbs}$ of ammonium nitrate and fuel oil.

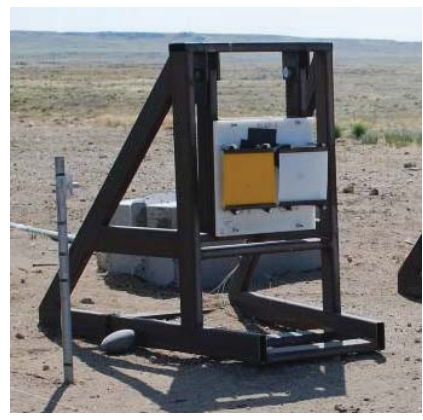

FIGURE 2. A re-designed blast test sled with two panels mounted on its front face. 
Test articles designed and fabricated for the third round of testing included granular materials bound and confined by various proprietary methods, hydraulic dissipative panels which featured proprietary core designs and fluids, impulse-elongating panels designed to deform large amounts before transmitting significant loads, and various geometries of porous core media. Figure 3 shows a few different technologies which were tested. In each blast, alongside the series of test articles, was a baseline panel intended to gauge "unprotected" performance.
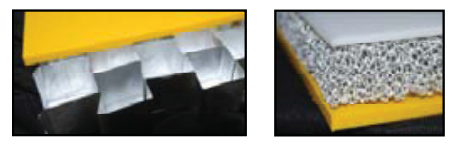

FIGURE 3. Four different technologies tested in phase three of blast testing. (From left to right: aluminum foil honeycomb cells, aluminum foam, corrugated-core sandwich panels).
Transmitted force-time histories were measured by a novel force measurement method that was developed specifically for this project. The method is a valuable contribution to the experimental capabilities of the laboratory, and exceeds the capabilities known to exist at other laboratories around the country. 


\section{Developing Resilient Control System Network Agents for Critical Infrastructure}

Dennis T. Vollmer

IC106

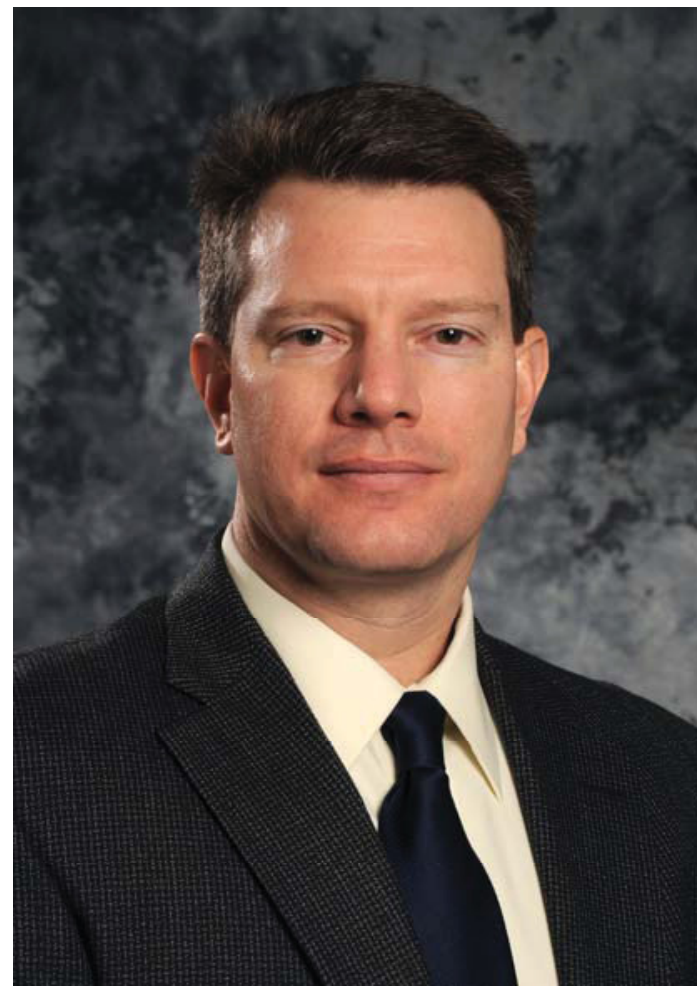

INL continues to play an important role in national and homeland security technology development for America. This LDRD project is contributing to INL's strong presence in securing critical infrastructure networks, especially control-systems cyber security.

In the second year of a three-year research project, INL's D. Todd Vollmer has been working with Milos Manic at the University of Idaho to research an Intrusion Detection Systems (IDS) mechanism to train anomalybased systems with knowledge gleaned from static-rule implementations.

Vollmer explained, "What this means is that we have developed a system which detects cyber security intrusions to critical infrastructure systems and reports these in understandable human terms."

He added, "The overall goal is to develop a system to minimize consequences of detrimental control-system network-based incidents that will safeguard new nuclear facilities with modern digital control designs."
The project is working to add intelligent detection schemes and response capability with fewer false positives to an intrusion-detection system at the controller level, instead of using a static rule-based system on the network segment. It focuses on changes that are related to security incidents, but is flexible enough to identify other, more general issues, as well.

An expert in system intrusion detection, Vollmer has worked to develop the ability to integrate that capability with other systems and recently presented three papers at international IEEE conferences, including:

- "Neural Network Based Intrusion Detection System for Critical Infrastructures," based on the original prototype was presented in Atlanta, GA. at the International Joint Conference on Neural Networks (IJCNN)

- "Human Interface for Cyber Security Intrusion Detection Systems" was presented at the HSI 2009 conference in Catania, Italy

- “Computationally Efficient Neural Network Intrusion Security Awareness," at the International Symposium on Resilient Control Systems (ISRCS) 2009.

Four specific milestones were achieved this year, including:

1. Refined development of the software prototype implemented in year one, to include machine intelligence algorithms.

2. Identify and procure development hardware capable of running the prototype algorithm identified in milestone one.

3. Targeted attack vectors more specific to control systems than used in year one development.

4. Determine response mechanisms and implement them in a prototype.

A new test system was completed at the end of the fiscal year. It consists of a Micrologix 1100 controller with a Moxa 505A IPv4/v6 managed-network switch. The system currently controls six LEDs, four push-button switches, two rotary potentiometers, two temperature sensors, a light source and a fan. All of this is packaged in a small, suitcase-sized portable 
container that has wheels and a pull handle. This portable system is roughly the size of a briefcase and will enable instrumentation and control research on real hardware. In addition, the researchers will no longer be confined to a fixed lab space. The physical lab can be moved at will and be exercised more efficiently in just about any location.
The plan for next year is to extend the prototype functionality to characterize the communication protocol data specific to command and control. This will enable detection of more sophisticated attacks, such as subversion of the command systems. Possible commercial applications for integration are being explored, including one software solution called Tofino being developed by Eric Byres. 


\section{Addressing the Spectrum of Nuclear-Related NDE Needs David Hurley \\ ST136}

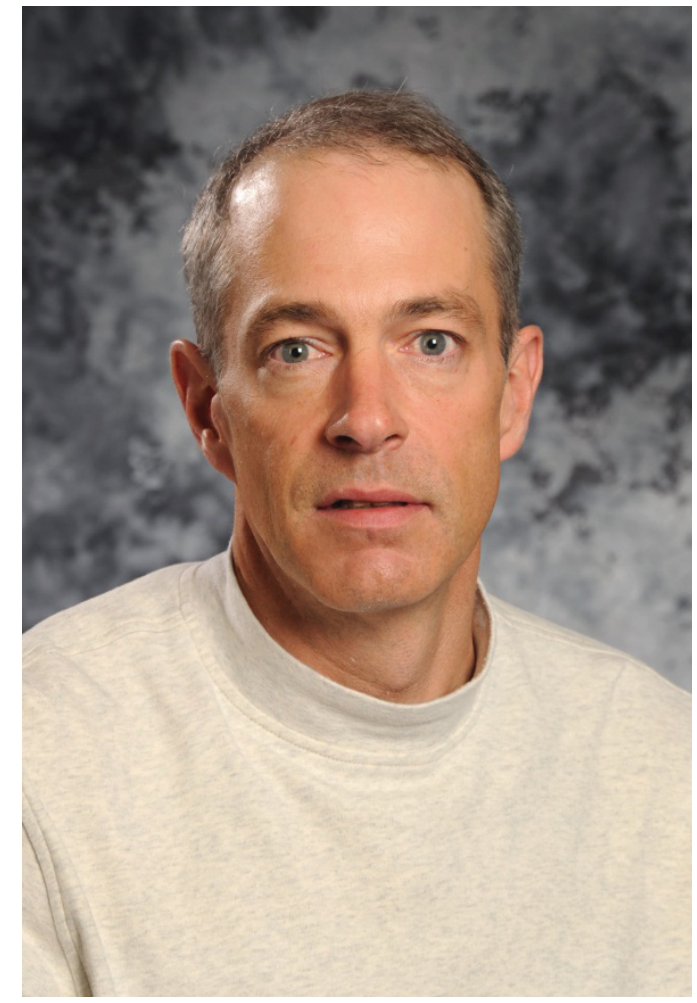

INL researchers are working to develop a new, hybrid non-destructive examination (NDE) system that could help extend the lifetime of existing nuclear plants and improve the design of future reactors.

In this LDRD project, principal investigator David Hurley and colleagues Dennis Kunerth and Zhandos Utegulov seek to increase the spatial resolution of NDE techniques. Better spatial resolution would allow better characterization of thin-film geometries - an important advance with many nuclear-related NDE applications.

"This work could improve our understanding of corrosion films, hydride rims and diffusion-barrier linings on fuel cladding," Hurley said. "And we could know more about grain boundaries, where corrosion often starts."

The researchers are investigating and integrating two different NDE technologies: ultra-high frequency laser ultrasound and pulsed eddy-current inspection. Both techniques evaluate material microstructural features, finding surface defects indicative of cracks, corrosion or other problems. Hurley, Kunerth and Utegulov are working to increase the spatial resolution of each technique, which would allow more and smaller imperfections to be found.

Ultra-high frequency lasers can generate surface acoustic waves (SAWs) in solid materials. Researchers analyze these SAWs to find defects or anomalies on materials' surfaces. Spatial resolution of this analysis is determined by the minimum acoustic wavelength.

As part of this LDRD project, the INL team has already devised a theoretical model of SAWs (Fig. 1) and determined that the necessary laser and electronic detection equipment should have a bandwidth greater than 3 gigahertz. With this theoretical underpinning firmly in place, Hurley, Kunerth and Utegulov have begun assembling the equipment needed for the next phase, coming soon: experiments in the lab that generate such ultra-high frequency SAWs.

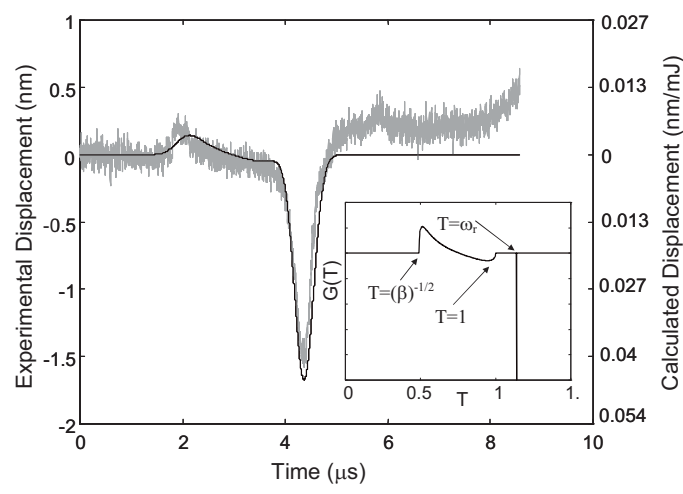

FIGURE 1. Comparison between surface-wave theory and experiments, using a single crystal zinc sample. Inset: Theoretical displacement, $u_{3}$, for surface waves generated with a line source in zinc.

The researchers are also trying to improve eddy-current NDE systems, which detect defects by pumping electrical currents through a sample and analyzing them. Traditional eddy-current probes use induction coils to generate and detect current. For high spatial resolution, very small coils are needed, which imposes serious limits on inductance and other electrical properties.

The INL team is evaluating an alternate sensor technology, giant magnetoresistive (GMR) devices, for use in eddy-current systems. GMRs could deliver higher spatial resolution; 
further, they have small physical footprints and a relatively broad bandwidth. Also, advanced microelectronic fabrication techniques mean that GMRs could possibly be combined into large arrays for increased area coverage during inspection.

The researchers' initial tests with GMRs have been promising, indicating that the technology is viable for detecting eddy-current responses (Fig. 2). However, more research is needed to understand the signal structure obtained with GMR sensors. Hurley, Kunerth and Utegulov are working on this now, and they're also trying to improve the integration of GMR sensors with other eddy-current equipment.
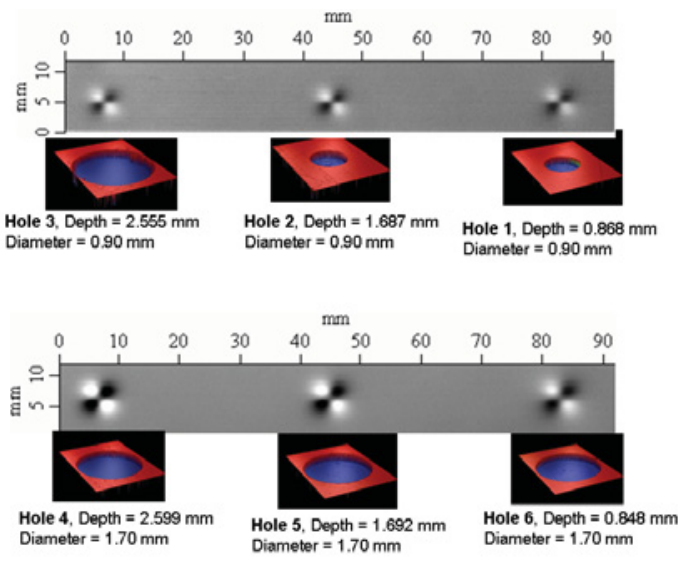

FIGURE 2. GMR response to flat-bottom holes in an aluminum plate.
"We hope to determine whether or not GMR technology could be used to develop a highresolution eddy-current sensor," Hurley said. "Combining that with improved laser ultrasonic techniques could lead to better on-line health monitoring of nuclear reactors." 


\section{Developing Numerical Tool to Aid INL Hybrid Energy Systems Testing (HYTEST)}

Donna Post Guillen

EI104

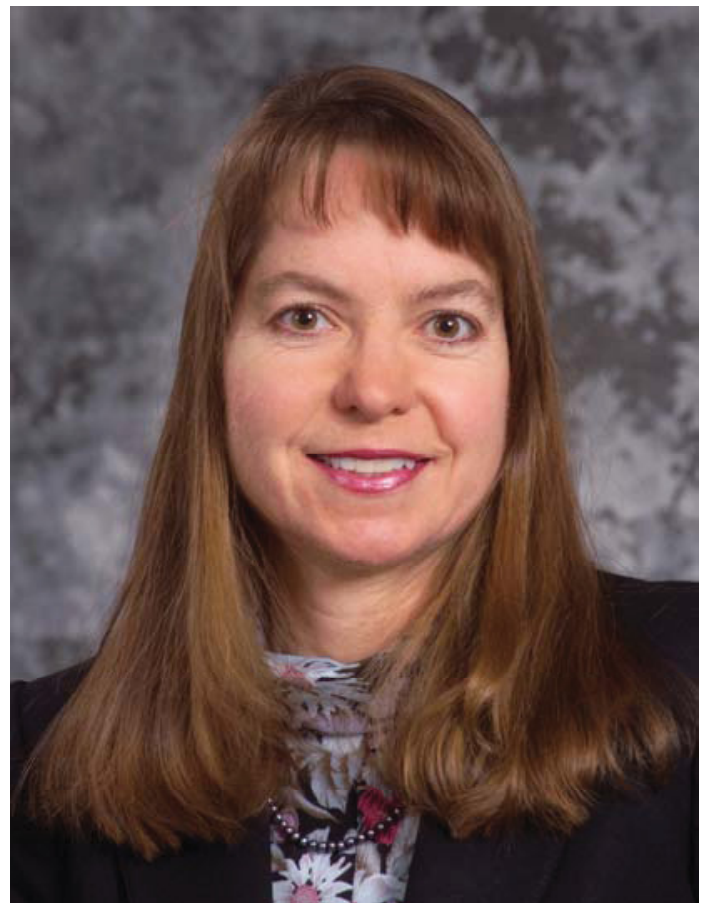

The INL is establishing the HYTEST Laboratory with sophisticated, state-of-the-art capability for testing hybrid energy systems, with a focus on process development, biomass gasification, catalysis research, alternative fuels, instrumentation and controls, plant modeling, emissions and economics studies, and computational fluid dynamics (CFD).

While there are many keys to assembling this complex testing system, Donna Post Guillen led a LDRD team in developing a practical modeling tool during the past three years. "The purpose of our research is to develop and validate a numerical methodology to simulate reacting gas-liquid-solid flows," she said.

The project, titled Computational Fluid Dynamics (CFD-Based) Simulation Capability of Fischer-Tropsch (FT) Reactors and Process Equipment included two technical objectives:

1. the development, implementation and validation of interfacial momentum transfer submodels to accurately describe the hydrodynamics in the SBCR, and

2. incorporation of mass transfer, heat transfer, and reaction kinetics submodels to simulate the chemical reaction process.
During FY07 to FY09, Guillen with four INL and three Rensselaer Polytechnic Institute researchers developed a robust methodology to couple reaction kinetics and mass transfer into multiphase CFD simulations for energy process applications. The modeling tool has the capability to assess and visualize fluid flow in a bubble column with chemical reactions, mass transport phenomena and heat transfer.

"Understanding the physics occuring within a Fischer-Tropsch reactor will help us improve system efficiencies and identify what new technologies may be needed," Guillen added. This effort has been to develop and validate a multi-field model of high gas holdup churnturbulent two-phase flows. A key factor for properly modeling the hydrodynamics of twophase flows is the formulation of consistent mechanistic closure laws that describe the dominant mass and momentum interactions at the fluid interfaces. It also reintroduces information lost as a result of ensembleaveraging the conservation equations. These closure laws account for the sub-scale mass and momentum transfer between the various fields and phases.

The interfacial momentum transfer submodels in the model were calibrated using the high quality experimental database for adiabatic air-water flow in a vertical pipe provided by our German collaborators at the TOPFLOW facility at Forschungszentrum Dresden-Rossendorf e.V. using a design optimization software package, Engenious iSight-fd. The bubbles were partitioned into four bubble size groups to better match the TOPFLOW data. Figure 1 shows some sample results from this study. The study confirmed that a minimum of two bubble size groups are necessary to adequately model the hydrodynamics of churn-turbulent flows. Bubble breakup and coalescence are important phenomena occurring in churn-turbulent flows, especially when attempting to predict flow evolution in a vertical riser. A straightforward, mechanistically-based breakup and coalescence model was exercised.

A chemical reaction model for the FischerTropsch (FT) process has been integrated into the SBCR hydrodynamics. Reaction kinetics for 
a cobalt catalyst are based upon values reported in the published literature. The initial SBCR model we developed tracked seven species:

- CO reactant in small bubbles, large bubbles, and the bulk fluid

- $\mathrm{H}_{2}$ reactant in small bubbles, large bubbles, and the bulk fluid

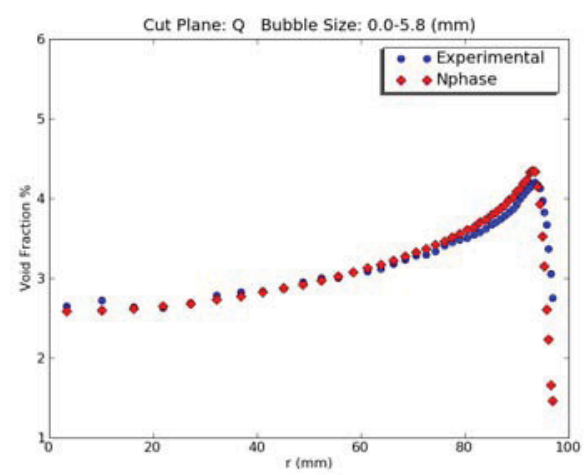

(a)
- $\mathrm{CH}_{2}$ product in the bulk fluid.

In this four-field model formulation, two of the fields are used to track the gas phase (i.e., small spherical and large slug/cap bubbles), and the other two fields are used for the liquid and the catalyst particles. Results of these simulations (Fig. 2) were presented at the 2008 AIChE Conference.

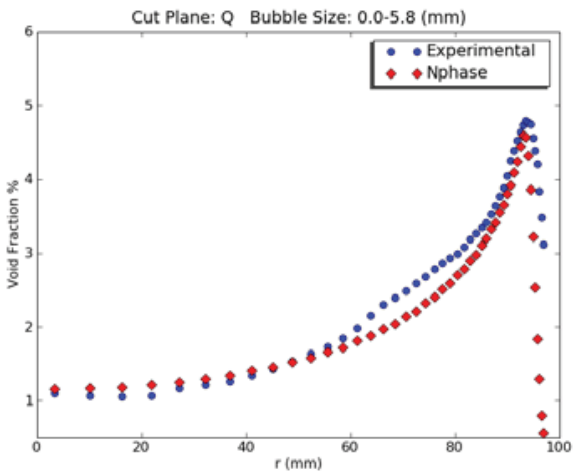

(b)

FIGURE 1. Comparison of computed vs. measured radial void fraction distribution for bubbles with diameters less than $5.8 \mathrm{~mm}$ (a) TOPFLOW case $118, j_{g}=0.22 \mathrm{~m} / \mathrm{s}, j_{l}=1.017 \mathrm{~m} / \mathrm{s}$; (b) TOPFLOW case $140, j_{g}=0.53 \mathrm{~m} / \mathrm{s}, j_{l}=1.017 \mathrm{~m} / \mathrm{s}$.

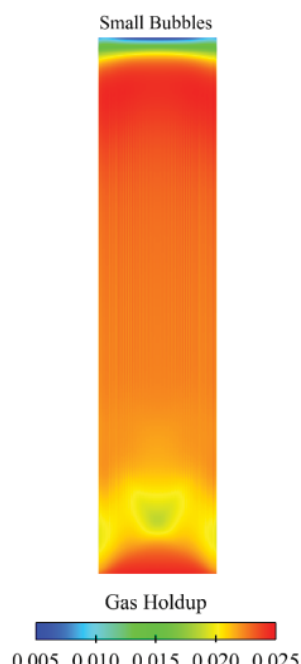

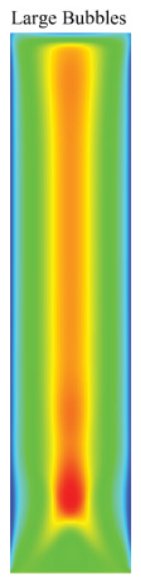

Gas Holdup

$\begin{array}{llllll}0.175 & 0.290 & 0.405 & 0.520 & 0.635\end{array}$

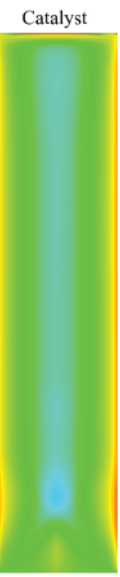

Volume Fraction

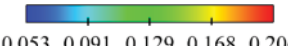

FIGURE 2. Computed volume fraction of small bubbles, large bubbles and catalyst.

In FY09, the model was expanded to represent the product distribution by representative liquid and vapor hydrocarbon (HC) products (based upon vapor liquid equilibrium) and water/steam. Simulation results included phase distribution, species concentration profiles, and local temperatures within the SBCR. This type of model can ultimately provide valuable information for process design, operation and troubleshooting of FT plants. 


\section{Working Toward a Better Understanding of Radiation Chemistry}

Bruce Mincher

NE147

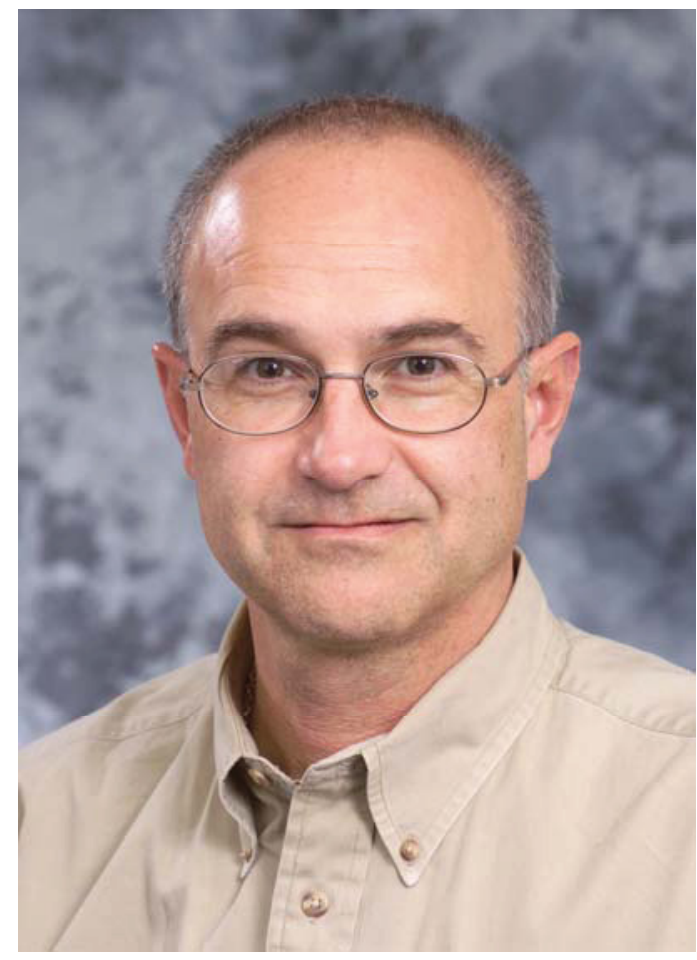

The nuclear industry commonly uses nitric acid as a solvent during spent-fuel reprocessing. However, the high radiation doses involved can split the acid into nitrogen-centered free radicals, extremely reactive compounds that degrade the other chemicals employed in the process. This chemistry is only partially understood, and knowledge gaps therefore impede efforts to design and develop the most efficient reprocessing methods.

Principal investigator Bruce Mincher, along with co-investigators Stephen Mezyk and Simon Pimblott, are working to remedy the situation. They're studying the mechanisms involved in nitric acid radiolysis, research that could improve fuel reprocessing and high-level waste treatment down the road. And the group's work should have other applications, too.

"Our results will also be significant to other disciplines interested in free-radical chemistry," Mincher said. The medical community, for example, could benefit, because free-radical reactions can lead to carcinogenesis and the degradation of lipids and nucleic acids. Nitrogencentered radicals also play roles in the creation of hazardous atmospheric pollution and in the treatment of drinking water by advanced oxidation technologies.

Mincher's group is trying to define the yield, type and behavior of free radicals produced in irradiated nitric acid. And they want to know how-or whether - these radicals $\left(\bullet \mathrm{NO}_{2}\right.$ and $\bullet \mathrm{NO}_{3}$ ) are interacting with aromatic functional groups in solution.

The researchers have made a great deal of progress. They have found, for instance, that nitrated organic species are indeed produced in irradiated nitric acid, as theory predicts. However, counter to expectations, direct nitration by $\bullet \mathrm{NO}_{2}$ or $\bullet \mathrm{NO}_{3}$ radicals isn't the main mechanism.

The team dissolved the aromatic compounds anisole and toluene in nitric acid, then irradiated the solution with cobalt- 60 . Nitration of anisole and toluene did occur, but by electrophilic aromatic substitution rather than direct radical reactions. Irradiation of nitric acid produced nitrous acid (Fig. 1), which enhanced these substitution reactions.

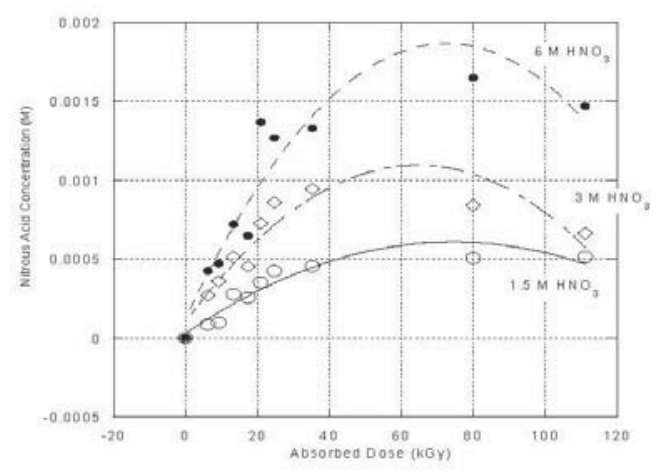

FIGURE 1. The ingrowth of nitrous acid in varying concentrations of irradiated nitric acid.

Work done under this LDRD project has already resulted in two peer-reviewed publications, with a third currently under review. But Mincher and his colleagues aren't done yet. They intend to perform more experiments with toluene to substantiate their findings and gain a better understanding of the mechanisms involved. The researchers are also collaborating with colleagues at California State University 
and the University of Manchester to model the reactions. Other experiments using pulseradiolysis equipment showed a remarkable absence of $\bullet \mathrm{NO}_{2}$ radical in irradiated nitric acid; Mincher hopes the modeling will shed light on this surprising result.

The work of Mincher's group could lead to better reprocessing methods - techniques that help recover fissionable materials for recycling, minimize the threat of proliferation and alleviate environmental concerns associated with waste disposal.

"Radiolytic reactions cannot be avoided in these systems," Mincher said. "It's important to elucidate their mechanisms and kinetics, so that we can better understand and mitigate their effects on solvent extraction." 


\section{Next-Generation Modeling for Next-Generation Reactors}

Dieter Wolf

SH100

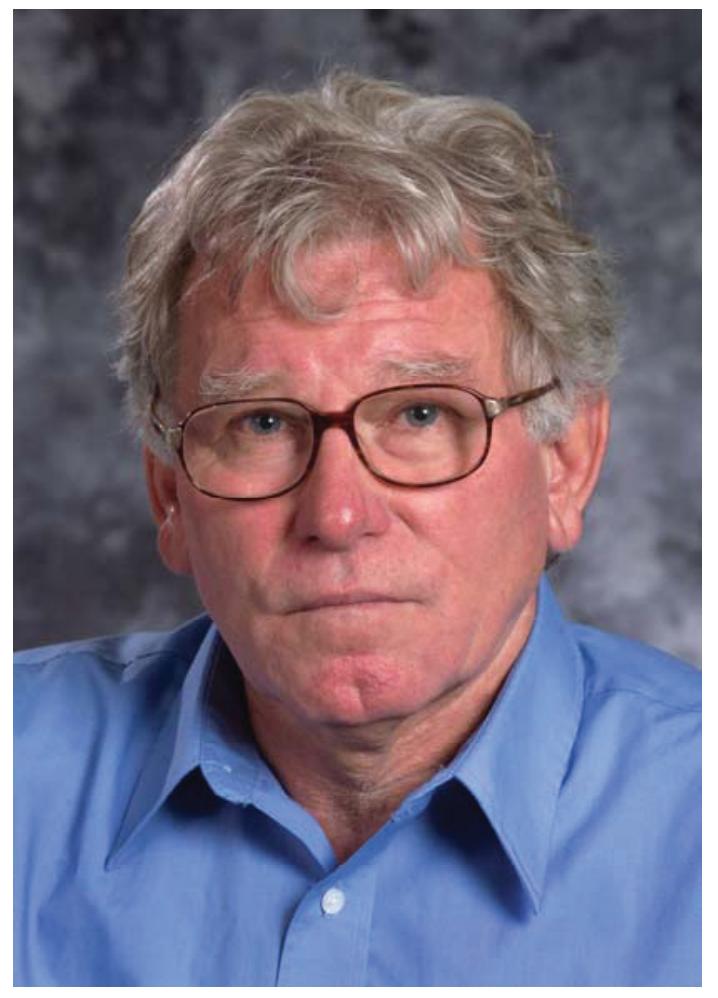

Modeling the behavior and performance of next-generation nuclear reactors will require next-generation computational tools.

In this LDRD project, Dieter Wolf and two young post-doctoral researchers, Paul Millett and Tapan Desai, are looking for ways to move beyond the empirically driven, applicationspecific modeling that nuclear engineers have relied on for decades. The team is developing simulations based on first principles - moleculardynamics (MD) models that can be scaled up to predict how fuel and cladding materials will behave in the core of a nuclear reactor.

"By contrast with conventional approaches, which are based on empirical observations, these first-principle methodologies are able to predict fuel behavior in situations - such as during transients - for which no empirical data base exists," Wolf said. "They could be a powerful tool for the design and validation of new and established nuclear systems."

The group's simulations focus on developing a fundamental understanding of the mechanisms behind radiation damage, and how these mechanisms affect the thermo-mechanical behavior of fuel and cladding materials. Wolf, Millett and Desai are looking at nanocrystalline uranium oxide (as a model fuel material) and body-centered-cubic molybdenum (model cladding). While computational limitations restrict the team's atomistic simulations to the nano-scale, insights gained from them can help develop models applicable at the engineering scale.

Wolf and his colleagues have made significant progress in three areas. First, they developed a novel approach to learn more about how irradiation-induced point defects evolve in uranium dioxide. Simulations found that point defects on the material's anion sublattice don't persist and spread unless there are defects on its cation sublattice, too. This insight revealed that uranium dioxide's cation sublattice is primarily responsible for the radiation tolerance (or intolerance) of the material.

The team also used MD simulations to characterize the migration of voids in uranium dioxide. A few hours after a nuclear reactor starts up, the temperature gradient within a fuel element causes voids, or fission-gas-filled bubbles, to move inward, forming a central hole. Understanding how these voids move is key for the accurate prediction of fuel-element performance. The researchers' simulations found that surface diffusion of the slowest-moving fuel-element component-uranium - was the dominant mechanism controlling void migration at the nano-scale. Extrapolations predicted that this should shift at larger scales: at the micrometer level, lattice diffusion should take over as the dominant mechanism.

Finally, Wolf and his team developed detailed, MD-informed simulations of void formation and growth in polycrystalline metals. Their quantified model captures many different relevant processes and demonstrates, among other things, that annihilation of voids at grain boundaries results in the formation of void-free zones nearby (Fig. 1). 

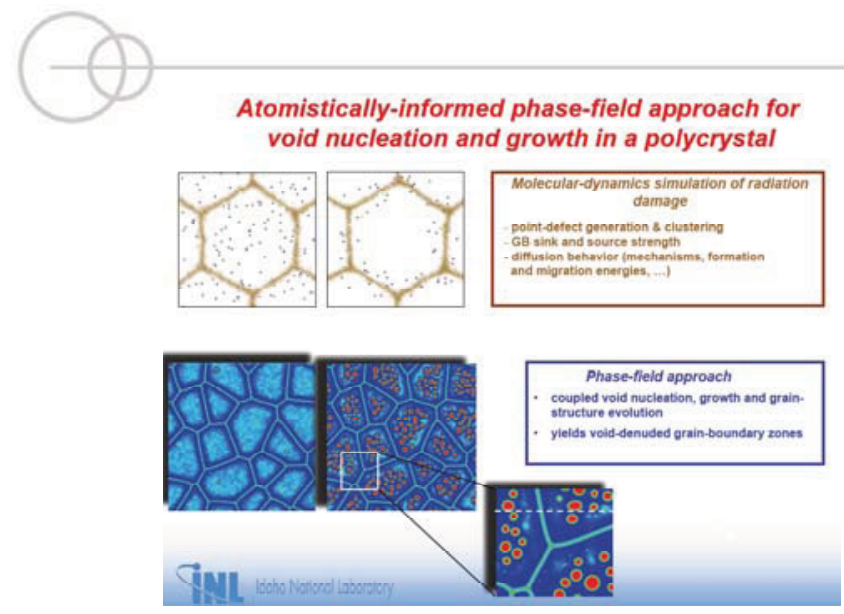

The team's various MD simulations provide valuable insight into the fundamental mechanisms controlling radiation damage in high-temperature environments. As such, the simulations give researchers a better idea of how such damage develops and progresses within reactor fuel - and clues as to how it might be slowed or prevented.

"These simulations could help spur the development of microstructurally designed fission-reactor fuel and cladding materials," Wolf said.

FIGURE 1. Concurrent void nucleation and growth in a polycrystalline metal super-saturated by continuous addition of irradiation-induced vacancies. The grain boundaries exhibit void-denuded zones that are formed because the grain boundaries act as vacancy sinks. 
PAGE FOR NUCLEAR ENERGY SCIENCE AND TECHNOLOGY DIVIDER

Nuclear Energy Science and Technology 


\section{Group Actinide Separation from SNF Using a Modified Universal Solvent Extraction Process}

Jack Law, Scott Herbst, Alena Paulenova

Development of a process to simultaneously extract and separate actinides from spent fuel

AE117

Advanced aqueous reprocessing of spent fuel affords a versatile method for reducing waste generation and significantly reducing proliferation potential in the nuclear fuel cycle. Current baseline flowsheets include individual processes for group extraction of actinides and extraction of cesium (Cs) and strontium $(\mathrm{Sr})$. Research is being performed to develop a process to simultaneously extract and separate actinides (as a group), Cs, and $\mathrm{Sr}$ from spent fuel. Such a process would significantly reduce the complexity and footprint, and thus reduce the overall cost of a spent fuel treatment facility. This project is based upon novel and expanded use of the Universal Solvent Extraction (UNEX) process solvent, originally developed to treat acidic waste solutions, to treat spent nuclear fuel. This work builds on work performed in FY-07 and FY-08 in which alternative actinide extractants were identified and tested.

\section{Technical Objectives}

This year's primary objectives were to:

- Evaluate the extraction of actinides with diamides of dipicolinic acid including thorium (Th), protactinium $(\mathrm{Pa})$, and neptunium $(\mathrm{Np})$

- Submit work for publication in journals.

Results and Accomplishments

The extraction of $\mathrm{Np}(\mathrm{V})$ by $0.2-\mathrm{M}$ solutions of ( $N, N$-diethyl- $N, N$-ditolyl-dipicolinamide (EtTDPA) isomers(ortho-o, para-p, meta-m) in FS-13 from increasing nitric acid conditions is shown in Figure 1 as a function of the nitrate activity. At lower acidities the meta-isomer

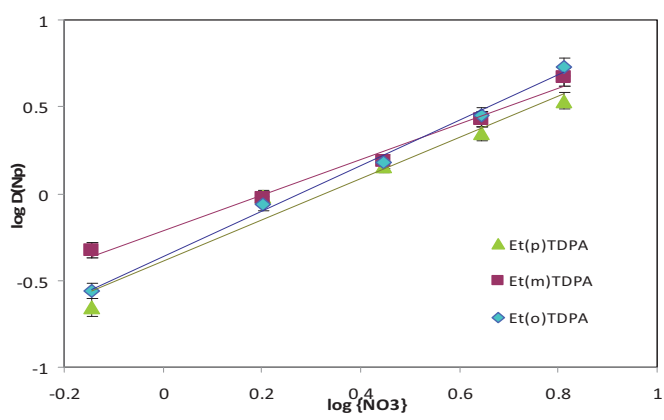

FIGURE 1. Nitrate dependence of $N p(V)$ extraction with 0.2$M$ EtTDPA in FS-13. shows the largest distribution ratios up to 3-M nitric acid $\left(\mathrm{HNO}_{3}\right)$, while Et(p)TDPA is the lowest across the entire range of acidities investigated, which was also observed in our previous study on americium extraction. This trend is different than that which was previously observed for uranium (U), where Et(p)TDPA exhibited the highest distribution ratios and Et(m)TDPA was nearly identical between 1 and 5-M nitric acid. However, in the studies of $\mathrm{U}$ (VI), Np (V), and americium (Am) (III), Et(o)TDPA begins with only slightly higher extraction capability than the lowest isomer at 1$\mathrm{M}$ nitric acid but then increases at a greater rate than either Et(m)TDPA or Et(p)TDPA with increasing acidity. The distribution ratios for Et(o)TDPA surpass that of both meta- and paraisomers at greater than $3-\mathrm{M} \mathrm{HNO}_{3}$.

Using log-log analysis of the nitrate activities and distribution coefficients of $\mathrm{Np}$ $\left(\mathrm{D}_{\mathrm{Np}(\mathrm{V})}\right)$ gives solvation numbers of $1.30 \pm 0.05$, $1.02 \pm 0.07$, and $1.19 \pm 0.13$ for ortho-, meta-, and para-EtTDPA respectively, again showcasing the greater ability of Et(o)TDPA for $\mathrm{Np}(\mathrm{V})$ extraction at higher acidities. Figure 2 displays the extraction of $\mathrm{Np}(\mathrm{V})$ with increasing Et(o)TDPA concentrations at different acidities. The first point of the 1-M $\mathrm{HNO}_{3}$ line was omitted due to the extremely low distribution of $\mathrm{Np}$ into the organic phase at these conditions, with count rates in the Np-237 window never being relatively larger than those observed as background. At all nitrate conditions the distribution ratios rose with increasing diamide

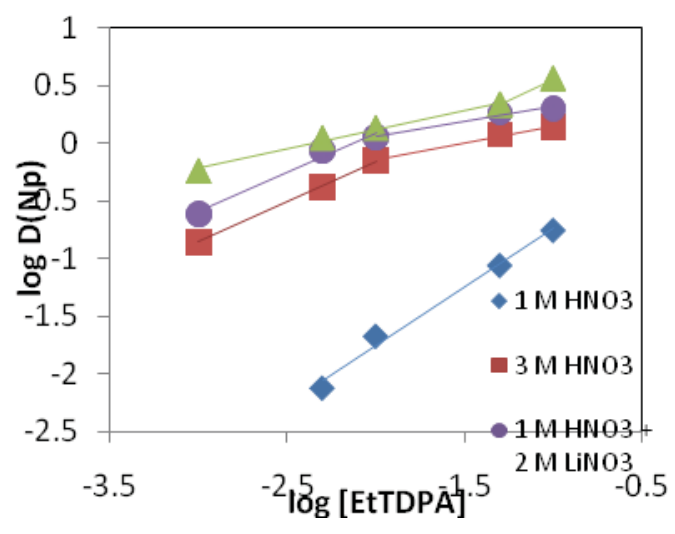

FIGURE 2. Ligand concentration dependence on $\mathrm{Np}$ extraction at various nitrate conditions. 
concentration. However, what is immediately noticeable from the figure is that only the data obtained at 1- $\mathrm{M} \mathrm{HNO}_{3}$ consists of a straight line, with a slope of $0.98 \pm 0.07$. A similar number at this concentration was also obtained for Et(p)TDPA with a value of $0.96 \pm 0.04$. Beginning at 3-M $\mathrm{HNO}_{3}$ the slope begins to decrease until the maximum concentration of diamide that could be easily solvated in FS-13 was reached.

A comparison of the extractions of both $\mathrm{Np}$ and $\mathrm{Pa}$ with increasing Et(p)TDPA is given in Figure 3. At 1-M nitric acid, both metals are nearly perfect parallels within standard deviation, with a slope of $0.98 \pm 0.03$ for $\mathrm{Pa}$ compared to the $0.98 \pm 0.07$ for $\mathrm{Np}$. However, $\mathrm{Et}(\mathrm{p})$ TDPA shows higher affinity for $\mathrm{Pa}$, with distribution ratios approximately 1.5 times larger than those obtained for $\mathrm{Np}$. At 3-M $\mathrm{HNO}_{3}$, one can immediately see the deviation that appears in the Np curve particularly after the third point in the trend. The Pa curve instead remains constant much longer, with a slope of $0.94 \pm 0.06$ until after the fourth point where linearity finally begins to break.

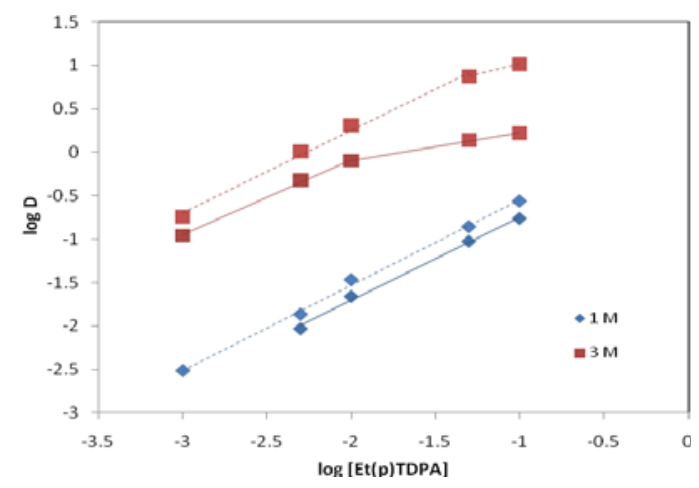

FIGURE 3. Extractions of $\mathrm{Np}$ (solid line) and $\mathrm{Pa}$ (dashed line) from increasing Et(p)TDPA concentration.

Figure 4 shows the distribution of Th into increasing EtTDPA concentrations from 3-M nitric acid. As expected, the extraction yields are extremely high compared to those in the previously studied penta-, tri-, and hexavalent cations. Thorium distribution ratios of greater than 1 are reached at $0.3 \mathrm{M}$ for both $\mathrm{Et}(\mathrm{m}) \mathrm{TDPA}$ and $\mathrm{Et}(\mathrm{p}) \mathrm{TDPA}$ compared to that of $\mathrm{Np}(\mathrm{V})$ which requires a nearly saturated solution of EtTDPA in FS-13 to achieve this level of separation. Et(o)TDPA shows the lowest capability for extraction, with Th distribution coefficient $\left(\mathrm{D}_{\mathrm{Th}}\right)$ values of approximately 78 times less than Et(m)TDPA and 4-5 times less than Et(p)TDPA for all identical concentrations. The solvation numbers obtained from slope analysis are $1.80 \pm 0.07,1.95 \pm 0.08$, and $1.92 \pm$ 0.03 for Et(o)TDPA, Et(m)TDPA, and Et(p)TDPA respectively.

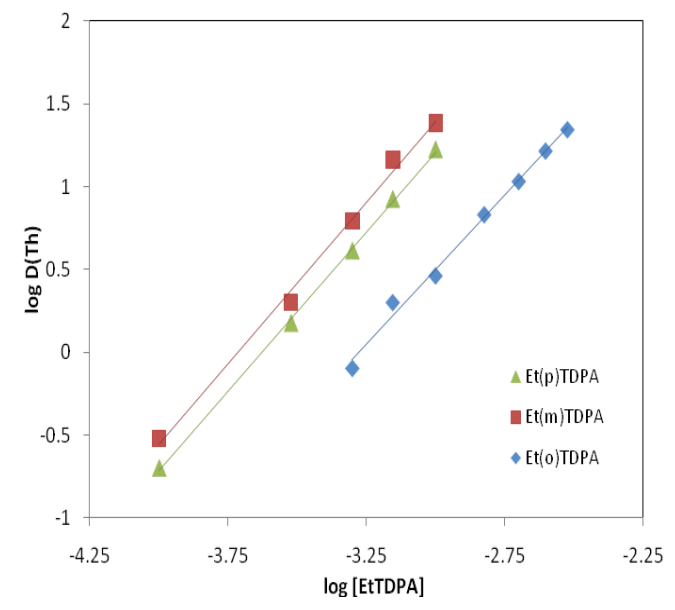

FIGURE 4. Extractions of thorium from 3- $\mathrm{M} \mathrm{HNO}_{3}$ with increasing EtTDPA concentration.

This high amount limited the range of conditions that could be studied, as lower amounts of EtTDPA could not contain a high enough metal-to-ligand ratio to ensure completion of the extraction mechanism, while higher concentrations of EtTDPA led to Th extraction beyond the sensitivity of the Arsenazo (III) spectrophotometric method. As such, for nitric acid dependence, only a concentration of 0.001-M EtTDPA was used for extraction rather than the 0.2-M EtTDPA used in all previous experiments. The distribution ratios obtained from these measurements are shown in Table 1. After 4-M $\mathrm{HNO}_{3}$, these values approach a maximum at 5- $\mathrm{M} \mathrm{HNO}_{3}$ and then begin to decline as EtTDPA begins to preferentially extract nitric acid over Th.

As was seen in the ligand dependence experiments, Et(o)TDPA again showed the lowest extraction capability for Th. Unlike the 0.2-M experiments, the ortho-derivative did not surpass the extraction capability of meta- or para-EtTDPA at higher nitric acid conditions. 
However, it did have the greatest solvation number of $2.90 \pm 0.12$, compared to $2.54 \pm 0.22$ for $\mathrm{Et}(\mathrm{m}) \mathrm{TDPA}$ and $2.69 \pm 0.25$ for $\mathrm{Et}(\mathrm{p}) \mathrm{TDPA}$. Based on the neutral extraction implied by Equation 2, it was expected that a nitrate value of approximately $\mathrm{x}=4$ would be obtained, but this was not observed. The obtained values lean towards the extraction of three nitrate groups plus an additional anion.

TABLE 1. $D_{T h}$ values from extractions with 0.001-M EtTDPA in nitric acid.

\begin{tabular}{lcccccc}
\hline $\mathrm{HNO}_{3}[\mathrm{M}]$ & 1 & 2 & 3 & 4 & 5 & 6 \\
\hline Et(o)TDPA & 0.06 & 0.74 & 3.8 & 11 & 18.8 & 18.6 \\
Et(m)TDPA & 0.55 & 6.25 & 25.5 & 51.5 & 57.3 & - \\
Et(p)TDPA & 0.25 & 3.40 & 15.0 & 30.6 & 35.6 & 29.3 \\
\hline
\end{tabular}

\section{Summary and Conclusions}

A collection of all the completed actinide extraction data with Et(p)TDPA can be seen in Figure 5. The exception is Th due to the differing extractant concentrations ( $0.001 \mathrm{M}$ versus $0.2 \mathrm{M})$ from the rest of the studied actinides. However, one can assume that based on the distribution

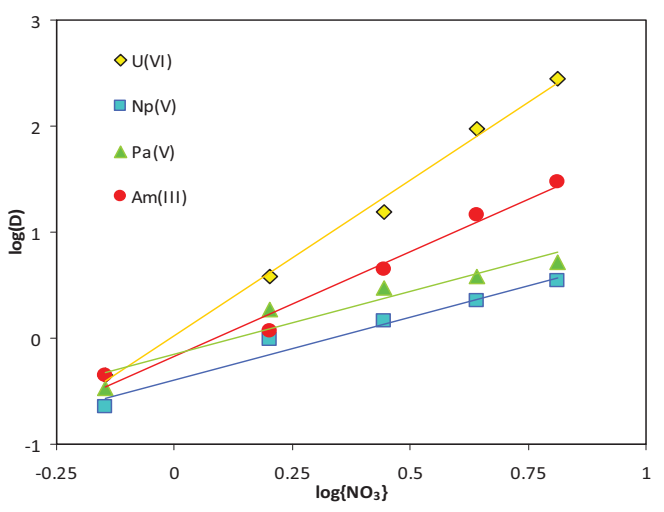

FIGURE 5. Actinide extraction with 0.2-M Et(p)TDPA from nitric acid. values for Th, if it were able to be displayed in this figure, it would be several orders of magnitude above the other metals. The increasing slopes in this plot confirm that extraction of actinides follows the order of effective cationic charge known as:

$\mathrm{An}(\mathrm{IV})>\mathrm{An}(\mathrm{VI})>\mathrm{An}(\mathrm{III})>\mathrm{An}(\mathrm{V})$.

This can clearly be seen above 1-M $\mathrm{HNO}_{3}$ from the best fit slope lines obtained. At higher acidities the separation factors between the wellextracted $\mathrm{U}$ and $\mathrm{Am}$ and the less-extracted $\mathrm{Np}(\mathrm{V})$ are significantly large, with $\mathrm{U}(\mathrm{VI})$ extracted nearly 100 times great that $\mathrm{Np}(\mathrm{V})$. Pentavalent protactinium is slightly better extracted than neptunium, because of its smaller ionic radius and slightly larger charge density than $\mathrm{Np}(\mathrm{V})$. 


\section{Exploration of Electrolyte Complexation and Pulse Deposition for Production of Dense Uranium}

Ken Marsden, Shelly Li

Investigation of methods to produce dense metallic deposits in a molten chloride system AE118

Current electrochemical processing techniques are limited by low-mass throughput and the necessity of high-temperature processing to separate electrorefined metal from the electrolyte. Current electrorefiner designs were created with the assumption that cathodic deposits must be grown at high current density on small cathodes, mechanically harvested, and processed in a high-temperature vacuum furnace to separate the metal from occluded salt and consolidate the fine metal pieces into a coherent ingot.

This work investigated methods to produce dense metallic deposits in a molten chloride salt system, avoiding the time-consuming and laborintensive steps of harvesting, vacuum distillation, and melt consolidation. A similar dense-deposition approach is commercially performed for less electropositive elements such as copper in aqueous electrochemical systems, and numerous studies have shown the ability to produce dense metallic deposits in molten fluoride salt baths. A complete electrolyte change from chloride to fluoride salts would likely alter all process steps in electrochemical processing and is not viewed as a viable option. Chloride salts are known to be a more difficult solvent for the creation of dense articles; nonetheless, it is used in the commercial fabrication of dense iridium crucibles and rocket nozzles. Deposition of coherent metallic uranium in a molten chloride electrolyte was examined in one study as an alternative to casting or machining of uranium, but this was and is not a competitive way to reshape already-purified uranium. The studies accomplished formation of dense uranium at a very low deposition rate in a few simple scoping studies and were terminated prior to development of an understanding of controlling mechanisms.

The intent of this work has been to develop an understanding of the science and methods necessary to create dense uranium deposits in a molten $\mathrm{LiCl}-\mathrm{KCl}$ electrolyte. For this end, three enabling objectives were pursued. The first objective was improvement of the fundamental understanding of lanthanide deposition in molten chloride. The lanthanide elements are chemically similar to the actinides, and the use of these nonradioactive surrogates allows a relatively rapid and inexpensive collection of data. In addition, previous fundamental studies of the lanthanide elements are sporadic and inconsistently performed. A broad study of lanthanide fundamental electrochemical properties is a useful stand-alone contribution to the literature.

The second objective was collection of data from electrochemical deposition in molten LiCl$\mathrm{KCl}$ modified with anions and cations that are known to affect deposition processes at high concentration. This includes the addition of the $\mathrm{F}^{-}$anion which is known to create baths with broader conditions from which dense deposits can be achieved. It is believed that the $\mathrm{F}^{-}$anion's small physical size and greater electronegativity allow the formation of a tight $\mathrm{F}^{-}$shell around the central cation, forming a molecular complex much more stable than that achieved with $\mathrm{Cl}^{-}$ anions alone. In addition to anion effects, cations of increasing size and decreasing polarization $(\mathrm{Cs}>\mathrm{K}>\mathrm{Na}>\mathrm{Li})$ improve the stability of complexes and the production of dense deposits. It is believed that their low polarization and large size allow this secondary cation to share a partial ionic bond with many anions in the $\mathrm{F}^{-}$or $\mathrm{Cl}^{-}$ shell, allowing them to be charge balancers that do not compete for a close bond with a particular anion. In this way, they are believed to contribute to the stabilization of the overall molecular complex.

The third objective to achieve dense deposition was developing the knowledge of how to set up and utilize electrochemical pulsing. As electrochemical deposition progresses, a concentration gradient or boundary layer develops. If a nucleus from the surface begins to penetrate through the boundary layer to a region of higher concentration, this asperity receives increased current density, which further accelerates its growth until it becomes a macroscopic dendrite. Electrochemical pulsing is a deposition method where metal is deposited from the electrolyte in conditions without a developed concentration gradient. To accomplish this, electrochemical pulsing pauses after a period of deposition to allow the concentration gradient to relax, and in some cases, the surface of the deposit may be periodically stripped to remove initiated dendrites. 


\section{Technical Objectives}

Only a small amount of funding was allocated to this work in Fiscal Year 2009, and the objectives for this final year were limited to evaluation of data obtained in previous years. The data obtained earlier included that from two or three temperatures for the elements zirconium, yttrium, lanthanum, cerium, praseodymium, neodymium, samarium, and ytterbium. Data was collected to (1) measure standard potential by at least two means, (2) obtain cyclic voltammetry at a range of scan rates and varying termination points, (3) determine species diffusion coefficients by at least two means, (4) determine exchange current density by the Tafel method, and (5) determine species valence by square wave and cyclic voltammetry methods, and (6) determine nucleation mode and characterize the response to basic electrochemical pulses. Data was also obtained with the rare-earth lanthanum at varying concentrations and with additions of $\mathrm{LiF}, \mathrm{CsCl}$, and $\mathrm{BaCl} 2$ to the electrolyte. These experiments were performed in a molten salt furnace in an inert-atmosphere glovebox.

\section{Results and Accomplishments}

Electrochemical data for the rare earths was obtained and evaluation begun in this work. Obtained electrochemical parameters are being assembled into several articles for peer-reviewed literature. Cyclic voltammetry is one of the most common diagnostic tools for electrochemistry, and Figure 1 shows a typical cyclic voltammogram obtained in this work for the lanthanum system. Lanthanum has a relatively clean deposition with the transfer of three electrons. Figure 2 shows a typical cyclic voltammogram for another rare-earth fission product, samarium. Samarium cannot be deposited from $\mathrm{LiCl}-\mathrm{KCl}$ eutectic and undergoes a one-electron valence change near the middle of the voltage window.

\section{Summary and Conclusions}

Electrochemical data for the rare earths, especially that pertaining to methods of dense deposition, was obtained and evaluated in this work. Data obtained for the rare earths is being grouped into several peer-reviewed articles for publication. Data was obtained and evaluation

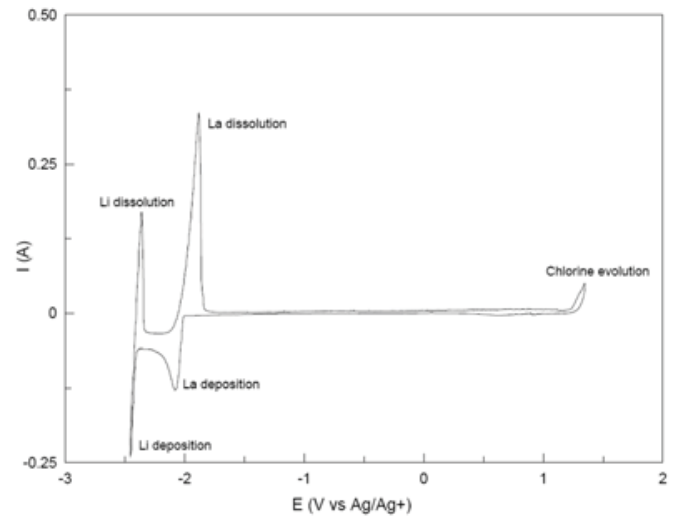

FIGURE 1. Typical cyclic voltammogram of $\mathrm{LaCl} 3$ in $\mathrm{LiCl}$ $\mathrm{KCl}$ eutectic at $500^{\circ} \mathrm{C}$, also showing voltage window of the electrolyte.

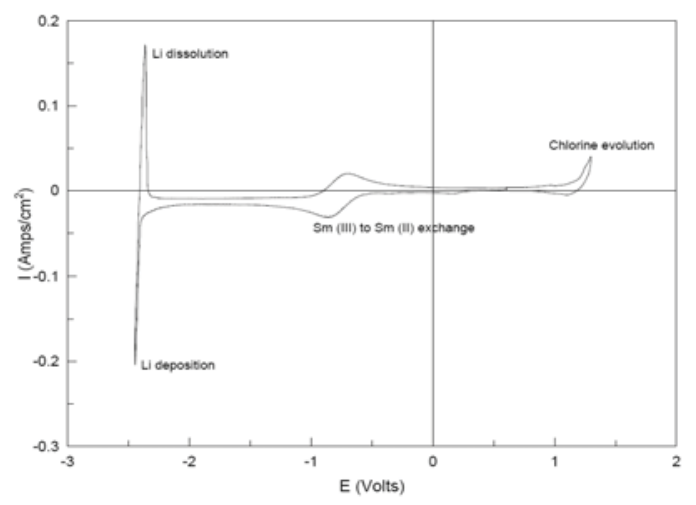

FIGURE 2. Typical cyclic voltammogram of Samarium rare earth in $\mathrm{LiCl}-\mathrm{KCl}$ eutectic at $500^{\circ} \mathrm{C}$ on a tungsten electrode, also showing the voltage window of the electrolyte.

partially completed with lanthanum at varying concentrations of electrolyte modifiers. While electrochemical changes are observed, it is not clear that minor amounts of modifying chemicals change electrochemical properties sufficiently to render a dramatic effect on deposit morphology. This data is also being assembled into one or two peer-reviewed articles for publication. Further directed studies are suggested to determine if electrolyte modifiers may contribute with assistance of other techniques. 


\section{A Strategy to Tightly Couple Neutronics and Thermal Hydraulics Models for Next Generation Reactor Analysis}

Christopher Newman, Benoit Forget (MIT)

Employing bond graph formalism to model coupled thermal and neutron transport phenomena BS104

The fundamental goal of this project was to tightly couple several physical phenomena in nuclear reactors. The important physics for reactor simulation include neutron transport, thermal hydraulics and mechanical response, which are all inherently strongly coupled. Most current efforts typically only model a single physics where the coupled data is determined externally. This is an imperfect approach, particularly for transient analysis, because the strong interdependence of the physics involved creates stability and accuracy issues. The inability to accurately predict transients under accident scenarios is the primary reason for highly conservative safety limits imposed on reactors. Due to computational limitations, this approach was employed for many years. Therefore, there is a need for faster, more accurate fully coupled codes capable of handling coupled transient systems. The introduction of such codes would allow reducing the safety margins on reactor operation and manufacturing and increase predictive capabilities for reactor simulations.

A similar single-physics approach was used in electro-mechanical systems until a new formalism was proposed and developed by Henry Paynter of the Massachusetts Institute of Technology (MIT) in the 1960s. This formalism is known as Bond Graphs, and it is a way of representing engineering systems as combinations of storage elements (capacitive and inertial), resistive elements, and junction elements, connected by bonds that transfer energy (or mass) between the elements. Bond graph modeling is summarized in its most basic form in Figure 1. Advantages associated with bond graph modeling include:

- $\quad$ Easy coupling of separately modeled systems

- Highly flexible models in terms of discretization schemes

- Graphical representation of a system which facilitates the understanding of multiphysics systems

- Many thermal-hydraulic components of power plants have been modeled using bond graphs and could be extended to model an entire plant

- Yields a system of first-order ODEs for which many robust algorithms exist.

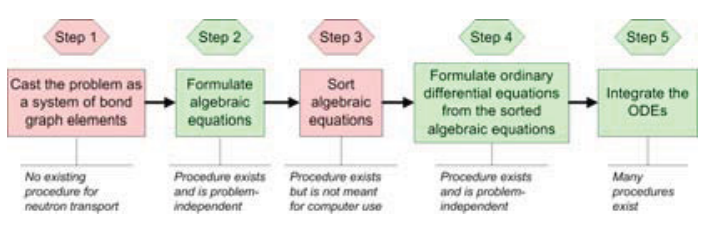

FIGURE 1. Bond Graph Formalism. Red steps required specific algorithm development for this project, green steps only required implementation.

The purpose of this project was to develop methods for modeling coupled thermal and neutron transport phenomena in a nuclear reactor using Bond Graph Formalism, and to develop a proof-of-concept code using these methods.

\section{Technical Objectives}

The technical objectives accomplished over the course of this project were:

- Developing a 1-D, one-speed neutron diffusion bond graph. The neutron diffusion bond graph was derived for both finite difference and finite element spatial discretization.

- Coupling of the 1-D neutron diffusion bond graph with a thermal diffusion bond graph. This step required the introduction of a twoport resistive element (R2) that couples the fission energy from the diffusion model to the heat source of the thermal model.

- Developing a bond graph processing code which required the introduction of bond graph file format. The code must also support the necessary algorithms for the R2 elements needed for the neutron and thermal coupling. Essentially, the code should automate Steps 2 to 5 of Figure 1, such that only a bong graph need be provided.

- Verifying the code using the Method of Manufactured Solutions (MMS) on a 1-D slab reactor.

- Determining the feasibility of using bond graphs for more complicated neutron transport approximations. 
- Assessing the benefits and problems of using the bond graph formalism for modeling coupled reactor physics and the feasibility of developing a full, parallelized reactor modeling code based on bond graph formalism.

All of these objectives have been successfully achieved. A brief summary of the main results are presented in the next section.

\section{Results and Accomplishments}

The 1-D, one-speed neutron diffusion bond graphs were shown to be nearly identical with the 1-D thermal diffusion bond graphs, which were known a priori. This is because the streaming terms in the neutron diffusion and thermal diffusion equations are very similar. The absorption and fission production do not have analogs in thermal diffusion and were handled via addition of resistive elements. These resistive elements were transformed into nonconservative couplers (two-port resistive elements) through which neutron and thermal bond graph systems were coupled.

The bond graph system descriptor (BGSD) file convention was developed to describe all the information about the bond graph system. A graphical user interface (GUI) for generating BGSD files was developed. The GUI is designed primarily for testing new types of discretization and for rapid creation of small BGSD files.

The bond graph processing code was written in MATLAB. MATLAB was chosen primarily because of its robust ODE integration suite, rich array-handling capabilities, and symbolic equation capabilities. The sorting procedure, Step 3 of Figure 1, is handled by algebraic manipulations via the MATLAB symbolic toolbox. The ODEs are formed from this reduced set of equations, Step 4 of Figure 1. The built-in MATLAB symbolic manipulation was chosen due to its ease of implementation, recognizing that it is not scalable and cannot be used on large problems. Therefore, one of the first steps in developing a more advanced bond graph processing code would be replacing symbolic sorting with a corresponding numeric procedure.

The code was tested using a method of manufactured solutions (MMS) on a 1-D slab reactor, modeling a rise to steady power. Figure 2 shows the results.
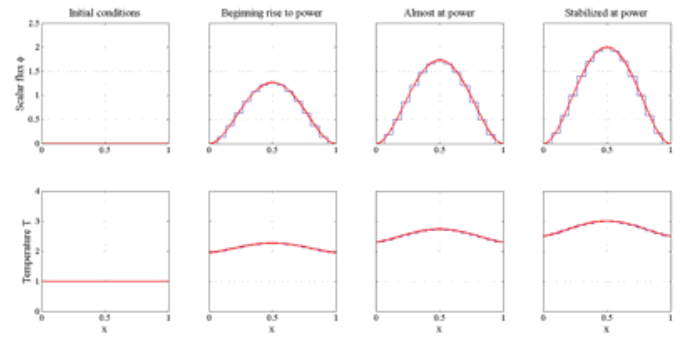

FIGURE 2. Verification results. Red curves show the exact solutions, blue curves show the computed solutions.

The MMS verification demonstrated that the code successfully coupled neutron and thermal diffusion, with the discretization error staying consistent both in transition and at equilibrium. MOOSE and TRILINOS were assessed as possible pathways to develop a code for much larger simulations. It was determined that the bond graph formalism has no need for the full set of capabilities provided by MOOSE. The ODE integrators of TRILINOS were evaluated for the time integration step in the algorithm and are a possible direction for large scale simulations.

This research benefits Idaho National Laboratory (INL) and the Department of Energy (DOE) as it provides an alternative approach for multiphysics strategies. This new flexible approach allows for various discretization schemes to be tested without any major modifications to the code.

\section{Summary and Conclusions}

In this project, a new architecture for reactor multiphysics codes was identified and assessed. The algorithm for applying bond graphs to coupled reactor physics problems was developed. A code for testing the algorithm was developed and tested via a MMS verification procedure. The test demonstrates the architecture's capability to accurately model tightly coupled transient problems.

The overall goal of this project is to develop a fast, flexible, fully coupled, accurate code for transient reactor multiphysics analysis. The next steps, based on what was accomplished so far, are proposed to be: 
- Replacing the symbolic sorting with an equivalent numeric algorithm built on sparse matrix-vector products

- Developing the algorithm for modeling multigroup 3-D neutron transport with bond graph

- Implementing the code using a faster language with parallelizable ODE integration capabilities, such as the TRILINOS integrators

- $\quad$ Benchmarking the code as needed.
This method is applicable to general multiphysics problems, meaning it is not restricted to nuclear applications; thus, future funding can target any agency that deals with complex multiphysics problems. To further its application to nuclear simulations, this work will target the upcoming Nuclear Energy University Program (NEUP) call as a possible source of funding. 


\section{Integrated Mesoscale Approach for the Simulation of Nuclear- Fuel Behavior}

Dieter Wolf

Enabling materials physics-based prediction of the thermo-mechanical behavior of polycrystalline fuel and cladding materials

FF104

The main goal of this work is to develop an integrated microstructure-based, mesoscopic modeling and simulation capability for nuclear fuels and structural materials that incorporates the critical role of microstructure and its evolution under irradiation, as well as stress and temperature effects. The integrated code aims to combine two main building blocks within a single computational platform: (i) an atomistically informed phase-field modelingand-simulation approach, and (ii) a finiteelement approach for the stress distribution within the evolving polycrystalline microstructure. These stresses will be used as input into the phase-field simulation approach. The combined simulation capability will enable materials physics-based prediction of the thermomechanical behavior of polycrystalline fuel and cladding materials under the effects of irradiation, including the complex interplay between fission-gas swelling and release, hightemperature creep and grain growth.

\section{Technical Objectives}

Traditionally, the prediction of swelling and fission-gas release behavior in both metallic and oxide fuel has been based on the classical rateequation approach that incorporates assumed key atomic mechanisms into an analytic framework. By their nature, such mean-field approaches ignore microstructural heterogeneity (e.g., distributions in the grain sizes, grain shapes, and anisotropic grain-boundary properties).

Nevertheless, they have been quite successful as evidenced by the existence of well-known software packages that capture the nucleation and growth of inter- and intra-granular bubbles under the simultaneous destructive influence of irradiation-induced gas-atom re-solution. However, uncertain - yet critically importantmaterials parameters of the model are usually adjusted by fitting to empirical data, rather than using a more rigorous materials physics-based simulation approach. These uncertainties raise questions about the validity in the physics that has been invoked in order to explain the observations.

The present effort aims to develop an integrated mesoscopic modeling capability that explicitly incorporates the critical role of microstructure and its evolution under the effects of irradiation and (internal and external) stresses. By combining a finite-element approach with a phase-field modeling approach, we will be able to model the thermo-mechanical behavior of both fuel and cladding in the presence of fissiongas bubbles and with explicit incorporation of microstructural discreteness and inhomogeneity. This approach represents a significant point of departure from the legacy models in that explicit consideration of the most critical microstructural processes in the fuel under irradiation makes possible the concurrent simulation of fission-gas behavior and fuel restructuring. Explicit consideration of the dynamic interplay between fission-gas bubble kinetics and microstructural processes, such as plastic deformation and grain growth, represents a critical aspect of this coupling. This then will enable prediction of the connection between bubble swelling and fissiongas release that naturally incorporates the effects of temperature and stress, and their gradients, on both fuel restructuring and fission-gas dynamics.

\section{Results and Accomplishments}

We have made significant progress in the following two areas.

Phase-field simulation of void nucleation and growth in irradiated metals. As a first step towards the development of a comprehensive phase-field model to capture the effects of defects generated as a result of fission processes, we have developed a phase-field model for void formation in irradiated metals with vacancy concentrations exceeding their thermalequilibrium values [1]. This model is motivated by the need to develop a spatially resolved theory of irradiation-induced microstructure evolution in metals. The model, which is phenomenological in nature, was cast in the form of coupled Cahn-Hilliard and Allen-Cahn type equations governing the dynamics of the vacancy concentration field and the void microstructure in the matrix. The model allows for a unified treatment of the effects of stress and cascadeinduced fluctuations of the vacancy field. In the model, void nucleation and growth in a supersaturated solution were investigated under the assumption that the void-phase dynamics is driven only by the super-saturation of vacancies. For simplicity, the effects of interstitials in 
conjunction with vacancies were not considered in this first study of its kind.

Stress calculation approach within the unified phase-field model. A critical component of an integrated mesoscale modeling capability is the prediction of the effects of internal stresses on microstructure evolution. We have initially developed a two-dimensional solution approach for the phase-field equations using the finite difference method coupled with a spectral method to calculate the stress fields in an inhomogeneous microstructure containing voids. To improve the numerical capabilities of the model and also integrate a more widely used computational approach into our comprehensive phase-field code, we have recently implemented a finite element approach for solving the phasefield equations within INL's MOOSE computational framework (in collaboration with Glen Hansen, INL). MOOSE is a parallel, nonlinear, finite element framework that facilitates modeling of complex geometries in 3D. The stress calculation can thus be carried out in 3D within the MOOSE framework and can include complicated boundary conditions not easily possible with the spectral method. In addition, the mesh-adaptivity capabilities built into MOOSE allow for higher resolution around areas of stress concentration, such as voids, bubbles, and grain boundaries.

\section{Summary and Conclusions}

We have taken important first steps towards developing a comprehensive conceptual and computational framework for a unified mesoscale approach that is capable of capturing the coupled, concurrently occurring microstructural processes that take place under irradiation within a single computational platform. Based on various considerations, we have converged on the phase-field approach for the microstructure-evolution simulations. The numerical solution of the Cahn-Hilliard and Allen-Cahn equations together with the corresponding free-energy functional has been mapped on to finite-element and adaptive-meshrefinement types of computational techniques. These are not only amenable to advanced, massively parallel computer architectures and algorithms but also facilitate scale-bridging algorithms that are consistent with the prevailing engineering-code approaches. 


\section{Process Modeling of Solvent Extraction Separations for Advanced Nuclear Fuel Cycles}

Dr. Troy Tranter, Dr. R. Scott Herbst

Defining and developing useable dynamic process models to simulate advanced solvent-extraction processes under transient and steady-state conditions

NE146

The U.S. vision of the next-generation advanced nuclear-fuel cycle for the commercial nuclear-power industry includes a series of aqueous separation steps based on solvent extraction technologies, which fractionate dissolved nuclear fuel into discrete product streams for storage, reuse, or disposal. Several options for this processing scheme exist, including various UREX options, and other process combinations for separating actinides, lanthanides, and fission products. The technical complexities (e.g., process upsets and perturbations due to feed and process inconsistencies) of operating such a complex system are as varied as the drivers for the separations themselves (e.g., energy recovery, repository capacity, and proliferation resistance). Development of dynamic process models is an important aspect of understanding and predicting the inherent transient behavior of such a complex series of unit operations and, ultimately, for the entire process from spent fuel receipt to waste treatment and disposal. The objectives of this project are to define and develop useable dynamic process models to ultimately simulate advanced solvent extraction processes under transient and steady-state conditions, thus providing a capability currently not available to the AFCI program in the United States.

Exponential cost increases in conducting experimental and pilot scale programs have forced researchers to work "smarter"; conversely, there have been dramatic enhancements in sophistication and reduced costs of available computer codes and computing power. Capitalizing on the latter can substantially augment the former by using computational methods to guide and minimize the amount of experimental and piloting work required to develop and understand complex systems or processes. This fact is exploited in many engineering fields and industry where computer simulations are routinely performed in lieu of pilot or semi-scale tests. However, the absence of any industrial-scale nuclear fuel reprocessing in the U.S. has precluded the necessary driver for developing the advanced simulation capability now prevalent in so many other industries. The U.S. must correct this deficiency quickly to allow advanced fuel-cycle processes to be tested and implemented.
Thus, a fresh process-modeling approach that incorporates efficient numerical-solution methods for the governing differential equations (in both temporal and spatial dimensions) is essential for simulating, understanding, and predicting the inherent transient behavior and feedback loops of such a complex series of unit operations. A capability for accurately simulating the dynamic behavior of advanced fuel-cycle separation processes will provide substantial cost savings and myriad technical benefits to include predicting and evaluating transient response to off-normal or upset conditions, establishing operational safety envelopes, evaluating product purities, minimizing waste volumes, or even examining proliferation scenarios.

\section{Technical Objectives}

Technical objectives for the second year of the project included:

1. Develop a dynamic model using gPROMS software to simulate dynamic conditions in an annular centrifugal contactor

2. Expand the unit operations model (from Objective 1 above) into a full simulations model to predict time-dependent changes in a 30-stage TRUEX flow sheet, from startup to steady-state conditions

3. Perform a pilot-scale test of a TRUEX flow sheet and compare the results to the model simulation.

Results and Accomplishments

An executable model was developed in the first year to successfully simulate dynamic conditions in a PUREX flow sheet using conventional mixer-settler solvent extraction equipment. This model was expanded in this year's work to include the governing equations for annular centrifugal contactors, which may be used to some degree in advanced fuel-cycle solvent-extraction flow sheets. To this end, a model was developed to simulate the timedependent changes in a 30-stage TRUEX flow sheet from startup to steady-state conditions. A pilot-scale demonstration was then performed according to the flow sheet shown in Figure 1 (next page). 


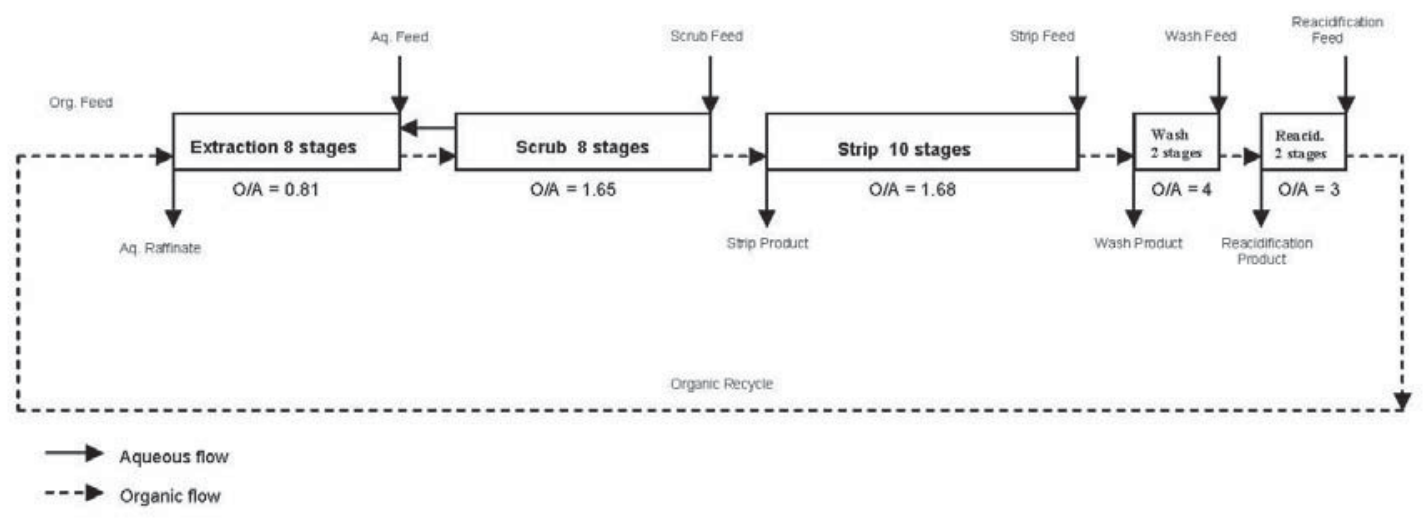

FIGURE 1. Flow sheet of 30-stage TRUEX test in centrifugal contactors.

Samples were collected from select stages at predetermined time intervals and analyzed for the extractable rare earth elements $(\mathrm{Ce}, \mathrm{Eu}, \mathrm{La}$, $\mathrm{Nd}$ ). The objective of the sampling scheme was to capture the changes in metal concentration from start up until the system reached steadystate operation conditions. The time-dependent concentration profiles obtained from the sampling effort were then compared to those predicted by the model in order to begin evaluation of model performance. The comparison of the model predictions to the dynamic data from one piece of the test equipment is shown in Figure 2. Figure 3 depicts the final concentration profile across all stages for cerium as calculated from the model simulation.

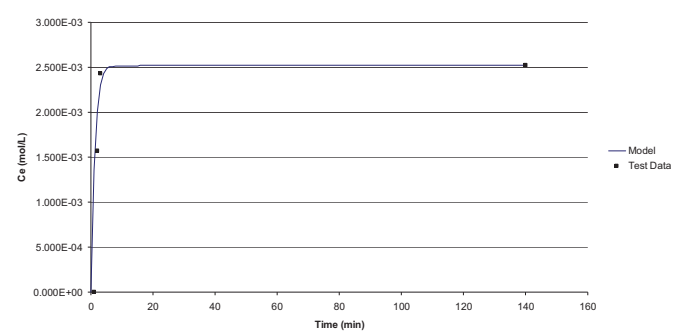

FIGURE 2. Pilot-plant data and model simulation results for time-dependent concentrations of aqueous phase cerium.

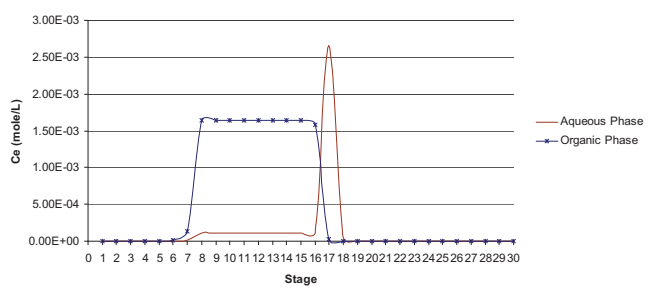

FIGURE 3. Model simulation of concentration profiles for cerium in aqueous and organic phases across 30 stages at steady-state conditions.

\section{Summary and Conclusions}

The model provides reasonable predictions of the extractable metal (Ce) as compared to the pilot plant data. It should be noted, however, that a number of problems were encountered with the analytical method used to determine metal concentrations in the pilot-plant samples. Thus, the pilot-plant data are suspect, and the analysis technique is being revised to provide more accurate data and close material balances across each stage. Nevertheless, the pilot-scale test did provide a preliminary validation of the model. It was also shown that the model simulation converges to a solution quite rapidly. The simulation predicts dynamic and steady-state concentration profiles that generally agree with expected system behavior, which indicates the structure and numerical solution of the model equations are correct. A number of additional data sets are, however, needed for further refinement and validation of the model. Work in the upcoming year will include the testing 
necessary to obtain additional data for refinement of mass transfer equations and axial mixing equations. This work is novel in that a functional model for centrifugal contactors does not currently exist. This type of simulation is needed to accurately simulate candidate flowsheets under AFCI and, therefore, would merit further programmatic funding following this initial proof of principle. 


\section{Reactivity of Radiolytically Produced Nitrogen Oxide Radicals toward Aromatic Compounds}

Bruce Mincher, Stephen Mezyk, Simon Pimblott

Defining the kinetics, thermodynamics, and mechanisms of the nitration of phenyl rings in irradiated, acidic, aqueous solution

NE147

The objective of this proposal is to define the kinetics, thermodynamics, and mechanisms of the nitration of phenyl rings in irradiated, acidic, aqueous solution. The use of nitric acid as a solvent is ubiquitous in the nuclear industry, especially in spent-fuel reprocessing. Understanding the mechanisms involved in nitric acid radiolysis is key to controlling the degradation of the compounds used in nuclearfuel reprocessing and high-level waste treatment. It was proposed here to determine the mechanisms and rates at which the principal nitrogen-centered radicals produced in irradiated nitric acid solutions react with model aromatic compounds. To determine this, a combination of steady-state irradiations coupled with stable product analysis, and electron-pulse radiolysis coupled with spectroscopic observation of shortlived intermediates was used to elucidate these reaction mechanisms. These results will also be significant to other disciplines interested in freeradical chemistry, including the community performing hazardous-waste treatment using advanced oxidation process technologies, the medical community interested in the free radical reactions leading to lipid and nucleic acid degradation and carcinogenesis, and environmental chemists interested in the production of nitrated species in the atmosphere.

\section{Technical Objectives}

- Understand the mechanism of nitration of aromatic groups in irradiated, aqueous nitric acid.

- Understand the yields and types of Ncentered radicals in irradiated, aqueous nitric acid.

- Publish these results in quality peerreviewed journals.

\section{Results and Accomplishments}

As we reported in Fiscal Year (FY) 2008, we initially hypothesized that the direct reaction of the N-centered radicals ${ }^{\circ} \mathrm{NO}_{3}$ and ${ }^{\circ} \mathrm{NO}_{2}$ produced by nitric acid radiolysis was responsible for the nitration of aromatic functional groups in irradiated, aqueous nitric acid. The literature has assumed that addition of $\mathrm{NO}_{2}$ radical to phenyl rings produces nitrated species. Similar reaction with ${ }^{\circ} \mathrm{NO}_{3}$ radical would produce nitroso-species, which could eliminate oxygen to produce a nitrated derivative.

However, the mechanism for such an elimination is unclear and, therefore, of research interest.

In our first year, we made kinetic measurements of the reactions of these two radicals with selected aromatic compounds to determine if the postulated mechanisms were possible. We developed techniques using $6 \mathrm{M}$ nitric acid or $0.1-1.0 \mathrm{M}$ sodium nitrite solutions to isolate the ${ }^{\mathrm{N}} \mathrm{NO}_{3}$ and ${ }^{\circ} \mathrm{NO}_{2}$ radicals, respectively. These techniques have since been used to assist the elucidation of reaction rates for $\mathrm{N}$-centered radicals in support of direct-funded programs, allowing INL to participate in Fuel Cycle Research and Development (FCR\&D) radiation chemistry research. Our results have revealed that the $\mathrm{NO}_{2}$ radical reaction is not an important source of nitration reactions in irradiated nitric acid. Although reactions with $\mathrm{NO}_{3}$ radical are fast, they are too fast to be addition reactions, and it is also not likely to be an important source of nitration.

However, we know that nitrated organic species are indeed produced in irradiated nitric acid. We irradiated anisole and toluene in aqueous nitric acid using our ${ }^{60} \mathrm{Co}$ source and measured the stable products. For nitroanisole products, only 2- and 4-nitroanisole were produced, at an ortho/para ratio of about 0.35 . The meta isomer was not detected. This substitution pattern, shown in Figure 1, suggests electrophilic aromatic substitution. Further, when unirradiated samples were maintained at the ambient irradiator temperature of $50^{\circ} \mathrm{C}$, lesser but significant amounts of the same products were created. In our last report we speculated that this might be due to nitronium ion reactions, with free-radical reactions as the source of nitronium ion:

$\cdot \mathrm{NO}_{3}+\cdot \mathrm{NO}_{2} \rightarrow \mathrm{N}_{2} \mathrm{O}_{5} \rightarrow \mathrm{NO}_{3}^{-}+\mathrm{NO}_{2}{ }^{+}$

However, we now know that the ortho:para ratio is more consistent with nitrous acid catalyzed nitration. The following reactions occur when small amounts of nitrous acid are found in nitric acid:

$\mathrm{HNO}_{2}+\mathrm{H}^{+} \rightarrow \mathrm{NO}^{+}+\mathrm{H}_{2} \mathrm{O}$ 
$\mathrm{ArH}+\mathrm{NO}^{+} \rightarrow \mathrm{ArHNO}^{+}$

$\mathrm{ArHNO}^{+} \rightarrow \mathrm{ArNO}+\mathrm{H}^{+}$

The nitroso product of Equation 4 is readily oxidized to form the corresponding nitro products (see Equation 5):

$\mathrm{ArNO}+\mathrm{HNO}_{3} \rightarrow \mathrm{ArNO}_{2}+\mathrm{HNO}_{2}$

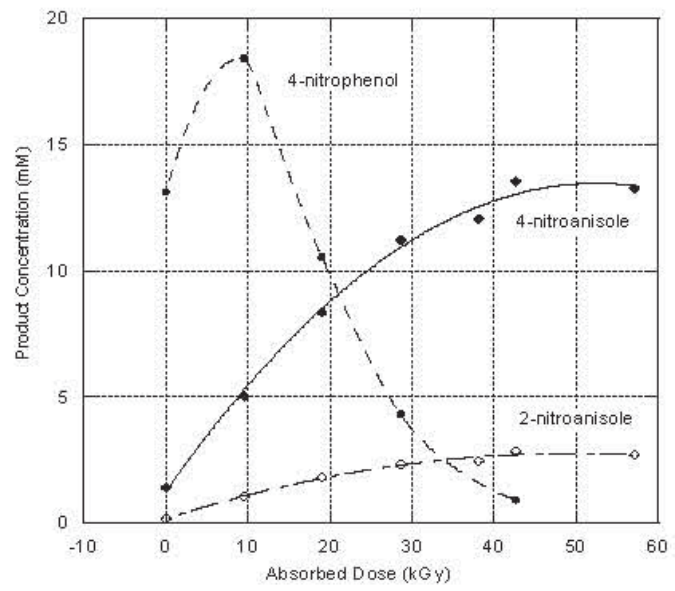

FIGURE 1. The ingrowth of 2- and 4- nitroanisole in irradiated anisole in $6 \mathrm{M}$ nitric acid solution. No 3nitroanisole was detected. Dinitrophenol is also present but cannot be quantitated.

Thus, radiolytically produced nitrous acid could be responsible for nitration reactions in irradiated nitric acid. Therefore, we measured nitrous acid production in irradiated nitric acid and obtained the results shown in Figure 2.

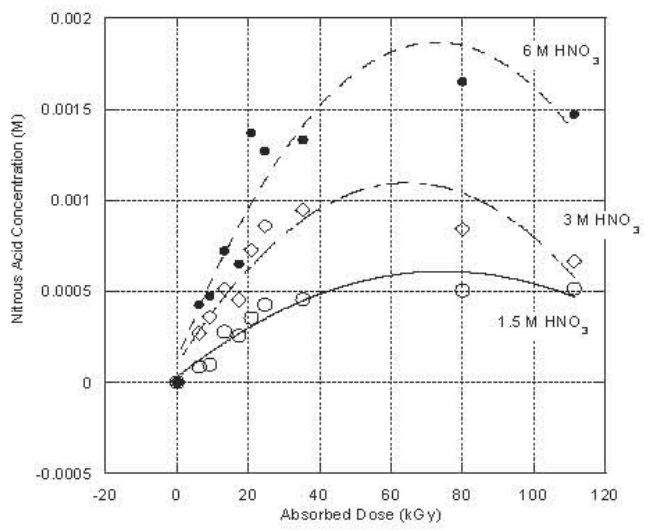

FIGURE 2. The ingrowth of nitrous acid in varying concentrations of irradiated nitric acid. 4-nitrophenol was also a major product in both irradiated and unirradiated anisole samples (Fig. 1). The formation of this product is consistent with a nitrous acid catalyzed mechanism. Further, when the nitrous acid scavenger hydrazine was added to samples, the nitrated products were not produced. We now propose that in irradiated nitric acid, nitration of phenyl rings is not caused by direct nitration via radical addition; rather, radiolytically produced nitrous acid results in enhanced electrophilic aromatic substitution reactions. We have prepared a manuscript summarizing our anisole results which is now in the review process at a high-impact peer-reviewed journal. INL's Gracy Elias' work on this project has been accepted as dissertation research to fulfill the University of Idaho's dissertation requirements.

Irradiation experiments using anisole were also conducted in sodium nitrite solution. In nitrite solution, only the ${ }^{\mathrm{N}} \mathrm{NO}_{2}$ radical is formed. Despite anisole's being a strong ortho/para director for electrophilic aromatic substitution, we found that meta-substituted nitroanisole was the major nitrated product resulting from $\bullet \mathrm{NO}_{2}$ radical reactions in irradiated sodium nitrite. This is further evidence that radiation-enhanced conventional thermal nitration is the mechanism of aromatic ring nitration in irradiated aqueous nitric acid. Nitrous acid reactions cannot occur in sodium nitrite, and the production of the meta isomer must be by reaction with the ${ }^{\circ} \mathrm{NO}_{2}$ radical, either through direct addition (unlikely due to the rate constant being $<10^{6} \mathrm{~L} \mathrm{Mol}^{-1} \mathrm{~s}^{-1}$ ) or by addition to carbon-centered radicals formed on the aromatic ring through other processes during irradiation.

During our pulse-radiolysis studies, we discovered a remarkable absence of ${ }^{\circ} \mathrm{NO}_{2}$ radical in irradiated nitric acid. We expected to see the absorbance of this radical in our solutions because it should be produced via electroncapture reactions with the $\mathrm{NO}_{3}{ }^{-}$anion, followed by hydrolysis of the resulting ${ }^{-} \mathrm{NO}_{3}{ }^{2-}$ radical product. At this time we are pursuing the modeling of this system with our California State University and University of Manchester collaborators to better understand why ${ }^{\circ} \mathrm{NO}_{2}$ radical is not appearing in irradiated acidic solution. The spectra for these transient species 
are shown in Figure 3, along with the reduction in absorbance versus time in the presence of the $\mathrm{NO}_{3}$ radical scavenger anisole.
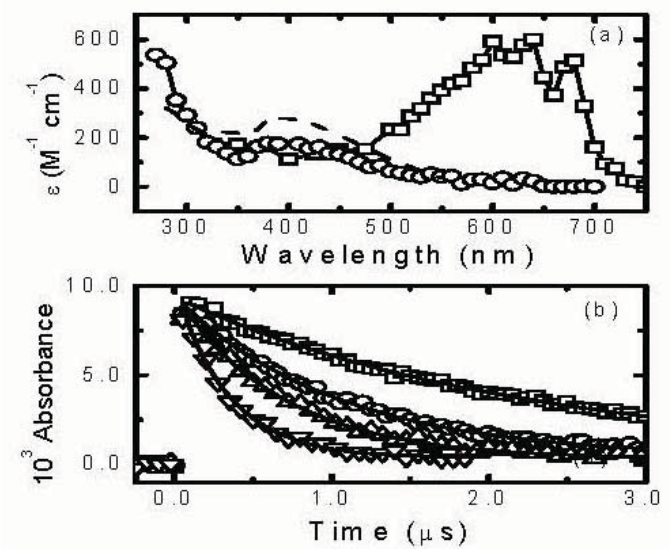

FIGURE 3. (a) Transient absorbance of the ${ }^{\circ} \mathrm{NO}_{3}$ radical in irradiated $6 \mathrm{M}$ nitric acid (boxes) and ${ }^{\circ} \mathrm{NO}_{2}$ radical in irradiated sodium nitrite solution (diamonds). Note the absence of ${ }^{\circ} \mathrm{NO}_{2}$ radical absorbance in irradiated acid. (b) The decay in transient absorbance versus time of the ${ }^{\circ} \mathrm{NO}_{3}$ radical at $640 \mathrm{~nm}$ for varying concentrations of anisole in 6 $\mathrm{MHNO}_{3}$. These data may be used to calculate the bimolecular rate constant for the reaction of ${ }^{\circ} \mathrm{NO}_{3}$ radical with anisole.

\section{Summary and Conclusions}

In contrast to initial expectations, the nitration of phenyl rings in irradiated aqueous nitric acid appears to be due to radiolytically enhanced nitrous acid-catalyzed nitration. This information should eventually allow us to predict the nature and yields of nitrated products in irradiated solution. In continued work, we intend to gather toluene data to substantiate this mechanism and to perform modeling in an attempt to understand the fate of produced ${ }^{\circ} \mathrm{NO}_{2}$ radical. This LDRD work has now led to two publications, with a third under review:

1. Mincher, B.J., G. Elias, L. R. Martin, and S. P. Mezyk, 2009, "Radiation chemistry and the nuclear fuel cycle," J. Radioanal. Nucl. Chem. DOI 10.1007/s 10967-009-0156-X.

2. Mincher, B.J., S. P. Mezyk, and L. R. Martin, 2008, "A pulse radiolysis investigation of the reactions of tributylphosphate with the radical products of aqueous nitric acid irradiation," J. Phys. Chem., Vol. 112, pp. 6275-6280.

3. Elias, G., B. J. Mincher, S. P. Mezyk, T. Cullin, and L. R. Martin, 2009 "Anisole nitration during gamma-radiation of aqueous nitrite and nitrate solutions: Free radical versus ionic mechanisms," Submitted to Environ Chem, August 9, 2009. 


\title{
Modernization/Optimization of the Advanced Test Reactor
}

\author{
D.W. Nigg, D. Scott Lucas, James Parry, George Griffith \\ Development of ATTILA models for the ATR \\ NE149
}

The primary objective of the Advanced Test Reactor (ATR) modeling and simulation upgrade is to modernize and standardize the computational methods and protocols used for engineering analysis of the coupled neutronic and thermal-hydraulic behavior of the ATR core under normal and postulated off-normal operating conditions, and for characterization of the neutronic environment in the various experiment irradiation positions. This is necessary because many of the computational techniques used to support ATR reactor operation and safety analysis are outdated to the point of being almost totally inconsistent with state-of-the-art practices and capabilities elsewhere in the nuclear industry. Furthermore, some aspects of the analysis process are highly empirical in nature (requiring very specialized experience to apply), and the overall process has become cumbersome to use, overly conservative, and difficult to validate according to modern practice. Finally, future users of the facility are anticipated to be experienced in the use of modern computational methods available for nuclear-systems modeling and are likely to expect modern computational support services.

INL efforts on this project were initially conducted in Fiscal Year (FY) 2009 under this laboratory-directed research and development (LDRD) and were completed mid-year. The results are summarized in this report. In the second half of the year, some parts of the effort were subsequently continued and, in fact, significantly expanded via direct program funding provided by several benefitting programs, including the Next Generation Nuclear Plant (NGNP) Program, the Reduced Enrichment Research, Training and Research Reactor (RERTR) Program, and the ATR Life Extension Program (LEP). Direct program support is expected to continue in FY 2010, with the final year of this LDRD project focused on a few remaining independent, computationalmethods-development activities that are needed to support the longer-term direct-funded effort.

\section{Technical Objectives}

The technical objectives of the LDRDspecific effort included continued demonstration of the feasibility of using modern computational neutron-transport methods for high-fidelity modeling of the ATR, with a focus on the ATTILA 3D transport code, but with an increased emphasis on cross verification of results against independent computations using the New Extended Step Characteristic (ESC)based Weighting Transport code (NEWT) and HELIOS transport codes. An additional objective included demonstration of the generation of spectrum and spatially weighted cross-section data for ATTILA, using the NEWT and HELIOS codes. Furthermore, in anticipation of transitionto-program support in FY 2010 and beyond, the development of a higher fidelity full 19-plate fuel element model for ATTILA was initiated. Finally, development of a depletion capability for the ATTILA code was initiated, although delays in some separately-funded support activities for the capability that are being conducted by Transpire (the commercial vendor for ATTILA) necessitated that a significant part of this activity be postponed until the third and final year of this LDRD project.

\section{Results and Accomplishments}

Progress on ATTILA Modeling Capability for ATR. ATTILA is a steady-state 3-D neutrontransport discrete-ordinates code. It uses the Linear Discontinuous Galerkin spatial integration scheme and an unstructured mesh, meaning that it is highly successful at meshing complex geometries and boundaries, as characterizes the ATR. The input model for ATTILA is built using SolidWorks' CAD-based software. ATTILA can use any set of crosssection libraries in DTF or AMPX format. At present, the SCALE libraries from Triton and NEWT are used. The ATTILA model (Fig. 1, next page) of the ATR was developed from the details of the ATR critical benchmark HEUMET-THERM-022 documented in NEA/NSC/DOC/(95)03/II, a publicly available document. It is an explicit 19-plate full-core 3-D model. The critical experiment was part of the nuclear requalification testing following the core internals changeout (CIC) completed in 1994. The model contained individual material designations for each fuel plate which can be used for depletion and also provide cross sections for each individual fuel plate in the ATR. An additional three region and seven plate models were built to allow faster execution and 
the computation of peak plate powers. ATTILA was exercised for the CIC-94, with both the 19plate and the seven-plate model. The plot of relative power is shown in Figure 2.

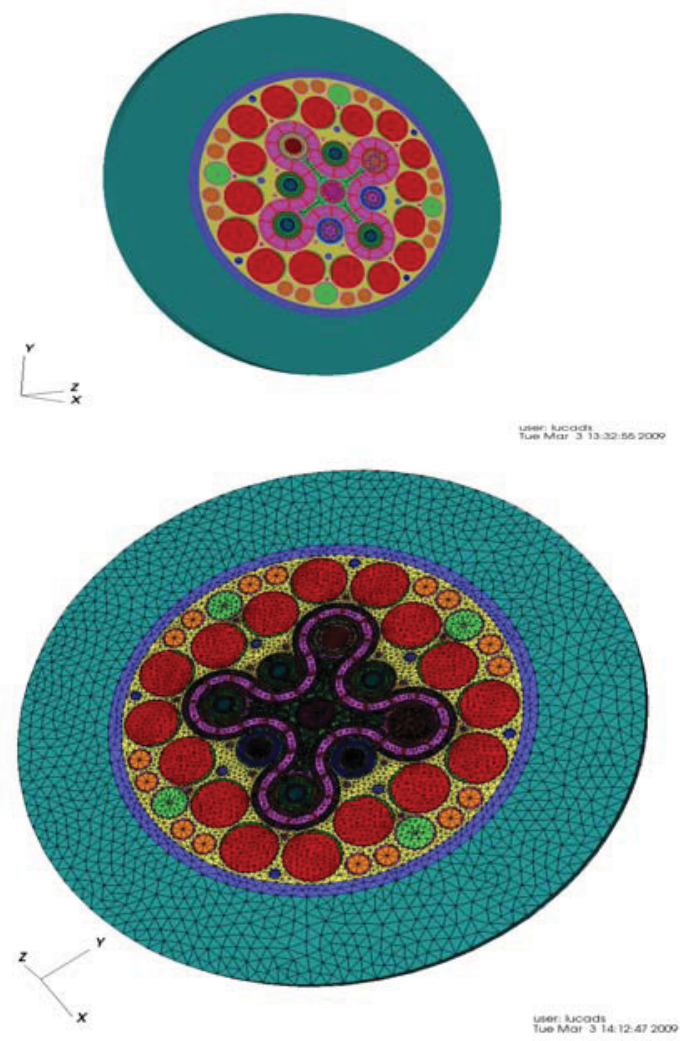

FIGURE 1. ATR 3-D ATTILA Model and 133-K Tet Mesh.

HELIOS Verification Model. HELIOS is a two-dimensional (2-D) lattice physics code that has been applied to a number of different reactor types. It was developed by Studsvik

Scandpower. Commercial and research reactors have been modeled. The solution method uses current coupling collision probabilities (CCCP). The HELIOS 1994 CIC model, created for the ATR reactor, was developed in collaboration with Studsvick Scandpower. The code produces accurate results that match previous MCNP calculations and measurements. The modified input deck and the HELIOS code also produced cross-sections suitable for ATTILA. Individual fuel-plate power edits were implemented this year, adding a new capability to the model (Fig. 3).

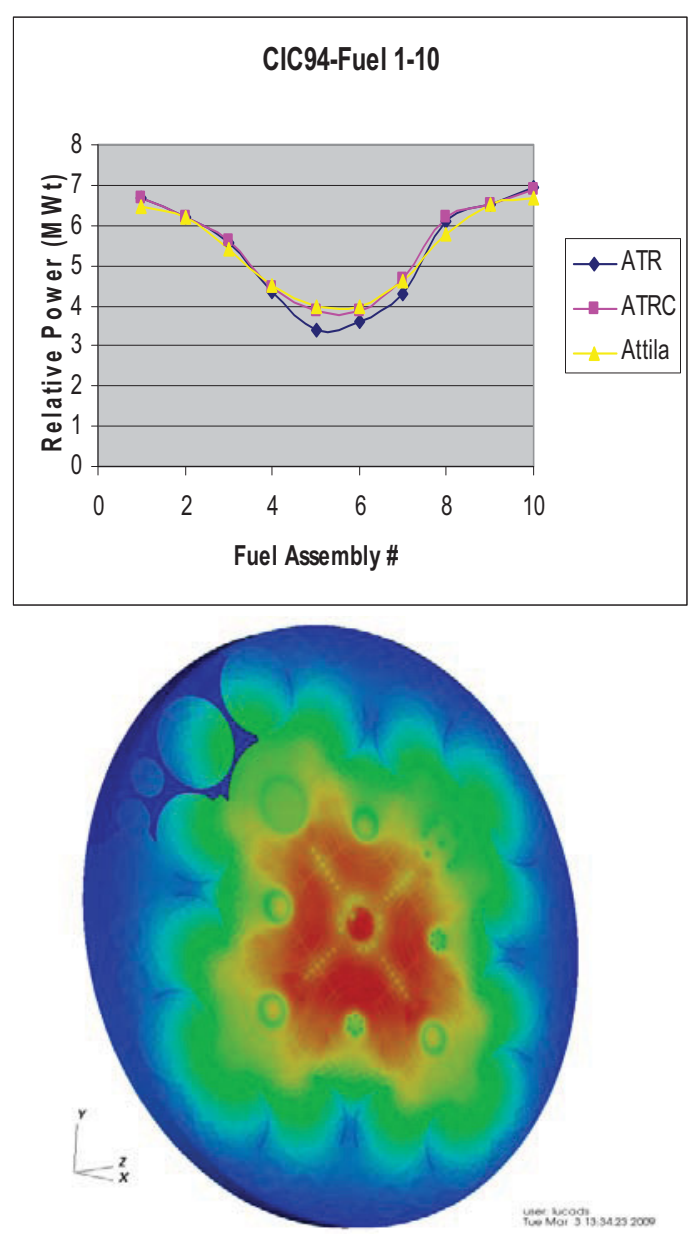

FIGURE 2. Fuel plate powers from ATTILA, averaged over ATR core for ten elements in a quarter-core model.

NEWT Verification Mode. NEWT is a 2-D discrete-ordinates transport code developed based on the ESC approach for spatial discretization on an arbitrary mesh structure. NEWT can also collapse cross-section libraries into specified group structures for each nuclide in each material mixture in the model. As part of the SCALE code package, NEWT is capable of performing fuel-burnup calculations using the ORIGEN-S module of SCALE. The NEWT model of the ATR is an explicit 19-plate fullcore 2-D model, again based on the 1994 CIC (Fig. 3). The NEWT model produces crosssections usable in the ATTILA code for each material collapsed to the desired number of groups. Run times for the current version of NEWT are somewhat long ( 24-36 hours). 
However, because NEWT is primarily viewed as a verification/validation and cross-section generation tool, this is acceptable, although an updated version that is anticipated to have considerably shorter execution times is currently under development at Oak Ridge.

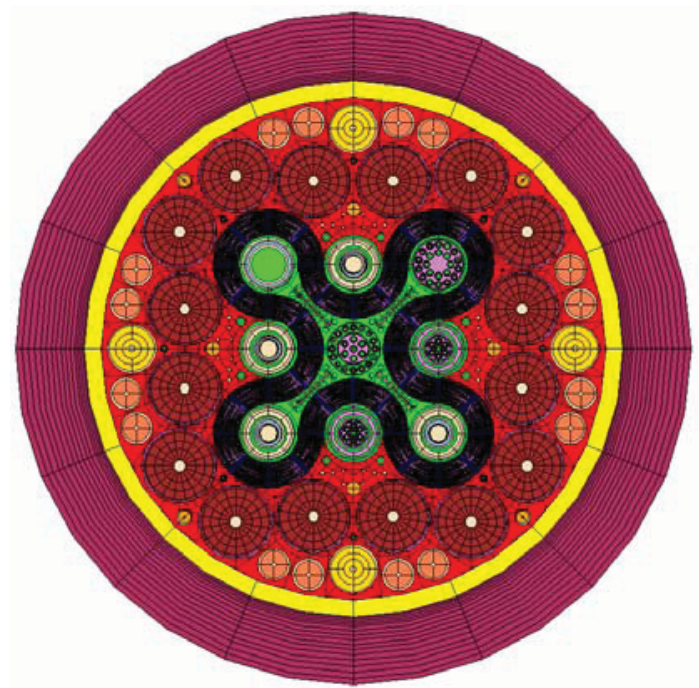

FIGURE 3. ATR 2-D HELIOS model.

\section{Summary and Conclusions}

Development of ATTILA models for the ATR continued during the report period, with significant progress on improved geometric fidelity and with the successful new development of supporting HELIOS and NEWT verification and cross-section-generation models.

Production-level ATR model development and verification and validation $(\mathrm{V} \& \mathrm{~V})$ activities initiated under this LDRD will be transitioned completely to direct program support after FY 2010. On-going work in FY 2010 under the LDRD will focus on the final ATTILA-code development deliverables, with emphasis on incorporation and testing of a fuel-depletion capability in collaboration with Transpire, supported on their side by a separate DOE Small Business Initiative (SBIR) grant. 


\title{
Evaluations of Linear Variable Differential Transformers for ATR In-Pile Instrumentation
}

\author{
J. Rempe, K. Condie, D. Knudson (INL); S. Solstad (Institute for Energy Technology/ \\ Halden Reactor Project) \\ Evaluating a sensor that can detect changes in length during high-temperature irradiation testing \\ NE150
}

New materials are being considered for fuel, cladding, and structures in next-generation and existing nuclear reactors. Such materials can experience significant dimensional and physical changes during irradiation. Currently, such changes are measured by repeatedly irradiating a specimen for a specified period of time and then removing it from the reactor for evaluation. The labor and time to remove, examine, and return irradiated samples for each measurement makes this approach very expensive. In addition, such techniques provide limited data, and handling may disturb the phenomena of interest.

This three-year project is evaluating the viability of Linear Variable Differential Transformers (LVDTs) as in-pile real-time sensors for detecting for detecting dimensional changes in specimens during high temperature (up to $500^{\circ} \mathrm{C}$ ) irradiations in the Advanced Test Reactor (ATR). Previous evaluations have shown that these sensors can detect dimensional changes in lower-temperature, non-irradiation environments. If evaluations proposed in this project are successful, LVDT techniques could offer much-needed high-temperature sensors for detecting dimensional changes during irradiation testing at the ATR and other materials and test reactors. Techniques investigated in this project offer the potential to eliminate the labor and time to remove, examine, and return irradiated samples for each measurement. In addition, these techniques offer the potential to significantly increase accuracy of in-pile measurements since they eliminate concerns about the impact of handling on phenomena of interest.

As shown in Figure 1a, LVDTs are electrical transformers with three coils placed end-to-end around a tube. The center coil is the primary, and the two outer coils are the secondaries. A cylindrical magnetically permeable core, attached to the object whose position is to be measured, slides along the axis of the tube. An alternating current is driven through the primary, causing a voltage to be induced in each secondary which is proportional to its mutual inductance in the primary. As shown in Figure 1b, when the core moves, these mutual inductances change, causing the voltages induced in the secondaries to change. The coils are connected in reverse series so that the output voltage is the difference between the two secondary voltages. When the core is in its central or "null" position, equidistant between the two secondaries, equal but opposite voltages are induced in these two coils, so the output voltage is zero. Many features of LVDTs (e.g., frictionless measurements, long lifetime, high resolution, etc.) make them ideal for in-pile applications.

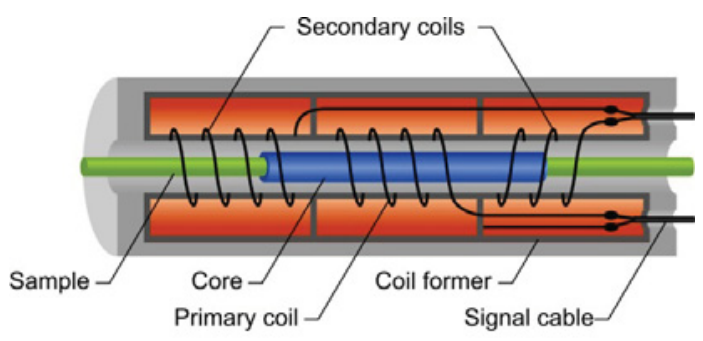

(a) LVDT

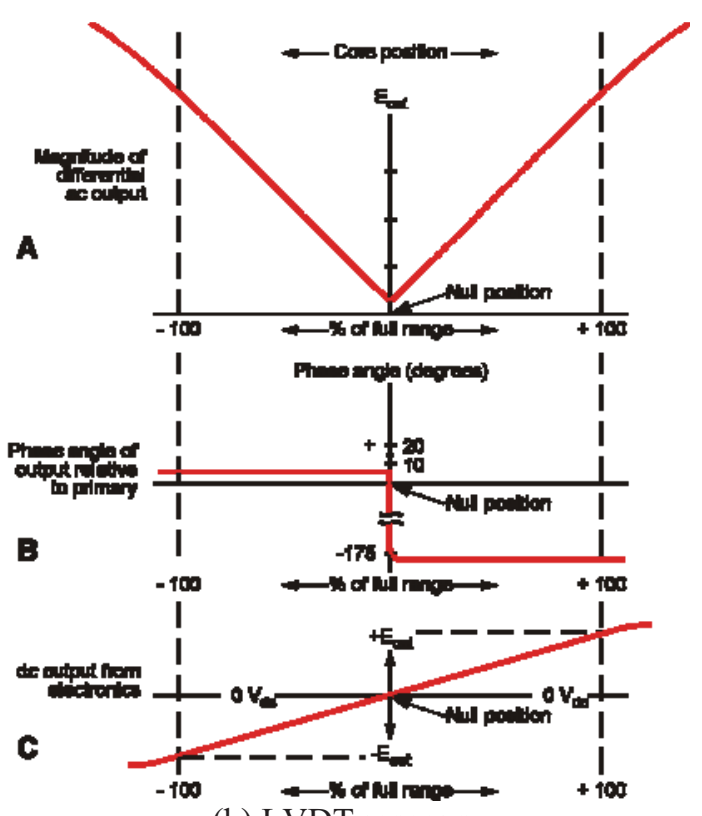

(b) LVDT response

FIGURE 1. Typical LVDT components and operation.

\section{Technical Objectives}

In this three-year project, the following technical objectives were identified for tasks to develop and demonstrate the viability of LVDT technology for ATR irradiations. 
Task 1: Commercially available nuclear-grade LVDT performance comparisons.

- Develop fixturing and data acquisition system for calibration comparisons

- Develop fixturing and data acquisition system for long-duration performance evaluations

- Procure commercially available nucleargrade LVDTs

- Complete calibration evaluations and longduration performance evaluations.

Task 2: Enhanced LVDT performance evaluations.

- Develop recommendations for enhanced high-temperature LVDT design

- $\quad$ Obtain prototype high-temperature LVDTs from vendors

- Complete calibration and long-duration performance evaluations of prototype high-temperature LVDTs

- Develop recommendations for enhanced high-temperature LVDT design for ATR irradiations.

By completing the above objectives, this LDRD will not only meet the proposed objectives for this project, but also significantly enhance instrumentation capabilities during ATR irradiation.

\section{Results and Accomplishments}

Scheduled workscope for the first two years of this three-year LDRD was completed as planned. INL evaluations completed in FY 08 identified two candidate nuclear grade LVDT vendor designs that could meet the ATR-specific customer requirements. Specifically, one supplier, identified as Vendor A, can currently provide LVDTs qualified to a maximum operating temperature of only $350^{\circ} \mathrm{C}$ while another supplier, identified as Vendor B, can currently provide only LVDTs with diameters exceeding listed ATR design limits. (Note that the Vendor-A temperature limitation was primarily due to instabilities associated with the sensor response as its coils pass through a material-specific Curie temperature at $\sim 350^{\circ} \mathrm{C}$.
Hence, evaluation of the Vendor-A LVDT was appropriate because alternate materials, which are not susceptible to Curie temperature effects, could be incorporated into their design. Likewise, Vendor B indicated that alternate components could be used in their sensor design so that it is smaller in diameter). In both cases, limited data were available to verify either manufacturer's claims regarding their performance at high temperature. During Fiscal Year (FY) 2009, INL completed several tasks to compare candidate design performance. First, laboratory calibration tests were completed to compare candidate LVDT response at temperatures up to $500^{\circ} \mathrm{C}$. Second, LVDT stability and reliability were assessed through long duration testing at $500^{\circ} \mathrm{C}$. After these initial evaluations, a superior design was selected for additional evaluations. First, finite element analyses were completed to determine the potential impact of gamma heating on LVDT performance. Second, additional assessments were completed between 300 and $420^{\circ} \mathrm{C}$ to evaluate any instabilities that may occur when the preferred LVDT design is exposed to its Curie temperature.

Calibration and long-duration hightemperature evaluations were completed using specialized fixtures for LVDT positioning, vendor-specific LVDT signal-conditioning equipment, and a computerized data acquisition system (DAS) to be used in conjunction with a high-temperature tube furnace (Fig. 2, next page).

Calibration results for the Vendor-A LVDTs remained linear and symmetric with respect to its null position for its design range of $+/-2.5 \mathrm{~mm}$ for the entire temperature range (e.g., up to $500^{\circ} \mathrm{C}$ ). However, results for the Vendor-B LVDT showed that its linear deviation was more than twice the Vendor-A LVDT maximum deviation. In addition, the linear deviation was not symmetric with respect to its null position. Evaluations also indicated that the Vendor-A LVDT sensitivity (e.g., $\mathrm{mV} / \mathrm{mm}$ ) was considerably higher than measured for the Vendor-B LVDT at $500^{\circ} \mathrm{C}$. Furthermore, the primary to secondary insulation resistance for Vendor B showed a sharp decrease after $300^{\circ} \mathrm{C}$. At $500^{\circ} \mathrm{C}$, primary-to-secondary insulation 

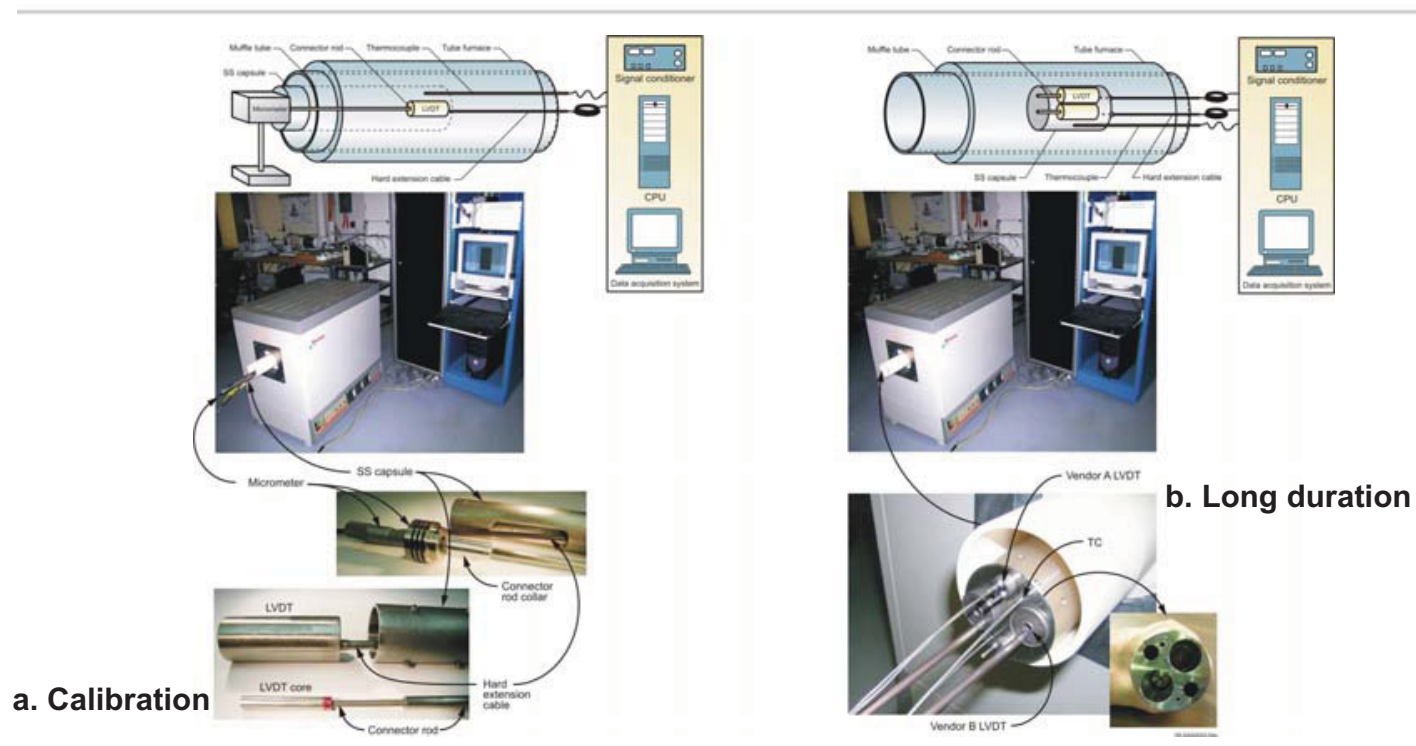

FIGURE 2. Setup for LVDT calibration and long duration performance evaluations.

resistances of $\sim 40$ ohms were measured for the Vendor-B LVDT, while the corresponding insulation resistance for the Vendor-A LVDT was measured in excess of $1 \times 10^{6} \mathrm{ohms}$.

Long-duration testing (Fig. 3) indicates that Vendor-A LVDTs remained very stable through $\sim 330 \mathrm{~h}$. Their maximum deviation during that period is equivalent to a displacement of $\sim 0.004 \mathrm{~mm}$ relative to their time 0 position. However, some periodic fluctuations in the response of one of the Vendor-A LVDTs began to appear after $\sim 330$ hours and dramatically

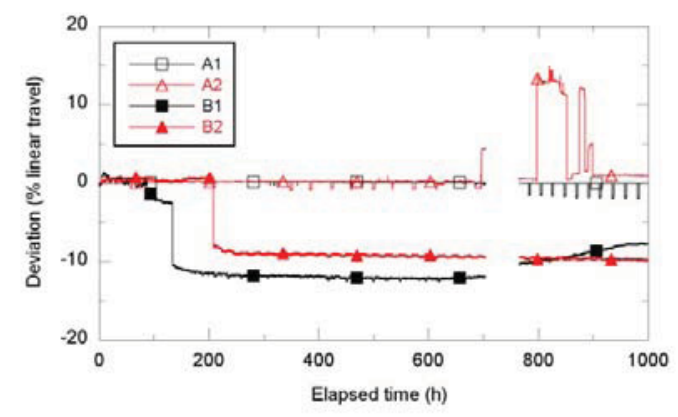

FIGURE 3. Comparison of LVDT response during long duration testing at $500^{\circ} \mathrm{C}$.

increased in both magnitude and duration after 700 hours. Shortly thereafter, the signal fluctuations of the second Vendor-A LVDT increased, with relatively frequent oscillations until the end of the 100-h test. Although the reasons for the behavior of Vendor-A LVDTs during the later part of the long-duration test are still under investigation, discussions with Vendor A indicate that they believe that tendencies for insulation degradation may be minimized by changes in their heat treatment/curing processes. Results for Vendor-B LVDTs showed substantial oscillation (starting at time 0 ) in addition to (as yet unexplained) dramatic step changes in indicated deviation in the first 200 hours of the test. In fact, the observed step change in the Vendor-B LVDTs is equivalent to a displacement of $\sim 0.6 \mathrm{~mm}$, indicating a reduction in stability by a factor of $\sim 150$, when compared to Vendor-A LVDTs, during the first $330 \mathrm{~h}$ of the test. After reviewing these initial results, Vendor-A indicated that it was acceptable for them to be identified (Vendor-A is the Institute for Energy Technology at the Halden Reactor Project). Vendor-B has not responded to requests for comments on these test results.

After these initial evaluations, the superior design manufactured by Vendor-A was selected for additional evaluations. INL finite-element analyses indicate that peak temperatures during irradiation due to gamma heating at the center of the core in an ATR flux trap will result in peak LVDT temperatures of $400^{\circ} \mathrm{C}$. Evaluations were also completed between 300 and $420^{\circ} \mathrm{C}$ to assess the instabilities that occur when Vendor-A LVDT designs were exposed to its Curie 
temperature. Results indicate that localized signal decreases of up to $50 \%$ may occur due to Curie temperature effects. However, these drops are typically limited to a $10^{\circ} \mathrm{C}$ range; and, as noted above, Vendor A is investigating several options to improve their LVDT performance at high temperature. INL will evaluate improved designs during FY 2010.

\section{Summary and Conclusions}

Results from this project are leading to an LVDT design suitable for ATR irradiations. Hence, project results increase ATR instrumentation capabilities, which support DOE's energy security mission by developing and demonstrating much-needed sensors to enhance the accuracy of data obtained from and reduce the costs of irradiations in materials and test reactors such as the ATR. Hence, project results provide much-needed sensors for obtaining key data required for key DOE-NE research programs, such as the Generation IV and Advanced Fuel Cycle Initiative (AFCI) programs, and enhance testing capabilities for the ATR National Scientific User Facility (ATR NSUF). Finally, recognition (e.g., publications, patents, and awards) from this research promotes INL's instrumentation capabilities, furthering INL efforts to develop the Instrumentation, Control, and Intelligent Systems Distinctive Signature. 


\section{Characterization of a Consolidated Electrochemical Technique for Separation and Recovery of Actinides from Fission Products}

Steven D. Herrmann, Shelly X. Li, Guy L. Frederickson

Improved single-step process for electrochemical treatment of oxide fuels NE157

Development of a sustainable closed nuclear-fuel cycle is a primary need within DOE's Fuel Cycle Research and Development (FCR\&D) program. The success of such a cycle depends on the effective and proliferationresistant separation and recovery of actinides from fission products in irradiated oxide fuels. One means of affecting such separation is by electrochemical processing. For over a decade, researchers at Idaho National Laboratory (INL) and Argonne National Laboratory have collaborated to investigate an integrated electrochemical process for treatment of oxide fuels based on a two-step oxide reduction and electrorefining process. A fundamental inefficiency in the integrated oxide-reduction and electrorefining processes is the need to pass current twice in two different salt systems in order to recover uranium metal from uranium oxide. Specifically, uranium oxide $\left(\mathrm{U}^{4+}\right)$ is electrolytically reduced to uranium metal $\left(\mathrm{U}^{\circ}\right)$ in an oxide-reduction process using a molten $\mathrm{LiCl}-$ $\mathrm{Li}_{2} \mathrm{O}$ electrolyte at $650^{\circ} \mathrm{C}$, after which the uranium is simultaneously re-oxidized (to $\mathrm{U}^{3+}$ ) and re-reduced (to $\mathrm{U}^{\circ}$ ) in an electrorefining process to recover uranium metal using a molten $\mathrm{LiCl}-\mathrm{KCl}-\mathrm{UCl}_{3}$ electrolyte at $500^{\circ} \mathrm{C}$. A significant improvement to the electrochemical treatment of oxide fuels could be realized by consolidation of the dissolution, separation, and reduction of uranium into a single-step process, which is the goal of this laboratory-directed research and development (LDRD). Specifically, uranium oxide feed material could be dissolved and simultaneously reduced to metal by electromotive force in a suitable salt system. Thus, uranium in oxide fuels could be separated from fission products and recovered as metal by passing current once in a single salt system. Furthermore, the proposed consolidated process is amenable to continuous operations in contrast to the two-step batch approach with the coupled electrolytic reduction and electrorefining processes.

\section{Technical Objectives}

The objective of this LDRD is to characterize the fundamental chemistry and electrochemistry of a novel molten-salt system for separation and recovery of actinides from fission products in oxide fuels. Specific objectives by fiscal year include the following:

- Fiscal Year (FY)-09: Design, fabricate, procure, and install the necessary equipment and materials to support the proposed experiments. Characterize the dissolution and electrolytic decomposition of dissolved uranium oxide in the proposed salt system via bench-scale experiments.

- FY-10: Introduce stable fission-product compounds into the proposed salt system and investigate the separation and recovery of uranium from fission products.

- FY-11: Introduce transuranic constituents into the proposed salt system and further investigate the separation and recovery of actinides from fission products.

\section{Results and Accomplishments}

In FY-09, equipment was designed, fabricated, and installed for use with an existing molten salt furnace in an argon-atmosphere, radiological glovebox at the Fuels and Applied Science Building (FASB) at INL's Materials and Fuels Complex (MFC) to support the first phase of this research. Specifically, the Molten Salt Furnace-III (MSF-III) was loaded with a fluoride-based salt and heated to $500^{\circ} \mathrm{C}$. Fifty grams of $\mathrm{UO}_{2}$ particulate was loaded into a permeable metal basket and immersed in the molten salt. Cyclic voltammetry was performed on select anode and cathode materials within the molten salt pool at varying temperatures between 500 and $900^{\circ} \mathrm{C}$. Depleted uranium fluoride was incrementally added to the molten salt pool to create three different uranium concentrations, and cyclic voltammetry was likewise performed at each of the prescribed temperature and uranium concentration settings. Salt samples were taken at each temperature and uranium concentration setting and analyzed for oxygen concentration. The molten salt was then subjected to constant current operations at 650 , 725 and $800^{\circ} \mathrm{C}$, during which uranium metal was deposited on a cathode rod and oxygen ions participated in reactions at an anode. Samples of uranium deposits were also analyzed. 
Cyclic voltammetry of the molten-salt electrolyte revealed potentials relative to a $\mathrm{Ni} / \mathrm{NiO}$ reference electrode at which reactions occurred on prescribed anode and cathode materials. Figure 1 shows cyclic voltammetry plots for a nickel wire cathode and a platinum wire anode in the fluoride salt at $650^{\circ} \mathrm{C}$ before and after uranium fluoride addition. These plots identified uranium reduction and salt decomposition potentials on the cathode and oxygen ion oxidation and platinum decomposition potentials on the anode.

The nickel and platinum wires were replaced with a nickel rod and platinum-wire coil, and a controlled current was applied to the cell. The current was controlled to produce the desired anode and cathode potentials and accompanying reactions as identified by the cyclic voltammetry runs. Figure 2 shows the uranium metal deposit ( $\sim 11 \mathrm{~g}$ on the cathode rod from the first run with this system) along with a cut-away view of the test setup and input materials.

The prescribed matrix of tests was repeated, using $\mathrm{U}_{3} \mathrm{O}_{8}$ particulate in lieu of $\mathrm{UO}_{2}$ particulate, producing similar cyclic voltammetry plots, salt samples, and uranium metal deposits. A third matrix of tests was performed that repeated conditions from the second matrix of tests with the addition of two alternate anode materials.

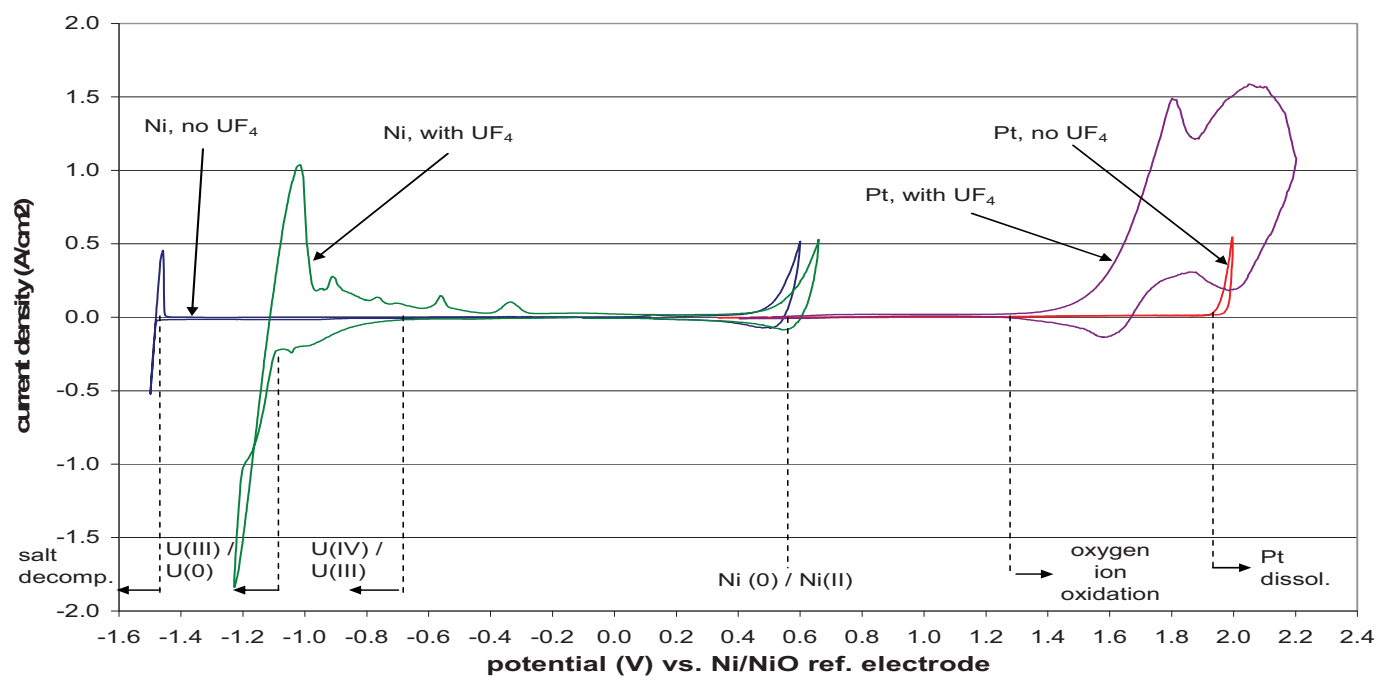

FIGURE 1. Cyclic voltammetry plot for nickel and platinum wire in fluoride salt at $650^{\circ} \mathrm{C}$.

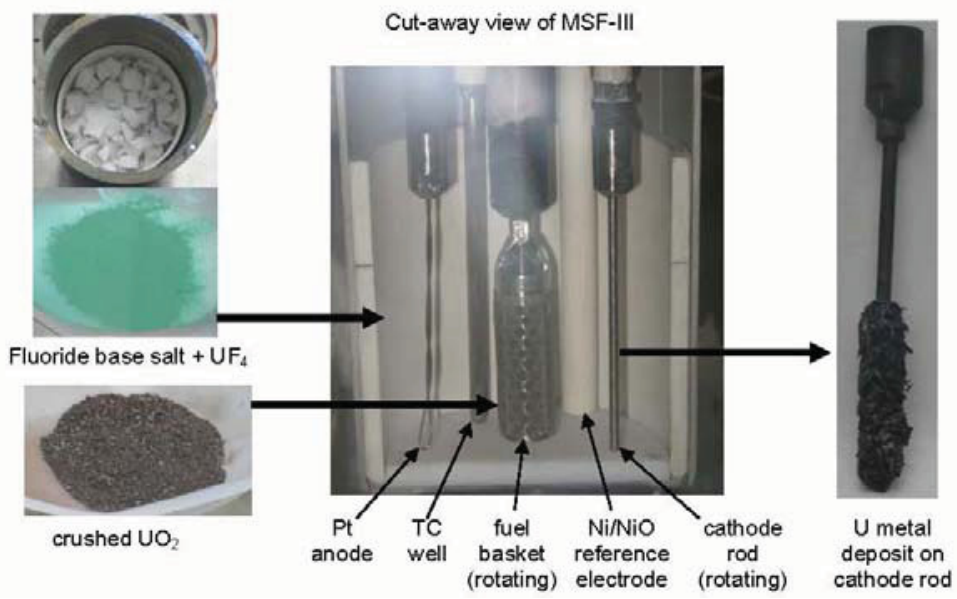

FIGURE 2. Cut-away view of MSF-III with input materials and uranium metal deposit on a cathode rod from the first run at $650^{\circ} \mathrm{C}$. 
Summary and Conclusions

The objectives in the first year of this LDRD were completed, which included a parametric study that characterized (1) the extent of uranium oxide dissolution and (2) the electrochemical potentials and current densities for uranium oxide decomposition as a function of system temperature and uranium fluoride composition. Other parameters assessed in this study were
$\mathrm{U}_{3} \mathrm{O}_{8}$ vs. $\mathrm{UO}_{2}$ as a feed material and alternate anode materials. Characterization of the prescribed technique is defining a pathway toward a novel and patentable process for a simpler and more cost-effective electrochemical treatment of spent oxide fuels than the state-ofthe-art approach. 


\section{Advanced Instrumentation for In-pile Detection of Thermal Conductivity}

Darrell Knudson and Joy Rempe, (INL); Heng Ban and Brent Stucker, Utah State University (USU); Steinar Solstad, Institute for Energy Technology/Halden Reactor Project (IFE-HRP) Developing an improved sensor detecting thermal conductivity during irradiation

NE158

Currently, changes in thermal conductivity of fuel or materials irradiated in the Advanced Test Reactor (ATR) are evaluated out-of-pile, where properties of materials are measured after samples have been irradiated for a specified length of time. The labor and time to remove, examine, and return irradiated samples for each measurement makes out-of-pile approaches very expensive. In addition, data may not be obtained under prototypical conditions because they only capture the sample's endstate, after it is removed from the reactor, and removal and reinsertion into the reactor may disturb the phenomena of interest.

To provide an in-pile method for detection of thermal conductivity, Idaho National Laboratory (INL) and Utah State University (USU), with assistance from the Institute for Energy Technology/Halden Reactor Project (IFE-HRP), are evaluating a two-thermocouple, steady-state approach and a transient hot-wire thermal method (THWM) for estimating thermal conductivity. Figure 1 shows schematically the key parameters used to estimate thermal conductivity in each approach. Using Fourier's Law in cylindrical coordinates, the steady-state thermal conductivity of a fuel rod, $\mathrm{k}$, can be calculated if the radial position from the sample centerline, r; volumetric heat generation, $\dot{q}$; and measured temperature difference, $\Delta \mathrm{T}$, are known (Fig. 1a). For in-pile applications, this approach is typically implemented with one thermocouple in the center of the fuel and one located outside the fuel cladding (in the coolant). The THWM or line heat-source method is applied by embedding a line heat source in the material, the thermal conductivity of which is to be measured. From a condition of equilibrium, the heat source is energized and heats the sample with constant power, $\mathrm{Q}_{\mathrm{w}}$. The temperature response of the sample is a function of its thermal properties, so the sample thermal conductivity is found from the temperature rise, $T_{2}-T_{1}$, during a specified time period, $\mathrm{t}_{2}-\mathrm{t}_{1}$, at a small distance from the heat source. It should be noted that in both approaches, in-pile degradation of fuel during irradiation due to swelling, cracking, etc., typically leads to results being presented as simply an indication of thermal-conductivity degradation (because the "effective" thermal conductivity of the fuel, cladding, and gap are measured and one is really detecting changes in the fuel that impact its thermal conductivity).

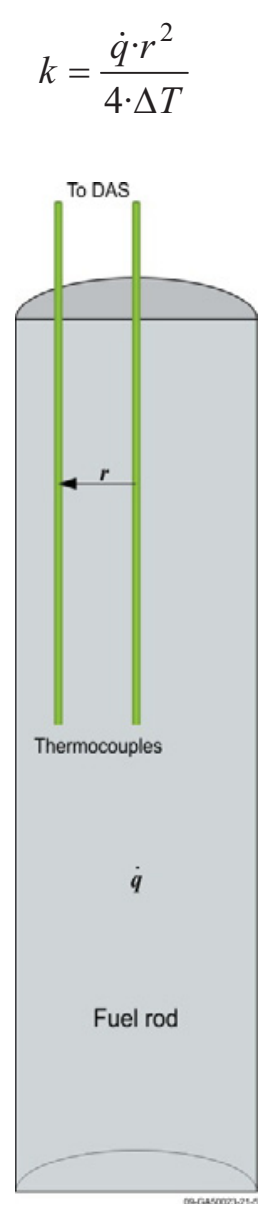

(a) Two
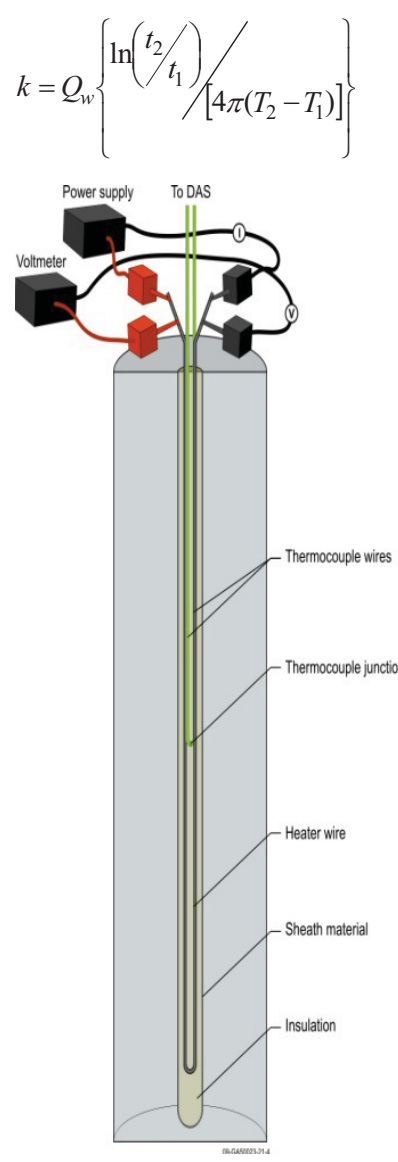

(b) THWM thermocouple

method

FIGURE 1. Key parameters and geometry used to estimate thermal conductivity.

\section{Technical Objectives}

During this three-year project, the following technical objectives will be completed:

- Explore the benefits and limitations of the two-thermocouple steady-state and transient hot-wire methods as in-pile effective thermal conductivity measurement techniques

- Quantify accuracy and limitations of techniques by comparisons with standard 
laboratory material property measurement systems and analyses from finite-element code predictions.

Completing these objectives will not only meet project objectives, but will also have the potential to provide ATR users a methd for detecting changes in fuel or material thermal conductivity during irradiation.

\section{Results and Accomplishments}

Initial Fiscal Year 2009 (FY-09) efforts focused on the two-thermocouple method. For in-pile applications, this approach is typically implemented with one thermocouple in the center of the fuel and one located outside the fuel cladding (in the coolant). In this project, evaluations focused on only estimating the thermal conductivity of a surrogate fuel rod using two thermocouples inserted in the rod, one to monitor centerline temperature and another to monitor temperature at a measured radial position within the rod. Although initial evaluations are performed using Type-K thermocouples, it is proposed to ultimately incorporate the use of INL-developed HighTemperature Irradiation-Resistant ThermoCouples (HTIR-TCs) because their doped molybdenum/niobium alloy thermoelements do not experience degradation during high-temperature irradiations. A hightemperature carbon structural foam was selected as the surrogate material in these evaluations.

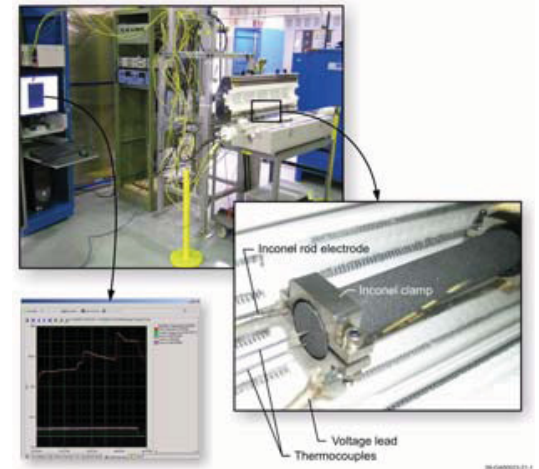

(a) Two thermocouple setup
Although several candidate surrogate materials were initially explored, FY-09 investigations indicated that this material was the only viable surrogate candidate identified with appropriate electrical resistivity, low thermal conductivity, and high-temperature resistance to oxidation and melting. Although the limited room-temperature data from its manufacturer were sufficient to select this surrogate material, more detailed temperature-dependent material-property data were needed for this USU/INL effort.

Temperature-dependent data were obtained using standard material-property measurement systems (e.g., laser flash diffusivity, pushrod dilatometry, and differential scanning calorimetry) available at INL's High Temperature Test Laboratory (HTTL).

In the test setup used for these evaluations (Fig. 2a), surrogate rod test samples are positioned inside a tube furnace with an argon cover gas to provide a controlled sample test temperature. Thermal conductivity testing began at INL's HTTL in January 2009, initially focusing on the $500-700^{\circ} \mathrm{C}$ temperature range. Results (Fig. 2b) were within $2-8 \%$ of the surrogate rod thermal conductivity values calculated from data obtained from material-property measurement systems. During FY-10, additional efforts will be completed to further assess the limitations and repeatability of this approach.

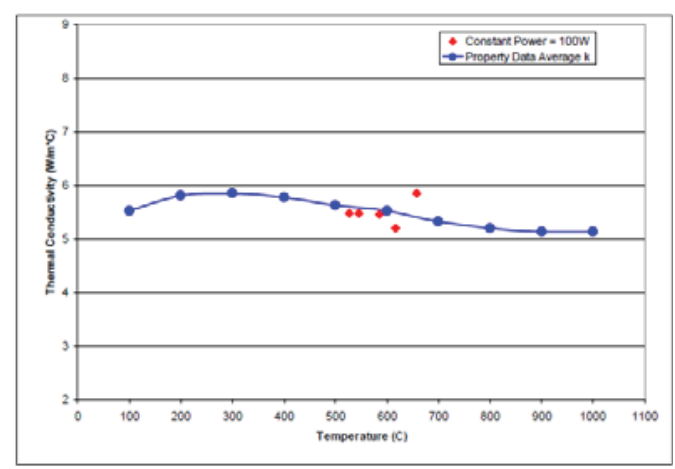

(b) Initial data (red) obtained from test setup

FIGURE 2. Setup and comparison of initial data from two thermocouple method evaluations with surrogate rod material data obtained from HTTL material property measurement systems. 
Using the Abaqus 6.8-2 ode, finite element analyses (FEAs) are being completed to provide additional insights and extend experimental results. Initial FEA calculations were performed to help bound the potential effects of non-idealcontact thermal resistance between the thermocouple and surrogate rod material in laboratory test conditions. Temperaturedependent material-property data for rod material and thermocouple constituents were input into Abaqus. Figure 3 shows results from analyses evaluating the change in surrogate rod thermal conductivity predicted when the assumption for gap coefficient varies from of 9376 to

$308 \mathrm{~W} / \mathrm{m}^{2} \mathrm{~K}$. As shown in Figure 3, this factor of 30 variation in assumed gap coefficient only affects surrogate fuel-rod thermal conductivity estimates by $9 \%$. During FY-10, FEA will be completed to gain additional insights about the accuracy and limitations of the two-

thermocouple steady-state and hot-wire transient methods.

In this project, a new hot-wire probe design, containing a resistance heater and thermocouple embedded in a single probe, has been developed (Fig. 1b), based on ASTM D 5334-05. Room temperature evaluations were completed at the HTTL using this probe in several materials (DELIN, particle board, quartz, etc., Fig. 4). Rod effective thermal conductivities are typically calculated to within $10 \%$ of values published in the literature for the materials evaluated. During FY-10, higher temperature evaluations will be completed to assess the viability of this probe for in-pile fuel irradiations.

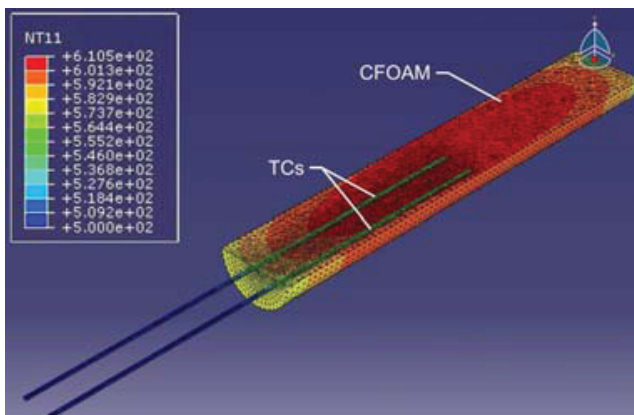

(a) gap coefficient of $308 \mathrm{~W} / \mathrm{m}^{2} \mathrm{~K}$

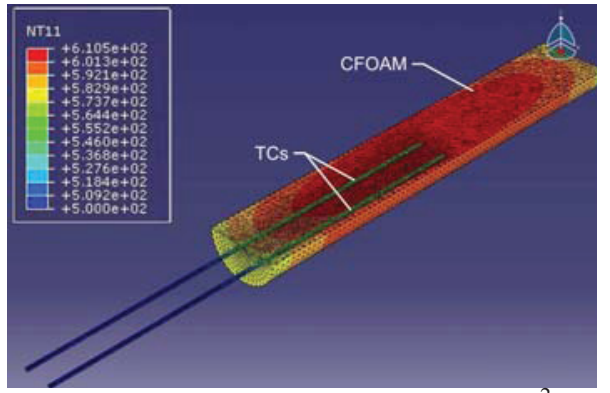

(b) gap coefficient of $9376 \mathrm{~W} / \mathrm{m}^{2} \mathrm{~K}$

FIGURE 3. Abaqus temperature predictions assuming upper and lower bound gap heat transfer coefficients.

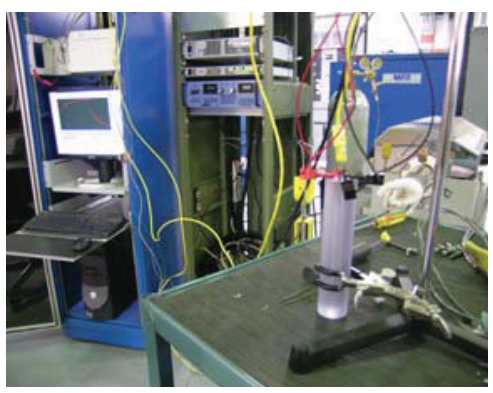

(a) THWM setup

\begin{tabular}{|c|c|c|c|}
\hline Material & $\begin{array}{c}\text { USU/NL Probe Average } \\
\text { Measured Effective Thermal } \\
\text { Conductivity } \\
\left(\mathbf{W m}^{2} \mathrm{~K}\right)\end{array}$ & $\begin{array}{c}\text { Average } \\
\text { Reported } \\
\text { Value } \\
\left(\mathbf{W}^{2} \mathrm{~K}\right)\end{array}$ & $\begin{array}{c}\text { Ratio of Reported to } \\
\text { USU/NL Measured Values }\end{array}$ \\
\hline $\mathrm{SiO}_{2}$ & 1.353 & 1.38 & 1.020 \\
\hline Delrin & 0.281 & 0.295 & 1.050 \\
\hline Lexan & 0.247 & 0.207 & 0.838 \\
\hline Acyylic & 0.215 & 0.216 & 1.005 \\
\hline Particle Board & 0.149 & 0.135 & 0.906 \\
\hline
\end{tabular}

(b) Initial room temperature data from test setup

FIGURE 4. Room temperature evaluation of THWM with needle probe imbedded in surrogate rod material. 
Summary and Conclusions

Initial project results suggest that both approaches under investigation could be deployed in the ATR as an in-pile method for detecting changes in thermal conductivity. Results from this project increase INL instrumentation capabilities, which support the Department of Energy's energy security mission by developing and demonstrating

high-temperature in-pile instrumentation needed to obtain key data required for key Department of Energy-Office of Nuclear Energy research programs, such as the Generation IV and
Advanced Fuel Cycle programs. Specifically, this research is enhancing INL's expertise for producing optimized, more reliable, and lowercost in-pile instrumentation. In addition, enhanced instrumentation from this research has the potential to increase testing capabilities of INL's ATR National Scientific User Facility. Finally, recognition (e.g., publications, patents, and awards) from this research promotes INL's instrumentation capabilities, furthering INL efforts to develop the Instrumentation, Control, and Intelligent Systems Distinctive Signature. 


\title{
Human System Simulation and Human Performance Assessment: Testing and Review of Digital Control Systems and Interfaces
}

\author{
David Gertman, Jeffrey Joe, Alan Mecham, William Phoenix \\ Combining virtual and physical testing for design, development, and review of digital control systems \\ and interfaces \\ NU100
}

Future control room design for nuclear facilities will incorporate advanced digital technologies, automation, and intelligent systems. Driven by these advances, the concepts of nuclear power plant (NPP) operations may be radically different for light water reactor (LWR) operation of nuclear power plants and at all levels of the fuel cycle. Design advances are necessary to meet complex control requirements, reduce staffing and maintenance costs, and reduce the burden of security costs. Prior to any change, however, research is needed to evaluate the impact of these new technologies and operational concepts for their effect on human performance and, ultimately, plant safety. This includes operator and crew performance in the presence of advanced instrumentation and control (I\&C) systems for abnormal and emergency operations.

The driving research issue at hand addressed by this LDRD is summarized in the following:
At this time, there does not seem to be an agreed-upon, effective methodology for designers, owner-operators, maintainers, and regulators to assess the overall impact of computer-based, human-machine interfaces on human performance in nuclear power plants. What methodology and approach should be used to assure proper consideration of human factors and human-machine interfaces? (National Research Council, 1997).

The proposed research will develop the much needed methodology: human cognition models, human performance metrics, and laboratory infrastructure to support the design and evaluation of advanced digital-control methods and interfaces. The goal of this research is to establish a sound technical basis for advanced control room technology selection and deployment, regulatory approval, system integration, staffing levels, and meeting safety requirements.

\section{Technical Objectives}

The objective of this research is to integrate NPP simulation with human-in-the-loop performance testing in order to develop capabilities and methods that establish a sound technical basis for new control room technology selection, regulatory approval, and system integration decisions. This is being accomplished by developing an iterative methodology that utilizes various conceptual tools and methods from which baseline human- and equipmentperformance measures in a technology-neutral framework can be derived.

Specific Objectives. The following are project milestones:

- Design Idaho National Laboratory (INL) human system simulation (HSS) laboratory to allow for collection of human-in-the-loop performance testing (Fig. 1)

- Perform underlying engineering analysis and construct/configure laboratory and integrate advanced equipment for conducting physiological recording of control room response (Fig. 1)

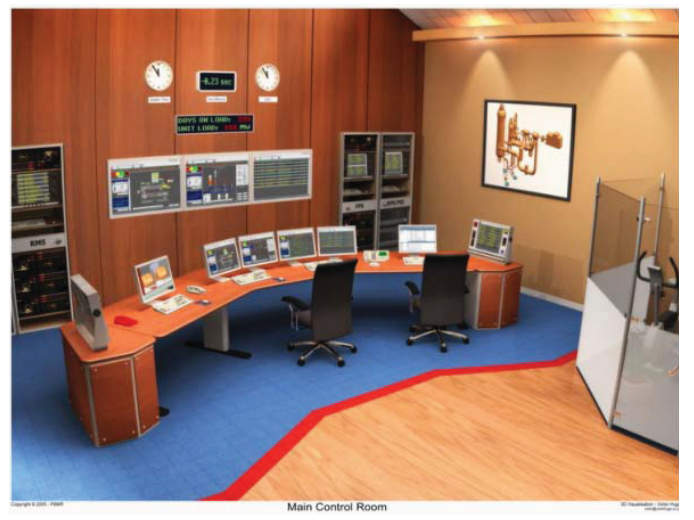

FIGURE 1. Advanced main control room design associated with control room operations.

- Train INL scientific and engineering staff in data collection for physical-recording equipment, including eye gaze, heart rate, breathing, galvanic skin response, and event logging (Fig. 2, next page, sample output from Fiscal Year (FY)-09 data collection efforts)

- Identify and obtain full-scope NPP pressurized water reactor (PWR) simulations for evaluation

- Conduct research studies evaluating human performance in advanced I\&C environments 
expected for NPPs as a function of light water reactor sustainability

- $\quad$ Present findings at peer-reviewed national and international conferences
- Establish strategic research relationships within Center for Advanced Energy Studies (CAES).

Heart Beat

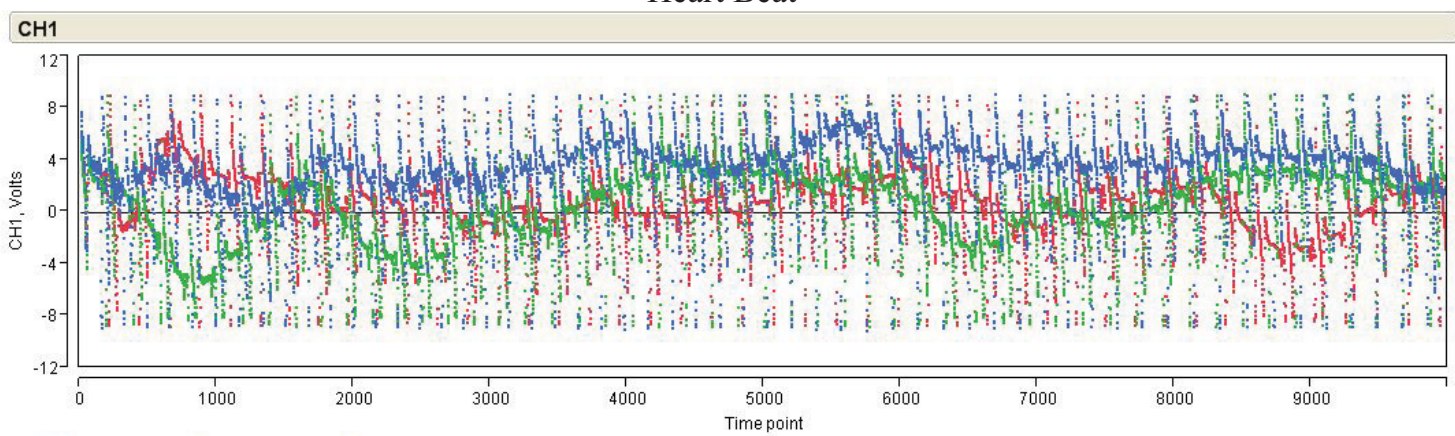

$Y \cdot \mathrm{CH}_{1}$ takeoff $\cdot \mathrm{CH} 1$ fllying $\cdot \mathrm{CH}{ }_{-}$Landing

Breathing

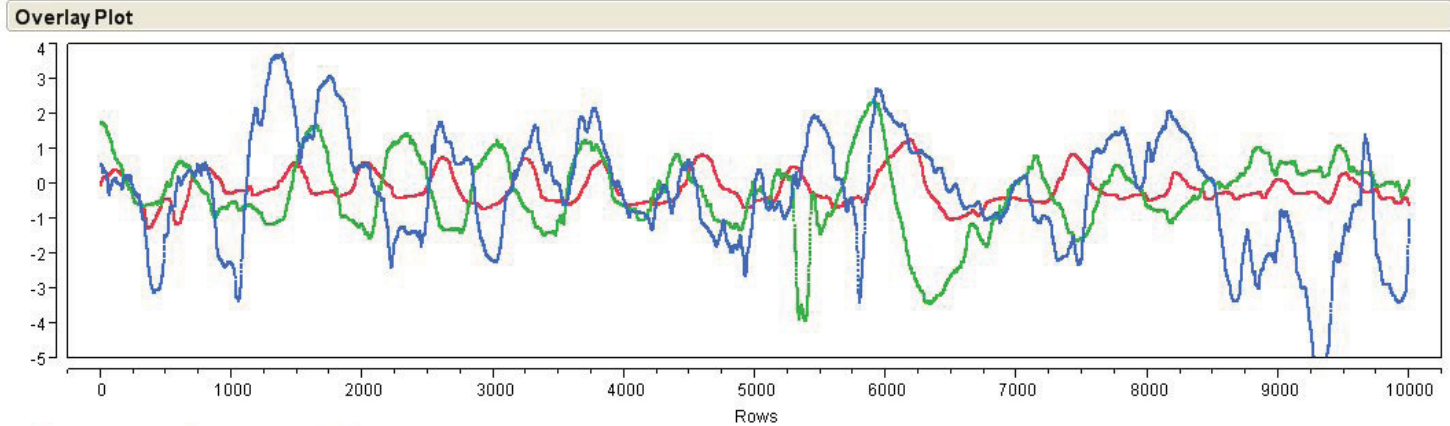

- $\mathrm{CH}_{-}$takeoff $\cdot \mathrm{CH}_{-}$flying $\cdot \mathrm{CH}_{-}$Landing

Skin Sensation

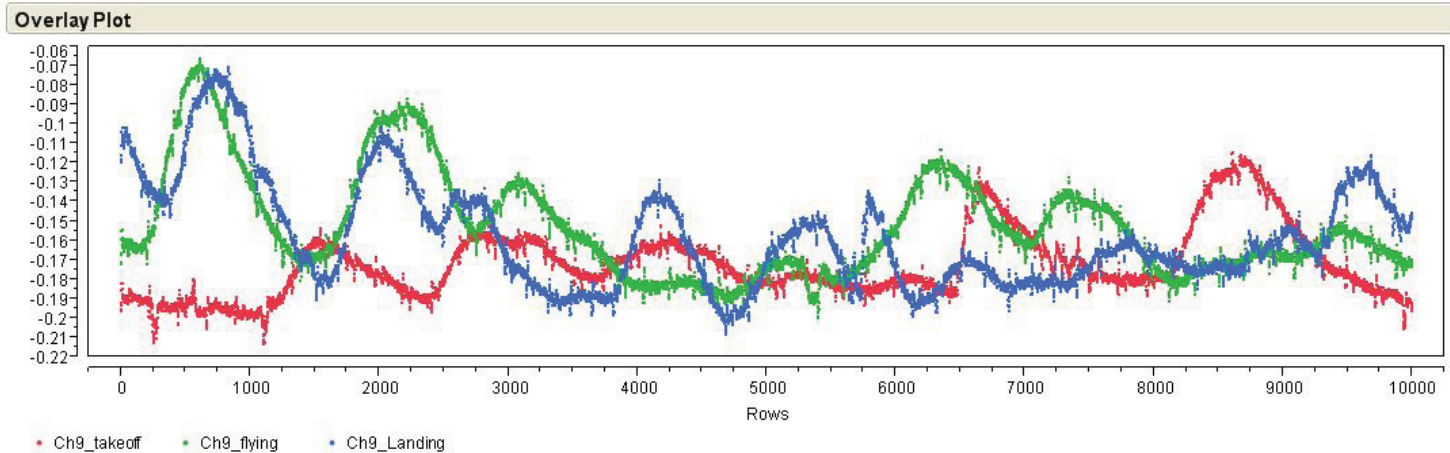

FIGURE 2. Sample of simulator-gathered physiological data for certified pilots.

\section{Results and Accomplishments}

For most of FY-09, activities focused on developing staff skills, defining requirements, and providing engineering analysis supporting the construction of the Human System Simulation facility located at the Center for
Advanced Energy Studies (CAES) in Idaho Falls, obtaining capital equipment supporting human-in-the-loop testing and physiological recording, and having researchers gain familiarity with recording equipment and programming in support of the test environment. The staff carefully considered present and 
anticipated research when developing the detailed specification for the CAES simulation laboratory. The specification addressed maximum noise levels, ambient and spot lighting, flexible placement of multiple video projectors and screens, a flexible room layout, including large observer galleries, flexible placement of physiological data gathering equipment, such as eye-tracking, microphones, electro-cardiogram, respiration rate and tidal volume, and galvanic skin responses, electrical power requirements, safety, and layout for a computer annex for the data-gathering and simulation-driver computers.

During this time, staff conducted a number of initial studies at the human factors laboratory in the Engineering Research Office Building (EROB) to fine-tune data-collection and analysis methods. INL researchers met with adjunct faculty from the University of Idaho to discuss cognitive modeling approaches, on-line datagathering techniques, and advances in neuroergonomics. INL staff also obtained two NPP PWR simulations for evaluation, one from the International Atomic Energy Agency (IAEA) and another from Western Services Corporation (WSC), a US full-scope simulation vendor. Staff supported the INL Instrumentation Control and Intelligent Systems (ICIS) research signature area in human factors, data fusion, and developing resilience engineering concepts. During the year, in parallel with staff experimentation with data recording and analysis methods, the research team conducted literature reviews on simulation, human-in-the-loop testing, and modeling human sensation, perception, and cognition.

During this year, INL staff also contributed to the development of visualization and modeling methodologies, including modeling human-system interactions in a dynamic realtime simulation environment. This second year's study of human cognition, visualization, and modeling resulted in a conference presentation at the Institute of Electrical and Electronics Engineers (IEEE)-sponsored Human System Interaction (HSI) 2009 conference. This presentation summarizes potential application of resilience concepts and models and has relevance to the next generation of advanced NPP control rooms.

Gertman, D. I., 2009, "Human Factors and Data Fusion as Part of Control Systems Resilience," in proceedings of $2^{\text {nd }}$ IEEE Annual Conference on Human Systems Interaction (HSI) 2009, April 15-19, Catania Italy.

Because of this research, INL received recognition in human systems simulation for advanced I\&C environments and in visual perception and cognition. The work carried out this year helps establish a facility and technical capability and adds to reactor safety by building a national capability to perform human-in-theloop studies of reactor safety in advanced control room settings. This work establishes INL and Department of Energy (DOE) human-factors expertise in modeling and simulation on a national and international level and will provide DOE with a models, methods, and data that can support LWR sustainability and next-generation reactors in this country. The next stage of study will address human-in-the-loop testing with an NPP PWR simulation. During FY-09, multiple studies supporting staff skill acquisition in online data gathering and analysis using flexible, repeatable simulation methods with certified pilots was achieved. These studies provided a focus to validate the laboratory protocol and control of testing. Additionally, interactive display equipment was designed and constructed to mimic the effects of secondary tasks on operator performance.

Direction for FY-10 includes establishing a human system simulation facility at the CAES that will allow university faculty, interns, and international researchers to support human factors research. In addition to literature surveys and small controlled studies, we have met with state-of-the-art military researchers at Camp Pendleton, California, who are instrumental in running a fully immersive CAVE system. This dialog is expected to help us in efforts to identify first principles for visualization and tasking and concepts for control in advanced control room environments. Studies will be conducted that provide basic data on human performance in the presence of advanced digital systems, including data on human performance related to navigation, search, use of automated procedures, 
and decision making. Talks continued with the Advanced Test Reactor (ATR) and a commercial nuclear facility on the integration of digital I\&C in reactors, and information gathered will be used to develop insights and craft research studies. Collaboration with the developing CAVE facility at CAES will allow us to evaluate a far greater range of displays and operational concepts than could have been achieved by LDRD resources alone.

\section{Summary and Conclusions}

The $2^{\text {nd }}$ year of the LDRD has been successfully completed, and the detailed project plan for the Laboratory, supported by requirements development and a number of supporting engineering analyses, is being implemented. Working relationships have been established with CAES researchers developing a 3D "CAVE" - an immersive visualization environment capability that will leverage existing research resources available through this LDRD program. Work in this area is being strengthened by developing relationships with advanced immersive visualization researchers at Camp Pendleton, California. Demonstrations of visualization and physiological recording approaches have also been developed and will continue to be refined. We expect that once human-in-the-loop studies are conducted with NPP simulations, the laboratory will provide a needed resource for human performance assessment supporting the ATR and Light Water Reactor Sustainability Program (LWRSP). Since no national simulation capability for crew performance in advanced control rooms exists, INL will be uniquely poised to offer research and analysis and data-collection capabilities. We expect that there will be non-LDRD funds of some magnitude available by the end of FY-10 that can be used to supplement the core capability developed under this LDRD. For example, the U.S. Nuclear Regulatory Commission (NRC) is actively seeking support in assessing the impact of computerized procedures upon crew and plant performance. Although no immediate intellectual property has been identified, INL is positioned to benefit from intellectual property related to methods, models, and data coming available from this research project. 


\section{Advanced Ceramic Nuclear Fuels}

Dr. Paul A. Lessing, Dr. Pavel Medvedev, Prof. W. R. Cannon

Develop advanced ceramic nuclear fuel forms that will withstand long-time service in fast-neutron reactors

NU101

The overall goal of this project is to develop advanced ceramic nuclear fuel forms (e.g., pellets or rods) that will withstand the rigors of long-time service in fast-neutron reactors. A supporting goal is to build expertise and facilities at Idaho National Laboratory (INL) in the area of advanced ceramic nuclear fuels. The focus during Fiscal Year (FY)-09 has been to develop advanced TRU-MOX fuel pellets and advanced processing development. The current process development work has utilized surrogate (nonradioactive) materials like $\mathrm{CeO}_{2}, \mathrm{HfO}_{2}$, and $\mathrm{Dy}_{2} \mathrm{O}_{3}$ to develop a ground-breaking "dry" process to produce very dense, "flowable" ceramic granules for green pressing fuel pellets. These developments have eliminated the need for the currently used "slugging" technology. These improvements were made using special "lab-scale" experimental processing and granulation equipment that was installed in MFC Lab 789 at INL's Materials and Fuel Complex (MFC). During FY-10 we plan to extend our capabilities to work with depleted uranium oxide $\left(\mathrm{DUO}_{2}\right)$ powders.

\section{Technical Objectives}

- Installed powder and granule processing equipment in MFC Lab 789. This processing "line" was brought into operational status.

- Produced rounded, "flowable" granules that were used to press excellent "green" pellets.

- Supported INL's funding opportunity announcement (FOA) project on spark plasma sintering by providing a series of pellets fabricated of many surrogate compositions using conventional "press and sinter" technology.

- Conducted research on several organic polymers for use as binders and pore-forming agents. This included green strength and radiation-damage resistance measurements.

- Prepared a journal paper covering the pellet green-strength results.

- $\quad$ Submitted a U.S. patent application (IDR BA-367) covering our special techniques to press and shape very homogeneous "green" pellets.

\section{Results and Accomplishments}

The production of "flowable" granules is of ultimate importance to produce ceramics that are homogeneous and strong (flaw-free). For this purpose, this laboratory-directed research and development (LDRD) project completed the installation of a modern surrogate ceramic fuel (e.g., MOX) processing line. Using this line we developed new techniques to dry-process ceramic powder, evaluate radiation resistant powder-binders, form "flowable" granules, press fuel pellets, and sinter fuel pellets. Example granules, as viewed using microscopy, are shown in Figure 1 (optical) and in Figure 2 (SEM).

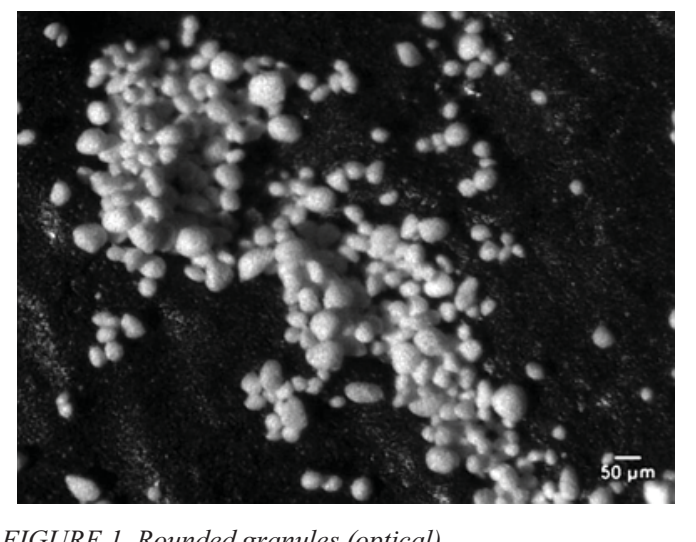

FIGURE 1. Rounded granules (optical).

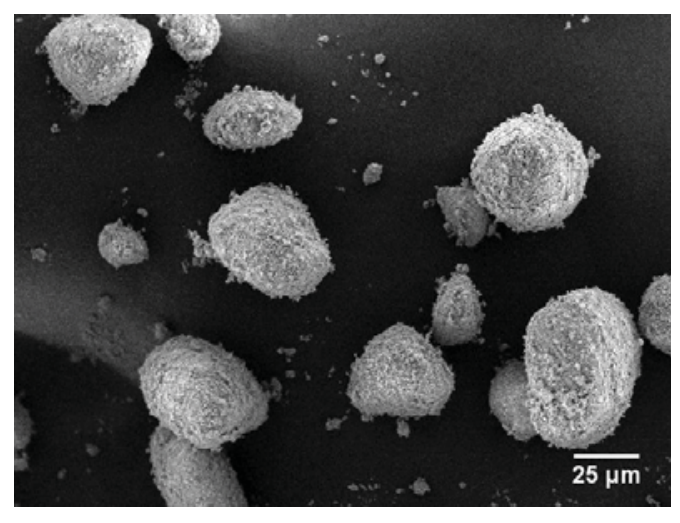

FIGURE 2. Rounded granules (SEM).

The flowability of various sieve-fractions of granules was measured; an example is shown using rounded $\mathrm{CeO}_{2}$ granules in Figure 3 (next page). The "pour" and "tap" densities measured for various sizes of $\mathrm{CeO}_{2}$ granules are shown in Figure 4 (next page). 


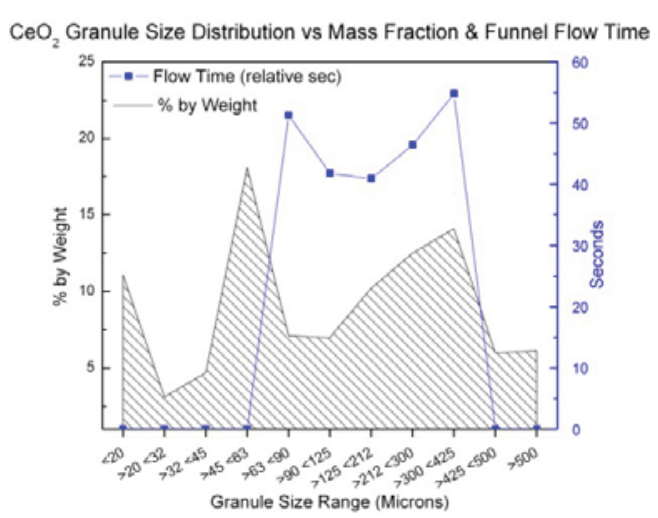

FIGURE 3. Flowability data for rounded granules.

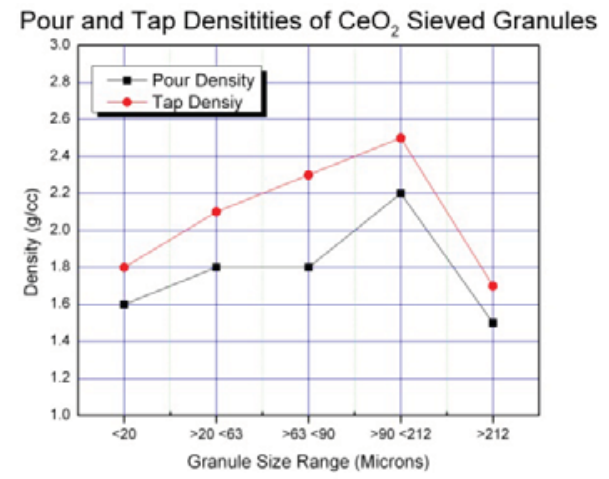

FIGURE 4. Packing density of rounded granules.

The project completed development and performance testing of a custom, computer-controlled gas handling system to control the oxygen partial pressure in a sintering furnace via the $\mathrm{H}_{2} / \mathrm{H}_{2} \mathrm{O} / \mathrm{O}_{2}$ equilibrium system. This system allows for the fine control of metal/oxygen $(\mathrm{M} / \mathrm{O})$ ratios during the fabrication of TRU-MOX ceramic fuel pellets. The performance of the control system is shown in Figures 5 and 6 where excellent agreement between measured and theoretical values (used in computer mixing program) is shown.

A paper submitted to the Journal of Nuclear Materials details the project's development work of green pressing with organic binders. An example of these results is shown in Figure 7 where fracture strength of green pellets pressed isostatically is markedly higher than those pressed uniaxially (in a metal die), and Figure 8 shows a large difference in the stress-strain

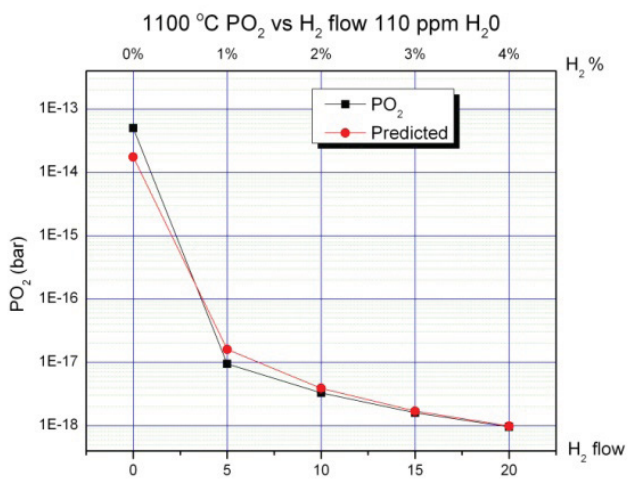

FIGURE 5. $\mathrm{PO}_{2}$ measured (zirconia cell) vs. values predicted by mixing program.

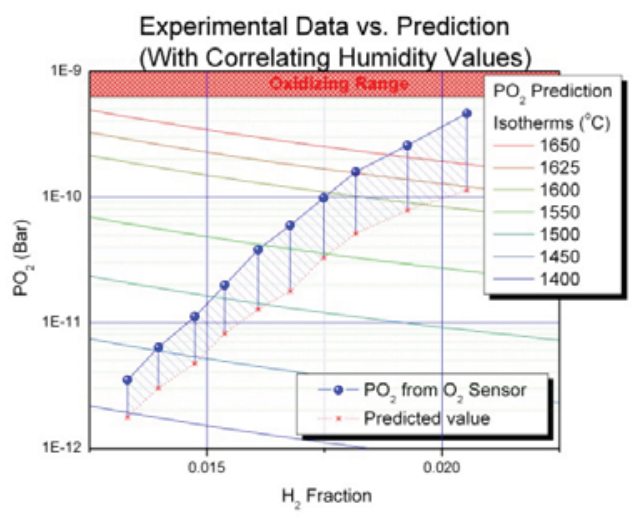

FIGURE 6. $\mathrm{PO}_{2}$ measured (zirconia cell) vs. values predicted by mixing program at high temps.

behavior of the two types of pellets. Other results measured properties (like strength) as a function of type and amounts of organic polymers added as binders and pore-formers. The various binders have also been evaluated with regard to their radiation resistance. Films were cast using selected binders. These films were then exposed to either alpha or gamma radiation. The damage sustained was measured using a positionannihilation technique. These results will be compared with pressed "green" pellets of TRUMOX composition (contain $\mathrm{AmO}_{2}$ and/or $\mathrm{NpO}_{2}$ ) that contain the same binders. Initial data on positron Doppler broadening measurements was recorded for polymeric fuel pellet binder materials that were exposed to high levels of gamma radiation $(48,260 \mathrm{R})$ and alpha exposure $\left(5.23 \mathrm{E}+9\right.$ alphas $\left./ \mathrm{cm}^{2}\right)$. Initial examination of the gamma irradiation data indicates little or no 
change induced by the gamma exposure with the exception of one binder. In contrast, the alpha irradiation induced statistically significant differences in all specimens measured. A tentative conclusion is that measurements should be performed using positron lifetime and the positron Doppler broadening coincidence system as they provide more information on structure. Irradiated binder films will be tested for changes in strength and TRU-MOX pellets fabricated from the most promising binder systems to test radiation resistance. When finished, the radiation resistance work will be submitted for journal publication.

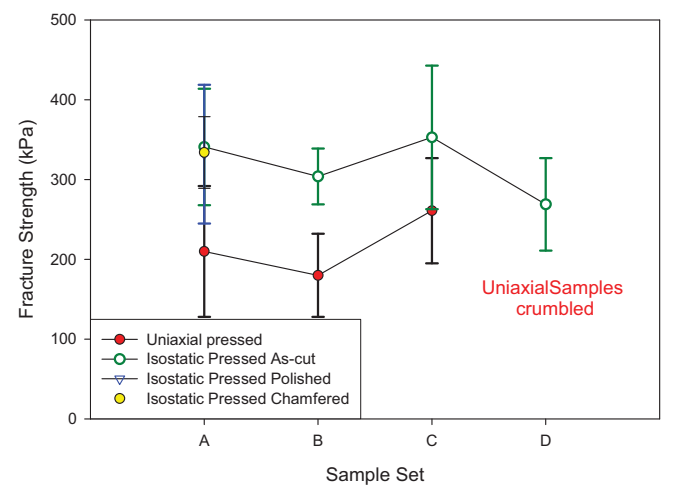

FIGURE 7. Fracture strength of green pellets pressured uniaxially versus isostatically

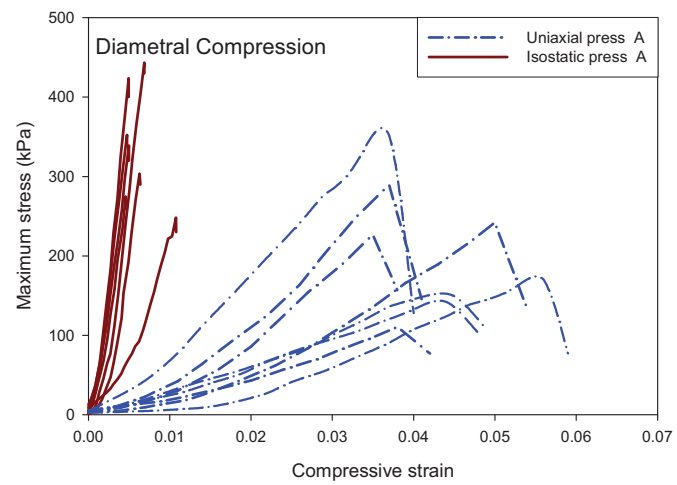

FIGURE 8. Stress-strain curves of green pellets pressed uniaxially versus isostatically.

Summary and Conclusions

During the second year of this project, excellent progress has been made in fabricating ceramic fuel pellets, building advanced capabilities at INL, and developing basic knowledge necessary for successful highperformance ceramic fuel fabrication. Work has resulted in the submission of a U.S. patent application and the completion of a paper for journal submission. The work has focused on developing methods and materials to control the density (porosity) and homogeneity necessary to enable ceramic fuels (TRU-MOX) to be used in a high-neutron-flux reactor (e.g. Advanced Recycling Reactor) where the fuel is to be irradiated to high burnup (beyond $100 \mathrm{GWd} / \mathrm{t}$ ). These reactors transmute transuranic elements (TRU) recycled from light water reactors (LWRs) to produce energy while reducing residual long-term radiotoxicity and decay heat for storage in a spent-fuel repository. The TRU material (Am and $\mathrm{Np}$ oxides) that we have added to $\mathrm{UO}_{2}$ and $\mathrm{PuO}_{2}$ ceramics are characteristic of re-cycled light-water reactor (LWR) fuel feedstocks that are being developed by the Advanced Fuel Cycle Initiative (AFCI). Variations on these new fabrication techniques will also prove innovative and valuable to existing LWRs that utilize ceramic fuel pellets, including MOX and IMF for LWRs. Therefore, commercial fuel manufacturers should be very interested in the results of this project. This interest will increase as we begin to utilize our new techniques to process $\mathrm{UO}_{2}$ (depleted) powder.

In addition, the project is now in a position to refine TRU-MOX properties for various reactor applications. Refinements could include: (1) investigate approaches to optimize microstructure to improve performance, (2) investigate effects of composition and microstructure on fuel thermal conductivity, (3) demonstrate a high melting point, (4) exhibit chemical and physical homogeneity, (5) show microstructure stability, and (6) maintain the characteristic of a controlled and stable stoichiometry (e.g. cation/anion ratio).

Acknowledgements: Dr. Gerald Egeland and Mr. Larry Zuck are acknowledged for their fine work in fabricating and testing surrogate ceramic pellets. 


\section{Characterization of Fluidized Beds via Pressure-Fluctuation}

\section{Analysis}

Douglas W. Marshall

A study of the influence that particle properties, fluid properties, and vessel dimensions have on the structure of the pressure fluctuations PH103

Fluidized beds are accumulations of solid particles that are levitated and mobilized by a flow of fluid, such as a gas, causing the particle bed to behave like a fluid. The excellent heat and mass-transport properties of fluidized beds make them a useful and popular technology in the chemical, pharmaceutical, and petroleum, industries. The movement of the levitating fluid and particles causes pressure fluctuations within the bed and associated piping that may reveal the onset of fluidization and the nature of particle movement and flow within the bed. The pressure fluctuations, when graphed as a function of time, display a periodic structure that is characteristic of the system (vessel design and properties of the particles and fluid).

Equipment has been designed, fabricated, and installed to begin studying the influence that particle properties (density, diameter, bed depth, etc.), fluid properties (density, viscosity, and flow rate), vessel dimensions (tube diameter, fluid orifice diameter, including cone angles, etc.) have on the structure of the pressure fluctuations (frequencies, amplitude, standard deviation, chaotic dimensions, etc.).

Relationships between structural characteristics of the pressure fluctuations and properties of the particles, fluid, and vessel can be established to provide an improved understanding of the bed dynamics and to follow changes in the particle properties. This is especially important when process constraints limit or prohibit the insertion of probes into the fluidized bed because pressure fluctuations can be monitored externally to the process.

Although this laboratory-directed research and development (LDRD) is not restricted to studying any one fluidization regime (quiescent, bubbling, slugging, spouted, or fast fluidization), initial efforts are focused on understanding spouted bed fluidization, which is used in the nuclear and other industries. Little has been published to date on the use of pressure fluctuation analysis to study spouted beds beyond identifying minimum spouting velocities for the levitating fluid. Understanding the "signatures" of the pressure fluctuations should enable researchers to improve the dynamics of their spouted bed and thereby improve process control and product quality.

\section{Technical Objectives}

The following items have been accomplished or are in process and scheduled to be accomplished before the end of Fiscal Year (FY) 2009:

- Relocated a high-temperature furnace and fluidization vessel from the Idaho National Laboratory Research Center (IRC) building to the Center for Advanced Energy Studies (CAES) building

- Established a task for the University of Idaho (U of I) to provide logistical support, experimental-design guidance, and fabrication of physical fluidized-bed models

- Designed two, two-dimensional spouted bed models that will enable particle and gas movements to be visualized and documented

- Designed, procured, and assembled dataacquisition equipment (pressure transducers, flow meters, etc.) and software to collect pressure-fluctuation data

- Obtained approximately 100 pounds of a variety of bed media over a range of particle densities and diameters

- Obtained sieve screens to subdivide the bed media and initiated sieving operations

- Started preparation of safety documents and laboratory instructions needed to enable studies to begin

- Obtained bids and awarded contracts (through CAES and the $\mathrm{U}$ of I) to route electrical, water, and compressed air utilities to the test equipment

- Obtained gas cylinder racks, cylinder manifolds, pressure regulators, and valves to enable studies to be conducted with compressed gases (for a variety of gas densities, viscosities, and temperatures).

Results and Accomplishments

The accomplishments this year are in the realm of preparations for conducting experiments and collecting data. CAES is a new facility with great potential, but limited existing resources and unproven administrative processes for installing 
equipment and setting up experiments. It is with the aid of the CAES facility that utilities are being connected and facility modifications are made to accommodate the experiments. Electrical power will be connected to the furnace on September 30, 2009. Other utilities are scheduled for completion by September 25, 2009.

No data can be collected until utility connections are completed. My hypothesis remains untested in FY-09.

INL, however, will have a functional laboratory that will enable data to be collected on spouted beds at temperatures in excess of $1200^{\circ} \mathrm{C}$, several hundred degrees hotter than has been documented in published literature. Furthermore, the equipment is configured to allow the use of $100 \%$ hydrogen gas. These aspects of the high-temperature furnace give the INL unique capabilities in the scientific community.

Data will be collected in FY-10 and a presentation will be made at the Sixth International Symposium on Spouted Beds to be held in Vancouver, British Columbia, Canada, this coming summer. Authors of the presented papers will be invited to publish their findings in a special issue of The Canadian Journal of Chemical Engineering.

\section{Summary and Conclusions}

Physical models of spouted bed equipment have been obtained for the purpose of collecting data relating attributes of the fluidized bed, particles, and levitating fluids to the structure of pressure fluctuations emanating from the fluidized bed. Final utility connections will be made in late FY-09, which should enable testing to begin in FY-10.

Data will be compiled, analyzed, and prepared for presentation and publication at the $6^{\text {th }}$ International Symposium on Spouted Beds, to be held in Vancouver, British Columbia, Canada, and published in The Canadian Journal of Chemical Engineering. 


\title{
Dissolution and Extraction Studies of Fission Products in Room Temperature Ionic Liquids (RTIL) and in Supercritical Fluid $\mathrm{CO}_{2}$ $\left(\mathrm{ScCO}_{2}\right)$ and Determination of Radiolytic Stability
}

\author{
Brian K. Harris, Bruce J. Mincher, William F. Bauer, Chien M. Wai (U of I)
}

Using ionic liquids as solvents in the nuclear fuel cycle PH104

Liquid-liquid extractions have historically been favored in nuclear-fuel-cycle processes, using relatively conventional organic solvents, many of which have toxic, flammable, volatile, or radiolytic properties that impose certain limitations on the processes. The recovery and reuse of the organic extractants is often limiting due to radiolysis. The cost of disposing spent conventional solvents can be a significant issue, particularly when they are contaminated with radioactive and unknown radiolysis components. The design, then, of an environmentally more favorable extraction process that is also more resistant to radiolysis would be advantageous to processing nuclear fuel and waste materials.

Interest is developing around the use of ionic liquids as solvents in nuclear-fuel-cycle industrial processes, remediation of wastes, and other environmentally relevant matrices. Within the last few years, supercritical fluids, e.g. carbon dioxide $\left(\mathrm{Sc}-\mathrm{CO}_{2}\right)$, have also received some attention in processing nuclear materials although the use of room-temperature ionic liquids (RTILs) and supercritical fluids together has not fully been investigated. The proposed research aims to study the properties of an RTIL extraction and $\mathrm{Sc}-\mathrm{CO}_{2}$ back-extraction system for radionuclides/actinides in several matrices and multiple nuclides with the ultimate goal of initiating the development of alternative methodologies for Environmental Management (EM) clean-up and advancing a cleaner nuclearfuel cycle.

\section{Technical Objectives}

- Investigate the parameters affecting the direct extraction of radionuclides from contaminated soil matrices and ores in order to bound the parameters for the backextraction:

- Arranged with the University of Idaho (U of I) to provide purified RTILs

- Performed direct extractions of reference contaminated soil materials with the RTIL 1-butyl-3-methylimidazolium-bis(trifluoromethylsulfonyl)-imide (BMIMTf2N) and the TBP/HNO3 complex that will be initially investigated in the $\mathrm{Sc}-\mathrm{CO} 2$ system. Since analytical procedures do not exist for elements and radioisotopes, only relative total activity was used as a measure of extraction efficiency as determined by liquid scintillation counting.

- Several sets of ${ }^{60} \mathrm{Co}$ irradiations of the RTIL were performed, and the samples examined by Raman spectroscopy and highperformance liquid chromatography (HPLC) as baseline radiolysis experiments.

- Built $\mathrm{Sc}-\mathrm{CO}_{2}$ extraction apparatus.

- Met with Dr. Chien Wai, U of I dissertation advisor, three times. The first visit was made to evaluate current and future research plans with Dr. Chien Wai, as well as meet with other committee and faculty. Second, a meeting was held in Idaho Falls to solidify milestones for completion of this research, and third, a meeting was arranged at the National American Chemical Society (ACS) Meeting in Washington, D.C., where Dr. Wai chaired a symposium on the Nuclear Energy Renaissance.

Results and Accomplishments

Initial direct extractions using the RTIL, modifiers, and matrices similar to what will be used in the $\mathrm{Sc}-\mathrm{CO}_{2}$ extractions were performed to create a baseline for the extraction parameters and to help develop optimized parameters for investigating the $\mathrm{Sc}-\mathrm{CO}_{2}$ back-extraction method parameters. Among the direct extraction parameters investigated at this point are reaction times to prepare the "super-acid" $\mathrm{TBP}\left(\mathrm{HNO}_{3}\right)_{1.8}\left(\mathrm{H}_{2} \mathrm{O}\right)_{0.6}$ when contacting TBP with an $\mathrm{HNO}_{3}$ solution, matrix/analyte combinations, and the desirable ratio of the super acid to the RTIL.

Extractions were performed by contacting the extractant $\left(\mathrm{TBP} / \mathrm{HNO}_{3}\right.$, or $\mathrm{TBP} / \mathrm{HNO}_{3}+$ RTIL) with the soil for thirty minutes, centrifuging, and aliquoting $0.5 \mathrm{~mL}$ into a liquid scintillation cocktail (LSC) for counting. LSC is attractive because it offers $100 \%$ counting efficiency. The alpha-particle emission, however, is given up to the cocktail in a short distance. The counts of these short-range 4-6 MeV 
particles are found in the energy window above, where most gamma and beta emitters are found.

Figure 1 shows that there is no significant difference across the range of TBP/ $\mathrm{HNO}_{3}$ reaction times for both $\mathrm{TBP} / \mathrm{HNO}_{3}$ used alone, and $\mathrm{TBP} / \mathrm{HNO}_{3}$ in the IL phase. When the $\mathrm{TBP} / \mathrm{HNO}_{3}$ was contacted by shaking, the reaction time was found to be instantaneous. This was verified qualitatively by using a thymol-blue indicator solution which showed the colored $\mathrm{HNO}_{3}$ was immediately transferred to the TBP phase, leaving the $\mathrm{HNO}_{3}$ phase clear. As the extraction values are consistent across each nuclide, a thirty-second vigorous contact is sufficient to produce the $\mathrm{TBP} / \mathrm{HNO}_{3}$ complex necessary for optimum extractions. Also, note that the RTIL is $\sim 1.6$ times more effective than $\mathrm{TBP} / \mathrm{HNO}_{3}$ alone for the extraction of $\mathrm{Pu}$ from soil.

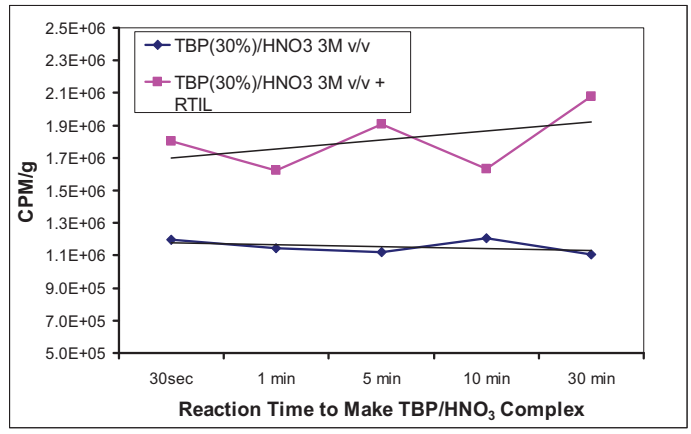

FIGURE 1. Effect of reaction time to make $\mathrm{TBP} / \mathrm{HNO}_{3}$ nuclides complex on the extraction of ${ }^{239}{ }_{\text {Pu from soil. }}$

Another important parameter is the effect of the percent TBP/nitric acid in the ionic liquid phase during extractions. The experimental design used the thirty-minute contact time between the sample and the RTIL with the $\mathrm{TBP} / \mathrm{HNO}_{3}$ to compare soil spiked with $\mathrm{Pu}$, soil spiked with Am, and a $0.87 \%$ U ore with different molar concentrations of $\mathrm{TBP} / \mathrm{HNO}_{3}$ in the IL. Figure 2 shows that, for all matrices, an extraction efficiency increases to at least $30 \%$ $\mathrm{TBP} / \mathrm{HNO}_{3} \mathrm{v} / \mathrm{v}$ complex in the ionic-liquid phase and that $\mathrm{Am}$ and $\mathrm{U}$ are likely more easily directly extracted than $\mathrm{Pu}$.

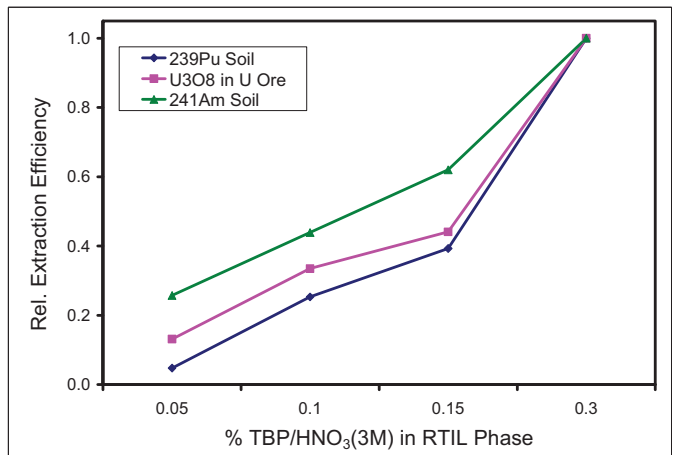

FIGURE 2. Relative extraction efficiency of nuclides over several ratios of TBP in the RTIL.

Interpretation of the results from the initial radiolysis experiments was difficult because optimal analytical methods need to be developed. However, some radiolysis chemistry occurred, as evidenced from color changes (Figs. 3 and 4) to the irradiated neat RTIL. HPLC analysis under less than optimal conditions indicates some retention-time shifts and possible UV-Vis spectral changes. Further analytical-method development will be required to determine the extent of the radiation damage and whether or not that damage affects extraction and recovery.

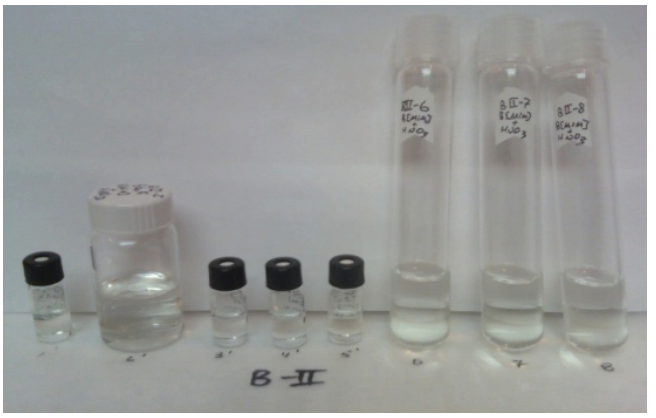

FIGURE 3. Pre irradiation with ${ }^{60} \mathrm{Co}(\mathrm{KGy})$.

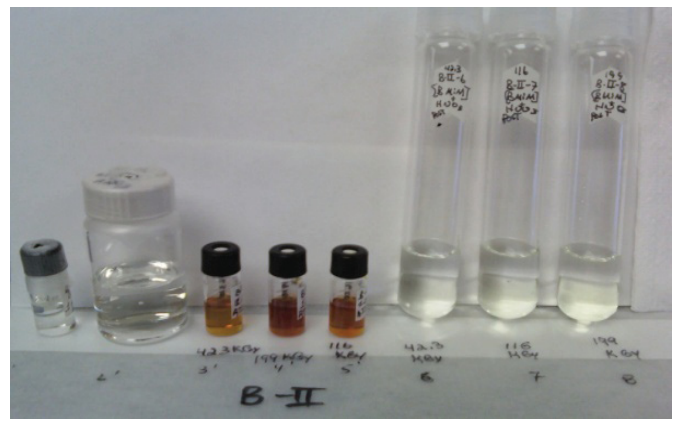

FIGURE 4. Post irradiation at 42.3 to $199 K G y$. 
A Sc- $\mathrm{CO}_{2}$ apparatus for working with radioactive samples has been constructed. This equipment has a $60-\mathrm{mL}$ cell and a $35-\mathrm{mL}$ cell, which will be used for direct visual and/or spectroscopic observation of the processes occurring in the SFC.

Summary and Conclusions

We have investigated several parameters which are necessary for moving from bench extractions to $\mathrm{Sc}-\mathrm{CO}_{2}$ extractions. Initial radiolysis experiments indicate that the reagents, in particular the RTIL's may not be as "radiolytically stable" as the literature suggests. Further analysis with HPLC and other analytical tools will aim to vet this out. Follow-on work will involve the development of processes where the solvents are recycled for a process that is less waste intensive. This research in RTILs is currently a very intensively researched topic and, because it is of such interest, our efforts will contribute to and position us for further development in this area through publications and invention disclosure records (IDRs). 


\section{High Temperature In-Pile Instrumentation Enhancements}

Joy Rempe, Darrell Knudson, Keith Condie, S. Curt Wilkins (Consultant),

John Crepeau (UI), and Ron Ballinger (MIT)

Optimizing sensors for in-pile irradiations

RP110

New materials are being considered for fuel, cladding, and structures in next-generation and existing nuclear reactors. However, there are insufficient data to characterize the performance of these new materials in high-temperature, oxidizing, and radiation conditions. To evaluate candidate material performance, robust instrumentation is needed that can survive proposed test conditions.

For example, commercially available thermocouples drift due to degradation at high temperatures (above $1100^{\circ} \mathrm{C}$ ) or due to transmutation of thermocouple components. Thermocouples were needed that could withstand both high-temperature and highradiation environments. To address this need, INL developed a High Temperature Irradiation Resistant ThermoCouple (HTIR-TC) design that contains commercially available doped molybdenum paired with a niobium alloy. Results from initial Idaho National Laboratory (INL) evaluations have demonstrated that these thermocouples function for long time periods (up to 4000 hours) at high temperatures (from $1200^{\circ} \mathrm{C}$ to at least $1500^{\circ} \mathrm{C}$ ) with minimal (less than $2 \%$ ) drift compared to commercial thermocouples that drift significantly $[>10 \%]$ due to high temperature or transmutation. Ongoing irradiations in the Advanced Test Reactor (ATR) for a test capsule containing gasreactor fuel specimens confirm the superior performance of HTIR-TCs.

The labor-intensive processes and materials used to fabricate HTIR-TCs, and other instrumentation designs proposed by INL that incorporate HTIR-TCs, can make such sensors significantly more expensive than commercially available thermocouples. Although these processes are required to impart proper configuration and prevent contamination and moisture absorption that could adversely affect instrumentation high-temperature performance, several options were identified that have the potential to reduce instrumentation-fabrication and material costs. In addition, alternate fabrication techniques were investigated that have the potential to enhance the performance of INL-developed instrumentation at high temperatures. All enhancement options were evaluated in INL's High Temperature Test
Laboratory (HTTL), which contains high-temperature furnaces, specialized thermocouple fabrication equipment, and high-temperature material-property measurement equipment required for this project.

\section{Technical Objectives}

During this three-year project, the following technical objectives were completed in tasks that explored options having the potential to reduce the cost, improve the accuracy, and enhance the reliability of INL-developed in-pile instrumentation that utilizes HTIR-TCs:

Task 1: Automated Fabrication Techniques

- Develop required fixturing for automating fabrication processes

- Evaluate the viability of automating fabrication on sensor performance and costs.

Task 2: Automated Calibration Techniques

- Develop required fixturing for automating calibration processes

- Evaluate the impact of automated calibration on sensor performance and costs.

Task 3: Alternate Heat Treatment Techniques

- Evaluate various sensor heat-treatment temperatures and durations

- Select optimized heat treatments for instrumentation performance at various operating conditions.

$\underline{\text { Task 4: Alternate Extension Cable Materials }}$

- Measure signal from various low-cost copper nickel alloys to identify a combination that duplicates low-temperature response of thermoelements used in INLdeveloped sensors.

Task 5: Alternate Fabrication Techniques

- Develop and design appropriate fixturing for fabricating alternate designs

- Fabricate sensors using alternate techniques and compare performance with standard designs. 
Task 6: Irradiation Performance of Enhanced Design

- Using insights from Tasks 1 through 5, develop optimized sensor designs for irradiations in the Massachusetts Institute of Technology (MIT) High Temperature Irradiation Facility (HTIF)

- Fabricate optimized sensors and ship to MIT.

By completing the above objectives, this LDRD not only met the proposed objectives for this project, but also significantly enhanced the capabilities and reduced the costs for hightemperature in-pile sensors.

\section{Results and Accomplishments}

During Fiscal Year 2007 (FY-07), several fabrication-process improvements for swaged HTIR-TCs (the use of fixturing that automatically rotates, lubricates, and feeds tubing into swagers at a controlled rate, automated calibration processes, and optimized heat-treatment temperatures and durations) were investigated in Tasks 1 through 3 . Results demonstrate that these options significantly reduce fabrication and calibration costs associated with INL-developed high-temperature instrumentation. Task 4 activities to explore the use of commercially available copper-nickel alloy wires of as lower cost extension cable for

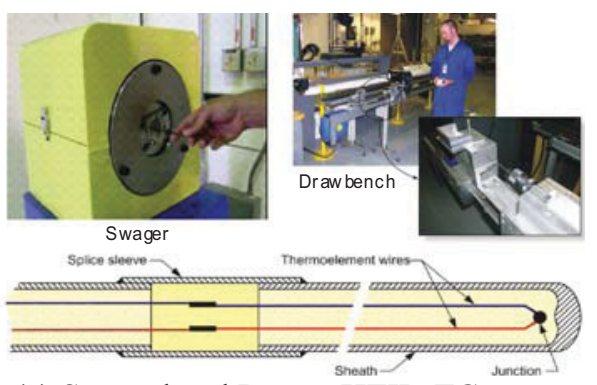

(a) Swaged and Drawn HTIR-TCs the expensive thermoelement wire materials used in INL-developed instrumentation were completed. Results indicate that a $\mathrm{Cu} 3.5 \% \mathrm{Ni} / \mathrm{Cu} 5 \% \mathrm{Ni}$ combination best duplicates

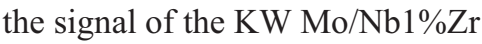
thermoelements used in HTIR-TCs between 0 and $150^{\circ} \mathrm{C}$ (the temperature range to which extension cable is typically exposed).

Task 5 activities to explore alternate fabrication processes were performed during FY08 and FY-09. Initially deployed HTIR-TCs rely on swaging fabrication techniques because of their simplicity and durability. As shown in Figure 1a, a swaged TC is fabricated by loading pre-formed, crushable insulator beads onto thermoelement wires and placing the insulated thermoelements in a sheath (tube) that is then swaged (compacted) to form a single, cohesive component. If desired, the thermocouple may be joined to hard extension cable using a splice sleeve as shown in Figure 1a. Drawn and looseassembly thermocouple designs each offer the potential for improved high-temperature performance over swaged thermocouples. Compared to swaging, the drawing process does not twist or distort HTIR-TC thermoelement wires during fabrication. In a loose-assembly HTIR-TC (Fig. 1b), higher temperatures may also be possible because thermoelement thinning and irregular deformation associated with swaging and drawing are avoided.
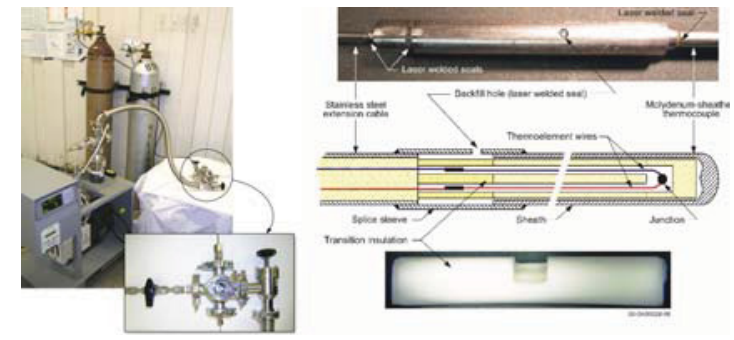

(b) Loose Assembly HTIR-TCs

FIGURE 1. Specialized equipment and components for alternate fabrication technique investigated.

Furthermore, the loose fit can accommodate some differential thermal expansion of thermocouple components without inducing thermoelement stress. A long-duration (1000hour) test was completed to compare the performance of swaged, drawn, and loose- assembly HTIR-TCs at $1500^{\circ} \mathrm{C}$ in a hightemperature vacuum furnace. Multiple swaged, drawn, and loose-assembly thermocouples were included in this test. As shown in Figure 2a, the signals from the swaged and drawn HTIR-TCs decreased by $2.3 \%$, primarily during the first 600 
hours of the test. The loose-assembly thermocouple displayed greater stability and resolution than either the swaged or drawn thermocouples. Although the swaged HTIR-TC response is consistent with the decalibration observed in prior 1200 and $1400^{\circ} \mathrm{C} 4000$-hour tests, the fact that most of the drift occurred during initial portions of this test suggests that longer duration heat treatments may be needed to stabilize the distortion associated with swaging or drawing processes in larger diameter

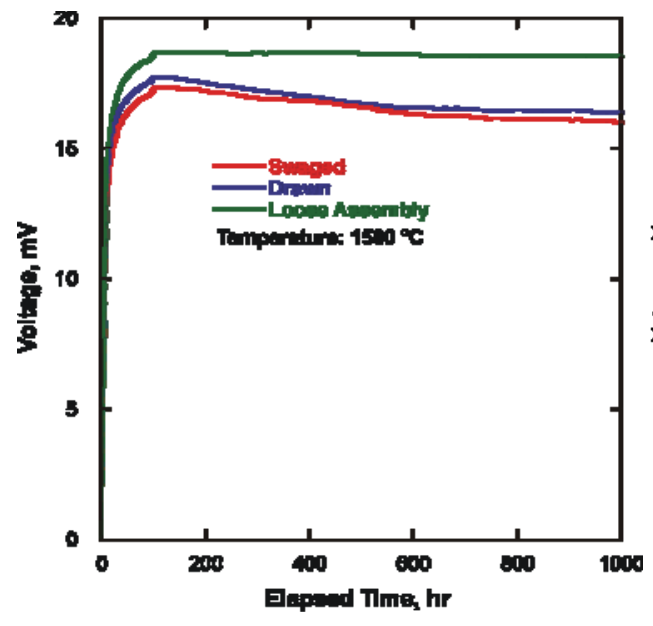

(a) $1500^{\circ} \mathrm{C}$ thermoelement wires (prior HTTL efforts to optimize heat treatment processes had focused on thermocouples' smaller diameter wires). Selected thermocouples from this test were tested at $1800^{\circ} \mathrm{C}$ without any additional heat treatment. Results, shown in Figure 2b, suggest that HTIR-TCs are also capable of functioning at these temperatures. The loose-assembly and drawn HTIR-TCs exhibited less than $1 \%$ drift and the swaged HTIR-TC exhibited approximately $8 \%$ drift.

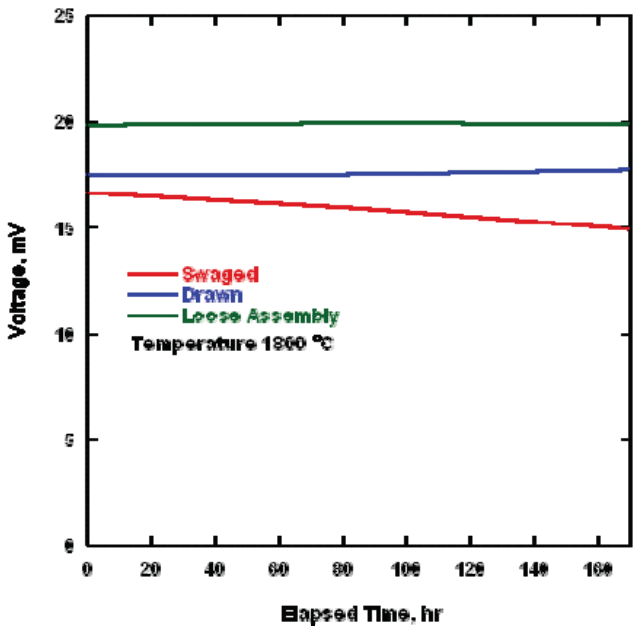

(b) $1800^{\circ} \mathrm{C}$

FIGURE 2. Loose-assembly and drawn HTIR-TCs exhibit superior stability in long-duration high-temperature tests.

In FY-09, insights from this LDRD were used to select an optimized HTIR-TC design for high-temperature irradiations at the MIT test reactor. Three optimized HTIR-TCs were fabricated and shipped to MIT for irradiation.

\section{Summary and Conclusions}

All proposed workscope for this laboratorydirected research and development (LDRD) project was completed as planned. Results from this project increase INL instrumentation capabilities, which support the Department of Energy's (DOE's) energy security mission by developing and demonstrating high-temperature in-pile instrumentation needed to obtain data required for key DOE-Office of Nuclear Energy (NE) research programs, such as the Generation IV and Fuel Cycle Research and Development programs. Specifically, this research is enhancing INL's expertise for producing optimized, more reliable, and lower-cost in-pile instrumentation. In addition, enhanced instrumentation from this research has the potential to increase testing capabilities of INL's ATR National Scientific User Facility. Finally, recognition (e.g., publications, patents, and awards) from this research promotes INL's instrumentation capabilities, furthering INL efforts to develop the Instrumentation, Control, and Intelligent Systems Distinctive Signature. 


\section{PAGE FOR NATIONAL AND HOMELAND SECURITY DIVIDER}

National and Homeland Security 


\title{
Multi-Stage Sequential Injection Gas Gun
}

\author{
Jeffrey M. Lacy, Henry S. Chu, Stephen R. Novascone, Warren F. Jones \\ Developing a device to characterize material behavior during high-strain-rate loading conditions \\ BS103
}

The multi-stage sequential injection gas gun invention was elected for patent pursuit by the BEST committee May 19, 2008. The motivation for the gas injection system is to have a device that can be used to characterize material behavior during high strain-rate loading conditions, such as when a projectile impacts a hard surface. Precise control of velocity and achieving very high velocity are also motivating factors. Developing this capability would attract potential customers in armor development and high-energy impact research.

The subject of development is a light-gas gun that injects high-pressure propellant at stages along the barrel, rather than from the breech alone. Sensors in each stage detect the location and velocity of the projectile, allowing the data acquisition and control system to calculate the correct charge duration for the desired final velocity and open the high-pressure injectors as the projectile passes. The fundamental concept here is that the projectile is subjected to low-jerk acceleration for a long period of time resulting in a predictable, very high velocity.

Where such a design was until recently infeasible, recent advances in electronic control, data acquisition devices and high pressure injectors make this approach to projectile launch possible.

\section{Technical Objectives}

- Perform valve timing tests

- Design/build control system

- Identify and procure all components

- Prepare LI-440 such that operation of the gun and take place at the National Security Test Range

\section{Results and Accomplishments}

Initial assessment of the selected hardware was accomplished via experiment. The purpose of the valve timing experiment was to quantify the time intervals between the application of voltage to a solenoid, valve opening, and time to peak pressure in an actuation valve, a typical result is shown in Figure 1. The result of this experiment was that the time between voltage application and valve opening was typically
$15 \mathrm{~ms}$, with a $+/-8$ millisecond repeatability. However, it was postulated that through some key component modifications this time could be improved.

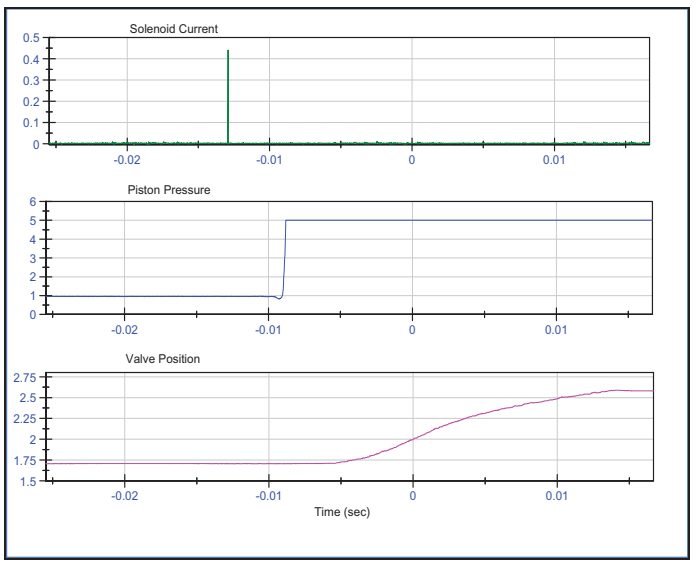

FIGURE 1. Typical valve characterization experimental data.

The initial design of the pressure distribution system has been completed, and is shown in Figure 2. All the components for the gas injection system have been procured. As noted previously, the research team has assembled the components to the point where valve timing studies were performed; however, the injection system is not in the final configuration stage.

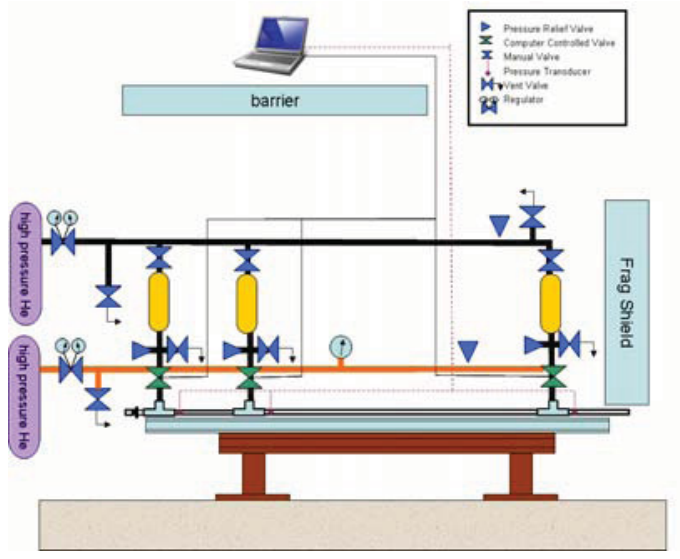

FIGURE 2. Pressure system.

The firing of the gun is accomplished via a combined software/hardware interface. The control logic and initial signals are formed in a commercial computer data acquisition environment, that gives the user the ability to 
modify the delays as required to generate the appropriate pressure pulses for a given test specimen. This is coupled to a signal amplifier which generates the voltage and current to
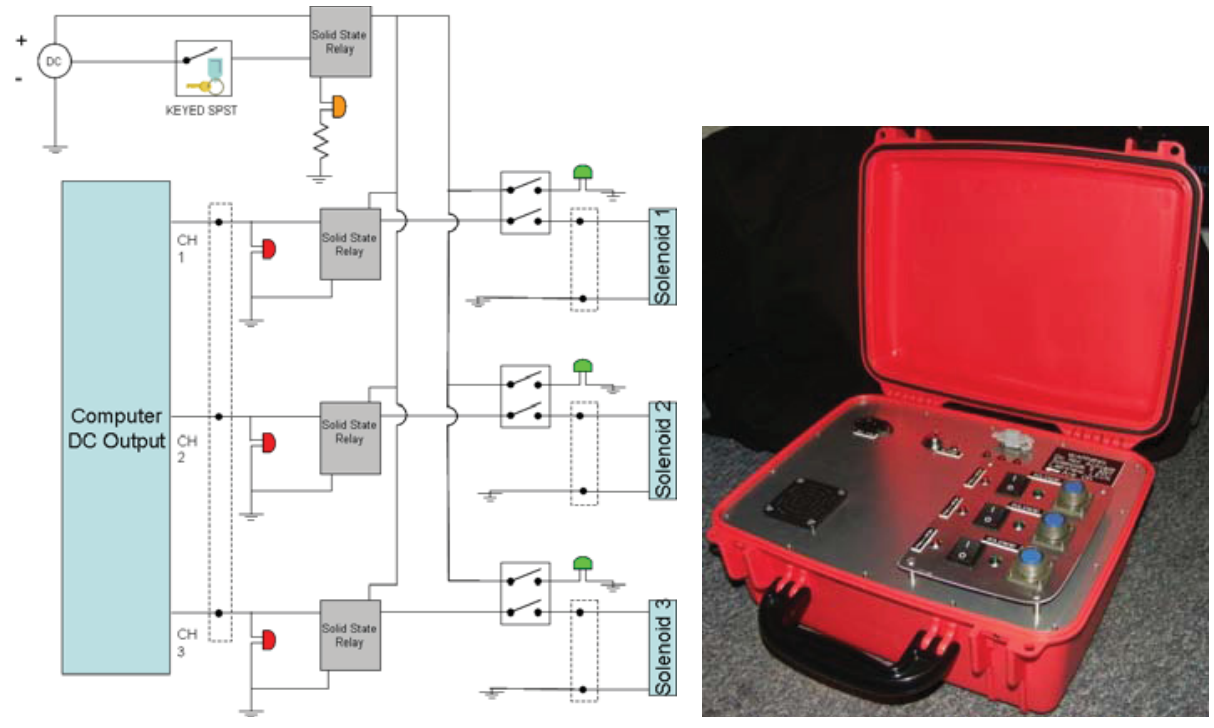

FIGURE 3. Control system.
During the development of the injection limit on projectile speed was the pressure wave speed of the gas. Barrel length and number of injection ports may not accelerate the projectile after the projectile approaches the pressure wave speed of the gas. As such, this is a topic of future investigation. However, the concept of applying acceleration to a projectile for a long period of time is still valid if the pressure wave speed of the gas can be increased. system, the research team hypothesized that the actuate the solenoids on the injector valves. The control system was fabricated and is shown in Figure 3 along with the control circuit diagram.

\section{Summary and Conclusions}

Once the components are assembled, the INL has the new capability of controlling projectile speed for precise material evaluation studies. This capability facilitates advanced material research and development as it allows comparison of candidate materials at relevant loading rates, and permits computational design studies of advanced engineering material concepts. 


\section{Enhanced Metal Ion Analysis}

James Delmore, Mark Stone, Darin Snyder, and Matthew Watrous

Improving the efficiency of ion production and simplifying sample preparation

FF107

Thermal ionization mass spectrometry (TIMS) is now a standard method for analysis of groundwater, geologic materials, isotope fingerprinting of pollutants, trace metals, medical measurements, and chemical metrology. The process of analysis involves collecting a sample and concentrating the analyte, typically either by evaporation or the use of some type of chromatography. Once the sample is in a suitable form, it is transferred to the filament of a TIMS system. The filaments are usually thin strips of very pure rhenium. In some cases the sample is applied by simply placing a drop containing the ions directly onto the filament and letting it dry. But in many cases, especially samples involving trace metals, the sample is transferred via ion exchange onto a very small ion exchange resin bead, and the bead is dried and then glued to the filament. Research has shown that if the bead containing the metal ions is then placed in a vacuum and heated until the resin bead is completely carbonized, the efficiency of ion production in the TIMS measurements is enhanced. This whole process is tedious and requires a few days to complete. This project conducted research to see if new methods can be developed that would enhance the efficiency of ion production and simplify sample preparation. Such improvements would have wide acceptance and applicability in many fields. It was reported that if a slurry was made of rhenium powder and coated the bead prior to it being carbonized, an improved efficiency was observed (David H. Smith and J. A. Carter, International Journal of Mass Spectrometry and Ion Physics, 40 (1981) 211-215). Despite the improved response, it added another preparation step.

\section{Technical Objectives}

This work will endeavor to improve ion production efficiency and simplify sample preparation. The general idea is to affix directly onto the filament a small pile of metal powder and then sinter it onto the filament. Then either the sample can be directly loaded onto the pile, or an ion exchange resin can be applied (simply by dropping a solution of it) to the powder pile and allowed to dry. After this, the sample can be added. This approach greatly simplifies sample preparation while keeping both the ion exchange resin and metal powders as the key components of the approach.

\section{Results and Accomplishments}

Several versions of the piles were tested, and one of the better combinations was a mixture of rhenium and platinum metal powders. These powders were mixed into hot glue extruded into tiny rods. Slices of the extruded rods were placed on the filaments and heated until the metal powders sintered together. Figure 1 shows different aspects of this. The top photo shows an early version of applying a drop of glue containing the powders onto the filament. The bottom left optical photo shows what the metal powder looks like after sintering and the bottom right shows the pile as seen by a scanning electron microscope (SEM).

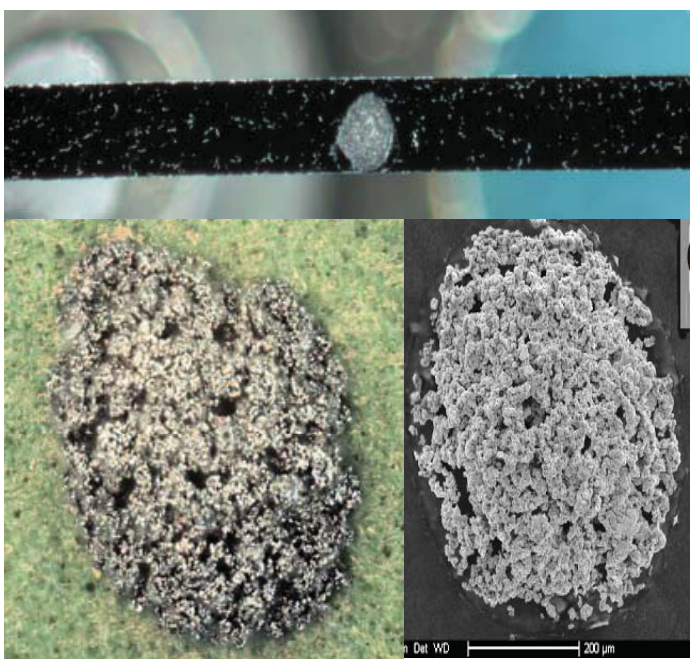

FIGURE 1. Photographs of metal piles. The top photo is a drop of glue containing the metal powder before sintering. The lower left shows the pile after sintering. The lower right is an SEM photo of a sintered metal powder pile.

This new method was tested on a solution containing praseodymium ions. When the solution was applied to beads in the normal procedure, the average efficiency for 7 samples was $3.8 \%$ of ions counted verses ions loaded. However, the same sample applied to the metal powder pile gave an efficiency of $35 \%$ for three samples tested. This is a remarkable result. Did this improved efficiency come just from the metal powder pile? Did it come from the platinum or the rhenium? Did the ion exchange resin play an important role? Some papers 
suggest that the metal ions need to be converted into carbides before they will produce charged metal ions. Many variables will need to be isolated and investigated.

\section{Summary and Conclusions}

A method was developed that improved ion production efficiencies in praseodymium metal ion samples by an order of magnitude. Efforts are underway to verify this result and to determine the values for the necessary and important parameters that will allow this method to be used for other ions. This is the direction that this LDRD will take in the next two years. 


\title{
Taylor Cylinder Determination of Impact Material Properties
}

\author{
Jeffrey M. Lacey, H. S. Chu, S. R. Novascone, W. F. Jones, Prof. Glenn Daehn
}

(Ohio State University)

Enhancing research related to the effects of blast, shock, and penetration on materials and material systems

NS156

Idaho National Laboratory (INL) is earning a reputation as a national asset for developing and fielding novel armor systems to defeat a variety of emerging threats. To further this evolution, we continue to enhance our capability to perform the basic and applied research related to the effects of blast, shock, and penetration on materials and material systems. A key element of this research is the capability to perform computational studies of ballistic and blast events to gain insight into the physics governing complex phenomena and to design armor elements and systems that take advantage of those phenomena.

Fundamental to the success of these computational studies is the representation of each material's response to its environment. Materials change volume due to pressure, shape due to deviatoric stresses, and strength and connectivity due to failure or change of state. Computationally, these responses are governed by models such as the equation of state, which relates pressure to specific volume and internal energy; the constitutive model, which relates deviatoric stress to distortion (or strain); and a damage model which describes the accumulation of damage, such as nucleation and growth of fractures in the material causing loss of strength.

This project has provided the Laboratory the capability to investigate the constitutive (strength) and failure properties of materials in the load regime of ballistic and blast phenomena, which may, in turn, be employed in computational design studies. This enables us to accelerate deployment of new armor and blastresistant technologies to the field by bypassing many of the build-and-test cycles traditionally employed in the development cycle.

\section{Technical Objectives}

At the end of the project's first year, we had designed, fabricated, fielded, and fired a new Taylor cylinder apparatus, shown in Figure 1. Building on this accomplishment, in Year 2, we

- Developed a novel 3-dimensional (volumetric) profiling technique for evaluation of the fired specimens
- Developed a novel computational optimization scheme to extract constitutive parameters from profile data

- Initiated a collaboration with the Ohio State University to study the feasibility of a new ballistic-rate material test, complementary to the Taylor device

- Designed and installed enhancements to the apparatus to support larger specimens and harder materials

- Are preparing an article for publication summarizing project findings

- Are submitting a collaborative proposal to the Department of Homeland Security for $\$ 1.5 \mathrm{M}$ to pursue further development.

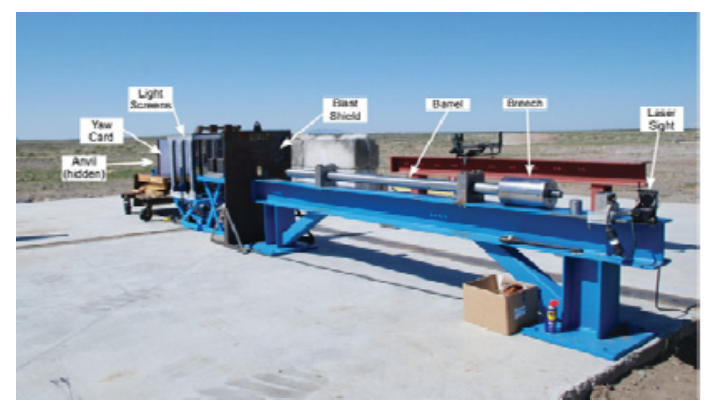

FIGURE 1. INL's Taylor impact experimental apparatus.

\section{Results and Accomplishments}

3-Dimensional Profilometry. One of the necessities of this project was the accurate extraction of the profiles from the deformed sample. This was accomplished via the combination of a consumer-grade 3D scanner and an INL-developed data reduction algorithm that aligns the surface data points relative to the original axis of symmetry of the sample and extracts the profiles at an angular spacing supplied by the user. To properly rotate and align the data, elliptical regressions are performed on various z-stations of the scanned data to determine approximate centers for each station. A regression is then performed on the centers and the data is rotated back to its original axis. Next, the data is re-centered and profiles are generated. 
Accuracy for each scan is determined via an examination of the un-deformed upper section of the cylinder to determine variance in the radius (Fig.2). This accuracy is assumed to be indicative of all vertical stations of the sample. Using this method, accuracies of $+/-0.0005$ in. have been achieved.
Noting that the profiles differ depending on the angle about the original axis of symmetry has led to an opportunity to investigate the sensitivity of the various coefficients to variations in the profile.
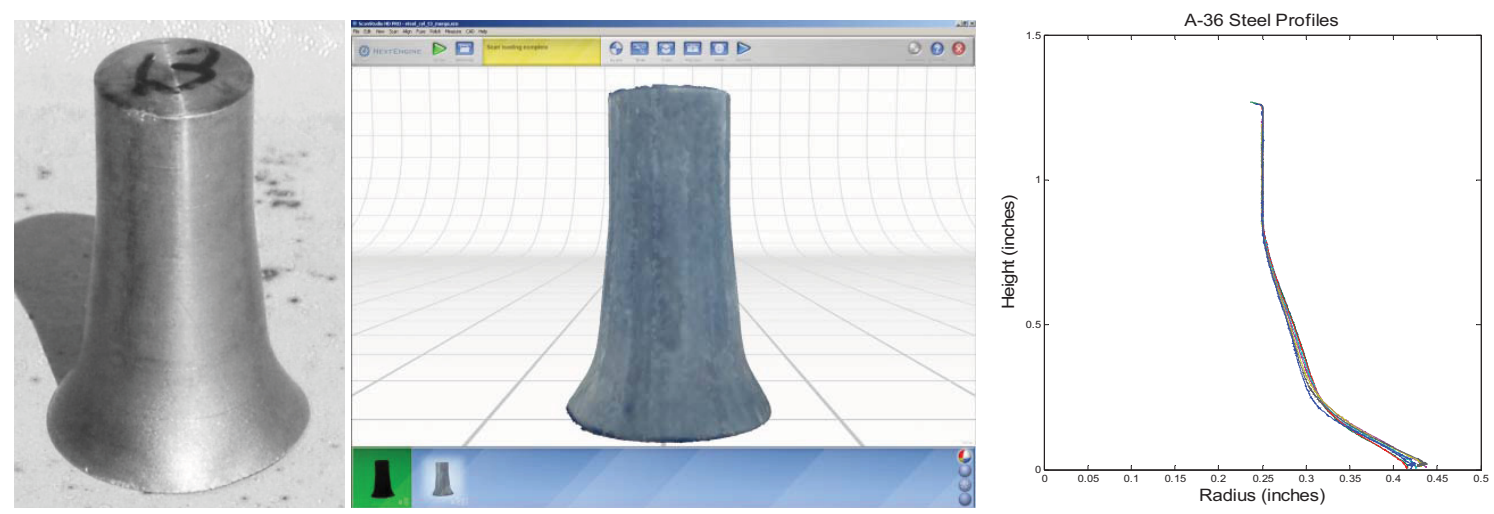

FIGURE 2. A-36 steel sample and scan data.

\section{Optimization-based Parameter}

Identification. A method was developed using statistical methods, such as Central Composite Design (CCD) and Latin Hypercube Sampling (LHS) to explore the parameter space for different material models. The CCD design is useful to obtain a second order equation with uniform error for the region sampled. The LHS design is useful when performing a CCD is not feasible. These statistical methods provide a structured approach to efficiently map out the parameter space.

To analyze each design point as specified by the statistical model, a hydrocode model (CTH in this case) was employed to evaluate cylinder deformation at different impact velocities and calculate the outer profile of the cylinder. These calculated profiles were then compared to the corresponding test profile via an RMS sum value - the optimization objective. A Kriging approximation model was then fit to the result, and the optimization space was evaluated to identify the optimum parameter set. In a case study, this method was shown to improve the accuracy of previously published materialparameter sets over the authors' original work.
Axisymmetric Dynamic Deformation Test. As the main purpose of this research was to enable advanced material characterization for use in computational studies, we initiated a collaboration with the Ohio State University to study the feasibility of the Axisymmetric Dynamic Deformation (ADD) test. This method employs an impulsive force to uniformly expand (or contract) a ring of specimen material. The radial location and velocity of the ring may be measured with high fidelity by Photon Doppler Velocimetry, yielding high temporal and spatial resolution of the stress-strain state of the specimen. Advantages of the method include a very high loading rate, uniform tensile (or compressive) stress state, simplicity of operation, and potential for standardization. This work led to a significant proposal to the Department of Homeland Security to standardize the method across the country.

\section{Summary and Conclusions}

At the conclusion of this project, INL has a new capability to extract material strength and failure characteristics for hard materials at high velocities with a high-velocity large bore Taylor cylinder device, novel, highly accurate 3-dimensional specimen profiling, and 
optimization-based parameter extraction techniques. This capability enables and

facilitates advanced armor and blast-resistant material research and development as it allows side-by-side comparison of candidate materials at relevant loading rates and permits computational design studies of advanced armor concepts. 


\title{
Development and Evaluation of Low Pressure Energy Absorbing Materials and Methods for Buildings
}

\author{
Mark Landon, Ben Langhorst, John Weathersby, Henry Chu, Theodor Krauthammer \\ (University of Florida)
}

\section{Low pressure energy absorbing materials and design methods for buildings}

NS161

In order to protect buildings and their contents from blast overpressures, material systems were developed for application on the exterior cladding. In an urban environment, the detonation of a large explosive device $(>500 \mathrm{lbs}$ TNT) could expose a few buildings to large overpressures and many more buildings to small overpressures on the order of 10-50 psi. Pressures of this magnitude are great enough to damage windows and façades and cause a hazardous debris field both in a building's interior and immediately outside the building. The application of robust cladding materials and systems could prevent damage to a building's exterior as well as dissipate the amount of blast energy transferred to the supporting structure.

Material systems were designed to affect two primary parameters of blast loading: peak load and impulse (analogous to energy) transmitted to a structure by a blast wave. Materials and systems were chosen according to their abilities to dissipate energy through various mechanisms, and three iterations of blast testing were used to refine panel design and incrementally improve dissipative performance. Experimental blast testing of 37 unique panels alongside 13 baseline unprotected panels revealed some very promising technologies, which not only reduce peak load and impulse, but are lightweight, inexpensive, and made of materials which are environmentally stable and readily available.

Existing instrumentation techniques applicable to this program precluded the measurement of time-histories and provided only a single value for total energy transferred throughout the event. Thus, in order to measure transmitted force-time histories behind each technology, custom-designed force sensors were created and used successfully. The force sensors were specially tuned to efficiently measure loads in the range at which testing occurred-peak incident pressures or approximately 10 psi.

This work also included the use of advanced modeling and simulation methods (such as SHAMRC coupled with LS-DYNA) to analyze conceptual absorption systems and the performance parameters needed for efficient absorption of energy. Computational fluid dynamics (codes such as FLUENT) were used to analyze the mechanistic behavior of concepts for high-viscosity fluid flow through restricted channels and porous media subjected to a pressure pulse. Based on analysis results, prototype mitigation concepts are being constructed, tested, and demonstrated at Idaho National Laboratory's (INL's) National Security Test Range using the arena test setup. Results from this testing are then introduced into the models to improve their performance. An example of a proposed mitigation system being tested includes granular porous material in a cement matrix to allow for future aesthetic assimilation into façades of conventional buildings. The pressure absorption ability of this layer is further supplemented by a second layer comprised of high-viscosity non-flammable fluid interspersed through special restricted channels. Other materials, such as fire-retarding polymeric membranes, are being evaluated to determine their potential for inclusion in this system.

Dr. Ted Krauthammer from the University of Florida investigated tunable, energyabsorbing, attachment mechanisms (panel to building) that could allow a controlled amount of energy to be passed from the energy-absorbing panel to the building.

This laboratory-directed research and development (LDRD) project also included a study of the applicability and enhancement of the modeling and simulation capability, Peridynamics, to the analysis methodology. Peridynamics is a fairly new formulation of continuum mechanics based on the integral equation of motion. It is a particle-based method with two very important features. First, it can handle material discontinuities - such as cracks - like any other deformation. Second, when the equation of motion is discretized, it looks exactly like the equation of motion for classical molecular dynamics simulations.

\section{Technical Objectives}

- Developed granular-filled protective panels that utilize a proprietary binding and confining methods that effectively mitigate blast effects on structures

- Developed a hydraulic-filled panel with a proprietary core design and unique hydraulic 
fill which effectively dissipates blast energy as it crushes

- Conducted 22 blasts to test a variety of different panel designs - collecting highspeed transmitted force data and correlated high-speed photography

- Developed a novel technique to measure transmitted force-time histories in specific load ranges

- Filed five invention disclosure records, wrote two technical papers to be submitted to refereed journals, and work will be presented at the annual Shock and Vibration Information Analysis Center (SAVIAC) conference in 2010.

\section{Results and Accomplishments}

The initial scope of this program included a multi-faceted research effort through which experimental data would be coupled with multiscale modeling to develop and validate a physics-based approach to modeling dynamic behavior of materials with energy dissipative potential. As this project evolved, the primary emphasis was on experimental development and the construction and blast testing of prototype test articles.

The first round of blast testing examined the following technologies:

- Granular material matrices

- Hydraulic dissipative panels

- Pyramidal truss core sandwich panels

- Deformation-induced impulse elongation panels

- Impedance mismatched stacks of typical construction materials.

These panels were tested at INL's National Security Test Range using full-scale explosions using commercially available transmitted force sensors and monitored by high-speed photography. The transmitted force sensors provided unreliable data because their piezoelectric nature caused them to return to non-zero loading - a common phenomenon that is often unwillingly accepted in the blast community. Observations through high-speed photography revealed some concepts that didn't warrant any future investigation and other concepts which could be refined to improve their performance.

The most promising classes of panels were those featuring granular materials and hydraulic dissipative mechanisms. To measure the incoming blast pressure on the front face of the sled to which test articles were attached, "sensor bolts" were used to house flush-mounted pressure gages. This idea was conceived at INL, but a patent search revealed that this concept had already been claimed by another inventor.

Figure 1 shows a pressure sensor housed within a bolt that is installed on the front face of the test sled.
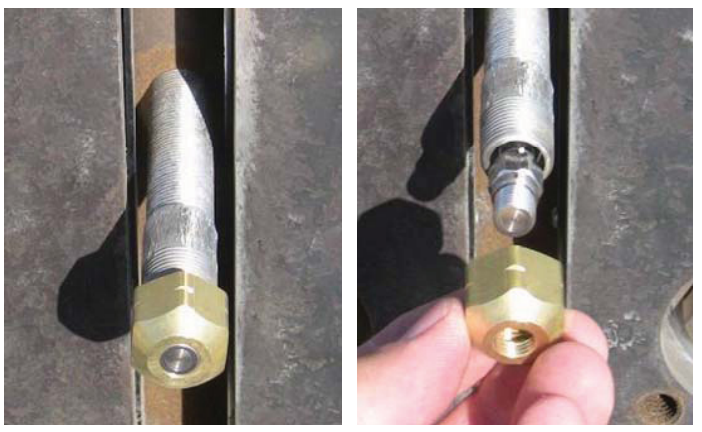

FIGURE 1. Sensor bolt technology: a pressure-sensor housed inside a homemade bolt to measure blast pressure on the front face of the test sled.

It was also observed that the light blast loading ( $\sim 10$ psi) was too little to cause deformation in some of the stiffer designs, so a second round of blast testing was conducted at increasingly higher blast magnitudes. Highspeed photography of these tests revealed that in some cases, the panels deformed as predicted, while in other cases, the deformation mechanisms were unexpected and ineffective at blast dissipation.

The lessons learned from the first two rounds of blast testing were incorporated into a new set of panel designs that were tested. In addition to revising the test articles, the blast test setup was improved in a number of ways. The test articles were positioned at higher elevations above the ground to eliminate inevitable boundary effects on the blast wave near the 
ground (Fig. 2). The test articles were also instrumented with custom-designed proprietary force transducers that measure force-time history reliably and accurately under the load range for which they were designed. These novel sensors were more accurate and less expensive than alternative sensor technologies that are commercially available.

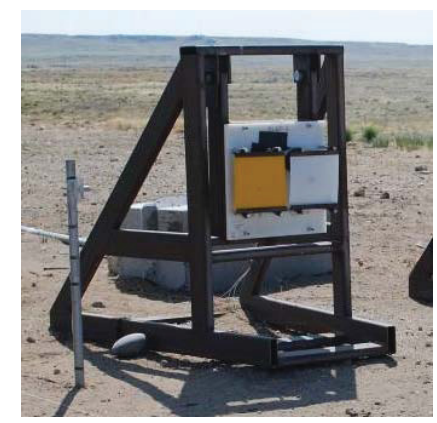

FIGURE 2. A redesigned blast test sled with two panels mounted on its front side.

Test articles designed and fabricated for the third round of testing included granular materials bound and confined by various proprietary methods, hydraulic dissipative panels which featured proprietary core designs and fluids, impulse-elongating panels designed to deform large amounts before transmitting significant loads, and various geometries of porous core media. Figure 3 shows a few different technologies that were tested. In each blast, alongside the series of test articles, was a baseline panel intended to gauge "unprotected" performance. Nine blasts were conducted and the force-time history acquired was analyzed and summarized for each test article relative to the baseline panel with which it was tested (Table 1).

\section{Summary and Conclusions}

Very promising technologies were identified and refined to enhance applicability, and fullscale blast testing revealed impressive blast dissipative performance of many technologies with efficient weights and costs. One promising design weighed $3.5 \mathrm{lb} / \mathrm{ft}^{2}$ and cost approximately $\$ 13 / \mathrm{ft}^{2}$ to fabricate a lab-scale sample; it performed superbly, reducing the peak transmitted force by $66 \%$ and reducing the transmitted impulse by $84 \%$. Further development is necessary to elevate the novel blast panel technologies to a higher technology readiness level. After protecting all intellectual property, the technologies developed in this program can be applied to solutions for externally funded projects.

The novel instrumentation method employed in this project is a valuable contribution to the experimental capabilities of the Laboratory and exceeds the capabilities known to exist at other laboratories around the country.
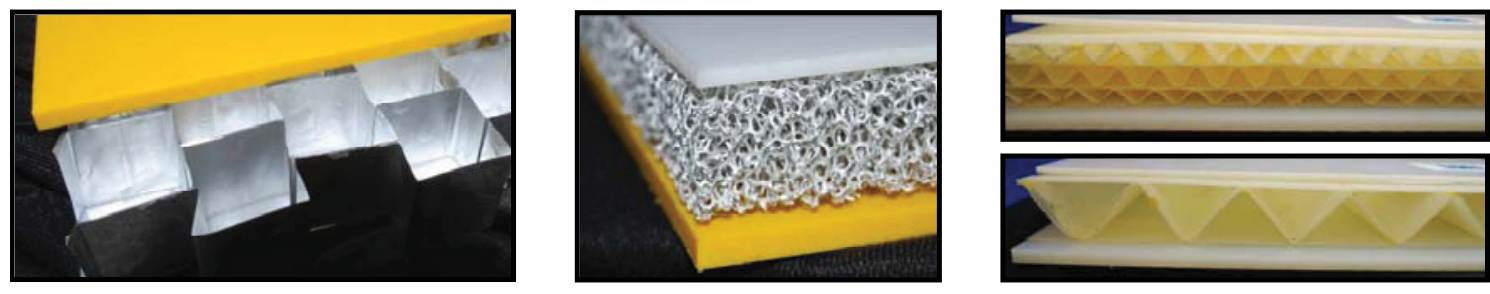

FIGURE 3. Four different technologies tested in Phase 3 of blast testing. (From right to left: aluminum foil honeycomb cells, aluminum foam, corrugated-core sandwich panels) 
TABLE 1. Array of panels tested during phase three of blast testing. The names and descriptions are hidden to protect proprietary information.

\begin{tabular}{|c|c|c|c|c|c|}
\hline \multirow[b]{2}{*}{ Panel ID } & \multicolumn{2}{|c|}{ Performance Statistics } & \multicolumn{3}{|c|}{ Panel Characteristics } \\
\hline & $\begin{array}{c}\text { Load } \\
\text { Reduction }\end{array}$ & $\begin{array}{l}\text { Impulse } \\
\text { Reduction }\end{array}$ & $\begin{array}{l}\text { Panel Thickness } \\
\text { (in.) }\end{array}$ & $\begin{array}{c}\text { Panel Weight } \\
\text { (lbs) }\end{array}$ & $\begin{array}{c}\text { Cost } \\
\text { (per sq. ft.) }\end{array}$ \\
\hline $1 \mathrm{~A}$ & $-66 \%$ & $-84 \%$ & 2.25 & 3.57 & $\$ 13.50$ \\
\hline 1B & $-37 \%$ & $-42 \%$ & 2.5 & 1.78 & $\$ 14.50$ \\
\hline $1 \mathrm{C}$ & $-32 \%$ & $-52 \%$ & 0.75 & 1.17 & $\$ 12.50$ \\
\hline $2 \mathrm{~A}$ & $-56 \%$ & $-72 \%$ & 1.875 & 5.09 & $\$ 7.00$ \\
\hline $2 \mathrm{~B}$ & $-66 \%$ & $-88 \%$ & 2.25 & 5.55 & $\$ 6.50$ \\
\hline $3 \mathrm{~A}$ & $-7 \%$ & $-66 \%$ & 1.5 & 4.66 & $\$ 7.50$ \\
\hline $3 \mathrm{~B}$ & $-37 \%$ & $-28 \%$ & 1.375 & 4.22 & $\$ 7.50$ \\
\hline $3 \mathrm{C}$ & $-32 \%$ & $-36 \%$ & 1.375 & 2.04 & $\$ 8.50$ \\
\hline $4 \mathrm{~A}$ & $-31 \%$ & $-67 \%$ & 1.625 & 2.56 & $\$ 24.00$ \\
\hline $4 \mathrm{~B}$ & $-31 \%$ & $-42 \%$ & 1.625 & 2.4 & $\$ 24.00$ \\
\hline $4 \mathrm{C}$ & $-36 \%$ & $-49 \%$ & 1.875 & 2.43 & $\$ 21.50$ \\
\hline $5 \mathrm{~A}$ & $-63 \%$ & $-66 \%$ & 1.625 & 1.63 & $\$ 40.00$ \\
\hline $5 \mathrm{~B}$ & $-37 \%$ & $-52 \%$ & 1.625 & 1.61 & $\$ 30.00$ \\
\hline $5 \mathrm{C}$ & $-56 \%$ & $-81 \%$ & 1.5 & 1.98 & $\$ 10.00$ \\
\hline $6 \mathrm{~A}$ & $-13 \%$ & $-29 \%$ & 1.625 & 2.33 & $\$ 100.00$ \\
\hline $6 \mathrm{~B}$ & $-22 \%$ & $-15 \%$ & 1.5 & 2.88 & $\$ 100.00$ \\
\hline $7 \mathrm{~A}$ & $-54 \%$ & $-92 \%$ & 2 & 4.5 & $\$ 50.00$ \\
\hline $7 \mathrm{~B}$ & $-38 \%$ & $-74 \%$ & 1.75 & 2.68 & $\$ 50.00$ \\
\hline $7 \mathrm{C}$ & $-43 \%$ & $-90 \%$ & 2 & 3.83 & $\$ 50.00$ \\
\hline $7 \mathrm{D}$ & $-37 \%$ & $-72 \%$ & 1.75 & 4.26 & $\$ 50.00$ \\
\hline $8 \mathrm{~A}$ & $-39 \%$ & $-60 \%$ & 1.375 & 1.92 & $\$ 200.00$ \\
\hline
\end{tabular}




\section{Modeling of Threats and Interdependencies on Critical Infrastructures}

Stuart Walsh, Barry Hansen

Cyber attack modeling of a representative portion of the electrical power grid, advanced metering infrastructure (AMI), and a natural gas pipeline NS162

The Idaho National Laboratory (INL) Cyber Attack Modeling tool is used to examine the possible effects of a cyber attack on critical infrastructures and what the introduction of a particular vulnerability might mean for those infrastructures. The problem was divided into three core areas: attack propagation, attack initiating actions, and attack consequences.

Attack propagation was concerned with answering these questions:

- From a particular starting point can a cyber attacker compromise a specific goal state (host and privilege) in the infrastructure network?

- How is the attack executed? Which specific vulnerabilities and hosts are used to achieve a goal?

- If an attack path does not exist, what is the minimum number of unknown configuration errors or vulnerabilities that must exist in order to achieve a goal?

- In the given network, where must each configuration error or unknown vulnerability exist in order to achieve a previously unattainable goal?

Attack initiating actions:

- What initiating actions can an attacker take to achieve damage in a particular infrastructure?

Attack consequences:

- Given a set of initiating actions, what are the physical effects of those actions on the critical infrastructure?

This effort focused on cyber attack modeling of a representative portion of the electrical power grid, advanced metering infrastructure (AMI), and a natural gas pipeline. The research did not place any emphasis on discovering actual new vulnerabilities but examined existing systems based on all known vulnerabilities, configuration information, and the introduction of currently unknown vulnerabilities. The penetration analysis was tied to a physics-based model of a natural gas pipeline and an electrical power grid to examine the potential consequences of such an attack.

\section{Technical Objectives}

- Build an attack graph of a representative AMI infrastructure, natural gas pipeline, and electrical power grid

- Build an attack graph analysis engine based on MulGraph

- Incorporate attack graph analysis with physics-based models of a representative natural gas pipeline and electrical power grid

- Integrate the attack graph analysis tool with Zigbee hardware as a representative AMI infrastructure model.

Results and Accomplishments

Attack propagation. A survey of cyber attack modeling tools was performed to assess the current state of the art. Of the tools surveyed two were found to provide the necessary basic functionality, MulVal and netSPA. Through collaboration with Kansas State University, MulVal was obtained and several attack graphs of representative portions of the infrastructures were built. An analysis engine was built on top of MulVal that will consume a network configuration and vulnerabilities definition, generate a formal attack graph, and determine the minimum number of configuration errors or vulnerabilities that must be introduced to compromise a target host.

An attack graph is defined as a graph that contains all possible exploit paths an attacker can follow to compromise a given node in a network. The graph information consists of all network, hosts, services (ports), routing configuration, and vulnerabilities for a given network. The graphs are created from three different nodes: an exploit condition leaf, a privilege increase goal (logical or), and a rule or set of conditions necessary to achieve a goal (logical and). In the example diagram leaves are boxes, goals (logical or) are diamonds, and rules (logical and) are ovals.

Figure 1 illustrates the path an attacker follows. The leaf nodes labeled "1" are the two necessary conditions for the attacker to achieve network access to the web server. The attacker obtains access to the web server through Rule 2 direct-network access. Rule 5 requires three 
conditions: network access, a specific vulnerability 'CAN-2002-0392', and that the apache web service be running. When all the conditions for Rule 5 are realized, the attacker has achieved Goal 6 and can execute arbitrary code on the web server.

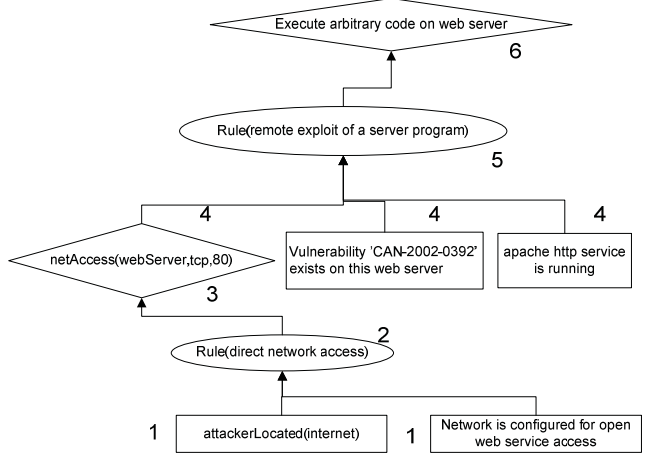

FIGURE 1. Simplified attack graph.

The actual attack graphs are more detailed but permit the INL Cyber Attack Model to determine with certainty the capability of an attacker to achieve a particular target. The analysis engine can be used to determine which assets are higher value targets based on the number of attack paths a node enables. For a target that appears to be unattainable, the analysis engine will examine a set of attack paths and generate a minimal set of unknown vulnerabilities and configuration errors that would be required to achieve a particular target. This capability enables prioritizing of assets, for a given attack, based on everything that is currently known about a network and what the effects of a future but currently unknown vulnerability might be.

Attack graphs permit the analysis engine to determine the attack points of a network. Attack point is defined to be the host and privilege at which an attacker stops penetrating a network and begins executing initiating actions to achieve the attack goal. In Figure 1, Nodes 3 and 6 are attack points.

Attack initiating actions. Initiating actions are those actions that an attacker executes once an attack point has been achieved. Referring to Figure 1, the attacker has reached Node 6 and has the capability to execute arbitrary code on the web server; initiating actions would be the code the attacker executes to achieve physical damage in the system. For a process control node in the electrical power grid, one initiating action would be to send a command to open a circuit breaker, preventing the flow of power.

Developing a methodology for determining initiating actions in an automated manner was beyond the scope of this project. Subject matter experts (SMEs) in AMI infrastructure, the electric grid, and natural gas pipeline were consulted to determine the initiating actions. The SMEs were asked to determine a set of initiating actions that were likely to lead to consequential results in the particular infrastructure. While the SME analysis is realistic and based on actual experience in the field, it is impractical for the analyst to examine thousands of possible attack points and determine possible initiating actions for each attack point. Hence, a small number of such actions were identified for a subset of the identified attack points.

Attack Consequences. The attack consequences area is primarily concerned with the physical effects of a cyber attack. The SMErecommended actions were then incorporated into scripts that were executed on the infrastructure models to examine consequences. The physics-based infrastructure models were developed previously in INL's CIPRSim environment.

\section{Summary and Conclusions}

The INL Cyber Attack model is an excellent tool for determining possible attack points, determining effects of introducing previously unknown vulnerabilities, and integrating the attack point information with the physics-based models for consequence analysis. However, the cyber model does not determine initiating actions in an automated fashion. An analyst is required to determine for a given attack point the attacker's initiating actions in the target control system that will lead to physical consequences. The work of developing an attacker profile and defense posture template for a given infrastructure point where the detailed attack graph is associated with a much more generalized attacker profile was begun but has not yet been completed. To determine the potential consequences in a thorough and reliable 
manner, an automated approach to investigating the attacker's initiating actions needs to be developed. The automated approach needs to include a mechanism for associating the attack graph and the physics-based infrastructure model with a template of the attacker's goals and capabilities. 


\title{
Fast Neutron Irradiation Capability
}

\author{
David L. Chichester, Jeff Sanders, Kevin Carney, Ed Seabury \\ Development of advanced nuclear forensics scientific techniques for evaluating analytical forensic \\ samples \\ NS163
}

Forensic determination of the origin of special nuclear materials (SNM) is a key part of nuclear nonproliferation and nuclear deterrence. Because of the ability to identify the origin of these materials prior to nuclear detonation or after detonation, the United States is capable of making a credible threat against the originator by knowing where the material comes from. This is important to deter the use of nuclear weapons against the U.S. by nation-states because it eliminates their confidence in acting secretly and avoiding retribution. Similarly, it forces those who might consider supplying SNM to terrorist organizations to reconsider their actions and the potential consequences since the U.S. may be able to identify them as the source of the material and therefore hold them accountable for the actions of their proxies.

Related to studies of the origin of SNM, this LDRD focuses on forensic studies of fission product distributions. This is important because fission product data can be used to deduce information about the conditions during which fission events take place; more specifically, the fractional distribution of fission-product isotopes is dependent upon the neutron energy spectrum that induces the fission events. Illustrating these differences, four fission product yield distributions are presented in Figure 1 for cases where U-235 and $\mathrm{Pu}-239$ are irradiated with either prompt fission neutrons (left) or monoenergetic neutrons at $14 \mathrm{MeV}$ (right). Significant differences are observed in the centroid locations and shapes of the high- $Z$ and low-Z fission product distributions for uranium and plutonium. Further, centroid region yields versus valley region yields are notably different when comparing fission induced by a fission neutron spectrum versus fission induced by 14$\mathrm{MeV}$ neutrons. In the first part of the project, we are developing equipment and facilities to support the nuclear forensics fission-product distribution research described here. Later, our research efforts will examine and optimize parameters related to preparation, irradiation, and treatment of fission product samples produced in the facility. In this area, we will consider issues including sample material matrix and composition, material handling, material radiochemistry, and sample preparation.
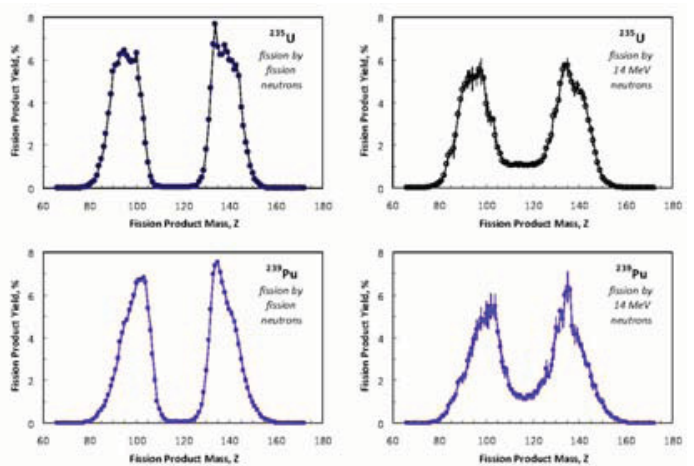

FIGURE 1. Fission product yields for U-235 and Pu-239 induced by irradiation with fission spectrum neutrons and induced by 14-MeV neutrons. (Report LA-UR-94-3106, Los Alamos National Laboratory, Los Alamos, NM (1994).)

\section{Technical Objectives}

The objective of this project is to explore scientific aspects of nuclear forensics science related to methods of analysis of fission product isotopic composition, the irradiation of fissionable material with fast neutrons, and to support the development of advanced nuclear forensics scientific techniques for evaluating analytical forensic samples. This research will take place at INL's Materials and Fuels Complex (MFC) and use a high-yield, 14-MeV electronic neutron generator (ENG) and exiting INL radiochemistry capabilities. Several complementary research efforts supporting nuclear forensics and other national security applications will take place within this scope, including:

- Research examining approaches and methods for irradiating samples of SNM with fast neutrons

- Research to develop irradiated SNM samples for training nuclear forensic scientists

- Development of new measurement techniques to support nuclear forensics

- Development of new methods for detecting and identifying ultra-trace quantities of uranium and plutonium based upon fission track analysis (FTA) in conjunction with thermal ionization mass spectrometry (TIMS) or secondary ion mass spectrometry (SIMS) 
- Research to develop techniques for producing short-lived nuclear materials for radiological emergency first responder training.

\section{Results and Accomplishments}

This year's primary focus was to evaluate the condition of an existing high-yield electronic neutron generator at MFC and to begin its refurbishment. Towards this goal we performed diagnostic analyses of the system in conjunction with professional technical staff from the manufacturer, worked to develop a refurbishment plan for the generator, started the process of dismantling and disposing of obsolete parts of the generator, and purchased advanced lead-time replacement parts for the generator. These activities were key towards re-establishing INL's capability for carry-out research using high-intensity $14-\mathrm{MeV}$ neutron radiation. However, this activity is not yet complete. During the next year, we will complete the refurbishment process and develop the work control, radiation safety, and laboratory instruction documentation needed for operating the neutron generator at MFC. This will entail the purchase of a replacement high-yield neutron tube to be used with the neutron generator and possibly construction activities to more optimally install the system in its operating vault.

On the research front this year, we studied diagnostic gamma-ray spectroscopy methods for analyzing short-lived fission products, laying the ground work for forensic research to be carried out in Year 3. This work was performed using a low-intensity, time-tagged Cf-252 neutron source in conjunction with a gated, high-purity germanium (HPGe) spectrometer. A photograph of the set-up together with representative data are shown in Figure 2 (Note: this data has been normalized). Clear differences are observed between the coincidence and anti-coincidence spectra, indicative of the decay of very shortlived fission products in the $\mathrm{Cf}-252$ source following individual fission events. Similar work to investigate short-lived spectroscopic fission product signatures will continue in Year 2 of the project.
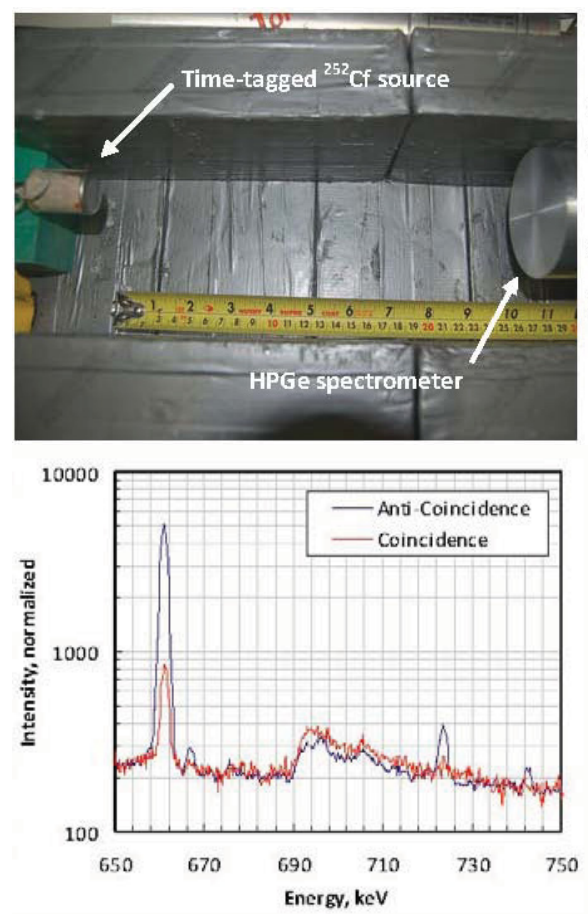

FIGURE 2. Test set-up (top) and representative date showing gamma-ray spectra in coincident mode () and anticoincident mode between the detector and the source bottom, coincidence is defined as $\sim 8 \mu$ s following a fission event).

\section{Summary and Conclusions}

Significant progress has been made this year towards rebuilding INL's high-intensity, fast-neutron radiation research and development capability. Upon completion this scientific tool will serve as the basis for a new area of nuclear forensic research at INL, allowing sophisticated studies of short-lived fast fission fission product distributions from SNM including uranium and plutonium. During Year 2 of the project, we will complete the refurbishment activities and transition to demonstration-scale irradiation operations. In parallel, exploratory research has been initiated to explore tools, techniques, and methodologies for assaying short-lived fission products to discern fission-product distributions. An ancillary benefit from this research will be a new nuclear physics research capability for studying fission through the examination of higher energy reaction channels (due to the use of 14-MeV neutrons) and the use of short-lived gamma-ray spectral data. 


\section{Trace Explosive Characterization on Materials}

Carla Miller and Timothy Yoder

The objective is to define trace explosive signature in an attempt to provide information that would help improve detection capabilities

NS164

It has long been understood that when someone comes in contact with surfaces after handling explosives there is a trace amount of material transferred from the hand to the material. Little work has been performed to identify what amounts of explosive traces are available for detection and how efficiently those residues can be harvested and transported to a detector. In the past, an explosive fingerprint was assumed to be about 50 microns, consisting of varying particle sizes and shapes that could readily be detected. This assumption was used in the development of explosive trace detection equipment, which was designed to detect a sample containing particulates of an explosive that had been obtained by physically swiping the surface of a substrate.

It is also understood in the explosive-trace community that some explosives are easier to detect than others, even from swipe sampling. This has always been attributed to the capabilities of the detection equipment, rather than giving consideration to what a fingerprint may look like on different surfaces. In addition, environmental factors were never considered in how the fingerprint may change over time. This laboratory-directed research and development (LDRD) project looked at the effects of environmental factors and time, as well as the effect of different surfaces on trace-explosive fingerprint morphology.

To accomplish the tasks, fingerprints of explosives were placed on 1-in. ${ }^{2}$ pieces of materials using consistent pressure on both on the explosive block as well as on the material. The substrates used were vinyl, leather, $t$-shirt ( $50 \%$ polyester $/ 50 \%$ cotton), canvas $(100 \%$ cotton), and metal. (The metal was obtained from a junked car, cut into pieces.) None of the materials was washed, so each contained oils, dirt, and sizing agents associated with the materials. The explosive particles were photographed using optical microscopy. Then the materials were placed in one of three environmental chambers (Tenney THRJ$\left( \pm 0.3^{\circ} \mathrm{C} / \pm 5 \% \mathrm{RH}\right)$, a Tenney T11RC-1.5 $\left( \pm 0.3^{\circ} \mathrm{C} / \pm 2 \% \mathrm{RH}\right)$, or a Thermotron model SE$\left.1200-3-3\left( \pm 0.2^{\circ} \mathrm{C} / \pm 2 \% \mathrm{RH}\right)\right)$ at varying temperature and humidity. The substrates were photographed using optical microscopy after set periods of time or until there were no explosive crystals visible on the surface.

The fingerprints were prepared in lots of ten. This allowed for different procedures — such as swabbing and sampling using explosive trace detectors such as GE Itemiser and Smiths 400B - to be performed at the same time. Colorimetric techniques, such as Exspray, were also used. Once the substrates were sampled for explosive trace detection, they were photographed using optical microscopy to determine the amount of explosive crystals that remained on the surface. Additional sets of samples were viewed with scanning electron microscopy and atomic force microscopy.

The focus of this LDRD was to determine how an explosive fingerprint's morphology is affected by different materials and what type of crystalline materials may be available for sampling with an explosive trace detector system. In addition, this LDRD looked at changes in these fingerprints over time due to environmental conditions such as humidity and temperature. This was a one-year LDRD project.

\section{Technical Objectives}

Technical objectives for Fiscal Year 2009:

- Determine the explosive fingerprint characteristics on such materials as suede, metal, cotton, denim, and vinyl.

- Determine the explosive fingerprint characteristics on the materials of three standard explosives: C-4, Semtex-A, and TNT.

- Determine how the fingerprints of each explosive type changes for each material substrate over time.

- Determine how the fingerprints for each explosive type and each material changes under the following environmental conditions: $40 \% \mathrm{RH} / 40^{\circ} \mathrm{F}, 40 \% \mathrm{RH} / 70^{\circ} \mathrm{F}$, $70 \% \mathrm{RH} / 70^{\circ} \mathrm{F}, 95 \% \mathrm{RH} / 70^{\circ} \mathrm{F}, 40 \% \mathrm{RH} / 95^{\circ} \mathrm{F}$, $70 \% \mathrm{RH} / 95^{\circ} \mathrm{F}$, and $95 \% \mathrm{RH} / 95^{\circ} \mathrm{F}$.

- Document how the explosive trace detectors respond to sampling after the substrate has been under the specified environmental condition for one week. 
- Use atomic force microscopy and scanning electron microscopy to determine in what ways explosives crystals are embedded in the substrate surface.

\section{Results and Accomplishments}

Three different explosives were used for the testing: C-4, Semtex-H, and TNT. The explosives on the substrates were photographed over specific periods of time using a Zeiss Discover V12 stereoscope with Axiocam ICc1 3-megapixel digital camera to determine the difference in the crystalline structure and surface contamination in an attempt to determine differences and similarities resulting from environmental factors, specifically humidity and temperature. The $\mathrm{C}-4$ and Semtex-H explosive crystals are held within different matrices which results in fewer noticeable changes due to the environmental conditions. However, in some cases, especially those of higher temperature and humidity combinations, there were some changes in the amount of trace explosive present on the surface after longer periods of time (30 days). Semtex-H generally has fewer crystals within a fingerprint than either of the other explosives, so one would expect it to be the most difficult to sample. With TNT, which is not bound in a matrix, there are more particles available for sampling, and the decrease in the amount of crystals on the surface was generally visible within the first seven days of being in the environmental chamber. It appears that the free crystals are removed more readily by temperature and humidity than is seen in those bound in a matrix. Figure 1 is a series of photographs over a period of time taken of TNT, fingerprinted onto vinyl; it shows a considerable loss of explosive occurs within the first four days.

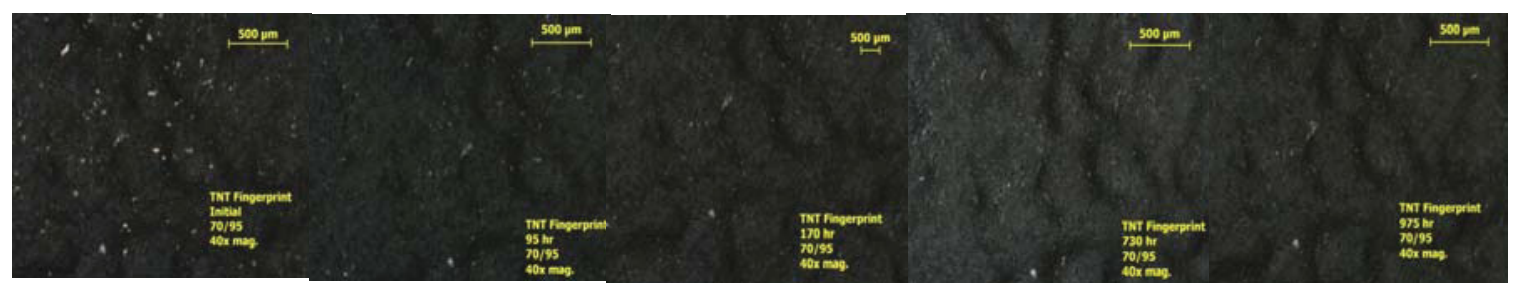

FIGURE 1. TNT fingerprints on vinyl over time at $70^{\circ} \mathrm{F}$ and $95 \%$ relative humidity.

In addition, more noticeable changes occur on cotton, leather, and vinyl substrates than on denim and metal. This may be a result of the explosives' wicking into the material over time or with increases in humidity. The crystals on denim are difficult to see from the very beginning because they tend to embed into the canvas fibers and cannot be discerned. Leather absorbs the most water as the humidity changes. The changes in crystals on leather are significant, but it is difficult to discern whether they are disappearing or sinking into the leather in response to the humidity. This makes the crystals more difficult to detect with the optical microscope. In addition, the crystals become flattened when they are placed on more rigid surfaces, such as metal, making them more difficult to see and more difficult to remove. This probably is a result of the amount of crystalline surface that bonds to the metal (i.e., a result of surface area).
In an attempt to discern whether the crystals are embedded and how the crystals may bind to the surface of the materials, atomic-force microscopy and scanning electron microscopy images were obtained. The atomic-force microscope (AFM) uses a cantilever lever to move across the surface of the material and take images of the various depths of the substrates. This technique could only be used on vinyl and metal samples due to the roughness of the other substrates. Even on the metal and vinyl surfaces, the atomic force microscope looks at such a small area in relation to the size of a crystal $(\sim 50-500 \mu \mathrm{m})$, that it was difficult to determine if there was any crystalline structure in the images. Therefore, it was determined that this technique was not useful for this application.

Scanning electron microscopy (SEM) uses a laser to create a signal from electrostatic forces that are measured as the laser moves across the surface of the substrate. It is an effective 
technique for determining the composition of the substrate as well as seeing the appearance of crystalline structure of the explosive on the surface of the substrate. The sample-preparation time for scanning electron microscopy is considerably longer than desired because the sample has to be mounted on a stub and then coated with gold to ensure that electrostatic forces are present. This is an effective technique for looking at all of the substrate surfaces. Figure 2 shows an optical photograph and an SEM image of C-4 explosive on vinyl. As can be seen in Figure 2, SEM provides more definition and depth than standard optical microscopy. Although it was not used in this project, other techniques are available to provide greater depth information using other SEM techniques.
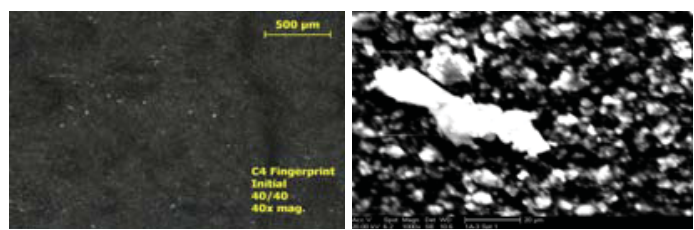

FIGURE 2. Optical photograph (left) and scanning electron microscope photograph (right) of $\mathrm{C}-4$ explosive on vinyl substrate.

\section{Summary and Conclusions}

Relative humidity and temperature do not affect the crystalline structure at low temperatures and low humidity. However, as temperature and the humidity are increased, the surface crystals, especially for free crystals such as TNT, decrease. The bound crystals do not decrease as readily. However, due to the techniques used, it was difficult to determine if the crystals are being removed from the surface (may be hydrophobic and disappear) or if they are wicking into the material with the moisture.
AFM is not a feasible technique for monitoring the crystalline structure on the rough surfaces that were used as substrates in these tests. SEM is a much better technique and could possibly be used to determine the effectiveness of sampling better than the current techniques of solvent extraction. In addition, the SEM provides depth information on how crystals are bound.

Based on looking at the optical microscopy pictures prior to swipe sampling and after swipe sampling, there is sufficient sample available to take multiple samples and still obtain results (i.e. if the explosive trace detector does not alarm, a second sample can be taken which may result in an alarm).

Last, after the samples had been in a moist chamber for $>30$ days, mold would grow on the substrate surfaces, especially denim and leather, and it was difficult to see the crystals through the mold growth. Further work could be performed to look at other technologies to determine binding properties of the explosives.

This information was presented to Department of Homeland Explosives Science and Technology Department. Interest was shown in the work. Currently, there is no external funding to continue this type of research although it appears to be of interest and value to the explosives community. In addition, explosives detection is one of the signature areas for the National and Homeland Security Directorate, and this type of research positions INL as a lead laboratory in explosives detection. 


\title{
Methodologies for the Design, Analysis, and Validation for Operation of Complex Resilient Networks
}

\author{
C. Rieger, V. Venkatasubramanian, Purdue; K. Daum, M. McQueen \\ Developing a rigorous mathematical and computational framework to model and analyze complex \\ resilient distributed control networks
} NS165

Complex networks are highly organized sets of flows of entities such as material, energy, information, money, etc., that have a goal to deliver service levels that maximize some performance metric, such as profitability, service availability, etc. Examples of such networks would be the electrical-power grid, transportation networks, supply chains, advanced nuclear fuel cycles (NFC), and so on. Such networks are national, even global, in scope. Highly regulated, they require traceability, are integrated with power systems, and have to adjust to supply, demand, and technological disruptions to ensure competitive products, services, and prices on timely deliveries. The ability to bring resilience to complex networks is, in general, an immature research area that is of growing interest. To provide a broader perspective on the research in this area, an attachment is provided that represents the "stateof-the-present" from the perspective of two conferences in the area to complement the more focused research presented in the remainder of this report.

In such an uncertain environment, the ability to identify and monitor complex interconnections and emergent properties and to assess the current state, diagnose anomalous or abnormal events, and autonomously reconfigure the network is vital to guarantee service and manage profitability. When these disruptions occur, strategic, operational, and tactical decisions involving engineering, management, and information layers will have to be executed. In the design and analysis of such networks, efficiency, robustness, and cost are critical performance metrics. Efficiency measures how well the network performs under given constraints. Robustness measures how well the network will perform when parts of it are taken down due to various disruptions, while cost considers not only the procurement cost of the system, but also the cost to keep the system performing up to the requirements. Resilient networks are both robust and efficient.

The goal here is to develop a rigorous mathematical and computational framework to model and analyze complex resilient distributed control networks so that one can systematically examine the effect of design and operational factors on performance metrics for various efficiency/robustness trade-off scenarios and their cost-benefit analysis. These descriptions, requirements, and metrics will be used by the proposed system toolbox to suggest, optimize, evaluate, and compare topological arrangements for implementing a given networked system.

\section{Technical Objectives}

The technical objectives and project deliverables for this 3 -year project will consist of developing and implementing:

- Understanding how the topological features of a complex resilient network affect performance metrics, such as efficiency, cost, and robustness

- Developing a general mathematical and computational framework for the design of complex resilient decentralized control networks

- Demonstrating these concepts and systems by implementing them in two testbeds that will simulate a real-life resilient network prototypes and scenarios

- Creating papers, presentations and reports to facilitate technology transfer to Idaho National Laboratory (INL).

Results and Accomplishments

This report summarizes the progress made in the first year of the project over a 6-month duration, from April 1 through Sept 30, 2009. We focused on the development of the graph theoretical mathematical framework for modeling and analyzing network topologies. We also developed the Purdue network (PurNETS) simulator testbed prototype.

Graph Theoretical Framework. It is useful to conceptualize a complex network or system as a graph of nodes and edges with various properties, such as costs and capacities. At its core, reconfiguration of a resilient network attempts to generate an optimal deployment of the nodes and edges to optimize performance given a description of current conditions. Reconfiguration can thus occur at the strategic, tactical, and/or operational levels, depending on the nature of the change in the environment. The 
following network metrics have now been implemented in the PurNETS testbed as described in "Complex Resilient Networks Testbeds," on page 4 of this document.

Efficiency of a network. The distance $d(i, j)$ between vertices $i$ and $j$ of a graph is the length of the shortest path between the two vertices. The average path length of a graph is the average distance between any pair of vertices (APSP - all pairs shortest paths):

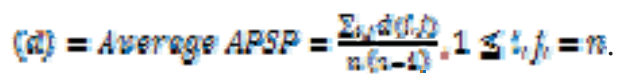

We define the absolute efficiency of a graph as the inverse of its average vertex-vertex distance:

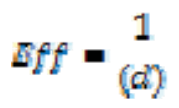

and the normalized efficiency as the ratio of its absolute efficiency to that of a 'Star' network with the same number of vertices:

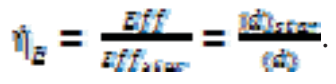

We use the above normalization since the 'Star' configuration, discussed in detail later, is known to give the minimum average path length for an MST.

Robustness of a network. A vertex $j$ is said to be reachable from node $i$ if there exists a path from $i$ to $j$. Let $n_{i}$ be the number of vertices in a strongly connected component $i$ of a graph. Henceforth, we refer to a strongly connected component simply as component. Then $\left(n_{i}-1\right)$ vertices are reachable from any vertex in this component. We define this number as the accessibility of the connected component $i$ :

\section{$\mu_{l}=n-1$.}

The effective accessibility of a graph is then defined as the sum of the accessibilities of its strongly connected components:

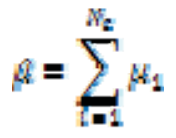

where $N_{c}$ is the total number of strongly connected components.

We define the structural robustness with respect to vertex $j$ of a graph as the ratio of the effective accessibility of the graph $S_{j}$ obtained by deleting vertex $j$ from the original graph to the maximum possible effective accessibility for $S_{j}$ :

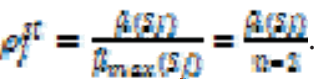

The average-case structural robustness of a graph is defined as the average of the structural robustness values computed over all the vertices:

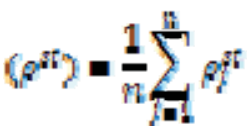

and the worst-case structural robustness as the minimum of the structural robustness values computed over all the vertices:

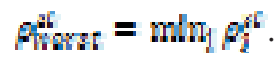

Cost. Now we introduce the notion of cost or resource for the network. In general, both the vertices and edges can have a cost associated with them. However, for a given system of $n$ vertices, the number of edges $e$ available is the amount of resource available to connect the nodes. Since all edges are equal (an assumption in this work, that can be generalized), the total cost is simply $e$ (assuming cost/edge $=1$ ). For a minimum spanning tree, the resource or cost needed is $e_{\min }=n-1$. Thus, when one uses more than the minimum edges needed to form a connected graph, one would incur an extra cost which is modelled as $C(\beta, k)$, where $\beta$ is redundancy. When $C$ is equal to zero, one is providing the system of $n$ vertices with the minimal number of edges required to stay connected, which is $n-1$ (i.e. $\beta=0$ ). When $e=$ $n(n-1) / 2$, one is providing maximum resources, which is the amount required to make a complete graph. This leads to maximum redundancy, with $\beta=1$. Of course, this added redundancy is achieved by incurring the additional cost. Thus, $C$ is also a measure of the economy of the design.

Designing Optimal Complex Networks. Under these conditions, the optimization 
problem may be formulated as follows: For a given environment $\alpha$ and cost functions $c_{1}(\beta, k)$ and $c_{2}(n)$, design a network by evolutionary adaptation that maximizes its survival fitness function $G$ given by:

$$
\max G=\left.\alpha\right|_{E}+(1-\alpha) \hat{q}_{A}-\sigma_{1}(\beta, k)-\sigma_{2}(n)
$$

where $\square$ is a constant, $0 \leq \square \leq 1 c_{1}$ is the cost function related to the addition of edges $c_{2}$ is the cost function related to the addition of nodes $k$ is the vertex degree of the node to which a new edge is being added $\square$ is the redundancy coefficient $n$ is the number of nodes.

This is a general formulation of the topology optimization problem. The $\alpha$ parameter models the environmental or selection pressure on the network. When $\alpha=1$, the survival of the system depends entirely on its efficiency, with no regard for robustness. Similarly, at the other extreme, when $\alpha=0$, the survival is determined entirely by its "worst case" robustness, with no regard for efficiency. For the intermediate values of $\alpha$, the environment demands that the system be both efficient and robust to varying degrees. Thus, by varying $\alpha$ from 0 to 1 , one can impose different selection pressures on the survival of the system and explore the properties of the emergent resilient structures.

These theoretical developments are focused on some specific failure scenarios using the two testbeds as discussed below.

Complex Resilient Networks Testbeds. We propose to use two testbeds, one at Purdue (being implemented) and one at INL (already existing) to test and validate the proposed approach. The Purdue testbed is a more conceptual and abstract environment, focusing on theoretical and algorithmic issues meant for developing and testing various network configurations and algorithms quickly, without getting burdened by domain-specific details. The other testbed will be a more realistic applicationoriented environment for testing, evaluating, and refining the results from the Purdue testbed. We plan to use the CIPR/SIM testbed environment at INL for this purpose.

The overall architecture of the PurNETS prototype that we have implemented is shown in Figure 1.

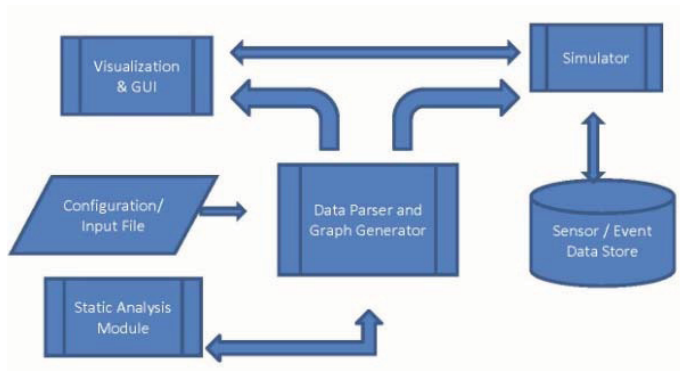

FIGURE 1. Architecture of the PurNETS prototype.

The various modules are:

- Configuration file: This file describes the metadata for the parser to understand. For example, it can suggest the list of acceptable node values, edge weights etc.

- Data Parser and Graph Generator: This module parses the input text file and populates the nodes and graphs based on the metadata filters acquired from the configuration file.

- Static Analysis: This module computes all the static data related to the graph generated by the parser. This analysis can include things like entropy, graph diameter, robustness measures, efficiency, etc.

- Sensor Event Data Store is a simulation event generation data set. Event and control sequences can be loaded into this dataset for the simulator to simulate.

- Simulator: This module uses the event data to run a simulation on the graph and do all the dynamic analysis associated.

- Visualization and GUI provide an interface to visualize the graph and tools to work on this graph and to run the simulation.

PurNETS is implemented in the PYTHON environment. This prototype has a basic user interface which can load a graph from a text file. The text file is self descriptive and has the graph stored as an adjacency list. The graph edges can be unidirectional or bidirectional and at present the only property of the edge is a weight. Different nodes are depicted using different colors. The screen shots in Figure 2 show some of the capabilities of this prototype. 


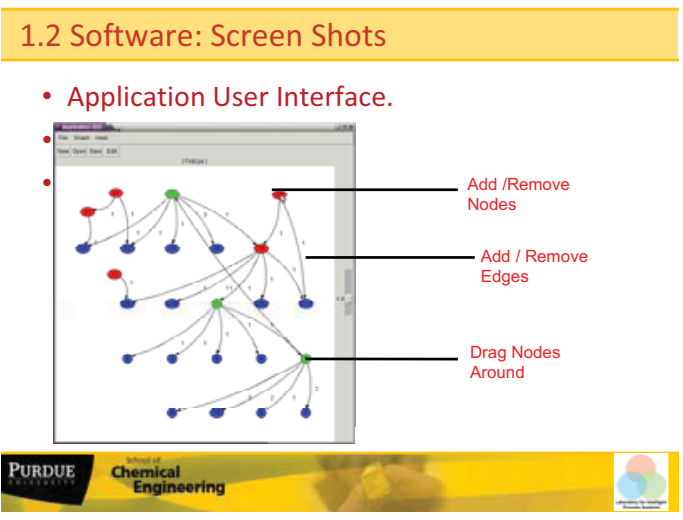

1.2 Software: Screen Shots

- Application User Interface.
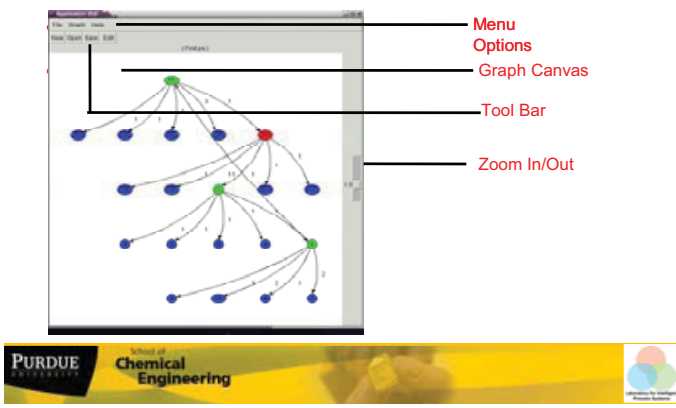

FIGURE 2. Prototype capabilities.

In the next two years of this project, with input from INL personnel, we plan to identify four failure scenarios that are of practical importance for testing and evaluating our resilient network design and control algorithms.

These would be first evaluated on PurNETS, and the promising designs will be then tested in the CIPR/Sim environment. Some candidate scenarios would be: (i) failure of power-station or warehouses or factories or airport-hub or control-system CPUs etc - i.e. node failures and (ii) failure of roadways, transmission lines, etc. i.e. edge failures. We would simulate various combinations of these, for various scenario probabilities, etc.

\section{Summary and Conclusions}

So far, we have accomplished the following:

- Developed the theoretical framework, using complexity science and graph theory concepts, to model, design, analyze, and control networked systems
- Developed the algorithms and representations needed to model and analyze the efficiency, robustness, cost and other metrics for networks

- Implemented the Purdue networks testbed prototype, PurNETS ver 1.0, in the PYTHON environment

- Gained familiarity with CIPR/Sim.

We believe that despite having only six months to work on this project in the first year, we have moved significantly towards the 12month, first-year objectives we had originally listed in the proposal. We are thus confident that we are well positioned to meet the second year objectives for this project. We are excited about the importance of this project and our collaboration with INL personnel. We look forward to developing and contributing new capabilities to INL in the form of mathematical models, computer algorithms and programs, and simulation testbeds for the design, analysis and control of resilient networks.

Attachment to FY 2009 Annual Report for NS165

ICIS Complex Systems Approach September 30, 2009

Miles McQueen and Keith Daum

Key aspects of the research plan for both the Instrumentation Controls and Intelligent Systems (ICIS) Signature Area as well as the N\&HS Applications Base LDRD for vulnerability assessment are to use a complex adaptive systems approach to maintain state awareness, provide a basis for resilient design, and also to assess vulnerabilities that can be caused by the complex nature of these systems. The purpose of attending this conference was to aid assessment of the current state of the art in complex networks and self-adaptive systems research, and evaluate whether these concepts could be applied to resilient control system research at INL. In order to (1) gain a cutting edge perspective of the current state of the art in complex networks and self-adaptive systems research, (2) evaluate whether these concepts could be applied to resilient control system research at INL, and (3) to make strategic contacts with internationally 
recognized researchers, INL staff participated in two significant symposia in these areas.

* Self-Adaptive and Self-Organizing Systems 2009

* International Workshop on Complex Systems and Networks 2009

This approach complements LDRD work being done by Purdue University in complex systems and assures that promising methods will be taken in future research.

General Thoughts:

1. There are two complex adaptive systems that need to be addressed.

a) The control system.

b) The system that the control system senses, monitors, and controls.

2. To maintain state awareness, the control system will need to constantly adapt to the changing conditions of the monitored system.

3. A resilient control system can absorb severe disturbance and still be adaptable to changing conditions of the monitored system.

Observations from: Self-Adaptive and SelfOrganizing Systems 2009

1. Adaptive systems will be a core part of any resilient electronic and intelligent system.

2. Self organization may be related to the adaptive capacity of a system but is a bit less directly connected to resilience.

3. Research work on self-adaptive systems ranges from application oriented with the intent of being field tested and delivered in short order, to highly theoretical, theorem proving, foundational work.

4. The research work which is focused on applications is particularly satisfying since it indicates a beginning maturity which may allow for real systems to more fully adopt self-adaptive mechanisms and the possibility of explicitly designing in adaptive processes so that a system will be inherently more resilient.

5. A simple instance of a self-adaptive system, which surprisingly had not been explicitly designed before, addressed the difficulty of tracking a moving vehicle. As the vehicle moves, there is noise in the environment and system sensors, and there may be momentary cover and obfuscation so a Kalman filter is used to help estimate the vehicle's location. Simple, elegant, adaptive, and useful implementation of a self adaptive system.

6. Some approaches to resilience and the design of complex networks might find value in mechanisms to reason backwards from system events to predict the location of adversarial compromises of the system and forward reasoning to then predict the components where the next set of compromises might take place.

7. Development of new, more resilient, consensus protocols and designs will aid the realization of robust adaptive control systems. A recent design of a more robust distributed consensus algorithm was accomplished through the judicious application of evolutionary computation (EC) techniques. Together with other engineering design problems this demonstrates the feasibility and value of using EC to develop new technological solutions to difficult problems. The application of EC to the development of new more resilient networks and associated functions appears to have merit and should be utilized when confronting known difficult problems that can be formed to fit within the EC construct.

8. The field of self-adaptive and selforganizing systems continues to mature and that with maturity the techniques are finding success in meeting the needs in a wide set of application domains. In particular the application of EC techniques does seem quite applicable to the design of complex networks for resilience and other related properties. 
9. Key contacts from the meeting:

a) Dr. Sukumar Ghosh Self-* properties, University of Iowa

b) Dr. Sven A. Brueckner IED Vector Research Center, a division of TechTeam Government Solutions, Inc.

Observations from: International Workshop on Complex Systems and Networks 2009

1. In the age of distributed information, a centralized system cannot control decentralized system. A distributed brain or organization is
a) More resilient
b) More likely to pick up weak signals distributed components.

2. In a resilient control system there is a role for the social networks that interact with both the control system and the monitored system.

3. Novel emergent properties of a control system result from multiple use of versatile conserved multifunctionary core components that allow them to be easily connected and recombined in many ways toward various ends. Novelty is usually not the invention of a new process.
4. Conserved processes are core processes.

a) Can facilitate changes in processes around them.

b) The system cannot live without a core process.

c) They are linked to other processes and can used in combinations.

d) Core processes are like logo blocks. Blocks don't change, but arrangement does.

5. In resilient multifunctionary systems, there are no stereotypical roles for producer and consumer. A diverse group of agents often fill both roles as a "prosumer."

6. Key contacts from the meeting:

a) Prof. David K. Arrowsmith, Queen Mary, University of London.

Responsible for EU initiative on grid resiliency and security, including the incorporation of intermittent low-carbon energy technologies.

b) Prof. David John Hill Australian National University. Responsible for EU initiative on grid resiliency and security, including the incorporation of intermittent low-carbon energy technologies. 


\section{Cognitive Network Engine and Simulation Framework}

Juan Deaton

Developing methodologies for creating cognitive networks

PH101

This Ph.D. candidate program seeks to develop methodologies for creating cognitive networks. Cognitive networks consider networklevel goals and coordination of multiple radios or elements to optimize network performance rather than links between pairs of radios. These networks have wide application to Department of Energy (DOE) and Department of Defense (DOD) applications.

In 1997, the President's advisors on science and technology concluded that wireless sensors could improve energy efficiency by $10 \%$ and reduce emissions by $25 \%$ or more. Additionally, a DOE-sponsored report highlighted the need for future industrial wireless systems to be become self-configuring, self-calibrating, selfidentifying, and self-reorganizing for optimal network performance and fault recovery. With the expected increase of wireless sensors in unlicensed spectra for applications such as smart grid, the need for cognitive networking techniques will become more important to manage dynamic spectrum access.

The DOD faces similar challenges as well. Next generation warfighter communications should be capable of dynamically adapting communications to compensate for spectrum availability and interference. This is why the Army's Communications-Electronics Research, Development, and Engineering Center (CERDEC) issued a call for proposals for cognitive network concepts. Additionally, DARPA's Next Generation (XG) and Wireless Network After Next (WNAN) are both programs which seek to advance cognitive networking concepts.

Therefore, leveraging the $\mathrm{Ph} . \mathrm{D}$. candidate at Virginia Tech, a lead university in cognitive networking research, and Idaho National Laboratory's (INL's) unique capabilities with the wireless test bed, this project seeks to identify methodologies for developing cognitive networks. The Ph.D. expertise in an important research area will have applications for the next 10 years or more in areas relevant to homelandsecurity missions with the Wireless Test Bed and Critical Infrastructure Protection. In terms of specific research, this involves researching a problem relevant to both DOE and DOD missions: channel assignment. Channel assignment is the problem of how to assign available spectrum channels and transmit powers to a wireless network such that the network topology can be formed.

\section{Technical Objectives}

The overall objective in Fiscal Year 2009 (FY-09) was to successfully complete classes and begin research outlined below:

Objectives in research are the following:

- Evaluation study of channel assignment algorithms

- One conference publication from research.

Results and Accomplishments

The channel-assignment study resulted in a novel evaluation technique for channelassignment algorithms and a simulation model with five comparative algorithms and a conference publication at the IC $3 \mathrm{~N}$ conference CogNets (Cognitive Networks) workshop. Additionally, an expansion of the work was requested for a special issue of the Springer Journal Wireless Personal Communications, "Cognitive Radio Networks and Communications." This journal publication should be completed early November 2009.

Summary and Conclusions

Cognitive-networks research is in line with INL, DOE, and DOD missions. The comparative analysis on channel-assignment algorithms will enable network developers to understand the tradeoffs when considering multichannel networks which have application to DOE and DOD missions. In terms of publications, one conference paper was published in FY-08, with two other papers expected to be completed this upcoming year. The invited journal paper and a paper on developing work using swarm intelligence and the mobile channel-assignment problem. The swarm-intelligence paper will address a more difficult problem of mobile channel assignment and is relevant to nationaland homeland-security missions. 


\section{Application of Dynamic Bayesian Networks to Systems with Ambient Intelligence}

Dana L. Kelly, Aarnout Brombacher

Demonstrate the practical application of dynamic Bayesian networks within an ambient intelligent system

PH102

The objective of this work is to demonstrate the practical application of dynamic Bayesian networks within an ambient intelligent system. Ambient intelligent systems possess the following attributes: First, they are contextaware, meaning they can recognize individuals within a particular situational context. Second, they are deeply customizable, tailored towards changing individual needs over time. Third, they are adaptive in their responses to inputs from the environment, allowing them to anticipate an individual's desires or intentions without conscious mediation. Finally, they are deeply embedded, integrated into the environment in a network context.

Major challenges to the development and deployment of practical ambient intelligent systems are the ambiguous, uncertain, and changing nature of sensor inputs; the changing state of the user; and constraints on available resources for making decisions. Dynamic Bayesian networks have the ability to model changing states of the environment and the user, and to provide appropriate inference about current states, along with predictions of future states, as an aid to decision-making. Thus, in principle, they can address all three of the major challenges listed above. However, to date, there has been only limited work done on practical application. This work will apply dynamic Bayesian networks to one or more prototype systems via a series of case studies. If this work is successful, it will demonstrate the capability of dynamic Bayesian networks as a practical tool for overcoming the challenges listed above and will further the application of ambient intelligent systems to solve difficult and vital problems in nuclear nonproliferation, facility security, patient monitoring, air traffic control, and electric grid reliability. In the vital arena of air traffic control, for example, ambient intelligent systems could provide a novel approach to reducing delays caused by the currently overloaded system. Sensors onboard planes, satellites, and ground tracking stations would provide real-time inputs to the system, which could then take action by providing signals to individual plane navigation systems, allowing problems to be circumvented before they can fully manifest themselves.

\section{Technical Objectives}

The direction of the research changed a bit from the original plan, as the Technische Universiteit (TU)/Eindhoven advisor became more interested in how to mine weak signals from a data-rich environment. We decided to explore an application of process mining to analysis of human-performance data collected at the Halden Reactor Project and explore how process mining of simulator data could enhance human-reliability analysis (HRA). Process mining uses Petri nets as the underlying model, rather than dynamic Bayesian networks, and the research utilizes open-source software for process mining developed at TU/Eindhoven.

The Idaho National Laboratory (INL) principal investigator (PI) met with researchers at TU/Eindhoven and the Halden Reactor Project to map out a path forward for this research. Data from a past experiment were supplied by Halden for a proof-of-principle analysis. This analysis was successful, the results were presented to TU/Eindhoven and Halden, and plans were made for follow-on research using data from additional experiments. The preliminary results will be presented at a Committee on Safety of Nuclear Installations (CSNI) workshop on the use of simulator data for HRA purposes, to be held in Budapest in November 2009.

\section{Results and Accomplishments}

As noted above, the research direction changed somewhat from the original hypothesis of applying dynamic Bayesian networks to applications in which data are sparse, to using process mining via an underlying Petri net model to those in which there is an over-abundance of data. The proof-of-principle study, using data from the Halden Reactor Project, was generally successful, and further studies with Halden have been planned for the coming fiscal year (FY). An immediate benefit of this work is that it has the potential for application in a recently inaugurated project with the Nuclear Regulatory Commission (NRC) and Electric Power Research Institute (EPRI) to conduct simulator studies at the South Texas Project, as an extension to the NRC's International HRA Benchmark Exercise. As a result of this research, INL has gained a new capability (process mining), which we were 
lacking before this effort. We now also have closer international ties with researchers at TU/Eindhoven and the Halden Reactor Project. The follow-on work planned for FY-10 has the potential to make a highly visible contribution to HRA.

\section{Summary and Conclusions}

At this point, we have accomplished a proofof-principle demonstration of the feasibility of mining simulator data in support of HRA.

Follow-on efforts will explore the application of process mining to larger and more complex datasets and to data collected in a recently inaugurated project with the NRC and EPRI to conduct simulator studies at the South Texas Project. The proof-of-principle results will be presented at a CSNI workshop on the use of simulator data for HRA purposes, to be held in Budapest in November 2009. Follow-on work will be presented in a follow-on workshop to be held in the U.S. and in a refereed article in Reliability Engineering and System Safety. 


\section{PAGE FOR SCIENCE AND TECHNOLOGY DIVIDER}

Science and Technology 


\title{
Thermal and Acid Activated In-plant Lignocellulose-degrading System for Eonomically Enhanced Industrial Processes
}

\author{
Jeffrey A. Lacey, David W. Reed, Thomas H. Ulrich \\ Engineering plant-produced enzymes to more readily degrade lignocellulose \\ BS105
}

The objective of this project was to engineer a plant that would produce its own lignocellulose-degrading enzymes that would help process the plant's biomass for fuel and chemical conversions. Current methods for preprocessing of biomass require that lignocellulose-degrading enzymes be added to make the cellulose available for downstream biochemical reactions. These added enzymes must be produced in a separate reactor, increasing overall costs. A plant-produced enzyme would be advantageous because it would be produced by the plant during its normal growth phase, and it would already be in the plant, ready to degrade the lignocellulose.

\section{Technical Objectives}

- Build a gene construct containing the proper gene promoter, signal peptide, cellulase/xylanase enzyme, and reporter gene.

- Insert this construct into the genome of the model plant, Arabidopsis thaliana.

- Monitor expression of the gene construct using fluorescence microscopy.

- Measure the enzyme's activity in plant tissue extracts.

\section{Results and Accomplishments}

The gene construct was assembled and inserted into a binary plasmid vector capable of inserting the construct into Arabidopsis (Fig. 1). This binary vector was introduced into Agrobacterium tumifaciens, a bacterium capable of inserting the vector into Arabidopsis. Plants were inoculated with this bacterium and seeds were collected and screened using antibiotic (hygromycin) selection. Thousands of potential transgenic seeds have been screened; however, only one seed was found to be hygromycin resistant, indicating that it may have the construct inserted into its genome. DNA was extracted from one of this plant's leaves, and the polymerase chain reaction (PCR) with primers specific to the designed construct were used to verify the presence of the gene construct in the plant (Fig. 2, next page). We have successfully inserted the gene construct into an Arabidopsis plant.

Seeds were collected from this plant, and they too were resistant to the antibiotic, indicating they had inherited the gene construct from the parental plant.

Microscopy was done to determine if the reporter gene was being expressed in the plant tissue; however, we were unable to detect the red fluorescent protein (RFP) in the leaf tissue due to high levels of autofluorescence. Root tissue was also screened. However, the RFP could not be identified in this tissue, and autofluorescence was still a problem.

Protein gels and Western blots were done with an RFP-specific antibody. While we were able to detect a bacterially produced RFP, we could not detect an RFP protein band in the plant samples. Since the plant-produced RFP could not be detected using fluorescent microscopy or Western blots, it was assumed that the cellulase enzyme was not being produced. No enzyme activity tests were done on this plant tissue.

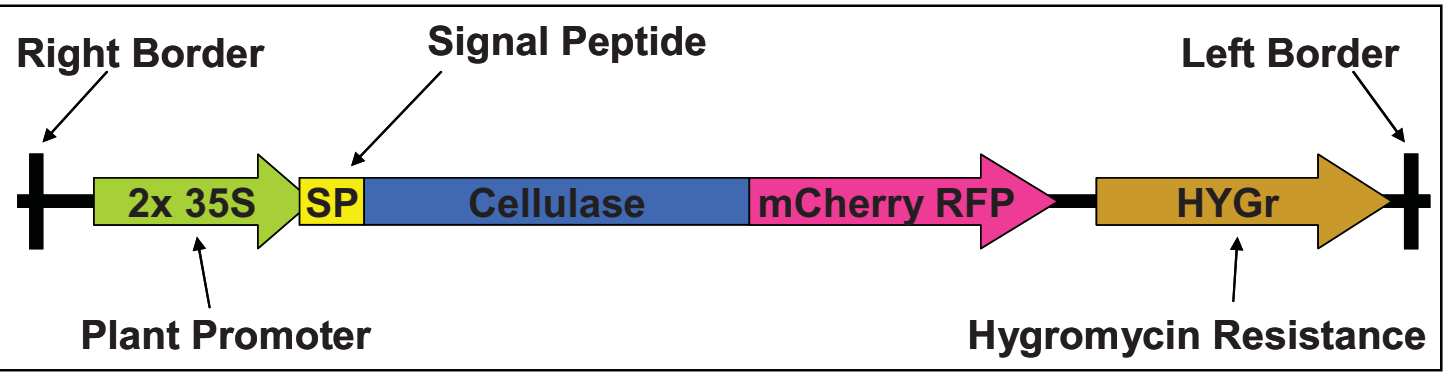

FIGURE 1. Gene construct designed to produce lignocellulose-degrading enzymes in plant tissue. Plant promoter was two copies of the $35 \mathrm{~S}$ califlower mosaic virus promoter, and the $m$ Cherry red fluorescent protein was used as a reporter gene as its optimal pH was better suited for the conditions in the apoplast. A hygromycin resistance gene was used to screen seedlings for construct insertion. 


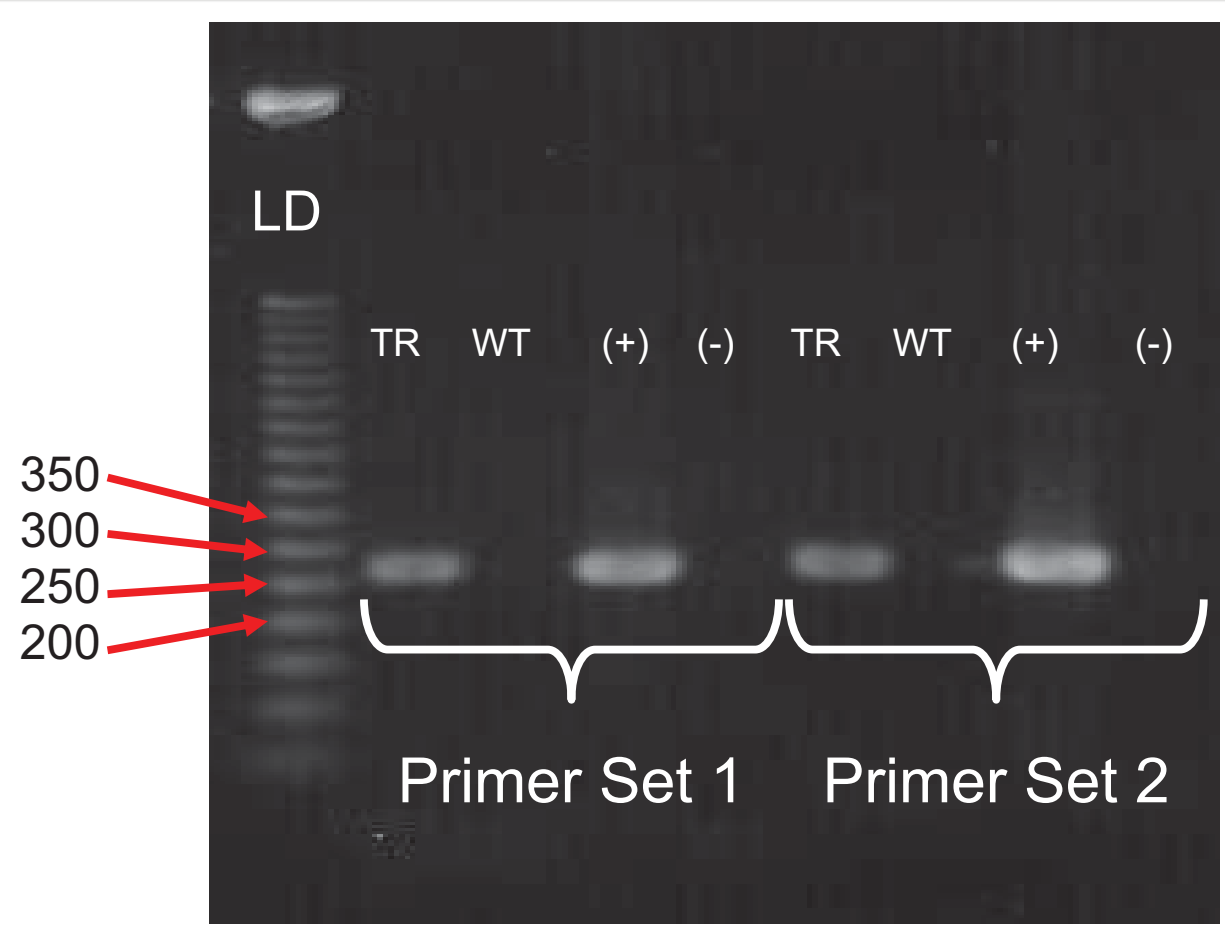

FIGURE 2. Agarose gel (0.7\%) showing results from the PCR verification of the presence of the designed construct in Arabidopsis DNA. PCR products were generated in lanes containing transgenic plant material and plasmid DNA containing the plasmid construct. $P C R$ products were not obtained using wild type plant tissue. $L D=50 \mathrm{bp} D N A$ Ladder, $T R=$ transgenic plant, WT $=$ wild type plant, $(+)=$ positive control (plasmid construct), $(-)=$ negative control .

\section{Summary and Conclusions}

While we were successful at introducing this gene construct into Arabidopsis, we were not able to get this plant to express the protein. We were also only able to get the gene into a single plant. When gene constructs are inserted into plant genomes, they can end up in places on the chromosomes where their expression is somehow inhibited. Ideally, we would have had several transgenic plants to work with, as this would have increased the chances of getting our proteins expressed. While our plant inoculation methods were good enough to get the construct into one plant, they were less successful than they should have been and will need to be improved for future work.
At this point we are working to identify a suitable industrial partner to advance this work. Two companies have been identified that have capabilities to introduce genes into a variety of model and agriculture plants. Plans are being made to submit a proposal to a joint Department of Energy (DOE)/U.S. Department of Agriculture (USDA) biomass call. 


\title{
Novel Catalysts for the Reduction of Biomass Pretreatment Severity
}

\author{
David N. Thompson, John R. Klaehn, Mason K. Harrup \\ Develop and synthesize templated hbrid organic-inorganic materials capable of selectively binding \\ water-soluble xylooligomers \\ BS106
}

Lignocellulosic residues are a valuable renewable biomass resource, yet billions of tons of utilizable lignocellulosic residues go to waste in the U.S. each year. Given our country's dependence on foreign oil and limited domestic oil reserves, development of alternate fuel and chemical sources is at the forefront of a number of initiatives to increase the impact of renewable, carbon-neutral fuels on the energy security of the U.S. Currently, hemicellulase enzymes are applied to assist in completing the hydrolysis of the soluble polysaccharide oligomers produced using reduced severity pretreatments. However, this requires lowering the temperature to levels compatible with commercial enzymes (ca. 50 ${ }^{\circ} \mathrm{C}$ ), and many of these enzymes have a high cost and can only be used once for this process. Therefore, reusable, oligosaccharide-templated materials that simulate these processes could help reduce the capital and operating costs for the transformation of hemicellulase to usable polysaccharide oligomers. Template-directed mesoporous silicates offer an alternative to achieve catalytic or binding materials. The resulting materials from this synthesis cast the shape and position of the functional groups of biologically- active molecules in a pliable form so that it has a high affinity for the target $\beta-1,4-$ linked polysaccharide oligomers. Overall, the resulting products are novel and have not been reported.

Our approach for such materials utilized current mesoporous organosilicate synthetic techniques that potentially enhance the xylooligomer binding (Fig. 1). Soluble xylooligomers or cellooligomers are bound to an organosilane metal-complex monomer, and the resulting complex used to surface template the pores of the silica as they are formed. The resulting periodic mesoporous organosilicate (PMO) macroscopic solid supports were synthesized which potentially recognize and improve binding of soluble xylooligomers and cellooligomers. Our synthetic method can enable surface-templating at the pores of mesoporous silicate resulting in robust binding with high activity per unit volume (large surface area). In general, these PMO materials should be specific and operate under conditions that would adversely affect the activity (i.e. high or low temperature, organic solvents). In addition, the mesoporous silicates should have a long shelflife without needing specialized storage and remain active after continuous use.

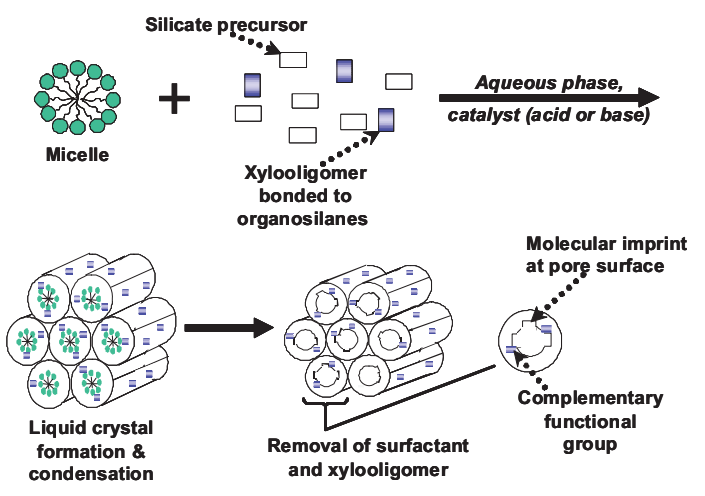

FIGURE 1. Surface Templating of Periodic Mesoporous Organosilicates (PMO).

\section{Technical Objectives}

The objectives of the work were limited to proof-of-principal.

- Develop and synthesize templated hybrid organic-inorganic materials capable of selectively binding water-soluble xylooligomers and can be recovered from the reaction mixture without the use of expensive membrane separations

- Test the resulting products for the binding of xylooligomers from aqueous solutions.

\section{Results and Accomplishments}

The focus of this Blue Sky project was the development of periodic mesoporous organosilicate (PMO) which have selective binding towards xylooligomers. The general synthetic procedure is depicted in Figure 1. In this method, soluble xylooligomers are bound to the organosilane metal-complex monomer, and the resulting complex used to imprint the pores of the silica as they are formed (in-situ). An imprint molecule-spacer complex (xylooligomer bonded to organosilanes) was thoroughly mixed with a surfactant while retaining an ordered phase. The imprint molecule was brought into contact with the pores by amino-functionalized alkoxysilanes as a similar copper metal-complex has been shown to strongly bind polysaccharides under alkaline conditions (same as the PMO's synthesis). This alkaline environment provided 
polysaccharide binding to the copper complex, therefore resulting in imprinted PMOs. The alkoxysilane end of the tether interacts with 1,2bis(triethoxysilyl)-ethane (BTEE; the bulk organosilicate precursor) while the functional group of the tether holds on to the imprint molecule. As the BTEE condenses around the imprint molecule at the surface, a functionalized imprinted binding site complementary to a specific substrate is formed. By imprinting the PMO pores and utilizing the pore sizes, the silicates can have controlled structures with a large number of binding sites. The imprint that is left behind in the PMO should have the size, shape and functional groups matching the specific substrate molecules. Some of the imprint molecule-spacer complexes will end up in the interior of the silicate but some will also end up at the surfaces of the silicate. Removal of the surfactant was carried out by acidic solvent extraction, which left behind the PMO with the imprinted sites without the bound copper. Copper ions were re-introduced later for the binding experiments. An interesting feature of these PMO reaction solutions are that they give an intense dark blue color when adding the copper bound amines, yet these colors change (to yellow) as the $\mathrm{pH}$ is altered by the acid solvent extraction. After isolation, the final PMOs are feathery, white powders.

Due to the complexity of the final PMO synthesis, it required the PMOs to be isolated at several different stages. The PMOs were split into two different categories using two surfactants, CTABr (cetyltrimethylammonium bromide) and ODTMA (octadecyltrimethylammonium bromide). The reason these surfactants were chosen was to determine if a longer alkyl chain will affect the isolated products. From these two categories the following PMOs were synthesized: plain PMO (no amine functional groups); $\mathrm{PMO}$ with 15 mol $\%$ of amine functional group; PMO with 15 mol\% amine functional group chelated (complexed) to copper; and PMO with $15 \mathrm{~mol} \%$ amine functional group complexed to copper which are bound to the xylooligomer (xylotriose and xylobiose). All of these PMOs were isolated under the same protocol without difficulty. This large array of PMO products was needed to provide evidence that there is a preferential binding behavior towards the xylooligomer(s) using the functionalized PMO complexed with copper AND templated with the xylooligomer. These reactions were completed in triplicate, therefore three sets of four PMOs for each surfactant was isolated. Lastly, 70-230 mesh chromatography silica was used as a control for these binding experiments. Sometimes, the high surface area found with chromatography silica exhibits similar binding behavior as the PMO products.

The synthesized PMO materials were analyzed by gas adsorption (BET) and scanning electron microscopy (SEM). The BET gives the surface area and pore size of the PMOs, and all synthesized PMOs had surface areas ranging between $1000-1300 \mathrm{~m}^{2} / \mathrm{g}$. These surface areas are very large, comparable to activated carbon. However, the pore diameter of these materials were small, between $25-30 \AA$. Nonetheless, the large surface areas of the PMOs provide a large number of binding sites. The SEM pictures, like Figure 2, supplied further evidence that the PMO materials are highly porous. Overall, the SEM pictures of the PMOs show irregular monoliths with textured surfaces. The SEM micrographs are similar to many other PMOs and mesoporous silicates found throughout literature.

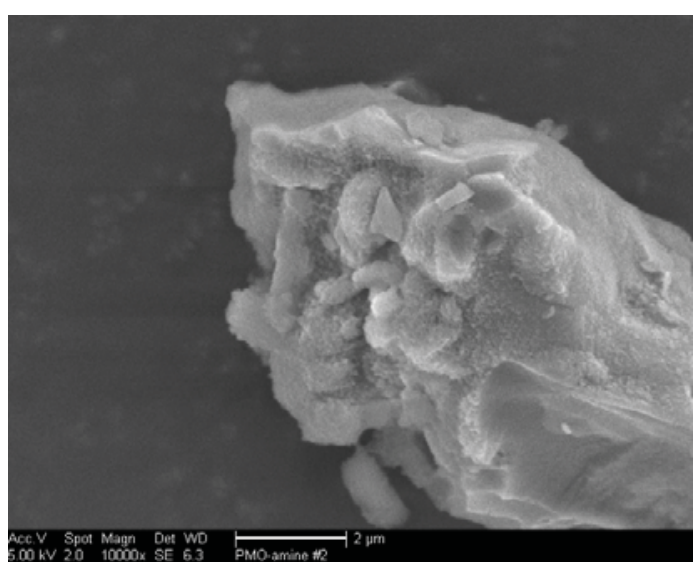

FIGURE 2. SEM of functionalized PMO with amine groups.

There were three different binding experiments done with the isolated PMO materials: 1) aqueous copper sorption/chelation (Fig. 3); 2) aqueous xylooligomer binding with PMOs without sorbed/chelated copper (Fig. 4; magenta columns); and 3) aqueous xylooligomer 
binding with PMOs that have sorbed/chelated copper (Fig. 4; beige columns). All of the PMOs and silica were pretreated with an aqueous solution of sodium carbonate $(\mathrm{pH}=\sim 8)$ to neutralize the acidic sites on the surface of the materials. The isolated materials were washed with nanopure water and air dried. 0.5 grams were added to $10 \mathrm{mLs}$ of a stock copper solution $(\sim 10 \mathrm{mg} / \mathrm{mL})$ and stirred for 24 hours. The aqueous solutions were isolated for copper binding analysis that was performed by Inductively Coupled Plasma (ICP). The PMO materials from these copper solutions were isolated for the xylooligomer binding analysis. The solids were washed with nanopure water and air dried. 0.25 grams were added to $10 \mathrm{mLs}$ xylooligomer solution $(\sim 5 \mathrm{mg} / \mathrm{mL})$ and stirred for 24 hours. This experiment was repeated with the neutralized materials without the copper present. The number scheme and labels for the samples tested are shown in Figure 3, and they are the same for Figure 4. PMO samples 2-7 and 13-15 used ODTMA as the surfactant, and PMO samples 8-12 used CTABr as the surfactant.

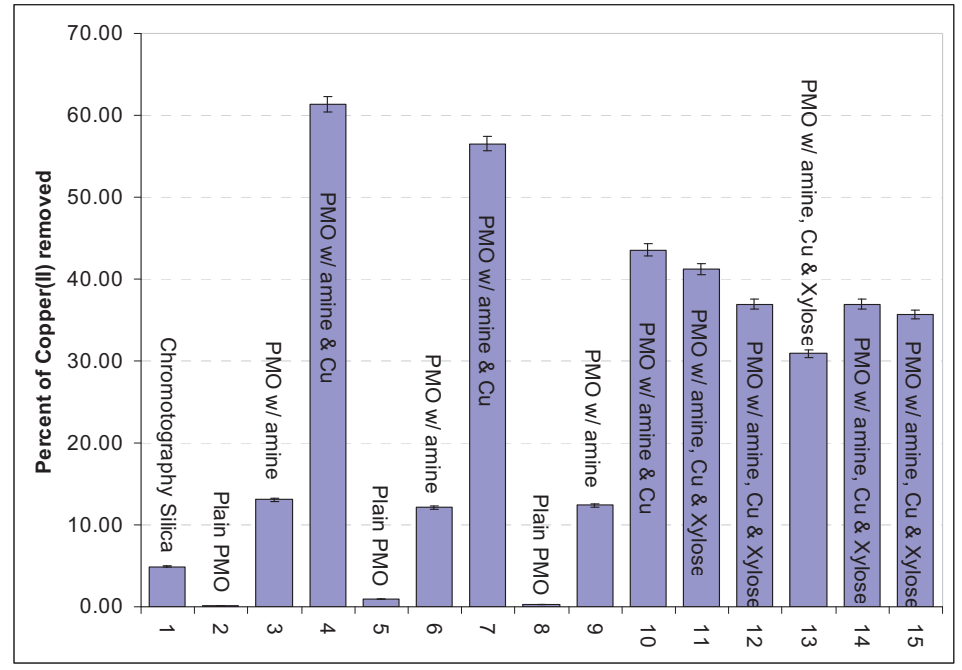

FIGURE 3. Percent of copper uptake from solution. (The number scheme (x-axis) for the samples is the same for both analyses.)

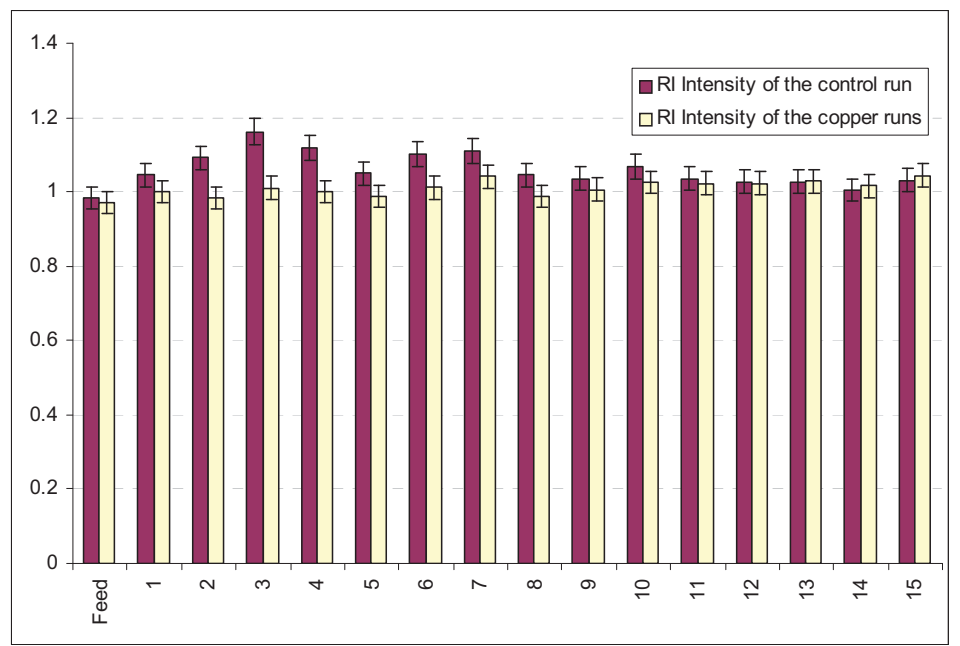

FIGURE 4. Xylooligomer Binding from Solution (magenta columns - no copper bound to PMOs; beige columns - copper bound to PMOs). (The number scheme ( $x$-axis) for the samples is the same for both analyses.) 
Figure 3 presents the percent of removed (uptake) copper relative to the stock solution. It is interesting to note that chromatography silica (1) sorbed copper, even though there were no amine binding sites present. PMO data (2-15) reveals that in-situ complexed copper on the amine functional group $(4,7$, and $10-15)$ is very important for accessibility and recognition of the binding sites at the surface of the PMO. This difference is unequivocal and provides almost complete copper site coverage at the PMO surface. However, the number of accessible copper sites decrease as the xylooligomer is bound (11-15) to the copper complex. This is possibly due to some fraction of the large xylooligomer complexes being incorporated into the PMO matrix reducing the number of accessible sites. Figure 4 shows the refractive index (RI) intensity of the PMO xylooligomer solutions relative to the stock xylooligomer solution (feed). When no copper is bound to the PMOs (magenta column), the RI actually increases compared to the stock solution. It is possible that the tested materials sorbed water from the xylooligomer solution causing the xylooligomer concentration to increase (instead of decrease). This phenomenon was also seen with the copper bound (complexed) PMOs. Overall, there are no real differences observed in the xylooligomer binding, whether the copper is bound or not. This could be related to the pore diameter size, suggesting $25-30 \AA$ is not sufficient area for the xylooligomer to bind, but it is large enough for copper ions to do so.

\section{Conclusions}

We conclude that current INL synthetic route can be used to produce functionalized PMOs that have a high capacity for binding copper ions. Our isolated products, $\mathbf{4}$ and $\mathbf{7}$, have great copper ion sorption compared to all of the others. It should be noted that products $\mathbf{4}$ and $\mathbf{7}$ can sorb/chelate nearly its theoretical maximum amount of copper ions from solution. The INL route to binding xylooligomers is novel; however, variation of the synthetic conditions is needed to yield PMOs with larger pore diameters, which in turn may bind the xylooligomers with greater efficiency. In addition, the current PMO synthesis should be investigated further for possible selective metal binding (e.g., $\mathrm{Cu}$ vs. Ni). A submission to the peer-reviewed journal Microporous and Mesoporous Materials is planned discussing the PMOs with high efficiency copper binding results. The patent literature is also being searched to determine whether there is sufficient novelty with this method to warrant submission of an Invention Disclosure Record. 


\title{
Particle-discrete Element Model Simulation of the Coupling between Material Failure/Deformation and Fluid Generation/Flow
}

\author{
Paul Meakin \\ Developing a better understanding of the generation of oil from oil shale
}

FF105

The purpose of this research is to develop a better understanding of the coupling between mechanical processes and other processes that control or are controlled by material deformation and fracturing. Although the theoretical and computational methods under development in this project could find a wide range of applications, the focus is on subsurface energy and environmental applications. In particular, development of a better understanding of the generation of oil from oil shale is a target for this research. On the order of a trillion barrels of oil could be produced from the United States oil shale resource, and a better understanding of processes that occur when oil is extracted from oil shale could improve recovery, reduce the amount of energy required to produce oil from oil shale, and reduce the environmental impacts. This research is being performed in collaboration with the Center for the Physics of Geological Processes at the University of Oslo (PGP), where the focus is on primary migration (the natural generation of oil in source rocks and its expulsion into secondary migration pathways). In the production of oil from oil shale, the natural primary migration process is accelerated by a factor of about one million by heating the organic-rich shale to temperatures that are higher than those encountered during primary migration.

In FY 2009, the collaboration with PGP allowed us to obtain information from preliminary high-resolution synchrotron X-ray tomography experiments at the European Synchrotron Radiation Facility (ESRF) in Villigen, Switzerland (the Swiss Light Source). These preliminary experiments demonstrated that synchrotron X-ray tomography can be used to characterize the micromorphology of oil shale and locate fractures that are generated by heating. Follow-on experiments at European Synchrotron Radiation Facility

(ESRF) in Grenoble, France, will provide key information that will be used to evaluate our computer simulations.

\section{Technical Objectives}

- In collaboration with PGP and Hai Huang (INL), develop computer code to simulate the coupling between fluid generation, geomechanics, and fluid flow
- Work with Idaho National Laboratory (INL) and PGP experimentalists and PGP field geologists to obtain information needed to validate computer codes

- Publish experiment and computational results in high impact geology, physics and energy journals.

\section{Results and Accomplishments}

The computational work has focused on a combination of discrete element models to simulate material failure and deformation with particle models for single- and multi-phase fluid flow and simple (first order kinetics) models for the production of hydrocarbon fluids by decomposing kerogen. Figure 1 (next page) shows preliminary simulations performed by a combination of a discrete element model (DEM) geomechanics model, a dissipative particle (DPD) model for fluid dynamics, and a simple decomposition kinetics model. I am in the process of developing a smoothed particle hydrodynamics (SPH)/DEM model.

An important goal for this project is to compare simulations and experiments, and high resolution $\mathrm{X}$-ray tomography is expected to play an important role in this endeavor. Figure 2 (next page)shows images of Mahogany Formation Green River Shale that was heated to generate fractures. These images were obtained by Francois Renard (one of my University of Oslo colleagues) and two of his graduate students at the Swiss Light Source (ESRF in Villigen, Switzerland) using Mahogany Formation Green River Shale. Shale (marlstone) from the Mahogany Formation is the most kerogen-rich component of the Green River Shale, and commercial development is most likely to begin with extraction of oil from the Mahogany Formation. Access to ESRF is obtained through a competitive proposal process. These preliminary studies indicate that high-resolution synchrotron $\mathrm{X}$-ray tomography can be used to investigate the microstructure of oil shale and microfractures formed by fluid generation. The samples were discs with diameters of $L \approx 1 \mathrm{~cm}$. The volex size is $L / 2048(\approx 4.9 \mu \mathrm{m})$, and resolutions below $4.9 \mu \mathrm{m}$ can easily be achieved by using smaller samples. 


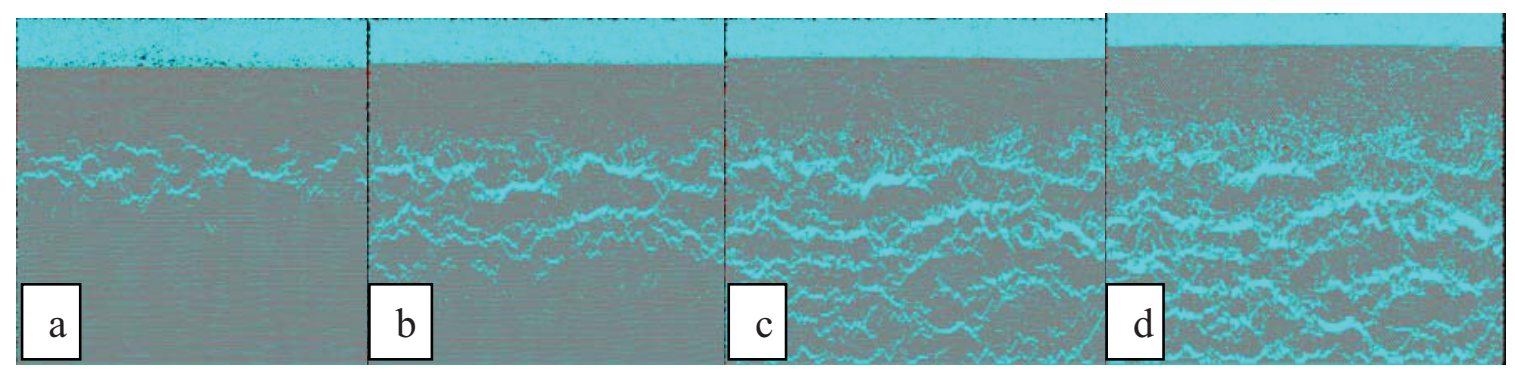

FIGURE 1. A two-dimensional DEM/DPD model for fracturing due to fluid generation. Gravity acts vertically; periodic boundary conditions are used in the lateral direction and the bottom boundary is closed. Parts a-d show different stages as the amount of fluid increases.
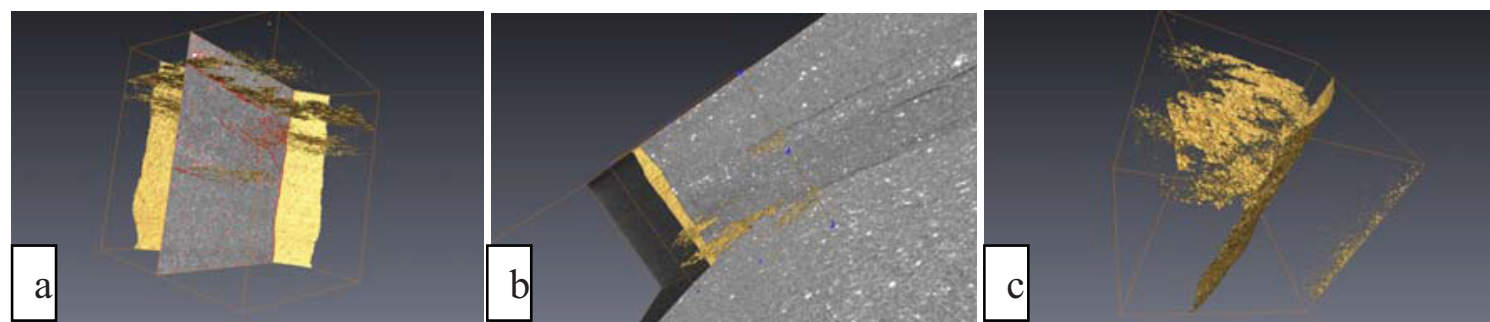

FIGURE 2. High resolution $(4.9 \mu \mathrm{m} \times 4.9 \mu \mathrm{m} \times 4.9 \mu \mathrm{m}$ voxel) synchrotron X-ray tomography images of Mahogany Formation Green River Shale that was heated to generate fluid and fracture the shale. From Francois Renard (Joseph Fourier University of Grenoble and $P G P)$.

A presentation titled "Coupling between fluid generation, fluid flow, deformation and fracturing in subsurface porous media: Discrete element, particle and continuum methods" by Paul Meakin, Hai Huang (INL) and Anders Malthe-Sorenssen (PGP) was delivered on December 17, 2008, at the American Geophysical Union Meeting in San Francisco.

A presentation titled "Fluid generation and flow in organic rich low permeability rocks" by Paul Meakin, Anders Malthe-Sorenssen(PGP) and Hai Huang (INL) was delivered on May 7 , 2009 , at the $22^{\text {nd }}$ Kongsberg Seminar,

Kongsberg, Norway.

\section{Summary and Conclusions}

This project is closely related to the computational research of Hai Huang (INL) and the experimental work of Carl Palmer (INL) and Earl Mattson (INL). In addition, I am the leader of a Norwegian Research Council

PETROMAKS project on "The mechanisms of primary migration," which supports the research of three Ph.D. students and a postdoctoral associate. We have been sharing computer code, ideas, and results. This work is helping INL to establish a strong position in the modeling and simulation of subsurface systems in which geomechanics plays a critical role. While others have been focusing on contaminate fate and transport (biogeochemistry and transport), we have been focusing on engineering applications in which rock fracturing and deformation is important. Apart from the application to oil shale and primary migration (with possible applications to basin modeling and exploration), many other opportunities exist. These include:

- Geothermal energy (controlled fracturing) carbon sequestration (reservoir integrity and coupling between fracturing and volume changing reactions)

- Shale gas (hydrofracturing and fracture proping)

- Heavy oil recovery (thermal and overburden stress in cyclic steam stimulation and overburden and flow-induced stress in cold heavy oil production with sand)

- In situ partial combustion for coal gas and bitumen production (thermal and mechanical stress) 
- Conventional oil reservoir stimulation (hydrofracturing)

- Reservoir evaluation (predicting barriers to flow)

- $\quad$ Mining. 


\title{
Scaling of Welding Processes
}

\author{
Herschel B. Smartt
}

Exploring deposition of metals in a manner suitable for rapid prototyping or production of relatively large metal components

FF106

Rapid prototyping is a new, quickly maturing technology field. The numerous processes included in the field may be loosely divided into additive and subtractive processes from the perspective of mass either being added or removed from a prototype object. Additive processes involve deposition or fusing of polymeric materials, with limited processes involving ceramics, metals, and other materials. Subtractive processes are actually conventional machining suitably packaged from a marketing standpoint and are not otherwise considered in this work. Additive rapid prototyping processes presently share a common limitation of being suitable for only relatively small components. An objective of this work is to explore deposition of metals in a manner suitable for rapid prototyping, or even production, of relatively large metal components. Specifically, this work is conducting fundamental research on metal transfer in the gas metal arc welding process as a starting point for future development of a metal deposition process for large metal objects.

We hypothesize that it is possible to scale (up or down) metal transfer from a gas metal arc welding process by independently controlling growth of a molten metal drop on the end of a welding electrode and detachment of such a molten metal drop. We will be able to do so by precisely controlling electrical current and voltage during operation of the process. The critical issue is ensuring that a drop does not detach from the electrode prematurely during growth and that the growth phase may be ended by ensuring drop detachment at a desired drop volume. To do so we desire a predictive model of drop detachment as a function of drop size. This requires identification of drop detachment mechanism(s) and quantification of the forces associated with the mechanisms. The perspective from which this work is being conducted is that of identifying and quantifying scaling laws for metal transfer in gas metal arc welding. Heat transfer from the process to the workpiece has previously been well characterized and will not be specifically addressed in this work even though it is obviously necessary to consider heat transfer as part of overall process development.
The gas metal arc welding process involves a continuously fed wire electrode, melted by an electric arc plasma, with molten metal drops transferred from the electrode to the workpiece by one of three transfer modes known as short circuiting, globular, and spray transfer. Early work by Kim, McEligot (Idaho National Laboratory [INL]), and Eagar [1] postulated a static force balance theory governing detachment of a drop from the electrode where detachment generally occurs when the sum of gravitational, aerodynamic, and electromagnetic forces on the drop exceed the surface tension force between the drop and the electrode. However, although they found that the static force balance theory held well for globular transfer, it did not adequately describe spray transfer. This later issue is important in the present work as spray transfer is the dominant transfer mode at high metal deposition rates and especially when deposition quality is of concern. There is also an issue associated with detachment of very small drops. Previous exploratory work at INL showed a minimum drop size independent of electrode diameter. This factor may limit the applicability of the process for fabrication of either small components or fine details on large components.

This work will formulate a dynamic model of the process around drop detachment, building on prior work both at INL and elsewhere, see [2, 3]. Specifically, this work will extend the static force balance theory into the spray transfer mode; this probably will involve including an inertia (or momentum) force associated with flow of mass from the electrode to the drop, a factor identified in previous work [2], but not yet included in the static force balance theory. The results of this work should provide a basis for a drop detachment controller design extending empirical work well into mass transfer rates requiring either very small drops or multiple drop, spray transfer to achieve. In addition to using gas metal arc welding for metal deposition as a rapid prototyping process, this work will provide a basis for extension of the process beyond practical welding limits.

\section{Technical Objectives}

- Design experimental apparatus for data acquisition and control law demonstration 
- Build experimental apparatus

- $\quad$ Formulate dynamic process model and derive control law

- $\quad$ Perform experimental studies to validate and characterize dynamic model and control law

- $\quad$ Present and publish results at the next ASM International Trends in Welding Research conference.

\section{Results and Accomplishments}

The goal of this work is to address net shape fabrication, specifically seeking a means of fabricating metal components. Our long-range programmatic goal is to develop a means of fabricating large metal components that would ordinarily require the investment of significant infrastructure funds and the high energy costs of a foundry. Specifically, we want to be able to fabricate components such as engine blocks, cylinder heads, and crankshafts in a stand-alone "cell." Conceptually, one may think of a very large 3-D printer. The cell will incorporate robotics, computer numerical controlled (CNC) machining, and one or more precision metal deposition processes. This project is specifically investigating potential use of gas metal arc welding as a metal deposition process for fabrication of component fine-scale features. To this end, we need to scale down metal deposition from the process while independently controlling heat transfer to the work piece from the welding arc. We may also use the gas metal arc welding process for high metal deposition rates, but that is yet to be determined.

At this point in the work, we have a concept of the fabrication cell, including technologies that will be incorporated into that cell. We have identified an existing robot, $\mathrm{CNC}$ milling machine, and gas metal arc welding equipment that we can use to assemble a small cell suitable for fabrication of hand sized components. We also identified a large robot and CNC milling machine suitable for fabrication of large components, but we have determined that it will be necessary to design and build suitable metal deposition equipment. The design and build of the large scale metal deposition equipment will not be undertaken by this project.
During this first year, we have built the experimental apparatus needed for the experimental studies. We have tested the apparatus and determined the initial operating conditions. The experimental apparatus provides a resource that will allow us to generate reliable data that is needed for success of the project. This next year, we will communicate project goals and results to potential industrial partners (probably in the U.S. automotive industry, but possibly including TACOM [U.S. Army]) and publish one or more papers on the work. We expect the technical direction of the research to continue as anticipated.

We also began model formulation and experiment design from the perspective of identifying our particular needs. This began with consideration of the static force balance theory which yields $F_{g}+F_{e m}+F_{d}+F_{s}=0$, where $F_{g}$ is the gravitational force, $F_{e m}$ is the electromagnetic force, $F_{d}$ is the aerodynamic drag force, and $F_{s}$ is the surface tension force. Prior work [2] added an inertia force $F_{i}$ associated with flow of mass into the drop from the electrode yielding

$F_{g}+F_{e m}+F_{d}+F_{i}-F_{s}=0$. The

gravitational force is $F_{g}=\frac{4}{3} \pi r^{3} \rho g$, where $r$ is

drop radius, $\rho$ is density, and $\mathrm{g}$ is gravitational constant. The electromagnetic force

is $F_{e m}=\frac{\mu_{o} I^{2}}{4 \pi} f$ and

$f=\ln \frac{R_{d} \sin \theta}{R_{w}}-\frac{1}{4}-\frac{1}{1-\cos \theta}+\frac{2}{(1-\cos \theta)^{2}} \ln \frac{2}{1+\cos \theta}$,

where $I$ is current, $\mu_{\mathrm{o}}$ is free space permeability, $R_{d}$ is drop radius, and $R_{w}$ is electrode radius. The aerodynamic drag force is $F_{d}=\frac{C_{d} A_{p}\left(\rho U_{b}^{2}\right)}{2}$ where $C_{d}$ is drag coefficient, $A_{p}$ is cross sectional area, $\rho$ is plasma density, and $U_{b}$ is plasma velocity. The surface tension force is $F_{s}=2 \pi R_{w} \gamma$ where $\gamma$ is surface tension.

We acquired various pieces of welding equipment, much from a recently completed Yucca Mountain Project, and used this 
equipment to build an experimental welding apparatus with precise computer control of the process parameters (voltage and thus current, electrode speed, and low-frequency pulsed-width modulator parameters) also incorporating suitable data acquisition. We tested this equipment and began experimental studies to identify the best starting parameters for our work. We also began formulation of a predictive model; the combination of these activities was complementary. We wanted to ensure that our selection of process control parameters and data acquisition capabilities properly supported our experimental needs. To this end we considered that drop detachment involves the following: A drop is attached to the electrode by surface tension; there is a liquid neck between the drop and the electrode. Opposing this attachment force is gravity and aerodynamic drag associated with the flow of arc plasma past the drop in a downward direction. This plasma flow is driven by the divergence of electrical current flowing in the arc. The liquid neck between the drop and the electrode is subject to pinch-off by the electrical current, which passes through the neck into the top of the arc. This pinching of the liquid neck changes the magnitude of the attaching force to some degree. Drop detachment will occur when the sum of the detaching forces exceeds the attachment force. Our task is to predict these forces for different electrode diameters and choose low-frequency, pulsed-width modulator control parameters to control a detachment event. We believe our apparatus and experimental design is appropriate for this work; we will continue with the majority of the model formulation and experimental validation studies this Fiscal Year 2010. A major contribution expected is extension of the static force balance criteria into a dynamic force balance criteria for detachment during spray transfer.
Summary and Conclusions

During this first year, we have built the experimental apparatus needed for the experimental studies. We have tested the apparatus and determined the initial operating conditions. The experimental apparatus provides a resource that will allow us to generate reliable data that is needed for success of the project. This next year, we will communicate project goals and results to potential industrial partners (probably in the U.S. automotive industry, but possibly including TACOM [U.S. Army]), and publish one or more papers on the work. We expect the technical direction of the research to continue as anticipated.

\section{References}

[1] Kim, Y. S., D. M. McEligot, and T. S. Eagar, 1991, "Analyses of Electrode Heat Transfer in Gas Metal Arc Welding," Welding Journal, Vol. 70, No. 1, pp. 20-s to 31-s.

[2] Watkins, A. D., H. B. Smartt, J. A. Johnson, 1993, "A Dynamic Model of Droplet Growth and Detachment in GMAW," International Trends in Welding Science and Technology, ASM International, pp. 993997.

[3] Bingul, Z., G. E. Cook, 2006, “A Real-Time Prediction Model of Electrode Extension for GMAW," IEEE/ASME Transactions on Mechtronics, Vol. 11, No. 1, pp. 47-54, February 2006. 


\title{
Effect of Glycosylation on the Activity and Stability of Bacterial Enzymes
}

\author{
William A. Apel, Brady D. Lee, and Vicki S. Thompson \\ Testing bacterial enzyme stability and activity profiles that may be favorably affected through \\ posttranslational modifications such as glycosylation \\ FF108
}

Based on results from our previous research and from the literature, we hypothesize that bacterial enzyme stability and activity profiles may be favorably affected through posttranslational modifications such as glycosylation. The goal of this research is to test this hypothesis using an enzyme, a catalase that we previously isolated and characterized from the extremophilic bacterium Thermus brockianus. Since we have the gene and amino acid sequence for this catalase, we can express it in both glycosylating and non-glycosylating systems (i.e., Pichia pastoris and E. coli, respectively). We also have strong indications from previous work with lignocellulose-degrading enzymes that different clones of transformed $P$. pastoris tend to produce enzymes with different stability and activity profiles. We believe this is due to different glycosylation patterns produced by these different clones. We plan to take advantage of this effect to produce a number of different clones of $P$. pastoris, transformed with the catalase gene. We expect these genes to possess different stability and potentially different activity profiles depending on their specific glycosylation patterns. We will compare these different enzymes among themselves, and also to non-glycosylated enzyme produced in $E$. coli, to begin to understand the effects of glycosylation on enzyme activity and stability profiles. If this effort is successful, it most likely will lead to intellectual property relating to the use of glycosylation to custom engineer bacterial enzymes having industrially desirable activity and stability profiles.

\section{Technical Objectives}

The objectives of the project are to:

- Express catalase in glycosylating and nonglycosylating expression systems

- Screen and characterize the resulting enzymes

- Analyze glycosylation patterns and determine their effects on enzyme activity.

Results and Accomplishments

In an effort to generate a thermophilic catalase enzyme for testing, an amino-acid sequence that was a hybrid developed from gene sequences from Thermus brockianus and Thermus thermophilus was submitted to DNA 2.0 for development of expression systems for $E$. coli and Pichia pastoris. The catalase enzyme from $T$. brockianus has been thoroughly characterized in our laboratories, and a partial sequence, lacking the terminal 5' and 3' sequences, is available from the National Center for Biotechnology Information (NCBI). Comparison of the T. brockianus catalase gene with a full-length catalase gene from $T$. thermophilus using the BioEdit program indicated a greater than $90 \%$ similarity. It also showed that 14 bases are missing at the 5' end and 36 bases from the 3 ' end of the $T$. brockianus gene. A hybrid catalase enzyme with the following amino acid sequence was submitted for gene optimization for codon bias, synthesis, and cloning into expression vectors:

MFLRIDRLQIELPMPKEQDPNA
AAAVQALLGGRFGEMSTLMN
YMYQSFNFRGKKALKPYYDLI
ANIATEELGHIELVAATINSLLA
KNPGKDLEEGVDPESAPLGFA
KDVRNAAHFIAGGANSLVMG
AMGEHWNGEYVFTSGNLILDL
LHNFFLEVAARTHKLRVYEMT
DNPVAREMIGYLLVRGGVHA
AAYGKALESLTGVEMTKMLPI
PKIDNSKIPEAKKYMDLGFHR
NLYRFSPEDYRDLGLIWKGAS
PEDGTEVVVVDGPPTGGPVFD
AGHDAAEFAPEFHPGELYEIAK
KLYEKAK.

DNA 2.0 supplied optimized genes for expression as $E$. coli clones carrying the $\mathrm{pBAD}$ expression vector for $E$. coli and the pPIC6 $\alpha \mathrm{A}$ for Pichia pastoris. Plasmids were extracted and transformed into their respective expression hosts, and recombinant enzymes were produced for testing.

Thermus thermophilus catalase, expressed in E. coli and purified by His tag, was tested for activity by adding suitably diluted enzyme to a $30 \mathrm{mM} \mathrm{H}_{2} \mathrm{O}_{2}$ solution at $70^{\circ} \mathrm{C}$ and $\mathrm{pH} 8.0$ and measuring the decrease in absorbance at $240 \mathrm{~nm}$. Although the enzyme successfully expressed in 
E. coli (Fig. 1), no activity was found. After examining the literature, we determined that two likely reasons for this result were either the formation of insoluble inclusion bodies during expression in E. coli or improper folding and assembly of the enzyme during expression (or both).

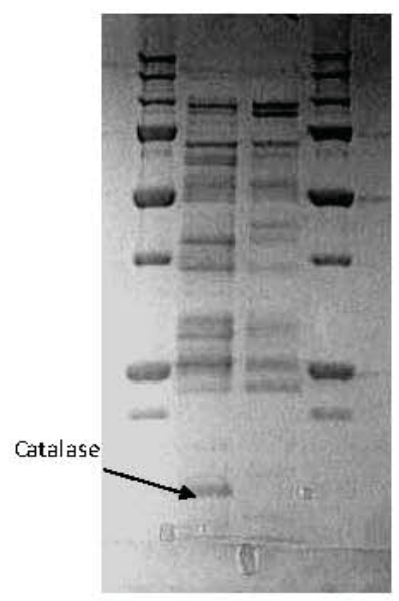

FIGURE 1. SDS-PAGE gel of expressed and non-expressed E. coli.

To test the inclusion-body hypothesis, the $E$. coli transformant was grown at sub-optimal growth conditions (i.e., lower temperatures, addition of a growth inhibitor, changing induction conditions, etc.). While these methods did reduce inclusion bodies in some cases, none of these attempts resulted in active enzyme. Next we examined the folding and/or improper assembly issue. This catalase is classified as a Mn-catalase and requires manganese for activity. This enzyme also has six subunits that have to assemble correctly for activity. We first tested expressing the E. coli transformant in the presence of $\mathrm{Mn}$ to see if that would facilitate proper folding and enzyme assembly. This did not result in active enzyme. Next we examined refolding/assembling the enzyme in vivo by denaturing the enzyme with guanidine and dialyzing the denatured enzyme against a Mn buffer. Unfortunately, the enzyme precipitated during this procedure, so a step-wise dialysis against gradually decreasing levels of guanidine was also examined. This also resulted in the enzyme precipitating at lower guanidine levels. The final method tried was to repeat these dialysis methods at higher temperatures with the hope that this would prevent enzyme precipitation. This also did not work.

We then went to the literature to find another expression system that would be better for thermophilic proteins; we determined that there is a commercial system, sold by Biotools B\&M Labs in Spain that is based on over expression of proteins in Thermus thermophilus. Given that this is the source of the catalase that we have been working with, the chances of success for this method are high. We have ordered a kit from this company and are currently working to insert the catalase gene into the correct vectors for the system. We will continue this work in FY-10.

\section{Summary and Conclusions}

We have worked with a commercial vendor to design and synthesize acceptable catalase genes for use in our work. When expressed in E.coli, the synthetic genes did not result in an active catalase. The expression of extremophilic enzymes by mesophilic expression systems can often be problematic, and this has led us to begin examining the use of a thermophilic expression system based on Thermus thermophilus. We anticipate the expression of active, nonglycosylated enzyme with this system, and this work will proceed in FY-10. 


\title{
A Systems Biology Approach to Understanding Lignocellulose- derived Carbon Metabolism
}

\author{
William A. Apel, Jeffrey A. Lacey, Brady D. Lee, Deborah T. Newby, David W. Reed, \\ David N. Thompson, Vicki S. Thompson \\ Understanding lignocellulose-derived carbon metabolism
}

GB103

An industrially important microorganism that can catabolize lignocellulosic compounds as sole carbon and energy sources is the focus of this research project. Our previous work and the work of others with this microbe has indicated it possesses a unique suite of enzymes that could be utilized for hydrolysis of lignocellulosic feedstocks to simple sugars that can be converted to fuels and value-added chemicals. Relatively little is known about how this microorganism controls the production of these enzymes and regulates its overall metabolism of lignocellulosic-carbon sources. The thrust of this proposal is to better understand lignocellulose metabolism by this microorganism. This will be accomplished by testing the following hypothesis: Specific suites of extracellular lignocellulose-degrading enzymes are produced in response to detection of a variety of soluble and insoluble lignocellulosic substrates; in turn, this affects internal regulation of additional gene activities related to lignocellulose-derived carbon metabolism by this microorganism. This hypothesis will be tested by using an integrated system of genomic, transcriptomic, proteomic, and metabolomic techniques to better understand the mechanisms this microbe uses to detect, degrade, and utilize lignocellulosic materials as metabolic carbon and energy sources. The results of this research will lead to: (1) increased fundamental understanding of this industrially relevant microorganism's lignocellulose metabolism, (2) the potential for new, lower-cost lignocellulose pretreatment systems based on this understanding, and (3) further building of Idaho National Laboratory's (INL's) capabilities to apply state-of-the-art genomic, transcriptomic, proteomic, and metabolomic techniques to address questions critical to development of the Nation's bioenergy industry.

\section{Technical Objectives}

The technical objectives of this project in the current fiscal year were to:

- Determine the batch culture growth rate characteristics of the target microorganism when grown on wheat arabinoxylan and on xylose

- Produce steady-state cultures of the target microorganism grown on wheat arabinoxylan and on xylose in continuous chemostat cultures, and harvest the cultures for systems biology analyses.

\section{Results and Accomplishments}

In Fiscal Year (FY) 2009 culture work continued on this project, with a focus on both simple sugars and more complex substrates. To this end, culture work was completed for the production of steady-state cultures of the target microorganism growing on xylose, arabinose, and arabinoxylan, a complex hemicellulose substrate. Substrate-limited continuous steadystate cultures were produced in mid-log growth for xylose and arabinose. Substrate-limited continuous steady-state cultures growing on arabinoxylan were provided in $\log$ phase 1 and mid-log phase 2, and an oxygen-limited culture was provided for mid-log phase 2 . These cultures were provided for proteomic, genomic, and metabolomic analyses.

Included in these analyses were RNA microarrays. The purpose of these microarrays was to better understand which physiological pathways are operating under various culture conditions. This information allows the simultaneous assessment of whether thousands of genes are expressing their respective proteins. To accomplish this, cells from $15 \mathrm{~mL}$ of each culture were collected. RNA was extracted from the cells and converted to cDNA. This cDNA was sent to Roche-Nimblegen for expression profiling. Microarrays and probe sequences were designed and manufactured by Roche-

Nimblegen, using all the genes identified in the organism of interest. The cDNA samples were then fluorescently labeled and hybridized to the microarrays. Fluorescent images were obtained (Fig. 1, next page), and gene-expression data were extracted from the array images. Raw intensity values for each gene that corresponded to the level of gene expression were sent to the INL for further analysis. Statistical analysis identified over one-thousand genes that were differentially expressed between the culture conditions. Many genes have been identified that are key to the organism's growth on specific substrates. Of particular interest in this large set of results were a few genes that showed the same expression pattern as a particular xylanase of interest, being expressed only during the second 
log growth phase when cultures were grown on arabinoxylan.

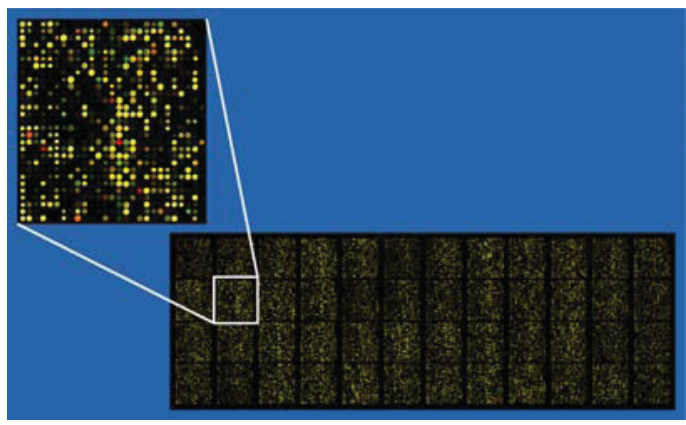

FIGURE 1. Example of a developed microarray.

For proteomic analyses, $40 \mathrm{~mL}$ samples were obtained from three replicate reactors for each substrate condition, quick-frozen in liquid nitrogen and lyophilized for storage. The samples were then rehydrated with lysis buffer, and the cells ruptured by passage through a
French pressure cell. The cellular debris was removed by centrifugation, and the protein supernatant was recovered. The protein samples from the various conditions were labeled with an 8-plex iTRAQ kit, as shown in Figure 2. This labeling scheme allowed our analyses to have three biological replicates and three technical replicates for each sample. The remainder of our focus for this year was converting our mass spectrometer from a MALDI source to an electrospray source. This required two service calls from the vendor to set up the system and to fix mechanical problems with the mass spectrometer. We also had to work out issues dealing with connection of our capillary liquid chromatography system to the electrospray source. This is not a trivial issue because of the extremely low flow rates utilized.

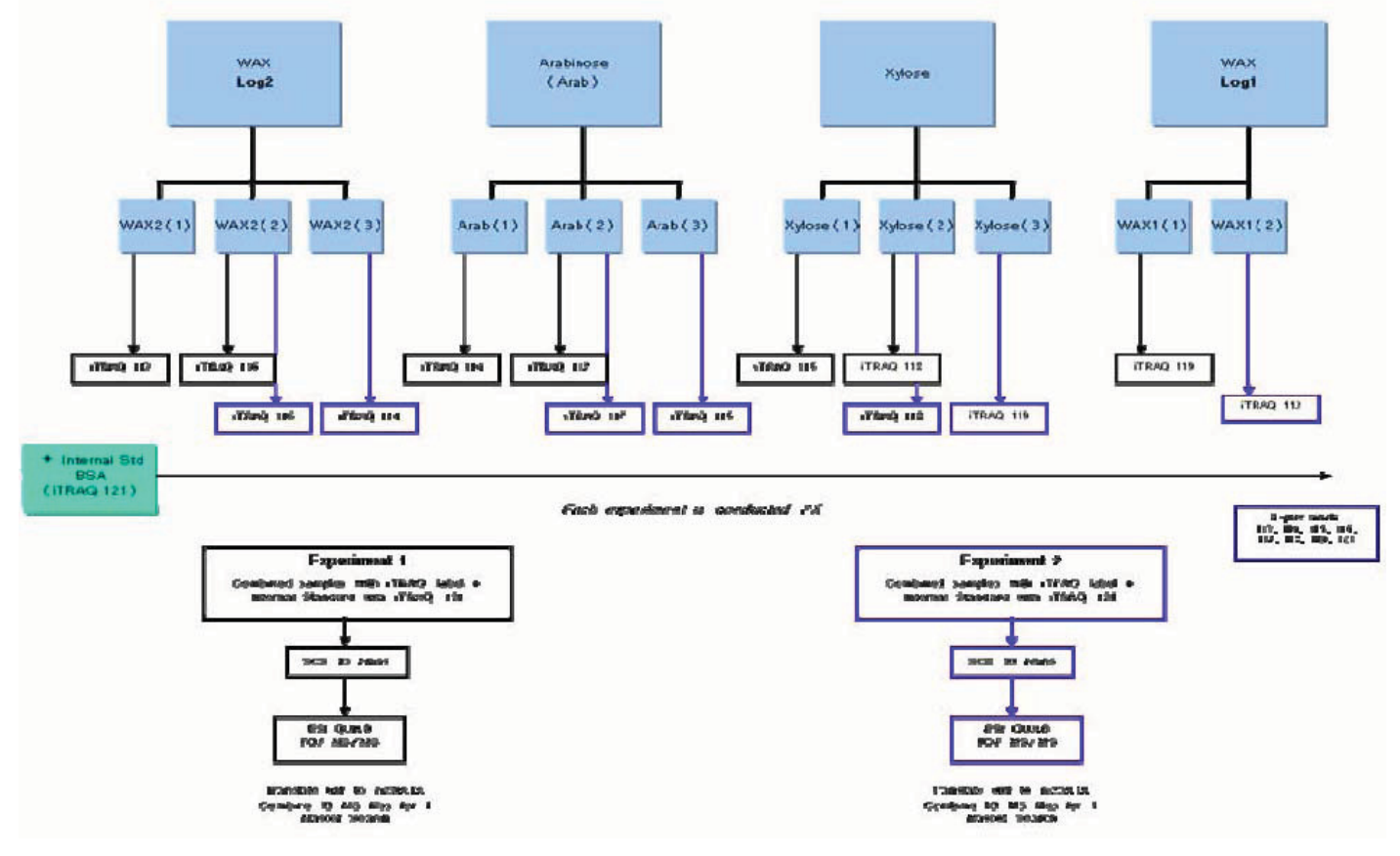

FIGURE 2. iTRAQ labeling conditions.

\section{Summary and Conclusions}

The organism has been successfully cultured under steady-state conditions, utilizing simple sugars and more complex carbohydrates as the sole carbon and energy source. Samples of these cultures were provided for physiological analyses, using a variety of techniques including microarrays and mass spectrometry. 8 plex iTRAQ labeling was effectively used as part of the mass spectrometry procedure. 


\title{
The Metabolic Engineering of a Gram-positive Thermoacidophile for Lactic Acid
}

\author{
Brady D. Lee, Frank F. Roberto (INL), Ross Carlson (Montana State University) \\ Developing the metabolic-engineering tools to genetically modify a bacterium for production of lactic \\ acid from glucose and xylose
}

GB104

This project is focused on the metabolic flux of a gram-positive thermoacidophile and the potential to alter this flux when growing on plant-derived monosaccharides for production of value-added chemicals. This bacterium produces glycosyl hydrolase enzymes that hydrolyze lignocellulosic-containing biomass to monosaccharides which are used as carbon and energy sources by the cell. Due to its thermoacidophilic nature and ability to hydrolyze both cellulose and hemicellulose to sugar monomers, this bacterium is an ideal candidate for consideration as a consolidated bioprocessing (CBP) microorganism. We hypothesize that redirection of pyruvate metabolism through targeted metabolic engineering of enzymes around the phosphoenol pyruvate (PEP)pyruvate-oxaloacetate (PPO) node which interconnects the major pathways of carbon metabolism, will allow for addition of the phenotype for metabolite production, specifically lactic acid.

The objective of this research project is to develop the metabolic-engineering tools for genetically modifying this organism for production of lactic acid from glucose and xylose. This objective is being accomplished by taking an interdisciplinary metabolic-engineering approach; which combines aspects of genetic engineering, bioprocess engineering and metabolomics to develop a genetically modified strain of the gram-positive thermoacidophile for production of value-added chemicals. The proposed research is important because it will increase fundamental understanding of carbon metabolism by this organism, provide the potential for a consolidated, cost-effective, more efficient bioprocess for the conversion of lignocellulosic biomass to value-added chemicals, and build the INL's capabilities to apply metabolic engineering techniques to address questions critical to development of the Nation's bioenergy industry.

\section{Technical Objectives}

Technical objectives of this project include

- Genetically modifying the gram-positive thermoacidophile for production of lactic acid from monosaccharides derived from plant cell walls
- Analyzing metabolic flux related to lacticacid production and associated intermediates of carbon metabolism by the gram-positive thermoacidophile

- Developing modeling approaches to study metabolic network analysis of the grampositive thermoacidophile.

Results and Accomplishments

During Fiscal Year 2009 (FY-09), progress was made toward meeting the objective of the proposed research. Accomplishments made during the year are as follows:

Metabolic Flux:

- Optimized methods for extracting metabolites from gram-positive thermoacidophile, building on techniques developed in FY-08

- Established growth characteristics and optimized chemostat parameters for optimum growth of test organism on xylose and glucose

- Continued use of capillary-electrophoresision trap-mass spectrometry method to look at anionic and cationic metabolites produced during growth of gram-positive thermoacidophile

- Added gene-expression-analysis research task to increase overall understanding of metabolic interactions in gram-positive thermoacidophile during transition between carbon-limited and nitrogen-limited growth

- Determined that ethanol is produced during carbon- and nitrogen-limited growth on xylose

Metabolic Modeling:

- Refined and tested metabolic model (Fig. 1, next page) developed during FY-08 using elementary flux mode analysis, determining that the gram-positive thermoacidophile has 3,865 unique elementary modes when grown on glucose, whereas only 1,871 modes are available when grown on xylose. 


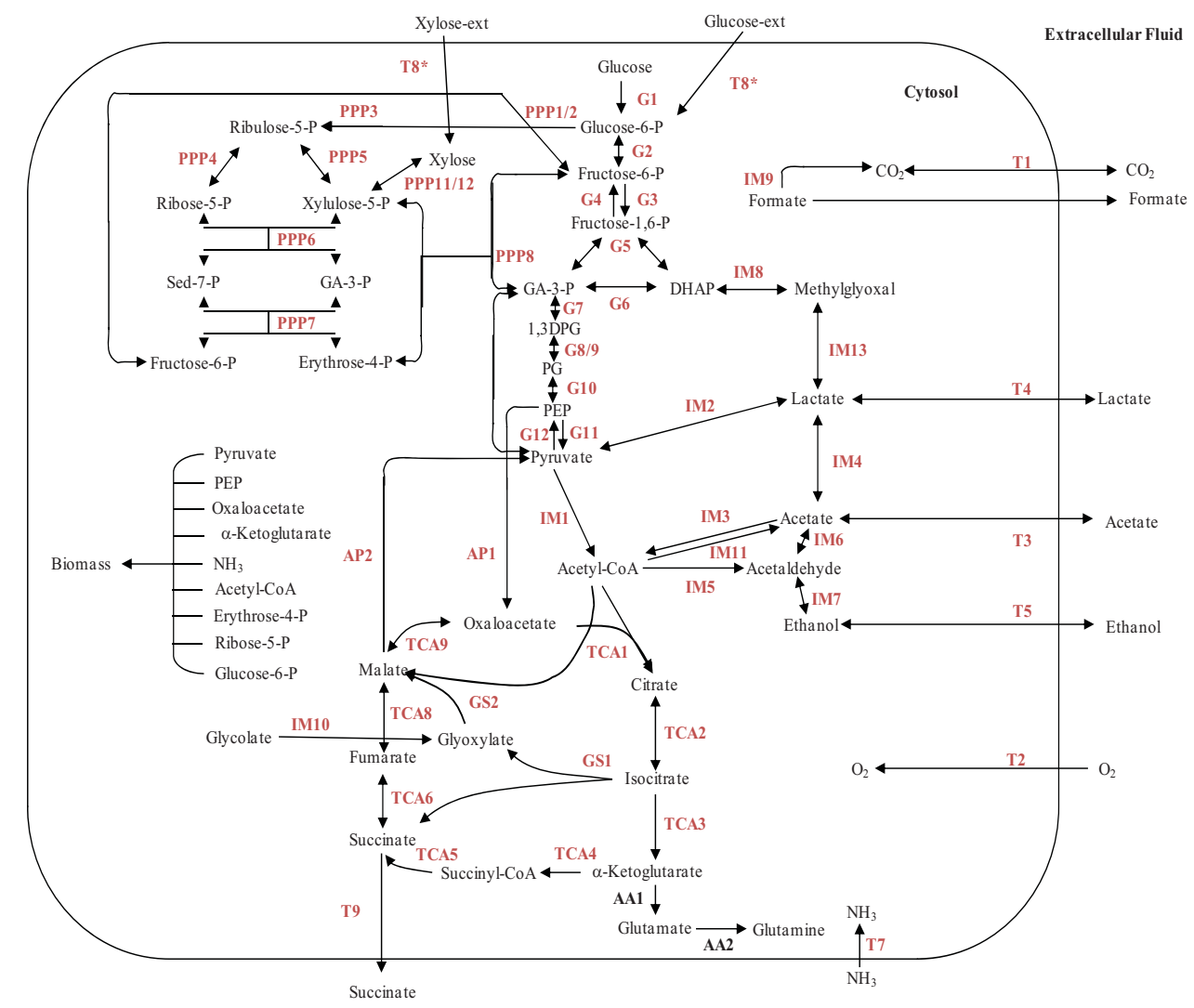

FIGURE 1. Graphical representation of the Alicyclobacilus acidocaldarius central metabolism. Model reaction numbers are listed next to substrates and products. Simulations considered either glucose or xylose as the energy source. Cofactors and electron transport components are not shown.

- Determined that the gram-positive thermoacidophile does have the potential to grow anaerobically-important because it had been classified as an obliagate aerobewhich would require expression of specific enzymes and production of organic acids or alchohols as redox sinks

- Determined growth rate dependent maintenance energy requirements which indicated a maintenance energy requirement of $9.55 \mathrm{mmole} \mathrm{ATP} / \mathrm{gcdw} / \mathrm{hr}$ (cdw = cell dry weight) using simulations in which 3 protons were generated per molecule of ATP utilized

- Project sponsored a 1.5-day hands-on short course titled "Metabolic Systems Analysis Workshop" at the INL - which was presented by Dr. Ross Carlson and PhD student Reed Taffs - attended by a regional university professors, post-doctoral researchers, graduate students and undergraduates.

Metabolic Engineering:

- Constructed kanamycin-resistant variant of broad host range vector pSUP104 using kanamycin nucleotidyltransferase gene from pUCD615 (kanamycin was chosen as a selective agent because of its increased thermostability)

- Initiated work with gram-positive broad host range vectors using the kanamycin resistance gene in plasmid pUB110, which is considered highly thermostable and may allow selection at the thermophilic growth temperatures required for growth of the gram-positive thermoacidophile 
- Isolated three kanamycin-resistant isolates from hot springs in Yellowstone National Park, which, together with numerous others, are thought to be closely related to the grampositive thermoacidophile being tested, so plasmids in these organisms may serve as cloning or shuttle vectors.

\section{Summary and Conclusions}

Research over the past year has demonstrated that, when grown on xylose and glucose, the gram-positive thermoacidophile being tested is able to produce a number of organic acids and ethanol. Production of these compounds was demonstrated under both carbon- and nitrogen-limited conditions.

Production of organic acids and alcohols by this bacterium appear to be factors in allowing anaerobic growth, which is interesting because the test organism is considered to be an obligate aerobe, requiring oxygen for growth. Progress is also being made in developing a genetic system that will allow metabolic engineering of this gram-positive thermoacidophile. Once developed, this system will be utilized to genetically modify the gram-positive thermoacidophile for production of organic acids, most likely lactic acid and ethanol.

Microarray experiments were added to the scope of the project to help determine genes up- or down-regulated during growth under carbon- or nitrogen-limited conditions. This analysis, along with the metabolic modeling, will allow for target-gene disruption for production of intermediary metabolites of interest.

This project is developing expertise in analysis of microbial metabolism as it relates to production of value-added chemicals from an industrially interesting gram-positive thermoacidophile. As part of this expertise, analytical methods for metabolome profiling of bacteria are being developed. This expertise will support genomic, transcriptomic and proteomic expertise currently being utilized at the laboratory. INL expertise currently exist for the genetic modification of gram-negative acidophiles; expertise on the current project will expand this knowledge for use with grampositive thermoacidophiles. Developments in this area will represent potentially patentable protocols that can be directly transferred to industry. In addition, this project is developing expertise in metabolic modeling through elementary flux-mode analysis. Expertise developed can then be used for future projects. 


\title{
Network Interaction in a Thermoacidophile In Response to Different Complex Carbon Sources
}

\author{
Brady D. Lee \\ Developing expertise in systems biology \\ GB105
}

The physiological response of a microbe to its growth environment is the product of complex interactions between networks of gene operons, proteins and metabolites. These interactions are especially important during growth on different carbon sources since the carbon source will govern the physiological response of the microbe to the growth environment. An important process that controls this physiological response is the expression of transcriptional regulators that control operonsencoding proteins that catalyze interactions with carbohydrates in the growth environment. Metabolites or the carbon source themselves will act as regulators or as signals for regulation of expression of many of these operons - a process called carbon-catabolite repression (CCR). Of specific interest to this research is CCR of a gram-positive thermoacidophile capable of growth on carbon sources ranging from simple sugars, such as xylose and glucose, to complex polysaccharides such as cellulose and hemicellulose. Growth on these substrates requires expression and regulation of different operons involved in carbon metabolism. The goal of the proposed research will be to understand carbohydrate-induced differential gene expression patterns by this gram-positive thermoacidophile during growth on a range of carbon and energy sources. Understanding the effect of CCR on regulation of genes during growth on different carbon sources, and the ability to control this regulation, represents the first step in optimizing growth and/or enzyme production. The research approach will be targeted toward carbohydrate-active proteins to explore issues related to utilization of saccharides by this bacterium, with a focus on regulation of gene expression.

The proposed research will develop expertise in systems biology at the laboratory. This expertise will then be available for projects relating to all aspects of microbiology, including bioenergy, environmental studies, homeland security research, and even medical microbiology. This research also will be used in support of a doctoral dissertation for the PI.

\section{Technical Objectives}

The technical objectives of this research will allow the research team:
- To understand the carbohydrate-induced differential gene expression by the grampositive thermoacidophile during growth on a variety of biomass derived mono-, oligoand polysaccharides

- To develop a genetic system to study operon promoter structure in the gram-positive thermoacidophile

- To develop a network interaction model using genome information and experimental data.

\section{Results and Accomplishments}

During FY-09, progress was made toward meeting the objective of the proposed research. Accomplishments made during the year are as follows:

Genome Analysis. During the year, an in silico approach was initiated to begin looking at genes and operons related to carbohydrate processing by the gram-positive thermoacidophile. As part of this task, additional curation and annotation of the genome information was performed. Of specific interest were genes and operons related to degradation of poly- and oligo-saccharides (i.e, glycosyl hydrolases). When present as part of operons, these genes, as is typical with other grampositive bacteria, are present with regulatory, sugar transport, and sugar utilization genes. In addition, some of the glycosyl hydrolases exist in distant locations on the genome.

During CCR, repressor proteins, such as carbon catabolite protein $\mathrm{A}(\mathrm{CcpA})$, bind to specific sequences in the promoter region or inside the actual gene near the promoter region to inhibit transcription of the associated gene or operon. The specific sequences are called catabolite responsive elements (cres). Genome analysis identified the presence of these genetic elements in the genome of our gram-positive thermoacidophile, indicating that CCR is active in this organism. CcpA, along with nearly 50 cre sequences, were found within the genome.

Network Interaction Modeling. As part of developing systems-biology expertise at the INL and for application to this project, bioinformatic and data-analysis tools were reviewed for 
mapping and monitoring network interaction during growth of the gram-positive thermoacidophile on various carbon sources. To this end, a network interaction modeling framework, based on a system used at the Institute for Systems Biology, will be utilized during the research. This bioinformatics system is based on a program called Gaggle that facilitates transport of data between modeling, statistical and other programs resident on a personal workstation, and through an application called Firegoose, which allows interaction with external databases containing information related to interaction networks of other microorganisms.

During the fiscal year, genome information was utilized to begin building a network-interaction model using the program Cytoscape. Annotated proteins (3554 proteins) from the gram-positive thermoacidophile were submitted to STRING, a database containing network interactions from numerous sequenced bacteria, and putative interactions in the organism of interest were built within the model. As would be expected many proteins yielded the highest homology with Bacillus subtilis or other bacilli, but there were numerous proteins that showed homology to distantly related bacterial species.

Gene/protein interaction networks deduced from information in DAVID will be validated during gene-expression analysis that will be initiated during year two of the proposed research.

Suppression of CCR. An additional goal of the proposal is to understand network interaction when genes related to CCR have been "knockedout" (removed so they no longer function). To this end a gene knock-out strategy was developed. This strategy will utilize a vector system developed for Geobacillus spp, and experiments were designed to look at transformation of the gram-positive thermoacidophile using protoplasts and electroporation. As part of this research, various experimental parameters will be compared to enhance the efficiency of moving foreign DNA into the gram-positive thermoacidophile.

Development of this system is important because it will allow verification of gene regulation in the gram-positive thermoacidophile and will allow for validation of gene interaction networks proposed above.

\section{Summary and Conclusions}

Research during the past fiscal year demonstrated the presence of many components of the typical gram-positive CCR system in the gram-positive thermoacidophile of interest to the research. In addition, initial interactions of these constituents with genes/proteins involved in carbohydrate processing by the bacterium were inferred using data from network interaction databases on the World Wide Web. In addition, a strategy for studying the effect of controlling CCR was developed during the fiscal year.

Initial experiments to monitor $\mathrm{CCR}$ in the gram-positive thermoacidophile will be initiated during the next fiscal year. Likewise, the strategy for controlling CCR will be tested.

Strategically, this project is designed to develop systems biology expertise at the INL. The INL currently has strengths in genomic, transcriptomic, proteomic and metabolomic analysis; systems biology methodology will provide the ability to begin looking at the interaction of these constituents in the grampositive thermoacidophile as well as other bacteria of interest. In addition, the research is supporting the development of an INL employee as a systems biologist through a $\mathrm{PhD}$ program. 


\section{Reducing CBM Water Discharge Volume}

Eric Robertson

Determining the viability of a WAG injection scheme in coalbed methane production operations GS101

The broad purpose of this work is to determine if a water-alternating-gas (WAG) injection scheme can be used to economically reduce or eliminate the discharge of produced water associated with the production of coalbed methane $(\mathrm{CBM})$ production operations. Specifically, the objective is to determine the viability of such a scheme by measuring gas production rates and total gas produced from a bench-scale model of a fractured coal seam. Results will be translated to field scale using a CBM simulator, and economic and technical viability will be determined.

Current options for disposal of water pumped from a coal seam to liberate the adsorbed gas include direct discharge to the surface watershed, lower the salinity through water treatment and then discharge to the surface, or re-injection into a deeper, more saline formation. The WAG process has been successfully used in enhanced oil-recovery methodologies, but has not been proposed for application to CBM production or to mitigate produced water discharge problems.

\section{Technical Objectives}

The objectives of this project for FY-09 were to:

- $\quad$ Build lab-scale model of a coal reservoir representing $1 / 4$ of a 5 -spot enhanced recovery pattern

- $\quad$ Test the lab-scale model under pressure

- Calibrate the equipment necessary to quantify future methane and nitrogen production from the model

- Conduct dry control tests on the model to determine methane production from depressurization.

\section{Results and Accomplishments}

The project began operations on May 1, 2008 , and is a three-year project with total requested funding of $\$ 150 \mathrm{~K}$ per year. In FY-08 (1/2 year), fresh coal was collected from two mines in Colorado and two mines in North Dakota, unique laboratory equipment was purchased, and experiments were designed.
We originally planned to use a larger rectangular block of coal $(1 \mathrm{ft} \times 1 \mathrm{ft} \times 0.5 \mathrm{in}$. $)$ for flow experiments. Due to Idaho National Laboratory (INL) regulations, we were unable to procure a vessel in which the block could be placed, but we were able to procure a smaller vessel that would allow the use of a smaller block $(0.5 \mathrm{ft} \times 0.5 \mathrm{ft} \times 0.5$ in. $)$ with internal pressure below 300 psig.

Coal seams are highly fractured formations. The natural fractures in coal, called cleats, are the means of transporting gas and water through the seam. To simulate or model a coal seam, the cleat system in our bench-top model was created by cutting 0.5 -in. cubes of coal using a diamondcoated tile saw. A block of coal 0.5-in. thick and 6 in. on a side was created by piecing together the $1 / 2$-inch cubes. The process of cutting the small coal cubes was more difficult than anticipated due to the friable nature of the coal. However, with patience we were able to develop the art and successfully cut the required small cubes to construct the rectangular coal block. The small irregularities between the cubes are designed to represent the natural orthogonal cleat system found naturally in coal seams. Figure 1 is a photograph of the 0.5 -in. coal cubes arranged into a 6-in. $\times$ 6-in. rectangular coal block.

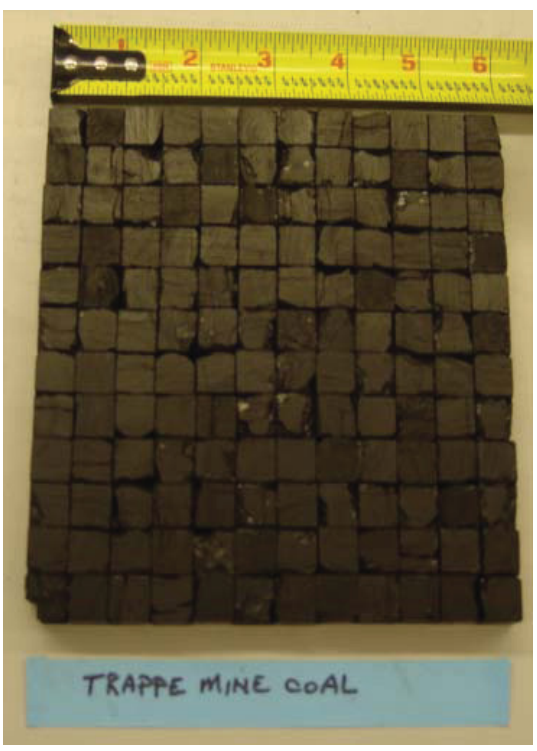

FIGURE 1. Photograph of 144 1/2-inch cubes of coal from the Trapper mine arranged into a $6 \times 6$-inch area. 
Five short lengths of stainless steel tubing, $1 / 16$ in. in diameter, were inserted into the 0.5 inch thick coal block at specific locations to a depth of $0.25 \mathrm{in}$. and used as ports to allow injection, production, and monitoring of pressure during the experiment. A non-sag epoxy coating of about $0.25 \mathrm{in}$. was applied to the entire outside surface of the bench-scale physical model. The epoxy coating acts as a pressure seal and also as a seal to contain the injected fluids.

The sealed coal block was placed inside a stainless steel pressure containment vessel rated to 14,000 psig. The $1 / 16$-in. injection, production, and pressure ports were threaded through a pass-through fitting in the pressure containment vessel to allow the pressure in the coal block to be controlled independently of the over-pressure maintained by liquid water within the pressure containment vessel. Figure 2 is a photograph of an epoxy-coated coal block showing the injection, production, and pressure monitoring ports.

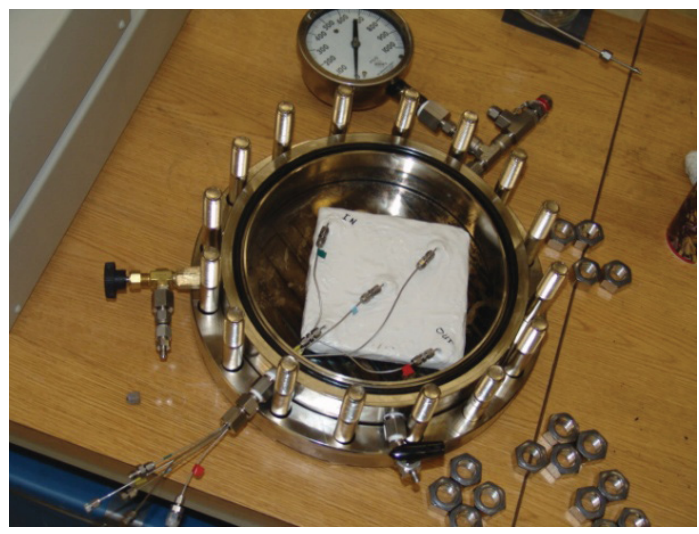

FIGURE 2. Photograph showing the coal block sealed with epoxy sitting in the pressure containment vessel. The five 1/16-inch ports used for injection, production, and pressure monitoring are also shown.

INL regulations impose a maximum pressure of 300 psig within the 14,000-psig-rated pressure containment vessel. Initial pressures within coal seams with commercial gas production can range from 500 psig to 6,000 psig, but because of safety regulations, we were limited to working with pressures below 300 psig in our laboratory experiments. The system was thoroughly tested under 300 psig pressure (maximum allowable) and found to operate free of leaks.

The flow meter was calibrated and used to calculate the initial pore volume and porosity of the coal block. Helium was used during this phase because it has a very low interaction with coal. Pore volume of the system was measured to be $21.16 \mathrm{~mL}$, bulk volume of the coal block was measured to be $294.96 \mathrm{~mL}$, and porosity was calculated to be $7.17 \%$. Porosities of coal seams in the field range from $1 \%$ to $5 \%$. The porosity of the bench-scale model was anticipated to be somewhat higher than typical field values because of the way the artificial cleats were made. The regularity of the cleats was necessary to develop an accurate reservoir description during the simulation phase of the project. The slightly higher porosity is not expected to present any difficulties during data interpretation.

Some unplanned experiments to measure sorption-induced strain were done to obtain data describing how coal from the Trapper mine reacts to the changing pressures and gas concentrations expected during the laboratory experiments. These data will be necessary to accurately model changes in coal permeability. Figure 3 is a plot of the sorption-induced axial strain as a function of pressure for four gases of interest.

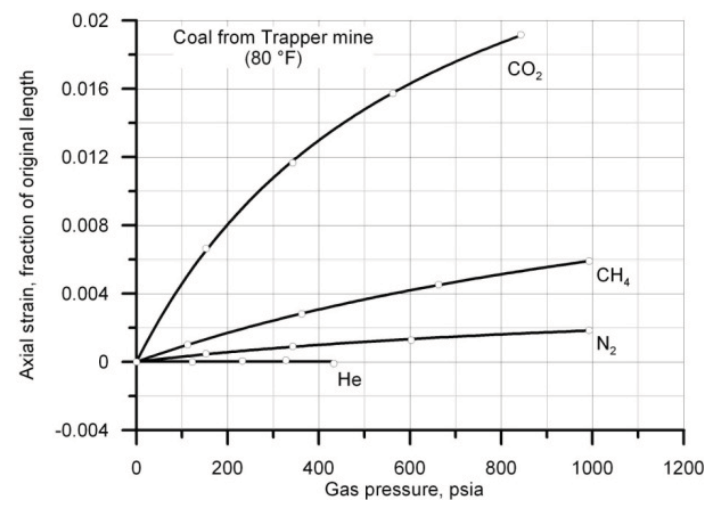

FIGURE 3. Axial strain of coal from the Trapper mine in Colorado caused by sorption of different gases as a function of gas pressure.

The research has led to discussions with colleagues from interested parties, including the University of Wyoming, Anadarko Petroleum, and mining companies from the collections sites 
of the coal used in the experiments. A proposal written jointly with Anadarko Petroleum was submitted to an industry-led forum (rpsea.org). Discussions and resulting proposals such as these have benefitted both the Idaho National Laboratory and the Department of Energy by keeping current on pertinent industry needs and providing opportunities for future external funding.

Capabilities gained include increased expertise in fluid flow through CBM fields, which is important not only for unconventional gas projection, but also for understanding and applying $\mathrm{CO}_{2}$ sequestration technologies in coal. We have expanded the state of the art for creating and working with bench-top coal-seam physical models from one-dimensional physical models to two-dimensional models. We have also developed and tested a new method for measuring internal volumes using a flow meter developed and patented from previous LDRD work at INL.
Summary and Conclusion

To date, we met the first three of four objectives for FY-09. In addition to these objectives, we obtained additional data on sorption-induced strain that is pertinent to future modeling exercises.

In FY 10, laboratory data will be collected on methane production from pressure depletion, as well as partial pressure reduction using dry nitrogen and flue gas. These data will be compared to methane production from injecting alternating slugs of nitrogen and water and flue gas and water.

Data generated from these tests will be used to calibrate a field-scale computer model of a full-scale CBM reservoir. Laboratory results will be modeled using a commercial CBM reservoir simulator (COMET3). The field-scale model will be used to evaluate the economic viability of implementing the process in the field. 


\section{Advanced Adaptive Algorithms in Phased Array Ultrasonics for Material Inspection}

Timothy R. McJunkin, Milos Manic (University of Idaho), Michael Moles (University of

Toronto)

Automating ultrasonic examination technology

PH105

Ultrasonic examination is a technology used to view the insides of objects, including the human body for medical applications or solid materials for characterization of structural integrity. Phased-array ultrasonic examination, as currently used, sets configuration for the examination entirely prior to use. This research is producing a novel, adaptive framework that reacts to the data available to automatically reconfigure an examination system. The hypothesis is that machine intelligence can provide both a more optimal collection and presentation of features contained within the examined subject at the current sensor location and instructions to the technician, or surrogate machine, for alternate locomotions to acquire better information or images. This improves on the paradigm of "collect as much data or information from as many points of view as achievable and then try to extract value" by turning the problem towards its inverse of evaluating a potentially small set of data and adjusting the point of view or depth of focus to arrive at more optimal information. Eventually, the algorithms will encapsulate a portion of the capabilities of a skilled technician in the art of ultrasonic examination and make the technician's task easier and results more consistent. Included in the research will be a novel automatedintelligence scheme for adaptively combining the entirety of the capabilities of phased-array ultrasonic instruments that have traditionally been used independently. Untapped capabilities of an instrument vital to the power-generation industry will be exploited, with potential benefits to efficiency, robustness, and accuracy in inspecting new and in-service components. Unresolved issues, such as imaged artifacts from off-angle reflectors and multiple propagation modes, are being put into reach with this research.

\section{Technical Objectives}

Objectives for this year would allow the researchers to:

- Develop interface software to use the available vendor software to allow real-time ultrasonic data to be made available to the algorithms and provide the mechanism for loading the adapted focal laws into the instrument
- Develop an algorithm for adaptive generation of focal laws for a stationary probe to best reveal the geometry of a crack

- Initiate design and development of software architecture for the system to provide locomotion directives to a technician and/or robotic interface

- Submit invention disclosures and manuscripts for peer-reviewed publications and presentations at conferences as appropriate.

These objectives have been achieved during the FY-09 effort. The primary logistically necessary task to accomplish the overall goalto develop the capability to iteratively read data from the instrument and modify the instrument setup through a software interface through which the algorithms can take effect - was accomplished with the cooperation of the instrumentation vendor. A beta version of the library necessary to interface with the vendor's phased-array system was provided to this project. With this cooperation, the framework was put in place to explore algorithms in benchtop experiments. A variety of cracks in weld specimens have been acquired to generate a level of adaptive adjustments at the hands of a human to serve as the baseline for the experiment to compare the results of algorithms as they are developed. Baseline data has been acquired from crack samples for comparison and originally proposed crack-investigation algorithms continue; however, this has taken an exciting turn towards a related, but more fundamental and possibly significant discovery, with potentially greater benefit. Methods for determining the explicit direction for readjusting the sensor location are a direct result of this new method. One article for a peer-reviewed journal is in preparation based on this result, one invited presentation on ultrasonic phased array was delivered, and several potentially patentable concepts will be offered early in FY-10.

\section{Results and Accomplishments}

Ultrasonic waves are most commonly generated by "pulsing" a piezo electric crystal with a pulse of current to cause it to contract and return to impart a mechanical wave into a gas, liquid, or solid. The mechanical energy will 
propagate through the medium as a wave until it meets a discontinuity, where it may reflect, transmit, and/or diffract. In solids that can support transverse or shear waves, the energy may convert its mode between transverse- and longitudinal-propagation waves, which travel at different speeds of sound. Ultrasonics are used in a variety of applications that range from the familiar imaging of unborn babies to inspecting materials, such as welds in pressure vessels, armor, and pipelines. Common in state-of-the-art ultrasonic equipment is the capability to drive piezoelectric transducers made up of many small elements that can be pulsed with individual timing such that the energy from each element, acting ideally as a point source, can be generated so that they superimpose with constructive interference at a specified point in space.

Figure 1 illustrates the instrumentation: the flexibility to electronically steer at different angles, focus and depths, and raster scan and the use of different apertures when the probe has length and even different effective apertures for transmit and receive. This current project is taking a fundamental step towards even stronger capabilities that may be applied to all uses of ultrasonics with potential applications such as phased radar. Whereas proposed methods for digesting the plethora of data generally encourage more acquisition [1], this work is determined to acquire the correct data from the appropriate position when feasible and suggests optimizations of hardware capabilities should be developed to make this feasible more often.

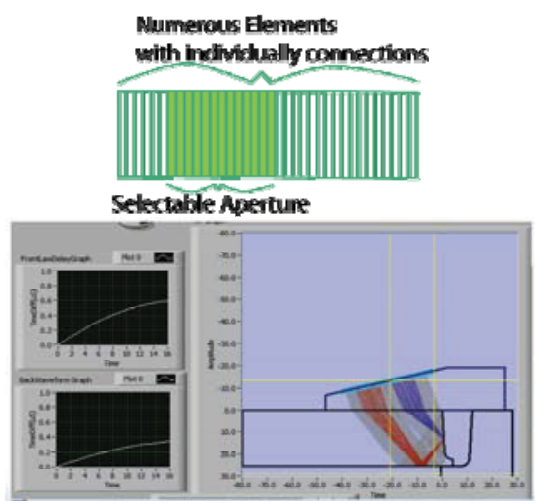

FIGURE 1. Top is an illustration of a linear phased-array transducer showing individually connected sections of a piezoelectric crystal. Bottom shows a screen capture from focal law calculator using two apertures and timing delays to achieve the desired focus.
Initially cracked weld samples were investigated for the capability to assign a measurement of depth, as shown in the original proposal of this work, the depth measurement can be in error when a vertical geometry assumption is made. As the data shows, adjustments can be made to more accurately image the extents of the feature.

In the process of developing the logistical tool to interface this type of algorithm to the phased array system, a related hypothesis was explored. Several possible artifacts can exist in image data produced by phased-array ultrasonics. Among these are off-angle energy returns simply from beam spread of from grating side lobes and alternate propagation mode returns, when longitudinal and shear waves are both present.

A genetic algorithm hypothesis [2] was formed for adaptively determining an optimized focal law. The setup for this experiment is shown in Figure 2. The phased array transducer is positioned in proximity to a side-drilled hole in a piece of stainless steel. A naïve (i.e., nonoptimized) random genetic algorithm was applied, initially to test the software interface. The amplitude of the signal within a prescribed time window was chosen as the measure of fitness. The window was chosen where a small amplitude signal was present.

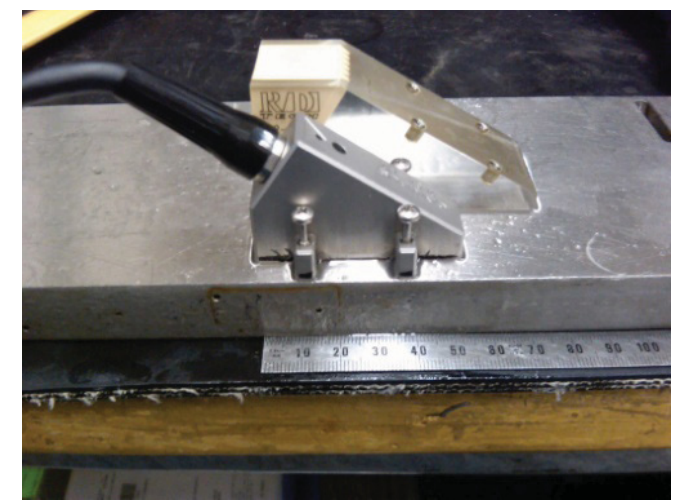

FIGURE 2. Photograph of bench setup for evaluation of adaptive interrogation of a feature. Shown is a 64-element phase array transducer place on a stainless-steel block with a standard side drilled hole.

The genes, in this case, are the delays assigned to each of the elements, which were each randomly perturbed by varying amounts. 
For each iteration, the delay patterns with the best fitness are chosen. Figure 3 shows that stronger and stronger candidates are found. This process is inefficient, but it establishes the inherent possibility of adaptively finding the optimal configuration. Figure 4 shows the result of a more efficient means of altering the parameters that generate the general form of a focusing delay pattern and leads to the next step of determining a more efficient algorithm early in the next year of this project. The significance is that indications that have small amplitude may not be completely described, and the resulting optimal-focal law allows for the determination of the location and mode of transit of the reflection, allowing for crisper and more accurate imaging in ultrasound (and possibly other) applications where phasing a superposition is applied.
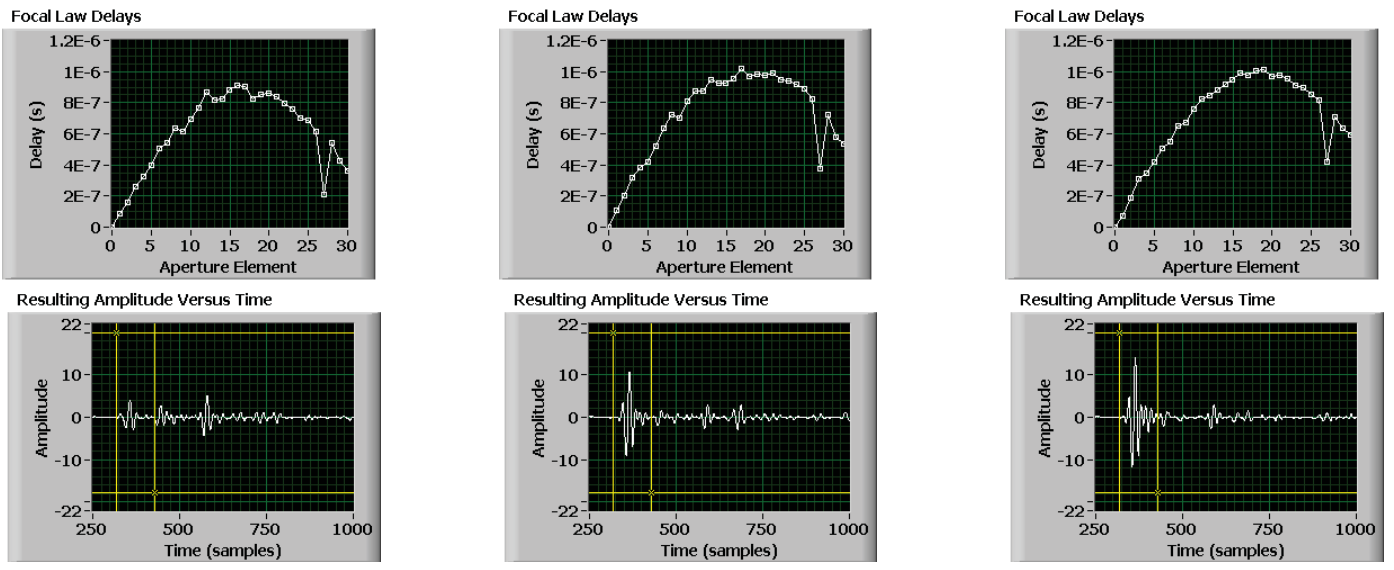

FIGURE 3. Illustration of result of the focusing result using a naïve genetic algorithm to adjust the individual delays of a focal law using the amplitude of a signal in with a specified time window as the measure of fitness. Sequence is increasing iteration from left to right. Top in each frame shows the focal law delays. Bottom shows the increasing amplitude of the echo of interest.

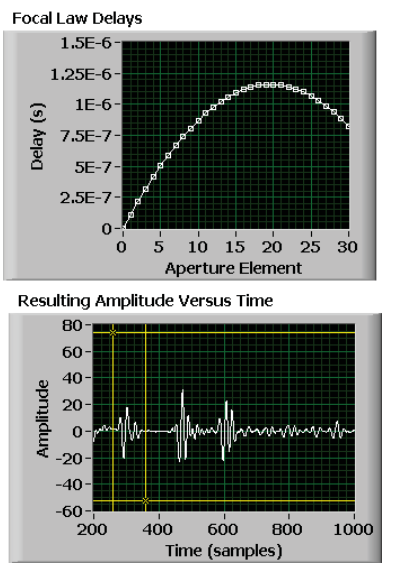

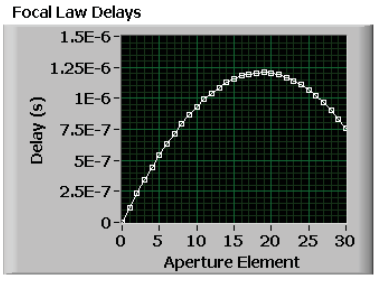

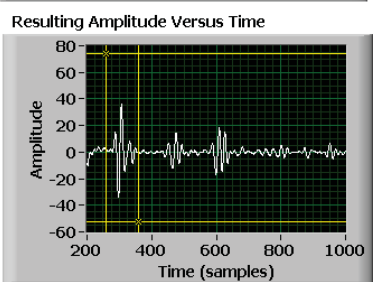

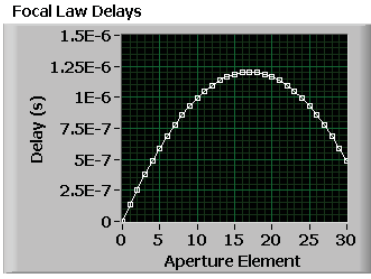

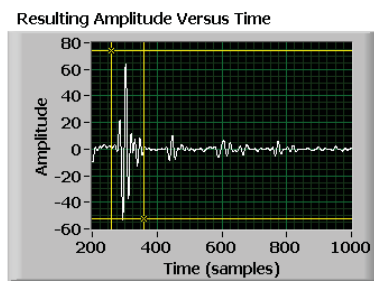

FIGURE 4. Illustration of result of the focusing result using a genetic algorithm where random mutations of parameters of the expected shape of a focal law curve is used. Convergence is an order of magnitude more efficient but requires a priori knowledge. Sequence is increasing iteration from left to right. Top in each frame shows the focal law delays. Bottom shows the increasing amplitude of the echo of interest. 
Summary and Conclusions

We have successfully created a framework for evaluating algorithms and a baseline for the potential for more accurately sizing cracks.

Additional, previously unexpected discoveries will contribute to the community a potential to be eventually worked into practice in the nondestructive examination field.
References

1. XE Gros, "Applications of NDT Data Fusion," Kluwer Academic Publishers, 2001.

2. Holland, J., "Outline for a Logical Theory of Adaptive Systems, ” JACM 9, 1962. 


\section{Investigation of Low-Temperature Performance in Membrane Materials and Processes for Gas Separations \\ Frederick F. Stewart, J. Orme, J. Douglas Way (Colorado School of Mines)}

Increasing the permeability of polymer membranes at low temperatures

ST130

One of the significant barriers to optimizing gas-separation membranes is the commonly observed trade-off in membrane permeability and selectivity. Many techniques exist for increasing the permeability of a polymer membrane to a targeted gas; however, improvements often come at the expense of selectivity. One parameter that has been poorly investigated and may be the key to significant improvements in gas permeability with little loss in selectivity is operating temperature. However, simply lowering the temperature at which a gas separation is performed without considering the nature of the polymer may not be the correct approach. The goal of this work is to investigate the interconnections between membrane structure, polymer glass transition temperature (Tg), and system operating temperature, which will lead to greatly increased membrane system performance, thus minimizing the costs of the permselectivity trade-off. Focus will be directed toward $\mathrm{CO}_{2}$ separations, which are becoming of greater importance.

\section{Technical Objectives}

The technical objectives of this project are as follows:

- Synthesize and characterize specific

- polyphosphazenes for enhanced

- gas-separation performance

- Determine $\mathrm{CO}_{2}$ permeability and solubility and for selected

- polyphosphazene membranes with respect to system operating temperature.

Results and Accomplishments

Synthesize and characterize specific polyphosphazenes for enhanced gas-separation performance. During Fiscal Year (FY) 2009, a novel phosphazene polymer (C) was synthesized that completed a series begun during FY 2008 (Fig. 1), where tert-butyl substituents have been added to the pendant groups systematically, with the goal of creating fractional free volume, or free space within the polymer membrane that will encourage greater gas diffusion. Polymer C was found to have a lower density as compared to polymers A and B. The synthesis of this material was expensive; thus, it is not expected that this polymer will have significant value other than for mechanistic study due to high cost. However, the purpose of this polymer was to provide a systematic determination of the factors in phosphazenes that affect gas transport. Preliminary gas transport studies have revealed greater permeability with respect to polymers A and $\mathrm{B}$, as expected. The study of this material will be completed in FY 2010. We expect that the inclusion of more tert-butyl groups per individual polymer unit will serve to create a membrane substrate that will encourage gas diffusion to a greater degree than the pendant groups shown for polymers $\mathrm{A}$ and $\mathrm{B}$. The question that exists is to what degree the performance is enhanced. A characteristic of these polymers that may work against the technique of adding bulkier substituents to create greater gas diffusion is the flexible nature of the polymer backbone. With a relatively stiff polymer backbone, configurations imparted by the pendant groups become "locked in" and, thus, greater fractional free volume is preserved. Phosphazenes, on the other hand, have flexible backbones, which can allow the polymer backbones to bend and twist into more dense structure, thus squeezing out free volume. This process generally occurs over a period of time and is often described as aging. The performance of polymer $\mathrm{C}$ with respect to temperature also will be completely characterized in FY 2010.
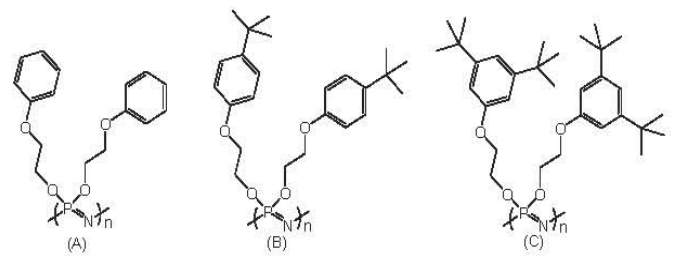

FIGURE 1. High-performance phosphazene polymers studied in this work.

Determine $\mathrm{CO}_{2}$ permeability and solubility and for selected polyphosphazene membranes with respect to system operating temperature. As a part of this project, a collaborative research effort with the Colorado School of Mines (CSM) was continued from FY 2008. During 2009, research concentrated on poly perfluoroalkoxyphosphazene (PFAP) membranes. A highly 
fluorinated structure (Fig. 2) gives this polymer a low $\mathrm{T}_{\mathrm{g}}\left(-60^{\circ} \mathrm{C}\right)$ and a high degree of resistance to chemical degradation. The advantage of this low $\mathrm{T}_{\mathrm{g}}$ polymer is that studies can be conducted over a range of temperatures without interference induced by the $T_{g}$. In this example, all experiments were conducted in the polymer's rubbery regime above $T_{g}$.

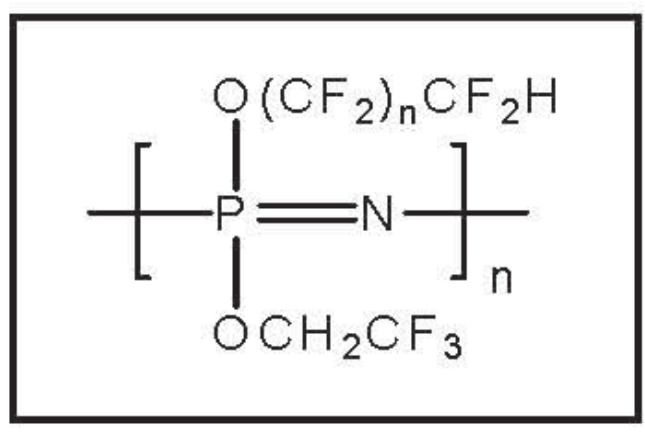

FIGURE 2. General structure of polyfluoroalkoxyphosphazene (PFAP) where $N=1,3,5$, and 7.

PFAP membranes were studied for permeability over a range of temperatures using both pure $\mathrm{CO}_{2}$ and $\mathrm{N}_{2}$ and various mixtures of these two gases. Additionally, $\mathrm{CO}_{2}$ and $\mathrm{N}_{2}$ solubility in this membrane was measured, which represents the first such measurement on PFAP. The significance of this study is that permeability and solubility are related by a simple relationship, where permeability is the product of solubility and diffusivity. Solubility represents the intimate chemical interactions between the gas and the membrane, while the diffusivity measurement characterizes the ability of a gas to move through the interstitial spaces within the polymer substrate. Thus, to understand the magnitude of the relative contributions to permeability, two of the variables must be measured. In this case, permeability and solubility were directly measured and diffusivity was calculated.

Pure gas permeability data for $\mathrm{CO}_{2}$ and $\mathrm{N}_{2}$ are shown in Figure 3 as a function of temperature. In general, the permeability of each gas decreases as the temperature is decreased; however, the more condensable $\mathrm{CO}_{2}$ decreases less, leading to an increase in the separation factor. This behavior was also shown in the mixed gas experiments (Fig. 4), which were performed using $80 \% \mathrm{CO}_{2} / 20 \% \mathrm{~N}_{2}$ and $20 \% \mathrm{CO}_{2} / 80 \% \mathrm{~N}_{2}$ gas mixes. Gas permeabilities, regardless of feed composition, were nearly identical. Comparison with the pure gas data reveals slightly lower $\mathrm{CO}_{2}$ permeability with the gas mixtures, which is not unexpected due to potential gas-gas interactions between $\mathrm{CO}_{2}$ and $\mathrm{N}_{2}$. Separation factors between the pure gas and

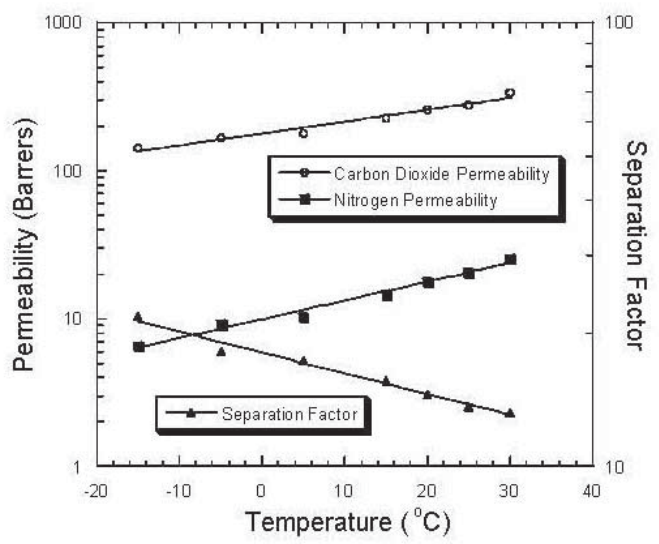

FIGURE 3. Pure gas performance of PFAP with respect to temperature.

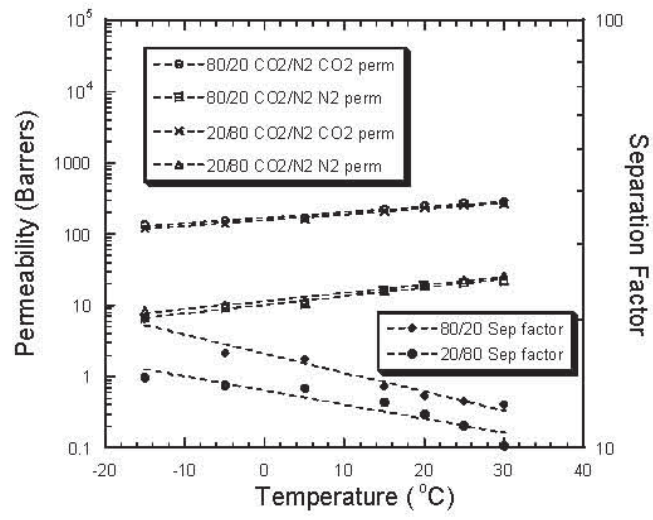

FIGURE 4. Mixed gas performance of PFAP membranes.

the two mixed gas experiments were roughly consistent across all temperatures studied. A more interesting result is the higher separation factors observed for the higher $\mathrm{CO}_{2}$ containing feedstream, which may be a result of the condensable $\mathrm{CO}_{2}$ blocking $\mathrm{N}_{2}$ transport, as shown by the slightly lower $\mathrm{N}_{2}$ permeability data. 
Sorption experiments were conducted over a range of temperatures, using $\mathrm{CO}_{2}$ as the analyte. $\mathrm{CO}_{2}$ solubility was measured to increase with decreasing temperature, as shown in Table 1. Using this and the permeability data, the diffusivity of $\mathrm{CO}_{2}$ through PFAP was calculated. As reflected in the permeability measurements, the diffusivity values are observed to decrease.
From this observation, we conclude that the decrease in the ability of the gas to diffuse through the polymer is a stronger influence on permeation than the increase in solubility. However, the increase in solubility also would tend to discourage nitrogen transport, as shown through higher separation factors.

TABLE 1. Solubility, permeability, and diffusivity of $\mathrm{CO}_{2}$ in PFAP.

\begin{tabular}{rccc}
\hline Temperature & Solubility Coefficient $(\mathrm{S})$ & Permeability & Diffusivity $\left(* 10^{6}\right)$ \\
\hline$\left({ }^{\circ} \mathrm{C}\right)$ & $\mathrm{cm}^{3} \mathrm{STP} \mathrm{CO}_{2} / \mathrm{cm}^{3}$ polymer .atm & Barrers & $\mathrm{cm}^{2} / \mathrm{sec}$ \\
\hline 30 & 0.95 & 336.35 & 2.71 \\
25 & 1.26 & 278.14 & 1.68 \\
15 & 1.95 & 226.87 & 0.88 \\
5 & 3.04 & 177.20 & 0.44 \\
-5 & 3.69 & 166.21 & 0.34 \\
-15 & 5.43 & 142.42 & 0.19 \\
\hline
\end{tabular}

\section{Summary and Conclusions}

This work has elucidated the relationship between the critical parameters in membrane transport - permeability, solubility, diffusivity, and temperature - using a perfluorinated rubbery phosphazene material as a model. Understanding these critical parameters in phosphazenes does not limit their utility to this class of polymer. Phosphazenes are important as models for other membrane systems; thus, this project has provided much enabling knowledge for the development of new and more effective membrane separation systems. Applications envisioned for the materials and concepts developed in this project potentially include the obvious $\mathrm{CO}_{2} / \mathrm{N}_{2}$ separation for $\mathrm{CO}_{2}$ capture, $\mathrm{CO}_{2} / \mathrm{CH}_{4}$ for natural-gas upgrading, and acid gas removal from both natural-gas and coalgasification systems. Another significant application is in $\mathrm{CO}_{2}$ separation from syn-gas ( $\mathrm{CO}$ and $\mathrm{H}_{2}$ ) that can facilitate the conversion of carbon feedstocks into either fuels or chemicals. During FY 2009, the project team produced its first peer-reviewed publication, accepted for publication in Journal of Membrane Science. 


\section{High-Performance Polymer Membranes for High-Temperature Gas Separations}

John R. Klaehn, Christopher J. Orme, William J. Koros

Improved membrane performance at high temperatures ST131

High-performance (HP) polymers are glassy polymers that include five polymer families (Fig. 1): polyazoles (polybenzimidazole [PBI]), phosphazenes (cyclomatrix derivatives), sulfur-containing polymers (sulfones, phenylsulfones), polyamides (Nylon, Nomex), and polyimides (Kapton, VTEC). HP polymers are known to have high thermal stabilities, with operating temperatures of $400^{\circ} \mathrm{C}$ that allow their use in applications unsuitable for most organic polymers. In addition, these polymers are resistant to acids, bases, and most organic solvents. They are mechanically robust, with high compressive strength. Even with such stability and strength, PBI and several polyamides are easily used (i.e., fabricated into parts, coatings, or free films) with properties that allow blending with copolymers and modifiers. At Idaho National Laboratory (INL), recent advances in PBI functionalization technology have facilitated further development of the other HP polymers. Exciting preliminary hightemperature gas separation results were obtained for two promising HP polymers, (PBI and polyimides).

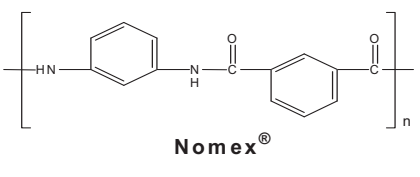

Polyamides (Nylon, Nomex ${ }^{\circledR}$ )

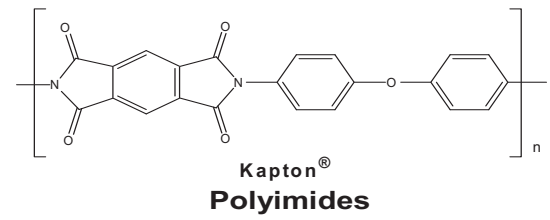

(Kapton ${ }^{\circledR}$, VTEC PI series)

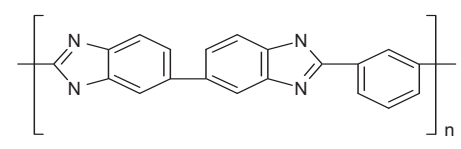

PB I Polyazoles (PBI, PBO)

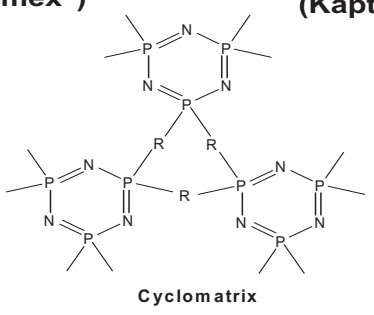

Phosphazenes (Cyclomatrix derivatives)

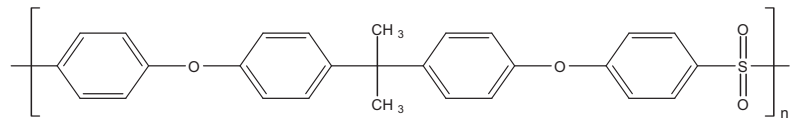

Poly (ether sulfone)

Polysulfones (Polyphenylsulfone)

FIGURE 1. High-performance (HP) polymer structures.

This project's goal is to increase membrane gas throughput while maintaining selectivity at high temperatures by developing new HP polymeric-membrane systems for gas-separation applications. Products include heat and environmentally treated polymer membranes that have desired carbon dioxide $\left(\mathrm{CO}_{2}\right)$ and/or oxygen $\left(\mathrm{O}_{2}\right)$ permeation properties. The proposed approach is based on "controlling" the polymer matrix, which includes heat and environmental treatments. The heat treatments were conducted by exposing the HP-polymer membranes to carefully controlled temperatures in a furnace.

Presently, this thermal treatment process is known to give highly selective membranes for gas separations under inert atmospheres (argon). The current literature lacks information regarding controlled heating under oxidizing conditions. We have carried out a suite of experiments in which we processed membranes using several different treatment methods in the hope that the membranes'gas transport properties are enhanced.

\section{Technical Objectives}

Technical objectives for the year included attempts to:

- Improve or stabilize the polymer matrix for better gas separations

- Control/enhance membrane gas transport properties using heat and environmental treatments

- $\quad$ Evaluate the resulting polymer membranes for gas transport via mixed gas and pure gas analysis. 


\section{Results and Accomplishments}

During FY 2009, we evaluated several high performance (HP) polymer membrane materials for this project: Kapton ${ }^{\circledR}$ (polyimide), VTEC polyimides, and polybenimidazole (PBI). These polymers were chosen for their high-temperature stability (greater than $250^{\circ} \mathrm{C}$ ) and their ability to provide gas separation. Our proposed approach was based on "controlling" the polymer matrix, which includes heat and environmental treatments. The heat treatments were conducted by exposing the HP polymer membranes to carefully controlled temperatures in a furnace. This thermal-treatment process is known to give highly selective membranes for gas separations under inert atmospheres (argon). However, current literature lacks information regarding controlled heating under oxidizing conditions.

We have processed multiple membranes under several different gaseous atmospheres, and our results show transport property improvements over the parent polymers, especially with Kapton. Using our processing methods, Kapton membranes provided excellent separations for carbon dioxide verses methane (Alpha $=34$ at $250^{\circ} \mathrm{C}$ ). As a result, we have successfully shown that the transport properties of a polymer can be changed using combined environmental and heat treatments, thus providing desired high-temperature HPmembrane transport properties. In addition, small molecule cross-linkers were found to greatly enhance the stability of these polymers. Certain polymer blends were shown to withstand very high temperatures (about $600^{\circ} \mathrm{C}$ ) without noticeable physical changes of the polymer blend. One particular polymer blend has shown desireable thermal properties with good gas throughput. Overall, it is thought that better synthetic and processing pathways that provide control (tuning) of the polymers' high-temperature gas transport properties $\left(>250^{\circ} \mathrm{C}\right)$ have been discovered.

The laboratory's high-temperature gaspermeability testing apparatus has been upgraded to provide mixed-gas permeability experiments of greater than $400^{\circ} \mathrm{C}$ (a new, unique INL capability). With this apparatus, Kapton membranes/films have been successfully tested at temperatures up to $450^{\circ} \mathrm{C}$. In addition, Kapton continues to give good carbon dioxide separation from methane for an extended period. This project's results, using a combination of environmental and heat treatments on polymeric films, are significant. Based upon results from Fiscal Year (FY) 2008, we postulated that using cross-linking agents with HP polymers might provide enhanced gas separations. During FY 2009, we found one cross-linked HP-polymer blend that forms stable high-temperature membranes. Remarkably, it provided even better gas separations than the parent polymer. This new polymer blend will be investigated next year using our treatment methods. These results demonstrate that a cross-linked polymer can give impressive high-temperature stability while achieving excellent gas separations.

We proposed to develop better working relationships with academia and industry and have done so. INL's enhanced relationships include: (1) Georgia Institute of Technology (Georgia Tech), for gas permeability analysis and development of small polymer-fiber membrane modules (contract research, supporting students and post docs) and (2) Texas Christian University (TCU), using positronannihilation-lifetime (PAL) spectroscopy for determining polymer-void volume of the membranes (contract with faculty member). We have developed a collaborative research and development agreement (CRADA) with Compact Membrane Systems for developing new blends of fluorinated polymers. Finally, new funding sources are being generated by this research through the Department of EnergyEnergy Efficiency and Renewable Energy's (DOE-EERE's) Industrial Technologies Program.

\section{Conclusions}

This research greatly benefits INL by enabling development of new approaches to membrane formation and processing for hightemperature $\mathrm{CO}_{2}$ separations as well as other industrial gases. Overall, this project provides methods that will benefit clean energy production at reasonable costs. This fiscal year, we have created key steps for future partnerships and targeted future funding opportunities for INL. We have developed collaborative relationships with several polymer 
manufacturers, including PBI Performance Products, Inc. (PBI), Compact Membrane Systems (CMS), and RBI, Inc. (VTEC) (polymer manufacturers). In addition, we are putting together collaborations with U.S. membrane manufacturers like Aire Liquide, Millipore, General Electric, Compact Membrane Systems (CMS), and Air Products. Also, INL will continue to collaborate with Georgia Tech and TCU. In addition, we have developed many working relationships/collaborations with industry and academia that will lead to development of commercial polymer membranes. The data and methods developed in this project were crucial for winning current projects that were awarded during FY 2009. The successes of the project will allow additional responses to expected DOE-Office of Fossil Energy (FE) and DOE-EERE solicitations in the upcoming years.

We have the unique technical understanding that makes possible chemical synthetic modification of several of the HP polymers and provides the basis for enhancing these polymers' performance. In addition, we have developed several scientifically cutting-edge polymerprocessing techniques and obtained gas separations data for both pure and mixed gases. In the future, the data obtained during this project may offer intellectual-property disclosures, as well as offering several publication and presentation opportunities. Overall, we are building an exceptional capability for gas-separation technologies that will continue INL's leadership in the area of membrane technology development for years to come.
Results and Tangible Accomplishments

- $\quad$ Funded proposal with Battelle Memorial Institute (BMI) for $\$ 240 \mathrm{~K}$

- $\quad$ DOE-EERE ITP (funded in FY 2010)

- $\quad$ Proceedings and presentations that will be published

- Klaehn, J. R.; Orme, C. J.; Luther, T. A.; Peterson, E. S.; "High temperature gas separations using high performance polymers," Divisions of Fuel and Petroleum Chemistry: Advances in Membrane Science and Technology, American Chemical Society, 2010 Spring National Meeting, 2010. (Proceedings)

○ Klaehn, J. R.; Orme, C. J.; Luther, T. A.; Peterson, E. S.; "High temperature gas separations using high performance polymers," Divisions of Fuel and Petroleum Chemistry: Advances in Membrane Science and Technology, American Chemical Society, 2010 Spring National Meeting, 2010. (Invited Presentation)

- Collaborations with Universities

- Georgia Institute of Technology (William J. Koros).

- Texas Christian University (Carroll A. Quarles).

- Future opportunities with collaborations and/or funding are forthcoming. 


\title{
Solid $\mathrm{CO}_{2}$ Capture Agents
}

\author{
Michael T. Benson
}

Elucidating the mechanism of $\mathrm{CO}_{2}$ binding in solid systems

ST132

The technical approach of this laboratorydirected research and development (LDRD) project is to investigate solid materials for $\mathrm{CO}_{2}$ capture, with the goal of elucidating a mechanism of $\mathrm{CO}_{2}$ binding. A great deal of information in the literature concerns the mechanism of $\mathrm{CO}_{2}$ binding in a liquid system, such as in commercial alkanolamines, but no information exists concerning similar mechanisms in a solid system.

\section{Technical Objectives}

The objectives of this LDRD were to synthesize materials to help elucidate the mechanism of $\mathrm{CO}_{2}$ binding to compounds containing amines. The materials could be discrete molecules, polymeric materials, and extended structures (through hydrogen bonding).

\section{Results and Accomplishments}

Reactions attempted previously, such as those shown in Schemes 1 and 2, were not successful in synthesizing the material of interest. Although the reaction in Scheme 1 was successful, the product was not able to be isolated from the reaction. The reaction shown in Scheme 2 was also successful, although when it was reacted with the support material, the imine bond $(\mathrm{C}=\mathrm{N}$ bond $)$ reacted, not the primary amine. A third protecting group was attempted, shown in Scheme 3, with the actual product, not the desired product. Instead of protecting one primary amine, leaving the second primary amine for attachment to the support material, both primary amines were protected, or neither reacted. This may be due to a cooperative effect between the amines. Regardless of the reason, the trifluoroacetate protecting group was not successful.

At this point, a different approach was attempted. Instead of protecting a primary amine, an amine precursor, a nitrile, was attempted. After attachment to the support material, the nitrile could be hydrogenated to the primary amine. The nitrile can be purchased as the aminoacetonitrile bisulfate salt. A range of reaction conditions were attempted for this substituent, with the hope of removing $\mathrm{H}_{2} \mathrm{SO}_{4}$, leaving behind aminoacetonitrile. Attempts to remove $\mathrm{H}_{2} \mathrm{SO}_{4}$ prior to reaction with the support material only yielded a red oil. Previous work has shown that a red oil is produced when the amino-nitrile reacts with itself. Attempts to remove $\mathrm{H}_{2} \mathrm{SO}_{4}$ in situ were also not successful; the red oil was again generated, and no substitution took place on the host material.<smiles>CC(C)C(=O)C(C)C(C)C</smiles>

Scheme 1. Protecting primary amine with 2,4-dimethyl-3-pentanone.

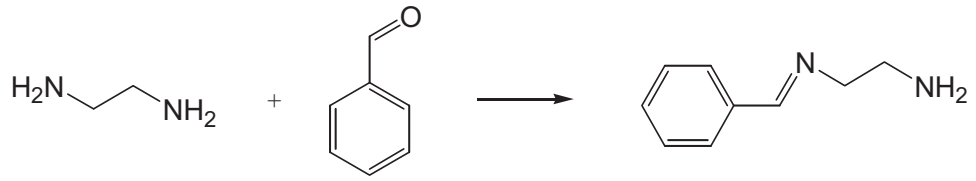

Scheme 2. Protecting primary amine with benzaldehyde.

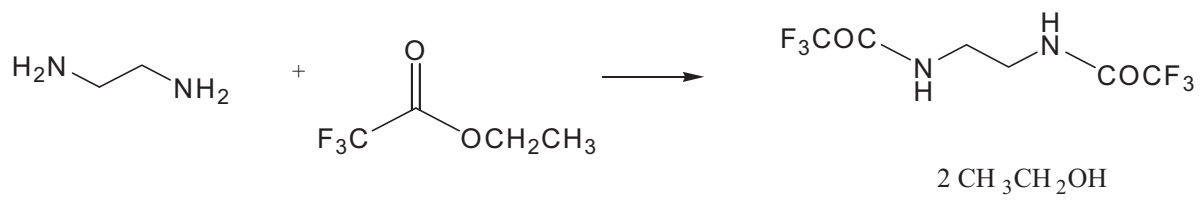

Scheme 3. Protecting primary amine with trifluoroacetate group. 
An analogous material, glycolic acid nitrile, was also attempted. To date, all of the reaction conditions attempted with this reaction have failed to produce the desired product, but this is a molecule worth pursuing. Once the nitriles are hydrogenated to primary amines, the substituents are basically ethanolamine, one of the commercial $\mathrm{CO}_{2}$ capture agents. The mechanism for $\mathrm{CO}_{2}$ capture by ethanolamines in solution has been extensively studied. Carbon dioxide forms a covalent bond with the primary amine in ethanolamine, making a carbamate. This covalent bond is the reason the energy requirements to regenerate the sorbent are so high. After attachment to the support material, the material will be a solid. It will be easy to discern using NMR spectroscopy if a carbamate is formed in the solid material as well. More work will be performed on this reaction.

Summary and Conclusions

A great deal of progress was made on the synthesis of these materials. These systems will help elucidate the mechanism for $\mathrm{CO}_{2}$ binding, which in turn will allow improvements to the system. 


\title{
Dynamic Impact Model and Information System to Support Unconventional-Fuels Development
}

\author{
Randy Lee, John Koudelka, Art Rood, Dan Ames (ISU) \\ Modeling the dynamic impact of unconventional fuel development \\ ST133
}

Development of unconventional

hydrocarbon resources has the potential to enhance the energy security of the United States, which possesses substantial resources in the Mountain West. For example, it is estimated that the equivalent of 1.5-1.8 trillion barrels of oil exist in oil shale deposits of the Uinta, Green River and Washakie Basins. Undoubtedly, locating, extracting, and processing these resources will draw people to the area and increase demand on water. Therefore, development of unconventional fuel sources, such as oil shale, commonly faces two principal challenges: (1) environmental, the most salient of which centers around water, and (2) socioeconomic, as it relates to rapid growth and development in rural areas. Each of these challenges has a broad range of temporal and spatial scales, with their solutions being highly interdependent with one another due to the interconnectedness of the entire system. Resource managers and stakeholders need tools and methods to identify, understand, anticipate, and mitigate the potential health and environmental effects of unconventional-fuel development.

The objective of this research is to develop a dynamic impact-modeling system to enable resource managers and stakeholders to understand and predict the potential environmental, social, and economic consequences of unconventional fuel development on the western United States across both spatial and temporal scales (Fig. 1). The foundation of this objective is to integrate existing and proven environmental models into a sophisticated state-of-the-art decision-support system that will provide better and more accurate predictions by allowing the data from each model to update the other. The system will contribute to a better understanding of the interdependencies between natural and human systems and will allow extensive evaluation of potential management options. A key capability is to provide a virtual test environment in which the user will interact with a time-based simulation and visually perceive the consequences and effects of various management options across the landscape. The initial focus of this research centered around the availability of water resources and the impacts associated with this resource by developing an oil-shale industry in the Piceance Basin in Northern Colorado.

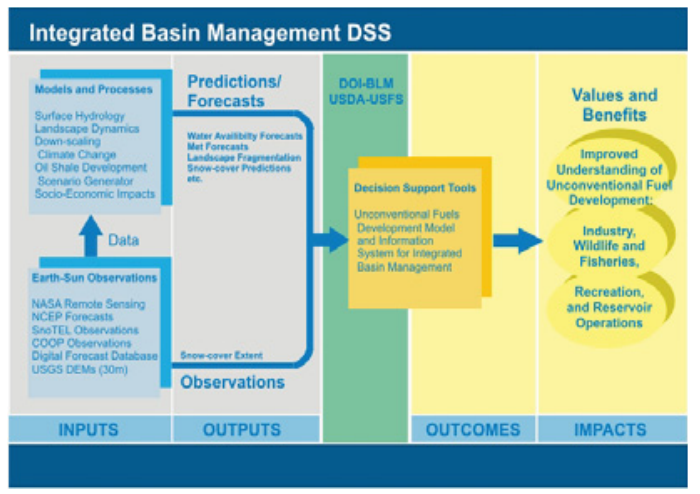

FIGURE 1. Integrated modeling systems approach.

\section{Technical Objectives}

Technical Objectives for Fiscal Year (FY) 2009 (second year of funding):

- Obtain spatial and tabular data for run-off and landscape change models

- Calibrate and run models

- Validate results against existing data

- Begin development of integration framework

- Choose global climate-modeling data and downscaling techniques.

\section{Results and Accomplishments}

During the previous year, we had selected two physically based water run-off models - the distributed hydrology soil vegetation model (DHSVM) and the regional hydro-ecological simulation system (RHESSys) model. After preliminary evaluation, based on model support and availability of spatial and tabular data, we determined that the high spatial and temporal resolution data required for these models were not available for the Piceance Basin. Difficulty in compiling, processing time, and user support were also issues with both of these models. Consequently, we decided to use a wellestablished and widely accepted model: snowmelt runoff (SRM). SRM runs on a PC/Windows platform and requires much less detailed information. We also decided to evaluate this model in a basin for which all the required data 
inputs exist. This well-instrumented basin is the upstream catchment to Jackson Lake, near Grand Teton National Park (Fig. 2).

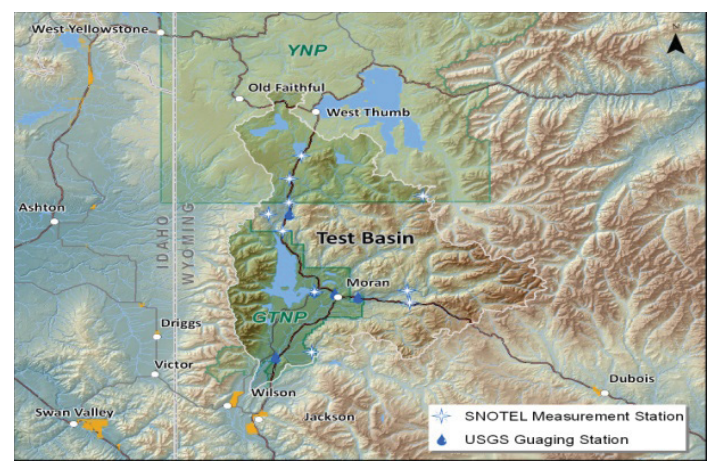

FIGURE 2. Test basin above Jackson Lake.

Along with the physical models chosen for the project, a change to the framework that connects the system's models was also made. In the first year, we had planned to utilize High Level Architecture ( HLA ) IEEE 1516 standard for developing our framework. After further evaluation of this standard, however, we decided an open-source framework would be better suited as many of the end-users of this product will likely require a low-cost or free modelintegration application. The open-source framework chosen is Open Modeling Interface (Open-MI), which is a standard interface that allows models to exchange data with each other and other modeling tools on a time-step by timestep basis as they run.

Specific results for the year include obtaining hydrology, hydrologic units, topography, vegetation, precipitation, and stream-flow data for the basin above Jackson Lake. We also successfully compiled, populated with data, calibrated, and ran SRM in the basin (shown in Figure 2) for individual years that were wet, normal, and dry (1996, 2000, and 2001 respectively [Fig. 3]).

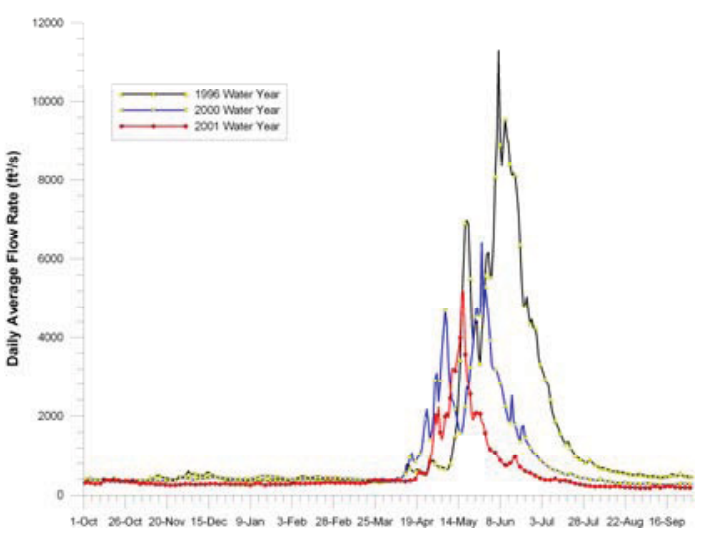

FIGURE 3. Stream flow plot for catchment above Jackson Lake.

A contract with Idaho State University (ISU) was established to fund a geography doctoral student to develop the framework-using OpenMI - which will connect the run-off and landscape dynamics models in a GIS-enabled environment. During the last few weeks of the fiscal year, ISU began to integrate OpenMI with Map Window 6. Map Window is an open-source GIS application that was developed under an LDRD project several years ago through INL and Utah State University. This integration will provide a graphical user interface that will allow easy execution of SRM in conjunction with geospatial and time series data.

We also successfully identified global climate-modeling data that will be used to run SRM and VDDT in a forecast mode, within the Open-MI framework, to test future impact scenarios regarding energy development.

\section{Summary and Conclusions}

In summary, the second year of this project has resulted in the final selection of models, framework-development platform, test basin, and data for developing a sophisticated state-of-theart decision support system for watershed mangers and stakeholders to understand and predict the potential environmental, social, and economic consequences of unconventional-fuel development across both spatial and temporal scales. 


\title{
Near-Field Impacts of In-Situ Oil-Shale Development on Water Quality
}

\author{
Thomas R. Wood, Carl Palmer, Hai Huang, Earl Mattson \\ Controlling and monitoring groundwater quality during oil shale development \\ ST134
}

Oil shale in the arid western states contains vast quantities of kerogen that could provide oil to significantly reduce the United States' dependence on foreign oil. However, many technical challenges remain to be solved before this important resource can be developed, including significant environmental concerns related to water. Available data to evaluate impacts to water resources are decades old and were collected under conditions that do not represent new oil shale in-situ technologies. Most current approaches are based on slow heating for long time periods at temperatures between 260 and $375^{\circ} \mathrm{C}$ to convert kerogen to oil. Formerly, oil-shale development involved fast heating to temperatures much greater than $600^{\circ} \mathrm{C}$, producing different and often more hazardous waste products. Groundwater models of the impact of oil-shale retorts on aquifer water quality will require knowledge of the contaminant fluxes, which are primarily controlled by the concentration of contaminants at the source and the permeability of the retort zone. The objectives of this research are to understand the oil and gas produced and the associated contaminants, to determine permeability during and after in-situ retorting, and to develop modeling and simulation tools to evaluate impacts on groundwater. Through careful laboratory testing of oil shale, we are defining the relationships between leachable contaminants and the applied heat and pressure. Additionally, we are developing quantitative relationships among the characteristics of permeability, retort heating history, and effective stress. These relationships are being incorporated into heat-flow models that will allow us to characterize the permeability field immediately surrounding the retort zone.

Controlling and monitoring groundwater during development is a key challenge for bringing unconventional fossil-fuel resources online to help secure the energy future for the United States (GAO, 2007). This proposal supports the DOE mission to accelerate development while simultaneously protecting groundwater resources. We will provide Idaho National Laboratory/Department of Energy (INL/DOE) with capabilities for environmental protection of water resources by creating the scientific models required to understand changes in groundwater flow fields, and predict contaminant release and migration during and after oil-shale retorting. These understandings, when coupled with INL cutting-edge computational tools, will provide stakeholders with a means of evaluating potential impacts on groundwater and designing appropriate monitoring systems. In addition, the potential for industry collaboration is high, as the end product will be beneficial for facilitating successful permitting of developments.

\section{Technical Objectives}

Our research is closely aligned with the Palmer, et al. (2007) LDRD, which is evaluating the character of shale oil recovered by modernday lower-temperature in-situ processes. The Palmer LDRD evaluates oil produced from oil shale heated across a range of temperatures, pressures, and water saturations. The waste material, or spent shale, from their experiments provides an ideal source of material for our leach tests and permeability tests of spent shale. Furthermore, equipment is shared between the two research projects. In addition to characterizing the leached contaminants from spent shale, this LDRD measures the permeability of oil shale that has been heated to temperatures below pyrolysis (the temperature required to produce oil from oil shale) to characterize the groundwater flow field adjacent to the retort chamber. Our project tasks are listed below.

\section{Task 1: Obtain oil shale - Complete.}

Task 2: Identify and determine concentrations of potential contaminants - Largely complete for all oil-shale samples under various retort-heating scenarios needed for environmental transport source terms.

Task 2a: Analysis of retort water and air fractions - Work continues with additional scenarios for release being developed based on industry interactions. We added $\mathrm{CO}_{2}$ this year, which was needed for an air source term.

Task 2b: Analysis of spent oil shale - Complete with the exception of several planned specialized tests; data are required for understanding the long-term potential of abandoned retort to act as source to ground water. 
Task 2c: Leach tests on spent oil shale after flushing - Work has begun and will be complete next year. Results are critical for ascertaining long-term environmental impacts.

Task 3: Permeability of oil shale - Several experiments conducted to assess the evolution of fracture permeability with heating. The results are needed for modeling near field release to aquifers.

Task 3a: Measure permeability of spent and unspent oil shale - About 50\% complete and will be finished next FY. The results are critical groundwater, air-flow and transport simulations.

Task 3c: Measure changes in fracture permeability with temperature - See Task 3 above.

\section{Task 4: Modeling.}

Task 4a: Fracture heat flow and geotechnical model - GeoFracFH code is in the process of being copyrighted. The model is critical for understanding the how tightly coupled processes evolve and affect contaminant transport during retort heating and post abandonment.

\section{Results and Accomplishments}

Several dozen oil-shale retorting experiments have been run this year to evaluate the toxic material produced by in-situ oil shale retorting and the toxicity of the spent oil shale remaining after retorting. The FY 2009 work advanced the laboratory testing initiated in FY 2008, primarily using instruments designed and built last year. Experimental conditions were systematically varied in terms of the maximum heat achieved during retorting, the duration of the retort, the grade (gallons per ton) of the oil shale ore, and the water (hydrous) content of the retort experiment to quantify relationships between retort conditions and contaminants produced. A suite of carefully controlled experiments using spent oil shale were also conducted to evaluate the chemical transformations, leaching characteristics and environmental water quality impacts from in-situ oil-shale retorting. A data set has been created that characterizes contaminants released under various retort conditions.
Retort experiments were conducted using intact cores of oil shale under compressive stress to evaluate the role of fractures and other geomechanical transformations on the mobility of contaminates at the boundary of in-situ retort zones. These experiments required engineering and machining specialized core holders to hold the core under confining pressure as a means of simulating in-situ conditions of pressure.

Additional experiments were conducted evaluating how fracture permeability evolves during heating due to plastic deformation of the oil shale. A surrogate fracture was created with a known permeability, placed in the core holder, and then retorted. After retorting, the permeability of the surrogate fracture decreased by three orders of magnitude due to plastic deformation/annealing of the fracture. This has important implications for contaminant transport by ground water away from spent-shale zones.

Laboratory experiments were conducted for the explicit purpose of considering vapor or gas transport to the atmosphere from in-situ oil shale retorts. These experiments were similar to the water phase experiments, with the addition of a sorption test of produced gases by raw oil shale. Basically, the exhaust gases were directed into a chamber filled with chipped oil shale material and allowed to equilibrate. Samples of the gas from the chamber were compared to samples from the retort to evaluate if sorption of contaminants will occur on the surrounding native rock. The results of the laboratory experiments will serve as input to a vadose-zone transport model and ultimately an air-transport simulation, which will be presented at the 29th Oil Shale Symposium.

The modeling and simulation activity has continued to advance the now-copyrighted computer code, initiated in FY 2008, by including plastic deformation and adding parallel computing capability. The GeoFracFH Model is able to simulate heat, fluid flow, and geomechanical rock deformation during in-situ retorting. Of considerable importance is the fact that these processes are very tightly coupled, and this code accurately simulates the feedback between heat, geomechanical deformation (fracturing), and fluid flow. This capability is not available from any other source, and it is 
required to simulate the evolution of rock permeability and porosity with heating, a critical factor for investigating the potential environmental impact to groundwater. A brief summary of the advancements made to the model in FY 2009 is provided below.

The GeoFracFH Model is a particle-based discrete-element model (DEM) that has been coupled with fluid flow and heat conduction/convection. The rock matrix material is represented by a network of DEM particles connected by mechanical bonds. During the simulation process, the mechanical bonds that have been stretched or bent beyond a critical strain - both tensile and shear failures are simulated - are broken and removed from the network in a progressive manner. Bonds can be removed from the network with rates or probabilities that depend on their stress or strain, or the properties of the discrete elements and bonds can be varied continuously to represent phenomena such as creep, strain hardening, and chemical degradation. Darcy flow and heat transport equations are solved on an underlying fixed-finite difference grid with evolving porosity and permeability for each grid cell that depends on the local structure of the discreteelement network (such as the DEM particle density). The fluid-pressure gradient exerts forces on individual elements of the DEM network, which then deforms and fractures the rock matrix. The deformation/fracturing, in turn, changes the permeability, which again changes the evolution of fluid pressure, thereby coupling the two phenomena. The intimate coupling between fracturing, fluid flow, and thermal transport makes the GeoFracFH model, rather than conventional continuum mechanical models, necessary for coupled hydro-thermalmechanical problems in the subsurface.

\section{Summary and Conclusions}

This LDRD has been successful on several fronts by (1) adding a technical capability to INL in line with energy development in the western U.S., (2) opening a new market niche for INL in unconventional strategic fuels that is highly leveraged with several ongoing and new national, regional, and local efforts, (3) generating and disseminating new scientific findings and results, (4) elevating INL's technical reputation in the oil-shale industry, and (5) making significant contributions to solving the nation's daunting problems with respect to imported fossil fuels. Many industry and university relationships have been developed over the year. Two competed research proposals have been awarded, and two industry contracts are in place as a direct result of this LDRD. 


\title{
Advanced Remote Sensing for Energy and Environmental Applications Using Unmanned Aerial Vehicles
}

\author{
Matthew O. Anderson, Ryan C. Hruska \\ Adapting UAVs to detect energy resources \\ ST135
}

Remote sensing technologies have been used to study and understand local and regional issues since the 1850s. However, the application of this technology to environmental studies was limited until advancements in multispectral imaging gained during World War II were applied to vegetation-health research in the 1950s. Since that time, rapid advancements in sensor technologies and satellite platforms have provided researchers and resource managers the ability to study ecosystem events and functions at both global and regional scales at everincreasing spatial and spectral resolutions. With the advent of unmanned aerial vehicles (UAVs) and miniature-sensor technologies, it is now possible to study and gain understanding of ecosystem functions and changes at scales never thought possible.

Idaho National Laboratory (INL) has built a nationally recognized UAV program through innovative work in command and control of these aircraft. Now, due to recognized data needs for defense, security, and environmental applications, INL is enhancing its UAV program by integrating remotely sensed data acquisition and processing systems. Autonomous and pilotin-the-loop UAV sensor platforms provide many advantages over traditional human-piloted aircraft and satellite sensor platforms. Such advantages include long loiter times, quick response, lower cost, high temporal and spatial resolution capabilities, and the ability to fly into areas deemed too dangerous for human-piloted aircraft. To date, UAVs have primarily supported military-style operations. However, there is a growing interest in the potential application of small, low-altitude UAVs to serve as remote sensing platforms for various environmental and scientific applications, including supporting forest-fire fighting, detecting invasive weeds, and tracking oil-spills, to name a few. Although UAV potential for environmental and scientific applications appears almost limitless, several hurdles must still be overcome to fully utilize these unique assets operationally. Challenges range from regulatory issues to system integration to data processing and analysis.

The overall technical approach of this research project is to develop an integrated UAV-based hyperspectral remote sensing system that provides the capability to detect, identify, and characterize changes to and functions of ecosystems, in addition to providing the technologies required to integrate into the national airspace. This will be accomplished by applying the INL low-altitude remote sensing workflow.

\section{Technical Objectives}

Technical Objectives for Fiscal Year (FY) 2009 were as follows:

- Hyperspectral-sensor selection, procurement, and calibration

- Arcturus T16 UAV airframe characterization, flight readiness, and sensor integration

- Flight tests to gather hyperspectral data over a control field.

\section{Results and Accomplishments}

\section{Hyperspectral Sensor Selection,}

Procurement, and Calibration. Initial evaluation of small hyperspectral-imaging (HSI) systems resulted in identifying two potential candidates suitable for integration into the UAV platform: Resonon and Novasol. One of the first objectives of this LDRD was to rent each of these systems and perform a head-to-head data comparison and analysis. However, the Novasol system rental was cost prohibitive for this project. As a result, a comparison was conducted using laboratory calibration results produced by Resonon and the Naval Research Laboratory, who funded the development of the Novasol system and agreed to share the resulting reports with the INL, a fellow government laboratory. Resonon was contacted and agreed to provide comparable data for the required data analysis activity. It was determined that the Resonon system could achieve objectives of this LDRD, based on availability, ability to integrate with the existing autopilot, system form factor, and cost. A capital-equipment request has been made to the Department of Energy (DOE) seeking opportunities to obtain the Novasol HSI system in order to perform a more rigorous evaluation.

Initial laboratory calibrations of the Resonon PIKA II imaging spectrometer were conducted at Resonon's laboratory utilizing a Labshpere, Inc., 
integrating sphere, which resulted in signal-tonoise ratios (SNR) as high as 250:1, peaking in the $600-\mathrm{nm}$ region (Fig. 1). Actual SNR achieved during ground and flight tests were closer to 100:1, which was expected based on environmental conditions and the need to increase the gain of the spectrometer. In addition, calibration coefficients were generated to enable the transformation from digital numbers $(\mathrm{DN})$ to radiance $\left(\mathrm{W} / \mathrm{m}^{2} / \mathrm{sr}\right)$.

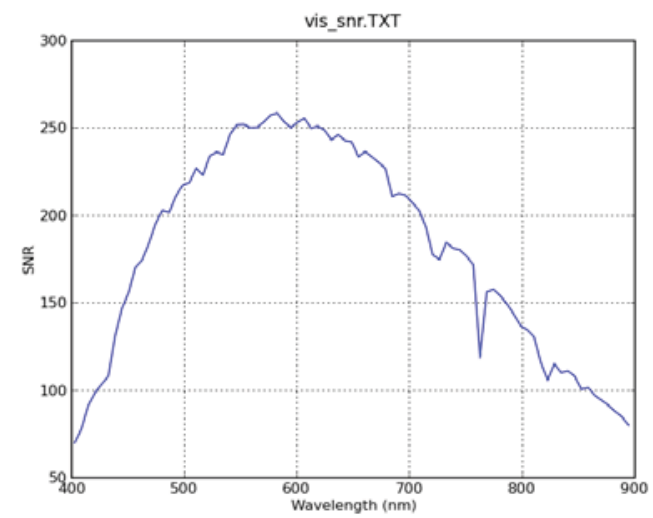

FIGURE 1. Laboratory calibrated signal-to-noise ratio.

\section{T16 UAV Characterization, Flight}

Readiness, and Sensor Integration. As required by INL's standard safety procedures, project Laboratory Instruction 1489-08-NBA "Unmanned Aerial Vehicle Research" was written and approved in November 2008, allowing development activities to proceed at RIC-613 NBA and B16-710 UAV Runway Laboratories. The T15 launcher was modified per the manufacturer's recommendations to accommodate the increased size of the T16 airframe. These modifications were completed using INL R\&D (Research and Development) Support Services, and the resulting system was successfully pressure-vessel and no-load tested. A new center-of-gravity balancer is required with the T16 wet wing configuration. This was manufactured, again by INL R\&D support services, and correct balancing was verified with Arcturus.

The T16 airframe was mechanically verified for flight worthiness, including the on-board power generator. Latest autopilot configuration files were obtained from the T16-airframe manufacturer and simulated via software-in-theloop autopilot software to verify appropriate gains and settings for INL flight conditions. A real-time kinematic differential global-position system (RTK DGPS) was procured and integrated into the T16 and UAV ground station. This allows for very accurate geo-rectification of the HSI data as well as autonomous landing of the UAV (a significant capability that will greatly improve the safety of UAV operations at the INL). All of these systems were flight tested in April using a dummy payload of the Resonon HSI system. Upon successfully completing the safety spiral flight test, the Resonon HSI system was integrated into the T16 (Fig. 2) and prepared for the initial data-acquisition flight tests.

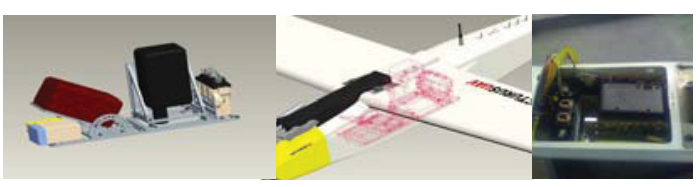

FIGURE 2. Integration of the Resonon HSI into the Arcturus T16.

Flight Testing. Four calibration flights were conducted over the INL UAV runway from May to July of this year. The purpose of these flights was to test geometric stability and radiometric performance of the Resonon airborne imaging system under operational conditions. Overall, geometric stability was good, with a resulting root-mean-square error of approximately 0.60 . However, differential vibrations resulted in a fine-scale saw-tooth pattern apparent in the resulting data. This issue is being investigated. In-flight radiometric calibration of the hyperspectral sensor was achieved using a NISTtraceable calibration standard and general-use calibration tarp targets. Initial evaluations are being conducted using an empirical linecalibration method. Preliminary findings indicate achievable SNR of 100:1, as reported above, using an 80-band configuration for $\sim 1000-\mathrm{ft}$ above-ground level (see Fig. 3, next page, for resulting initial data geo-referencing). 


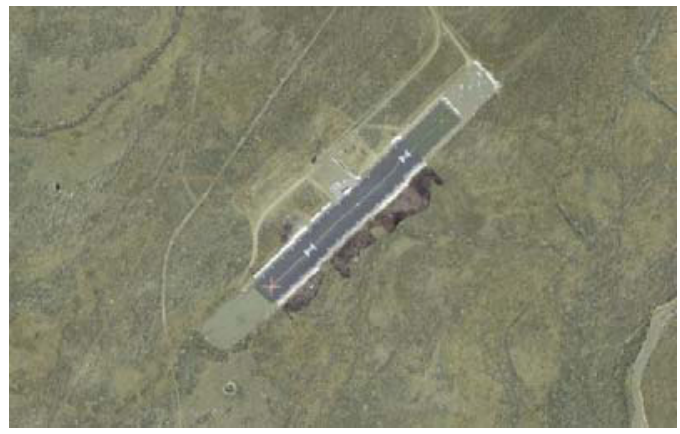

FIGURE 3. Initial geo-referenced Resonon PIKA II data.

In addition, subcontracts were placed with Idaho State University (ISU) Geosciences, including work by Professor Nancy Glenn and $\mathrm{Ph}$.D. student Jessica Mitchell and use of a fielddeployable radiometric calibration unit (FieldSpec Pro, analytical spectral device). ISU supported field tests in May and July by developing a data-collection plan targeted at comparing ground versus aerial hyperspectral data aimed at determining leaf nitrogen content of sagebrush at the INL UAV Research Park (Fig. 4). Radiometric measurements were taken at 35 GPS-located sample points during the solar noon test flights in May and July. Leaf samples were also taken and will be dried and analyzed for nitrogen content. Correlations between nitrogen content and band absorption features in the field-radiometer data will be explored statistically. The field-radiometer data will then be compared to the hyperspectral data from Resonon to identify the capability of using the Resonon data for nitrogen-content detection in sagebrush. Preliminary results indicate detection of nitrogen content in the field-spectrometer data. Relevant wavelength intervals are: 596-631 $\mathrm{nm}, 632-730 \mathrm{~nm}, 1855-2008 \mathrm{~nm}, 2009-2220$ $\mathrm{nm}$, and $2221-2421 \mathrm{~nm}$. This project will address whether the SNR and spectral bounds (400-900 nm) of the current Resonon sensor are sufficient for estimation of foliar nitrogen concentration in sagebrush. For this study, ISU has donated matching time and travel for Glenn and Mitchell. Other resources include donation of the FieldSpec Pro from Bonneville County, Idaho.
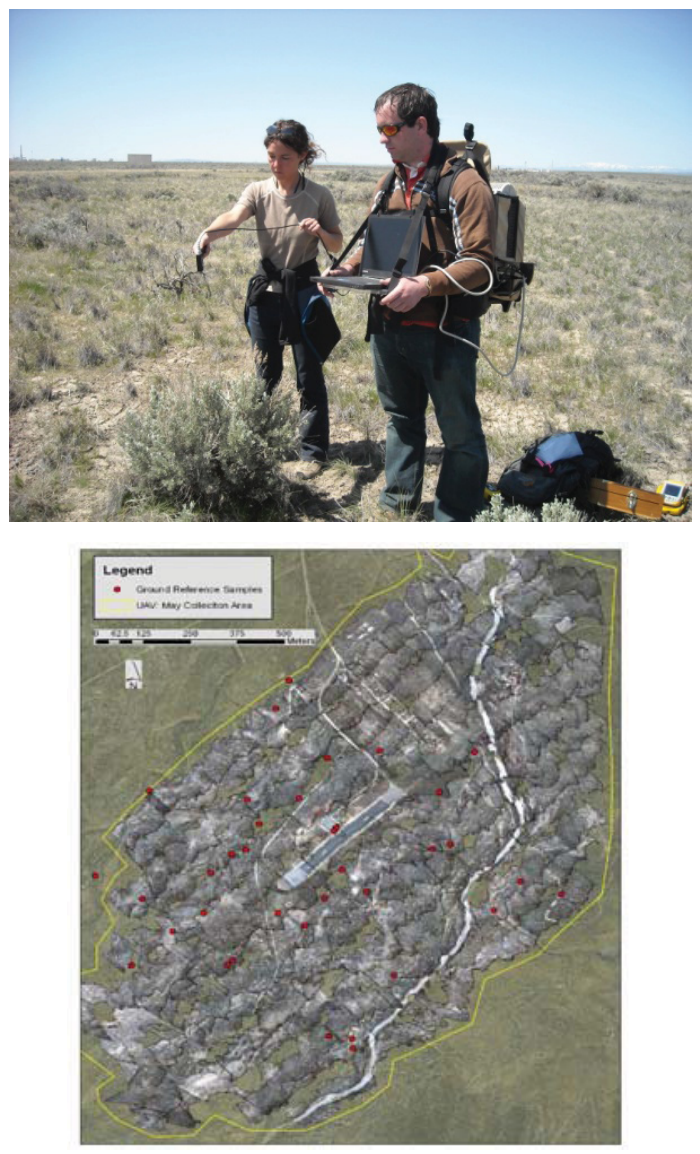

FIGURE 4. Geo-referenced ground data collection versus esonon PIKA II UAV-based data collection.

\section{Summary and Conclusions}

Year 1 resulted in the successful selection and integration of the Resonon PIKA II hyperspectral-imaging system on board the Arcturus T16 UAV airframe, four fully autonomous flights (including take-off and landing), and hyperspectral geo-referenced imagery of the INL UAV Research Park. These flights demonstrated the ability to collect fine spatial-resolution hyperspectral imagery from small, Class-II UAVs. The resulting imagery is being evaluated by both INL and Idaho State University. Also noteworthy is Resonon's Phase II SBIR, in which they include INL as a partner in deploying a short-wave version of their HSI system. This SBIR response is due in the third quarter of 2009. 


\section{Addressing the Spectrum of Nuclear-Related NDE Needs}

David Hurley, Dennis Kunerth, Zhandos Utegulov

Developing ultra-high-frequency ultrasonic lasers for nondestructive examination

ST136

This laboratory-directed research and development (LDRD) project involves the development of the theoretical and experimental underpinnings of a hybrid nondestructive examination (NDE) approach that incorporates both laser-ultrasound and pulsed-eddy-current inspection. The specific problem of characterizing the mechanical, thermal, and dimensional properties of a thin layer on an infinite half space is addressed in detail. This problem has many nuclear-related NDE applications. For a vertically stratified geometry, potential applications include characterization of a corrosion film, a hydride rim, or a diffusionbarrier lining on fuel cladding. For a laterally stratified geometry, potential applications include characterization of isolated grain boundaries and stress-assisted intra-grain boundary corrosion.

\section{Technical Objectives}

The overall intent of this project involves increasing spatial resolution in order to adequately characterize thin-film geometries. The first objective deals with the initial design and development of a ultrahigh-frequency laser ultrasonic system. The emphasis is placed on generation and detection of $\mathrm{GHz}$ frequency surface acoustic waves. In this case the spatial resolution is determined by the minimum acoustic wavelength. The second objective involves the preliminary design and development of a high-frequency, small-length scale eddycurrent system. A giant magneto-resistance (GMR) device is used to detect perturbations in the eddy-current signal due to small surface imperfections.

\section{$\underline{\text { Ultra-high-frequency Laser Ultrasonics }}$}

A theoretical model of laser-generated surface acoustic waves (SAW) is presented. The predicted temporal waveform will be discussed in detail. The experimental parameters that determine the acoustic bandwidth are individually identified. Last, a list of experimental equipment for generation and detection of ultrahigh frequency SAW is presented.

Model development involves specifying a set of stress boundary conditions that are equivalent to a thermoelastic line source:

$$
\begin{aligned}
& \left.\left(\sigma_{23}\right)\right|_{x_{3}=0}=\widetilde{F} \delta^{\prime}\left(x_{2}\right) H(\tau), \\
& \left.\left(\sigma_{33}\right)\right|_{x_{3}=0}=0, \\
& \widetilde{F}=\left(F_{3}(1-\kappa)+F_{2} \alpha\right), \quad F_{2}=\frac{B_{22} T_{0}}{\alpha}, \quad F_{3}=\frac{B_{33} T_{0}}{\alpha},
\end{aligned}
$$

For displacements along bounding plane, the Cagniard integration path is along the imaginary $\omega$ axis. Performing the Cagniard inversion gives

$$
\begin{aligned}
& u_{3}\left(x_{2}, 0, \tau\right)=\widetilde{F} G(T), \\
& G(T)=\frac{1}{\widetilde{F}}\left(\bar{\psi}_{r} \delta\left(T-\omega_{r}\right)+\frac{g(T)}{C_{44}\left|x_{2}\right| \pi}[H(T-1 / \sqrt{\beta})-H(T-1)]\right), T=\frac{\tau}{\left|x_{2}\right|},
\end{aligned}
$$

The details of the model are presented by Hurley and Spicer.[1] A typical displacement waveform generated using Equation 2 is shown in the inset of Figure 1 (next page). The initial disturbance, referred to as the surface-skimming bulk wave, arrives at the longitudinal velocity and vanishes at the shear wave velocity. The later arriving SAW travels as a Dirac Delta function. It should be noted, according to Eq. 2, the SAW travels without geometric attenuation, while the surface-skimming bulk wave, represented by $\mathrm{g}(\mathrm{T})$, decreases in amplitude inversely with the distance from the source. 


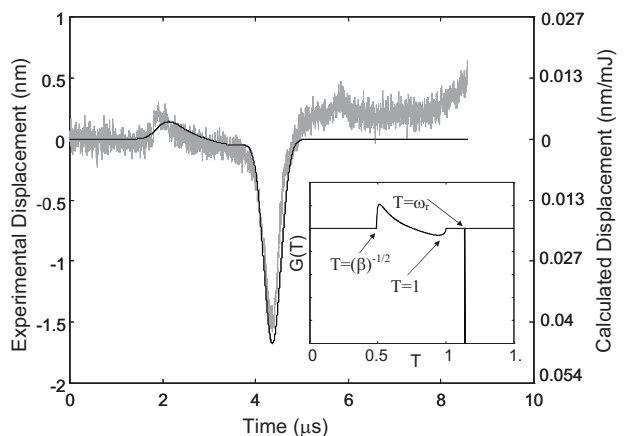

FIGURE 1. Comparison between experiment and theory for the surface wave case. The sample is single crystal zinc with the c-axis perpendicular to the free surface. Inset:

Theoretical displacement, $u_{3}$, for surface waves generated with a line source in zinc.

Because the SAW travels as a delta function, line generation of SAW provides an excellent platform to assess the overall experimental bandwidth due to acoustic generation and detection. The ultimate limitation on bandwidth is related to the acoustic transit time across a particular dimension of the optical absorption volume. For Rayleigh waves the lateral dimension of the absorption volume (parallel to surface) is the dimension of interest.

Conventional optical focusing techniques lead to SAW wavelengths that are restricted by the diffraction-limited optical-spot size $(\sim 1 \mu \mathrm{m})$. This corresponds to upper bandwidth limit of $\sim 3$ $\mathrm{GHz}$ for most materials. Achieving this limit requires that the generation laser and the electronic detection equipment have a bandwidth in excess of $3 \mathrm{GHz}$.

We have begun assembling experimental equipment for the purpose of generating ultrahigh frequency SAWs. A continuum leopard pulsed laser with pulse duration $\sim 200 \mathrm{ps}$ will be used for acoustic generation. A 14-GHz photodetector, in combination with a $4-\mathrm{GHz}$ oscilloscope, will be used to record the interferometric signal. With the assembled system, the upper bandwidth limit will be determined by the diffraction-limited generation spot size as describe above.

\section{Eddy Current Inspection}

Eddy-current inspection techniques are typically used to interrogate metal components for surface breaking or near surface defects. The technique is based on the generation of electrical currents in a test sample via magnetic induction. Defects or material anomalies of interest are detected by the character of electrical current generated or a disruption in flow. Traditional eddy-current probes use induction coils for generation and detection that define both the test-frequency bandwidth and spatial resolution. For high spatial resolution, very small coils/inductors are required. This imposes limits on inductance and the associated properties of the electrical circuitry, i.e. frequency and loading. Alternate magnetic sensor technologies, such as giant magnetoresistive (GMR) devices, are available that provide possible advantages for high spatial resolution eddy-current testing. They are solid-state microelectronic devices having high sensitivity, physically small footprints, and a relatively broad bandwidth ( $\approx$ DC-1 MHz). Microelectronic fabrication techniques also provide the possibility of making large arrays for increasing area coverage during inspection. An evaluation of the GMR technology is being performed to determine their value in developing a high resolution eddy current sensor that can be combined with laser ultrasonic techniques to evaluate material microstructural features.

An aluminum-alloy plate containing six flat bottom holes with varying depths and diameters was used to test the viability of a GMR sensor (NVE, Inc. AAH002-02) to detect and image surface breaking anomalies via eddy currents (Fig. 2). The eddy current sensor is comprised of a copper plate with a GMR chip mounted on the surface. The sensing axis of the GMR is $90^{\circ}$ to the magnetic-flux lines generated by a current sheath induced in the copper plate. When placed next to a metal test sample, eddy currents are generated in the sample parallel with the current sheath. The eddy currents produce a secondary magnetic field that is also aligned $90^{\circ}$ to the sensing axis of the GMR. Only defects that deflect the path of the eddy currents and their associated magnetic fields $90^{\circ}$ will be sensed by the GMR. 


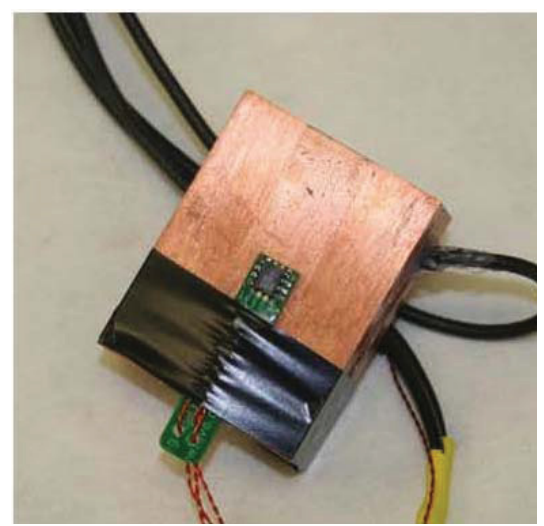

(a)
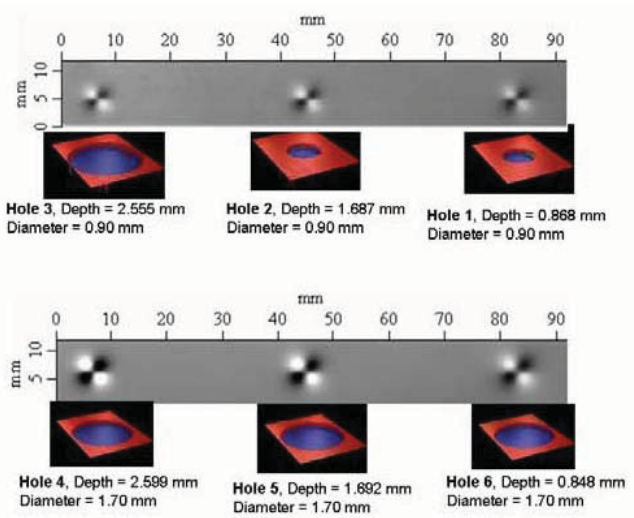

(b)

FIGURE 2. (a) GMR-based eddy current sensor; (b) GMR response to flat-bottom holes in an aluminum plate.

The responses presented in Figure 2 were obtained at a test frequency of $7.2 \mathrm{kHz}$. As expected, the strongest signals (highest $\mathrm{S} / \mathrm{N}$ ratio) were measured for the largest spatial defect, Hole 4 (Fig. 3). The signals decrease with increasing frequency because the magnetic flux lines are contracted closer to the copper plate and drive-coil reactance increases, thereby reducing sheath-current amplitude. Typically, an increase in the test frequency will concentrate the eddy currents closer the surface, thereby improving the signal to noise of the measurement.

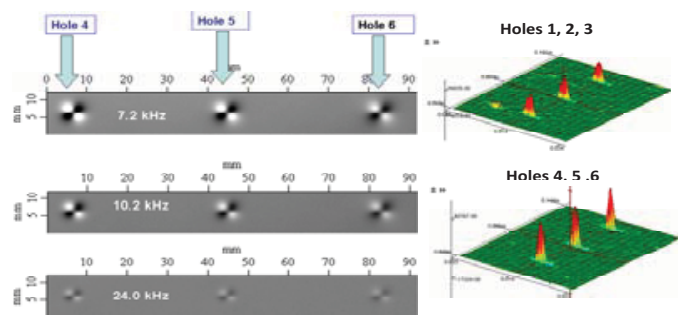

FIGURE 3. Measurements at 7.2, 10.2, and $24 \mathrm{kHz}$. (Left): GMR response to different test frequencies. (Right): Eddycurrent responses to flat bottom holes obtained using an induction coil probe at $500 \mathrm{kHz}$.

This demonstrates the need to better integrate the GMR sensor with the source drive into a more coplanar arrangement. Illustrated in Figure 3 are the responses to the flat bottom holes obtained using a set of 2-mm-diameter transmit-receive induction coils. Comparison of the GMR and induction coil responses indicates that GMR sensors are viable for detecting eddycurrent responses and similar signal-to-noise ratios can be achieved. However, different structures were obtained for the two responses that need to be understood in order to implement the GMR technology for high-resolution measurements. Future work will be directed at improving the integration of the drive and GMR sensor as well as understanding the signal structure obtained.[2,3]

\section{References}

1. Hurley, D. H., J. B. Spicer, 2004, "LineSource Representation for Laser-Generated Ultrasound in an Elastic Transversely Isotropic Half-Space," Journal of the Acoustical Society of America, Vol. 116, p. 2914.

2. Obeid, S., F. M. Tranjan, and T. Dogaru, 2007, "Eddy Current Testing for Detecting Small Defects in Thin Film," Review of Quantitative Nondestructive Evaluation, Vol. 26, Ed. D. O. Thompson and D. E. Chementi, pp. 340-345.

3. Marchand, B., F. Vacher, G. Gilles-Pascaud, J.M. Decitre, and C. Fermon, 2008, "High Resolution Eddy Current Probes for Nondestructive Testing," Review of Quantitative Nondestructive Evaluation, Vol. 27, Ed. D. O. Thompson and D. E. Chementi, pp. 313-320. 


\title{
Advanced VHTR Core Components
}

\author{
W.E. Windes, T. Roney (INL); B. Marsden, P. Mummery (University of Manchester) \\ Studying pore microstructures and how they may change after irradiation/thermal exposure \\ ST137
}

This project's primary objectives were (1) technical and (2) capability enhancement. The primary technical objective focused on mechanisms and microstructures of nuclear-grade graphite to assist in understanding fundamental mechanisms during irradiation. This work focused on utilizing the most modern characterization tools available to produce experimental data on microstructure of nuclear graphite types, specifically focusing on the pore microstructure, which is a key parameter in how the graphite behaves under irradiation. Such experimental data was recently identified as a major objective to be pursued at a recent International Atomic Energy Agency (IAEA) Consultancy meeting on irradiation creep of graphite. The second primary objective was to form strong collaborations with the University of Manchester in the United Kingdom. University of Manchester is considered one of the foremost authorities on nuclear graphite and ceramics in the world and has extensive experience in researching nuclear graphite. The experimental work conducted at Manchester on Idaho National Laboratory (INL)-produced nuclear graphite lead to Manchester joining INL and Boise State University (BSU) in a U.S.-sponsored Nuclear Energy University Programs (NEUP) grant.

\section{Technical Objectives}

The primary technical objective of this study was to focus on the mechanisms and microstructures of nuclear-grade graphite to assist in the understanding of fundamental mechanisms during irradiation such as irradiation creep. However, this proved too broad for only six months of funding, so we narrowed the work to a more manageable study of pore microstructures and how they may change after irradiation/thermal exposure. As such, INL acquired the newest nuclear grades of graphite that are of interest to the new Generation IV research program, PCEA (GrafTech International) and NBG-18 (SGL Inc.). INL then used a variety of traditional studies to ascertain the pore structure within graphite samples machined from these two new varieties of graphite.

Traditional intrusion methods (mercury, water, and BET) were used to determine the pore size distribution in the structure along with optical micrographs of the graphite samples (Fig. 1). Our intention was to provide a comparison study of the different techniques used to detect pore distribution in graphite and the results from Manchester's state-of-the-art 3D computer tomography (CT) technique. The 3DCT data provided a definitive map of the pores in the solid material as well as the pore distribution. This data was then compared to the more traditional intrusion techniques as well as the destructive optical methods often used to obtain this data.

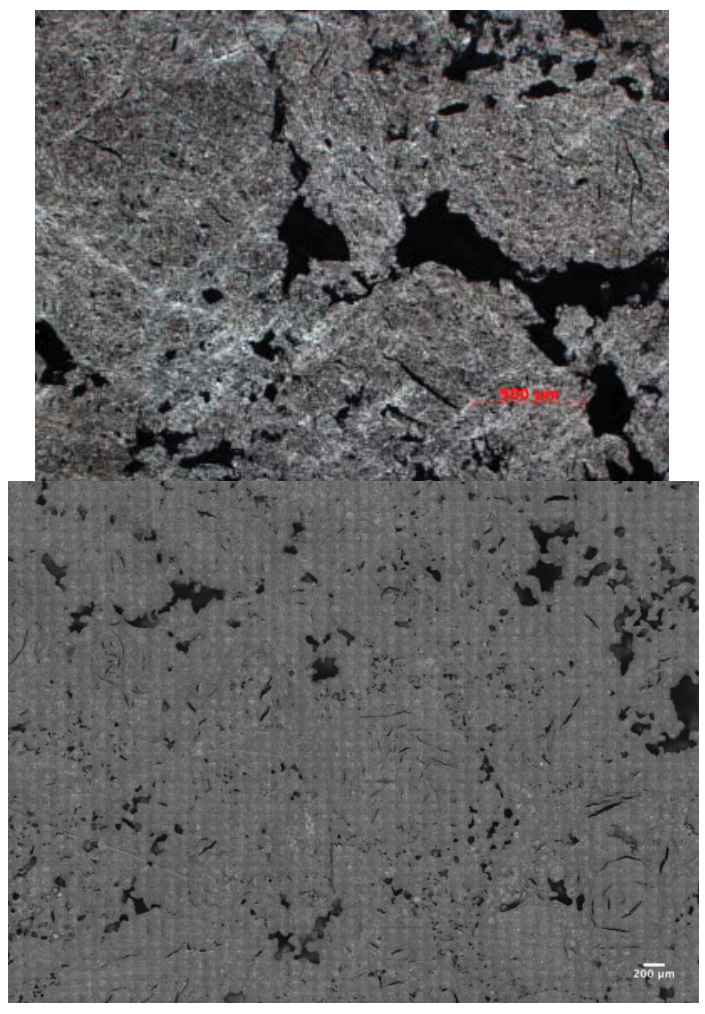

FIGURE 1. Optical micrograph of NBG-18 grade nuclear graphite.

The 3D-CT data clearly illustrates the large pore structures within a typical nuclear-grade graphite (Fig. 2). Depending upon the acquisition time and sample size, pores down to 1-2 microns are readily detectable. However, while the complete pore structure is visually discernable a quantitative analysis of the pore size distribution is not. INL developed a computer algorithm to calculate the size, number, and orientation of the pores within each 3D-CT image to provide the quantitative data necessary to compare this 
technique to the intrusion methods. A computergenerated pore structure based upon this algorithm is presented for comparison (Fig. 3).

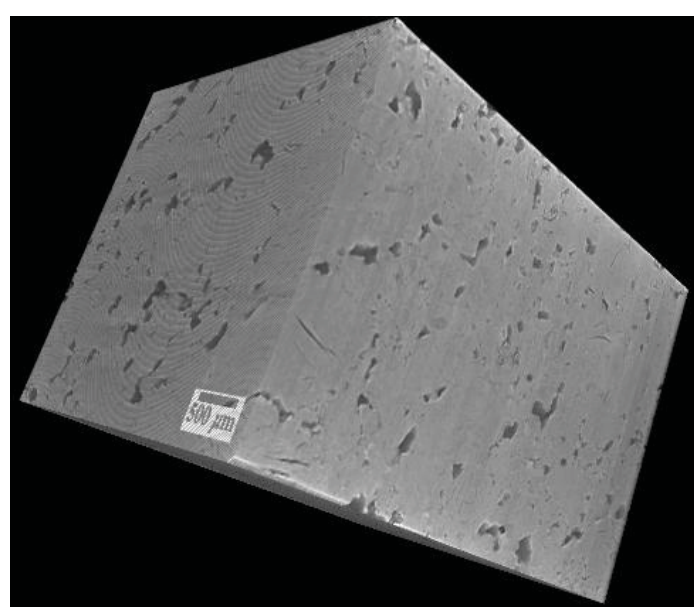

FIGURE 2. A 3D representation of PCEA nuclear graphite. Image is constructed from over $10002 D$ slices providing up to 5-micron resolution of the microstructure.

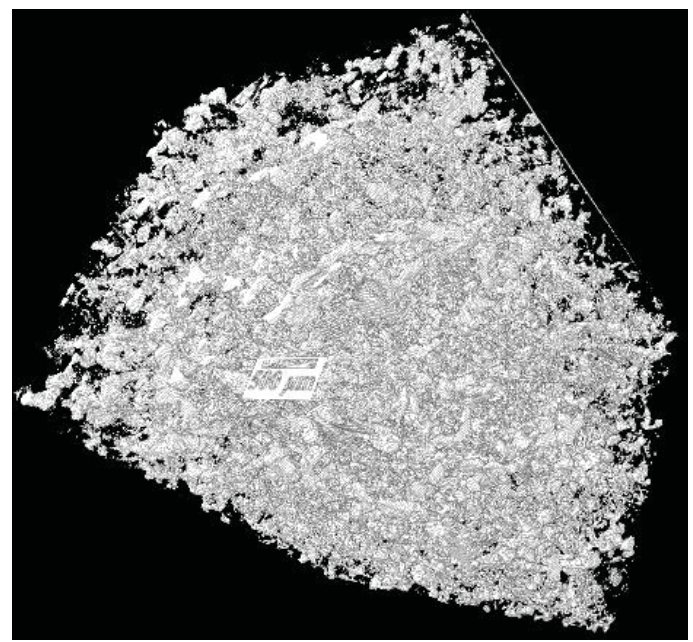

FIGURE 3. An image representing the total distribution, size, and orientation of the pores within the PCEA nuclear-grade graphite image shown earlier.

The data gathered from these studies are being compiled into a manuscript for submission to the Journal of Nuclear Materials. The data from all pore structure analysis techniques are being compared to allow a greater understanding of the limitations for each technique. For instance, while 3D-CT can provide definitive pore structure information the technique is sample size limited, only small samples can be analyzed. Therefore, by comparing 3D-CT results to other data from techniques that can analyze large sample sizes, we can determine the accuracy of those different techniques.

Manchester professors will be co-authors on this journal submission.

This work is the first step in establishing a method to quantitatively determine the changes to the pore structure in nuclear-grade graphite during high-temperature irradiation. We have obtained outside funding to pursue future research activities based upon this unique characterization method.

\section{Results and Accomplishments}

The major accomplishments fall into two main areas: technical accomplishments and collaboration accomplishments. Technical accomplishments include:

- Acquired 2 new nuclear graphite types for analysis: PCEA (GrafTech) and NBG-18 (SGL)

- Conducted optical microstructure and porosity measurements using traditional methods

- Conducted 3D CT on the two different graphite types

- Preparing manuscript for Journal of Nuclear Materials utilizing these results.

Collaborative accomplishments include:

- Collected 3D-CT data in collaboration with University of Manchester

- Entered into a new collaboration agreement to study irradiation creep mechanisms, leading to a new NEUP between BSU, Manchester, and INL

- Mapped out and agreed upon a new 3D-CT data session utilizing advanced siliconcarbide ( $\mathrm{SiC}$ ) composites to be used in nuclear applications.

\section{Summary and Conclusions}

The nuclear graphite's large pore structure is a key element to understanding the behavior of the material during both thermal and neutron exposure. The pore structure (i.e., pore sizes and 
orientations) changes during exposure to high-temperature neutron environments. This work is the first step in establishing a method to quantitatively determine the changes to the pore structure during high-temperature irradiation. Manchester, INL, and Boise State University have extended this work into thermal and irradiation behavior studies to build on these initial successes. Manchester is machining and characterizing similar graphite samples which will then be exposed to high thermal temperatures $(<2000 \mathrm{C})$ at INL. This will allow us to map the changes to the pore structure as a result of high temperatures.

BSU and INL will head a similar samplecharacterization program for an irradiation study. Under a new Nuclear Engineering University Program (NEUP) grant, a new irradiation creep capsule will be designed to irradiate and test new graphite and composite materials. This capsule is strictly for basic science testing such as determining fundamental mechanisms for irradiation creep in graphite, thermally enhanced irradiation creep, fiber-matrix damage mechanisms under load in ceramic composites, and irradiation dimensional change mechanisms in both graphite and advanced composites. This new capsule will provide the opportunity to quantitatively understand the fundamental mechanisms behind many of the irradiation behavior in these unique materials.

Finally, based on the initial success of our graphite analysis, Manchester is eager to use this technique to analyze the new advanced $\mathrm{SiC}$ composites developed for the NGNP program. The work will utilize the same characterization technique on $\mathrm{SiC}$ composite samples but will add the additional complexity of tracking a crack propagating through the fiber-matrix microstructure of these composites. Manchester currently has a large program for characterizing advanced structural composites, and this work would complement their program. 


\section{Development of a Sustainable Ecological Engineering Approach to Support Hybrid Energy System Development in the Western Inland Energy Corridor}

Robert P. Breckenridge, Robert Cherry, Thomas Wood, Richard Boardman

Modeling the synergies of hybrid energy systems

ST138

Hybrid energy systems combine several technologies in a synergistic manner to potentially address the limitations of individual energy technologies, increase power reliability, minimize the environmental footprint, and reduce energy costs. To date, there is no approach capable of evaluating the environmental and socioeconomic impacts of a hybrid energy system. This project developed a decision-analysis tool for a coal-fired power plant and/or an oil-shale mining and processing plant within the Uinta Basin of Utah by advancing a method developed by a multi-stakeholder group called the Sustainable Rangeland Roundtable (SRR 2008). A Uinta Basin site was identified by the research team consisting of scientists and engineers from Idaho National Laboratory (INL) and Utah State University (USU). Using realistic development scenarios, the impacts to water, air, wildlife, infrastructure, socioeconomic regimes, and other natural-resource capital were predicted for two scenarios: (1) a conventional energy system and (2) a hybrid energy system that integrates renewable (e.g., wind) and fossil energy resources. A conceptual model that incorporates ecological engineering and socioeconomic concepts was developed (following examples from the National Research Council, 1990) for a power plant using both renewable and fossil energy. The model has two different states: the current state $\left(T_{0}\right)$ and the future state $\left(T_{1}\right)$. Four different sets of conditions are evaluated for both $\mathrm{T}_{\mathrm{o}}$ and $\mathrm{T}_{1}$ : (1) biophysical (air, water, soils, plants and animals); (2) natural-resource capital (total biomass and biodiversity); (3) social capacity and economic capital (economic assets/liabilities, social opportunities and constraints); and (4) current human condition (values and norms, income, health and security). The model is a powerful tool for considering net benefits and impacts for both the conventional and hybrid energy approaches.

This research evaluated how hybrid synergies can address many environmental and socioeconomic concerns; for instance, how would net reduction in water consumption and air releases correlate with long-term economic benefits to the region. Industry and local and state governments have all voiced an interest in development of this approach because it will allow them to conduct improved impact assessments and provide the scientific information required for proactive regional planning. An important secondary benefit of the research was creation of teaming relationships internal at INL and between INL and USU scientists and engineers to collaborate on development of various components of the decision-analysis approach.

\section{Technical Objectives}

- Design a decision-analysis tool to evaluate impact from hybrid energy systems

- Develop ecological and socioeconomic indicators for the evaluation of the difference in impacts between conventional and hybrid energy systems

- Perform an analysis of how conventional and hybrid systems impact ecological and socioeconomic systems in the Uinta Basin in Utah

- Present results at the annual meeting of the Sustainable Rangeland Roundtable, held in Portland, OR.

\section{Results and Accomplishments}

This research started late (August) in Fiscal Year 2009. The results showed good potential for application of this approach to assess how both conventional and hybrid energy systems impact selected ecological and socioeconomic indicators for the Uinta Basin in Utah. The following progress was made towards meeting the stated objectives:

1. Design a decision-analysis tool to evaluate impact from hybrid energy systems - A decision analysis tool was designed based on information generated during a meeting between INL staff and collaborators from USU. The analysis tool is a combination of indicators developed as part of Objective 2 and a set of design requirements identified as appropriate for energy development activities in the Uinta Basin of Utah.

2. Develop ecological and socioeconomic indicators for the evaluation of impacts between conventional and hybrid energy systems - A set of indicators was developed 
based on an approach developed by the SRR (2008). The criteria and indicators cover ecological processes, such as the conservation and maintenance of soil, water, air, plants and animals, as well as socioeconomic benefits for both current and future generation. In total, 64 indicators were selected for the decision-analysis assessment. The criteria were selected with assistance for collaborators at both the University of Wyoming and USU. The criteria considered the status of ecological and socioeconomic conditions at both predevelopment as well as post-development stages.

Perform an analysis of how conventional and hybrid systems impact ecological and socioeconomic systems in the Uinta Basin in
Utah - Design configurations for both energy systems were developed for the Uinta basin. The designs were developed with collaborators from USU's Uinta Basin Regional Campus to ensure a high degree of accuracy (Fig. 1). For both cases inputs and outputs for power generation and oil shale plants were developed. Estimates of land disturbance and human demographics were determined and compared against the criteria developed under objective two. In general, the biophysical indicators showed low to moderate negative impacts from both energy systems with the hybrid system being slightly less negative. For the socioeconomic indicators, the hybrid system has a moderate to high positive impact, and the conventional, a low to moderate positive impact. Overall, the hybrid system has fewer negative and more positive impacts than the conventional energy system.

\section{Hybrid Configuration - Uinta Basin Oil Shale Plant}

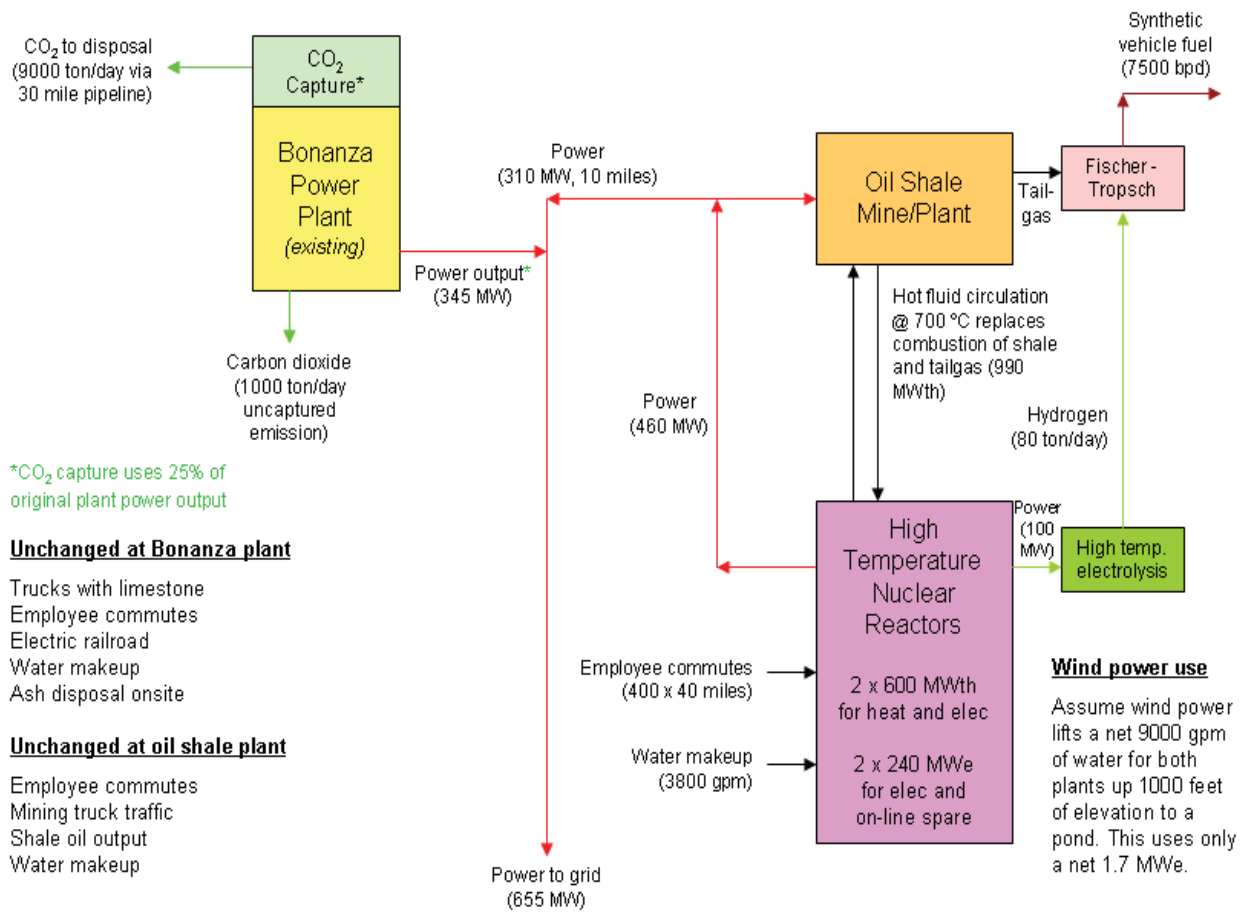

FIGURE 1. Hybrid energy system configuration. 
3. Present results at the annual meeting of the Sustainable Rangeland Roundtable, September 22, 2009, Portland, OR - A presentation of the decision-analysis tool and indicator assessment was made to an interagency group of scientist and policy maker gathered at the SRR annual meeting. Participants were pleased with the use of the indicators and encouraged INL staff to publish results in the near future.

\section{Summary and Conclusions}

The decision-analysis tool and indicator assessment developed as part of this LDRD positioned INL to improve relationships with regional universities and governmental agencies. The assessments provided a broader basis for evaluating how both conventional and hybrid energy systems impact both environmental and human systems in western inland basins. This work should help position INL and its university partners to secure future funding related to development of energy resources in western Basins.

\section{References Cited}

National Research Council 1990, Managing Troubled Waters, Commission on Engineering and Technical Systems. National Academy Press. 125 pp.

SRR 2008, Maczko K. and L. Hidinger (eds.). Sustainable Rangelands Ecosystem Goods and Services. Sustainable Rangelands Roundtable, Mountain View Press. Fort Collins, CO. http://sustainablerangelands.warnercnr. colostate.edu/ 


\section{Development of 3D Multiphase Flow and Reactive Transport Codes and their Applications to Reactive Flow in Porous Media and Fracture Apertures}

\section{Hai Huang}

Detailed $3 D$ simulations of reactive flow in porous media

TM106

The main objective of this project is to develop three-dimensional (3D), coupled, multiphase fluid flow and reactive transport simulation codes based on the various types of interface-tracking techniques, including the volume of fluid, level set, and phase field methods. The developed codes can be applied to a number of important problems such as immobilization of metal contaminants in the subsurface, enhanced oil recovery, and geological sequestration of $\mathrm{CO}_{2}$.

\section{Technical Objectives}

The following are the technical objectives for this project:

1. Perform detailed 3D simulations of reactive flow in porous media using the 3D parallelized level set method.

2. Develop and apply phase-field model to model reactive flow in porous media.

3. Prepare manuscripts for peer-reviewed journals.

\section{Results and Accomplishments}

For this laboratory-directed research and development (LDRD) project, three important research accomplishments were achieved in Fiscal Year 2009 (FY-09).

The first accomplishment of FY-09 was applying the 3D level set simulation code developed in FY-08 to study coupled flow, reactive transport, and precipitation/dissolution in porous media. Previous years' research indicates that direct simulation of reactive transport in porous media is challenging due to the difficulty of tracking the dynamics of evolving solid-fluid interfaces. Also, 3D geometry (Fig. 1) significantly increases the complexity of the problem.

The second major accomplishment of FY-09 was the development of phase field code to model multiphase flow and reactive transport in porous media. Figure 3 shows the simulation result of a classic Rayleigh Taylor instability problem, a coupled diffusion-mineral surface precipitation problem, and the comparison against the level set simulation result.

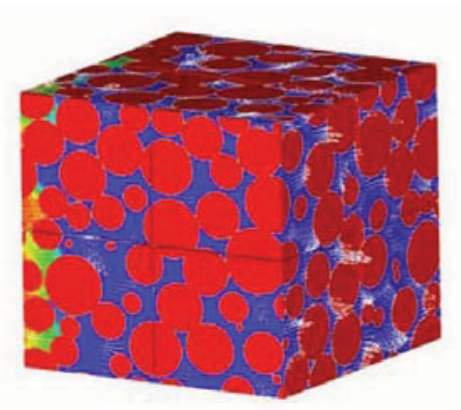

FIGURE 1. MPI-based parallel level set simulation of reactive transport in $3 D$ random porous media.

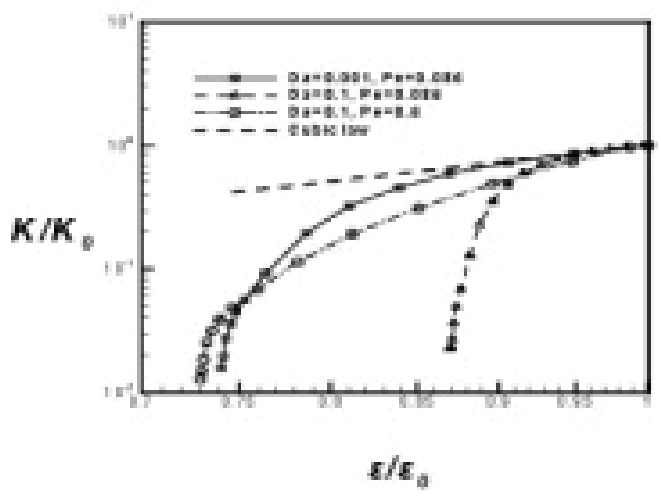

FIGURE 2. Simulated permeability-porosity.

Besides code development, we simultaneously performed a number of computational studies using the developed codes and prepared a number of research manuscripts for publication in peer-reviewed journals. By the end of FY-09, a total of four manuscripts directly supported by this laboratory-directed research and development (LDRD) project have been prepared. One was published in Water Resources Research in December 2008; two manuscripts were submitted, and one is finished and ready for submission.

By the end of FY-09, we successfully accomplished the technical goal of this LDRD: develop and apply computer codes using three different interface tracking approaches - volume of fluid (VOF), level set, and phase field - to study the fundamental physics that govern flow, transport, and reactions in fractured and porous media. 


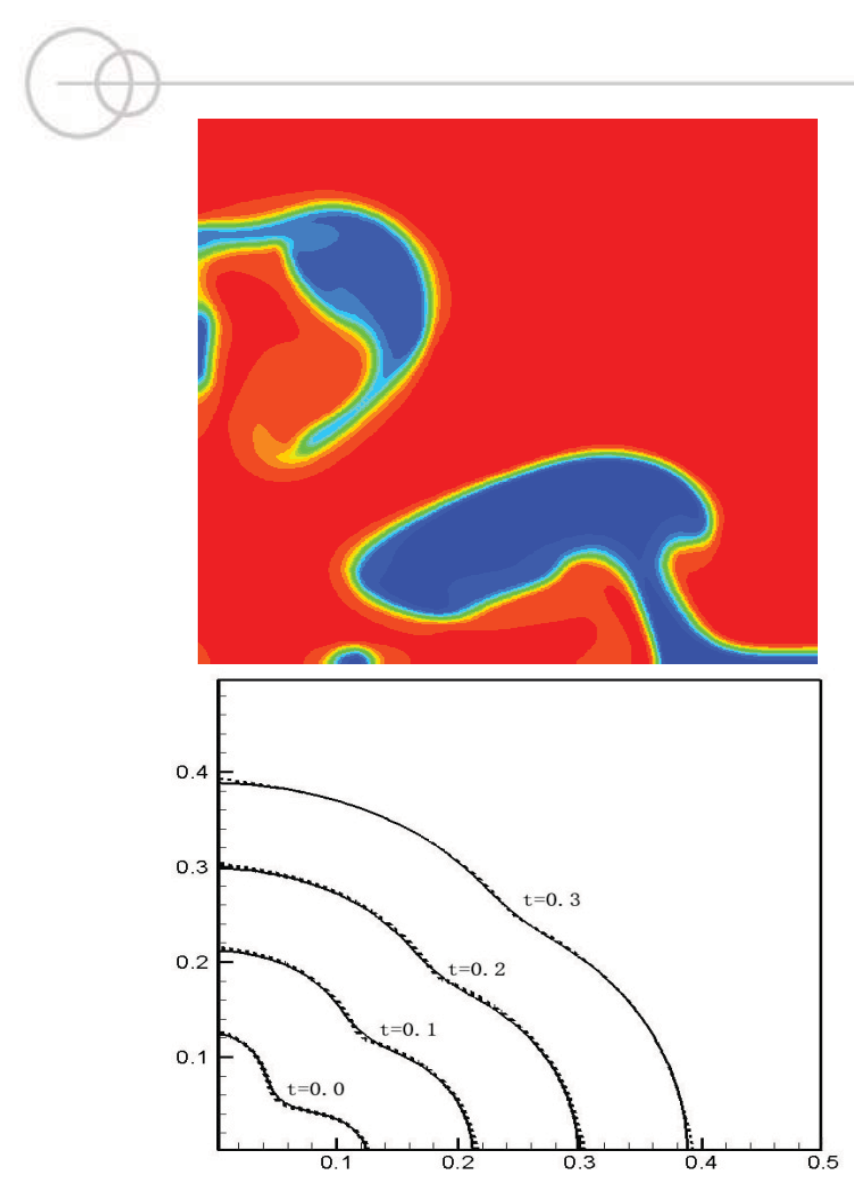

Idaho National Laboratory (INL) developed a unique set of pore-scale multiphase flow and reactive transport codes using different approaches. The list of pore-scale capabilities INL now has includes:

- Particle-based methods (supported by a previous EMSP program, led by Paul Meakin)

- Smooth particle hydrodynamics (SPH)

- Dissipative particle dynamics (DPD)

- Lattice Boltzmann simulation (LB)

- Grid-based methods (supported by this LDRD)

○ Volume of Fluid Method (VOF)

- Level Set method (LS)

- Phase Field method (PF)

All these codes have complementary strengths and weaknesses. INL's subsurface modeling group is in a very unique position nationwide because it is the first group that successfully coupled computational fluid dynamics with various interface tracking techniques to study such fundamental physics in the subsurface places. The simulation results and findings of this project directly supported a successful proposal development in the early spring of 2008, which targeted the Department of Energy's Environmental Remediation Science Program. This newly awarded 5-year project, called INL's Scientific Focused Area, is worth $\$ 6,000,000$. Hai Huang is one of the principal investigators for the above project. INL's subsurface science group has started to gain recognition in pore-scale modeling. predictions. We are the first group that successfully coupled computational fluid dynamics and various interface tracking techniques to study such fundamental physics in the subsurface.

\section{Summary and Conclusions}

We have developed the capabilities to use high-performance computing to understand single-phase reactive transport and multiphase flow in complex porous media and fracture networks. One future work is to incorporate more complex chemistry into the reactive transport simulations. By the end of FY-09, 


\section{PAGE FOR ADVANCED NUCLEAR ENERGY INITIATIVE DIVIDER}

Advanced Nuclear Energy Initiative 


\title{
Multi-Reactor Design and Analysis Platform
}

\author{
Joshua Cogliati, Rodolfo Ferrer
}

The Multi-reactor design and analysis platform

AE119

The Multi-Reactor Design and Analysis Platform (MRDAP) is designed to simplify the creation, transfer, and processing of data between computational codes. MRDAP accomplishes these objectives with three parts:

- Allows each integrated code, through a plug-in, to specify the required input for execution and the required output needed

- Creates an interface for execution and data transfer

- Enables the creation of graphical user interfaces (GUI) to assist with input preparation and data visualization.

Reactor physics calculations, or in general any reactor design calculation, often requires the use of multiple analysis codes. In many cases, data generated by one code is subsequently used as input by another code. Several methods have been used to transfer the data between multiple codes. The most rudimentary method is to simply transfer the data manually by copying certain sections of the output file and inserting them in the input to the next code. This is timeconsuming, inefficient, and prone to errors. Furthermore, the manual transfer of data makes tasks that require repetitive execution and/or making small changes to the input (such as in the case of parametric studies) time-consuming and unreliable. An alternative approach is to use shell scripts to automate sequential code execution and input preparation. While this simplifies the transfer of data and enables the user to prepare input more readily, the nature of shell scripts constrains their applicability to other analysis codes. This is because, typically, the paths to certain files and libraries are hard coded into the script. Also, because scripts primarily execute a series of predefined commands, if used by an inexperienced user, they can fail in several ways when applied outside their predefined scope.

The lack of robustness of a scripting system can also waste resources, such as in the case of a failed code execution that generates necessary input for subsequent codes, in which case the automated scripting may very well continue, or may fail later, giving the wrong location for the error. Second, if some of the upstream data has not changed, the script will still recalculate the data at that location. While scripts can be created that do not have those problems, robust scripts are time-consuming, complex to create, and usually not user-friendly. The aforementioned methods of connecting codes lack the robustness that one can achieve by implementing modern practices, languages, and data management systems to automate sequential input preparation and code execution.

MRDAP embodies an attempt to simplify reactor physics calculations by streamlining data transfer between codes in a robust, transparent, and modular fashion. MRDAP provides a GUI for creating and visualizing the hierarchical data connectivity between codes. This GUI also provides input/output data storage containers with the ability to sense data dependencies between codes. This dependency sensing allows preventing the redundant recreation of downstream data for instances where upstream data has not changed from a previous run. The dependency sensing also allows for controlled parallelization of upstream codes such that downstream code execution is queued until all prerequisite dependencies are met.

Ultimately, the main motivation of this work was to enable reactor designers and analysts by providing a tool that increases efficiency and minimizes the potential for errors and failed executions. The overall platform allows the user to interact with one code and input file for the entire workflow as opposed to multiple codes, input files, and data handling scripts.

\section{Technical Objectives}

- Improve the usefulness of MRDAP

- Prepare for MRDAP release.

Results and Accomplishments

This year's focus was on improving the usefulness and polish of MRDAP and preparing for a MRDAP software release. Improving the usefulness was accomplished by multiple items including the improvement and addition of plugins and improvements to the base program. New nuclear code plug-ins were created for the NJOY cross section processing tool and the DRAGON neutron transport code. A shell plug-in library was created that allows new plug-ins to be 
created that can easily switch at runtime from running locally to running by sshing to remote computers. The MCNP5 plug-in uses that capability and can run remotely now. In addition, the MCNP5 plug-in now supports using alternative cross-section directories. Other plugin improvements include increasing the functionality of the floating point expression plug-in and adding the ability to save to the database all the values generated in a loop instead of just the most recent. Multiple polish enhancements were added to the core MRDAP program. It is now easier to rename and delete workflows. Temporary directories created for running codes can either be automatically removed or kept for debugging. An improved system for creating and editing preferences was created. A generic string conversion plug-in was created and a string concatenator plug-in was created. This combination makes creating input files during workflow running possible. The MRDAP workflow editor has been improved (Fig. 1); it now allows the workflow to be zoomed in or out to change the amount and detail of information shown on the screen. Connecting lines now can have bends, which allows them to be routed around other units.

The MRDAP program was prepared for more widespread use. User documentation and a zip file distribution have been created. In the distribution, a jar file that can be executed directly has been created. This makes installation require only installing the Java runtime environment and unzipping the MRDAP zip file. MRDAP has been reviewed by the export control process. With these tasks accomplished, MRDAP is ready for distribution to other users.

\section{Application and Testing}

The MRDAP code was used to automate coupling of the nuclear cross-section generation process with full core design and analysis of sodium-cooled fast reactors and accompanying fuel cycle. The nestable loop feature of MRDAP was used to autoregenerate cross-section data as a function of the evolving actinide isotopic composition due to multirecycle of the fuel. This is an example of connecting support codes with design codes. Unlike most scripting systems, MRDAP was able to run multiple cross-section calculations in parallel, collect the data-files from these calculations and combine the results into a format that the higher level code can interpret. Most script systems would not have been able to run parallel executions of the same lower level code while at the same time sense when all of the parallel jobs had been completed before running the higher level code.

The unique aspect of MRDAP is that the entire design process can be easily built and visualized using drag-and-drop icons on the computer screen, each icon corresponding to a code calculation. Therefore, the entire design process can be built all in the same workflow (i.e., worksheet) or broken into multiple workflows of the same project. The core and fuel

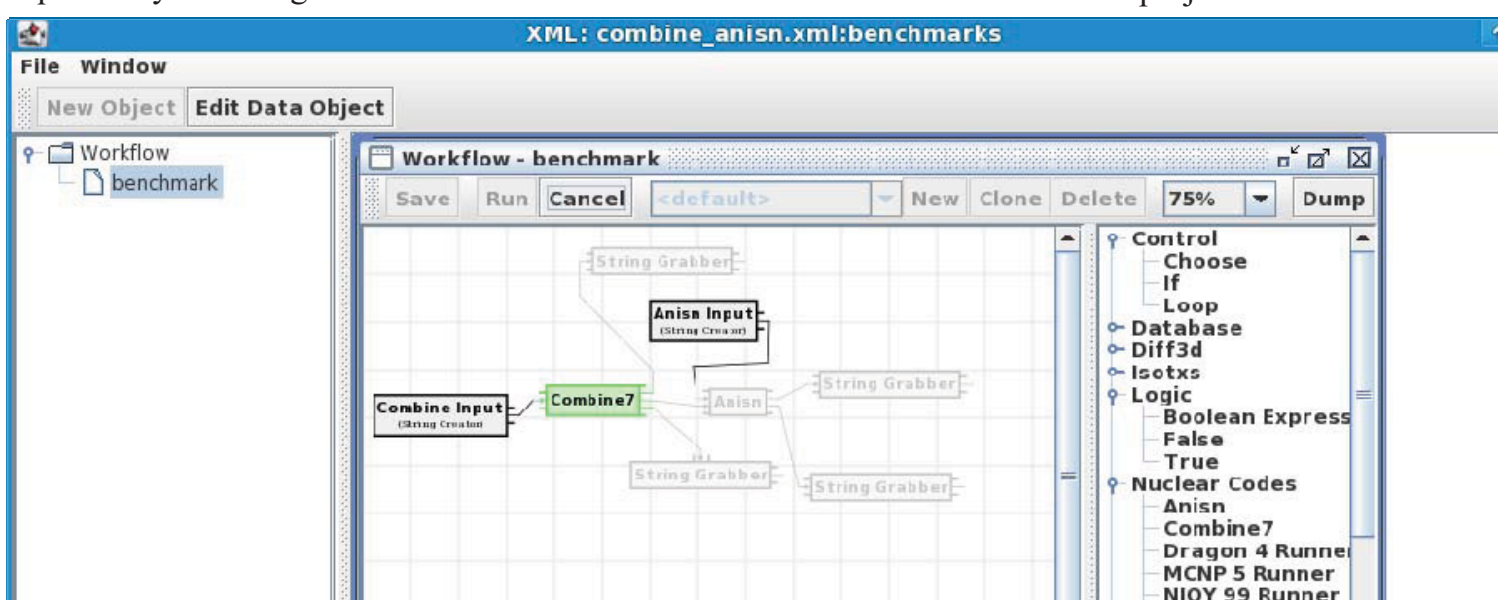

FIGURE 1. MRDAP user interface. 
cycle workflow of the previous example was expanded to include calculations detailing the core design's safety (Doppler, void) reactivity worth parameters. This is an example of how MRDAP can be used to not only connect various codes, but also to connect various phases of the design process.

An effort was undertaken to show coupling of different types of physics in reactor design. The neutron physics performance of the core is dependent on the thermal-hydraulic performance of the reactor system. When carrying a reactor idea from novel concept all the way through to finished point-design, varying levels of calculation fidelity are required. In practice, this level of fidelity is somewhat governed by the code user's expertise, either being physicist or thermal-hydraulicist. To expand the limitations bounded by a user's comfort zone, a set of coupling codes and file architectures was created for performing quick and appropriately accurate preliminary neutronic and thermal-hydraulic design. These homegrown computational tools and file formats were created in a way that allows modular swapping with production codes, either neutronic or thermal-hydraulic or both, depending on the level of fidelity required. As a concept evolves into a more mature design phase, these scoping calculation tools can ultimately be replaced in a MRDAP workflow with higher fidelity codes.

These application demonstrations have resulted in a published preliminary fast reactor core design. Due to the flexibility of MRDAP to facilitate code connectivity, other applications are foreseeable. Examples include: modeling the time dynamic isotopic evolution in the fuel cycle and the resulting impact on waste form, fuel handling and transport cask design, multiobjective optimization of key features of a reactor design, automating large numbers of physics calculations for populating perturbation matrices used in uncertainty analysis, and ensuring quality control of follow-on calculations pertaining to new experimental rigs of the Advanced Test Reactor.

\section{Summary and Conclusions}

The MRDAP program has been made more useful and polished this year. It is now ready for wider distribution and use. The original goal of producing a plug-in-based code to link together multiple nuclear codes has been accomplished. 


\section{A General Framework for Simulating Fully Coupled Mass and Energy Transport}

Richard Martineau, HyeongKae-Park

Developing next-generation, high-fidelity simulation tools to analyze nuclear systems AE121

This laboratory-directed research and development (LDRD) project focuses on developing next-generation, high-fidelity simulation tools to analyze nuclear systems. These tools will enhance our predictive capabilities and will be used for the design, optimization, and safety assessments of future advanced nuclear reactors. Current simulation tools for conventional light water reactors (LWRs) employ a number of parameters that are tuned over years of operational experiences. The lack of operational experiences for very hightemperature reactors (VHTRs) and significant design differences between LWRs and VHTRs makes it difficult to apply traditional methodology and requires high-fidelity simulation solving more fundamental governing equations. For example, higher thermal loadings are expected in VHTRs versus traditional reactor concepts. In this operational condition, incompressible flow assumption with the Boussinesq approximation for buoyancy is proven to be inadequate $[1,2]$, thus requiring the conservative form of the Navier-Stokes equations to accurately represent physics. However, in this very low-Mach number flow regime, the Navier-Stokes equation exhibits a numerical stiffness due to the large time scale separation between acoustic and advective modes. An efficient numerical algorithm solving this flow regime helps significantly, simulating accurate fluid flows and heat transfers of VHTRs in both operational and accident scenarios.

A traditional method of coupling physics for reactor simulation is explicit "code coupling" or first-order operator splitting among physics. While this approach can produce answers, questions of accuracy and stability are usually left unanswered. Therefore, development of reliable tightly coupled simulation tools are desirable. To accomplish this, we have a developed a multiphysics framework using the Jacobian-free Newton-Krylov (JFNK) method [3] for implicit coupling of nonlinear physics. One of the challenges in solving a nonlinear equation system implicitly when using Newton's method is the formation of an expensive (if not impossible) Jacobian matrix. The JFNK method alleviates this difficulty by taking advantage of Krylov methods that only require Jacobianvector products, not the Jacobian matrix itself.
Finite differencing the nonlinear residual functions approximates the Jacobian-vector product in a matrix-free approach. Nonlinear residual functions can be seen as the discretized PDEs; therefore, new physics can be readily incorporated to the existing set of equations. An efficient semi-implicit, operator-splitting approach can be used as an efficient physicsbased preconditioner to accelerate the convergence of a Krylov method.

\section{Technical Objectives}

- Design and test an "all-speed" flow algorithm to solve low-Mach number flows with high-heat flux (continued from FY 2008)

- Develop a three-dimensional, parallel finite element VHTR reactor simulation code that exceeds the performance of traditional tools

- Publish the "all-speed" flow algorithm manuscript (accepted for publication in Journal of Computational Physics [4])

- $\quad$ Publish a comparative manuscript discussing the difference between compressible and incompressible flow (accepted for publication in Nuclear Engineering and Design [2])

- $\quad$ Present preliminary results of tightly coupled VHTR code at the International Conference on Advances in Mathematics, Computational Methods and Reactor Physics (M\&C 2009) [5]

- Submit a coupled solution algorithm manuscript on pebble-bed type VHTR benchmark results with an extension to the prismatic VHTR concept (in preparation).

Results and Accomplishments

In FY 2009, we developed a flow algorithm that efficiently solved very low-Mach number flow with significant heat flux. As a next step, we focused on developing a multiphysics HPC framework, the Multiphysics Object Oriented Simulation Environment (MOOSE) [6], and a VHTR simulator, PRONGHORN. MOOSE is the foundation for all of INL's multiphysics methods groups software efforts. It is a programming environment for the solution of 
multiphysics systems that involve multiple physically dependent models or multiple simultaneous physical phenomena. The multiphysics systems are generally represented (modeled) as a system of fully coupled nonlinear partial differential equation systems (a simple example of a multiphysics system is magnetohydrodynamics where, in the strictest description, the conservative form of the NavierStokes equations is coupled to Maxwell's divcurl system for electromagnetism). Inside MOOSE, the JFNK method is implemented as a parallel nonlinear solver that naturally supports effective coupling between physics equation systems (or kernels). The physics kernels are designed to contribute to the nonlinear residual that is then minimized inside of MOOSE. MOOSE provides a comprehensive set of finite element support capabilities (libMesh) [7] and provides for mesh adaptation and parallel execution. The framework uses the nonlinear solver capabilities in either the Portable, Extensible Toolkit for Scientific Computation (PETSc) project [8] or the Trilinos project [9].

PRONGHORN was started as a specific simulation tool for the gas-cooled pebble-bed VHTR concept. The current capabilities of PRONGHORN include transient and steady simulations of fully coupled porous fluid flow, solid-state heat conduction, and multigroup neutron diffusion models (i.e., fixed-source, criticality, and time-dependent). The coupled physics can be solved in three-dimensional Cartesian coordinates and two-dimensional cylindrical $(r-z)$ coordinates with precursor and adiabatic thermal feedback models. Recently, a nonlinear acceleration scheme for the criticality problem and a simple thermal-fluid model for the prismatic reactor concept were added. Future capabilities include a more advanced flow model (to study thermal boundary layer effects) and a radiation transport model. This code has been validated against the PBMR400 (pebble bed modular reactor) benchmark and several neutronics benchmarks (pebble bed reactor [PBR], light water reactor [LWR], and SFR), and has also been compared with the Reactor Excursion and Leak Analysis Program (RELAP5) solution for the prismatic reactor concept.

Figures 1a-1e (next page) show the result of the PBMR400 thermal-fluid benchmark [10], and Figures $1 \mathrm{f}$ and $1 \mathrm{~g}$ show the comparison between PRONGHORN and THERMIX. Our code matches well compared THERMIX, a wellvalidated thermal-fluid simulation tool for the pebble-bed type VHTRs. Figure 2a (next page) exhibits the code-to-code comparison of the PBMR400 neutronics benchmark. Figure $2 b$ shows that the PRONGHORN result matches well with other codes. Figure $2 \mathrm{c}$ shows the convergence of $k_{\text {eff }}$ using first- and second-order finite elements. It clearly shows the advantage of using second-order elements. Applications developed on the MOOSE framework can utilize first- and second-order elements without changing a line of the code. Figures $3 \mathrm{a}-3 \mathrm{~d}$ (next page) show the result of the three-dimensional coupled thermal-fluid neutronics problem. Figure $3 \mathrm{e}$ shows the computed $k_{\text {eff }}$ and maximum solid temperature with a prescribed reactor power.

\section{Summary and Conclusions}

Because of this year's LDRD project, we have successfully developed a flexible multiphysics framework and preliminary simulation tools for VHTRs. The multiphysics framework allows rapid development of parallel, implicit three-dimensional codes, and a resulting application code, PRONGHORN, has shown competitive results and the potential to replace the legacy codes. 


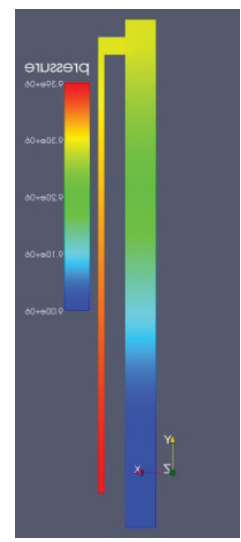

(a)

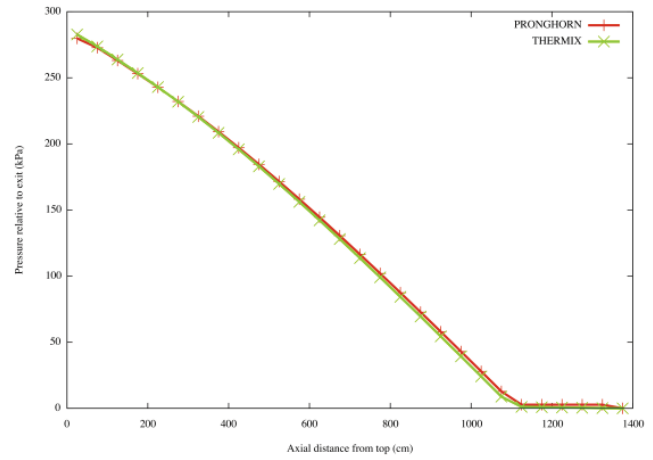

(f)

FIGURE 1. Pronghorn solution of the PBMR400 thermal-fluid benchmark.

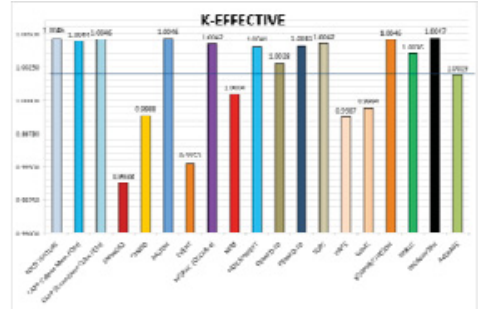

(b)

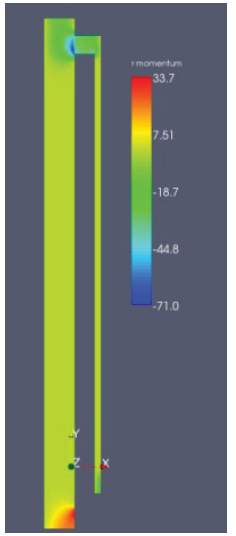

(d)

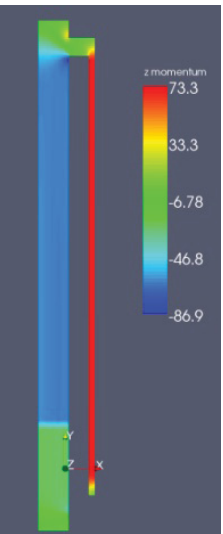

(e)

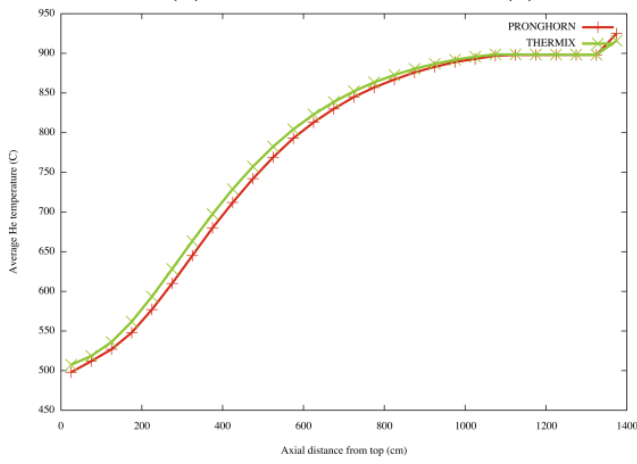

(g) (a)

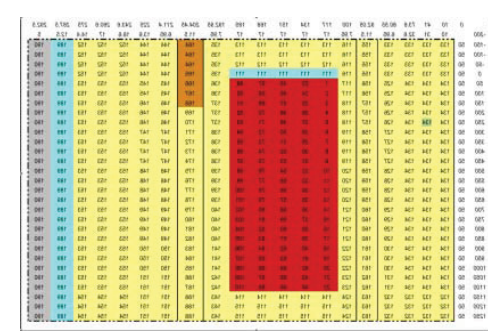

FIGURE 2. Pronghorn solution of the PBMR400 neutronics benchmark. 


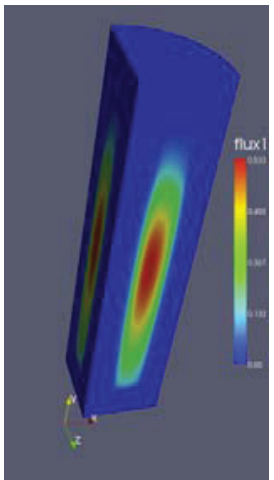

(a)

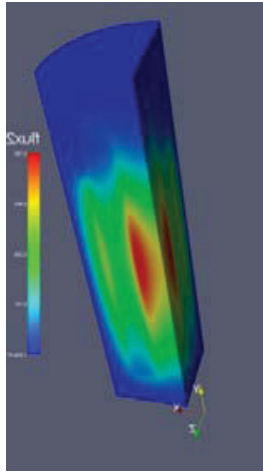

(b)

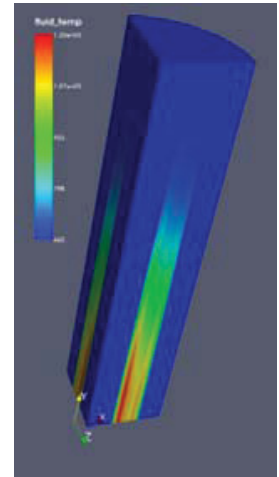

(c)

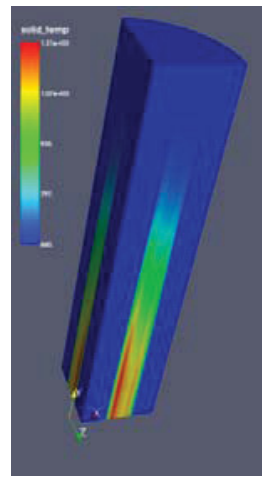

(d)

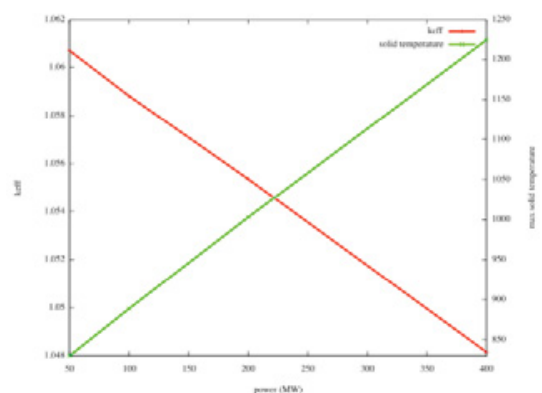

(e)

FIGURE 3. Pronghorn Solution of the simplified coupled thermal-fluid neutronics problem.

\section{References}

1. Darbandi, M., and S. F. Hosseinizadeh, 2007, "Numerical study of natural convection in vertical enclosures using a novel non-Boussinesq algorithm," Numer. Heat Tr. A-Appl., Vol. 52 pp. 849-873.

2. Martineau, R., et al., 2009, "Comparative analysis of natural convection flows simulated by both the conservation and incompressible forms of the Navier-Stokes equations in a differentially-heated square cavity," accepted for publication in $\mathrm{Nucl}$. Eng. Des.

3. Knoll, D. A., and D. Keyes, 2003, "Jacobian-free Newton-Krylov Methods: A Survey of Approaches and Applications," J. Comput. Phys., Vol. 193, pp. 357-397.

4. Park, H., et al., 2009, "On physics-based preconditioning of the Navier-Stokes equations," accepted for publication in $J$. Comput. Phys.

5. Park, H., et al., 2009, "Tightly coupled multiphysics simulations for pebble bed reactors," International Conference on Mathematics, Computational Methods \& Reactor Physics (M\&C 2009), Saratoga Springs, New York, on CD-ROM.

6. Gaston, D., et al., "MOOSE: a parallel computational framework for coupled systems of nonlinear equations," Nucl. Eng. Des., Vol. 239, pp. 1768-1778.

7. Kirk, B. S., et al., 2006, "libMesh: a C++ library for parallel adaptive mesh refinement/coarsening simulations," Eng Comput-Germany, Vol. 22, 3-4, pp. 237254.

8. Balay, S., et al., 2001, "PETSc Web page," http://www.mcs.anl.gov/petsc.

9. Heroux, M. A., et al., 2005, "An overview of the Trilinos project," ACM Trans. Math. Softw., Vol. 31, 3, pp. 397-423.

10. Reitsma, F., et al., 2007, "PBMR Coupled Neutronics/Thermal Hydraulics Transient Benchmark The PBMR-400CoreDesign," Tech.Rep.NEA/NSC/DOC(2007)DraftV07,OECD/NEA/NSC. 


\title{
System Analysis for Reactor Applications with High Fidelity (SARAH)
}

\author{
Vincent Mousseau, Haihua Zhao, and Hongbin Zhang \\ Basic research demonstrated in a set of pilot codes to influence the next-generation nuclear reactor \\ safety simulation code \\ AE 122
}

This project provided basic research demonstrated in a set of pilot codes to influence the next-generation nuclear reactor safety simulation code. The project successfully demonstrated some of the key concepts that will be employed in the now-funded R7 project. These include parameter, numerical, and model uncertainty quantification. The project has also helped to recruit a new strategic hire, Nam Dinh, who is now a lab fellow. In addition, this project served as a stepping stone for the larger and very successful R7 laboratory-directed research and development (LDRD) project. The $\mathrm{R} 7$ project is now a major milestone of the laboratory. This project also helped to bring visibility to Idaho National Laboratory (INL) through the principal investigators' (PIs) and co-PIs' involvement in the "Verification and Validation for Nuclear Systems Analysis Workshop" in Idaho Falls, July 21-25, 2008. Although this project was small at only 1 FTE, it influenced the lab's strategic direction through the R7 project.

\section{Technical Objectives}

1. Marco Delchini, a graduate student from Texas A\&M, has completed initial work on model sensitivity analysis for single-phase flow. This work is documented in INL technical report INL/EXT-09-16995, “A Preliminary Study to Assess Model Uncertainties in Fluid Flows." In this work Marco coded up a general solution that allowed multiple models to be solved. Because the same spatial and temporal discretization was used, the difference in the solutions was caused solely by the difference in the models. This is the basis for his master's thesis.

2. Vijay Mahadevan, a Ph.D. student from Texas A\&M, continued his work on the KARMA code. His current work is to couple his 3-D neutron diffusion with his 3-D thermal conduction to multiple 1-D fluid flow channels. He is benchmarking this software. This work has been presented at conferences, in INL technical reports, and in a Nuclear Engineering and Design paper and is the basis of his Ph.D. dissertation.

3. Suneet Singh, a post doc from Washington State University, has worked on three important projects in two-phase flow modeling. This work has been presented at conferences and is documented in an INL technical report INL/EXT-08-14878, "Effects of Compressibility on Hyperbolicity and Choked Flow Criterion of the Two-phase Two-fluid Model."

a) Work on the well posedness of the twophase model. A Nuclear Engineering and Design paper on this work is in preparation.

b) Work on the two-phase choked flow model. A Nuclear Engineering and Design paper on this work is in preparation.

c) Work on the phase appearance and disappearance problem in two-phase flow modeling. This work will be continued by a new post doc.

4. Haihua Zhao continued his work on uncertainty quantification; this work was documented in INL technical report INL/EXT-08-14847, "Extended Forward Sensitivity Analysis for Uncertainty Quantification.” This work shows how to extend the traditional parameter based uncertainty quantification work to numerical uncertainty caused by grid spacing and time step size. A Nuclear Engineering and Design paper on this work is in preparation.

5. Nam Dinh, Robert Nourgaleiv, and Vincent Mousseau worked on support for the R7 project to help get it started and initiate planning.

Results and Accomplishments

The main work of this project focused on uncertainty quantification and reduction methods for the R7 project to implement into a production code. Although parameter uncertainty quantification is very mature, the augmentation of uncertainty quantification to include numerical uncertainty and model uncertainty are the main accomplishments of this project. Haihua Zhao's work to extend the forward sensitivity analysis algorithms to address spatial and temporal truncation error allows one to directly compare parameter uncertainty with numerical uncertainty. Marco Delchini has 
extended this work to address model uncertainty. By building a single piece of software that uses the same spatial dicretization and the same temporal discretization, Marco has created a code that allows direct comparison of different single-phase fluid models. Again combining this with the parameter uncertainty work and the numerical uncertainty work, one can clearly see where the largest source of uncertainty is coming from and can now start the harder job of uncertainty reduction that follows uncertainty quantification. If one measures numerical error and parameter error separately, there is no way of knowing which uncertainty is the largest. This can result in large amounts of money and time invested in reducing a small uncertainty and leaving the largest one untouched.

Suneet Singh's two-phase flow addressed three of the key problems in two-phase flow. First is the ill-posed nature of the two-phase flow equations resulting in a divergence of solutions under mesh refinement. This divergence prevents any verification study and makes quantification of numerical error impossible. Suneet calculated the minimum correction required to make the two-phase flow equations stable. Because the two-phase flow equations are ill-posed, the speed of sound is imaginary. This complex sound speed has a negative impact on choked flow where the two-phase mixture is supposed to exit the pipe at the real sound speed. Finally, one of the hardest problems in two-phase flow occurs when the fluid goes from single-phase (three equations) to two-phase (six equations) or from two-phase to single-phase. This phase appearance/disappearance problem results in slow execution times and large mass errors. Suneet's investigation into the older homogeneous equilibrium model (HEM) may be the key to eliminating the phase appearance/disappearance problem since the HEM model is always three equations.

This project also addressed how to accurately model coupled neutronics and thermal hydraulics. This is documented in two journal papers, one by Mike Pope in Nuclear Engineering and Technology and one by Vijay Mahadevan in Nuclear Engineering and Design. This work is based on extensive research by the PI in the Jacobian-free Newton-Krylov method with physics-based preconditioning. As the two papers (and their references) show, the key to accuracy is tight coupling of the neutronics and thermal-hydraulic physics, and the key to efficiency is preconditioning.

\section{Summary and Conclusions}

This work provides the basic research for uncertainty quantification and reduction for the R7 project. By addressing the problems of twophase flow and uncertainty quantification first, this LDRD has paved the way for the R7 project. The success of this project is mainly through its support of the larger, successful R7 project. Although this LDRD project is small - only one FTE for two years - it has provided a stepping stone for the R7 project and has thus influenced the strategic direction of the lab. 


\title{
Acquisition and Improvement of a Modern Lattice Physics Capability
}

\author{
Cristian Rabiti, William F. Skerjanc
}

Development of a 2-D lattice physics code capable of meeting future DOE programmatic missions AE123

The technical purpose of this research is to develop a lattice physics code capable of modeling, analyzing, and producing cross sections for the prismatic very high temperature reactor (VHTR) design. The HELIOS code suite, developed by Studsvik Scandpower, Inc. for light water reactor (LWR) modeling, has been under development to improve the HELIOS neutron cross section library for graphite moderated reactors and to treat the challenges associated with double heterogeneous (DH) fuel (TRISO-coated fuel particles dispersed in a graphite compact, which are inserted in a graphite fuel assembly).

HELIOS is a general geometry 2-D neutrongamma lattice physics code. The original HELIOS library was developed for LWR modeling and cross section generation. The characteristics of a graphite moderated reactor are much different than that of a typical LWR core (e.g., flux spectrum). As such, a VHTR graphite-specific HELIOS library is necessary to accurately model and produce cross sections for the prismatic design. In addition, treatment of the $\mathrm{DH}$ fuel is important to account for the spatial effects associated with TRISO particles embedded in compacts. The method selected for the DH treatment in HELIOS is the statistical method of Sanchez and Pomraning as implemented for the method of characteristics.

Currently, only a select number of lattice physic codes are capable of treating $\mathrm{DH}$ fuel associated with the prismatic VHTR design. Further, the neutron cross section library for this type of reactor is not vey well defined. This project's research advances Idaho National Laboratory's (INL) capability to accurately model the prismatic VHTR, perform fuel cycle analysis, and develop cross sections for auxiliary 3-D depletion codes (e.g., REBUS, PARCS). The results of this research will be an in-house 2D lattice physics code capable of meeting future Department of Energy (DOE) programmatic missions.

\section{Technical Objectives}

The terminal objective of this research is to enhance the HELIOS code package to more accurately model the prismatic VHTR to support reactor safety analysis, fuel cycle analysis, and cross section generation. The success of the terminal objective is accomplished with the following enabling objectives:

- Modification of the original HELIOS LWR library to produce a VHTR graphite-specific HELIOS library

- Treatment of DH fuel associated with the prismatic VHTR design.

Results and Accomplishments

HELIOS Library Modifications. The original HELIOS LWR library has evolved throughout this project to produce a VHTR-specific HELIOS library that more accurately captures graphite-moderated reactors. First, resonance integral (RI) data was calculated for a VHTR pin-cell to capture the effects from neutrons slowing down in graphite. Primary emphasis of the new calculated resonance integral data was on U-238. This modification to the library improved accuracy for homogeneous and heterogeneous calculations. The second iteration of the HELIOS library involved infinite dilution of $\mathrm{Pu}-240$. This affects the cross section value in resonance shoulders and has $\sim 400$-pcm effect (as compared to MCNP) in pin-cells at $80 \mathrm{GWd} / \mathrm{T}$.

A typical heterogeneous prismatic VHTR pin cell is illustrated in Figure 1, complete with graphite block, helium coolant gap, and compact (TRISO particles homogenized in the graphite matrix). The results of the evolution of the HELIOS library are summarized in Table 1 for a VHTR pin-cell at $600 \mathrm{~K}$. Version 2 of the HELIOS library incorporates the VHTR-specific RI data. Version 3 of the HELIOS library reflects the VHTR-specific RI data and Pu-240 infinite dilution. Similar results between the three libraries and MCNP were obtained at $300 \mathrm{~K}$ and $1200 \mathrm{~K}$.

As Table 1 shows, significant improvement in the HELIOS library was accomplished for a VHTR pin-cell when compared to MCNP (all MCNP calculations had an estimated $\mathrm{k}_{\mathrm{eff}}$ standard deviation of 0.00008 ). The absorption reaction rate eigenvalue worths of selected isotopes are summarized in Figure 2. The new RI data significantly reduced the discrepancy between HELIOS and MCNP throughout the entire depletion. Infinite dilution of $\mathrm{Pu}-240$ 
greatly improved the accuracy of HELIOS as the pin-cell was depleted. Combined, these library modifications greatly improved the accuracy of HELIOS when compared to MCNP.

Double Heterogeneity Treatment in HELIOS. The method selected for the DH treatment is the statistical method of Sanchez and Pomraning as implemented for the method of characteristics. This method calculates an effective stochastic cross section for the fuel regions, accounting for the average track lengths in the various microsphere layers and the fuel matrix, obtained from an analytic solution of the renewal equation. This provides shielded cross sections for the transport equation. The existing subgroup method in the HELIOS code was also augmented in this fashion.

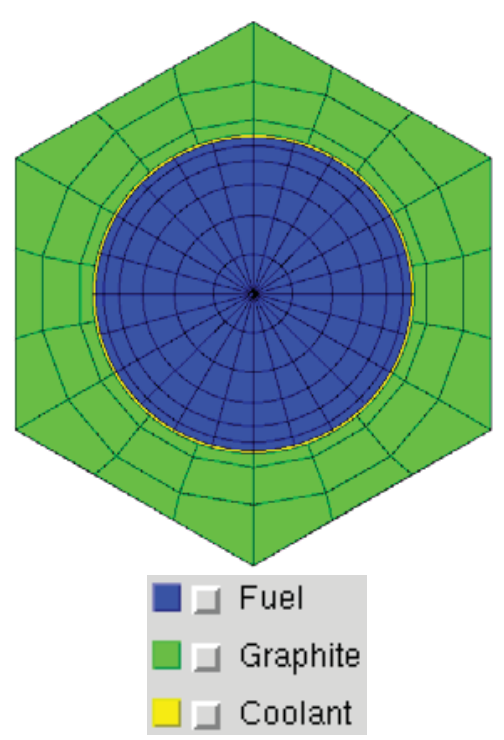

FIGURE 1. Heterogeneous hexagonal VHTR pin-cell.

TABLE 1. MCNP vs. HELIOS pin-cell results with HELIOS library modifications.

\begin{tabular}{|c|c|c|c|c|c|c|c|c|c|}
\hline \multirow{3}{*}{$\begin{array}{c}\text { Burnup } \\
(\mathrm{GWd} / \mathrm{T})\end{array}$} & \multicolumn{9}{|c|}{ MCNP5v1.51 vs.HELIOS- $2.0-600 \mathrm{~K}$} \\
\hline & \multicolumn{3}{|c|}{ Original LWR HELIOS Library } & \multicolumn{3}{|c|}{ VHTR HELIOS Library $\underline{\text { Version } 2}$} & \multicolumn{3}{|c|}{ VHTR HELIOS Library Version 3} \\
\hline & HELIOS-2.0 & MCNP5v1.51 & $\mathrm{pcm}$ & HELIOS-2.0 & MCNP5v1.51 & $\mathrm{pcm}$ & HELIOS-2.0 & MCNP5v1.51 & $\mathrm{pcm}$ \\
\hline 0.0 & 1.27628 & 1.26680 & 948 & 1.26745 & 1.26680 & 65 & 1.26635 & 1.26680 & -45 \\
\hline 0.5 & 1.23330 & 1.22412 & 918 & 1.22479 & 1.22410 & 69 & 1.22372 & 1.22419 & -47 \\
\hline 5.0 & 1.19990 & 1.19039 & 951 & 1.19146 & 1.19025 & 121 & 1.19022 & 1.19033 & -11 \\
\hline 10.0 & 1.16545 & 1.15594 & 951 & 1.15710 & 1.15535 & 175 & 1.15558 & 1.15542 & 16 \\
\hline 20.0 & 1.09908 & 1.08913 & 995 & 1.09099 & 1.08866 & 233 & 1.08908 & 1.08888 & 20 \\
\hline 40.0 & 0.99951 & 0.98927 & 1024 & 0.99235 & 0.98872 & 363 & 0.99055 & 0.99038 & 17 \\
\hline 80.0 & 0.88675 & 0.87643 & 1032 & 0.88164 & 0.87725 & 439 & 0.88075 & 0.88004 & 71 \\
\hline 120.0 & 0.82130 & 0.81100 & 1030 & 0.81816 & 0.81304 & 512 & 0.81774 & 0.81646 & 128 \\
\hline
\end{tabular}
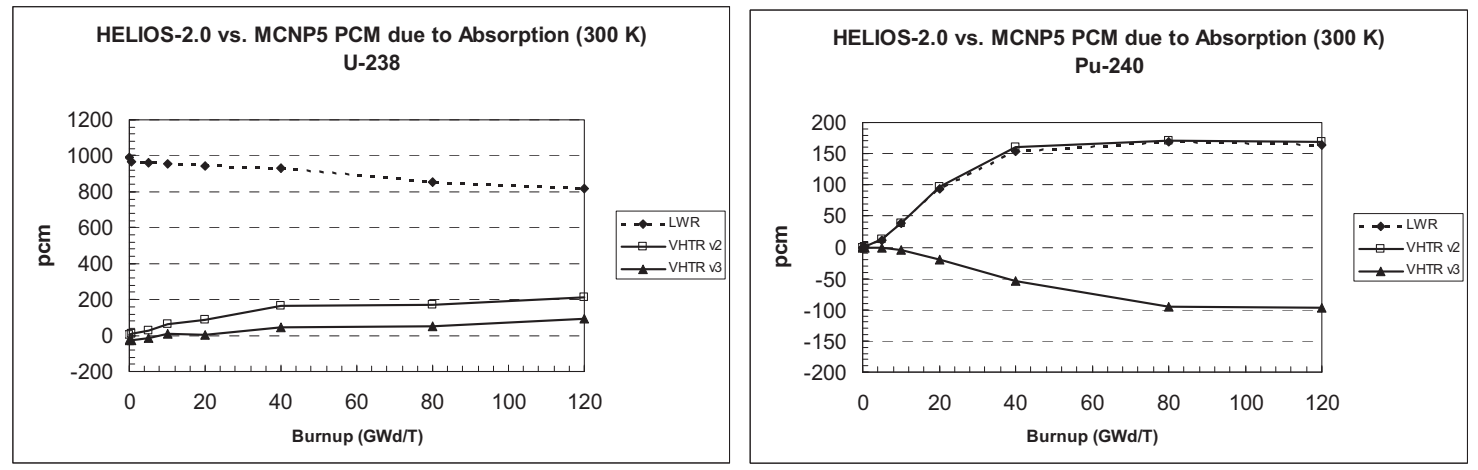

FIGURE 2. Reaction rate eigenvalue worth due to absorption of U-238 and Pu-240 as compared to MCNP. 
A MCNP random TRISO dispersion model was obtained courtesy of courtesy of Han Gyu Joo, Department of Nuclear Engineering, Seoul National University, and is illustrated in Figure 3. This particular model has a TRISO packing fraction of $25.016 \%$. An equivalent HELIOS DH model was created and the results versus MCNP are summarized in Table 2 at various temperatures.

\section{Summary and Conclusions}

INL now has an in-house developed lattice physics code capable of treating the DH fuel associated with the prismatic VHTR. The research gained in this project will support DOEfunded programs including Next Generation Nuclear Plant (NGNP), Advanced Fuel Cycle Initiative (AFCI), and Deep Burn. In addition, INL now has expertise in performing reactor physics calculations using HELIOS and modeling the prismatic VHTR. These capabilities will make INL a national resource for future prismatic VHTR modeling and analysis to support future DOE missions.
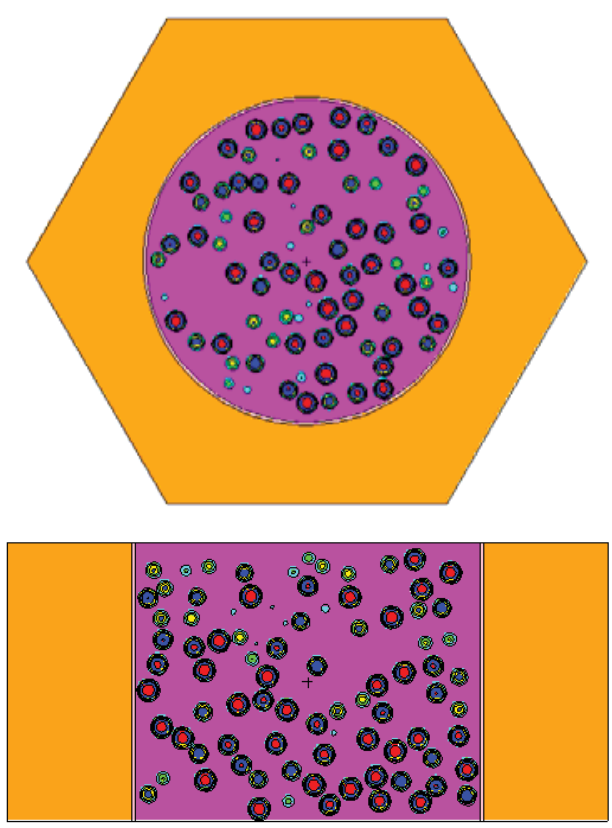

FIGURE 3. MCNP random TRISO dispersion model (courtesy of Han Gyu Joo, Department of Nuclear Engineering, Seoul National University).

TABLE 2. HELIOS DH treatment vs. MCNP at $0 \mathrm{GWd} / \mathrm{T}$.

\begin{tabular}{cccccccc} 
& & \multicolumn{3}{c}{$\begin{array}{c}\text { Original LWR HELIOS } \\
\text { Library }\end{array}$} & \multicolumn{2}{c}{$\begin{array}{c}\text { VHTR HELIOS Library } \\
\text { Version 2 }\end{array}$} & \multicolumn{2}{c}{$\begin{array}{c}\text { VHTR HELIOS Library } \\
\text { Version 3 }\end{array}$} \\
\cline { 2 - 9 } $\begin{array}{c}\text { Memperature } \\
(\mathrm{K})\end{array}$ & $\mathrm{k}_{\mathrm{eff}}$ & $\mathrm{k}_{\mathrm{eff}}$ & $\mathrm{pcm}$ & $\mathrm{k}_{\mathrm{eff}}$ & $\mathrm{pcm}$ & $\mathrm{k}_{\mathrm{eff}}$ & $\mathrm{pcm}$ \\
\hline 300 & 1.39499 & 1.40328 & 829 & 1.39659 & 160 & 1.39578 & 79 \\
600 & 1.34019 & 1.34913 & 894 & 1.34302 & 283 & 1.34136 & 117 \\
1200 & 1.26831 & 1.27849 & 1018 & 1.27378 & 547 & 1.27021 & 190 \\
\hline
\end{tabular}

Future work for this project as it relates to the prismatic VHTR includes further modifications of the HELIOS library to produce even more accurate results. These modifications tentatively include increasing the number of energy groups, improving accuracy at higher burnups and temperature, and resolving resonance integral data. In addition, INL is currently participating in a code-to-code hightemperature gas-cooled reactor benchmark.
Work performed next year will also extend the capabilities of HELIOS to fast reactors. Combined, these efforts will put INL in position to market a multi-purpose 2-D lattice physics code capable of modeling LWRs, VHTRs, and fast reactors to the industry for reactor safety analysis, fuel cycle analysis, and cross section generation. 


\title{
Unified Two-phase CFD Modeling of Boiling, Cavitation, and Bubble Collapse
}

\author{
Ray A. Berry \\ Accurately predicting flow dynamics, regime transitions, and heat transfer in two-phase flows \\ AE124
}

In nuclear reactor optimization and safety, there are key issues that rely on in-depth understanding of basic, compressible two-phase flow phenomena with heat and mass transfer. Within the context of multiphase flows, two bubble-dynamic phenomena-boiling (heterogeneous) and flashing or cavitation (homogeneous boiling), with bubble collapseare technologically very important to nuclear reactor systems. The main difference between boiling and flashing is that bubble growth (and collapse) in boiling is inhibited by limitations on the heat transfer at the interface, whereas bubble growth (and collapse) in flashing is limited primarily by inertial effects in the surrounding liquid. The flashing process tends to be far more explosive (and implosive), and is more violent and damaging (at least in the near term) than the bubble dynamics of boiling. Moreover, other problematic phenomena, such as crud deposition, appear to be intimately connecting with the boiling process. In reality, these two processes share many details, and the fundamentals of these phenomena mark the core of this research.

Because modern, high-resolution numerical methods/computational fluid dynamics (CFD) codes divide the flow space into a much finer computational grid with many more computational cells, it is imperative that we not:

- Utilize traditional multiphase models which are mathematically ill-posed

- Rely on traditional "flow regime maps" used in subchannel thermal-hydraulics codes to evaluate the interface topology.

Traditional six-equation, single-pressure, two-phase mixture models have a domain in which the square sound speed is negative, which produces ill-posedness, non-hyperbolicity, wrong wave dynamics, and inappropriate transient solutions. Such equations have been used in the past because first order numerics were employed on course grids that produced large enough artificial viscosity to render a solution. However, grid convergence with such a scheme is not possible - it is an untenable situation. Using such equation systems negates the possibility of utilizing modern highresolution methods (second order or higher) - for example, finite volume methods based on
Godunov methods with approximate Riemann solvers.

Phase interactions in multiphase fluids depend on both the area and the topology of the interface. Traditional "flow-regime maps" are used in subchannel thermal-hydraulics codes to evaluate the interface topology from crosssection-averaged flow parameters. Because CFD codes divide the flow space into many more computational cells, they need not rely on the traditional subchannel flow regimes. Instead, they must evaluate the local interface surface topology. The advantage realized with this approach is that the ensemble of many computational cells, with relatively simple interface surface topologies, can provide complex global topologies that include all the traditional subchannel flow regimes. With these simplified flow regime topological maps, which will necessarily depend upon computational cell size, only a few parameters need be used to determine the interface surface topology in each cell. These parameters will be based on experimental data and direct numerical simulation (DNS) of highly resolved phenomena on a smaller scale.

With the above motivation, the appropriate research direction for this project was established, aimed at developing well-designed physical/mathematical models along with highresolution numerical solution methods for general multiphase flows. Specifically, research for this project is directed towards a unified physical/mathematical and numerical model development of compressible, all-speed multiphase flows spanning the following levels:

1. General mixture level (true multiphase)

2. Resolved interface level (DNS-like)

3. Multi-scale methods to resolve both (1) and (2) automatically, depending upon specified mesh resolution.

We have demonstrated [2] that a welldesigned two-pressure, two-velocity, sevenequation, two-phase mixture model (as in 1) can be systematically reduced analytically to produce a one-pressure, one-velocity, five-equation model (as for 2) capable of a DNS-like resolved interface solution. If such a systematic reduction 
was accomplished numerically on a local spatial level, a general algorithm (such as 1) could be made to reduce locally where appropriate to the interface resolved model (2), effectively giving an automatic multiscale treatment (as 3).

Research is needed to lay the foundations necessary to acquire the capability to simultaneously solve fluid dynamic interface problems as well as multiphase mixtures arising from boiling, flashing (or cavitation) of superheated liquid, and bubble collapse, etc., in light water reactor systems. The effective nonlinear system of first order partial differential equations, along with their closure conditions, must: (1) be hyperbolic and well-posed, (2) be in conservative form, if phase interactions and dissipative terms are neglected, and (3) exhibit correct wave dynamics.

Such two-phase flow phenomena occurring inside light water nuclear reactors includes, especially with departure from nucleate boiling (DNB) and film boiling instability (boiling crisis), coolant phase changes and multiple flow regimes that directly influence coolant interaction with the fuel elements/assemblies and, ultimately, the reactor performance. Because of the inherent coupling, an understanding of these phemonena, along with subcooled boiling and bubble collapse, is also key to gaining an understanding of crud depostion in these systems.

The ultimate goal of this research is to provide models giving highly resolved details where necessary, simultaneously with large scale vessel/component simulation by providing a well-posed, multiscale model that will (1) resolve interfaces for larger bubbles (direct numerical simulation, DNS-like) with singlevelocity, single-pressure treatment, and (2) average (or homogenize) the two-phase flow field for small bubbles with two velocities and two pressures - that is, to lay the foundations for developing the capability needed to simultaneously solve fluid dynamic interface problems as well as multiphase mixtures arising from boiling, flashing or cavitation, bubble collapse, etc., in light water reactor systems. The key is that in these domains wave propagation is of fundamental importance, and mathematical formulations as well as numerical methods must be perfectly clean. To solve compressible multiphase flows, even for weak wave problems, well-posedness is essential and correct wave dynamics (correct, eigenvalues, eigenvectors, dispersion relations, etc.) is imperative. Our collaboration with Professor Saurel and his group in Marseille is particularly ideal because of their complementary capabilities with INL researchers.

After working so hard during the first year of this LDRD to build a strong relationship of joint research with Professor Richard Saurel and his SMASH group at École Polytechnique Universitaire de Marseille (France), the relationship was suspended during the current year due to large funding reductions.

Because this work had received some notoriety, it attracted the interest of Professor Sergei K. Godunov, Sobolev Institute of Mathematics of the Russian Academy of Sciences in Novosibirsk, who asked to join Professor Saurel as a member of our Ph.D. student's jury. Professor Godunov is internationally famous for Godunov's Theorem and Godunov's Method, which forms the basis of virtually all modern methods in Computational Fluid Dynamics. Fortunately, our student was still able complete his research and was granted a Ph.D. in December 2008 for his contributions to this effort.

Also, subsequent to FY 2008 reporting, the PI and Professor Saurel visited Scott Bowman, Vice President, Methods and Software Development, GE Hitachi Nuclear Energy, a collaborator in this work in its first year, and made detailed presentations of their works. GE Hitachi Nuclear Energy remains committed to collaboration on this research, as evidenced by their recent submission of an ARPA-E proposal that included INL as primary collaborators.

Because of the significance of the first year's research efforts, a considerable effort was expended in FY 2009 to document these technical developments. A comprehensive INL external report was written, with each of its seven chapters addressing a major component of our efforts to advance the numerical simulation of compressible multiphase flows [1]. 
We continued our research on diffuse interface methods (DIM), from the previous year, for direct numerical solution (DNS) of compressible multiphase flow fields, but to a lesser extent because of the funding reduction. There still remains the task of refining mass transfer between phases, surface tension, and adding efficient implicit time integration. During this fiscal year, we have produced publications and presentations based upon our research on the DIM method [2,3].

This research was highlighted (by invitation) in the ASME Fluids Engineering Division (FED) Spring Newsletter [4]. The PI was also invited to present and discuss this research at Los Alamos National Laboratory [5], which was well received by several researchers there (including Burton Wendroff, a retired, but still active and world renowned, mathematician who is best known for development of the Lax-Wendroff method for numerically solving hyperbolic partial differential equations).

With this year's reduced funding, we emphasized more heavily the development of the discrete equations method (DEM) for fully compressible two-phase flows in ducts of spatially varying cross-section.

Typically, multiphase modeling begins with an averaged or homogenized system of partial differential equations (which are traditionally illposed) then discretizes this system to form a numerical scheme. This presents problems for the numerical approximation of non-conservative terms at discontinuities as well as the difficult treatment of fluxes with seven waves. To solve interface problems without conservation errors and to avoid this questionable determination of average variables and the numerical approximation of the non-conservative terms in conjunction with two-velocity mixture flows, the PI and collaborators employed a new homogenization method known as the discrete equations method (DEM) [6]. Contrary to conventional methods, the averaged equations for the mixture are not used, and this method directly obtains a (well-posed) discrete equation system from the single-phase system to produce a numerical scheme which accurately computes fluxes for arbitrary numbers of phases and solves non-conservative products. The method effectively uses a sequence of single-phase Riemann equation solves. Phase interactions are accounted for by Riemann solvers at each interface. Flow topology can change, with changing expressions for the fluxes. Nonconservative terms are correctly approximated. Some of the closure relations missing from the traditional approach are automatically obtained.

Lastly, we were able to identify the continuous system induced by the discrete equation, which may prove very useful from a theoretical standpoint. In this research, we constructed a DEM model for 1-D flow of two compressible phases in ducts of spatially varying cross-section, with realistic equations of state and with realistic interfacial area functions, to test this approach. For the first time, we demonstrated on a converging-diverging twophase nozzle that this well-posed two-pressure, two-velocity model can be integrated to a meaningful steady-state with both phases treated as compressible. The model exhibits complicated, but realistic and very interesting, two-phase flow choking behaviors. In this first attempt, we included a simple mass transfer model and postponed investigation of general mass transfer between multiple compressible phases through development of a truly reactive Riemann solver (RDEM) [7] to a later date.

To illustrate this, consider a nozzle with area distribution shown in Figure 1 (next page) in which a two-phase mixture of steam and water flows. Each phase is treated as compressible and each is governed by its own stiffened gas equation of state, $P=(\gamma-1) \rho(e-q)-\gamma P_{\infty}$, where $\gamma, q$, and $P_{\infty}$ are material (fluid) constants. Here $q$ is a "binding" energy which for two-phase flow will effectively represent a reference state.

Simple, efficient and robust algorithms are needed to solve the well-posed models. The various ingredients employed in the methods to be developed should be general enough to consider future extensions to problems involving complex multiphysics. Using the fractional step methods typically applied (and often misapplied) to calculate multiphysics phenomena exhibiting 
multiple time-scales requires some precautionary measures to avoid splitting and conditioning

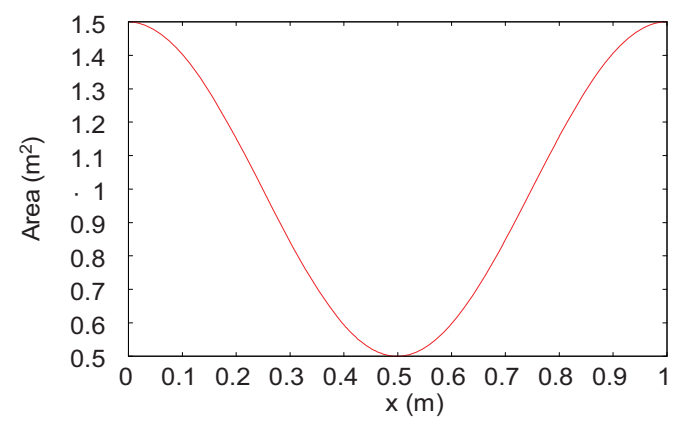

FIGURE 1. Nozzle area.

errors. For our initial testing we utilized explicit time integration. In the future, to relieve time step restrictions due to stiffness and to achieve tighter coupling of the equations, a fully implicit treatment may be necessary. An appropriate fully implicit approach will allow integration over the fast time scales for slow-transient flows. (We anticipate that using a Jacobian-Free NewtonKrylov method with physics-based preconditioning will allow tightly coupled solutions of the multiphysics phenomena inherent in nuclear reactor core applications.) Toward that objective, a significant effort was expended, along with Tami Grimmett from the CAMS group, toward development of a method of lines (MOL) or semidiscrete method approach to the integration of the equation system. With the MOL we discretize separately the space and time domains, effectively converting to a large system of ordinary differential equations (ODE) that can be integrated with previously developed, highly refined special-purpose software. Our initial explicit model, in addition to being very interesting in its own right, serves as a necessary beginning for this implicit methods development, an ongoing effort.

For this 1-D, volume-centered, finite volume method, the HLLC approximate Godunov solver [8] is used to determine the fluxes at the cell faces. Explicit time stepping is used to integrate the solution to steady state. For this example, constant pressure and velocity relaxation coefficients are maintained with a specific interfacial area of 100, and a simple mass and heat transfer model incorporated. Figure 2 shows the liquid and gas variable distributions at steady state. (For more details, see Ref. 9, produced during our research based on this DEM method.)

\section{References}

1. Berry, R. A. (PI), R. Saurel, F. Petitpas, E. Daniel, O. Le Métayer, S. Gavrilyuk, N. Dovetta, and R. C. Martineau, 2008, "Progress in the Development of Compressible, Multiphase Flow Modeling Capability for Nuclear Reactor Flow Applications," Idaho National Laboratory external report INL/EXT-08-15002.

2. Saurel, R., F. Petitpas, and R. A. Berry, 2009, "Simple and efficient relaxation methods for interfaces separating compressible fluids, cavitating flows and shocks in multiphase mixtures," Journal of Computational Physics, Vol. 228, March 2009, pp. 1678-1712.

3. Berry, R. A., R. Saurel, and F. Petitpas, 2009, "A simple and efficient diffuse interface method for compressible twophase flows," International Conference on Mathematics, Computational Methods \& Reactor Physics (M\&C 2009), Saratoga Springs, New York, May 3-7, 2009.

4. Berry, R. A., R. Saurel, and F. Petitpas, 2009, "Unified two-phase CFD modeling of boiling, cavitation, and bubble collapse," Fluids Engineering, Spring 2009, p. 10-11.

5. Berry, R. A., 2009, "A Simple and Efficient Diffuse Interface Method for Compressible Two-Phase Flows," Presentation to Los Alamos National Laboratory, June 2009.

6. Abgrall, R. and R. Saurel, 2003, "Discrete equations for physical and numerical compressible multiphase mixtures," Journal of Computational Physics, Vol. 186, 2003, pp. 361-396.

7. Le Métayer, O., J. Massoni, and R. Saurel, "Modelling evaporation fronts with reactive Riemann solvers," Journal of Computational Physics, Vol. 205, 2005, pp. 567-610. 
8. Toro, E.F., 1999, "Riemann solvers and numerical methods for fluid dynamics," $2^{\text {nd }}$ Edition, Springer-Verlag, Berlin, 1999.

9. Berry, R. A., R. Saurel, and T. Grimmett, 2009, "The discrete equation method (DEM)
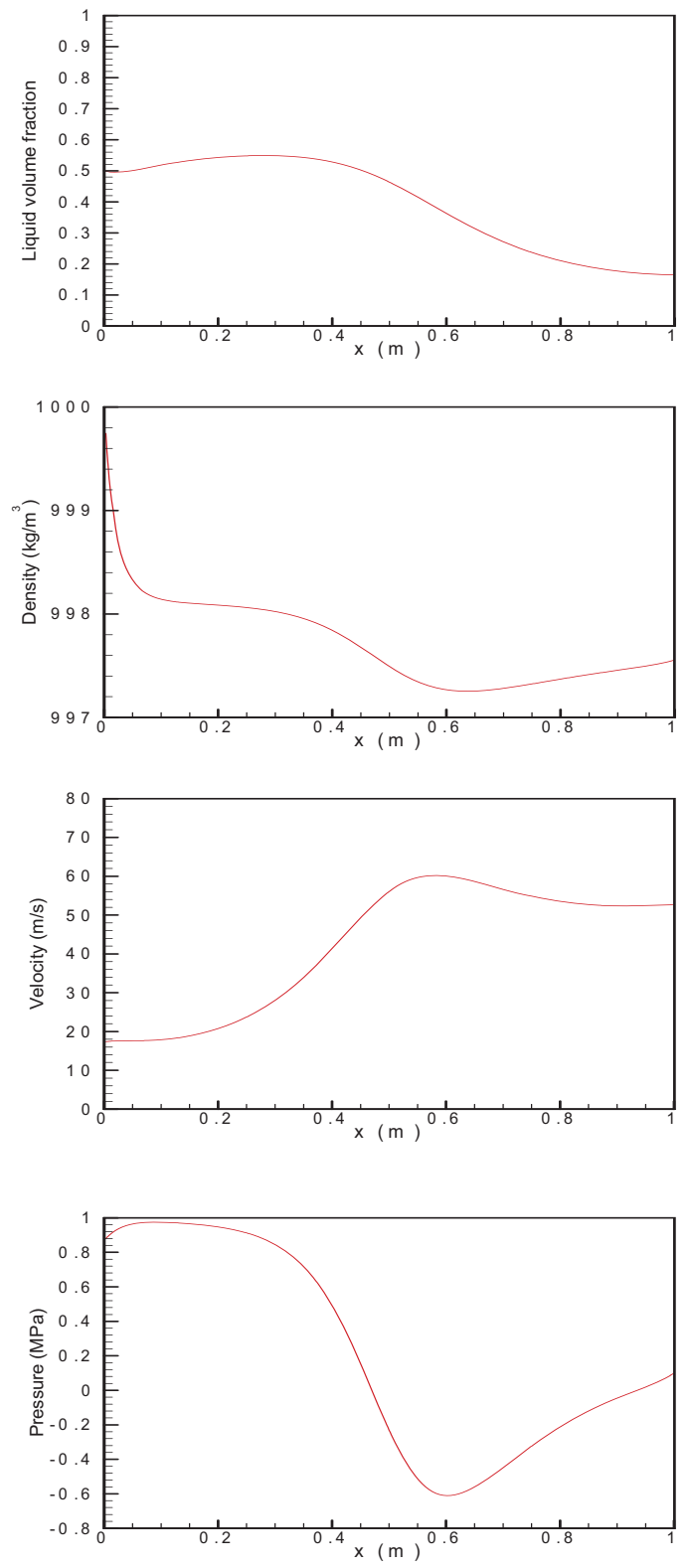
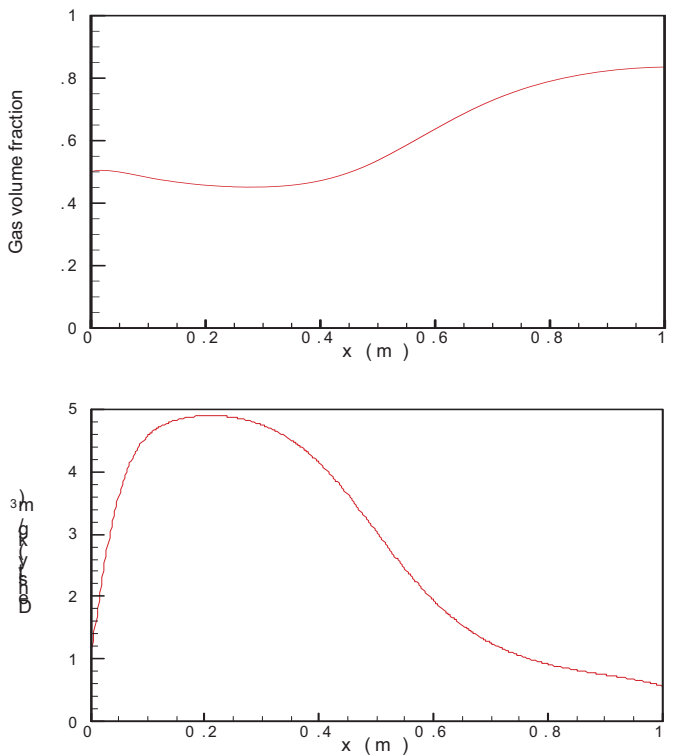

for fully compressible two-phase flows in ducts of spatially varying cross-section," Nuclear Engineering and Design, In review, August 2009.
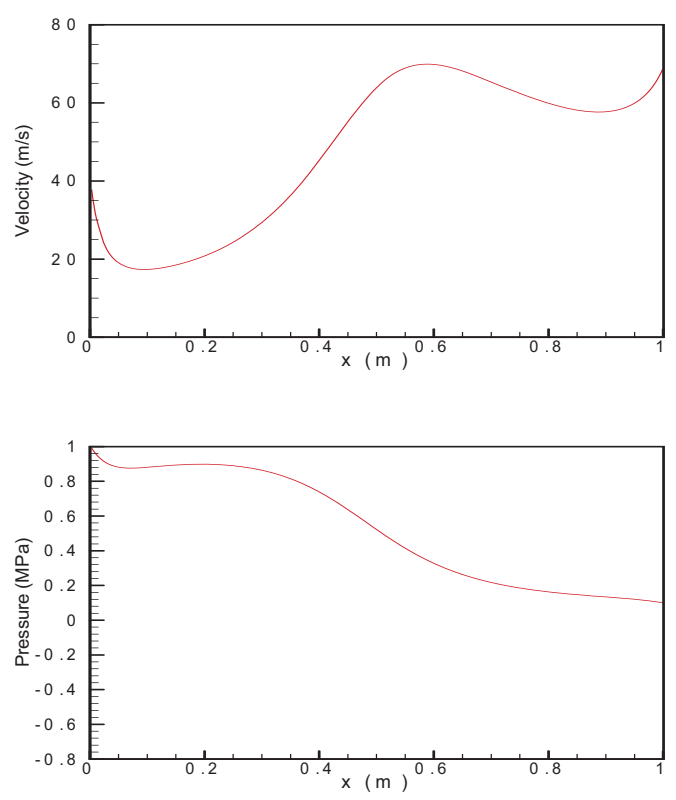

FIGURE 2. Two-phase solution. Left colum is liquid, right column is gas. 


\section{Reactor Physics Sensitivity Analysis, Uncertainty Quantification, and Data Assimilation Capability}

C. Rabiti, G. Palmiotti, Y. Wang, M. Assawaroongruengchot, H. S. Abdel-Khalik, W. C. Proctor Development of software tools for sensitivity analysis, uncertainty quantification, and data assimilation and application of the validation methodology NE153

Sensitivity analysis and uncertainty quantification are essential tools in reactor physics design. They allow the quantification of margins, perform optimizations, define target accuracy requirements, evaluate relevance of experiments, and carry out nuclear data adjustments [1]. Many of these tasks play a significant role in the validation process. Moreover, studies have pointed out that present uncertainties in nuclear data should be significantly reduced in order to get full benefit from advances in modeling and simulation $[2,3$, 4]. Only a parallel effort in advanced simulation and in nuclear-data improvement will be able to provide designers with more general and wellvalidated calculation tools to meet design target accuracies. Idaho National Laboratory (INL) lacks tools to use for these types of studies as well as a clear, established strategy and methodology to apply in the validation process. The goal of this laboratory-directed research and development (LDRD) is to fill this gap by (1) developing the needed new software tools, (2) defining a science-based methodology for validating existing codes and data, (3) applying the tools and the methodology using available experimental data, and (4) creating both expertise and a culture among the INL reactor physicists that could be eventually propagated to other fields (e.g. thermal-hydraulics, thermal mechanics, fuel behavior, etc.) for a multiphysics application.

\section{Technical Objectives}

Sensitivity analysis and uncertainty evaluation are the main instruments for dealing with the sometimes scarce knowledge of the input parameters used in simulation tools. For sensitivity analysis, sensitivity coefficients are the key quantities that have to be evaluated. They are determined and assembled, using different methodologies, in such a way that when multiplied by the variation of the corresponding input parameter, they will quantify the impact on the targeted quantities whose sensitivity is in question. Sensitivity coefficients can be used for different objectives, like uncertainty estimates, design optimization, determination of targetaccuracy requirements, adjustment of input parameters, and evaluations of the representativity of an experiment with respect to a reference design configuration.

In uncertainty evaluation, the sensitivity coefficients are multiplied by the uncertainties of the input parameters in order to obtain the uncertainty of the targeted parameter of interest. The origin and quality of the uncertainties of the input parameters can be different and vary significantly. In some cases, they are provided by the expert judgment of qualified designer. In some other cases more useful information is available - for instance, from experimental values - and they are cast in more rigorous formalism. This is the case, for instance, of covariance matrix for neutron cross-sections, where correlations in energy and among the different input parameters (reactions, isotopes) are also provided.

Target accuracy assessments are the inverse problem of the uncertainty evaluation. To establish priorities and target accuracies on datauncertainty reduction, a formal approach can be adopted by defining target accuracy on design parameter and finding out required accuracy on data. In fact, the unknown uncertainty data requirements can be obtained by solving a minimization problem where the sensitivity coefficients in conjunction with the existing constraints provide the needed quantities to find the solutions.

Sensitivity coefficients are also used in input parameter adjustments. In this case, the coefficients are used within a fitting methodology (e.g. least square fit, Lagrange multipliers with most-likelihood function, etc.) in order to reduce the discrepancies between measured and calculational results. The resulting adjusted input parameters can be subsequently used, sometimes in combination with bias factors, to obtain calculational results to which a reduced uncertainty will be associated.

A further use of sensitivity coefficients is a representativity analysis of proposed or existing experiments, in conjunction with a covariance matrix. In this case, the calculation of correlations among a target reactor design and small experiments allows a determination of how representative the experiments are of the design, and consequently, to optimize the experiments 
and to reduce their numbers. Formally, one can reduce the estimated uncertainty on a design parameter by a quantity that represents the knowledge gained by performing the experiment.

Two main activities are performed under this proposed LDRD:

- Development of software tools for sensitivity analysis, uncertainty quantification, and data assimilation

- Application of the validation methodology. Products that are delivered this year include:

- Acquisition and implementation of sensitivity and uncertainty analysis tools of the ERANOS code system

- Initial implementation of the Effective Subspace Method (ESM) methodology for deterministic codes

- Assimilation data code base on global statistical adjustment methodology

- Application of validation strategy on an initial limited set of experiments and reference systems.

\section{Results and Accomplishments}

During this fiscal year all promised milestones have been achieved and products delivered. The main achievements include:

1. Acquisition and implementation of sensitivity and uncertainty analysis tools of the ERANOS code system.

The code system and the sensitivity and uncertainty capabilities are fully operational on different clusters and computers. It is now possible to perform sensitivity coefficient computation for many integral parameters including multiplication factor $\left(\mathrm{K}_{\text {eff }}\right)$, reactionrate ratios (slope and attenuation of neutron propagation), reactivity coefficients (e. g. coolant void, Doppler temperature effects, control-rod reactivity), $\beta_{\text {eff, }}$ etc. When covariance data (i.e. uncertainties and their correlation on crosssections) are available the related uncertainty quantification can also be carried out. These capabilities have been largely used in different applications of programmatic work packages as uncertainty quantifications of several integral parameters of interest for the neutronic design of an advanced reactor, as well for the sensitivity coefficients needed in the adjustment of nuclear data for improved margins.

\section{Initial implementation of the ESM} methodology for deterministic codes.

The efficient subspace method (ESM) has been implemented on the INL HELIOS cluster with the help of NCSU, the original proposer of this technique. A student from NCSU was resident at INL for the ESM implementation. Subsequently, this method has been tested and compared against other techniques as the standard adjoint approach and the forward stochastic uncertainty propagation (DAKOTA code) for a case of an advanced reactor system as a sodium-cooled fast burner reactor. This comparison was performed in the framework of an Advanced Fuel Cycle Initiative (AFCI) programmatic work package.

3. Production of assimilation data code based on global statistical adjustment methodology.

Two codes have been written. The first one is a tool for sensitivity coefficient selection. As a byproduct, it can also perform uncertainty evaluation. The second one performs the statistical adjustment using the discrepancies between the observed experimental results, the covariance data on the parameters to be adjusted, and the related sensitivity coefficients. It is possible that these two codes, depending on future requirements, could subsequently be merged. The use of these two codes has been essential in formulating a proposal for a funding opportunity announcement (FOA) from the Department of Energy (DOE) Office of Science. The proposal on an application of a consistent data assimilation that allows use of the information coming from integral experiments for improving basic nuclear parameters has been funded. This methodology, which is embedded in the two codes, allows them to span over an unprecedented range of 13 orders of magnitude, going from the meter scale to femtometers. 
4. Application of validation strategy.

The validation strategy has been applied using uncertainty quantification of many design parameters (including multiplication factor, coolant void reactivity coefficients, Doppler temperature effect, power peak, etc.) using a set of newly released covariance data associated to the ENDF/B-VII cross section files. Further application has been carried out, studying reflector effects in reactor systems, where uranium blankets are replaced by stainless steel reflectors. The data assimilation has allowed the identification of improvements needed in structural material cross-sections in order to reduce discrepancies previously observed in experiments devoted to the study of this effect. Finally, a study, which will be finished in Fiscal Year (FY) 2010, is being performed on the impact of the content of minor actinides in the fuel of advanced nuclear systems. This study, using the appropriate sensitivity coefficients, quantifies the effect of the different types of minor actinides (fertile or fissile) on several reactivity coefficients, like coolant void, Doppler, and $\beta_{\text {eff. }}$

\section{Summary and Conclusions}

INL has directly benefitted from the work carried out in this LDRD by establishing its reputation in verification and validation (V\&V) activities that are of significant interest for DOE in the AFCI program and in particular for the NEAMS (Nuclear Energy Advanced Modeling and Simulation) project. The developed capabilities can eventually be used in the certification process for licensing future reactors with NRC.

Moreover, the sensitivity- and uncertaintyevaluation tools, as well as the validation strategy that is developed in this LDRD, could be directly applied to quantify and reduce margins in nuclear reactor designs of interest to DOE (AFCI, GEN-IV, Next Generation Nuclear Plant [NGNP], and advanced light-water reactors [LWRs]).
References

1. G. Palmiotti, M. Salvatores, et al., "Methods in Use for Sensitivity Analysis, Uncertainty Evaluation, and Target Accuracy Assessment," 4th NEMEA Workshop on Neutron Measurements, Evaluations and Applications - Nuclear Data Needs for Generation IV and Accelerator Driven Systems, October 16-18, 2007, Prague, Czech Republic.

2. G. Palmiotti, et al., "Requirements for Advanced Simulation of Nuclear Reactor and Chemical Separation Plants," ANLAFCI-168, Argonne National Laboratory (2006).

3. G. Palmiotti, M. Salvatores, et al., "Validation of Simulation Codes for Future Systems: Motivations, Approach and the Role of Nuclear Data," 4th NEMEA Workshop on Neutron Measurements, Evaluations and Applications - Nuclear Data Needs for Generation IV and Accelerator Driven Systems, October 16-18, 2007, Prague, Czech Republic.

4. M. Salvatores, et al., "OECD/NEA WPEC Subgroup 26 Final Report: Uncertainty and Target Accuracy Assessment for Innovative Systems Using Recent Covariance Data Evaluations," OECD-NEA Report in print, 2008. 


\section{Fracture Methods for Reactor Fuel Performance Analysis}

Richard L. Williamson, Dana Knoll

Development and demonstration of state-of-the-art computational methods to simulate fractures in nuclear fuel

NE154

Idaho National Laboratory (INL) is currently developing state-of-the-art capabilities to model nuclear fuel performance. A crucial issue not adequately addressed by the current effort is fuel cracking, which, for oxide fuels, occurs during the first rise to power and continues during essentially all operations (Fig. 1).
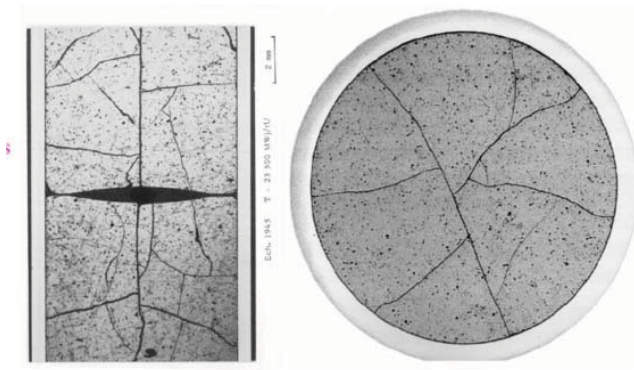

FIGURE 1. Typical oxide fuel fractures.

Fractures have a very strong influence on fuel performance, controlling critical phenomena such as fission-gas release, fuel creep, pellet-clad mechanical interaction (PCMI), and clad failure. Accurate multidimensional fracture modeling is essential for predictive fuel performance simulation.

The objective of this research is to develop state-of-the-art fracture-mechanics computational methods, both for immediate use in the INL near-term nuclear-fuel performance code (ABAQUS), and for eventual implementation in the next-generation 3D code currently under development (BISON). The first year effort involved development, implementation, and testing of modern smearedcrack and cohesive-zone $(\mathrm{CZ})$ fracture models, providing INL with state-of-the-art fuel performance modeling capabilities.

\section{Technical Objectives}

The major tasks and first-year milestones for this project were:

- Develop and test an elastic smeared-crack material model for oxide fuels

- Develop and test cohesive-zone fracture models for oxide fuels
- Publish early results at applicable reactorfuels meetings.

\section{Results and Accomplishments}

For the first year of this project, all fracture models were implemented and tested in ABAQUS, a powerful multidimensional finite element thermomechanics code in common use throughout the world. ABAQUS has been selected as the development platform for the INL near-term fuel performance code.

Initial efforts focused on the implementation of smeared-cracking models, which locally modify the elastic material behavior to approximate the effects of fracture. These models are reasonably straightforward to implement, since they do not involve the complexities associated with tracking of discrete cracks; only the local constitutive behavior in terms of stresses and strains is modified in any region which undergoes cracking. Fortunately, during Fiscal Year 2009 (FY-09) a collaborative relationship was developed with ANATECH, Inc. (developers of the state-of-the-art code FALCON fuel performance code for EPRI) which proved very beneficial in this area. Joe Rashid, company president and inventor of the smeared-crack theory, provided considerable assistance in the development of this capability within ABAQUS. Although additional testing and validation is warranted, smeared-cracking capability is now available for INL fuel performance analyses.

Discrete fracture methods, such as the $\mathrm{CZ}$ approach, are attractive from a mechanistic and physical standpoint, since they reflect the localized nature of cracking. The precise locations where fractures initiate, as well as the crack evolution characteristics, are determined as part of the solution. Cohesive zone models also offer the possibility to simulate important phenomena such as crack closure during power reduction and crack healing (re-sintering) in high-temperature regions. Powerful CZ analysis capability was recently included in ABAQUS and was used for this research. To evaluate $\mathrm{CZ}$ capabilities, a simple cylindrical fuel pellet geometry was assumed, and test cases were explored in both 2D (axisymmetric and planestrain geometries) and 3D. Fully coupled 
thermo-mechanical behaviour was employed, including the effects of thermal expansion and fission product swelling. Significant effort was also expended to parametrically study and document numerical sensitivities, which are known to exist with $\mathrm{CZ}$ methods. Typical numerical results for the three geometries considered are shown in Figures 2-4.

Figure 2 shows the computed maximum inplane principal stress for the 2D axisymmetric model, displaying predicted cracking behavior at various times in fuel life. Cracks tips are easily identified by the focused high-stress (red) regions. In the first three figures, plot times were selected just after crack initiation and display rapidly propagating fractures. The first crack forms on the axial symmetry plane at a fuel center-to-edge temperature difference of only 80 $\mathrm{K}$, and results in significant local stress relief and a shift in the location of the peak stress along the pellet surface. A second set of cracks initiates at $136 \mathrm{~K}$, resulting in a total of three transverse cracks at the end of pellet heat-up step. The final set of cracks is not observed until 456 days of operation, and occurs due to a gradual rise in the pellet temperature gradient due to reduced thermal conductivity with burnup. No further cracking is predicted during further steady operation as the temperature gradient continues to increase. Once fractured into eight pieces, very large temperature gradients are possible while the maximum principal stress remains well below the $\mathrm{UO}_{2}$ fracture strength.

Figure 3 compares the maximum principal stress and predicted cracking behavior for the $2 \mathrm{D}$ plane-strain model at the end of the heat-up step, assuming crack initiation (or "seeding") at eight, four, three, and one circumferential locations. The 8- and 4-seed cases show symmetric cracking, as expected, with only radial cracking in the 8-seed case, but both radial and circumferential cracking in the 4-seed case. Even a single crack results in immediate stress relief along the pellet periphery, with no further crack initiation from the outer surface. Instead, further radial and circumferential cracking occurs from inside the pellet.

Figure 4 shows the maximum principal stress and predicted cracking behavior for the $3 \mathrm{D}$ model, at three times during initial heating. Fracture begins at a temperature difference of $88 \mathrm{~K}$, with cracks forming in both the radial and transverse directions (Fig. 4a). As the temperature difference increases, the radial cracks converge on the symmetry axis at the pellet top and bottom (Fig. 4b), and, with further heating, result in the formation of new radial cracks that propagate outward (Fig. 4c).
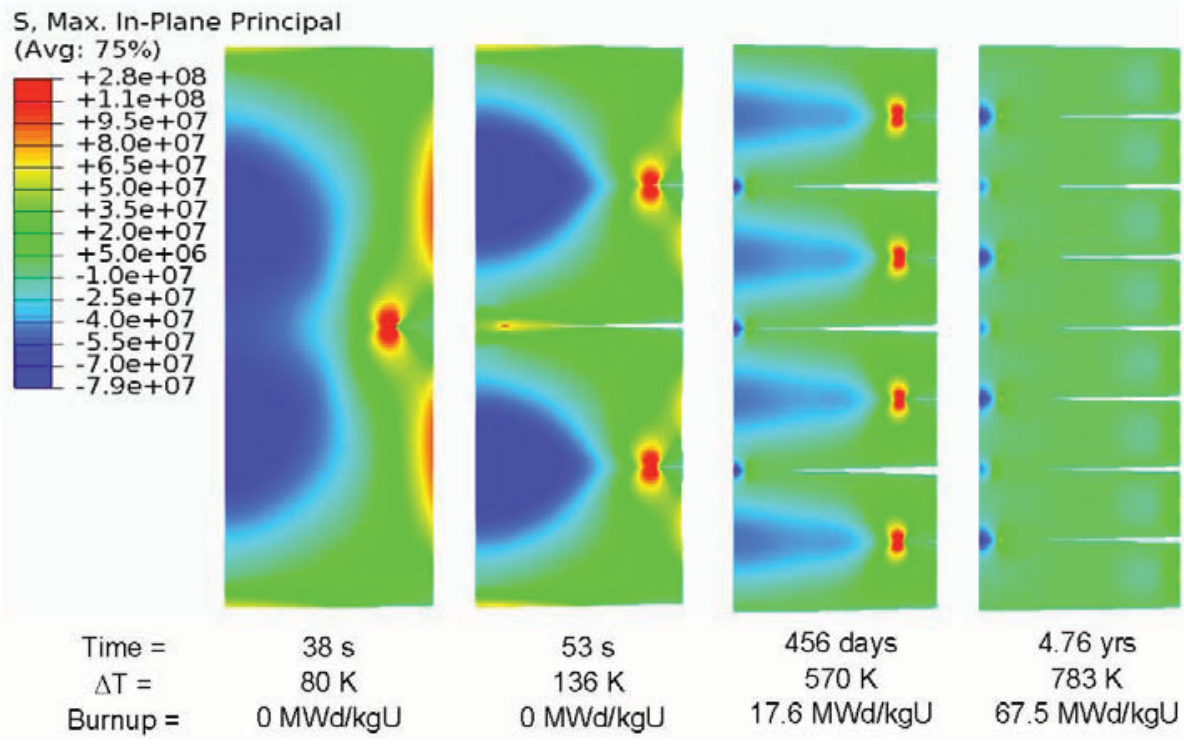

FIUGRE 2. Maximum in-plane principal stress (Pa) from the axisymmetric calculation, showing the predicted cracking sequence. Displacements are magnified by $10 x$ to better visualize fractures. 

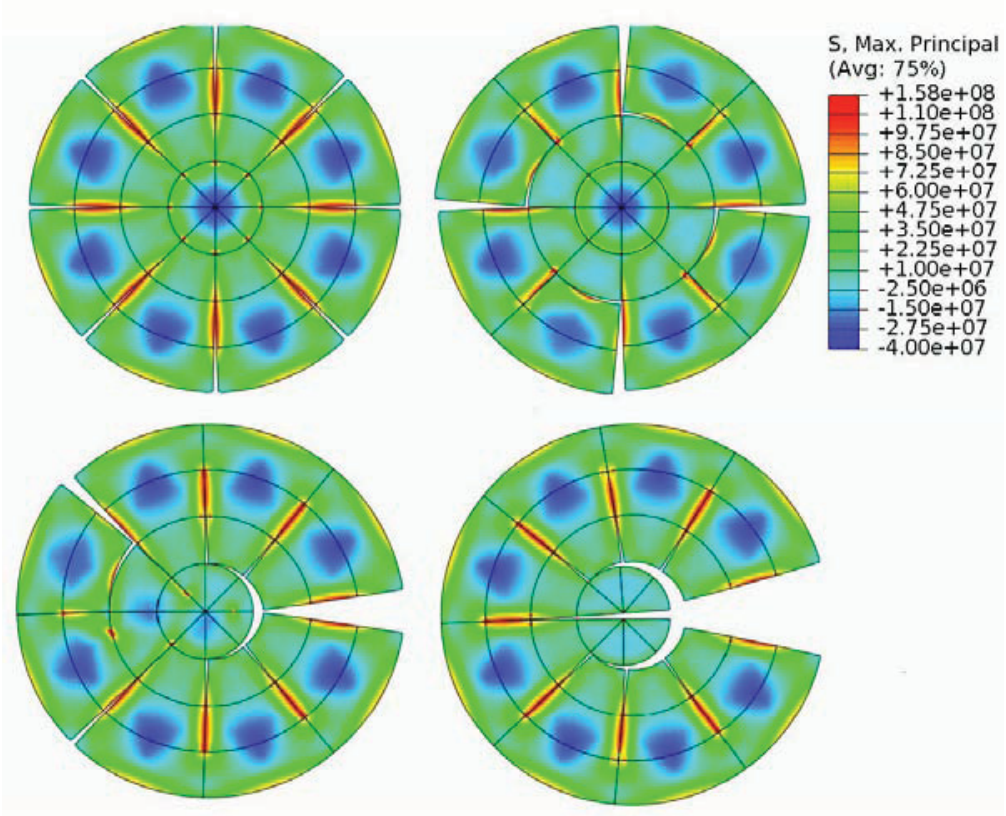

FIGURE 3. Maximum principal stress (Pa) from the plane-strain model, showing predicted cracking for (a) 8-seed, (b) 4-seed, (c) 3seed, and (d) 1-seed assumptions. Displacements are magnified by 10x to better visualize fracture.
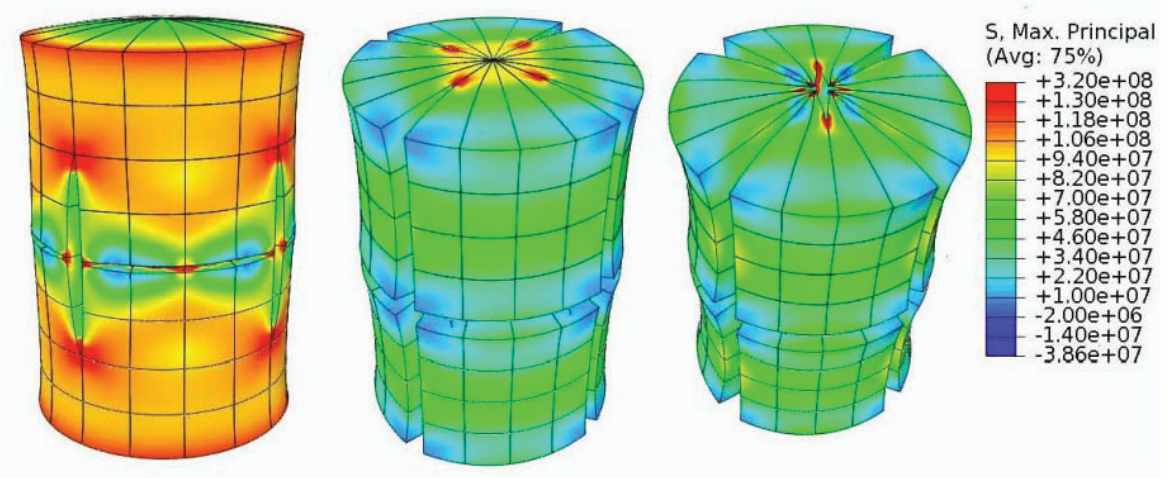

FIGURE 4. Maximum principal stress and predicted cracking behavior for the 3D model, at three times during initial power-up. Displacements are magnified by $200 x$.

To achieve rapid dissemination of the research and demonstrate new INL fuelsmodeling capability, research results were documented in papers at two key technical conferences in the area of nuclear-fuelperformance modeling during 2009. Both papers were peer-reviewed and well-received at the meetings and are published in the conference proceedings. Paper references are:

R. L. Williamson and D. A. Knoll, "Simulating Dynamic Fracture in Oxide Fuel Pellets
Using Cohesive Zone Models," Proceedings of the $20^{\text {th }}$ International Conference on Structural Mechanics in Reactor Technology (SMIRT 20), Espoo, Finland, August 9-14, 2009.

R. L. Williamson and D. A. Knoll, "Enhancing the ABAQUS Thermomechanics Code to Simulate Steady and Transient Fuel Rod Behavior," Proceedings of Top Fuel 2009, Paris, France, September 6-10, 2009. 
Both papers will be expanded into journal articles as the research continues. All first-year objectives and milestones were achieved.

Summary and Conclusions

Accurate multidimensional fracture modeling is essential for predictive fuelperformance simulation. Results from this research indicate that highly complex fuel fractures will likely be best simulated using a combination of smeared and discrete fracture methods. Results provided in the above referenced conference papers demonstrate that the INL is now at the forefront in fuel-fracture modeling capability. Such results place the laboratory in a good position to compete for external funding in this area. 


\title{
Use of Ice Thermal Storage Systems to Improve LWR Plant Efficiency
}

\author{
Haihua Zhao, Hongbin Zhang (INL), Wei Yan, Blaise Hamanaka, WoonSeong Jeong \\ Using ice thermal storage systems at LWR plants to reduce cooling water requirements and boost \\ thermal efficiency \\ NE155
}

Availability of sufficient cooling water for large nuclear power plants has been one of the major issues for the nuclear power plant site selection. Cooling water issues have frequently disrupted the normal operation at some nuclear power plants during heat waves. The issues become more serious due to the new round of nuclear power expansion and global warming. The cooling water issue not only depends on where a plant is located, but also depends on when the cooling requirement becomes peak. During hot summer days, cooling water leaving a power plant may become hot enough to threaten aquatic life so that federal or state regulations may force the plant to reduce power output or even temporarily to shutdown. For new nuclear power plants to be built in areas without enough cooling water, dry cooling has to be used to remove waste directly into atmosphere.

However, dry cooling will result in much lower thermal efficiency when the weather is hot. An Electric Power Research Institute (EPRI) study shows that dry cooling imposes a heat rate and lost-capacity penalty on a plant that can range up to $25 \%$ during the hottest hours of the year and exceed $8 \%$ for over 1,000 hours at a hot and arid site.

One potential solution to these issues is to use thermal storage systems that reduce cooling water requirements and boost the plant's thermal efficiency in hot hours. Ice is suitable to store "cold energy." The heat of fusion for ice is equivalent to heating water by $80^{\circ} \mathrm{C}$ for the same amount of mass. Therefore, ice has at least 10 times the cooling ability as the same amount of water. Some gas-turbine plants already use daily ice making and thermal storage (ITS) systems to increase thermal efficiency during peak hours in summer. ITS systems (Fig. 1) have also been widely used for building cooling to save energy cost. Another similar idea is the seasonal storage of ice for a large power plant. The increased thermal efficiency and higher peak electricity sale price could generate large revenues while reducing the thermal pollution and water consumption. The primary objective of this laboratory-directed research and development (LDRD) proposal is to perform the preliminary scoping and feasibility study to prove the viability of the proposed approach. Using ice thermal storage/making systems for nuclear power plants is a new idea and could potentially have large economic impact on the nuclear industry.

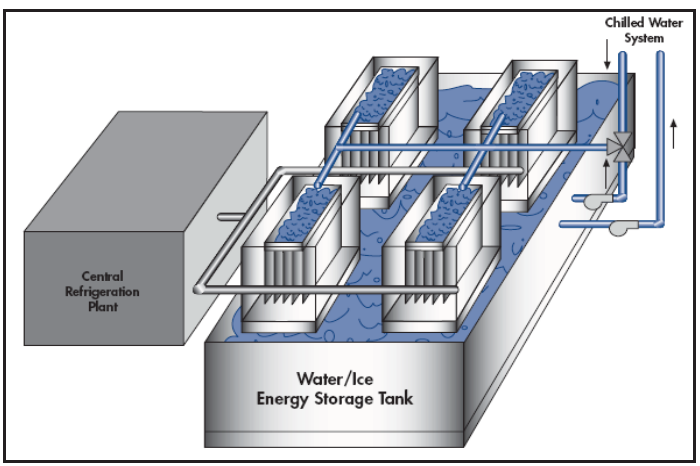

(a)

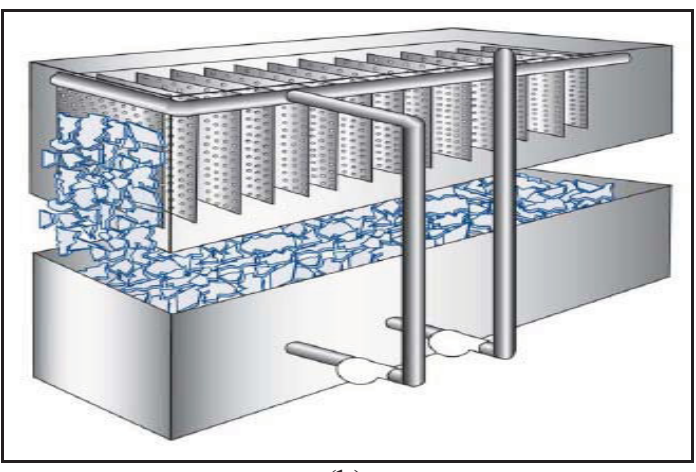

(b)

FIGURE 1. Dynamic ice thermal storage system. (a) Refrigeration plant configuration (Mueller Company 2009. (b) Organization of the refrigeration plant (Mueller Company 2009).

\section{Technical Objectives}

The primary objective of this one-year LDRD project is to perform the preliminary scoping and feasibility study to prove the viability of the proposed approach. The following tasks have been completed:

- Literature review on U.S. nuclear power plant cooling issues and ITS systems

- Thermodynamics study on the effect of cooling temperature to provide insights about the relative benefits of once-through cooling, wet cooling, and dry cooling with ice thermal storage options 
- Identification of possible U.S. sites based on review of nuclear power plant weather and environmental conditions

- A conceptual design of the ice storage building or structure, and a preliminary cost estimate

- Preliminary study on the economic impact.

Results and Accomplishments

A light water reactor (LWR) power plant's net power output depends on cooling temperature through three aspects, as shown in Figure 2; simple steam Rankine cycle efficiency depends on cooling temperature, turbine efficiency depends on cooling temperature, and thermal discharge temperature limits are set by

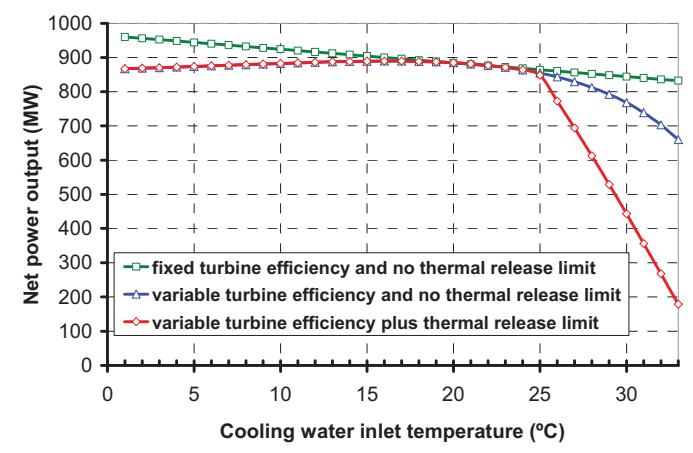

FIGURE 2. A once-through cooling LWR plant net power output depends on cooling water inlet temperature when a regulatory limit of $35^{\circ} \mathrm{C}$ is respected for cooling water outlet temperature.

environmental protection regulations. If fixed turbine efficiency is assumed and there is no thermal discharge temperature limit, the electric power will decrease with cooling water inlet temperature as shown in the green line, which can be expected from simple thermodynamic analysis. However, a steam turbine is optimized at fixed pressure ratio. When the condensation temperature is far above the reference value, the turbine efficiency decreases rapidly. The blue line shows this effect. For a plant with a dry cooling system, a hot weather efficiency penalty follows the same two reasons. Water protection regulations typically set strict limits on how hot the discharge cooling water from a power plant can be. When that limit cannot be met, a thermal power decrease (derate) is the only choice without violating the regulations. The red line shows this effect.

Among three cooling methods-oncethrough, wet cooling tower, and dry cooling tower-once-through cooling systems near a large water body like an ocean or a large lake and wet cooling tower systems can maintain the designed turbine backpressure (or condensation temperature) $99 \%$ of the time; therefore, adding ITS to those plants would not generate large benefits. For dry cooling systems and oncethrough cooling systems near a small water body, like a river or a small lake, the ITS could add significant economic benefits and avoid forced derating and shutdown during extremely hot weather as shown in Figure 3. Depending on plant location, the availability of empty land, electricity sale price, water price, and other factors, an ice making/storage system, a seasonal ice storage system, or a combination of both systems may achieve the best economics. These ideas work for both existing plants and new units.

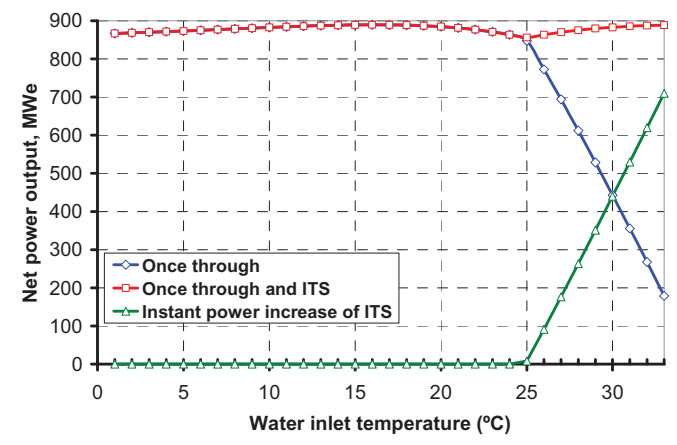

FIGURE 3. Avoiding derating with ITS for an once through cooling LWR during heat wave.

Consider a typical LWR plant with insufficient once-through cooling for only a short time. Assume that ITS provides about $50 \%$ of total cooling for 100 hours to prevent $50 \%$ derating loss due to thermal discharge violation by reducing cooling temperature by $5^{\circ} \mathrm{C}$ (see the case at water inlet temperature $30^{\circ} \mathrm{C}$ shown in Figure 3). Similar events happened at the Browns Ferry Nuclear Power Plant in 2007 and 2008 and during European heat wave events during 2003. Preliminary analysis shows that the increased electricity generation with ITS is 50 
million kw-hr, or 2.7 times the electricity consumed to make the needed ice. The capital cost for such an ITS could be much less than the capital cost for a mechanical draft wet cooling tower system providing similar cooling capability.

This idea can be compared with pumped storage hydroelectricity, a type of hydroelectric power generation used by some power plants for load balancing. Pumped storage hydroelectricity needs a large amount of water and height difference between two natural bodies of water or artificial reservoirs. These two requirements limit its range of application, as is typical of hydroelectric power generally. However, ITS systems can be more widely used than the pumped storage hydroelectricity.

\section{Summary and Conclusions}

LWR nuclear power plants have the highest power and the lowest thermal efficiency among all the baseload power plants. Consequently, the cooling requirement for LWR plants and their thermal and water consumption impact on the environment are the highest. The day-night and summer-winter swings provide a unique opportunity to use thermal storage systems to regulate cooling and water needs while increasing revenues and reducing negative impact on the environment. The use of ITS systems could avert building expensive cooling towers for some new plants, especially for those co-located with existing plants with a limited cooling water source during extremely hot weather. For the new plants using dry cooling towers, adding the ice thermal storage systems can effectively reduce the efficiency loss and water consumption during hot weather so that new LWRs could be considered in regions without sufficient cooling water.

This one-year feasibility study clarifies LWR cooling issues and shows the feasibility and great potential of ITS systems to address such issues. Follow-on works should perform more detailed studies, such as ITS system design and cost analysis, especially on ice storage structure/building cost, and specific LWR plants performance with once-through cooling plus ITS and with dry cooling plus ITS. 


\section{Development of a Next-Generation Production Code for Nuclear Reactor System Analysis and Safety Margin Quantification}

T.-N. Dinh, R. Nourgaliev, V. Mousseau, P. Bayless, H. Zhao, J. Ragusa, Y. Hassan, B. Smith, Q.

$W u$ and $J$. Buongiorno

Aligning the system analysis code development with the emerging concept of risk-informed safety margin characterization (RISMC)

NF.156

The R7 code project aims to develop and demonstrate the scientific basis and computational technology for the next generation of system codes that enable the nuclear-power industry to effectively perform numerical simulation and engineering analysis of a nuclearpower plant's transients and accidents with high confidence. As designed, the R7 code is an instrument for plant risk-informed safety decision making and one of the critical elements in moving towards a new paradigm in nuclearsystem safety design, licensing, and oversight. The main engine for this paradigm shift is a formal process for risk-informed, simulationdriven, performance-based safety case development, evaluation, and acceptance that aims to facilitate design optimization, and make the licensing process more effective and predictable, both for licensees and regulators.

More specifically, the $\mathrm{R} 7$ project builds on (1) the knowledge base, success, and legacy of the Reactor Excursion and Leak Analysis Program (RELAP5) system thermal-hydraulic analysis code developed at Idaho National Laboratory (INL); (2) advanced methods in computational science and engineering, and (3) the emerging formulation of risk-informed safety margin characterization (RISMC). The RISMC framework is envisioned to bring together the now-classical methods of deterministic safety analysis and probabilistic risk analysis (PRA) for an integrated risk model. The PRA-based risk insights help focus the analysis on risksignificant transients and postulated accidents. Modern methods of sensitivity analysis (SA) and uncertainty quantification (UQ) incorporated into the R7 code will enable a quantitative phenomena identification and ranking table (PIRT) process that will effectively guide the $R \& D$ towards uncertainty reduction and render the much sought-after robustness and predictability in safety-issue resolution and plant licensing.

\section{Technical Objectives}

The R7 project's technical objectives are (1) to establish the architecture, foundations, basic components and infrastructure for the R7 computational engine, (2) to develop and demonstrate a methodology for R7 code verification and validation (V\&V), and (3) to demonstrate the code's intended capabilities through selected case studies in a reactor system.

The project's first-year focus is to:

- Investigate requirements for the reactorsystem safety-analysis tools,

- Investigate advanced solution algorithms for thermal hydraulics

- Develop a system code version that can be used to test different solution methods.

Results and Accomplishments

This project execution followed the original plan that focuses the activity on investigating a set of requirements to support architectural design of a next-generation system code for safety-margin characterization with uncertainty quantification. Because two companion proposals (on coarse-grain modeling [CGM] and on adaptive model refinement [AoMR] — both presenting critical technologies for R7 and counted on as parallel projects) were not funded in Fiscal Year 2009 (FY-09), the CGM and AMoR scoping activities were supported within $\mathrm{R} 7$ project. The project also reached out to a broader team of experts and university collaborators as it has become clear during the project implementation that the next generation of system safety codes would need to go well beyond an improved RELAP5 as thermal hydraulics code, but to include neutronics, materials (aging), and fuel performance.

During the year, the project benefitted from an international workshop ("R7 Verification and Validation, Sensitivity Analysis, and Uncertainty Quantification Workshop") organized by the R7project team and held at INL in January 2009, specifically addressing the core technology for the R7 code. It brought together more than 40 experts from 15 institutions. Upon insights and recommendations from the workshop, the project stresses further the importance of having in R7 a diverse set of methods to support (safety margin) uncertainty quantification, and long-term planning for experimental validation. This has also led to interactions with leading experimentalists and risk-analysis experts, to incorporate their insights in developing R7 code requirements. 
According to the plan, the project was pursued in four parallel areas (research, code development, demonstration, and validation) as planned. In each area, progress was made with respect to the original hypothesis:

- Research was carried out on selected key areas in high-order-accurate numerical methods in all-Mach compressible flow equations for single and two-phase flow, uncertainty quantification. The results confirmed the hypothesis about potential for significant improvement of computational efficiency (in time stepping and grid size) while maintaining solution accuracy by means of fully implicit JFNK algorithm and rDG scheme. Two papers for Journal of Computational Physics are prepared on this advancement. The work on Lagrangian Averaged Navier Stokes (LANS) equation as a coarse-grain fluid-flow model also provided evidences to confirm that the LANS can be extended to compressible and thermal flows of importance for reactor safety analysis. The research on two-fluid equations identified the gap in having a physically consistent and mathematically well-posed model, pointing that a consistent homogenization (of equation and closure laws) is required to effectively model plant transient processes.

- Code development produced a developmental version of fully parallelized computational engine in object-oriented computational framework that was used to test the performance and impact of different computational fluid dynamics (CFD)originated discretization schemes for their suitability for a system analysis with complex requirements. This task produced insights that are key to update the requirements and the design of the computational framework and engine.

- Demonstration was performed in a limited scope this first year, primarily to demonstrate selected aspects of the code development and verification and validation $(\mathrm{V} \& \mathrm{~V})$ procedure, e.g. verification of computational engine (numerical methods) through method-of-manufactured solutions.
A review of safety needs in different reactor systems led to a suggestion to focus the demonstration program on light-water reactor (LWR)/advanced light water reactor (ALWR), which can subsequently be supported by Department of Energy (DOE) programs on LWR life extension and ALWR safety and performance.

- Validation planning was started, including laying the foundation for experimental design procedure and supporting efforts to establish a program on validation (test facility) at Utah State University, Oregon State University, and Ohio State University. The work confirms the original hypothesis that R7 development — due to a broadened scope, new focus on risk-informed space, passive-safety transients, and new modeling methodology - will require a new approach to experimental validation, and demonstration of a consistent modeling, experimentation and validation strategy will require an "in-house" (which, although it may not be in the same organization, will nevertheless necessarily be tightly coupled) team of experimentalists and set of facilities, which are either designed and built from scratch or the R7 team has significant "control" power over the facility modification and operation.

The project team has made an effort to disseminate the ideas, techniques and insights developed in the project. The principal investigator (PI) will deliver a keynote speech ("A Next Generation of Nuclear Plant System Codes to Support Risk-Informed Safety Margin Characterization") at the $13^{\text {th }}$ International Topical Meeting on Nuclear Reactor Thermal Hydraulics (NURETH-13, Japan). The PI and co-PIs lectured on the R7 concept at International RELAP User Group meetings $(2008,2009)$ and the first Nuclear Engineering Summer School "Modeling, Experimentation and Validation" (Idaho Falls, July 2009).

By integrating capabilities in deterministic and probabilistic analysis, the project advanced a technical basis needed to formulate utility, architectural, and developmental requirements for a next generation of nuclear reactor safety simulation, a more comprehensive and accurate 
characterization of safety margins in nuclear power plants. Synergy achieved in this project through interactions and collaboration of experts from different fields (from computational science, experimental thermal hydraulics, fuels/materials, to risk assessment, etc.) presents the uniquely new capability. The work on the code architecture and computational method had produced a simulation engine for simplified system that is used to test different hypotheses and establish operational envelope of different numerical-solution techniques.

\section{Summary and Conclusions}

Research conducted in the R7 project has significantly improved a basic understanding of objectives and functionality of a future reactorsystem safety-analysis tool. This was made possible by bringing in a team of diverse expertise in system-safety analysis, risk assessment and management, computational fluid dynamics and multi-physics analysis. Together with insights derived from selected investigation of modeling, numerical-solution and sensitivity/uncertainty-analysis methods, the improved understanding of the simulation tools as a means to safety decision making paves way to formulate R7 as an engine for a RISMC process and greatly influences the R7's software requirements. The requirements will help define the work scope and prioritize follow-on tasks in developing methods, models, and data to support the production code implementation and assessment. Research on R7-critical methods (e.g., adaptive-model refinement, coarse-grain modeling, forward sensitivity analysis, integration of PRA and DSA) and R7 models (e.g. consistent set of two-phase flow models with time and length scales) identified by the requirements must be carried out.

Internally, the research performed in this project created a technical ground to formulate "safety analysis" as a strategic area in modeling and simulation R\&D in INL's Nuclear Science and Technology (NS\&T) Program. The NS\&T Peer Review Committee's final report “strongly endorses" the R7 project.

Externally, insights from the investigation of technical framework for a next generation of system-safety codes have benefitted the formulation of R\&D scope and plan for the Department of Energy's "LWR Sustainability" (LWR-S) Program's risk-informed safety-margin characterization pathway. This pathway also started in FY-09 was elevated by the LWR-S Technical Integration Office (TIO) from a "seed" position to a top priority. The LWR Sustainability Program's steering committee recommended the program to support R7 development and accelerate it to cope with the schedule for LWR plant-life extension decision making. The funding for RISMC (including R7 production code work) in FY-10 is $\$ 2.1 \mathrm{M}$ and projected by the TIO to grow to $\$ 5.4 \mathrm{M}$ in FY-11 and to a steady level of $\$ 15 \mathrm{M}$ in FY-14. The project also plays a critical role in establishing INL's position as a lead player to the DOE's Nuclear Energy Innovation Hub, should the NE's Modeling and Simulation Hub mission be formulated with safety emphasis.

The project activities have brought excitement to nuclear engineering's academic and laboratory community. Through its network of university partners and collaborators, and support for Ph.D. students and faculty, this project in its first year has already created a nation-wide team of talents (including the Massachusetts Institute of Technology, North Carolina State University, Oregon State University, Ohio State University, Utah State University, Texas A\&M University, University of California and others) which is critical for a long-term and large-scale effort required to advance safety analysis capabilities.

To achieve its objectives, the $\mathrm{R} 7$ project necessarily expands in FY-10 and subsequent years. The software development team needs also to acquire talent with experience in largescale software development and large-scale data processing techniques. The physics-modeling team needs to bring in expertise in structural mechanics and material-degradation modeling and modeling of nuclear-fuel/cladding behavior in operational, transient, and accident conditions. Test problems should also be devised to verify solution algorithms of integrated deterministic/stochastic and tightly coupled multi-physics treatment. As a part of the project execution plan, it is proposed to develop a simplified model of a plant system and use it to 
test and demonstrate a broad range of intended simulation/analysis capabilities required for riskinformed safety margin characterization. 


\title{
Multi-physics Simulation Methods for Advanced Reactor
}

\section{Analysis}

\author{
Dana Knoll, Glen Hansen, Chris Newman, Samet Kadioglu \\ Advancing computational nuclear engineering \\ $\mathrm{SH} 104$
}

This project has been divided into three major supporting thrust areas: multi-physics coupling, multi-physics grid generation, and direct numerical simulation of boiling two-phase flow. Within each of these areas prototype software is being developed, with advanced algorithms, in order to establish expected benefits in computational nuclear engineering applications.

\section{Technical Objectives}

The primary objectives of this project are to aid in the advancement of computational nuclear engineering and to make Idaho National Laboratory (INL) the leader in computational nuclear engineering.

\section{Results and Accomplishments}

In 2009, we continued to make progress in general multiphysics algorithms, multiphysics mesh generation, and implicit interface tracking for DNS two-phase flow. The primary focus was on general multiphysics algorithms research with some additional emphasis on multiphysics fuel performance simulation. The results are exemplified by the following partial list of peerreviewed journal articles, conference sessions organized, and conference presentations.

Kadioglu, Knoll, and de Oliveria, 2009, "Multiphysics Analysis of Spherical Fast Burst Reactors," Nuclear Science and Engineering, Vol. 163. pp. 132-143.

Newman, Hansen and Gaston, 2009, "Three dimensional coupled simulation of thermomechanics, heat, and oxygen diffusion in UO2 nuclear fuel rods," Journal of Nuclear Materials, Vol. 392, pp 6-15.

Kadioglu and Knoll, 2010, “A Fully Second Order Implicit/Explicit Time Integration Technique for Hydrodynamics plus Nonlinear Heat Conduction," Journal of Computational Physics (accepted) 2010.

Knoll, Park and Smith, "Application of the Jacobian-Free Newton-Krylov method in Nonlinear Transport Acceleration," Nuclear Science and Engineering, (in review) 2010.

Knoll, Park and Newman, 2010, “Acceleration of k-Eigenvalue/Criticality Calculations using the Jacobian-Free Newton-Krylov Method," Nuclear Science and Engineering, (in review) 2010.

In addition, this laboratory-directed research and development (LDRD) project supported the work which produced four peer-reviewed conference papers at the American Nuclear Society topical meeting on Mathematics and Computation in Saratoga, New York, in May 2009 (papers authored by Knoll, Newman, Hansen, and Kadioglu).

As a part of this LDRD, Dana Knoll organized a symposium at the SIAM Computational Science and Engineering conference in Miami, Florida in March 2009, with eight speakers from industry, national labs, and academia.

This LDRD lead to a number of invited presentations on the same topic of algorithms and software for multiphysics computational nuclear engineering: two by Knoll and Hansen at the SIAM Annual Meeting in Denver, Colorado (July 2009); two by Knoll at Oregon St. University (October 2009) and University of Michigan (November 2009); one by Newman at Los Alamos National Laboratory (LANL) (Feb 2009); and one by Hansen at the Massachusetts Institute of Technology (MIT) (April 2009).

As the most solid measure of this LDRD's recognition across the Department of Energy (DOE) lab complex, Knoll was invited to give a presentation and write a peer-reviewed journal article for the highly prestigious Office of Science conference Scientific Discovery through Advanced Computing (SciDAC 2009) in San Diego, California in June 2009.

Gaston, Hansen, Kadioglu, Knoll, Newman, Park, Permann, and Taitano, 2010, "Parallel multiphysics algorithms and software for computational nuclear engineering," Journal of Physics: Conference Series, Vol. 180.

Additionally, it is important to note crossfertilization with other LDRD efforts. The core algorithmic concepts put forward by this LDRD are physics-based preconditioning and the JFNK method. Through the guidance of Knoll on this LDRD, these algorithmic concepts have been demonstrated in additional muliphysics 
computational nuclear engineering applications by Park on another LDRD (principal investigator: Martineau) resulting in three conference presentations and two invited presentations by Park (Oregon St. University and LANL), as well as the following:

Park, Nourgaliev, Martineau, and Knoll, 2010, "On physics-based preconditioning of the Navier-Stokes equations," Journal of Computational Physics, Vol. 228, pp 91319146.

Park, Knoll, Gaston, and Martineau, 2010, "Tightly coupled multiphysics algorithms for pebble bed reactors," Nuclear science and Engineering (in review).
Summary and Conclusions

This LDRD has clearly demonstrated a successful modern algorithmic approach to the next generation of multiphysics computational nuclear engineering. We have provided clear demonstrations for steady-state and transient reactor problems and multiphysics nuclear fuel problems. This general work will be seen as the defining effort in the next generation of multiphysics computational nuclear engineering. Our invitation to present our work at the prestigious SciDAC 2009 conference is a clear statement of the impact of this LDRD outside of INL and outside of DOE Office of Nuclear Energy (DOE-NE). 


\section{PAGE FOR ENERGY SECURITY INITIATIVE DIVIDER}

\section{Energy Security Initiative}




\title{
Chemical Separations and Process Research to Enable Biorefinery Systems
}

\author{
Frederick F. Stewart, Mason K. Harrup, David N. Thompson, and G. Glenn Lipscomb \\ (University of Toledo) \\ Investigating a process to remove enzymatic poisons from biomass hydrolysate
}

EI101

In the conversion of low-value biomass feedstocks, chemical and thermal steps are employed to break down cellulosic materials into simpler saccharides, which are then biochemically converted to ethanol. In many cases, these chemical and thermal steps generate the desired saccharides and other organic compounds (such as acids, furfurals, and furans) that interfere with and retard downstream biochemical processing, resulting in lower conversion efficiencies. To address the problem of process toxins, it was proposed in this work to design and test catalytically active membranes that will both separate and degrade these toxins selectively. For this initial development effort, organic acids (such as acetic acid) were the targeted poisons. In general, it was envisioned that an extraction system would consist of an aqueous phase, which is derived from the hydrolysis process, and an organic phase, which could be separated from the aqueous phase with a membrane. Thus, in addition to synthesis of catalytically active membranes, an organic phase was identified.

Membranes were fabricated at Idaho National Laboratory (INL) and delivered to the University of Toledo for testing. Following the catalyst/membrane testing, the University of Toledo worked on converting INL materials into commercially applicable hollow tube modules for characterization against actual biomass hydrolysates, which was supplied by our collaborators at the National Renewable Energy Laboratory. The importance of this work is evident in the ever-increasing demand for ethanol as a "green" fuel and the realization that corn cannot be the sole source for ethanol. A greater supply of ethanol must be made from non-food commodities such as corn stover or switch grass. This proposal directly addresses pressing research needs that will enable the economical use of these resources.

\section{Technical Objectives}

The technical objectives of this work for Fiscal Year (FY) 2009 are shown below.

- Development of the technology to load polyoxometalate $(\mathrm{POM})$ catalysts into membranes selectively
- Formation of microporous membranes

- Characterization of catalytic activity

Results and Accomplishments

FY 2009 was the third and final year of this project. To assure the completion of the desired workscope, a critical analysis of the transport mechanism of contaminants through the catalyst-containing membranes was performed. To this point, the project has been conducted with the goal of using sulfonated poly(ether ether ketone) (SPEEK) membranes as the substrate for loading of the catalyst. Although this is an attractive route, there were concerns that the amount of catalyst loaded into inaccessible regions of the membrane would be excessive and that using a microporous membrane could potentially be attractive in maintaining a high level of catalyst activity. To address the potential issue of inactive catalyst, microporous membranes based on polyimides were formed and delivered to the University of Toledo.

\section{Develop the technology to load} polyoxometalate catalysts into membranes selectively. SPEEK membranes are phaseseparated materials in which the polymer selfsegregates into hydrophilic and hydrophobic domains. Loading of the membrane with catalyst into these dense (non-porous) materials is performed by including the POM catalyst into the casting solution, followed by conventional membrane casting. A disadvantage of this procedure is that significant quantities of POM will reside in the hydrophobic domains, which is expected to be inaccessible to the selected penetrants. This serves to reduce the overall activity of the catalytic membrane. To rectify this behavior, a novel pervaporation method was investigated to selectively load the hydrophilic channels without loss of catalyst into the hydrophobic domains.

Pervaporation is a membrane separation technique in which solubility of the permeant within the membrane provides the selectivity. The feed does not need to be pressurized; however the feed side of the membrane is operated at subambient pressure to assure removal of permeant. Thus, permeants undergo a phase change from a liquid to a vapor at the 
surface of the membrane permeate side. The water will diffuse readily, especially under pervaporation conditions, through the hydrophilic domains of phase separated membranes, such as Nafion and SPEEK, while penetrating minimally into the hydrophobic domains. Membranes were initially conditioned through the pervaporation of pure water to a steady-state flux (Fig. 1). Replacement of the feed with a POM-water solution was expected to lead to penetration of the POM into the

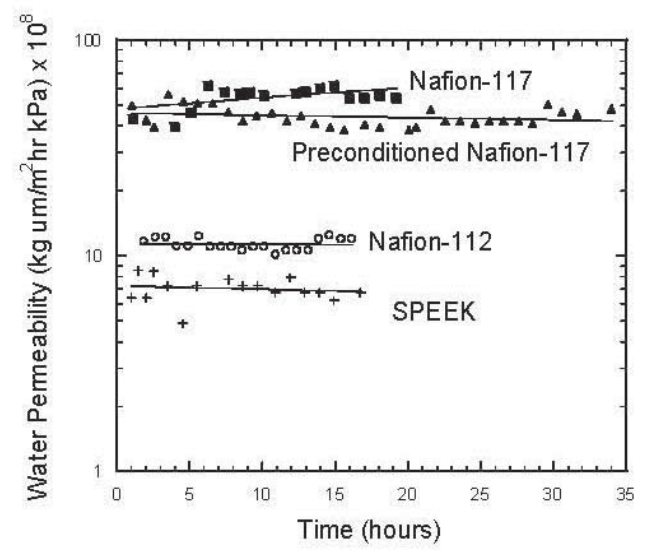

FIGURE 1. Water permeability data through selected membranes.

hydrophilic channels. Active transport of POM through the membrane will not occur since it cannot vaporize off of the membrane backside; however, the POMs are expected to be carried into the membrane by the active transport of water. Pervaporation experiments were conducted over a period of time and water was removed from the feed supply. Membranes studied using this new method include Nafion$112 \AA$, Nafion-117®, acid preconditioned Nafion-117®, and SPEEK. Materials have been sent to the university collaborator for characterization.

Formation of microporous membranes. Microporous membranes based on polyimides were formed using a phase inversion technique. Standard solution casting involves removal of solvent by evaporation to yield a thin dense film. Porosity can be introduced into the same solution-cast membrane by immersion of the wet membrane into a solvent for which the solvent is miscible but the polymer is not. Polyimide phase inverted membranes were formed from N,Ndimethylacetamide and the inversion solvent was water. Membranes formed using this technique were dried and delivered to the university collaborator for characterization. The advantage of the phase inversion technique is that pores can be introduced into the polymer into which catalyst can be placed. Porous membranes generally have high permeabilities as compared to dense structures. Thus, these new materials may offer the potential for high-performing materials.

Characterization of catalytic activity. Previous efforts have identified co-solvents and process conditions for the esterification of acetic acid with 1-octanol yielding extractable 1octylacetate. Partitioning coefficients for acetic acid between water and 1-octanol phases were measured at 0.486 and 0.482 at $25^{\circ} \mathrm{C}$ and $60^{\circ} \mathrm{C}$, respectively. Additional work demonstrated that $60 \%$ conversion could be achieved in approximately 15 hours using $\mathrm{H}_{3} \mathrm{PW}_{12} \mathrm{O}_{40}$. Furthermore, 30 hours were required to complete conversion of the acetic acid to the acetate. $\mathrm{H}_{4} \mathrm{PV}_{2} \mathrm{~W}_{10} \mathrm{O}_{40}$, a more acidic polyoxometalate catalyst, was more active because the $60 \%$ conversion mark was reached in approximately 60 hours.

Catalyst activity between these two POMs was characterized to 0.840 grams of acetic acid converted per gram of catalyst $(\mathrm{g} / \mathrm{g})$ for the $\mathrm{H}_{3} \mathrm{PW}_{12} \mathrm{O}_{40}$ and $0.632 \mathrm{~g} / \mathrm{g}$ for the $\mathrm{H}_{4} \mathrm{PV}_{2} \mathrm{~W}_{10} \mathrm{O}_{40}$. The difference in activity was surprising because the stronger acid was less active. In a comparison, a non-POM commercial solid acid catalyst, Amberlyst-15, was obtained. Activity for this material was measured at $1.208 \mathrm{~g} / \mathrm{g}$, which is substantially more active that either POM.

In a final experiment, catalyst durability was studied. In this experiment, catalyst materials were recycled to determine if there was any drop off in performance. Both POM materials showed significant losses in performance over time. For example, the $\mathrm{H}_{4} \mathrm{PV}_{2} \mathrm{~W}_{10} \mathrm{O}_{40}$ catalyst lost a majority of its activity (Fig. 2) as did $\mathrm{H}_{3} \mathrm{PW}_{12} \mathrm{O}_{40}$. Amberlyst-15 did not show any appreciable reduction in performance. Using this 
and the activity data, the POMs appear to be inferior catalyst materials for this application.

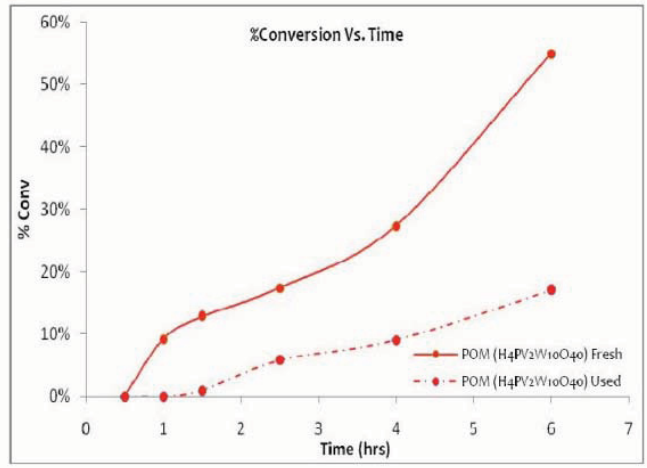

FIGURE 2. Performance of POM catalyst with respect to time.

\section{Summary and Conclusions}

In this project, a process for the removal of enzymatic poisons from biomass hydrolysate was investigated. The concept involved the use of a membrane mediated liquid-liquid extractor to draw organic acids from an aqueous feed stream. The solvent systems and catalyst were identified for the esterification of acetic acid with 1-octanol. Furthermore, methods for developing catalytic membrane materials were developed. Polyoxometalate catalysts were found to have low activity and poor durability in which they were reduced and deactivated during repeated use. However, a solid acid catalyst, Amberlyst15 , was found to be highly active and resisted deactivation. The results of this project suggest that catalytic and separation systems based on membranes to address organic acid contamination is a valid concept and plans are in place for the INL team to continue this work in collaboration with the University of Toledo team that will lead to the further development of this technology. 


\section{Advanced Predictive Condition Monitoring and Control for Modern Energy Systems: Gasification-based Processes}

Humberto E. Garcia, Wen-Chiao Lin, Lee Shunn, Bing Liu, and Larry Baxter (BYU)

Online modeling, monitoring, and controlling of the coal gasification process EI102

Our project aims at modeling, monitoring, and controlling the coal gasification process in a more rigorous and comprehensive manner. The main goal of this project is twofold: (1) develop online predictive condition monitoring and control (CMC) technologies to estimate equipment conditions and regulate the total system, including load following and feed changes, and (2) develop algorithms and software code for the modeling and simulation of entrained-flow slagging gasifiers. Consequently, our technical approach (illustrated in Fig. 1) is divided into completing two main groups of tasks. The first group includes tasks for modeling and simulation of entrained-flow gasifiers, as illustrated in Figure 2. The integrated gasifier model is necessary to produce (simulated) data needed to synthesize and evaluate the proposed predictive $\mathrm{CMC}$ algorithms, with subsequent validation on actual plant data. The second group, also illustrated in Figure 2, includes tasks for development of CMC capabilities to achieve the predictive operational performance proposed. Monitoring algorithms are developed to estimate gasifier operating conditions including the remaining useful life of the gasifier refractory, while control algorithms are developed to balance the syngas production rate and the degradation of the gasifier refractory. The developed monitoring and control algorithms will optimize gasifier maintenance and operations.

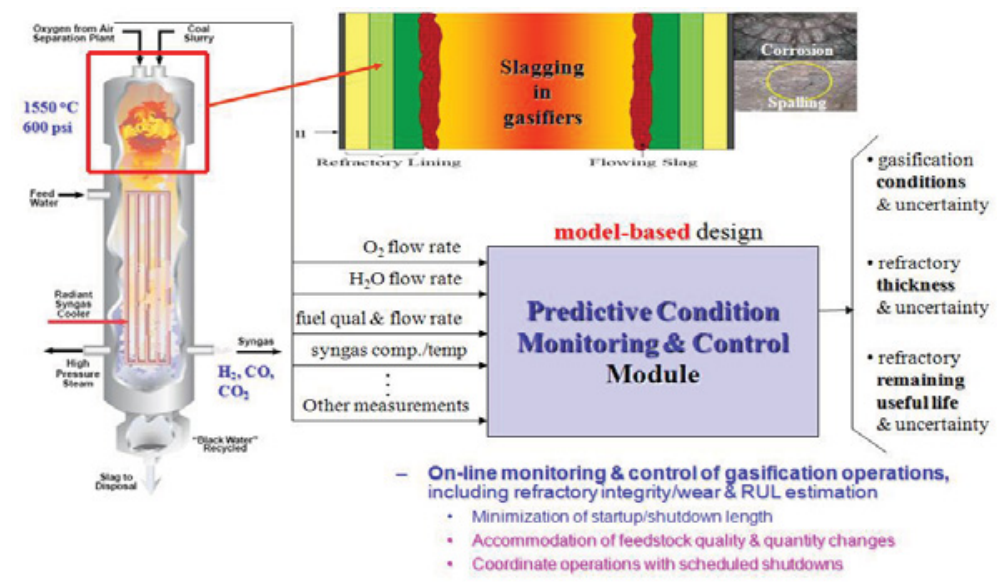

FIGURE 1. General description of the project's condition monitoring and control approach.

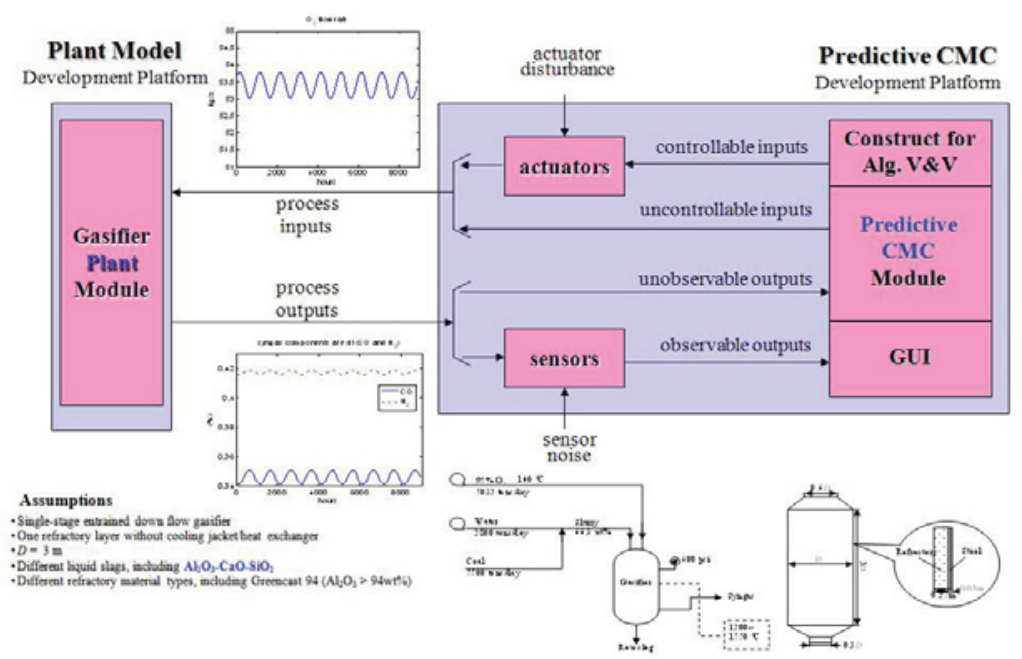

FIGURE 2. Development verification and validation (V\&V) platform. 


\section{Technical Objectives}

Technical objectives for Fiscal Year (FY) 2009 were as follows:

- Complete and verify model of entrainedflow slagging gasifiers

- Model kinetic phenomena

- Model fluid dynamics

- Model refractory degradation

- Complete architecture and development of predictive CMC algorithms

- Monitor and control architecture

- Monitor and control algorithms.

This third and last year is aimed at completing and verifying a comprehensive model for slagging, entrained-flow, coal gasifiers and developing methods for predictive CMC of gasifier operations. The gasifier model was divided into four submodels: (1) gasification model, (2) wall model, (3) slag model, and (4) refractory wear model. The integrated program is expected to produce data needed to synthesize and evaluate the proposed predictive CMC algorithms. Similarly, software infrastructure was developed to host the predictive CMC unit.

\section{Results and Accomplishments}

During FY 2009, the following tasks were accomplished:

Task 1: Complete and verify gasification model package

A generalized computer model for entrained-flow coal gasification (EFCG) has been developed and verified. The EFCG model provides estimates of gasifier performance that are suitable for monitoring and control of pilot and commercial gasification systems. It also provides reasonably detailed estimates of gas phase, particle, and wall properties, including deposition and slag formation, chemical dissolution (corrosion), and mechanical fracture, spalling, and erosion. The model is designed in unified modeling language (UML) and written primarily in $\mathrm{C}++$ according to object-oriented design principles.
The model describes the gas-phase

composition, temperature, and velocity in an Eulerian framework with one independent spatial dimension and as a function of time. The flow field is more accurately described as axisymmetric and radially invariant (no gradients in either the tangential or radial directions) than one-dimensional. That is, velocities are defined in all three dimensions, but there are no gradients in velocity in the tangential or radial directions. Thus, flows expand from a central inlet and contract to a central exit and may swirl, but the only dimension that shows variation in the expanding, contracting, or swirling flow is the axial dimension. Figure 3 illustrates the model's simplified representation of a down-fired EFCG reactor. $\mathrm{N}$ computational nodes are used to compute the reaction's evolution in the primary flow direction. The nodal colors in Figure 3 roughly correspond to temperatures in a typical gasifier - beginning with cool inlets, the volatiles combine with oxygen to produce hightemperatures, then endothermic gasification reactions and heat-loss from the vessel lead to decreasing temperatures at the outlet.

The model also describes coal particle temperatures, compositions, and trajectories within the gaseous flow field. The particle trajectories are three-dimensional and depend on residence time and Eulerian time. A single particle trajectory is indicated by the dotted line in Figure 3. A typical simulation would include multiple trajectories representing different particle sizes, starting locations, and/or particle types.

Finally, the model includes a twodimensional, time-dependent Eulerian wall model. As shown in Figure 3 (next page), $M$ wall nodes are allocated at each of the $\mathrm{N}$ axial positions. The coloration of the wall nodes represent typical temperature variation within the wall, with the inside being the hottest and a monotonic (but non-linear) temperature decrease with radial distance. The inside boundary of the wall is located at the edge of the deposit or slag layer, and the wall extends through the ceramic refractory liner to the outer edge of the metallic containment vessel. The wall model describes deposit accumulation and physiochemical 


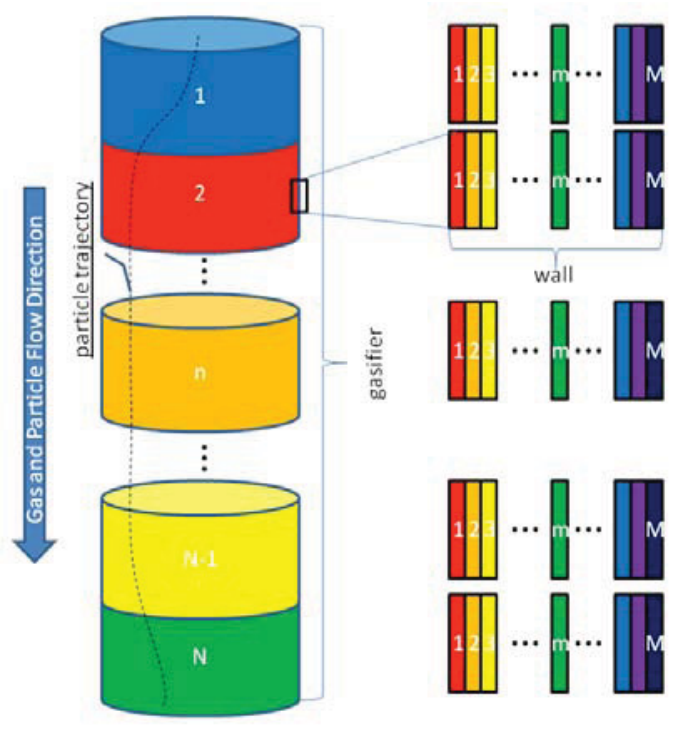

FIGURE 3. Schematic representation of the EFCG model domain.

interactions with the gasifier liner, including its dissolution and spalling. Three main types of input define the reactor operation: (1) time of operation, (2) reactor design (size, materials, etc.), and (3) reactor operating conditions (inlet flow rates, coal type, oxygen purity, etc.). Material properties and their dependence on temperature and pressure are numerically estimated from database information.

The EFCG model comprises gas, particle, and wall submodels. These are interdependent and must be solved by iteration. The gas submodel estimates particle mass source terms and wall conditions and calculates the gas-field properties such as velocity, temperature, composition, and turbulence parameters. The

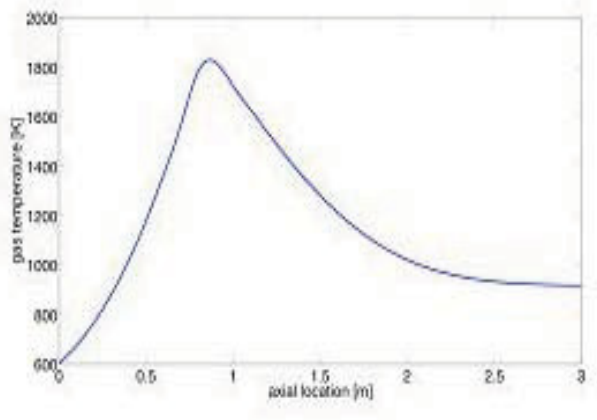

particle submodel estimates particle mass loss, heat transfer, temperature, trajectories, and related information using the results from the gas model. These results are used to update the gas source terms and Eulerian representations of the Lagrangian particle properties. The particle model also provides information required to estimate local deposition rates on the reactor wall. The wall model uses results from both the gas and particle models to estimate temperature, composition, dissolution rate, and fracture characteristics as a function of axial and radial position in the wall. The wall model also estimates surface slag flow rate and emissivity. Iterative solution of these three models continues until simultaneous convergence is achieved.

The entire model is advanced in time at intervals specified by the user. At each time step, all components of the model are exercised. However, only the wall submodel includes transient terms. The gas and particle submodels assume pseudo-steady operation, meaning that transport equations involve average values of velocity, temperature, and composition, with instantaneous values represented only in the mean as root-mean-square turbulent fluctuations. These steady-state properties depend on the (transient) wall conditions such as emissivity, temperature, and (to a much lesser extent) slag flow rate. The wall deposit thickness is often trivial compared to the overall reactor dimensions, so the average gas velocities exhibit negligible dependence on the deposit thickness. Typical gas phase temperature and composition profiles from the EFCG model are shown in Figure 4, with corresponding distribution of wall temperatures shown in Figure 5.

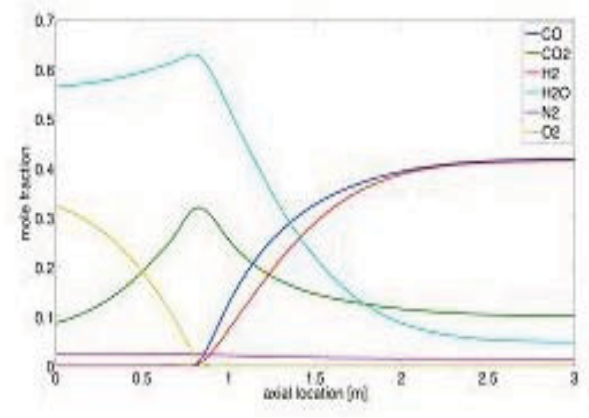

FIGURE 4. Gas phase temperature (left) and chemical composition (right) from the EFCG model at the baseline operating conditions. 


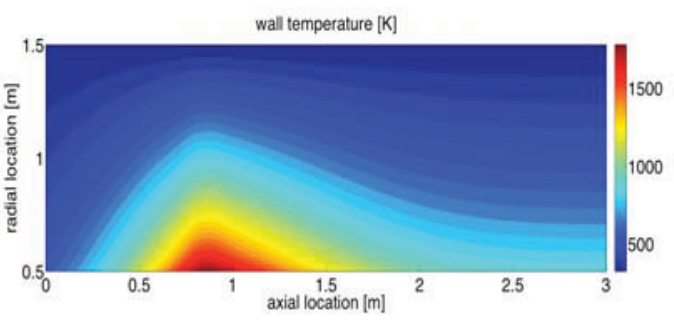

FIGURE 5. Wall temperature profile predicted by the EFCG model at the baseline operating conditions.

Auxiliary models for anomalous or "upset" operating conditions have been developed to aid the development and testing of monitoring and control algorithms for EFCG systems. These disturbances are turned on or off in the computer model by passing various parameters to the EFCG code that govern the effectuation and magnitude of each anomaly condition. The specific disturbances that were implemented for this work include an upset in coal feedstock properties, an undetected irregularity in the coal/water slurry flow rate, and a random spalling event in the refractory liner. The individual effect of each of these disturbance conditions on the gas phase temperature is depicted in Figure 6.

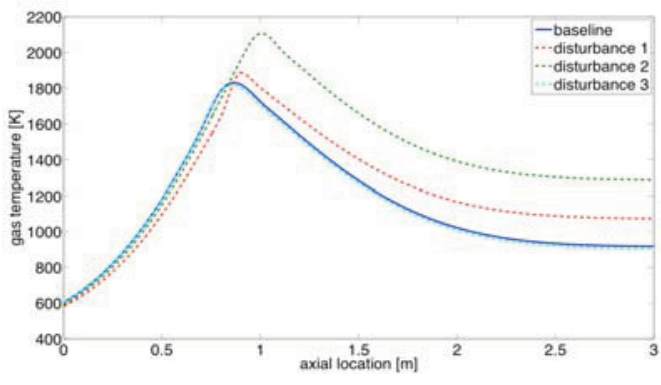

FIGURE 6. Gas temperature 30 minutes after the initiation of a disturbance: "disturbance 1" is a change from Illinois \#6 to Kentucky \#11 coal, "disturbance 2" is a 20\% decrease in the coal slurry flow, and "disturbance 3" is the spallation of $5 \mathrm{~cm}$ of refractory.

Task 2: Develop and evaluate predictive CMC algorithms

The main accomplishment for this year was the development of an adaptive control algorithm for balancing competing requirements for the gasifier. A conceptual diagram of the algorithm is shown in Figure 7.

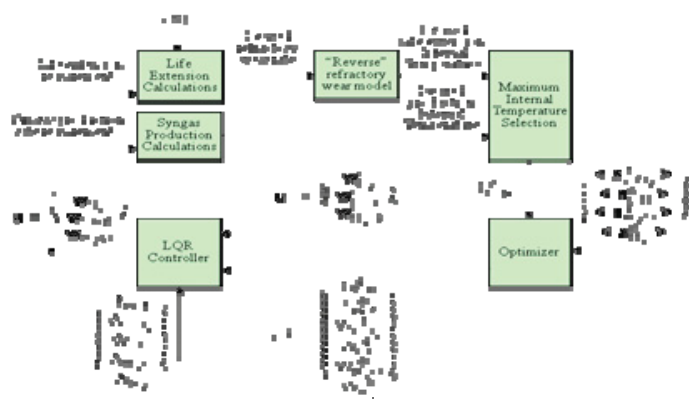

FIGURE 7. Adaptive control algorithm.

In this architecture, the competing requirements for the gasifier are the life extension and syngas production rate requirements. The life extension requirement is determined by the time until the next scheduled maintenance. This requirement and the estimation on the remaining useful life of the gasifier refractory determine the desired refractory wear rate. A reverse refractory wear model determines the desired maximum internal temperature of the refractory for the life extension requirement. For the syngas production rate requirement, it is assumed that the syngas production should be pushed as high as possible. Hence, the desired maximum internal temperature of the refractory is set to a reasonably high number for this requirement. The desired maximum internal temperature of the refractory for the optimizer to calculate the control set points is selected from those for the above two requirements. Specifically, if the life extension is emphasized, the desired maximum internal temperature of the refractory for life extension requirement is chosen. Otherwise, the desired maximum internal temperature of the refractory for syngas production requirement is chosen.

An optimizer is designed to calculate the optimal control inputs (i.e., flow rates of the oxidizer, water, and coal) using a model of the gasification model obtained from a linear regression process. In particular, the optimizer takes as inputs the desired maximum internal temperature of the refractory and the desired upper and lower bounds of the compositions of the major syngas components (i.e., $\mathrm{CO}, \mathrm{CO}_{2}, \mathrm{H}_{2}$, $\mathrm{H}_{2} \mathrm{O}$ ). The optimizer calculates the control inputs 
that maximize the flow rates of the major syngas components subject to the following:

1. The internal temperature of the refractory does not exceed the desired maximum.

2. The flow rates of the inputs are within boundaries for the gasification process.

3. The compositions of the major syngas components are within desired upper and lower bounds.

The optimizer employs a nonlinear programming algorithm to carry out the optimization. Once the optimal control inputs are calculated, the set points for the system states (i.e., maximum internal temperature of the refractory, temperature at gasifier exit, flow rates of $\mathrm{CO}, \mathrm{CO}_{2}, \mathrm{H}_{2}$, and $\mathrm{H}_{2} \mathrm{O}$ ) can also be calculated. The gasification model is then linearized at the set points calculated from the optimizer. An LQR controller is then designed based on this linearized model to regulate the control inputs so that the gasfier operates around the calculated set points.

As an example, consider a gasification operation of time length 60 seconds divided into three 20 -second time segments as follows. In the first 20-second time segment, the operation requires maximum syngas production rates without considering the life extension of the gasifier. In the second 20-second time segment, the operation takes into account the life extension requirements. Finally, in the last 20second time segment, the operation requires maximum syngas production rates without considering the life extension of the gasifier (as in the first 20 seconds). Suppose the desired maximum internal temperatures of the refractory for maximum syngas production and for life extension are $2200 \mathrm{~K}$ and $1780 \mathrm{~K}$, respectively. The optimizer calculates the optimal inputs and the corresponding set points of the system states at the beginning of each time segment. The LQR controller (augmented with an integral control) regulates the system around these set points to reject disturbances. Simulations of the gasification process are conducted using a regression model learned by data obtained from extensive simulations of the gasification model. Moreover, it is assumed that step disturbances in the coal flow rate are present during the latter half of each of the 20-second time segments considered. The step disturbance is assumed to be a $15 \mathrm{~kg} / \mathrm{sec}$ increase in the coal flow input (this is roughly $25 \%$ of the coal flow input). In the simulation results that follow, it is assumed that the gasifier reacts fast enough to the changes in the optimal inputs so that each time segment begins with the desired set points. Assuming no LQR control, Figure 8 shows the maximum internal temperature of the refractory as a function of time, while Figures 9 and 10 show the production rates of the syngas and its composition, respectively.

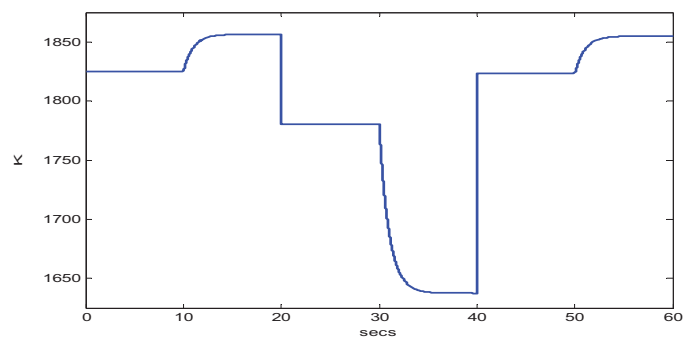

FIGURE 8. Maximum internal temperature of refractory without control.

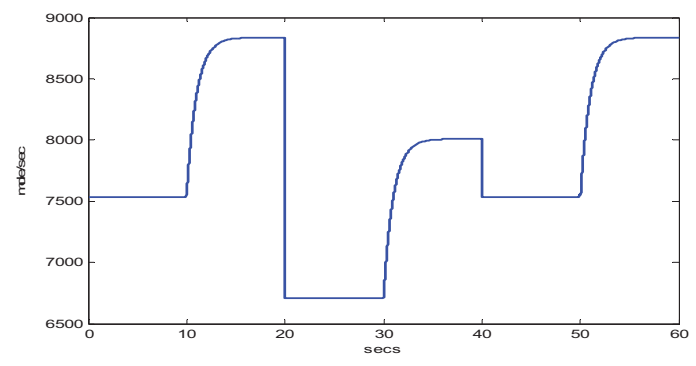

FIGURE 9. Syngas production rate without control.

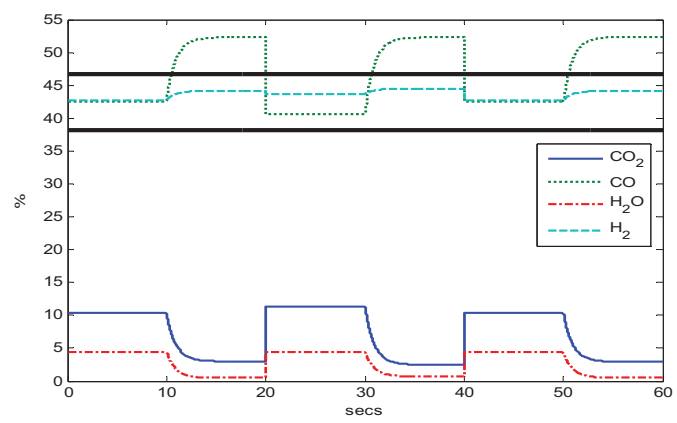

FIGURE 10. Syngas composition without control. 
Note that without the LQR control, the maximum internal refractory temperature, syngas production rate, and syngas composition are not able to be maintained at the desired level calculated by the optimizer as the step disturbances appear during the latter half of each time segment. On the other hand, Figures 11, 12, and13 plot the time progress of these same parameters but when the developed LQR control approach is present.

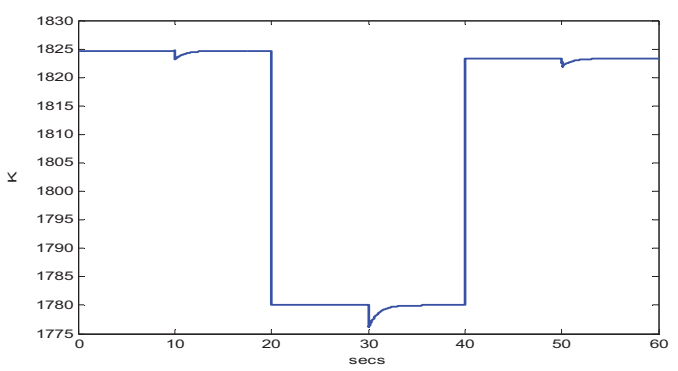

FIGURE 11. Maximum internal temperature of refractory with control.

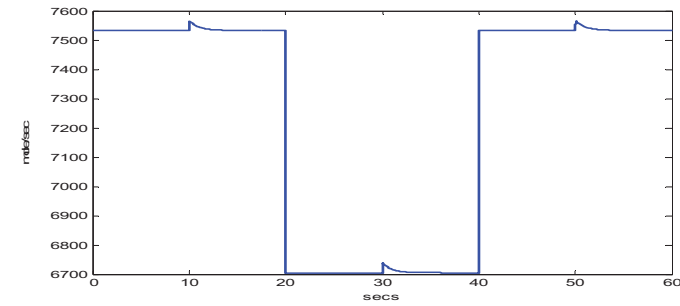

FIGURE 12. Syngas production rate with control.

Note that, in accordance with the requirements, the maximum internal temperatures of the refractory are higher during the first and third hour, while the temperature is lower for the second. Similarly, the production rate of the syngas is higher during the first and third hour, while the rate is lower for the second. The syngas composition is kept almost the same throughout. To illustrate, the thick black horizontal lines indicate the required upper and lower bounds of the percentage of $\mathrm{CO}$ production given to the optimizer. Despite the disturbances and changing requirements for the maximum internal refractory temperature, the syngas production rate remains with the required boundaries. Note that the LQR control of the system keeps the effects of the step disturbances, which occur during the latter half of each hour, small.

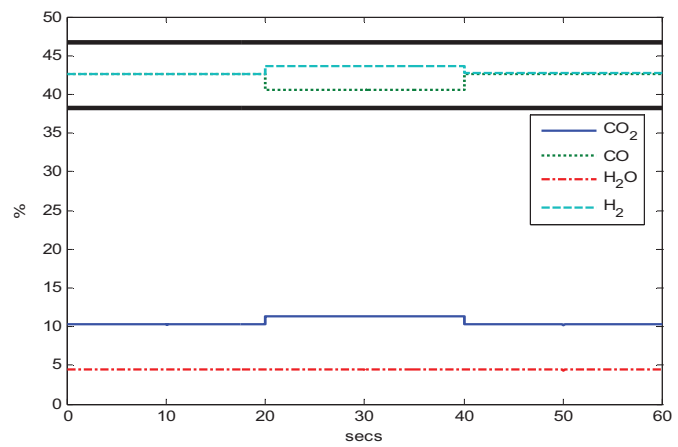

FIGURE 13. Syngas composition with control.

As another example, we consider the effect of a change in feedstock on gasifier operation with and without compensating controls. Suppose that the gasifier coal input is disturbed by a sudden switch in coal type. Within a window of 1 second, simulations using the developed coal gasifier model were performed assuming that the mentioned disturbance enters at 0.5 seconds. The syngas production rate and syngas compositions are shown in Figures 14 and 15 (next page), respectively, for the case when there is no control. Notice that without control, these process variables are not held at the desired set points. For example, the syngas composition for $\mathrm{CO}$ becomes lower than the desired value after the coal disturbance enters.

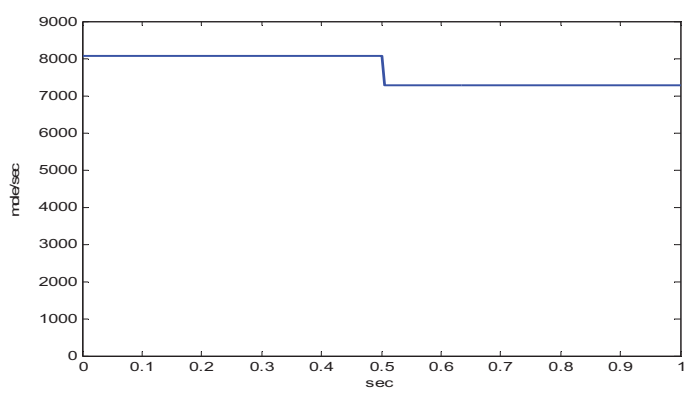

FIGURE 14. Syngas production rate without control when coal type changes. 


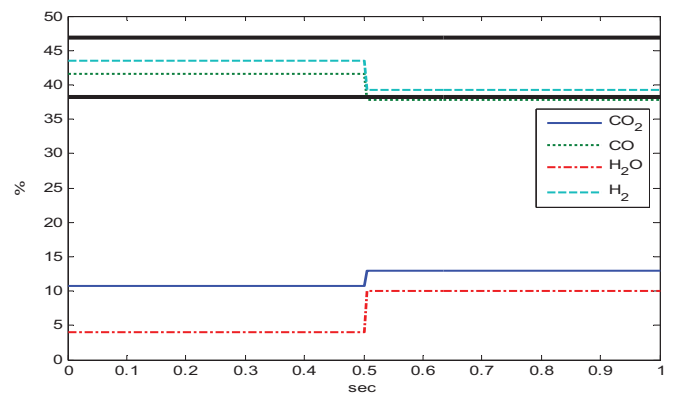

FIGURE 15. Syngas composition without control when coal type changes.

On the other hand, Figures 16 and 17 show the simulation results when the synthesized control approach is present. With this control, both the syngas production rate and syngas compositions are kept at the desired set points.

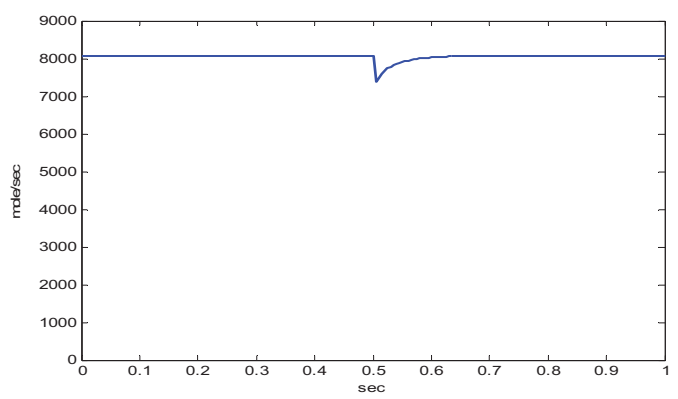

FIGURE 16. Syngas production rate with control when coal type changes.

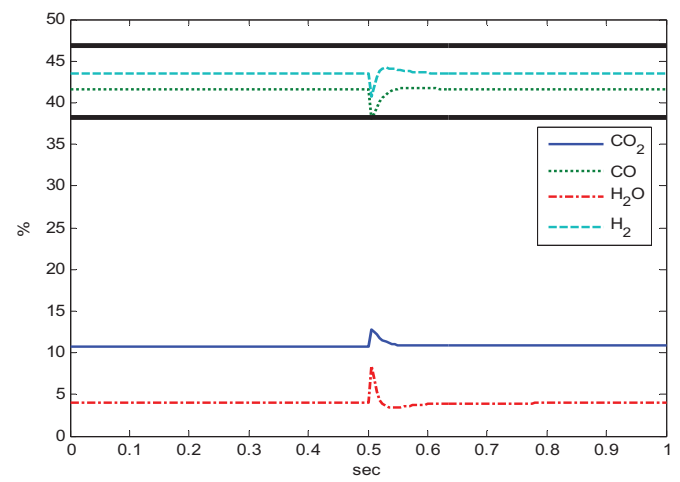

FIGURE 17. Syngas composition with control when coal type changes.

\section{Summary and Conclusions}

This project has delivered the following main capabilities:
- A software package that can simulate the whole coal gasification process, which includes, but is not limited to, gasification reactions, heat and mass transfer, ash deposition, chemical and mechanical refractory wear, gasifier design, and process flowcharts that are only partially available in most current gasification packages.

- $\mathrm{CMC}$ algorithms that allow meeting competitive operational objects; i.e., syngas production and refractory life extension.

- A plant-CMC integrated development platform that can be applied to monitor and control other industrial processes where predictive CMC technologies are needed.

- Personnel involved in this project have become knowledgeable and aware of gasification processes, challenges, and technologies.

Entrained-flow gasifiers are most often operated at high temperatures where molten slag is formed. Because of harsh operating conditions, direct online measurements are difficult and still a challenge. No acceptable model or approach exists to describe this process in addition to the difficulty of analyzing refractory wear caused by the harsh operating environment. Even though our models have yet to be refined and tested in industry, the developed package offers a unique function to comprehensively simulate and evaluate an industrial coal gasification process. Incorporation of the code within predictive CMC algorithms will also be a unique asset compared with publicly available gasification research. What we have developed can not only quantitatively and qualitatively describe the gasification process and estimate refractory wear, but also help set up an online predictive $\mathrm{CMC}$ module to prolong operating period and minimize operations cost.

Additionally, many of the technologies developed under this effort are transportable to other INL/Department of Energy programs. For example, the computer models that are being developed under this effort can be incorporated with other process modeling capabilities also being developed at INL under different projects. Similarly, the developed online predictive CMC 
algorithms can be used in other industries. These predictive capabilities with simplified refractory wear calculations can be used in actual industrial processing units. This benefits the Department of Energy and the nation by improving the reliability, availability, maintainability, and safety of energy systems to which our developed capabilities may be applied. 


\section{CFD-Based Simulation Capability of Fischer-Tropsch Reactors and Process Equipment \\ D. Guillen, A. Gribik, T. Grimmett, J. Shelley, D. Wendt, INL; S. Antal, M. Podowski, E. Tselishcheva, Rensselaer Polytechnic Institute \\ Development of CMFD-based mechanistic modeling and simulation capabilities for Fischer Tropsch reactors}

EI104

The Idaho National Laboratory (INL) Hybrid Energy Systems Testing (HYTEST) Laboratory is being established to develop and test hybrid energy systems in order to safeguard U.S. Energy Security by reducing dependence on foreign petroleum. This project is part of an integrated suite of interdisciplinary projects supporting the Energy Security Initiative that includes biomass gasification, catalysis research, geophysics, instrumentation and controls, plant modeling, emissions and economics studies, and computational fluid dynamics (CFD). Advances in process development, control, and modeling are the unifying vision for Hybrid Energy Systems. INL's contribution to energy security will hinge on the ability to transition ideas to models to working prototypes in order to anticipate and solve tomorrow's problems.

The purpose of this work is to help industry understand and improve the complex physical processes occurring in the slurry bubble column reactor (SBCR) used to produce synthetic fuels. The model we are developing can be used as a numerical testbed to study custom problems that concern industry and gain a better understanding of the mechanisms that govern reactor performance. To date, much of the understanding of these types of reactors has been obtained by limited studies using correlations that are applicable to operating conditions and geometries tested. It is our goal to develop a fundamental, mechanistic-based model that is more broadly applicable and can scale to prototypic sizes and operating conditions.

\section{Techinical Objectives}

The objective of this research project is to further develop and validate a numerical tool to analyze reacting gas-liquid-solid flows. Progress toward meeting this objective includes: (1) developing, implementing, and validating interfacial momentum transfer submodels to accurately describe the hydrodynamics in the SBCR, and (2) incorporating mass transfer, heat transfer, and reaction kinetics submodels to simulate the chemical reaction process.

\section{Results and Accomplishments}

In Fiscal Years (FY) 2007-2009, our team has developed and demonstrated a robust methodology to couple reaction kinetics and mass transfer into multiphase CFD simulations for energy process applications. The modeling tools have the capability to assess and visualize fluid flow, chemical reactions, mass transport phenomena and heat transfer. Chemical reaction submodels tailored to the Fischer Tropsch (FT) process have been developed. The platform for this development is the NPHASE-CMFD computer code provided by our research partner, Rensselaer Polytechnic Institute, and installed on the INL high-performance computing enclave. NPHASE-CMFD is a computational multiphase fluid dynamics (CMFD) code for the prediction of turbulent, multifield flows with mass, momentum, and energy transfer.

To date, the focus of this effort has been developing and validating a multi-field model of high gas holdup churn-turbulent two-phase flows. Hydrodynamic predictions of fully developed flow have been validated using the high quality experimental database for adiabatic air-water flow in a vertical pipe provided by our German collaborators at the TOPFLOW facility at Forschungszentrum Dresden-Rossendorf e.V. Two TOPFLOW datasets with low and high superficial gas velocities were used for model validation. The study confirmed that a minimum of two bubble size groups are necessary to adequately model the hydrodynamics of churnturbulent flows. However, the bubbles were partitioned into four bubble size groups to better match the TOPFLOW data. Figure 1 shows results from this validation study.

A key factor for properly modeling the hydrodynamics of two-phase flows is the formulation of consistent mechanistic closure laws that describe the dominant mass and momentum interactions at the fluid interfaces. Closure relations are needed to reintroduce the information that was lost as a result of ensembleaveraging the conservation equations. These closure laws account for the sub-scale mass and momentum transfer between the various fields and phases. The closure models provide the functional form expected for the interfacial forces. The interfacial momentum transfers considered important for churn-turbulent flows include the interfacial drag force as well as interfacial non-drag forces, such as the lift, wall, 

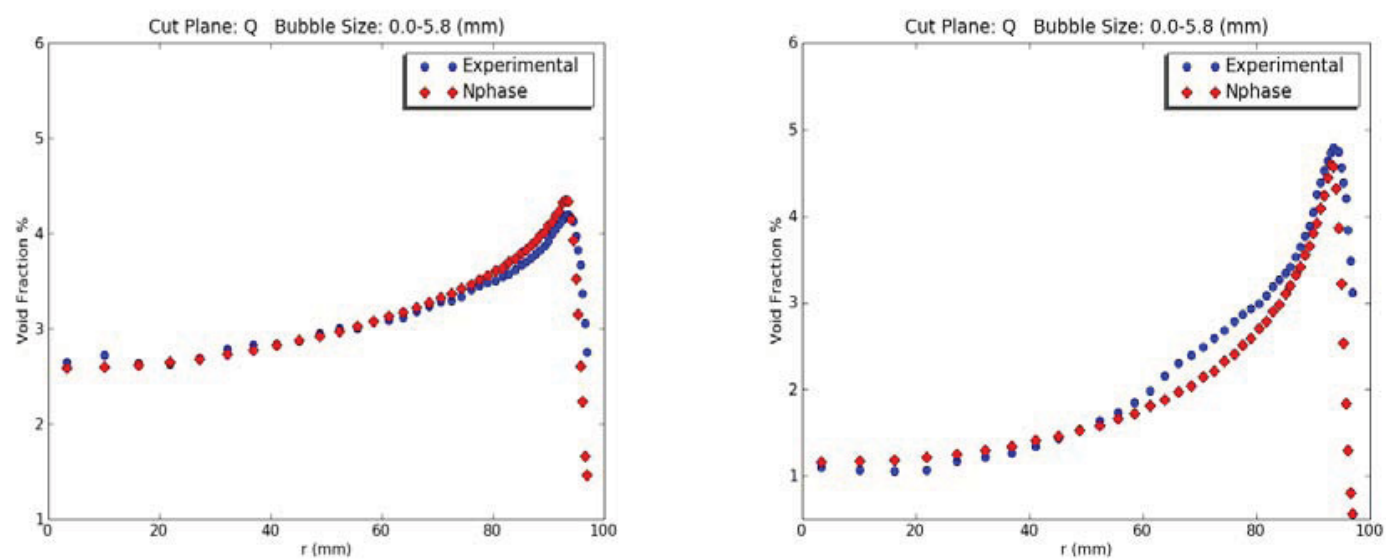

FIGURE 1. Comparison of computed vs. measured radial void fraction distribution for bubbles with diameters less than $5.8 \mathrm{~mm}(\mathrm{a})$ TOPFLOW case $118, j_{g}=0.22 \mathrm{~m} / \mathrm{s}, j_{l}=1.017 \mathrm{~m} / \mathrm{s}$; (b) TOPFLOW case $140, j_{g}=0.53 \mathrm{~m} / \mathrm{s}, j_{l}=1.017 \mathrm{~m} / \mathrm{s}$.

turbulence dispersion, and virtual mass forces. However, local condition-dependent coefficients are expected since the closure models are derived for ideal bubbly flow conditions and applied to churn-turbulent flows. A design optimization software package, Engenious iSight-fd, was used to calibrate the coefficients in the interfacial momentum force submodels.

Bubble breakup and coalescence are important phenomena occurring in churnturbulent flows, especially when attempting to predict flow evolution in a vertical riser. A straightforward, mechanistically based breakup and coalescence model was exercised. Work to accurately predict the developing churn turbulent flow in a vertical pipe was begun in FY 2009.

A chemical reaction model for the FT process has been integrated into the SBCR hydrodynamics in the NPHASE-CMFD code. Reaction kinetics for a cobalt catalyst are based on values reported in the published literature. The initial SBCR model we developed tracked seven species using a four-field model:

- CO reactant in small bubbles, large bubbles, and the bulk fluid

- $\mathrm{H}_{2}$ reactant in small bubbles, large bubbles, and the bulk fluid

- $\mathrm{CH}_{2}$ product in the bulk fluid.
In this four-field model formulation, two of the fields are used to track the gas phase (i.e., small spherical and large slug/cap bubbles), and the other two fields are used for the liquid and the catalyst particles. The absorption and kinetic models, specifically changes in species concentrations, have been incorporated into the mass continuity equation in NPHASE-CMFD. In addition, the model includes heat generation in the bulk fluid due to the exothermic chemical reaction as well as heat removal from a constant temperature heat exchanger. Results of these simulations (Fig. 2, next page) were presented at the 2008 AIChE Conference.

In FY 2009, the model was expanded to represent the product distribution by representative liquid and vapor hydrocarbon (HC) products and water/steam. A property method approach was developed that incorporates vapor-liquid equilibrium. This results in modeling 12 species:

- CO reactant in small bubbles, large bubbles, and the bulk fluid

- $\mathrm{H}_{2}$ reactant in small bubbles, large bubbles, and the bulk fluid

- HC product in small bubbles, large bubbles, and the bulk fluid

- $\mathrm{H}_{2} \mathrm{O}$ product in small bubbles, large bubbles, and the bulk fluid. 

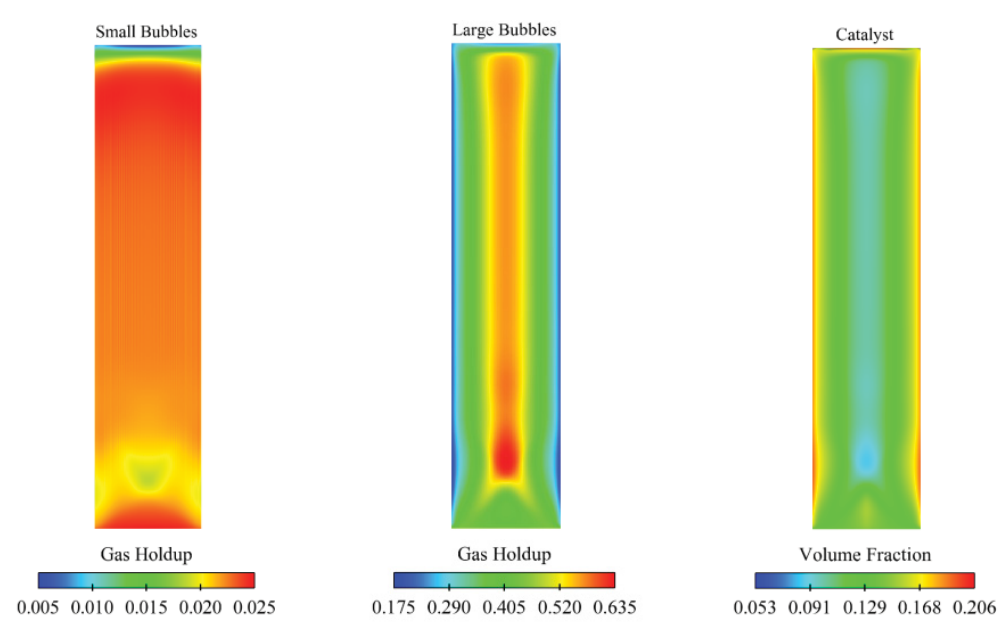

FIGURE 2. Computed volume fraction of small bubbles, large bubbles and catalyst.

\section{Summary and Conclusions}

This research project has developed a CMFD model of the FT process in a SBCR. The model has been exercised for a prototypic SBCR to demonstrate viability of the overall solution scheme. Simulation results include phase distribution, species concentration profiles, and local temperatures within the SBCR. This type of model can provide valuable information for process design, operation, and troubleshooting of FT plants.

Publications and presentations made during FY 2009 include:

1. "Optimization of a Numerical Model of Churn-Turbulent Flow in a Vertical Pipe," Proceedings of the ASME Fluids Engineering Division Summer Meeting, August 2-5, 2009, Vail, CO.
2. "Optimization of a Two-Fluid Hydrodynamic Model of Churn-Turbulent Flow," Proceedings of ICONE17, Paper No. ICONE17-75113, $17^{\text {th }}$ International Conference on Nuclear Engineering, July 12-16, 2009, Brussels, Belgium.

3. "Development And Validation of a Multifield Model of Churn-Turbulent Gas/Liquid Flows," Proceedings of ICONE17, Paper No. ICONE17-75210, $17^{\text {th }}$ International Conference on Nuclear Engineering, July 12-16, 2009, Brussels, Belgium

4. "Incorporation of Reaction Kinetics into a Multiphase, Hydrodynamic Model of a Fischer Tropsch Slurry Bubble Column Reactor," AIChE Annual Meeting, November 16-20, 2008, Philadelphia, PA. 


\title{
Biomass Feedstock Assembly to Gasification Process Computational Interface Development
}

\author{
Kevin L. Kenney, Jared M. Abodeely, David J. Muth, Stephen. P. Gent, Mark Bryden \\ Expanding the feedstock assembly system model's capabilities, simplifying the design process, and \\ developing methods and models for insertion systems
} EI105

Biomass has become one of the nation's most important forms of renewable energy and the only form of renewable energy that can be converted into liquid transportation fuel. For this reason, along with high efficiencies, low emissions, carbon neutrality, and local energy sources, biomass gasification has seen increased interest over the last decade. Several challenges remain for the large-scale implementation of biorefineries for ethanol production including the logistics of getting the biomass from the field to the throat of the conversion reactor and the development of effective and efficient feed handling and insertion systems for the conversion reactor. The objective of this third year of the project is to expand the feedstock assembly system models capabilities through framework integration to improve and simplify the design process as well as develop the methodologies and models for dilute solid phase and dense solid phase insertion systems for various feedstocks into the conversion reactor.

The feedstock assembly system model encompasses the individual operations necessary to move biomass from the field to the throat of the reactor of the biorefinery. Process and equipment decisions made within each operation of the feedstock assembly system determine the intermediate biomass properties which subsequently influence process and equipment decisions further in the system and ultimately the final biomass format and properties received by reactor. One of the tasks addressed by this project enhances system design by providing intelligent and intuitive operational interfaces to interact with the assembly model and provide economic operational analysis and feedstock characteristics.

For the feedstock assembly system to operate and be assessed on an operational basis with such design flexibility as mentioned earlier requires a design and analysis framework. Integration of the modularized feedstock assembly system model into such a framework creates an environment where these operations can exchange operational information as well as intermediate feedstock properties to enhance operational decision-making throughout the system. This dynamic framework also provides the means for complete design and analysis of the entire ethanol conversion process from harvest to ethanol conversion. The modularized feedstock assembly system model, feed handling and insertion models, and process simulation models can be coupled to give operational and final economic data for converting the biomass into ethanol.

The feed handling and insertion system for the conversion reactor remains one of the key challenges for large-scale implementation of biomass gasification systems. The design challenges are due to the strict performance parameters for these systems which include continuous and consistent flow rates for a generally non-flowable material across highpressure differentials. Biomass insertion into the conversion reactor can be broken down into two general methods: dilute solid phase insertion and dense solid phase insertion. Dilute solid phase systems usually suggest a pneumatic conveyance system where a fluid is used to transport the biomass material. Dense solid phase flow generally requires a mechanical system to transport the biomass material. This project works to develop and utilize existing numerical modeling methods to analyze these systems' abilities to meet the feed handling and insertion system criteria listed above. These models must be capable of accurately representing and capturing particle-to-particle interactions, high pressure differentials, and active transport systems.

\section{Technical Objectives}

The main objectives of this work are to develop a framework to create a comprehensive design and analysis environment through model integration and interfaces as well as to model gasifier feed systems - both bulk solid flow, and pneumatic conveying systems. Work over the past year has focused on initial integration of system models and development of methodologies for dense flow and pneumatic feed systems. Continuing and building upon this research, the objectives for the third and final year of work are listed below:

- Enhance framework communication between integrated design and analysis models 
- Develop interfaces for modularized feedstock assembly system

- Develop initial model of dense phase flow feed system

- Construct a pneumatic conveyance system for examining biomass flow properties

- Validate dilute solid phase flow numerical modeling methodologies.

Results and Accomplishments

Framework Integration. The INL feedstock assembly system model provides a highly detailed economic evaluation of a vast set of feedstock supply scenarios based on available technologies for existing local feedstock resources and infrastructures. Optimizing the system requires certain flexibility and understanding of local feedstock supplies, equipment, and infrastructure restrictions. The feedstock assembly system model provides this flexibility to design systems within the local constraints. The feedstock assembly system model represents the unit operations necessary to take the biomass from the field to the throat of the conversion reactor which includes harvest and collection, storage and queuing, preprocessing, and transportation and handling. Each unit operation involves decisions about how the operation will be completed and the equipment necessary to complete the operation. These decisions influence the biomass format and properties and operational decisions later in the system. A modularized system model provides a means to interact with the unit operations individually through integration into a dynamic framework.

Modularization of the feedstock assembly system involves parsing the model into discrete operations based on modifications to the biomass' format, properties, and location through the life of the system. This breaks the model down into the most basic operations and creates a more manageable design environment. Interfaces for each module were developed to handle operational inputs such as equipment selection and biomass format and properties. These inputs are passed back to the model for analysis and return an operational economical evaluation and the resulting biomass format and properties. Information exchange between modularized operations is facilitated through an object-based design and analysis framework. Over the past year a large effort has been put forth in improving this framework to simplify and enhance model integration and communication. The framework facilitates data transfer between integrated modularized operations allowing data aggregation as each operation is analyzed, resulting in a highly detailed economical analysis of the entire feedstock assembly system as well as the individual operations and the final biomass format along with the process intermediate biomass characteristics.

Previous framework development within this project dealt with the integration of a thermochemical process model developed in Aspen Plus and its ability to perform system operations such as opening and closing files and running simulations through the framework. The Aspen Plus module was able to receive inputs through customized code which limits the interaction it can have with other integrated modules within the framework. In a true objectbased framework, each module should be able to receive and send data to other modules within the framework without restriction. Further framework development has enabled Aspen Plus models to be fully integrated similar to any other module. Framework enhancements over the past year facilitate full design and analysis of the entire biomass conversion system from biomass production through ethanol conversion. The feedstock assembly system model in its modularized form along with the feed handling and insertion system model and process simulation model can be coupled within the object-based framework to perform detailed design and analysis. This type of simulation environment displays flexibility within the design process and demonstrates how a single design change can impact the entire system.

Feed Handling and Insertion Systems. The feed handling and insertion system for biomass gasification remains one of the more difficult challenges for the ethanol conversion process. These systems must be able to accommodate various types of biomass and biomass formats, which is challenging due to the feedstock 
flowability characteristics. This project explored current technologies available for feed handling and insertion systems as well as existing numerical methods capable of modeling biomass flowing through these types of systems. Two approaches were taken in investigating possible options for the feed handling and insertion system for biomass gasification: (1) dilute solid phase flow and (2) dense solid phase flow. Dilute solid phase transport systems use pneumatic pressure and vacuum conveying systems to handle the movement of material while solid bulk transport systems use mechanical systems such as hoppers and rotary pumps to meter and transport material across high-pressure differentials.

Prior work examined the use of existing technologies for solid bulk transport systems and the advantages and disadvantages associated with these feed systems. After investigating all available options, a decision for the Fiscal Year (FY) 2009 work was to model a conceptual rotary pump that addresses some of the issues associated with current solid bulk transport systems. The rotary pump is modeled using an Eulerian multiphase CFD solver package. The CFD model is constructed through a series Java scripts; this includes geometry, boundary conditions, solver options, and conversion criteria. This enables the ability to address different design questions outside of the CFD software package. Through the same framework utilized above, an initial interactive design tool has been developed to address the various design questions during the early stages of the design process. The tool allows the designer to modify rotary pump design parameters and operating conditions and initialize the simulation.

During the past year, INL constructed a pneumatic conveyance system to test biomass flowability through various pieces of geometry. The system consists of a VersiFeeder that delivers the biomass to a piping system with multiple bends driven by a vacuum system. Pressure taps were placed along the piping system to monitor pressure drops as the biomass material was pulled through the system along with a high-speed video camera. This provided valuable data for development and validation of the multipahse numerical methods chosen during the FY 2008 work to describe dilute solid phase flows. The approach for modeling these types of flows is an Eulerian-Eulerian multiphase method through a commercial software package. The software's Eulerian-Eulerian multiphase solver capabilities allow user intervention through exterior user-defined functions or subroutines. These subroutines handle the inter-particle and particle-fluid interactions which result in momentum exchange due to the drag of the particles. The model takes into account the unique properties of the biomass material including its size, non-spherical shape, and low bulk density. These attributes result in momentum exchanges between the phases and between the particles.

The developed models and numerical methods were developed for ground corn stover, but the principles governing the model for dilute solid flow of corn stover can be applied to additional biomass feedstocks. Upon completion, the developed models were able to predict general flow characteristics of the biomass entrained in the flow. This includes the reduced air volumetric flow rate due to the presence of the biomass, the rapid deceleration and accumulation of biomass as it travels through a bend in the pneumatic conveyance system, and the reacceleration and re-entrainment of the biomass as it exits a bend.

\section{Summary and Conclusions}

The past year's work has been successful in enhancing the current design and analysis framework being utilized by the INL Bioenergy Team to improve the coupling of disparate models. Through this framework, the entire process for ethanol conversion can be simulated from harvest through the conversion process. Economical information and biomass properties can be traced throughout the system, giving designers the ability to dynamically interact with each of the integrated models. The framework's ability to integrate and couple disparate models makes it applicable for future projects and has generated interest particularly for its ability to interact and couple to process simulation models like Aspen Plus.

Efforts towards establishing methods for feed handling and insertion systems led to the 
investigation of two methods for delivering biomass to the gasifier. Numerical methods for dilute multiphase flow were developed and validated through data obtained from the pneumatic conveyance system constructed at the INL. The principles that guide the numerical modeling method are were developed for ground corn stover but are applicable to other feedstocks. Work completed for solid bulk transport systems includes an initial model of a conceptual rotary pump and an interactive tool for rotary pump design. The design tool facilitated by the design and analysis framework provides a means to dynamically interact with the rotary pump and choose design parameters, operating conditions (boundary conditions), and convergence criteria. The numerical methods developed for this work aided in the completion of a dissertation and the work completed throughout the project's duration has led to several in-progress peer-reviewed journal articles. 


\title{
Generation and Expulsion of Hydrocarbons from Oil Shale
}

\author{
Carl D. Palmer, Earl D. Mattson, Hai Huang
}

An effort to develop more efficient extraction processes and improve the nation's ability to meet future energy demands

EI107

A potential solution to meeting some of the increasing demand for energy is to develop unconventional fuels. In the U.S., shale-oil resources are a substantial portion of this unconventional resource. Oil shales are rocks containing relatively high amounts of insoluble organic matter known as kerogen. As kerogen is heated, it can be converted to petroleum products that can be further upgraded and refined. The world-wide, in-place resource of shale oil is approximately 2.83 trillion barrels (Dyni, 2006). About 2.09 trillion barrels, nearly $74 \%$ of the world's resource, are found in the United States with 1.47 trillion barrels, i.e., $70 \%$ of the U.S.' resource and $52 \%$ of the world's resource, located in the Green River Formation in Colorado, Utah, and Wyoming (Dyni, 2006). Approximately $80 \%$ of the U.S.' oil-shale resource is located on public lands and the BLM will be demanding that energy developers attempt to extract most of the resource rather than high-grading the extraction. Better understanding of processes controlling the generation and expulsion of shale oil can help energy companies develop more efficient extraction processes and improve the nation's ability to meet future energy demands.

We have begun to focus on key geochemical factors that affect the rate of pyrolysis of oil shale and the quality of the generated shale oil. We will identify geochemical signatures that will provide information about the performance of the in-situ retorting processes and on the physical mechanisms governing the expulsion of product from the oil-shale rock and the transport of that product to an extraction well.

\section{Technical Objectives}

- Develop an expression for the rate of pyrolysis that accounts for the effects of temperature and accounts for the role of water

- Develop a better understanding of how confining stresses affect the expulsion of oil from the shale matrix

- Identify "performance signatures" that will permit one to determine the temperature and pressure regimes present in the subsurface

- Incorporate thermogeomechanical effects of the oil shale during heating to understand the stability of fractures and the recovery of product.

\section{Results and Accomplishments}

Thermal Properties of Oil Shale. The literature was reviewed and the thermal properties of oil shale as a function of temperature were estimated. Heat capacity was estimated using Kopp's Law of Additivity where the heat capacity of the oil shale is the weighted sum of the components of the oil's shale. The heat capacity of the oil shale is assumed to be

$$
C_{p}(\text { oil shale })=f(\text { minerals }) * C_{p}(\text { oil Shale })+f(\text { kerogen }) * C_{p}(\text { kerogen })+f(\text { char }) * C_{p}(\text { char })
$$

where $\mathrm{f}$ (minerals), $\mathrm{f}($ kerogen), and $\mathrm{f}($ char) and the mass fractions of minerals, kerogen, and char in the oil shale and the $C_{p}$ are the heat capacities of the components. The heat capacity of the mineral fraction is based on individual mineral values reported in the literature and the mass fractions determined in our hydrous retort tests. The heat capacity of the mineral fraction has a quadratic dependence on temperature with a zero intercept. The heat capacity of char was estimated using the values for coke over the temperature range of $25-700^{\circ} \mathrm{C}$ reported by Hanrot (1994) and follows a general quadratic form. Heat capacity for kerogen was obtained by assuming the values for coal obtained by Hanrot (1994) but shifting the curve upward so that the heat capacity at $20^{\circ} \mathrm{C}$ was $1500 \mathrm{~J} \mathrm{~kg}^{-1} \mathrm{~K}^{-1}$ as suggested by Waples and Waples (2004). The heat capacity of the kerogen between 50 and $400^{\circ} \mathrm{C}$ follows

$C_{p}($ kerogen $)=a+b T+c / T^{2}+d T^{2}$

where $\mathrm{T}$ is the temperature in kelvins. The thermal conductivity $(\lambda)$ of oil shale was taken from Wang et al. (1979) and Nottenburg et al. (1978) and combined with thermal conductivity for limestone (with assumed grade of zero) and fits the general form 
$\lambda=1 /(a+b T+c G)$ where $\mathrm{G}$ is the grade in gallons per ton.

Design and Construct an Axial-Stress Retorting Apparatus. An axial stress retorting apparatus for use in our PARR retorting vessels has been built and tested. Our testing demonstrates that we can produce oil from the samples. Visual observations of the collected oil suggest an improvement of the quality of the oil perhaps from additional catalysis reactions with the porous stainless steel. Because of the small oil shale mass used in these experiments, we cannot assess this hypothesis at this time. Testing on artificially fractured oil shale samples showed a three order-of-magnitude decrease in the fracture permeability with retorting of the oil shale sample.

Design and Testing of Permeameter. A permeameter that will allow for continuous measurement air permeability of oil shale samples and changes in strain under uni-axial stress conditions at elevated temperatures was designed and built. The system has been tested up to $250^{\circ} \mathrm{C}$, i.e., temperatures that are below those at which pyrolysis begins to occur. With a step increase in temperature, we initially observed thermal expansion of the sample followed by consolidation. A walk-in fume hood has been acquired so that experiments can be run to higher temperatures where pyrolysis is occurring. This fume hood will be installed in the laboratory early in Fiscal Year (FY) 2010. The apparatus should allow us to measure the permeability and strain over the temperature range of ambient to $400^{\circ} \mathrm{C}$ and loads from 0 to $11,000 \mathrm{lb}(\sim 6000 \mathrm{psi}$ stress with a typical sample).

\section{Design and Construction of New Retorting} System. We have designed and constructed a new retorting system that will allow us to better simulate in-situ retorting conditions. The new system is open but with a back-pressure that can be set. We are currently considering backpressures in the range of 100-300 psi. The system utilizes our existing 1-liter retort vessels in which the shale will be retorted after purging with Ar. As pressures exceed the set backpressure, the gases will bleed via stainless-steel tube to a condenser which is kept at sub-ambient temperature. The non-condensable fraction will be collected in a tedlar bag for subsequent analysis. We will be conducting retorting experiments in this new system early in the next fiscal year.

Develop Pore-Scale Models of In-Situ Oil Shale Retorting. We have continued to develop a computer code that couples fluid flow, heat transport, and rock deformation/fracturing during the in-situ retorting process. This code supports our study of the evolutions of rock permeability and porosity due to rock deformation and fracturing induced by thermal expansion and fluid pressure buildup and diffusion, which is a critical factor for understanding fluid generation, oil expulsion, and residual oil content in spent oil shale. We successfully developed a rock mechanistic model based on a discrete element method (DEM). The DEM model is able to accurately simulate the deformation/fracturing process due to external and internal stress. We then successfully coupled this DEM model with a fluid-flow model and heat conduction model. The in-situ generation of fluid (mixture of oil and gas) inside the rock and the consequent buildup of fluid pressures acting on the rock matrix change the effective stress of rock matrix and cause rock to deform and eventually generate fractures once the rock deformation exceeds some threshold values (critical strain). The deformation/fracturing of rock undergoing in-situ retorting will, in turn, change the porosity and permeability that govern fluid flow paths inside the rock. Our simulation results qualitatively agree with experimental observation, but more quantitative comparisons between simulation and experimental results need to be performed. Our next improvements to the model will be to incorporate plastic deformation and include the temperature dependence of the mechanical properties.

\section{Presentations and Publications}

1. Huang, H., P. Meakin, A. Malthe-Sorenssen, T. Wood, C. D. Palmer, and E. Mattson, 2008, "Modeling deformation and fracturing of oil shale induced by in-situ fluid generation," Abstract, $28^{\text {th }}$ Oil Shale Symposium, October 13-17, 2008, Colorado School of Mines, in Golden, Colorado. 
2. Mattson, E., and C. D. Palmer, 2008, "Influence of water vapor pressure on oilshale product recovery." Abstract, $28^{\text {th }}$ Oil Shale Symposium, October 13-17, 2008, Colorado School of Mines in Golden, Colorado.

3. Palmer, C. D., E. Mattson, and R. B. Perkins, 2008, "Dissolved constituents in water from hydrous retort experiments," Abstract, $28^{\text {th }}$ Oil Shale Symposium, October 13-17, 2008, Colorado School of Mines in Golden, Colorado.

4. Palmer, C. D., and E. Mattson, 2008. "Oil Shale Research: Geochemistry,” Alberta Research Council, September 28, 2008.

5. Mattson, E. and C. D. Palmer, 2008, "Physical Changes During Oil Shale Retorting," Alberta Research Council, September 28, 2008.

6. Palmer, C. D., and E. Mattson, 2008, "Oil Shale Research: Geochemistry," University of Calgary, September 29, 2008.

7. Mattson, E. and C. D. Palmer, 2008, "Physical Changes During Oil Shale Retorting," University of Calgary, September 29, 2008.

8. Palmer, C.D., 2008, "Unconventional Oil Resources: INL Capabilities and R\&D Challenges," University of Utah, July 11, 2008.

9. Perkins, R. B., G. H. Grathoff, C. D. Palmer, and E. D. Mattson, 2008, "Mineralogical changes in oil shale from hydrous retorting," 2008 Goldschmidt Conference, Vancouver, Canada, July 13-18, 2008, Geochimica et Cosmochimica Acta, 72, 12S (July, 2008): A737.

10. Mattson, E., C. D. Palmer, E. Johnson, H. Huang, and T. Wood, 2009, "Permeability changes of fractured oil shale cores during retorting," Abstract, $29^{\text {th }}$ Oil Shale Symposium, October 19-21, 2009.
11. Huang, H., T. Wood, C. D. Palmer, E. D. Mattson, and Z. Xu, 2009, "Massively parallel modeling of coupled thermal-hydromechanical processes during in-situ shale retorting," Abstract, $29^{\text {th }}$ Oil Shale Symposium, October 19-21, 2009.

\section{Summary and Conclusions}

We have pulled together information from the literature about the thermal properties of oil shale and were able to establish simple equations to describe the heat capacity and thermal conductivity of oil shale a function of temperature and grade. A device was designed and built that allows us to retort oil shale under stress in our 1-L retort vessels. We observe decreases of about three orders of magnitude in the permeability of fractures retorted in this device. We have also constructed a permeameter that allows for continuous measurement of permeability and strain on an oil shale sample at elevated temperatures (ambient to $400^{\circ} \mathrm{C}$ ) and uniaxial stress $(0-6000 \mathrm{psi})$. Tests at temperatures $\leq 250^{\circ} \mathrm{C}$ show thermal expansion of the sample followed by consolidation. A new back-pressure retorting system has been designed and built. This system should provide us with improved simulation of in-situ retorting conditions. We have developed a geomechanics model for oil shale based on the discrete element method. This geomechanics model has then coupled to continuum heat- and fluid-flow equations. Results of our work have been provided in presentations at professional meetings and to small technical groups. In the next fiscal year, we will continue to develop experimental capabilities, build collaborations, and produce peer-reviewed publications. 


\title{
Nuclear Pathways to Energy Security
}

\author{
Craig Cooper, Robert Cherry \\ Building secure, affordable energy systems that operate within the carbon and water constraints imposed \\ by the regional and global environment \\ EI109
}

The purpose of this work is to develop a better understanding of how to build secure, affordable energy systems that operate within the carbon and water constraints imposed by the regional and global environment. The U.S. and the rest of the world are at a crossroads. We do not have the resources for 6.5 billion people to consume energy and dispose of waste in the environment as we have had for much of the past century, and the problem will only get worse as global population grows to a projected 9 billion by 2050 . We have to change our approach to energy and build fundamentally new kinds of systems whose operating parameters and environmental impacts are largely unknown today. Furthermore, these systems have to be optimized to accommodate factors other than cost and security of supply. Energy systems must also accommodate carbon emissions, water demand, other waste emissions, resource availability, land use impacts, and public perception. This project seeks to advance our knowledge and capabilities in this area by conducting systems design studies and analyses of the carbon and water impacts of transportation fuels systems - with a focus on identifying ways nuclear power can mitigate those impacts.

Many analyses propose various ways to improve the security of U.S. energy supplies and/or reduce U.S. carbon emissions in a costeffective manner. Few studies agree, almost none of them use the same basis for assessing capabilities, and none currently factor in the impact of these systems on water resources. This work will quantify the water impacts of energy systems in a rigorous manner and then use this quantification as a basis for contrasting the carbon and water impacts of various strategies for improving U.S. energy security. We anticipate that this work will further demonstrate that nuclear-driven energy systems can cost effectively provide a secure, low-carbon, watersmart source of energy for all market sectors.

\section{Technical Objectives}

This project uses systems modeling techniques to address two nested problems. First, we seek to improve our understanding of the complex links between energy and environmental systems by demonstrating how innovative new sustainable energy systems can be designed on the basis of environmental constraints on greenhouse gas emissions and water availability. Second, within the context of this overarching goal, we will also determine whether nuclear energy can provide cost-effective options for producing secure, lowcarbon transportation fuels from North American feedstocks between 2010 and 2030. Fiscal Year (FY) 2009 technical objectives of the work were to:

- Complete development of initial lifecycle water quantity model for U.S. energy systems in Excel. Use both SEDS and GREET (ANL, lifecycle carbon model) capabilities as a guide for what energy systems to include in the initial model.

- Develop a framework for comparing water quantity, water quality, carbon, security, and other "sustainability" parameters on a consistent basis to facilitate clearer comparisons.

- Begin to transfer framework for model from Excel to Analytica ${ }^{\circledR}$ (systems modeling software) to improve model capabilities.

- Submit 1-2 peer-reviewed publications on lifecycle water impacts. Give 1-2 presentations on energy and water; at least one should be at a national technical conference.

A generalized lifecycle water model has been completed in Excel, though the data sources for the model are preliminary and subject to many caveats. A framework for comparison has been developed using the concept of energy and environmental services and expressed in terms of dollars per unit energy. Different energy technologies have different requirements for carbon, water, security of supply, and other sustainability services and thus can be compared on the basis of the total cost to provide sustainable energy. These sustainability costs will exceed the market price of energy services (e.g. heating, electricity, transportation), but comparing energy options on the basis of these costs will provide a consistent methodology for evaluating different energy options and system configurations. The challenge in doing this comparison is that many of these costs are 
extremely hard to quantify - with much of the error in the calculation being in the value of the parameters. Given this uncertainty and the relatively small added value from building complex calculations around uncertain variables (thereby creating more uncertainty), efforts were directed in achieving greater certainty in the underlying data rather than transferring Excel calculations into more advanced models. We are currently working on manuscripts for two papers. One describes the framework for analysis, and the other will quantify the water implications for different plans for meeting U.S. energy needs in a carbon-constrained economy. These papers will be submitted for publication in FY 2010.

\section{Results and Accomplishments}

This work's primary accomplishment is that it provided a better understanding of how to evaluate the limits of deployment of secure and sustainable energy systems. Such evaluations are difficult, because they must consider a wide range of disparate issues - everything from carbon to oil imports to water impacts and land use. All energy resources are physically limited in some way, with the limit being expressed in terms of the cost of energy services. As resources become more scarce, costs rise- unless governmental subsidies hide the cost as an externality. Thus, physical limits can be expressed as a cost curve with an asymmetrical U shape whereby costs go down with increasing production for a time, but then begin to rise again as the physical limits on those resources are approached.

Provision of energy services impacts a broad range of resources and environmental services. For a given energy technology deployed in a given region, each environmental and/or security service will have a different impact on the integrated cost curve for providing energy to that region's society. Technologies that have lower total costs (e.g., energy + environment + security services) will be cleaner, safer, more secure, and more sustainable. Comparison of these integrated cost curves for sustainable energy can provide insights into which technologies or sets of technologies will best provide clean and secure energy for society. With regard to the hypotheses originally posed, we have made the following advances:

- Hypothesis \#1: We can create a better understanding of the relationship between energy and environmental systems by building systems models that start from environmental constraints and "work backwards" to design new energy systems that are more sustainable.

- Completed an initial, simplified lifecycle water model for major energy resources and developed a consistent methodology for comparing water limitations to other sustainability issues such as carbon, security, resource surety, reliability, land use, and ecosystem impacts.

- Hypothesis \#2: Nuclear energy can provide new options for producing clean, secure transportation fuels from North American feedstocks.

- Currently working on a spreadsheet model that compares nuclear energy with other energy options across a subset of sustainability services that are both important and easier to quantify (climate maintenance, water resource availability, energy security, energy resource surety).

\section{Benefits and New Capabilities}

In addition to the benefits and new capabilities achieved in FY 2008, this work has contributed significantly to the development of new programmatic work with the U.S. Department of Energy (DOE) Climate Change Technology Program (CCTP). In FY 2009 Idaho National Laboratory (INL) received $\$ 365 \mathrm{~K}$ in new funding for three projects: (1) economic analysis of the climate impacts of U.S. energy policies, (2) limits analysis of the limits to deployment of low-carbon technologies, and (3) gap analysis of key research and development (R\&D) needs for developing nations and members of the Major Economies Forum (MEF) in order to meet greenhouse gas (GHG) reduction goals established via the United Nations Framework Convention on Climate Change (UNFCC). 
This work has also contributed significantly to assessing how nuclear reactors can potentially replace low-temperature coal-fired heating for U.S. industrial facilities that are dependent on coal for heat and cannot effectively compete internationally in a carbon-constrained U.S. economy in the absence of new low-carbon heating technologies that do not involve carbon capture and sequestration. Specifically, results from this laboratory-directed research and development (LDRD) provided information that INL researchers used to demonstrate the potential value of nuclear energy to Eastman Chemical Corp., and subsequent work funded by business development funds has led to detailed discussions of how INL research in nuclear energy can help Eastman adapt to a low-carbon economy while remaining competitive internationally.

Information from this LDRD has contributed significantly to the development of a value proposition for nuclear energy integration and helped lay the groundwork for future R\&D efforts within DOE-Office of Nuclear Energy (NE) for investigating new and innovative applications of nuclear energy within hybrid systems.

\section{Summary and Conclusions}

The end result of the work to date is that INL is actively developing a new, programmatically funded capability to describe how environmental limits constrain energy development and how nuclear energy can produce more energy for electricity, transportation fuel, and process heating markets than current systems while staying within those constraints. We have developed a method to compare energy technologies and/or options across a range of sustainability parameters in a comprehensive way and have begun preparing the publications. We expect that INL will provide continued support to DOE-CCTP and envision that this work could lead to establishing a DOE-funded strategic energy analysis group at INL that focuses on the strategic value of nuclear energy to U.S. energy priorities. Creation of such a capability at INL would enable DOE-NE to make stronger value cases for investment in nuclear R\&D and place nuclear on equal footing with fossil energy and renewable energy in the national energy debate. This LDRD will not conduct this programmatic work but does provide the value argument for investment in nuclear-driven systems for secure, sustainable fuels. Integration of the results of this LDRD, with ongoing LDRD investigations into hybrid systems and the Next Generation Nuclear Plant project, can lead to the establishment of this envisioned new capability in strategic energy analysis. 


\title{
Investigation of Fischer Tropsch catalyst deactivation
}

\author{
Lucia M. Petkovic, Daniel M. Ginosar
}

Examining the deactivation kinetics of FT catalysts over a wide range of carbon monoxide and hydrogen concentrations and temperatures

EI110

The Fischer Tropsch (FT) process is a catalytic process to produce hydrocarbon fuels from synthesis gas (a mixture of carbon monoxide and hydrogen). The synthesis gas may be produced from a number of sources including biomass and coal. The products may be upgraded using traditional petroleum refinery operations. In particular, a combination of biomass gasification and FT synthesis is a very promising route to produce renewable transportation fuels and diminish the U.S.' dependence on foreign and dwindling petroleum supplies. The objective of this research project is to study the deactivation kinetics of FT catalysts over a wide range of carbon monoxide and hydrogen concentrations and temperatures. This research work is essential because deactivation rate parameters, which are lacking in the open literature, are necessary to reliably predict and analyze FT reactor behavior (e.g., reactor conversion and exit composition) and in turn produce better designs (e.g., reactor internal configurations, required separations, recycle) of complete biomass- or coal-to-liquid processes. This project will provide substantial improvement to the existing practice of reactor modeling and simulation and will narrow the wide gap usually found when trying to predict reactor behavior based only on reaction rates without considering deactivation.

\section{Technical Objectives}

This project was planned for three years, with Fiscal Year (FY) 2009 as its first year. The technical objectives include FT reaction tests, FT deactivation tests, and catalyst characterization analyses. In FY 2009, the tasks were focused on FT reaction tests on a cobalt catalyst.

\section{Results and Accomplishments}

The catalyst studied was a $0.3 \% \mathrm{Ru} 25 \% \mathrm{Co}$ $\mathrm{La} / \mathrm{Al}_{2} \mathrm{O}_{3}$ catalyst completely synthesized in our lab following an aqueous preparation route suggested by the Brigham Young University Fischer Tropsch Consortium. This catalyst was characterized at different stages of the synthesis protocol to determine the best set of conditions and temperatures for calcination and reduction.
A set of preliminary blank tests were performed to compare the performance of a continuous stirred tank reactor with catalyst basket (CSTR) and a straight tube stainless steel reactor. The results indicated that the CSTR reactor was not appropriate because the reactor material displayed catalytic activity. Thus the straight tube stainless steel reactor was selected for the reaction tests. Cold and hot traps were designed, built, and connected to the straight tube reactor. The reaction tests were conducted under conditions for which not only deactivation but also heat and mass transfer limitations were negligible to avoid masking true catalytic rates. Two hundred and fifty milligrams of pre-reduced and passivated catalyst were mixed with 1 gram of quartz and re-reduced in situ. Experiments to study the effect of hydrogen partial pressure were performed at a constant total flow rate using nitrogen as the balancing gas. The total pressure was always maintained at $300 \mathrm{psi}$. Temperatures studied were $225^{\circ} \mathrm{C}$ and $250^{\circ} \mathrm{C}$.

\section{Summary and Conclusions}

An experimental setup for bulk (up to about 30 grams of solid) catalyst preparation was assembled in IF $603 \mathrm{Lab}$ A-17. This setup allows for catalyst calcination and reduction under controlled temperature ramps and controlled gas flow rates. Experimental analyses to determine reaction rates on cobalt FT catalysts were performed under different sets of reaction conditions.

This project has developed expertise in cobalt FT catalyst synthesis and laboratory-scale study of Fischer Tropsch reactions, which increases the applied science capabilities for studying syngas-to-fuels processes at Idaho National Laboratory. 


\section{Altering Wettability by Chemical Amendments to Improve Gas Production from Tight Sands}

Eric Robertson

Determining feasibility of enhanced gas deliverability from tight (very low permeability) gas sands EI111

The purpose of this work is to determine if chemicals can be designed and used in conjunction with other well completion fluids to enhance gas deliverability from tight (very low permeability) gas sands. This project will test certain chemicals for their effectiveness in altering wettability in gas/water systems. Chemicals will be prescreened for effectiveness using a system of glass capillary tubes to represent rock surfaces. The most effective prescreened chemicals will be used in spontaneous imbibition tests and fluid flow tests using reservoir cores to estimate the wettability change and to evaluate the change of the gas deliverability before and after the chemical treatment. Ultimately, a field pilot is planned to verify lab results.

Tight gas sands hold vast quantities of natural gas but are difficult to produce in many cases because of water encroachment into the near well bore region, which effectively curtails the flow of gas to production wells. This project will attempt to find or design cost-effective chemicals that can be used to modify the wetting state of the near well bore region to lessen the impact of encroaching water on gas flow rates through tight sands.

\section{Technical Objectives}

- Conduct tests on small samples of the field cores to characterize the rock on a pore scale. These tests will include scanning electron microscopy, x-ray diffraction, and thin section analysis.

- $\quad$ Perform the initial screening of potential chemicals using glass capillary tubes.

- $\quad$ Characterize the whole field cores in laboratory by measuring permeability.

- Complete relative permeability corefloods using methane and simulated field brine and selected chemical(s).

\section{Results and Accomplishments}

2009 is the first year of this proposed threeyear project. This LDRD project will test certain chemicals for their effectiveness in increasing gas production from tight sand formations. The selected chemicals can be added to fluids used in drilling and well completions to enhance gas production by altering the near wellbore wettability state. Since the wettability is dependent on mineral composition and pore geometry, the rock was first examined using thin section analysis and scanning electron microscopy (SEM).

From the thin section, SEM, and x-ray diffraction (XRD) analysis, we determined that the Wamsutter tight gas sands contain quartz, rock fragments, feldspar, carbonate (dolomite replacing feldspar), kaolinite, and quartz cement (quartz overgrows).

Analysis of the thin sections shows that porosity of the rock is dominated by the secondary porosities formed from partial feldspar dissolution, micro-porosity from clay minerals and chert. The primary porosity from the intergranular pores is minimal due to quartz overgrowth and lithofragment cementation. The porosity of the core samples ranges from 2 to $13.5 \%$.

Cores from the Wamsutter tight gas reservoir - a tight gas field in the Greater Green River Basin in Wyoming - were donated by BP and were used in the experimentation. The permeabilities of five different field cores were measured using equipment developed and patented by INL researchers for measuring very low gas flow rates. Because of the lack of primary porosity and pore connectivity, the rock permeability for the cores is quite small, ranging from 0.5 microdarcy $(\mu \mathrm{d})$ to $500 \mu \mathrm{d}$. The very low permeabilities of these cores resulted in long times needed to measure this rock characteristic. The measured permeabilities of the cores will be used to select appropriate matching sets (one used as a control and one used with the chemical variable) for relative permeability and imbibition studies.

Four of the five cores showed typical Klinkenberg (gas slippage) effects, i.e., positive linear slope of gas permeability as a function of inverse average flowing pressure. However, the gas permeability of core $68 \mathrm{D}$ decreased with increasing inverse average flowing pressure meaning that this core was highly sensitive to changes in net pressure. Figure 1 shows typical permeability results for the Wamsutter cores. 


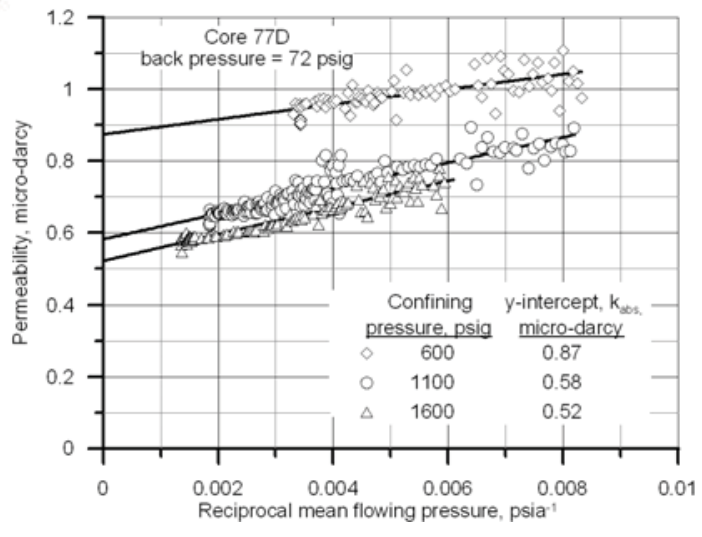

FIGURE 1. Results of permeability measurements for core $77 D$ showing typical Klinkenberg effects for three confining pressures.

Results of permeability analyses for all the cores provided except core $68 \mathrm{D}$ are summed up in Figure 2, where absolute permeability is plotted against reciprocal confining pressure. Of the four cores shown, cores $184 \mathrm{H}$ and 126D have significantly higher permeabilities than cores $74 \mathrm{D}$ and 77D. The permeability of the two latter cores is roughly the same value $(\sim 0.75 \mu \mathrm{d})$. These two cores, therefore, have been selected for further experimentation for wetting state changes with one as a control.

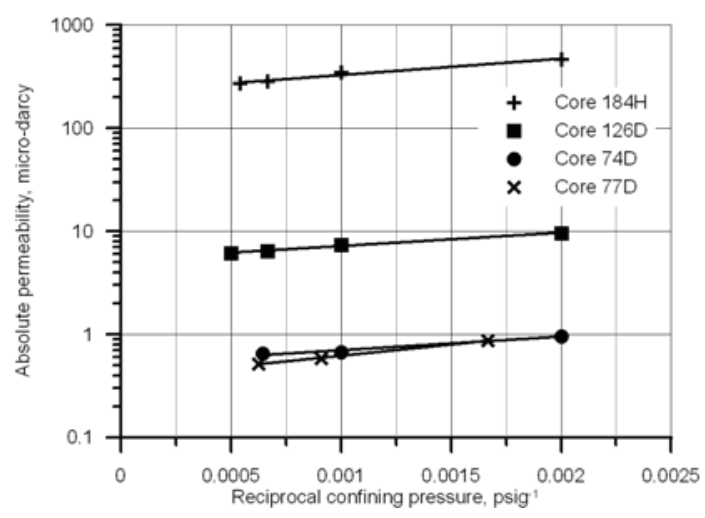

FIGURE 2. Results of permeability analyses of four Wamsutter cores showing absolute permeability as a function of reciprocal confining pressure.

The Wamsutter field cores are strongly water wet in their native state. Because reservoir cores are scarce and tests of wettability alteration on cores are time consuming, the candidate chemicals were prescreened using capillary rise method. The most effective chemical or chemicals will be verified with tests on rock samples. A group of nine water-soluble (or partially soluble) chemicals and one oil-soluble chemical were selected for screening (Table 1). Three different concentrations of the active ingredients $(0.1 \mathrm{wt} \%, 0.3 \mathrm{wt} \%$, and $0.5 \mathrm{wt} \%)$ were used for each chemical. Cylindrical capillary tubes with diameters of $0.11 \mathrm{~cm}$ and $0.04 \mathrm{~cm}$ were prepared to be strongly water-wet to mimic the strongly water-wet Wamsutter cores.

TABLE 1. Chemicals screened by capillary rise method.

\begin{tabular}{lcl}
\hline \multicolumn{1}{c}{ Product } & $\begin{array}{c}\text { Active } \\
\text { ingredient, } \\
\text { wt } \%\end{array}$ & Manufacturer \\
\hline Amphosol CG-50 & 30 & Stepan \\
Amphosol LB & 30 & Stepan \\
Petrostep SB & 50 & Stepan \\
Stepanquate 3300 & 33 & Stepan \\
Stepanquate 8358 & 80 & Stepan \\
Petrostep Q50S & 50 & Stepan \\
Petrostep Q-80T & 80 & Stepan \\
Tomadry N-4 & 25 & Tomah Prod. \\
Arquad 2C-75 & 75 & AkzoNobel \\
Redicote AP & 100 & AkzoNobel \\
\hline
\end{tabular}

Cleaned capillary tubes were soaked in the synthetic formation water (composition listed in Table 2, next page). While one tube was kept submerged in formation water, the rest of the tubes were soaked in different concentrations of surfactant solutions for 24 hours at $60^{\circ} \mathrm{C}$ and then briefly rinsed with formation water. One end of each of the capillary tubes was then inserted into the synthetic formation water and the capillary rise in the tubes was measured using a cathetometer. The lower the rise of the water into the tubes (signifying lower water wetness), the more effective the chemical was for altering the wettability of the strongly waterwet surface to a less water-wet state. 
TABLE 2. Composition of the formation water taken from a field well.

\begin{tabular}{lc}
\hline \multicolumn{1}{c}{ Ion } & Concentration, $\mathrm{mg} / \mathrm{L}$ \\
\hline $\mathrm{Fe}^{3+}$ & 19.1 \\
$\mathrm{Ca}^{2+}$ & 92 \\
$\mathrm{Mg}^{2+}$ & 39 \\
$\mathrm{~K}^{+}$ & 1,500 \\
$\mathrm{Na}^{+}$ & 745 \\
$\mathrm{Cl}^{-}$ & 400 \\
$\mathrm{SO}_{4}{ }^{2-}$ & 110 \\
$\mathrm{HCO}_{3}^{-}$ & $3,965^{*}$ \\
$\mathrm{TDS}^{-}$ & 6,870 \\
Because bicarbonate salt could not dissolve in the \\
mixture, $\mathrm{HCO}_{3}{ }^{-}$was replaced by $\mathrm{Cl}^{-}$in the synthetic \\
formation water.
\end{tabular}

Results of the capillary rise experiments suggest that Tomadry N-4 has the best opportunity to alter the wettability of the strongly water-wet cores to an intermediate water-wet state. Higher surfactant concentrations resulted in lower capillary rises for all surfactants except for Amphosol CG-50 (Fig. 3). Compared to the other tested surfactants, Tomadry N-4 suppressed the capillary rise most at all three concentrations, demonstrating that Tomadry N-4 most effectively alters the wetting state from strongly water-wet to an intermediate water-wet state.

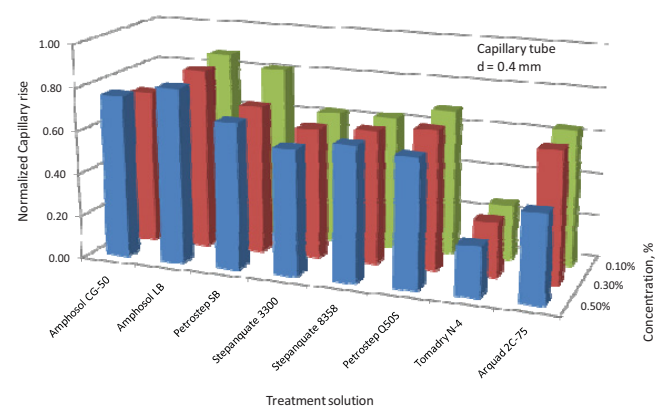

FIGURE 3. Normalized capillary rise data for all the surfactant-treated capillary tubes with diameter of $0.4 \mathrm{~mm}$.
We believe that this change in wetting state can suppress the imbibition of the formation water into the treated area near the well bore, decrease the connate water saturation, increase the gas phase permeability, and improve gas deliverability.

The stability of the wetting state of the treated rock surface will determine the actual improved gas deliverability after the treatment. Corefloods using reservoir cores will be performed next year at reservoir conditions to determine the stability of the wetting change.

\section{Summary and Conclusions}

Results of this first year of research point to successful results in this coming year and also to a successful field trial of the process in Year 3 of the project. The very low permeability characteristics of the field cores have stretched the core flooding and measurement capabilities of the lab and put the project a little behind schedule. However, much progress has been made and we feel confident that we can accomplish those tasks scheduled for Year 2 of the project.

In the coming year we plan to perform the relative permeability tests on cores $74 \mathrm{D}$ and 77D, which entail saturating one core with the surfactant (Tomadry N-4) solution and the other with plain formation brine, injecting wet gas ( $100 \%$ humidity) under constant pressure to displace the formation water from the core to determine the irreducible water saturation. A decrease in irreducible water saturation and an increase in gas relative permeability will indicate an improvement in the gas deliverability. We also plan to perform long-term imbibition studies designed to determine the stability of the chemical treatment and to begin the process of designing a field test with our industry partner, BP.

We anticipate that our good working relationship with the University of Wyoming and with BP will lead to future research funding in this area from industry and DOE. 


\section{Adaptive Process Modeling Using Parameter Estimation \& Mechanism Sensitivity Analysis}

Lee Shunn, Richard Boardman

A new approach for adaptive modeling of highly coupled, interdependent chemical processes to assist in state assessment, system prognostics, and automatic control

EI112

The scarcity of fossil energy resources and a growing concern for the environment are driving a shift in the way that energy systems are designed and operated. In the midst of these changes, a new vision for energy production systems is emerging. The new paradigm emphasizes coupled networks of energy processes that are tightly linked by inter-process exchanges of heat and mass. These energy systems, which combine various types of resources and operate reactor subsystems in close concert, are termed hybrid energy systems (HES). By effectively allocating resources and managing reaction by-products, HES have the potential to drastically improve efficiency and reduce environmental impacts compared with existing energy processes. These benefits can only be realized, however, if the very complex subsystems in the HES can be operated in close coordination with one another. This prospect raises a host of challenges for existing control systems, which have historically been designed and configured to operate subsystems at nominally steady conditions. Modern controls must maintain stability while adapting to dynamic interactions that occur amongst subsystems. In order to anticipate and respond to these complex interactions, new modeling concepts and methods are needed.

The objective of this project is to develop and demonstrate a new approach for adaptive modeling of highly coupled, interdependent chemical processes to assist in state assessment, system prognostics, and automatic control. A hybrid synthetic fuels production process has been selected as the target application of this effort. In this process, a high-temperature steam electrolysis (HTSE) reactor produces hydrogen gas from water using electricity and process heat from a nuclear reactor. The hydrogen is combined with a carbon source (such as gasified coal or biomass) in a catalytic synfuels reactor to produce high-quality liquid fuels suitable for transportation applications.

Hybrid synfuels production is an interesting application due to several technical challenges in modeling. Reaction and transport phenomena in the synfuels reactor are highly dependent on instantaneous conditions within the reactor. For example, reaction rates are a strong function of temperature, pressure, and reactant composition. In highly coupled systems, where multiple reactors are networked together by recycle streams, disturbances in one subsystem can rapidly propagate throughout the entire network. Furthermore, heterogeneous catalysts are notoriously sensitive to deactivation due to chemical fouling, hydrothermal sintering, mechanical wear, and other process complications. This means that chemical reaction rates, activation energies, rate coefficients, and even reaction mechanisms can change over time. This presents serious challenges to predictive models that are built on global estimates of key transport and reaction parameters, and makes it difficult to construct simple models that can be used in control systems. A solution to this challenge is to use real-time process data from reactor operations to dynamically adjust model parameters as the system evolves in time.

Any adaptive modeling approach must be thoroughly vetted by experimentation. Consequently, the first step in the execution of this project was the construction of a laboratoryscale facility for testing hybrid synfuels production. Parallel with this activity, computational models for the key system components have been developed. Separate models for a synthetic fuels (methanation) reactor, steam methane-reforming reactor, hightemperature water-gas shift reactor, and $\mathrm{CO}_{2}$ membrane separation reactor have been implemented. These models have been validated using transient and steady-state measurements from the laboratory experiments.

\section{Technical Objectives}

- Complete design and construction of a laboratory-scale hybrid synfuels production facility, including:

- synthetic fuels (methanation) reactor

$\circ$ methane-reforming/water-gas shift reactor

- $\mathrm{CO}_{2}$ separation membrane module

○ compressed hydrogen collection and storage tanks

$\circ$ process monitoring and controls instrumentation 
- Operate hybrid synfuels facility in concert with INL HTSE experiment and collected over 60 hours of process data.

- Develop MATLAB-based transient models for mass transport and chemical reaction in general catalytic packed-bed reactors. Applied the reactor model to: synthetic fuels production (methanation), steam methane reforming, high-temperature water-gas shift reactions.

- Develop a transient model for membrane separation of arbitrary multi-component gas mixtures. Applied the membrane model to $\mathrm{CO}_{2}$ separation from simulated flue gas.

- Validate computational models against process data from the integrated laboratory-scale experiments.

\section{Results and Accomplishments}

A laboratory-scale hybrid synfuels production system was constructed in Bay 9 of the Bonneville County Technology Center (BCTC). This facility utilizes hydrogen produced from the HTSE reactors in Bay 9 (not funded under this LDRD) to synthesize methane gas. A series of tests was conducted to demonstrate integrated operation of the synfuels system with the HTSE reactors. A photo of the completed synfuels production unit is shown in Figure 1.

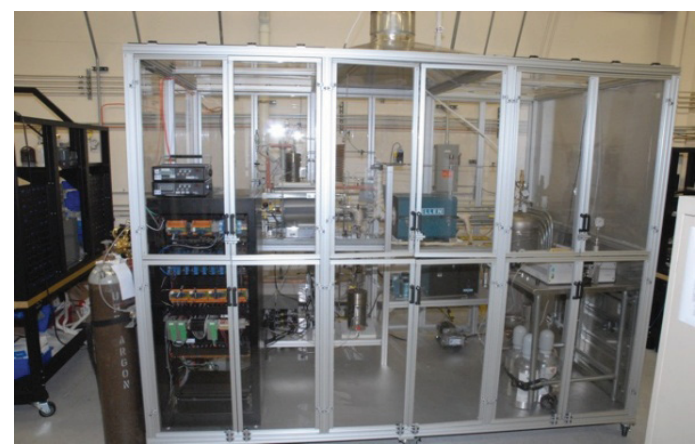

FIGURE 1. Laboratory-scale hybrid synfuels production facility in BCTC Bay 9.

As a representative example, results from the synfuels testing and modeling are presented. The synfuels reactor was operated for more than 30 hours of integrated testing. Figure 2 shows an example of data output from a typical day of testing (September 9, 2009). The outlet pressure and reactor gas temperature are shown in blue and red, respectively. During operation, the data acquisition computer recorded temperature and pressure data at 5-second intervals. Stream composition data were measured with the GC at a maximum frequency of approximately 3 minutes per sample. GC sampling times are indicated by the black "+" symbols near the bottom of Figure 2. During this particular day of testing, data was collected at two nominal pressures and three temperatures. Information from the separate data collection systems is reconciled through the digital timestamp on the files.

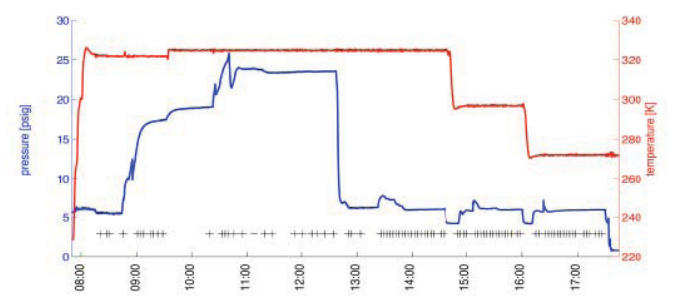

FIGURE 2. Temperature and pressure history for methanation reactor on September 9, 2009.

The test conditions and results from the methanation experiments are displayed in Table 1, with outlet concentrations of $\mathrm{CH}_{4}$ reported on a dry product basis. The temperature and pressure values represent time averages over the duration of each test. Two pressure ranges (roughly 25 psig and 5 psig) and three reactor temperatures $\left(325,300\right.$, and $\left.275^{\circ} \mathrm{C}\right)$ were investigated. The inlet concentrations of reactants were held constant, excepting the addition of moisture during Test 8 . Tests 9 and 10 explored the effects of reactor residence time by increasing the base flow rate. In general, the fractional conversion of $\mathrm{CO}$ to $\mathrm{CH}_{4}$ was greater at higher pressures and lower temperatures (compare Tests 2, 3, and 7 with 4, 6, and 5). This is consistent with Le Chatelier's principle for the exothermic methanation reaction where the total number of moles decreases with the extent of reaction. Likewise, the addition of the "product" $\mathrm{H}_{2} \mathrm{O}$ to the inlet flow suppresses the formation of methane (compare Test 7 with 8). Interestingly, reducing the residence time resulted in a slight increase in the methane yield (compare Test 4 with 9,10 ). 
TABLE 1. Methanation test conditions and outlet methane concentration.

\begin{tabular}{ccccccccc}
\hline Test & $\begin{array}{c}\mathrm{T}_{\text {inlet }} \\
{\left[{ }^{\circ} \mathrm{C}\right]}\end{array}$ & $\begin{array}{c}\mathrm{T}_{\text {reactor }} \\
{\left[{ }^{\circ} \mathrm{C}\right]}\end{array}$ & $\begin{array}{c}\mathrm{P}_{\text {inlet }} \\
{[\mathrm{psig}]}\end{array}$ & $\begin{array}{c}9 \% \mathrm{CO} \\
{[\mathrm{slpm}]}\end{array}$ & $\begin{array}{c}45 \% \mathrm{H}_{2} \\
{[\mathrm{slpm}]}\end{array}$ & $\begin{array}{c}100 \% \mathrm{~N}_{2} \\
{[\mathrm{slpm}]}\end{array}$ & $\begin{array}{c}\mathrm{H}_{2} \mathrm{O} \\
{[\mathrm{slpm}]}\end{array}$ & $\begin{array}{c}\text { Outlet } \\
\mathrm{CH}_{4} \mathrm{~mol} \%\end{array}$ \\
\hline 2 & 301.5 & 325.1 & 23.7 & 0.65 & 0.48 & 0.88 & 0 & 2.23 \\
3 & 299.6 & 325.1 & 6.5 & 0.65 & 0.48 & 0.88 & 0 & 2.11 \\
4 & 299.2 & 297.6 & 6.22 & 0.65 & 0.48 & 0.88 & 0 & 2.28 \\
5 & 296.8 & 272.6 & 5.95 & 0.65 & 0.48 & 0.88 & 0 & 2.35 \\
6 & 307.1 & 272.7 & 24.2 & 0.65 & 0.48 & 0.88 & 0 & 2.55 \\
7 & 299.7 & 297.6 & 23.6 & 0.65 & 0.48 & 0.88 & 0 & 2.48 \\
8 & 295.0 & 321.5 & 26.2 & 0.65 & 0.48 & 0.88 & 1.06 & 0.16 \\
9 & 313.1 & 297.6 & 5.61 & 0.97 & 0.72 & 1.28 & 0 & 2.45 \\
10 & 306.5 & 297.7 & 6.39 & 1.30 & 0.96 & 1.76 & 0 & 2.44 \\
\hline
\end{tabular}

The experimental data were compared with results from the one-dimensional packed bed model. Figure 3 shows the steady-state spatial profiles of temperature, velocity, and mass fraction for $\mathrm{P}_{\text {inlet }}=23.7 \mathrm{psig}$ and $\mathrm{T}_{\text {reactor }}=325^{\circ} \mathrm{C}$. These conditions roughly correspond to Test 2 in Table 1 . The symbols along the various curves indicate the locations of the computational grid points, which adaptively evolve to resolve solution features.

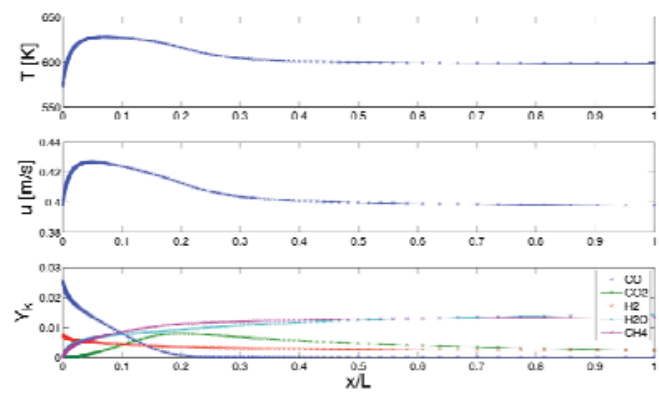

FIGURE 3. Steady-state spatial profiles of (top-to-bottom) temperature, velocity, and species mass fractions in the synthetic fuels reactor. $T_{\text {reactor }}=325^{\circ} \mathrm{C}, P_{\text {inlet }}=23.7 \mathrm{psig}$

Transient measurements of species concentrations for Test 2 are compared with predictions from the model in Figure 4. The steady-state values from the model agree well with the experimental data. The general trends and qualitative behavior of the experiment are also reasonably captured in the computational model. Quantitatively, however, advancement to the steady-state condition in the simulations is much faster than what was measured in the experiment.

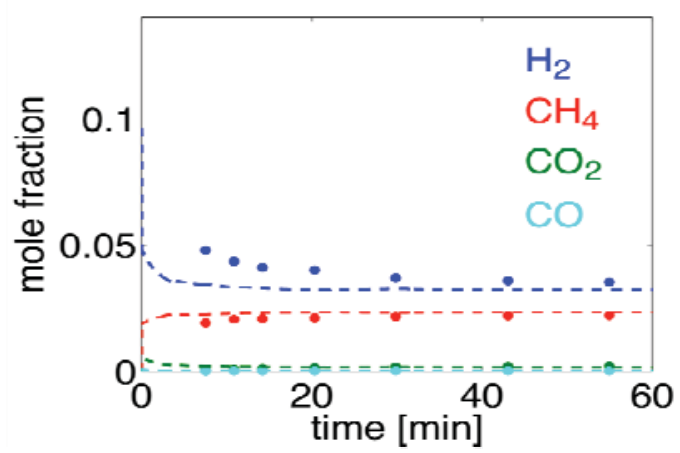

FIGURE 4. Transient species concentrations at the exit of the synthetic fuels reactor. $T_{\text {reactor }}=325^{\circ} \mathrm{C}, P_{\text {inlet }}=23.7 \mathrm{psig} . \cdot=$ Experimental data, and - - = Model prediction.

Several factors likely contribute to the apparent disagreements. First, there are differences between the catalyst used in the experiment and the catalyst used to deduce the model's kinetic mechanism and reaction rates, and differences in the adsorption rates and activation energies between the two catalysts are likely.

The exact initial conditions and "start time" of the experiment were also difficult to define and replicate in the model. For example, in the experiment there was likely significant dispersion and axial mixing of reactants in the 
complicated piping upstream of the synfuels reactor. This would present quite a different initial condition than the "plug flow" condition that was applied in the model. This could lead to significant changes in the early evolution of the flow. Other differences such as temperature boundary conditions and the exclusion of "dynamic" pressure and temperature fluctuations could also play a role.

In addition, evidence suggests that the GC data exhibited non-negligible "memory" effects when many samples were tested in rapid succession. For instance, at the end of a test after the flow of $\mathrm{CO}$ was discontinued, the GC would still detect $\mathrm{CH}_{4}$ for up to 15 minutes of sampling. This hysteresis is caused by time lags as materials flow from the reactant source locations, through the process piping and reactor volumes, and to the GC sampling locations. During sustained operations without sufficient time to purge the $\mathrm{GC}$, material can also deposit within the GC columns, causing test results from one sample to "bleed" into the next. Given the uncertainties of the current experimental setup, it is too soon to draw a conclusive appraisal of the performance of the current model.

\section{Summary and Conclusions}

A laboratory-scale hybrid synthetic-fuels facility was constructed and operated in conjunction with the INL HTSE experiment for over 60 hours of integrated testing. During operations, the synfuels reactor, $\mathrm{CO}_{2}$ membrane reactor, high-temperature shift reactor, and methane-reforming reactor were operated in several configurations to demonstrate various aspects related to hybrid energy systems and synfuels production. This facility represents a major accomplishment within the Energy Security Initiative, as it provides a flexible and reconfigurable test-bed for laboratory-scale demonstrations of hybrid-energy concepts and technologies.

Computational models for key reactor components in the synfuels production unit were developed. The models have been validated using transient and steady-state measurements from the experiments. General agreement between the model results and the measured data was achieved; however, further efforts are needed in both modeling and experimentation. In particular, improved instrumentation for monitoring and controls is needed in order to provide consistent, accurate, and useful data to inform and direct future models. On the other hand, more sophisticated models will be needed as the laboratory facilities expand in scope and capability. Undergirding both of these efforts is the development of tractable approaches to collect, analyze, and utilize process data. These activities are underway for the coming fiscal year. 


\section{PAGE FOR NUCLEAR NONPROLIFERATION INITIATIVE DIVIDER}

Nuclear Nonproliferation Initiative 


\title{
Chemical Signatures of Nuclear Proliferation on Particles
}

\author{
Gary S. Groenewold, Garold Gresham
}

Measurement of organic extractants on surfaces using secondary ion mass spectrometry

NN110

An activity of concern to the nonproliferation community is fuel reprocessing for the purpose of recovering fissionable radionuclides. The dissolution of fuel, followed by liquid extraction, uses an ensemble of organic chemicals that constitute a highly diagnostic chemical signature for specific reprocessing activities. In most instances, acquiring samples of these organics from facilities suspected of reprocessing is difficult, if not impossible, because the overwhelming tendency of the reprocessing chemicals is to partition to surfaces. However, chemical signatures may adhere to the surfaces of particulates, and fugitive particles from such facilities may represent an important source of samples. A priori, the problem with utilizing samples of this type is that current collection and detection approaches are not adequate. In this research project, it is hypothesized that the tendency of the reprocessing chemicals to adsorb to surfaces can be exploited by collecting particulates that have come into contact with the interior or effluent atmosphere of a facility and then detecting the reprocessing compounds using high-sensitivity surface analysis (Fig. 1).

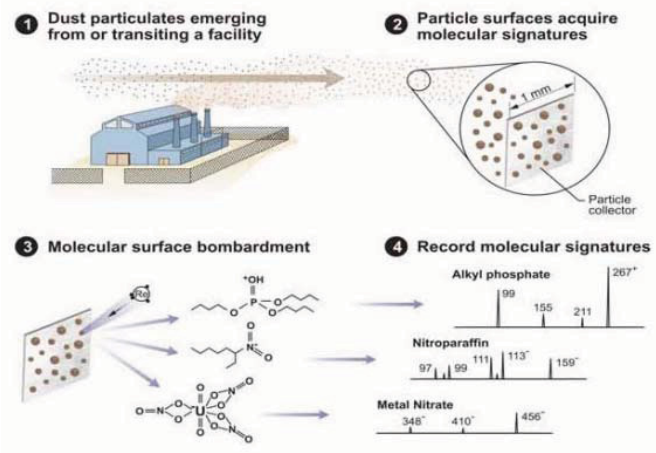

FIGURE 1. Conceptual diagram from detection of reprocessing signatures on surfaces.

Fuel dissolution using nitric acid is followed by liquid separations that involve formation of organic-soluble, actinide-ligand complexes. The organic complexants that are used are highly diagnostic for the separation process, and information on these compounds would ideally complement conventional compositional data. However, analysis of complexant chemicals has not received widespread attention because they have low volatilities and tend to bind strongly to nearly all surfaces they contact. Consequently they have not been seriously considered as potential signatures for identifying reprocessing activities. However, the tendency to bind to surfaces would result in their presence on particulate materials that would be part of fugitive releases from facilities. Thus, it may be possible to acquire contaminated particulates in opportunistic sampling scenarios.

Surface binding also influences signature detectability as shown by analysis of different types of particles exposed to multiple chemical vapors. The range of detectable compounds, their concentrations on the surfaces of the particles were evaluated in the beginning years of this laboratory-directed research and development (LDRD) project, using highly sensitive surfaceanalysis technology adapted for handling particulate samples.

Experiments conducted in the final year of the project focused on understanding signature partitioning, which involves both adsorption and desorption processes, understanding the rates of these processes. These studies are important because they have implications for sampling strategies and data interpretation. This will enable evaluation of the critical issue of signature persistence on surfaces.

\section{Technical Objectives}

- Exposure studies, phosphoryl-bearing organics on natural particulates

- Surface detection studies, multicompound/multi-surface

- Persistence evaluation

- Environmental studies of indigenous surface contamination

\section{Results and Accomplishments}

1. Exposure studies, phosphoryl-bearing organics on natural particulates.

Investigations in 2007 focused on mineral particles, typical of dusts found in arid environments. Particles were contaminated via a direct spike and vapor exposures, such that surface concentrations were $\leq 1$ molecular layer. In 2008, vapor exposures were conducted for 
lower molecular weight phosphoryl compounds that would simulate products formed from radiolysis of larger extractant compounds. This research showed that the soil particles were effective for collection of signatures from both liquids and vapors.

\section{Surface detection studies, multiple compounds and surfaces.}

In 2007 detectability studies emphasized characterization of next-generation diphosphoryl complexing agents [1]. The mass spectra were influenced by the surface chemistry of the mineral particles, but this could be understood and, in fact, used to verify contaminant identification. Research studies emphasized analysis of more complicated mixed functional-group molecules, such as the carbamoylmethyl phosphine oxide (CMPO) class of extractants (Fig. 2a). Subsequent studies emphasized more conventional extractants, specifically tri-n-butyl phosphate (TBP). Research showed a readily detectable signature arising from a) formation of the protonated molecule $[\mathrm{TBP}+\mathrm{H}]^{+}$followed by $b$ ) elimination of three $\mathrm{C}_{4} \mathrm{H}_{8}$ neutrals to form an abundant ion at $m / z$ 99. This spectral signature is easy to observe and, together with several lower abundance ions, provides unequivocal identification.

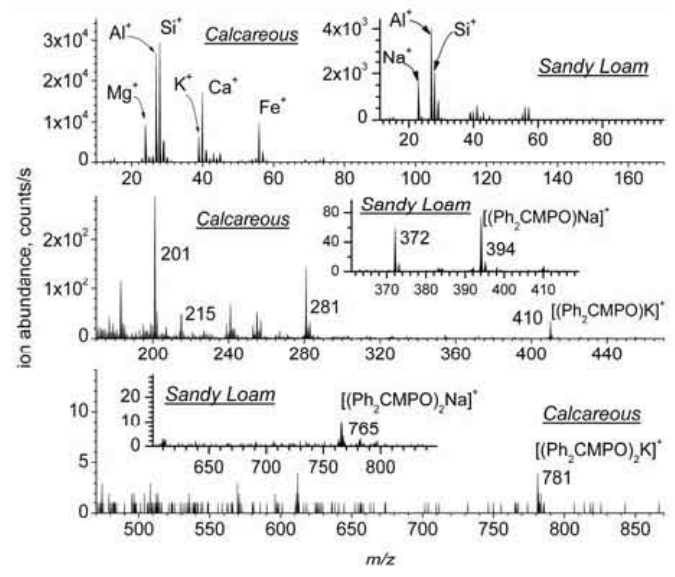

FIGURE 2. Surface mass spectra of a diphenyl CMPO extractant adsorbed to a calcareous soil, relatively high in potassium (K). The inset shows partial spectra of the same compound adsorbed to a sandy loam, higher in sodium ( $\mathrm{Na}$ ).

\section{Signature Persistence Studies.}

A key aspect of the research effort in 2010 centered on understanding the persistence of the signatures on particles, so a number of studies were initiated to determine how long a signature might be observed. Exposure of a large soil sample to TBP droplets within a covered chamber initially showed only low abundance of TBP-derived ions at $\mathrm{m} / \mathrm{z} 99$ and 137 (the latter, the kalliated version of $m / z$ 99) after one day; normalized ion intensities $<1 \%$ of the total (Fig. 3). However, upon standing, the TBP signature began to increase as a result of partitioning of the compound from the chamber to the surface of the soils. The summed intensity of the TBP-derived ion signature exceeded $10 \%$ after 7 days, at which point the chamber was uncovered. Within one day, the fractional abundance had decreased to $6 \%$ but, surprisingly, remained at this level for the remainder of the experiment, $\Delta t_{\text {exposure }}>100$ days. The abundance vs. $\Delta \mathrm{t}_{\text {exposure }}$ plot shows that the TBP concentration builds up on the surface with increasing time. A fraction of this volatilizes and is dispersed once the exposure chamber is uncovered, which probably represents loosely bound TBP; however, a large fraction, which must be very strongly bound, remains behind.

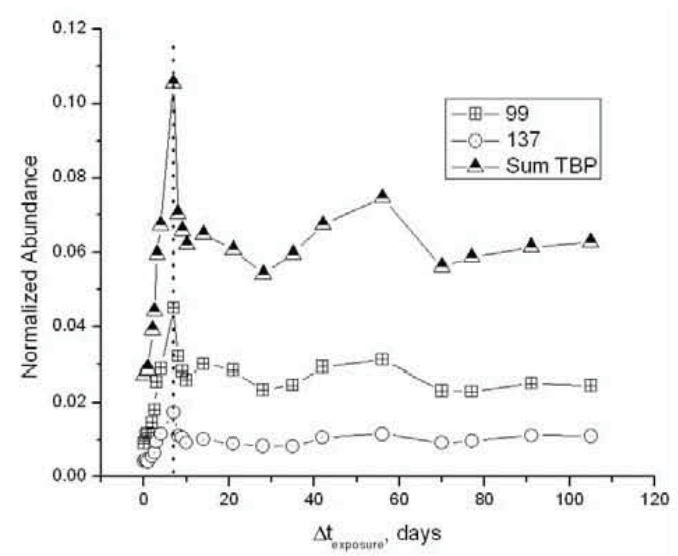

FIGURE 3. TBP ion abundance plotted versus exposure time, for a calcareous soil, showing little change in the TBP abundance to $>100$ days.

\section{Environmental Studies.}

Several environmental studies were also conducted to evaluate the ability to measure surface contaminants from indigenous particles 
where chemical exposure was known to have occurred. Ongoing studies at the Idaho National Laboratory (INL) Research Center showed detection of cyclohexylamine on particles for long periods of time after initial release [2]. Particulate samples were collected from a receiving facility where TBP was handled, and the mass spectra showed elevated intensities of $\mathrm{m} / \mathrm{z} 99$, consistent with the presence of persistent TBP in this locale, years after use.

\section{Summary and Conclusions}

Ensuring non-proliferation is an absolute necessity if nuclear energy production is to achieve its ultimate potential. National security sponsors are actively seeking potentially "transformational" approaches for identifying proliferation activities. The strategy of detecting organic reprocessing signatures would ideally complement ongoing compositional detection approaches, and the preliminary results achieved thus far have shown that detection of surfacebound organics used in separations processes has the potential to greatly augment the information that can be acquired from opportunistically acquired samples.
The experiments validate the hypothesis that surface contaminants persist on particulate samples, and can be detected using surface analysis long after their initial use. Future efforts will focus on development of programmatic support for expanding this research area.

References

1. Groenewold, G. S., G. L. Gresham, R. Avci, and M. Deliorman, 2009, "Characterization of Bidentate Phosphoryl Compounds on Soil Particulates using SIMS," Surf. Int. Anal., Vol. 41, pp. 244-250.

2. Groenewold, G. S., M. M. Cortez, A. K. Gianotto, G. L. Gresham, J. E. Olson, R. V. Fox, B. M. White, W. F. Bauer, R. Avci, M. Deliorman, and E. Williams, 2007, "Surface Analysis of Laboratory Hood Exhaust Manifold Particulates," Surf. Int. Anal., Vol. 39 , pp. 547-553. 


\section{Technetium Signature Analysis}

Troy Tranter, Tedd Lister, Nick Mann, Doug Jenson

Closing technical holes in the analysis and demonstrating the technology on groundwater specimens

NN112

The objective of this program is to deliver a collection and analysis package that will allow technetium-99 $\left({ }^{99} \mathrm{Tc}\right)$ to be used as a key identifier of possible nuclear-fuel reprocessing or uranium-enrichment processes. The ability to accurately detect and quantify these processes, whether declared or undeclared, is of primary importance in determining and assessing potential nuclear-proliferation activities. Many types of signatures can be used to paint a picture regarding the existence and magnitude of these operations. Most of these signatures, taken alone, do not determine with certainty that nuclearprocessing activities are taking place, but are instead used with additional confirmatory data to make a decision regarding the probability of these events. A notable exception to this is the detection of key radioisotopes present at concentrations above the fallout amounts resulting from past nuclear weapons testing. The detection of these radioisotopes significantly above fallout levels indicates with certainty that some type of nuclear activity is being performed,(i.e., reactor operations, fuel reprocessing, or possibly enrichment).

The technical approach involved developing several technical steps and linking them to develop a highly sensitive detection method for ${ }^{99} \mathrm{Tc}$. The method involves: (1) developing ionexchange columns for isolation/concentration of Tc from a groundwater specimen, (2) electrodepositing Tc as a small metal spot on filament materials, (3) performing thermalionization mass spectrometry (TIMS) on filament to detect ${ }^{99} \mathrm{Tc}$ isotope.

\section{Technical Objectives}

For Fiscal Year 2009, the project objectives were to close remaining technical holes in the analysis and demonstrate the technology on groundwater specimens.

Specifically, these objectives were

1. To complete development and validation of ${ }^{99} \mathrm{Tc}$ electroplating technique

2. To prepare multiple filaments and determine TIMS ultra-trace detection capability for ${ }^{99} \mathrm{Tc}$

3. To validate method with contaminated groundwater from INL-Site plumes.
Additionally, plans to promote and continue this work were

1. To seek national security customers

2. To publish and present data at international conferences.

Results and Accomplishments

Recent work has focused on electrodeposition and TIMS analysis. Some technical difficulties were overcome by developing a deposition method that was compatible with the column elution chemistry. Developing the TIMS analysis was challenging due to infrequent openings in the heavily scheduled Idaho National Laboratory (INL) instrument. Despite these challenges, the project appears to have made progress in the last two months and may lead to future work. Below are specific technical descriptions of the work performed in the last year.

\section{Electrodeposition of ${ }^{99} T c$ from dilute} standards. In order to deposit dilute Tc onto the filaments, a unique flow-deposition cell was designed and built (Fig. 1). This cell allows Tc to be deposited on a very small area (filament width is $\sim 1 \mathrm{~mm}$ ) of the front side of the filament ribbon. TIMS analysis benefits from a small spot versus a broad one. Using the flow deposition cell, Tc was deposited from $\mathrm{TcO}_{4}{ }^{-}$onto $\mathrm{Pt}$ ribbon from stock solutions and solution from ionexchange column concentrating experiments. In order to maximize the loading, deposition was performed for 24 hours to provide adequate time for as much Tc from the solution to deposit onto the filament. Figure 2 shows a voltage-time curve for deposition from $5 \mathrm{~mL}$ solution containing $1.47 \mathrm{ng}$ of ${ }^{99} \mathrm{Tc}$ from a stock solution. The noise in the voltage signal is due to $\mathrm{H}_{2}$ gas generated at the electrode surface. LOM of a deposit formed under these conditions is shown in Figure 3. The Tc deposits were black, but had some variability in texture from deposit to deposit. To determine the effectiveness of Tcmetal deposition on the filament, the postdeposition solution was collected and analyzed by GC-MS. The results of those analyses are that only about $10-15 \%$ of Tc is being loaded during the electrodeposition step. This loading efficiency can be increased with smaller solution volume and cell changes. 


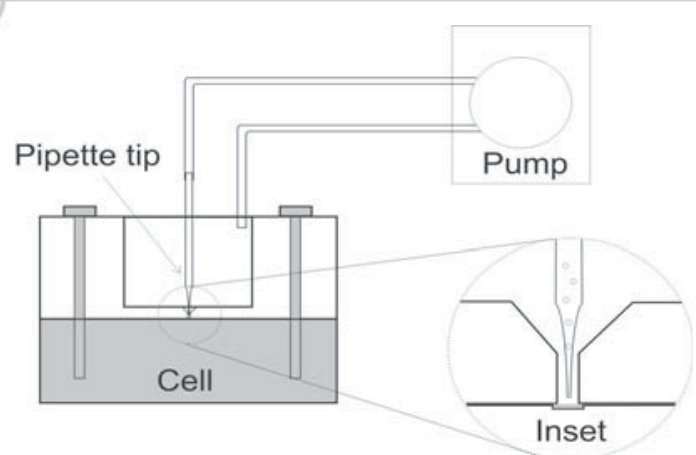

FIGURE 1. Diagram of micro-electrodeposition cell.

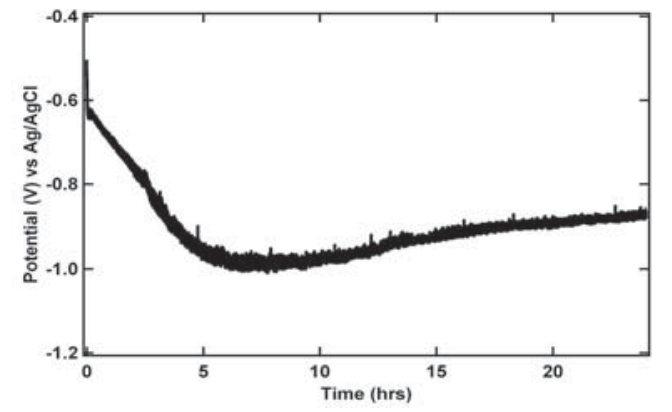

FIGURE 2. Voltage-time curve for deposition of Tc from 11.1 $D P M / m L$ solution.

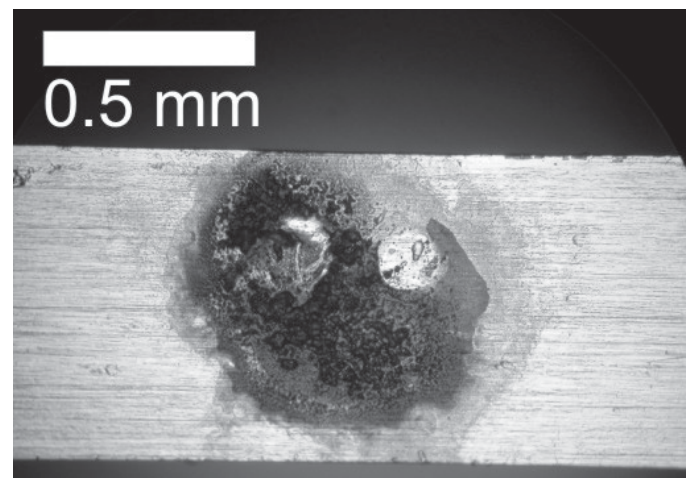

FIGURE 3. Tc metal deposit formed on Pt filament.

TIMS analysis of electrodeposits of ${ }^{99} T c$. In order to develop the method of analysis, ${ }^{99} \mathrm{Tc}$ was deposited from standard solutions and analyzed on the TIMS instrument. Ions were collected using a Faraday cup positioned between the electrostatic sector and the first magnetic sector. This was performed by varying the filament current while monitoring the signal. The ion current proved extremely sensitive to filament position, which is thought to be due to the small deposit area. After tuning the ion beam at low filament current, the ion current was monitored at mass 163 while increasing the filament current in small increments. ${ }^{99} \mathrm{TcO}_{4}{ }^{-}$ ions were initially observed at a filament current around $1.3 \mathrm{~A}$ and irreversibly exhausted by 1.6 A. For a filament loaded from $5 \mathrm{~mL}$ of solution, containing a total of $1.47 \mathrm{ng}$ of ${ }^{99} \mathrm{Tc}$, a maximum ion current of $26 \mathrm{pA}$ was measured. This provided an ion beam well suited for quantitative analysis. Scanning the mass resulted in a flat-topped peak at mass 163 as shown in Figure 4 taken at $1.4 \mathrm{~A}$ before the maximum filament current had been achieved. The small hum at 265 is likely the contribution of ${ }^{18} \mathrm{O}$ in the pertechnetate. The detection limit is estimated to be roughly 2 orders of magnitude lower $(\sim 15$ pg). Improvements in electrodeposition efficiency could lower this further.

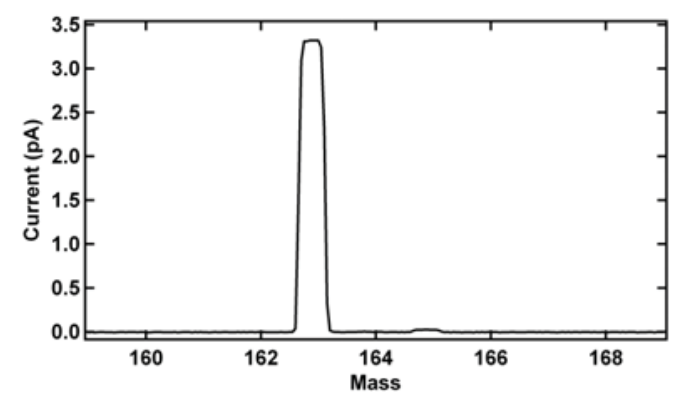

FIGURE 4. Mass sweep of pertechnetate ion region. Peak at mass 163 is $^{99} \mathrm{TcO}_{4}^{-}$.

Groundwater analysis. Groundwater water (USGS Well 67) was concentrated onto ionexchange columns and eluted using a 2-M $\mathrm{NH}_{4} \mathrm{OH}$ solution. This solution was acidified using concentrated sulfuric acid to provide a final concentration of $0.5-\mathrm{M} \mathrm{H}_{2} \mathrm{SO}_{4}$ as used in the standards above. This resulted in solutions which were expected to contain $0.218 \mathrm{ng} / \mathrm{mL} \mathrm{Tc}$. Three filaments were prepared using 24-, 48-, and 96-hour deposition times from $5 \mathrm{~mL}$ of solution. A four filament was prepared where $50 \square \mathrm{L}$ of the above solution was added to $5 \mathrm{~mL}$ of $0.5-\mathrm{M} \mathrm{H}_{2} \mathrm{SO}_{4}$ and deposited 96 hours. In all cases, well-defined deposits were obtained. The peak ion beam current for ${ }^{99} \mathrm{Tc}$ filaments were $0.33 \mathrm{pA}, 0.52 \mathrm{pA}$, and $0.20 \mathrm{pA}$ for $24-, 48-$, and 96-hour depositions, respectively. Thus no correlation with deposition time was observed, and the signal is an order of magnitude lower 
than observed for standards with slightly higher amounts of Tc in the solution. The interesting aspect was that the $1 / 100$ diluted Tc filament (10.8-pg ${ }^{99} \mathrm{Tc}$ ) had a peak current of $0.22 \mathrm{pA}$, not significantly lower than the above filaments. It is possible that some contamination occurs in the eluent of which the 1/100 dilution specimen contained less. The diluted filament had plenty of signal to noise to be measured analytically. Thus, a very promising result for analysis of trace Tc has been achieved. A further set of filaments with Tc from groundwater will be analyzed in the near future to complete the project.

\section{Summary and Conclusions}

This project has been successful in developing a method to measure trace quantities of Tc using TIMS. The focus of this last part of the work has been to develop electrochemical methods of isolating Tc on a small TIMS filament and perform quantitative measurements using TIMS. This has been demonstrated. Yet to be performed, but scheduled, is the analysis of groundwater samples. TIMS time has tentatively been allocated for the last week of the fiscal year. It is anticipated that this will achieve the final goal for the project. There is still more optimization to be performed and follow-up funds are anticipated based on these promising results. A paper using data presented above is in progress and will be submitted to a peerreviewed journal after the final data has been collected. The ability to perform this type of analysis is unique to INL and will be an important capability. The electrochemicaldeposition method could also be applied to other metals for trace analysis as well. 


\title{
Active Interrogation Die-Away Assay Development Program
}

\author{
David L. Chichester, Augustine J. Caffrey, Edward H. Seabury
}

Developing a prototype portable system capable of detecting shielded SNM hidden inside moderate-sized objects

NN113

Our nation urgently needs a portable analytical system capable of detecting the presence of shielded special nuclear material (SNM). This capability is needed by federal and state law enforcement agents, all branches of the U.S. military, the U.S. Coast Guard, and the U.S. intelligence community in order to have a rapidly deployable asset for field-response emergency situations to either (a) confirm a field assay of suspect SNM materials or (b) perform a field assay to identify the presence of SNM in a suspect location.

Today only passive assay systems are readily available to detect the presence of SNM in field situations. Unfortunately, since most of these instruments are based upon low-energy gamma-ray spectroscopy and gross neutron detection, they are ineffectual in reasonable time periods for detecting highly enriched uranium (HEU) hidden behind moderate thickness shields (for the gamma-ray detector systems) or for detecting plutonium when hidden within legitimate shipments of spontaneous fission neutron-emitting radioisotopes (for the neutron detector systems). Indeed, with less than 2 inches of lead, the passive gamma-ray signatures from HEU (not reprocessed) are very difficult to measure by these devices in reasonable assessment times.

This laboratory-directed research and development (LDRD) project is directed towards the development and demonstration of a prototype portable assay system capable of detecting the presence of shielded SNM. We are working to develop a system exceeding currently defined requirements as outlined in NIST ANSI Standard N42.41 (2007) for active interrogation systems as well as recent Department of Homeland Security technology goals in this area. To achieve this we are building upon previous research in active interrogation detection and assessment (AIDA) techniques at Idaho National Laboratory (INL) to detect, identify, and characterize SNM, and we are incorporating more recent INL research related to electronic neutron generators and advanced SNM detection techniques. Our work for this project incorporates simulation and modeling to investigate radiation transport issues effecting active interrogation die-away measurements and other AIDA techniques, as well as laboratory experiments to study instrumentation and engineering issues. Experiments for this project take place at the Active Neutron Interrogation Laboratory and at the Materials and Fuels Complex (MFC) Zero-Power Physics Reactor (ZPPR) facility. A key INL asset supporting these efforts is our accessibility to significant quantities of SNM at ZPPR including varying enrichment HEU samples and different grade plutonium samples.

\section{Technical Objectives}

The AIDA project is focused towards the development of a prototype portable system capable of detecting shielded SNM hidden inside moderate-sized objects. Specific project goals and milestones outlined in the original proposal include the following:

- Test ideas for the AIDA approach including delayed neutron, delayed gamma, and prompt/delayed

- Neutron/gamma data fusion

- Decide on an appropriate interrogating neutron source

- $\quad$ Select components for constructing a prototype instrument

- Test prototype equipment using SNM in shield configurations under laboratory conditions

- Develop an SNM detection decision analysis tree

- Test prototype equipment using SNM in shield configurations in field environments

- Develop an AIDA system ROC curve

- Design of a final concept system ready for deployment.

\section{Results and Accomplishments}

This year we focused on understanding the signatures associated with active neutron interrogation in portable configurations. This work included the collection of additional benchmark data during an experimental campaign at the ZPPR facility and the development of more sophisticated models for 
simulating our measurements. An example of the higher fidelity modeling achieved during Fiscal Year (FY)-2009 is seen in Figure 1, where excellent agreement is seen in a comparison between data collected using an INL photonuclear neutron detector (PND) module and simulations of the measurement.

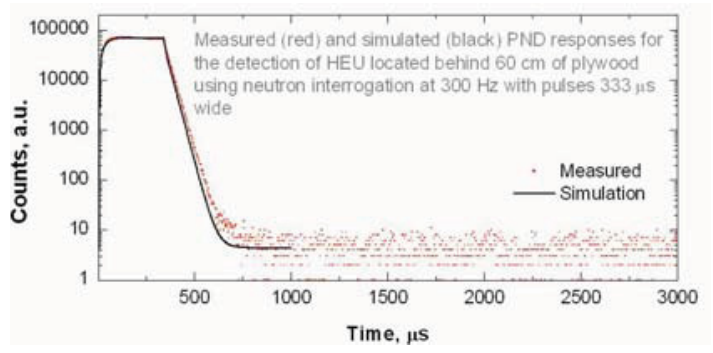

FIGURE 1. Comparison of experimental data and the simulated response for the time-dependent decay of an INL $P N D$ measuring fission neutrons generated in a plywood shield assembly containing $a>1-\mathrm{kg}$ sample of HEU.

Throughout FY-2009 we used new simulation and modeling tools to carry out an exhaustive parametric design study to develop a ${ }^{3} \mathrm{He}$-based portable detection system to use in the AIDA program. This work has considered individual detector module designs as well as and the impact arrays of detector modules have on one another. Up until now we have used INL's photonuclear neutron detector (PND) modules designed for use in bremsstrahlung-based active interrogation with delayed-neutron detection. Moving forward with the AIDA project a more optimized, higher efficiency neutron detector is needed. Key factors in this analysis are the fast-neutron detection sensitivity and the fast-neutron die-away time in the detectors. Large polyethylene moderated detector modules have better overall detection efficiencies but suffer from longer die-away times. The results of this design trade study are near completion as we approach the end of FY-2009 and will be presented at the 2009 Institute of Electrical and Electronics Engineers (IEEE) Nuclear Science Symposium.

Along this line, we have also made significant progress to further our understanding of the other active interrogation signatures available in portable, neutron-based interrogation scenarios detecting shielded fissionable material. An example is shown in Figure 2 where data from the measurement of a post-irradiation delayed neutron signature from HEU is compared with a theoretical decay fit for ${ }^{235} \mathrm{U}$. In prior work usually only one SNM signature is considered, very rarely are two or more considered. Based on our work in this LDRD we have systematically considered potential nuclear signatures from active interrogation and have identified 16 time-correlated signatures that can potentially detect SNM, using both neutron and gamma rays from both prompt fission and beta-delayed fission products.

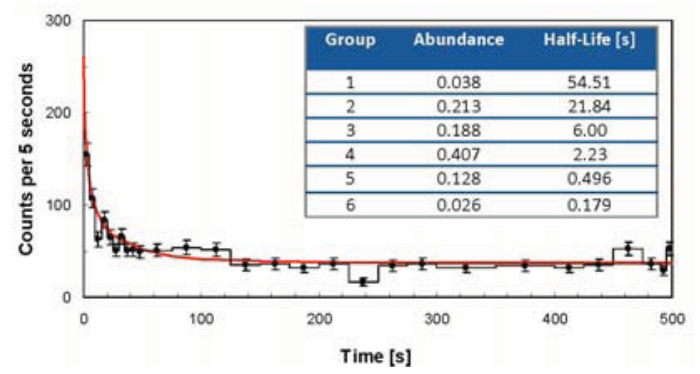

FIGURE 2. Post-irradiation delayed-neutron signature measured using a cadmium/boron shielded ${ }^{3} H e$ neutron detector following a 120-second irradiation of HEU. Data was collected in 5-s time bins for 500 seconds. The red line represents the theoretical delayed-neutron signature using 6 delayed-neutron groups.

During FY-2009, this project generated one peer reviewed publication: Chichester, D. L. and Seabury, E. H., 2009, “Using Electronic Neutron Generators in Active Interrogation to Detect Shielded Fissionable Material," IEEE Trans. Nucl. Sci. Vol. 56, pp. 441-447. Based on the presentation of that paper at the 2008 IEEE Nuclear Science Symposium, an invitation was extended to attend and prepare a talk at the International Atomic Energy Agency. That talk was developed into a proceedings report for the meeting: Chichester, D. L. and Seabury, E. H., 2009, "Active Neutron Interrogation to Detect Shielded Fissionable Material," Proc. International Topical Meeting on Nuclear Research Applications and Utilization of Accelerators, International Atomic Energy Agency, Vienna, Austria, May 4-8, 2009, $\mathrm{SM} / \mathrm{EN}-02$. Building upon these efforts, David Chichester has been invited to chair a session titled "Advances in Safeguards Technology," 
and to give an invited presentation at a session titled "Nuclear Analytical Methods for the 21st Century-Role of Neutron Sources from Nonreactor Facilities" at the 2009 American Nuclear Society Winter Meeting.

Summary and Conclusions

This project serves as a foundation building effort for developing a new research thrust area of portable SNM active interrogation screening and supports INL's nuclear nonproliferation initiative. Primarily we are investigating the use of active interrogation die-away assay measurements to detect shielded SNM, an effort which both leverages and supports other related efforts at the Laboratory related to SNM detection. Indirectly, this LDRD is also laying groundwork to support future work related to testing and evaluating active interrogation technology at INL using SNM. Over the next two years we will continue to refine the AIDA detection technique through detector and parameter optimization, and we will expand our experimental testing activities using SNM at ZPPR, maintaining our original plan of developing a prototype instrument set-up for test work at INL and elsewhere. Looking towards the completion of the project, we have already begun long-term planning to develop industrial partnerships and to transition the work from LDRD funding to external support. 


\title{
${ }^{135} \mathrm{Xe}$ recovery from the spontaneous fission of ${ }^{252} \mathrm{Cf}$
}

\author{
J. D. Baker, C. A. McGrath, K. P. Carney
}

Using fission products generated in the spontaneous fission of ${ }^{252} \mathrm{Cf}$ as a source of ${ }^{135} \mathrm{Xe}$ for use as a standard

NN114

${ }^{135}$ Xenon $\left({ }^{135} \mathrm{Xe}\right)$ is a good indicator that fission has occurred and is a valuable isotope that helps enforce the Comprehensive Test Ban Treaty. Due to its rather short half life and minimal commercial interest, there are no known sources from which ${ }^{135} \mathrm{Xe}$ can be purchased. The nuclear forensic community would like to have available standards of this isotope for calibrating collection and analytical techniques. ${ }^{135} \mathrm{Xe}$ has a half life $\left(t_{1} / 2\right)$ of $9.14 \mathrm{hr}$ and one of the highest thermal neutron capture cross sections of any isotope. Thus it is difficult to produce by activation of ${ }^{134} \mathrm{Xe}$ or from fission products resulting from thermal fission. ${ }^{135} \mathrm{Xe}$ could be produced in a fast reactor where ${ }^{135} \mathrm{Xe}$ neutron absorption cross is negligible.

We propose to use the fission products generated in the spontaneous fission of ${ }^{252}$ californium $\left({ }^{252} \mathrm{Cf}\right)$, which we presently have, as a source of ${ }^{135} \mathrm{Xe}$ for use as a standard. ${ }^{252} \mathrm{Cf}$ has a spontaneous fission rate of $6.14 \times 10^{8} \mathrm{f} \mathrm{s}^{-}$ ${ }^{1} \mathrm{mg}^{-1}$. The cumulative yield from the spontaneous fission of ${ }^{252} \mathrm{Cf}$ is $4.19 \%$, and the competing neutron-capture reaction that depletes ${ }^{135} \mathrm{Xe}$ in thermal reactor systems is negligible because the neutron-capture cross section is low for fast-fission neutrons. Also, we believe that using ${ }^{252} \mathrm{Cf}$ as a source of ${ }^{135} \mathrm{Xe}$ is advantageous because there is no need for a fast nuclear reactor or to fast-neutron irradiate ${ }^{235}$ uranium $\left({ }^{235} \mathrm{U}\right)$ or ${ }^{239}$ plutonium $\left({ }^{239} \mathrm{Pu}\right)$ using a D,T neutron generator. Since ${ }^{252} \mathrm{Cf}$ spontaneously fissions, all that is necessary is to develop a technique to separate the fission products from the ${ }^{252} \mathrm{Cf}$ so it can be continually "milked."

\section{Technical Objectives}

- Design and build a chamber to contain ${ }^{252} \mathrm{Cf}$ and thermalize fission fragments

- Fabricate a ${ }^{252} \mathrm{Cf}$ source to optimize emission of fission fragments

- $\quad$ Perform preliminary fission product collection and analyze collection.

\section{Results and Accomplishments}

We electroplated a 2-ng ${ }^{252} \mathrm{Cf}$ source (which was all that was available) in order to study both the transmission of fission fragments through various foils and to capture fission fragments.
Figure 1 shows the electroplated source and Figure 2 the fission spectra of the bare ${ }^{252} \mathrm{Cf}$ source and the same spectra after the fission fragments have passed through various thin films.

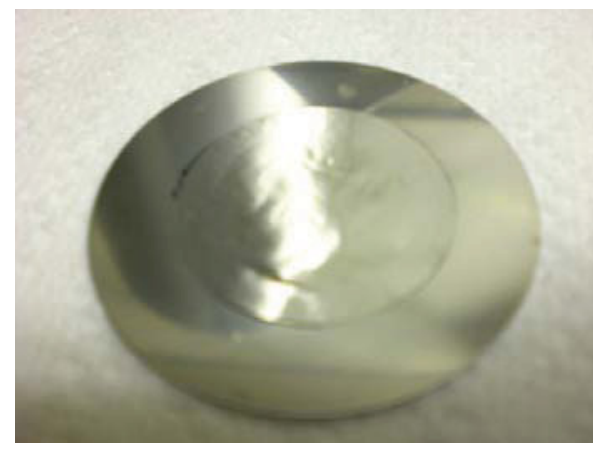

FIGURE 1. Electroplated ${ }^{252} C f$ source.

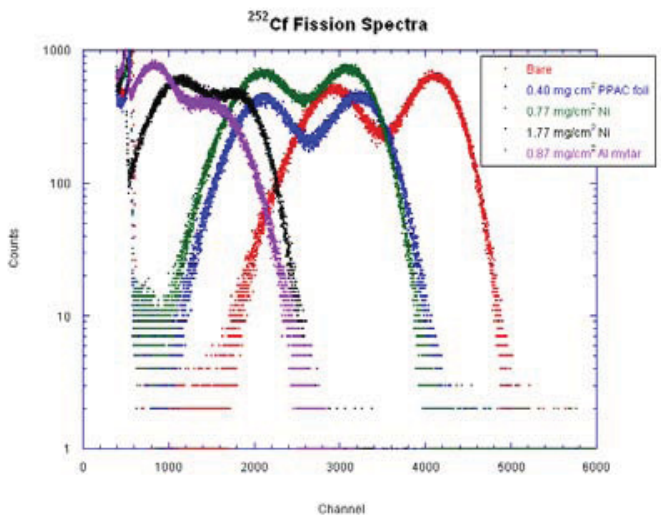

FIGURE 2. ${ }^{252}$ Cf fission spectra for the bare source and after transmission through various thin foils.

It is quite apparent that as the foil thickness increases, the energy spectrum is shifted to lower energies and gradually the lower energy peak is increasingly populated. However, it is obvious that we can cover the ${ }^{252} \mathrm{Cf}$ source with a foil thickness of up to $\sim 1.8 \mathrm{mg} / \mathrm{cm}^{2}$ and still get most of the fission fragments to transmit.

We designed plans for a chamber and ancillary equipment to contain ${ }^{252} \mathrm{Cf}$ sources; this will allow us to capture fission fragments on foils when the chamber is evacuated. In another mode, we can thermalize the fission fragments in a pressurized gas (He [helium]) and transport either the fission gases or all of the fission products. A picture of the actual chamber is shown in Figure 3. The design is shown in the 
appendix. We plan to operate the chamber at 30 psi, even though it is rated to 4000 psi. Using $\mathrm{He}, 30 \mathrm{psi}$ will thermalize the fission fragments within $~ 3 "$.

Finally, we used the 2 -ng ${ }^{252} \mathrm{Cf}$ source to collect fission fragments and gamma-ray counted the Al collection foil. A gamma-ray spectrum from this collection showing the ${ }^{135} \mathrm{Xe}$ gammarays is shown in Figure 4, and Table 1 (next page) lists some of the more dominant gammaray emitting isotopes observed as the fission products decayed.

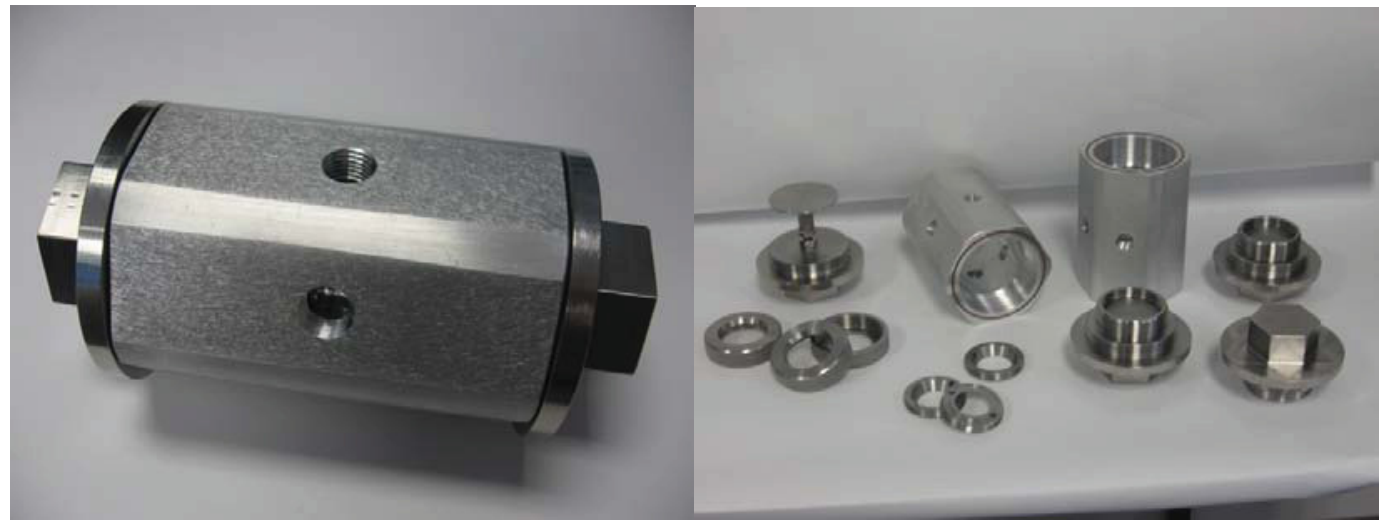

Figure 3. Chamber to contain ${ }^{252} \mathrm{Cf}$ and collect fission fragments.

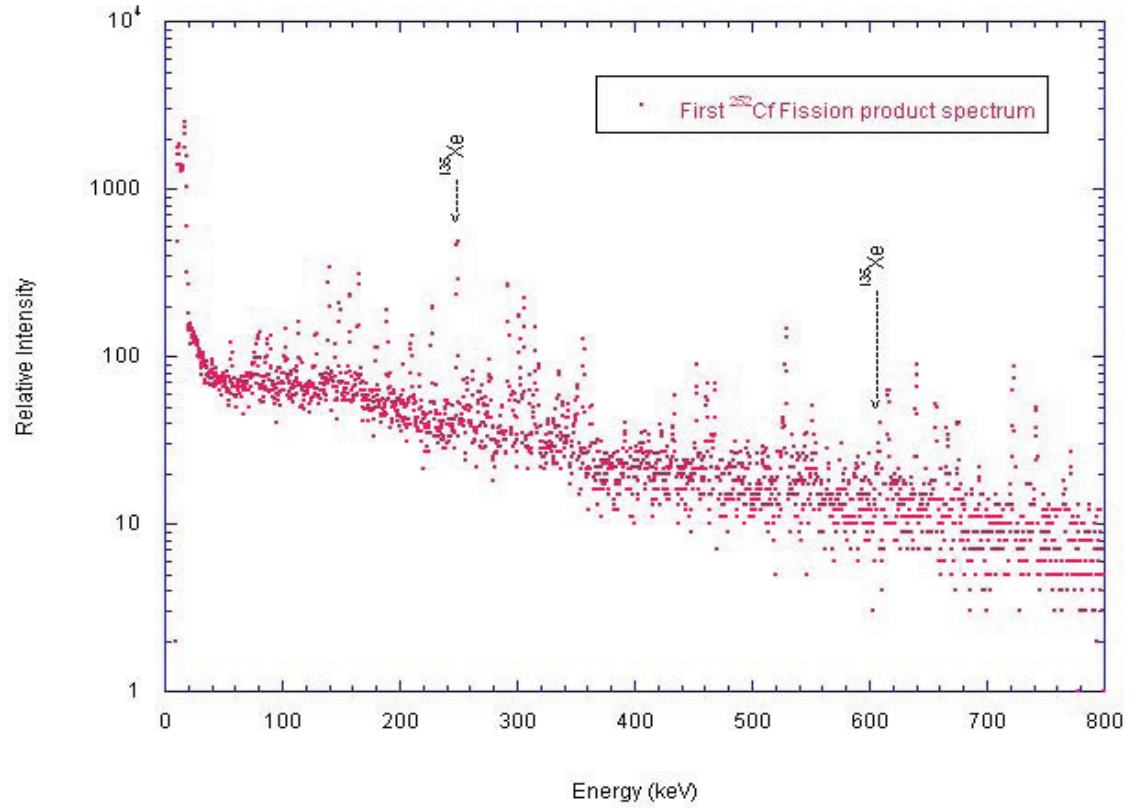

FIGURE 4. A gamma-ray spectrum of collected fission products from spontaneous fission of ${ }^{252} \mathrm{Cf}$. 


\begin{tabular}{|c|c|c|c|c|c|c|c|}
\hline Isotope & ${ }^{99} \mathrm{Mo}$ & ${ }^{99 m} \mathrm{Tc}$ & ${ }^{105} \mathrm{Rh}$ & ${ }^{132} \mathrm{Te}$ & ${ }^{133} \mathrm{I}$ & ${ }^{135} \mathrm{Xe}$ & ${ }^{143} \mathrm{Ce}$ \\
\hline $\begin{array}{c}\text { Half-life } \\
\text { gamma-rays }\end{array}$ & $\begin{array}{c}65.9 \mathrm{hr} \\
140.5,181.1\end{array}$ & $\begin{array}{l}6.0 \mathrm{hr} \\
140.5\end{array}$ & $\begin{array}{c}35.4 \mathrm{hr} \\
129.6,306.8\end{array}$ & $\begin{array}{c}3.2 \mathrm{~d} \\
116.4,228.3\end{array}$ & $\begin{array}{c}20.8 \mathrm{hr} \\
529\end{array}$ & $\begin{array}{l}9.1 \mathrm{hr} \\
249.8\end{array}$ & $\begin{array}{c}33.0 \mathrm{hr} \\
293.3\end{array}$ \\
\hline \multicolumn{8}{|l|}{ Count time } \\
\hline 3/11/08 10:39 AM & 3.91E-05 & 4.10E-05 & $5.41 \mathrm{E}-03$ & 7.54E-05 & $1.88 \mathrm{E}-04$ & $2.73 \mathrm{E}-04$ & $2.35 \mathrm{E}-04$ \\
\hline 3/11/08 1:30 PM & 3.97E-05 & 4.16E-05 & 1.76E-04 & 4.71E-05 & 1.34E-04 & 2.90E-04 & 1.96E-04 \\
\hline 3/11/08 2:01 PM & 4.54E-05 & 4.76E-05 & 1.46E-04 & 4.57E-05 & $1.94 \mathrm{E}-04$ & 2.74E-04 & 2.29E-04 \\
\hline 3/11/08 3:01 PM & 4.66E-05 & 4.90E-05 & 1.93E-04 & 4.62E-05 & $1.54 \mathrm{E}-04$ & $2.90 \mathrm{E}-04$ & 2.20E-04 \\
\hline 3/11/08 3:52 PM & 4.86E-05 & 5.10E-05 & $1.75 \mathrm{E}-04$ & 5.26E-05 & $1.44 \mathrm{E}-04$ & $2.73 \mathrm{E}-04$ & $2.50 \mathrm{E}-04$ \\
\hline 3/11/08 4:43 PM & 5.23E-05 & 5.49E-05 & 1.72E-04 & 3.86E-05 & 1.33E-04 & 2.80E-04 & 2.02E-04 \\
\hline 3/12/08 8:21 AM & 5.18E-05 & 5.44E-05 & 1.31E-05 & 4.17E-05 & 6.47E-05 & $1.55 \mathrm{E}-04$ & 1.70E-04 \\
\hline 3/12/08 9:19 AM & 5.04E-05 & 5.29E-05 & 1.37E-04 & 3.81E-05 & 7.41E-05 & 1.34E-04 & 1.26E-04 \\
\hline 3/12/08 9:52 AM & 4.81E-05 & 5.05E-05 & $1.65 \mathrm{E}-04$ & 3.33E-05 & 7.33E-05 & 1.39E-04 & $1.48 \mathrm{E}-04$ \\
\hline 3/12/08 11:04 AM & 5.55E-05 & 5.76E-05 & 1.34E-04 & 4.01E-05 & 7.37E-05 & 1.23E-04 & 1.57E-04 \\
\hline 3/12/08 11:39 AM & 5.53E-05 & 5.81E-05 & $1.41 \mathrm{E}-04$ & 3.46E-05 & 6.84E-05 & $1.05 \mathrm{E}-04$ & 1.28E-04 \\
\hline 3/12/08 1:12 PM & 5.37E-05 & 5.64E-05 & 1.32E-04 & 2.93E-05 & 7.36E-05 & 1.13E-04 & 1.38E-04 \\
\hline 3/12/08 2:45 PM & 4.41E-05 & 4.63E-05 & 1.32E-04 & 4.16E-05 & 8.30E-05 & 1.03E-04 & 1.18E-04 \\
\hline
\end{tabular}

\section{Summary and Conclusions}

This laboratory-directed research and development project started on March 31, 2008. We accomplished everything except for the transport of the fission gases from the collection chamber. This will happen early in Fiscal Year 2010. Our laboratory was shut down and we had to move our operation to the Materials and Fuels Complex (MFC).

We believe that the results of this work will be beneficial to Idaho National Laboratory (INL), the nation, and the Department of Energy (DOE). The ability to capture ${ }^{135} \mathrm{Xe}$ is important because it is used as an isotope for nuclear weapons treaty verification. This isotope is not produced commercially, and INL is pursuing isolating this isotope in sufficient quantities for instrument calibration for these programs.

We can in the near future generate a needed radioisotope that is necessary for nuclear nonproliferation studies. Additionally, since we will have access to nearly all of the shorter halflived fission products, we will be able to develop specific chemical purification, if necessary, for any desired isotope. These isotopes are important to the nuclear forensics community.

Furthermore, this apparatus will be an invaluable teaching tool for future nuclear and radiochemists. This device can provide numerous isotopes efficiently at low cost that can be used for isotope trace experiments. 


\section{Developing a Next Generation, Risk-Informed Approach for the Physical Protection of Nuclear Facilities}

J. Phillips, J. Alexander, R. Bean, T. Bjornard, D. Gertman, D. Hebditch, P. McCabe, B. Meppen, C. Smith, D.Tingen

Development of a risk-informed approach to physical security

NN115

The Design Basis Accident (DBA) for the Three Mile Island Reactor was considered to be the instantaneous, double-ended guillotine rupture of a main coolant pipe at the inlet to the reactor. In 1979, however, the reactor experienced what amounted to a relatively small leak that resulted in meltdown of the reactor core. This accident was far from the design basis event and demonstrated in very dramatic fashion a shortcoming of the DBA approach to safety. A risk-informed approach has since become standard in nuclear safety analysis, as well as in reactor operations and maintenance, with dramatic positive impact on plant performance.

In physical protection of nuclear facilities, the Design Basis Threat (DBT) approach (moderately changed to be the Graded Security Protection approach) continues to be the reference approach in the United States. The investigators of this laboratory-directed research and development (LDRD) project hypothesize that adaptation of a risk-informed approach to physical security design and assessment has the potential to cost effectively improve physical security. This was partially confirmed by a project called RAMCAP (Risk and Management of Critical Asset Protection), examined by the LDRD team, that used some risk-informed techniques to assess the security of all 104 operating nuclear power plants. An important outcome of the RAMCAP study was that the risk-informed technique identified vulnerabilities in all of the plants, and all plant operators found value in the process - this supports the value of going forward with this LDRD.

Further, the Nuclear Regulatory Commission (NRC) is convinced of the value of the risk-informed process and states as much in their NUREG 1860, "Feasibility Study for Riskinformed, Performance Based Regulatory Structure for Future Plant Licensing." NRC expects that the risk-informed, performancebased process used for safety should also be used for the physical protection in the licensing process for future plants. The NRC expects the risk-informed tools to be used in the design process for the next generation plants to create more safe and secure plants. This LDRD is expected to provide important insights into how to incorporate such tools.
This LDRD will use Idaho National Laboratory's (INL's) Probabilistic risk analysis (PRA) strengths to efficiently and effectively apply PRA-type techniques to augment the DBT and GSP processes for physical protection. The technical approach in year one of the LDRD was to study the current approach to vulnerability assessment, identify all of the methodological elements, and assess how risk-informed elements could either replace or augment them. This resulted in identification of a proposed INL methodology that is documented in a separate report, INL/LTD-09-17450, "Developing a Next Generation, Risk-Informed Approach for the Physical Protection of Nuclear Facilities."

\section{Technical Objectives}

The following are the technical objectives for this LDRD:

- Define and identify the methodological elements of the current approaches to vulnerability assessment

- Determine which elements of the methodology would be potential candidates for replacement or augmentation by riskinformed techniques

- Define a proposed, risk-informed approach to physical protection

- Apply the proposed methodology on an example problem (Year 2 - Fiscal Year 2010 [FY-10]), and identify benefits and shortcomings of the proposed approach.

- Refine the methodology appropriately in FY-11.

\section{Results and Accomplishments}

The first year's work focused on two areas. The first was to thoroughly examine the methodological elements of the DBT approach to physical security design and assessment to identify points at which a risk-informed approach could be useful. Based on that, a proposed risk-informed methodology was developed. Also, the University of California (UC) Berkeley team performed some analysis of the DBT approach; they used game theory to determine that the DBT approach does not effectively allocate physical security resources 
when viewed from a risk/consequence perspective.

Both of the above support further development in Year 2, which is planned to be a demonstration application of the risk-informed approach to physical security, for a real problem. UC Berkeley will develop the game theory application further to influence the risk analysis quantitatively. Two reports have been prepared summarizing this work. The INL-led methodological development is reported in INL/LTD-09-17450, "Developing a Next Generation, Risk-Informed Approach to the Physical Protection of Nuclear Facilities."

\section{Summary and Conclusions}

The work performed to date has reviewed the state of the art in physical protection, partially confirmed the expectation that a riskinformed approach to physical protection will be useful, and identified a number of risk-informed methodological elements in a proposed methodology. This work is documented in the report INL/LTD-09-17450, "Developing a Next Generation, Risk-Informed Approach to the Physical Protection of Nuclear Facilities."

Planned for FY-10, a trial application is required to test and further refine the methodology, and to gain some real-world experience that will help to determine the expected benefit and define the path forward for FY-11. 


\title{
Neutron Spectrometer Development
}

\author{
David L. Chichester, Edward H. Seabury, Jim Johnson, Scott Watson
}

Studying the technology and application of fast neutron spectrometry for detecting, identifying, and characterizing fissionable material for nuclear nonproliferation and counterproliferation and for nuclear forensics

NN116

The detection, identification, and characterization of highly enriched uranium (HEU) and plutonium inside objects or in difficult-to-reach locations is a technical challenge that currently has a limited and generally unsatisfactory set of solutions. Passive detection of shielded fissionable material relies on the detection of radiation emitted from the material. With HEU the characteristic 0.186$\mathrm{MeV}$ gamma-ray line may be used as the basis for analyses if there is minimal shielding, but it may be difficult to measure or obscured in some cases by container shielding. HEU that has spent time in a reactor (e.g., reprocessed naval reactor fuel) contains isotopic contamination with U-232 that produces $2.614-\mathrm{MeV}$ gamma-rays during its decay. However, pure HEU derived directly from isotopic enrichment does not contain this impurity and remains easy to shield and difficult to detect using passive photon screening. Neutron emissions from HEU are low in intensity and are straightforward to shield to reach background levels. Plutonium generally possesses a significant spontaneous neutron emission signature $(\sim 5.6 \times 104 \mathrm{n} / \mathrm{s} / \mathrm{kg})$ and is typically accompanied by an easily measureable yield of 2.22-MeV photons resulting from neutron capture in surrounding hydrogenous materials. However, these signatures may be masked within shipments of standard commercial neutron source instruments including Cf-252 sources used in bulk material analyzers, $\mathrm{PuBe}$ and $\mathrm{AmBe}$ radioisotope well-logging sources, and hand-held industrial moisture gauges.

Research in the area of radiation instrumentation for nuclear nonproliferation and counterproliferation primarily focuses on gamma-ray spectrometers and gross thermal-neutron counting detectors; together these present a useful suite of tools for passive screening and active interrogation. One area that has received comparatively less attenuation has been instrumentation for neutron spectroscopy. Neutron spectra contain valuable information about the source material from which they are emitted. For example, $(\alpha, n)$ neutron spectra tend to be highly structured; this structure can provide insight into the material absorbing the alpha particles and generating the neutrons (e.g., Be, B,
$\mathrm{O}, \mathrm{F}$, etc.). Spontaneous and induced fission produce a smoother continuum of neutron energies that is distinctive from $(\alpha, n)$. For both types of sources the presence of intervening materials which scatter neutrons can be investigated using fast neutron spectroscopy by assessing the perturbation of the source neutron spectra. For example, neutron spectroscopy may be used as a tool for nonproliferation transparency and verification measurements by providing data to determine if plutonium in a test object is in a metallic or an oxide form.

This laboratory-directed research and development (LDRD) proposal is directed towards the study and development of high-resolution fast neutron spectroscopy equipment exploiting the unique kinematic behavior of the He-3(n,p)H-3 reaction. The ultimate goal of this project is to develop neutron instrumentation for performing fast neutron detection and neutron spectroscopy in the $0.1-$ $15-\mathrm{MeV}$ range. As part of this objective a project goal is to build and test a gas electron multiplier (GEM) based He-3 proportional counter suitable for active interrogation neutron counting of prompt and delayed neutron signatures. Digital Pulse Shape Discrimination (dPSD) is being used to perform leading-edge pulse shape discrimination to resolve the full-energy neutron capture signals of the neutron capture reaction from competing background clutter signals; these techniques have the potential to improve upon previous-generation analogue signal analyses approaches. This new detector will be ideally suited for integration into large detector arrays and have the ability to report neutron spectroscopic results in real time without the need for spectral unfolding.

\section{Technical Objectives}

The Neutron Spectrometer Development Project is studying the technology and application of fast neutron spectrometry for detecting, identifying, and characterizing fissionable material for nuclear nonproliferation and counterproliferation and for nuclear forensics. Specific project goals and milestones outlined in the original proposal relevant to firstyear activities for this LDRD include the following: 
- Examine the leading-edge signal phenomenon in $\mathrm{He}-3$ gas proportional counters to develop a baseline understanding

- Assemble bench-top equipment for highspeed signal digitization and digital signal processing for proportional counters

- Develop computational methods for analyzing and using leading-edge pulse shape discrimination from He-3 proportional counters

- Assemble a test fixture for testing He-3 proportional counters.

Results and Accomplishments

This year we performed neutron spectroscopy tests with several He-3 proportional counters to evaluate electronic component requirements. We tested large-area, 10-cm diameter, $\mathrm{He}-3$ proportional counters with varying fill gas compositions ( $1 \mathrm{~atm} \mathrm{He}-3+4$ atm krypton, 2 atm He-3 +4 atm krypton, and 4 atm He-3 + 4 atm krypton) and small area, 2.54-cm diameter, He-3 counters ( 4 atm He-3 and $10 \mathrm{~atm} \mathrm{He}-3$ ). We evaluated several different preamplifier electronic systems including ORTEC 142AH, 142IH, and 142PC model preamplifiers. We also began developing dPSD methods for analyzing signals from these sensors. From these tests we have identified optimal experimental conditions for analyzing He-3 risetime signals and have successfully shown that "standard" He-3 proportional counters used in many homeland security applications have the potential to perform neutron spectroscopy. An example neutron spectra from a $2.54-\mathrm{cm}$ diameter $\times 76.2-\mathrm{cm}$ long, $10 \mathrm{~atm}$ He-3 proportional counter is shown in Figure 1.

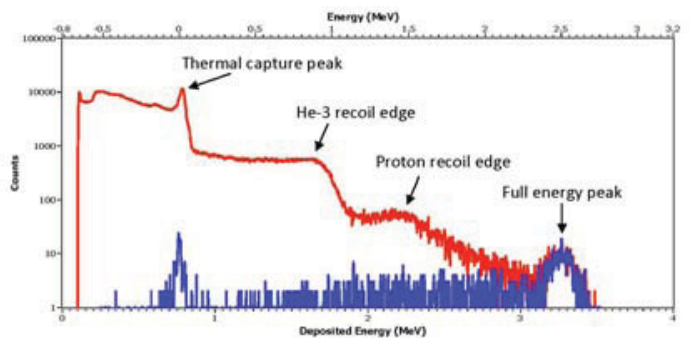

FIGURE 1. Comparison (log-scale) of raw pulse-height data (red) and dPSD analyzed pulse-height data (blue) from a 2.54-cm diameter $\times 76.2-\mathrm{cm}$ long, $10 \mathrm{~atm} H \mathrm{He}-3$ proportional counter exposed to a $2.55-\mathrm{MeV}$ neutron source.

\section{Summary and Conclusions}

A key finding from first-year studies is the important role that proper experimental techniques play in being able to collect good-quality spectra from these instruments. The primary signal from these proportional counters is very small, $\mathbf{O}(\mathrm{nC})$, and highly susceptible to both lab-originated radiofrequency noise as well as microphonic noise from vibrating equipment (e.g., cooling fans). To overcome these challenges special care must be used to reduce signal-line lengths and to vibration-isolate the detector. Looking towards the next fiscal year, we will continue to develop the dPSD algorithms for these spectrometers, begin testing with a special-purpose $\mathrm{He}-3$ proportional counter spectrometer, and begin bench-scale tests with gas electron multiplier (GEM) detector components. 


\title{
Nuclear Material Detection Using Neutron Time-of-Flight
}

\author{
Mathew T. Kinlaw (INL), James T. Johnson (INL), Scott M. Watson (INL), Alan W. Hunt \\ and Scott J. Thompson (Idaho Accelerator Center) \\ Examination of the efficacy of utilizing traditional time-of-flight and/or pulse height analysis for \\ detecting the presence of nuclear material within an adverse, active inspection environment \\ NN117
}

This research represents a collaborative effort between the Idaho National Laboratory (INL) and Idaho State University's Idaho Accelerator Center (IAC). The primary focus of the experiments presented here was an initial assessment of two specific neutron detection systems' abilities to effectively perform in a high-background, active-inspection environment. In terms of nuclear signatures of interest, both systems were focused on the detection of highenergy prompt fission neutrons as an indicator for the presence of nuclear material. Previous work completed at the IAC has already demonstrated this detection technology, performing similar measurements in a lowbackground, isolated environment.[1,2] However, many possible end-use applications of such technology, including field-deployable, outdoor, and/or standoff detection scenarios, may require operational capabilities for minimally controlled, high-background radiation environments.

The governing rationale in developing a detection technology based on prompt fission neutrons is their relatively large abundance and energies compared with alternative signatures (e.g. delayed neutrons). Prompt neutrons' higher emission rates can provide several advantages, including decreased inspection times, increased sensitivities, lower minimal detectable limits, etc. Furthermore, prompt neutrons are emitted with an average energy of $\sim 1-2 \mathrm{MeV}$, which is two to four times larger than the average delayed neutron energy ( $400-500 \mathrm{keV})$, leading to greater target shielding penetration and increased standoff detection distances.

\section{Technical Objectives}

- Performed two neutron detection systems/methodologies (plastic scintillatorbased time-of-flight spectrometer and liquid scintillator-based pulse height analysis system) evaluations for operations in a highbackground, active inspection environment (i.e. inside main accelerator hall, adjacent to linear electron accelerator). Detection efforts focused on the identification of high-energy neutrons from photon-induced fission reactions.
- Performed an experimental assessment of high-energy neutron detection capabilities in an outdoor active inspection environment, focusing on an initial assessment of standoff capabilities and applicability of current transportable, high-energy Linac.

- Presented experimental results during the Non-Intrusive Inspection Technologies session of the 2009 SPIE Defense, Security, and Sensing Conference.

\section{Results and Accomplishments}

Traditional time-of-flight measurements require a precise measurement of the flight time of the detected particles. However, since the signature of interest in this case is prompt neutrons from photon-induced fission, the temporal characteristics of the detected neutrons must be recorded relative to when the reactions were induced, not when the Linac's photon pulse occurred. Hence, $t_{0}$ is defined as the time at which the bremsstrahlung photons from the accelerator impinge upon the target of interest. In a well-isolated or low-background environment, this measurement is relatively trivial; however, the active inspection environment in which this research occurred contained multiple sources of background which needed to be understood and characterized. The Linac used during this phase of experimentation has several large magnets for steering and/or focusing the electrons. A substantial number of background photons, which are generated when the electron beam passes through the magnets, interfered with the identification of the target-scattered photons needed for establishing $\mathrm{t}_{0}$. Hence, additional photon shielding was incorporated into the existing detector shielding on the Linac side of the detector housing. Additionally, each electronic photomultiplier tube's base (plastic scintillator) was terminated to further reduce low-energy photon detection and minimize electronic noise pick-up.

With the active background reduced, several data sets were collected in order to identify the time at which the bremsstrahlung beam impinged upon the target. Figure 1 (next page) shows the resulting timing spectra from these data sets. Of particular interest is the peak that appears at $\sim 10$ ns. This is the target peak used to calibrate the 
timing and, hence, the energy of the detected neutron events.

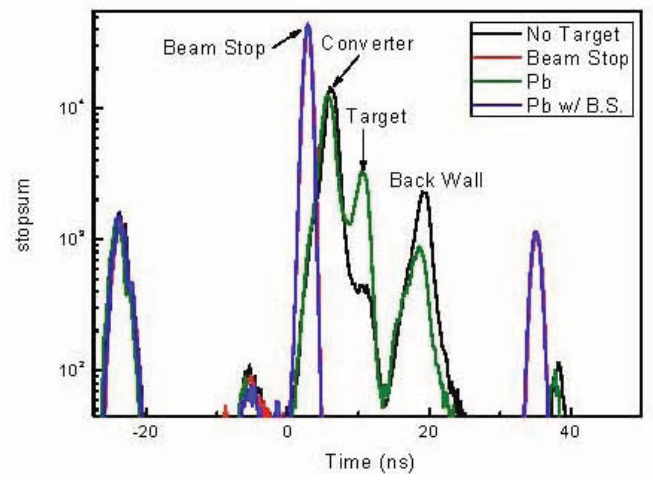

FIGURE 1. Various $\gamma$-ray peaks were produced as a result of bremsstrahlung beam scattering. The first two peaks originated from the electron-bending components of the Linac. The peak of primary interest is the target peak, which allows $t_{0}$ to be set for data analysis.

Figure 2 (top plot) presents the neutron time-of-flight energy spectra, detected with plastic scintillators, emitted from ${ }^{238} \mathrm{U}(\square)$ and ${ }^{\text {nat }} \mathrm{Pb}(\mathbf{\square})$ targets irradiated by $12-\mathrm{MeV}$ endpoint energy bremsstrahlung. As can be seen in the figure, the neutron energies from the $\mathrm{Pb}$ target experience a maximum around $5.3 \mathrm{MeV}$, which results from $E_{\max }=E_{\gamma}-E_{t h(\gamma, n)}$, where $E_{\gamma}$ is the energy of the incident photon and $\mathrm{E}_{\mathrm{th}(\gamma, \mathrm{n})}$ is the neutron separation energy of the target isotope. This equation neglects the effects of conservation of momentum; however, these effects are usually negligible, with the exception of very low- $Z$ materials. The elevated detection rates for the ${ }^{238} \mathrm{U}$ target from neutrons above $\sim 5.3 \mathrm{MeV}$ demonstrate the ability to discriminate nuclear from non-nuclear materials.

With traditional time-of-flight, particle energies are determined based on the time of the detected event; relatively large timing uncertainties necessarily result in large uncertainties in the calculated energies. A significant challenge arises when utilizing an accelerator pulse width that is long relative to the high-energy neutrons' flight time. Inclusion of standard uncertainties influenced by the timing uncertainty makes the nuclear and non-nuclear spectra quite difficult to discriminate from one another statistically. Hence, to overcome this challenge, data were analyzed by PHA with the liquid scintillators. Since the pulse height of the detected event is proportional to the energy transferred to the scintillator material by the incident radiation, this method can be utilized to discriminate nuclear from non-nuclear material, despite a relatively large bremsstrahlung pulse width.
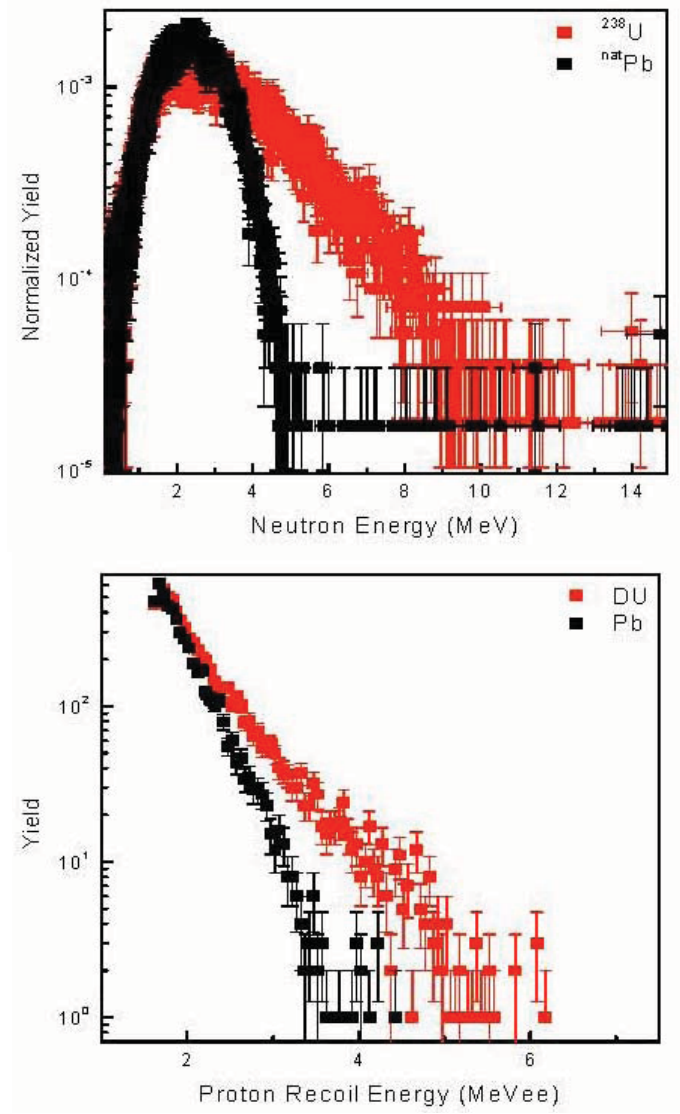

FIGURE 2. Top plot: energy spectra (nToF) for neutrons,

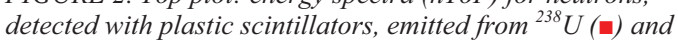
${ }^{n a t} \mathrm{~Pb}$ (@) targets irradiated by a 12-MeV bremsstrahlung beam. Bottom plot: pulse height spectra for neutrons, detected with a liquid scintillator, emitted from ${ }^{238} U(\square)$ and ${ }^{n a t} \mathrm{~Pb}$ (ロ) targets irradiated by a 12-MeV bremsstrahlung beam.

Figure 2 (bottom plot) shows the pulse height spectra for neutrons, detected with a liquid scintillator, emitted from ${ }^{238} \mathrm{U}(\square)$ and ${ }^{\text {nat }} \mathrm{Pb}(\mathbf{\square})$ targets irradiated by $12-\mathrm{MeV}$ endpoint energy bremsstrahlung (35-ns pulse width). Because the absolute energy of each detected event is not obtainable, this method is less efficient than the traditional nToF method; however, in the absence of precise timing 
resolution and/or known target-to-detector distances, PHA provides a reliable method for discriminating nuclear from non-nuclear material.

The next phase focused on neutron detection capabilities in an outdoor active inspection environment, with a transportable, high-energy Linac. This Linac was designed primarily for the detection of delayed fission neutrons, and thus is typically operated with a significantly longer pulse width $(\sim 4 \mu \mathrm{s})$ than would be desirable for nToF. Hence, the pulse forming network was modified to minimize the width of the electron and subsequent bremsstrahlung pulse. The goal was to decrease the total pulse width to less than $50 \mathrm{~ns}$, which initial measurements supported. However, during preliminary operations, significant electronic reflections from the primary transformer were observed. As can be seen in Figure 3, there were at least two observed reflections interfering with the neutron regions, regardless of the target-to-detector distance. It is quite likely that several additional reflections are present within the neutron region. To completely separate these from the neutron region would require a large increase in the number of detectors or detector-to-target distance (requiring large increases in data collection time), both of which were outside the available resources.

Due to the presence of the electronic reflections, the original transformer in the pulse forming network was re-installed, and the pulse width returned to $\sim 4 \mu \mathrm{s}$. With this setup, data were collected with the liquid scintillator-based acquisition, providing pulse height distributions from fissionable $\left({ }^{238} \mathrm{U}\right)$ and non-fissionable $(\mathrm{Pb})$ targets. With this data, the bremsstrahlung endpoint energy can be estimated to be $\sim 16$ $\mathrm{MeV}$, with a radiofrequency grid drive voltage of $17 \mathrm{kV}$ (Linac klystron). Furthermore, although the spectra from the two targets appear to be very similar, there are 148 detected neutron counts above $9 \mathrm{MeV}$ from the ${ }^{238} \mathrm{U}$ target, compared with only 24 detected neutrons from the $\mathrm{Pb}$.
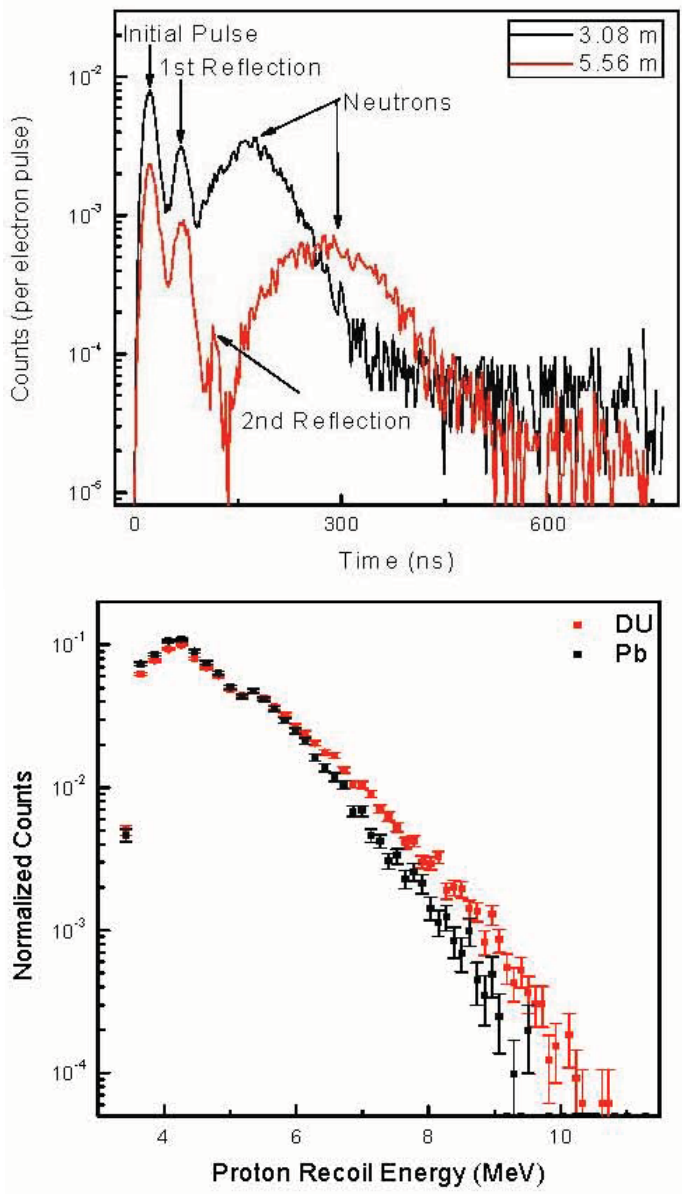

FIGURE 3. Top plot: timing spectra for two detector-totarget distances. By increasing this distance, additional reflected electron pulses are evident, extending well into the neutron region. Bottom plot: pulse height spectra for neutrons, detected with a liquid scintillator, emitted from ${ }^{238} \mathrm{U}(\mathbf{\square})$ and ${ }^{\text {nat }} \mathrm{Pb}(\mathbf{\square})$ targets.

\section{Summary and Conclusions}

The detection of nuclear materials using neutron time-of-flight has been demonstrated in an adverse, active inspection environment with plastic scintillator-based and liquid scintillatorbased acquisition systems. While short Linac pulse operations allow for traditional time-offlight measurements, longer pulse operations can utilize pulse height analysis methods with liquid scintillators to achieve similar detection capabilities. Based on the conclusion of the research presented here, several areas were identified for continued work. To continue to advance the efficacy of utilizing traditional nToF 
for outdoor, standoff material detection, further modifications to the Linac are needed. Designing and constructing a new electron gun hot deck for the Linac should allow for a significant reduction in the electron pulse width, without the multiple reflections experienced in the prior effort. An enhanced hot deck would also potentially provide a several factors increase in the available electron beam current, subsequently decreasing minimum detectable limits and necessary inspection times. Furthermore, the current nToF spectrometer contains eight $2 \times 2$ in. plastic scintillators. This system should be capable of supporting up to sixteen $4 \times 4$ in. detectors. The increased detector solid angle afforded by this improvement would allow greater detection efficiency, decreased inspection times, and/or greater standoff detection distances.
References

1. S. J. Thompson, M. T. Kinlaw, J. F. Harmon, D. P. Wells, E. B. Farfan, A. W. Hunt, Appl. Phys. Lett. 90, 074106 (2007).

2. S. J. Thompson, M. T. Kinlaw, J. F. Harmon, D. P. Wells, A. W. Hunt, Proc. VII Latin Amer. Symp. Nucl. Phys. App., 63 (2007).

3. Assuming the highest energy photon produced at this Linac setting is $16 \mathrm{MeV}$, the largest neutron energy possible from ${ }^{207} \mathrm{~Pb}$ is $16 \mathrm{MeV}-6.7 \mathrm{MeV}=9.3 \mathrm{MeV}$.

Hence, a significant detection of neutrons with energies above this value indicates the presence of nuclear (fissionable) material. 


\section{On-line monitoring of Actinide Concentrations for Advanced Aqueous Separations}

Shelly X. Li, Jan-Fong Jue, Scott Herbst, Steven Herrmann, University Participator: Prof. Srikanth Gopalan (Boston University)

\section{Advanced Separation Process Monitoring}

NN118

The main goal of this project is to design and develop an online actinide-monitoring system that can be applied to aqueous-based SX reprocessing applications. The work will initially focus on developing and characterizing a sensor that can be used directly to discern actinide concentrations in aqueous streams, primarily representative of the important product streams associated with PUREX processing. Ultimately, these representative aqueous streams will be acidic media (nitric acid, $\mathrm{HNO}_{3}$ ) containing various combinations and concentrations of $\mathrm{Pu}$ (either as $\mathrm{Pu}^{+3}$ and/or $\mathrm{Pu}^{+4}$ ), $\mathrm{UO}_{2}^{+2}, \mathrm{~Np}$ (in numerous oxidation states), $\mathrm{Am}^{+3}$, and $\mathrm{Cm}^{+3}$. Consequently, these streams would represent product streams (U product with some $\mathrm{Pu}$ contamination or Pu product with some $\mathrm{U}$ contamination) associated with classical PUREX processing, or modifications for potential nextgeneration-reprocessing flowsheet aqueous products (e.g. mixed $\mathrm{Pu} / \mathrm{U}$ products or mixed actinide, combinations of $\mathrm{Pu} / \mathrm{U} / \mathrm{Np} / \mathrm{Am} / \mathrm{Cm}$ ). Once data have been generated to underscore the technical validity and operational characteristics of the sensors, application aspects of implementing such systems becomes practical.

Solid-state-ionic sensors, which utilize ionic-conducting ceramics, have been used as sensors in molten media for over 40 years. The advantages of these types of devices are simplicity, reliability, and high in-situ sensitivity. It is hypothesized that the solid-state ionic sensor technology will be applicable to on-line monitoring of actinide activities (concentrations) in acidic $\mathrm{HNO}_{3}$ solutions in the PUREX flowsheet.

There are several basic criteria in selecting actinide-sensor materials for the aqueous separation process: (1) room temperature trivalent ionic conductivity must be high (ionic conductivity needs to be $>10^{-6} \mathrm{~S}-\mathrm{cm}^{-1}$ [siemens per $\mathrm{cm}]),(2)$ must be corrosion resistant in acidic aqueous solution (desired that the material function for $\sim 1$ week (or longer) before corrosion influences the measurement or necessitates sensor replacement), (3) must be radiationdamage resistant (desired that the material/sensor function for $>1$ week before radiation induced damage influences the measurement or necessitates replacement), and (4) must respond quickly to concentration changes in the solution (desired measurement time constant of $\sim 10$ minutes or less). Once the solid electrolyte materials are identified and become available, design of the sensor assembly and performance evaluation can be conducted.

\section{Technical Objectives}

The technical objective of this laboratorydirected research and development (LDRD) project is to develop an in situ electrochemical sensor technology for on-line monitoring of actinide concentrations for advanced aqueous separations. Specific objectives for Fiscal Year 2009 (FY-09) are

- Develop a strategy for a real-time actinidemonitoring technique for aqueous-based separation processes

- Synthesize and characterize surrogate trivalent ion sensor materials.

\section{Results and Accomplishments}

A literature search in FY-09 identified sodium super-ionic conductor (NASICON)based ceramic ionic conductors and poly-vinyl chloride (PVC)-based polymer ionic conductors as candidate materials for aqueous actinidesensor development. Polymer ionic conductors have been successfully used in monitoring $\mathrm{Ce}^{3+}$ ion concentrations in aqueous solutions with reasonable selectivity. NASICON-based ceramic conductors are also expected to have acceptable conductivity of trivalent ions at low temperatures.

Synthesis efforts have started on rare-earth ion-conducting NASICON. Phase identification by $\mathrm{X}$-ray diffraction (XRD) has been completed and characterization of low-temperature ionic conductivity has been performed. In parallel, modeling work on PVC-based ionic conducting polymers has started. The purpose of this modeling work is to identify the best polymer compositions for trivalent ion sensing.

An XRD pattern of a mixed and calcined cerium ceramic-ionic conductor is shown in Figure 1 (next page). 


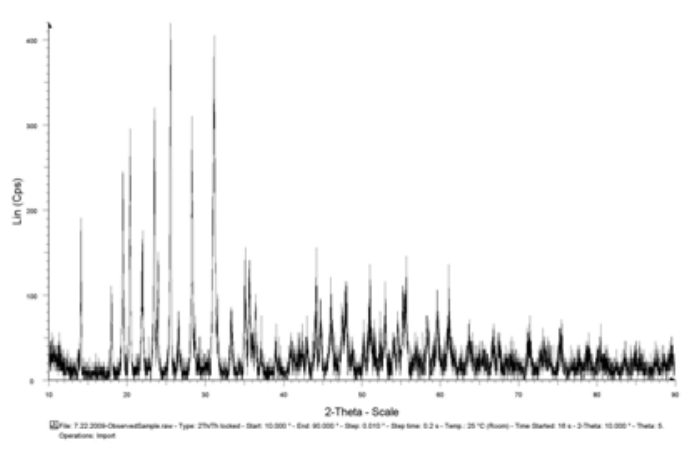

FIGURE 1. X-ray diffraction pattern of $\left(\mathrm{Ce}_{x} \mathrm{Zr}_{1-x}\right)_{4 / 4-}$ x) $\mathrm{Nb}\left(\mathrm{PO}_{4}\right)_{3}$.

The XRD pattern shows the formation of the NASICON-structured phase with a small amount of an as yet unidentified second phase. Efforts to eliminate the second phase by modifying the process conditions are underway.

The electrical conductivity of this material at different temperatures was measured. The conductivity of the material at the temperatures of interest $\left(<80^{\circ} \mathrm{C}\right)$ for employment in ceriumion sensors is relatively low $\left(\sim 10^{-8} \mathrm{~S} / \mathrm{cm}\right)$. However, the contribution of the unidentified second phase to the low electrical conductivity needs to be determined. A modification to the chemical composition may also improve the conductivity. These will be the main subjects of research for FY-10. It is anticipated that the low temperature conductivity of NASICON can be improved to meet the $10^{-6} \mathrm{~S} / \mathrm{cm}$ criterion.

In the last decade, ion-selective electrodes, based on polymeric membranes with an ionophore incorporated directly into the polymer, have played a major role in the development of potentiometric sensors. Examples include PVCbased 1,3,5-trithiane-coated graphite electrodes for the determination of $\mathrm{Ce}^{3+}$ ions in an acidic aqueous medium.

Gupta et al. [1] developed a potentiometric membrane which was capable of detecting cerium $\mathrm{Ce}$ (III) ions with high sensitivity, low detection limit, fast response time, good Nernstian slope, and a wide linear range. The overall performance of the membrane indicated that the $\mathrm{Ce}(\mathrm{III})$-ion transport mechanism was significantly enhanced over other kinetic processes. Figure 2 shows the potentials generated by various ions as measured by Gupta's group using their membrane. The high selectivity towards $\mathrm{Ce}$ (III) can be seen by the significantly higher potentials relative to the other ions. Also, $\mathrm{Ce}$ (III) has the best detection limit as indicated by the concentration at which the potential begins to increase. Lanthanum (La), which is only one atomic number away from $\mathrm{Ce}$, is the second-highest potential in the graph. Interestingly, although Terbium ( $\mathrm{Tb}$ ) is also a rare-earth element and also has a +3 valency, it is one of the poorest performers in this graph (fifth from the bottom up).

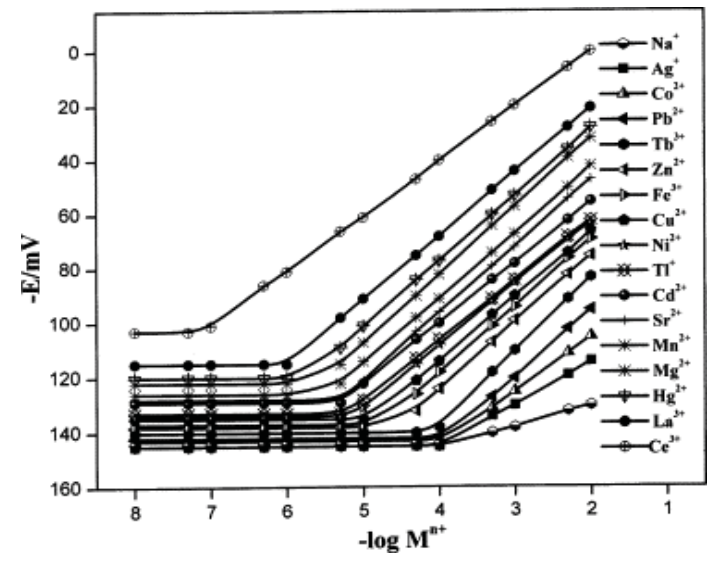

FIGURE 2. Performance of the proposed poteniometric membrane (from Gupta et al.) The proposed membrane has an excellent Ce(III)-ion selectivity.

In the Gupta et al.'s study, high-density PVC was used as the structural component of the $0.3-\mathrm{mm}$ membrane. Although a plasticizer was not strictly needed, the detection limit is improved by one or more orders of magnitude through its use. Since plasticizers essentially increase the distance between the polymer chains, they improve the mobility of the ionophore molecules. Depending on the plasticizer molecule, it may also influence the state of the ligands and the dielectric constant of the membrane. As an additive, which was used in every sample prepared by Gupta et al., oleic acid (OA) improved the performance of the membrane the most. Since the ionophore is highly lipophilic, a fatty acid such as OA can form a phase within the membrane through which the ionophore is mobile. Gupta et al. also believed that due to the optimal molar ratio of OA to ionophore of 1:4, the additive contributes 
to the complexation mechanism. The best membrane composition is found to be PVC:NPOE:OA:ionophore in the ratio of $150: 250: 20: 5(\mathrm{w} / \mathrm{w})$. Computer simulation is underway at Boston University to determine the best composition for high selectivity.

Summary and Conclusions

Two candidate materials, namely NASICON-based ceramics and PVC-based polymers, have been identified for aqueous actinide-sensor applications. Synthesis efforts have started on NASICON-based ceramics using a process reported in the literature. Early results are encouraging. Based on the modeling work at Boston University, PVC-based polymer with good selectivity will be synthesized for a trivalent ion sensor. An invention- disclosure record, entitled "Sensors for On-line Monitoring of Actinide Concentration for Advanced Aqueous Separation Processes," was filed earlier this year.

Successfully identifying two ionic conductors that can be used for aqueous actinidesensor applications is a very important first step for this research project. Sensor-material synthesis will be the next crucial step. By successfully developing this technology, sensorbased actinide monitoring for safeguard and nonproliferation applications will become reality.

References

[1] Gupta, V. K., A. Singh, and B. A. Gupta, 2006, Analytica Chimica Acta, Vol. 575, pp. 198-204. 


\section{Development of a Safeguards Approach for Pyroprocessing}

Robert Bean, Richard Metcalf, Clint Baker, Phillip Casey Durst, David Gerts, Thomas

Johnson, Shelley Li, Mark Schanfein, Michael Simpson, Charles Solbrig

The development of a standard approach to the safeguards of pyroprocessing facilities is a vital step in the adoption and development of this technology

NN119

Large-scale investment in pyroprocessing facilities will only become acceptable if comprehensive safeguards are established to meet the International Atomic Energy Agency’s (IAEA) standards for detecting the diversion of significant quantities of nuclear material in a timely manner. Without these safeguards, proliferation concerns will impede the commercial adoption of pyroprocessing and limit the development and expansion of fast reactor technology.

The primary objectives of this laboratorydirected research and development (LDRD) project are to determine the validity of existing safeguards approaches for pyroprocessing facilities and exploit the unique attributes of the pyroprocess to identify new safeguards methods. Idaho National Laboratory's (INL) development of these pyroprocessing safeguards will constitute a new sub-field in reprocessing safeguards and therefore will bring INL recognition as the preeminent experts in international safeguards applied to pyroprocessing facilities. INL's unique pyroprocessing facilities create an ideal opportunity to take the lead on this topic.

The present-day lack of a comprehensive safeguards approach that can meet the IAEA goal quantity and timeliness criteria for pyroprocessing facilities is the fundamental driver of this project. Without it, the IAEA is unable to determine that the state is in compliance with its treaty obligations for peaceful use of the nuclear material. The National Nuclear Security Administration's (NNSA) Next Generation Safeguards Initiative (NGSI) contains a requirement to develop a safeguards approach for pyroprocessing facilities.

In support of the development of a comprehensive safeguards approach for pyroprocessing, this report summarizes the nuclear safeguards goals, objectives, diversion scenarios, and mitigations for a hypothetical pyroprocessing facility that would be subject to international nuclear safeguards as implemented by the IAEA. Special attention is placed on evaluating the existing international safeguards perspective and exposing/researching new opportunities for pyroprocessing that have not been thoroughly examined by previous efforts. These prior efforts relied on several assumptions that have not been tested. Some of these assumptions include homogenous salt mixtures, a perfect understanding of the $\mathrm{Cm} / \mathrm{Pu}$ ratios, consistent $\mathrm{Cm} / \mathrm{Pu}$ ratios, development of a frontend destructive analysis technique to determine transuranic isotopic ratios, and non-destructive assay (NDA) equipment which functions to set specifications when measuring high-density salts. In this work, these assumptions will be verified or rejected by comparison to actual operating experience and a safeguards approach including the unique diversion detection techniques that can be developed for an actual pyroprocessing facility.

Furthermore, the previous work did not outline specific diversion scenarios. The approaches were generic and did not consider specific threats. In light of this deficiency, this report contains a detailed review of pyroprocessing-specific potential diversion scenarios. However, this report is provided in support of further discussion regarding the implementation of international nuclear safeguards at a pyroprocessing facility, and at this stage, only limited evaluation has been made regarding the credibility or attractiveness of the scenarios indicated. The development of pyroprocessing safeguards also provides a basis for specifying IAEA safeguards equipment for the pyroprocessing facility, and examples of relevant equipment are provided herein. Ultimately, the IAEA-type safeguards equipment will be installed in the pyroprocessing Fuel Conditioning Facility (FCF) at INL as a center for training, testing, demonstrating, and optimizing safeguards equipment and methods for pyroprocessing.

\section{Technical Objectives}

This year the project was oriented toward establishing a knowledge base of the threats associated with the pyroprocess, leading to several parallel technical objectives:

- Determine the context under which the safeguards engineer would require the assumptions previously mentioned 
- Evaluate the safeguards assumptions from existing technical records

- Determine the specific threats to each of the material balance areas (MBAs) of an example pyroprocessing facility

- Develop mitigation strategies within the existing safeguards techniques commonly employed to meet these specific threats

- Evaluate new techniques and technologies that could be applied to electrometallurgical refinement.

\section{Results and Accomplishments}

Research began with a safeguards red-team effort in discussions with the operators of the INL pyroprocessing facility, with a concurrent effort to address the known vulnerabilities. This lead to the following final conclusions:

- $\quad$ Fundamentally, the safeguards approach would be comparable for pyro and aqueous reprocessing plants and is dictated by the IAEA Safeguards Criteria for reprocessing plants, irrespective of which process is used.

- The safeguards approach for the pyroreprocessing plant should be easier to implement, less complex, and less costly because the reference facility is far smaller and the throughput is $1 / 25^{\text {th }}$ that of an aqueous reprocessing plant.

- Verification of spent fuel needs to be improved in both cases to detect the undeclared removal of spent fuel rods upon receipt. Consequently, there is a need to verify the uranium, U-235, and plutonium content of spent fuel to an accuracy of approximately $\pm 3.0 \%$.

- The uranium, U-235, and plutonium content in spent fuel dissolver (electrorefiner) solution needs to be verified in both cases, using on-line methods.

- Methods need to be developed and optimized to verify the uranium, U-235, and plutonium content in the electrorefiner solution by destructive analysis (DA) or nondestructive analysis (NDA) to the level of accuracy for spent fuel dissolver solution in an aqueous reprocessing plant.

- Methods need to be developed and optimized to verify the uranium, U-235, and plutonium content in the casting furnace solution by DA or NDA to the level of accuracy for MOX product powder in an aqueous reprocessing plant.

- NDA methods need to be optimized to verify the uranium, U-235, and plutonium content in uranium/plutonium buttons by NDA to the level of accuracy for MOX product powder in an aqueous reprocessing plant.

- There is a need to verify the uranium, U235 , and plutonium content in the hulls and HAW removed from the pyroprocess. There is a comparable need for verifying these wastes from the aqueous process as well. Techniques have been applied for this purpose, but are still being optimized. It is likely that similar systems could be adapted for assaying the waste produced by the pyroprocess.

- The metallic form of the product button from the pyroprocess is no more amenable to weaponization than the MOX product from an aqueous reprocessing plant. Consequently, the metallic form of the product is not more attractive to diversion.

- If residual radioactivity were left in the product button from the pyroprocess, it would make the material harder for a wouldbe diverter to handle, but the uranium- and plutonium-bearing material would still need to be subject to rigorous safeguards.

- International safeguards could be applied to a development-scale pyroprocess with existing safeguards measures and tools. However, the development and optimization of analytical methods noted above would ensure that future safeguards at such facilities are effective and efficient. Without optimized analytical tools, it would be challenging for the IAEA to independently and efficiently verify safeguards sample taking, treatment, and analysis, in a large-scale commercial facility. A 
comparable problem exists in efficiently safeguarding aqueous reprocessing plants.

This set of known vulnerabilities can now be targeted with specific technologies and provides the base document from which INL can suggest exploration of pyroprocessing. Without these conclusions, the sponsor would not be able to define what is and what is not vulnerable in pryoprocessing. Furthermore, the parallel technologies are being reoriented to address these specific concerns; INL has the primary vulnerability knowledge and is able to aggressively address these impediments to a more complete fuel cycle.

\section{Summary and Conclusions}

To date this LDRD has been able to explore the weaknesses and strengths of pyroprocessing from a safeguards perspective. Through a rigorous red team analysis of the mitigations, the major remaining impediments to safeguardability have been determined. The majority of these concerns can be addressed using existing IAEA technology, which INL is purchasing and will be able to demonstrate the first truly safeguarded pyroprocessing facility.

We are therefore in the best of situations: we have the primary capability, the sponsors are interested in the research, and we are building and transferring institutional knowledge. After this equipment is installed and we begin addressing the other significant pyroprocessing concerns, this should open an entirely new subportfolio for INL. 


\section{Radionuclide Collection-Detection Device for the in situ Remote Monitoring of ${ }^{99} \mathrm{Tc}$ as a Proliferation Indicator}

Troy J. Tranter, Mitchell R. Greenhalgh

Develoment of an integrated radioisotope-collection-detection device capable of concentrating and detecting specific beta- or alpha-emitting radioisotopes in situ

NN120

The objective of the research is to produce the experimental data necessary for the development of an integrated radioisotopecollection-detection device capable of concentrating and detecting specific beta- or alpha-emitting radioisotopes in situ and transmitting the data to offsite locations via telemetry and satellite link. The device will enable the target radioisotope to be concentrated inside the actual detector, providing very high counting efficiencies and the ability to passively collect the radionuclide while the device is in sleep mode. More specifically, the work under this proposal will be focused on a device for concentrating and measuring the fissionproduced radionuclide technetium-99 $\left({ }^{99} \mathrm{Tc}\right)$, which can be used as a key identifier of nuclear fuel reprocessing, to recover ${ }^{239} \mathrm{Pu}$, or uranium enrichment, to produce highly enriched ${ }^{235} \mathrm{U}$. There are several reasons that make ${ }^{99} \mathrm{Tc}$ a compelling choice as a signature for accessing potential proliferation programs. In addition to a long half-life $\left(2.13 \times 10^{5} \mathrm{y}\right)$ and a high fission yield $(\sim 6 \%)$, the most notable characteristic is that ${ }^{99} \mathrm{Tc}$ exists mainly as the pertechnetate anion, ${ }^{99} \mathrm{TcO}_{4}^{-}$, in natural waters. This chemical characteristic results in ${ }^{99} \mathrm{Tc}$ being much more mobile in natural waters than most fission products since it is not easily exchanged onto sediment or geological surfaces. The ability to accurately detect and quantify ${ }^{99} \mathrm{Tc}$, is of primary importance in determining, monitoring and assessing declared or undeclared nuclear proliferation activities.

Most of the current work on selective uptake of technetium has been centered on contaminated water sources with relatively high concentrations of ${ }^{99} \mathrm{Tc}$. According to our calculations, a selective-extractive resin must be able to achieve concentration factor of about $1 \mathrm{E}^{5}$ to $1 \mathrm{E}^{6}$ of $\mathrm{Tc}$ relative to what is in the water. These calculations are based on an earlier Savannah River study conducted to determine Tc concentrations in groundwater at select distances from fuel reprocessing activities. Previous work has utilized commercially available anionexchange resins that are macro-reticular polymer supports loaded with quaternary amine extractants. These resins have been loaded from 15-40 weight percent extractant, and the surface area of these resins has been compromised due to this loading. The measured nitrogen BET surface area of these resins was $<5 \mathrm{~m}^{2} /$ gram.

Stoichiometrically, the 15-40 weight percent loaded resins provide several orders of magnitude more extractant than is needed for the amount of Tc present in natural waters. Our approach is to prepare several Tc extractive resins that have a much lower extractant loading and higher surface areas in comparison to the commercially available resins. It is expected that this lower extractant loading will allow the resins to have a much higher surface area and will improve the mass transfer efficiency and equilibrium capacity for Tc. Equilibration experiments on the newly prepared resins will help elucidate the best combination of extractant loading and surface area to achieve the highest concentration factors necessary for trace level detection of ${ }^{99} \mathrm{Tc}$.

\section{Technical Objectives}

1. Prepare new high surface area resins for selective extraction resin testing.

2. Perform equilibrium testing of new resins with technetium.

3. Prepare equilibration isotherms of new extractive resins with technetium.

Results and Accomplishments

Numerous extraction resins were prepared using a dry impregnation technique, which involves loading quaternary amine extractants onto several different support materials with varying surface areas. The prepared resins were analyzed for surface area, utilizing nitrogen BET, and it was found that high-surface-area resins were successfully made with surface areas ranging from $500 \mathrm{~m}^{2} /$ gram to $950 \mathrm{~m}^{2} / \mathrm{gram}$. This analysis proved the hypothesis that by lowering the extractant loading of the support material, higher surface area resins loaded with Tcselective quaternary amines could be synthesized. It is expected that these highersurface-area resins will have improved masstransfer efficiencies and will increase the Tcconcentration factors proportionally. Figure 1 (next page) is a picture of the synthesis of these new resins. 


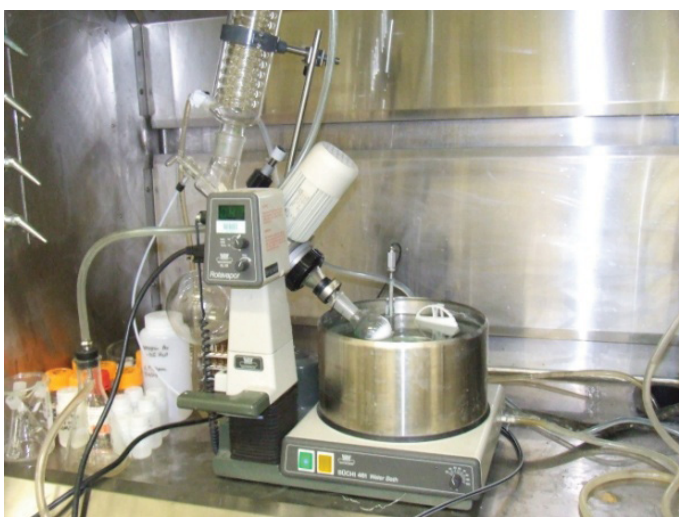

FIGURE 1. New resin being prepared in a rotary evaporator.

Preliminary equilibration experiments were performed using the newly synthesized extraction resins to begin developing equilibration isotherms. Initial data demonstrated that with very low extractant loading (e.g., 0.1$1.0 \mathrm{wt.} \%$ ), mass transfer efficiencies were quite low. It is expected in Fiscal Year 2010 (FY-10) that experiments will be continued to elucidate the optimum extractant loading vs. surface area for the best achievable concentration factors of Tc. The equilibration isotherms will be used to determine this information. During the course of these equilibration experiments, it was also found that the resins extract $\mathrm{Tc}$ much better in the presence of some form of ionic strength in the water (i.e. concentration factors were much better with the resins in ground water than in deionized water). This is a very positive result as the ultimate goal of the project is to create a detector that can concentrate Tc from natural water with little or no pretreatment necessary.

\section{Summary and Conclusions}

The experimental results to date have proven that by varying the extractant loading on the polymer support, the characteristic supportsurface areas can be retained. The retention of these surface areas is expected to provide better mass transfer efficiencies than the commercially available resins and concentrate $\mathrm{Tc}$ to levels that can be detected in situ. Further experimental work to determine the optimum loading vs. surface-area concentration factors and the development of a radioisotope-collection-device is anticipated in FY-10.

This work will fill a niche in the proliferation detection area that has not been previously exploited to its fullest potential. The capability gained from this work would add a needed tool for the detection of undeclared activities as well as the monitoring of declared activities. The expertise and capability gained from this work will strengthen the Idaho National Laboratory's reputation among the various national security agencies and will ensure that we continue to play a growing and pivotal role in the nuclear nonproliferation arena. Science and engineering advances gleaned from this project will very likely merit funding from sponsors such as the National Nuclear Security Administration and the International Atomic Energy Agency for further research and development work. 


\title{
PAGE FOR CENTER FOR ADVANCED ENERGY STUDIES DIVIDER
}

\author{
Center for Advanced Energy Studies
}




\title{
Microstructural Evolution During Spark Plasma Sintering of High-Temperature Fuels and Coatings
}

\author{
Mark Carroll, INL; Darryl Butt, BSU; Megan Frary, BSU; Batric Pesic, U of I; \\ Alan Hunt, ISU \\ Spark plasma sintering capabilities \\ CA104
}

Spark plasma sintering (SPS) is an approach for densification of powder compacts through rapid application of a pulsed DC current. While moderate pressures are induced, the compaction of the premixed powders occurs largely due to the reaction between proximate particles under the applied high current. Theories differ over whether this sintering action is due to actual plasma formation or relatively simple joule heating, but in either case powders that would otherwise be extremely difficult to form into compacts via casting (due to high melting temperatures) can be densified, and at a rate that is rapid enough to limit grain growth effects or elemental stratification. Additionally, the limited cycle times for producing compacts allows for functionally graded materials (FGMs) to be formed with discrete layers with limited intermixing. The applications for the types of high-temperature materials with highly refined compositions that can be formed through spark plasma sintering vary widely, but the technology was not available through Idaho National Laboratory (INL) prior to the initiation of this laboratory-directed research and development (LDRD).

\section{Technical Objectives}

- Coordinated the efforts of the three Idaho universities and INL to fund and operate the SPS-515S in the INL Research Center (IRC)

- Combined the skill sets of each of the co-PIs to initiate and foster numerous graduate student projects leading to degree advancement at all three universities

- Successfully sintered and evaluated microstructures in numerous powder compositions under project- and studentspecific experimental matrices in order to optimize sintering conditions for specific applications

- As was originally intended, moved the SPS$515 \mathrm{~S}$ and supporting equipment to the newly established Center for Advanced Energy Studies (CAES), opening up the opportunity for widespread future use in the Advanced Materials Laboratory for any of the participants in the CAES consortium.

\section{Results and Accomplishments}

During Fiscal Year (FY) 2009, the major objective for the INL-based principal investigator was to facilitate the relocation of the equipment to CAES. Use of the equipment at IRC had progressed during the previous year, but the implications of bringing new students into the program was hampered by the requirements associated with student access to INL laboratories. Relocating the SPS capabilities to CAES (Figs. 1a and 1b) allowed the equipment to be located in a facility designed to be cohabitated by a large number of member institutions, with the largest footprint being occupied by INL and the three Idaho universities that were already partnered in this LDRD.

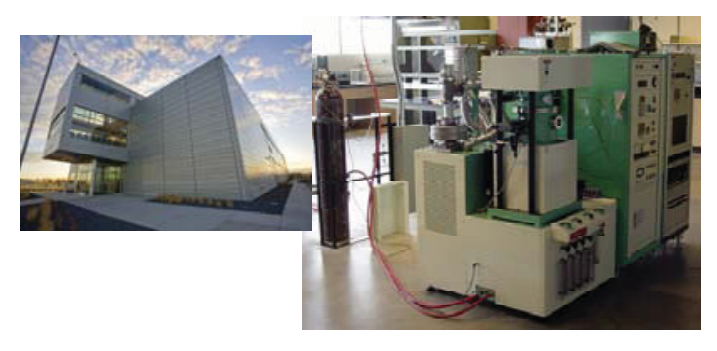

(a)

(b)

FIGURE 1. a: CAES stock photo-www. CAESenergy.org. $b$ : The new location for the SPS-515S at the CAES building in the Advanced Materials Lab. Shown from left to right are the vacuum sintering chamber, DC pulse generator, and computer control system - all integrated into a single unit.

The move to the CAES facility was challenging, especially dealing with the growing pains associated with occupation of a new facility. The SPS was the first major piece of equipment to begin operations at CAES not long after the building's official dedication. With the requisite modifications to the lab, certification of the equipment to state code, re-energizing of the SPS system, and adoption of CAES-based procedures and policies, the use of the equipment could be re-instigated by the operators that had been qualified prior to the move from the IRC building - two from INL's Center for Space Nuclear Research, two from Boise State University, one from the University of Idaho, and one from Idaho State University. Training programs were put in place to qualify new users, both through CAES orientation policies and 
equipment operating procedure establishment. To date, one new user has been qualified from the University of Idaho and four from Boise State University.

Interest in the capabilities of this SPS system has largely centered on development of nuclear fuels, providing the motivation to expand the capabilities available for SPS use. Precompaction of "hot" materials - particularly $\mathrm{UO}_{2}$ powders - requires a controlled atmosphere for powder handling and graphite mold assembly. Through the efforts of a number of collaborators, funding was generously added to the original LDRD for the purchase of a large glove box through Premier Technologies. Premier was also a valuable contributor to the project, providing the glove box presently being assembled at CAES at a substantial discount. The acquisition of this equipment through the associated benefits to this LDRD will further expand the unique capabilities of the SPS equipment for INL and energy-based research in nuclear fuel development.

The focus of this LDRD was clearly not the straightforward purchase and setup of equipment. The students from each of the member universities made considerable gains in the overall understanding and optimization of the densification of various materials and the understanding of associated microstructural evolution of the powders following varying sintering cycles. Figure 2 shows an example of the types of densified compacts that resulted from SPS processing. As seen in the micrographs, the individual grains or powder granules can remain largely intact, while the
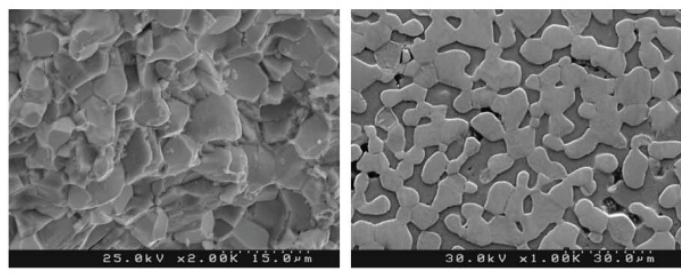

FIGURE 2. Sintering of $\mathrm{Ni}-20 \% \mathrm{~W}$ (left) and $\mathrm{Ni}-10 \% \mathrm{~W}$ (right) powders shows the appreciable level of densification and sintering achievable through optimization of the SPS process (Courtesy Jeffrey Perkins, Ph.D. candidate, Boise State University). resulting material shows almost complete densification and clear evidence of cross-particle sintering. The co-continuous nature of the alloy shown on the right demonstrates the impressive "alloying" effect of the process while retaining a clear phase-based microstructure. Figure 3 demonstrates a direct comparison between the resulting microstructure in two ceramics - one using SPS processing (left) and one using conventional hot press technology (right). The specific tasks of each of the individual university subcontracts were largely divergent in the actual materials being investigated, but carried the common goal of optimizing the process for specific applications - from powder production to microstructural evaluation. A large degree of cooperation was also encouraged between the universities in order to take advantage of the individual talents that each university brought to the table, with specific regard to material handling, fuels development, and electron microscopy.

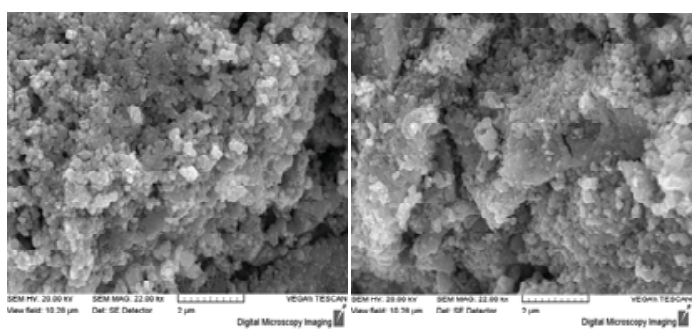

FIGURE 3. Doped ceria powders comparing SPS (left) and hot press (right). Note uniform SPS structure (Courtesy Frank Battick, M.S. student, University of Idaho)

\section{Summary and Conclusions}

The purchase, use of, and resulting research to date using the SPS and ancillary equipment has resulted in a highly successful LDRD project. The applications for which this equipment is the only viable option for material production, both through nuclear fuel-based materials and beyond, are formidable. The SPS$515 \mathrm{~S}$ is one of the few spark plasma sintering systems in the United States that is capable of sintering materials at microbar vacuum levels, increasing the potential for compaction of ceramic or composite materials that simply cannot be produced elsewhere. While the location and intended use of the equipment at CAES limits the specific INL-based proprietary 
use, numerous research projects will continue to make the availability of this equipment beneficial for INL projects. Several projects continue in the Center for Space Nuclear Research and will benefit from SPS material production, and at least one NEUP proposal has been funded that specifically requires SPS processing for highly advanced alloy development. In addition, the member universities (Idaho State, the University of Idaho, and Boise State University) continue their work as present students work through their respective degree programs, and at least one new university (the University of Wisconsin) has been granted access to the equipment for two students to visit Idaho Falls and carry out research specifically on nuclear fuels development. 


\section{Suitability of Layered Basalt as Targets for Industrial Carbon Dioxide Sequestration}

T. McLing, R. Podgorney (INL); J. Fairley (UI), S. Mooney (BSU), S. Hughes (ISU),

K. van Wijk (BSU)

Developing a systematic methodology by which the potential for geologic sequestration of $\mathrm{CO}_{2}$ for a given mafic rock site can be estimated

Carbon sequestration in mafic (basalt) lava

flows in various igneous provinces, such as the

Columbia River Basalt Group, the High Lava

Plains of eastern Oregon, the basalts of the

Snake River Plain in Idaho, and mafic units in general, require the development of a systematic methodology to estimate the potential for geologic sequestration of greenhouse gasses. Variations in geologic setting and lithology within and between basaltic provinces require that each system be considered uniquely in terms of the viability for carbon sequestration. Volcanic and structural complexities present local and regional problems, although the overall characteristics of basalt formations may translate to other mafic systems. Flood basalt provinces are generally considered more applicable to storage, in principle, due to relative uniformity of flow thickness and lithology. However, volcanic complexities in some provinces, such as in the Eastern Snake River Plain (ESRP) of Idaho, provide challenges related to this assumption as well as an opportunity to evaluate how complexities can be addressed in practical situations.

The current trend in geologic carbon sequestration is moving towards large-volume injection projects in deep saline aquifers. However, transportation of $\mathrm{CO}_{2}$ to the nearest suitable saline aquifer may not be economically feasible to small- and medium-scale $\mathrm{CO}_{2}$ producers, as well as to $\mathrm{CO}_{2}$ producers in developing countries. This project is developing a methodology for evaluating the suitability of a layered fractured basalt reservoir, examining the costs of injection with the volume of $\mathrm{CO}_{2}$ injected, allowing for evaluation of on-site or near-field sequestration. This will offer the best opportunity for a scalable carbon sequestration technology - that is, one that is economically viable for small producers of $\mathrm{CO}_{2}$ as well as for large producers.

\section{Technical Objectives}

The primary objective of the research is to develop a systematic methodology by which the potential for geologic sequestration of $\mathrm{CO}_{2}$ for a given mafic rock site can be estimated, based on physical, chemical, technical and economic considerations. In order to meet the main objective, a number of diverse technical objectives have been developed based on geologic, geophysical, numerical modeling, and economic research activities with the various tasks being preformed by co-principal investigators (co-PIs) from Idaho State University (ISU), the University of Idaho (UI), Boise State University (BSU), and Idaho National Laboratory (INL). The type locality for the research is the central ESRP, where the PIs involved have considerable experience and recognized expertise.

\section{Results and Accomplishments}

\section{ISU_Lead Dr. Scott Hughes. Dr. Hughes} advised a graduate student for the completion of an ESRP study in order to identify the major parameters needed to assess the sequestration potential of a site. This work resulted in the publication of a master's thesis and a master's degree for Ms. Desire Staires. The work identified five key parameters needed to assess the appropriateness of the reservoir (specifically the ESRP) as a potential storage site: (1) porosity and permeability and available pore volume, (2) the depth and pressure necessary to inject and maintain $\mathrm{CO}_{2}$ in its supercritical phase, (3) the mineralogy and geochemistry of basalts, (4) the morphology and size of volcanic features, and (5) subsurface stratigraphy with an emphasis on sedimentary interbeds as a potent trapping mechanism.

Of particular importance in the results of ISU's activities is a scaling relationship between the surface expression (area) of a low-angle shield volcano and the volume of sequestration capacity that is possible. This information is critical for capacity estimates at a potential sequestration site and will be used by others on the research team in FY-09.

\section{UI-Lead Dr. Jerry Fairley. The locations} of potential target zones for $\mathrm{CO}_{2}$ injection are thousands of feet below land surface and cannot be inspected directly; therefore, this problem must be approached through the statistics of basalt property distributions. Dr. Fairley and $\mathrm{Ph}$.D. candidate Ryan Pollyea gathered data on the hydraulic properties of basalt flows for outcrops exposed near Arco, Idaho. The data were analyzed to develop statistical models of basalt properties to estimate the volume available 
for $\mathrm{CO}_{2}$ storage and the interconnectivity of injection horizons. Similar data have been gathered from boreholes in previous studies (e.g., Welhan and Reed, 1997; Welhan and Wylie, 1997; Gégo et al., 2002), but the long distances between wells (hundreds of meters to kilometers) proved insufficient for a model of spatial variability at the resolution required for geologic $\mathrm{CO}_{2}$ sequestration (i.e., meter-scale resolution).

The key result of U of I's activities is the small- to large-scale spanning statistical distribution model of basalt hydraulic properties. While significant in itself, this model will be combined with the results from ISU to form the basis for more accurate capacity estimates and the backbone for large-scale reservoir modeling with statistically generated property distributions. The work completed to date was presented at the 2008 annual meeting of the American Geophysical Union in San Francisco, CA, by graduate student Ryan Pollyea. This work resulted in the publication of a master's thesis and a master's degree for Ms. Desire Staires. Ryan is currently working on an article for publication describing the use of spatial statistical methods in planning and executing a sampling campaign for heterogeneous (fractured) rock domains. In addition, the work completed and currently in progress is being submitted to an international conference on $\mathrm{CO}_{2}$ sequestration in Trondheim, Norway.

INL_Lead Travis McLing and Robert Podgorney. INL's primary accomplishments included:

1. Building the numerical framework for largescale computational evaluation of carbon sequestration capacity in plains-style volcanic terrains. The numerical framework model was used initially to develop parameter sets for inclusion into economic models. A paper on the reservoir-economic model was presented at the 2008 CCS conference in Pittsburgh, PA, in May.

2. Planning and hosting a workshop titled "Carbon Management in Idaho," at which a number of invited presentations involving carbon management were made to an audience of Idaho legislators, regulators, academics, and the public at large. The workshop was conceived to be an educational and awareness-raising venue for the State of Idaho. Workshop attendees sent positive feedback.

3. INL researches are also responsible for project management activities.

In the final phase of this project, a numerical modeling framework will incorporate the results for ISU's and U of I's Year 2 results for largescale simulations of supercritical $\mathrm{CO}_{2}$ flow and transport in ESRP's fractured basalts.

BSU Geophysics - Lead Dr. Kasper van Wijk. Dr. van Wijk numerically investigated changes in the general character of seismic waves before and after an injection of $\mathrm{CO}_{2}$ in the reservoir. The goal was to establish whether seismic waves can remotely monitor sequestered $\mathrm{CO}_{2}$ in flood basalts. To do so, numerical elastic wave equation simulations were performed to model surface-to-surface (i.e., reflection) seismics, surface-to-borehole (i.e. vertical seismic profiling, or VSP), and down-hole seismics (where source and receivers are in or near the reservoir). The code we used is a Spectral Element code by Komatitsch and Tromp, well-established in basin and whole earth seismology. The geologic model is digitized based on well logging information in an area of flood basalts, characteristic of the northwestern USA. Since it is currently poorly understood what effect $\mathrm{CO}_{2}$ will have on the elastic properties of the reservoir (both short- and longterm), we researched different scenarios in which the $\mathrm{CO}_{2}$ imposed different degrees of elastic changes on the reservoir.

This work is currently ongoing and will be integrated with the large-scale numerical modeling activities during the final phase of the project. The basalt property sets will be replaced with statistical model results from ISU and U of I, and be based on the modeled distribution used in the reservoir models. In this way, a realistic monitoring scheme can be tested.

BSU Economics — Lead Dr. Sian Mooney. Dr. Robert Podgorney (INL) and Dr. Sian Mooney developed broad parameters for a hypothetical $\mathrm{CO}_{2}$ sequestration site in Idaho. Dr. Podgorney ran some preliminary models that 
could demonstrate how $\mathrm{CO}_{2}$ would move through a given type of simplified basalt geology. These joint activities resulted in a presentation at the CCS annual meeting. While these scenarios were being completed, Dr. Mooney continued to work on using secondary economic data to estimate the economic impacts of environmental changes on the value of natural resources in Idaho using the example of open space provision as a test case. This activity resulted in a working paper from the Department of Economics at Boise State University. This experience at valuing economic changes as a result of some external policy change was a good learning tool for continuing to focus on the possible economic changes as a result of sequestering $\mathrm{CO}_{2}$ in geologic formations.

Business prospects:

- U of I: In January 2009. PI Fairley traveled to Trondheim, Norway, to meet with representatives of StatoilHydro's research group on geologic carbon sequestration. We are currently waiting on word regarding funding from StatoilHydro for future research on $\mathrm{CO}_{2}$ migration in vertical faults.

- BSU: Murari interned with Shell last summer, teaming up to run fluid flow simulations for $\mathrm{CO}_{2}$ sequestration. Shell is working on this problem. Potentially, there are options for collaboration between INL and Shell.

- INL

- Contract nearly complete for Shell WFO (\$750K) FY-09-11.

- Abledo Technologies CCS and Biofuel project $(\$ 3000 \mathrm{~K})$ performance period still being determined

- Zurich Financial Site characterization, still in negotiations.
Accomplishments and Awards

- ISU: Developed a reasonable assessment of the overall potential for carbon storage in ESRP basalts based on the morphology of eruptive centers

- Uof I: Developed a statistical/mathematical properties model for a generic basalt flow for input to numerical simulations of $\mathrm{CO}_{2}$ injection

- INL: Developed numerical physical multiphase model framework and methods to interface with statistical properties and economic models

- BSU: Developed the procedure to create a synthetic model for basalt of the ESRP for MMV activities

- BSU: Developed theoretical framework for economic analysis

- Two PIs have been appointed to the Idaho Carbon Issues Task Force (McLing and Mooney)

- $\quad$ Paper submitted to Hydrology: Pollyea, J.P. Fairley, R.K. Podgorney, and T.L. McLing, "Geostatistical characterization and sampling of fracture heterogeneity in lowvolume pahoehoe basalts, East Snake River Plain, Idaho." 


\title{
Understanding Apomixis: The Basis for a Robust Trait Delivery and Containment Platform for Bioenergy Crops
}

\author{
Thomas H. Ulrich, Jeffrey A. Lacey \\ Exploring the potential of using a barley stripe mosaic virus as a VIGS vector in sorghum \\ CA108
}

Natural apomictic plants, e.g. dandelions, Kentucky bluegrass, and many varieties of crab apples, blackberries, citrus and others, produce seeds that are genetic replicas of the maternal plant. In such plants the male gender is effectively abolished, and embryos form from somatically derived cells. Understanding and harnessing apomixis could revolutionize crop genetic engineering and seed production systems including those of bioenergy crops. With this technology, trait delivery and containment platforms could be developed for "delivering" quality and productivity traits while "containing" the delivered traits in conventional and dedicated bioenergy crops so they do not escape into other crop plants or weeds through cross pollination.

Results from gene expression studies comparing sexual sorghum plants to apomictic sorghum plants have shown several genes that are differentially regulated. These genes represent candidate apomixis-causing genes. A virus-induced gene silencing system (VIGS) using barley stripe mosaic virus (BSMV) was used to attempt to manipulate gene expression in sorghum. Our collaborators at the University of Idaho have used this system to successfully silence gene expression in wheat. Our goal was to use this system in sorghum to manipulate the expression of the candidate apomixis-causing genes to affect the development of apomictic sorghum ovules to gain a better understanding of the molecular mechanisms that control apomixis.

\section{Technical Objectives}

- Develop barley stripe mosaic virus for use as a VIGS vector

- Show that the up or down regulation of candidate apomixis-causing genes in sorghum affected the development of the early embryo sac.

\section{Results and Accomplishments}

We began this research using BSMV virus because the literature indicates that sorghum is susceptible to BSMV, and our collaborators at the University of Idaho suggested this virus as good candidate for our research. Despite our best efforts, we were unable to infect sorghum with BSMV; however, we were able to infect wheat, indicating that our methods were adequate, at least for wheat (Fig. 1). There are two possible reasons that we were unable to infect sorghum. First, our methods may have been inadequate and require further refinements. We applied the viral genome in its RNA form directly to the surface of the leaves. RNA is highly susceptible to degradation, and the sorghum we worked with may have degraded it to a higher degree than the wheat. If the RNA genome is degraded, infection would be inhibited. Normally, the virus has a protein coat that protects its RNA genome. The lack of the protein coat may be the reason that we were unsuccessful. In future experiments, we could obtain the complete virus from an infected wheat plant and use that to inoculate sorghum.

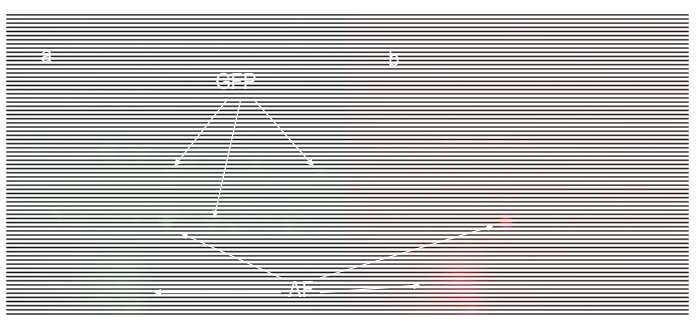

FIGURE 1. Fluorescent microscopy imaging of the same inoculated wheat leaf surface using a GFP optimized filter set (a) and a longer wavelength filter set that excludes GFP fluorescence (b). Note that autofluorescence is visible in both images. The fluorescence that only appears in image " $a$ " was determined to be due to the presence of GFP.

The second reason that we were unable to infect sorghum could be that the literature is incorrect, and sorghum is not susceptible to BSMV, or the specific strain of the virus used was incompatible with the cultivar of sorghum used. All of these possibilities will require further testing. The intent of this work was to develop BSMV as a VIGS vector in sorghum to characterize putative apomixis-causing genes; however, we may need to consider other viruses for development, or completely different methods, such as knock-out mutants or overexpressing mutants.

The results of this research have been included as a chapter in Jeff Lacey's Ph.D. dissertation, which he successfully defended on September 15, 2009. The final draft was approved by Jeff's advisor, Bob Zemetra.

In order to publish our findings, the microarray results needed to be validated. To do this, quantitative polymerase chain reaction 
(qPCR) was performed. New ovule samples from both apomictic and sexual sorghum were collected. PCR primers were designed to amplify a short sequence in six highly up-regulated genes and six highly down-regulated genes. Initial testing of these primers on sorghum genomic DNA showed that 10 primer pairs provided good PCR products. qPCR was performed on the RNA extracted from the ovule samples. Nine genes on the microarray were validated, as six genes found to be up-regulated in the microarray results were also up-regulated in the qPCR results, and three genes found to be downregulated in the microarray results were also down-regulated in the qPCR results (Fig. 2). The magnitude of the results differed in some genes; however, the up or down regulation of the genes matched between methods. These results validated our microarray results and have enabled us to publish our results in a variety of journals.

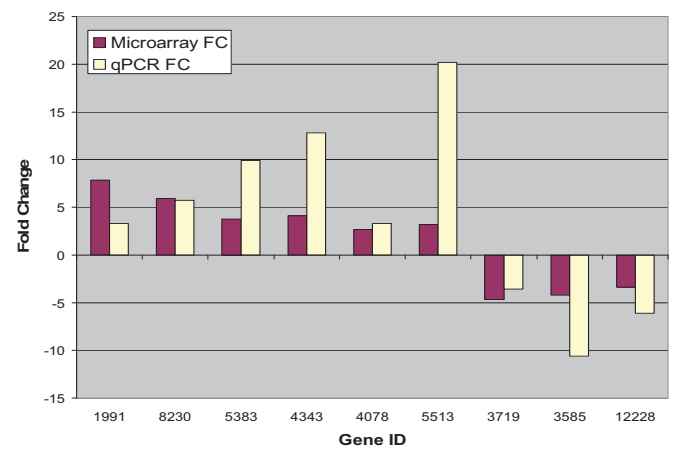

FIGURE 2. Bar chart showing the correlation of fold change $(F C)$ between results obtained with microarray gene expression profiling, and quantitative polymerase chain reaction $(q P C R)$. While the magnitude between the two methods differed for some genes, genes found to be up or down regulated were consistent between methods. The qPCR results validate the results obtained using the microarrays.

\section{Summary and Conclusions}

Further development will be needed in order to use BSMV as a VIGS vector in sorghum. It is possible that BSMV is not compatible with sorghum. Other methods for the characterization of the putative apomixis-causing genes should be considered. While not likely to be published in the literature, these results were published in Jeff Lacey's dissertation.

The microarray experiment has been validated using qPCR, and this lends strength to all of the findings of this research. Adding to this strength is the fact that the samples used for qPCR were collected at a later date than those used for the microarray research. 


\title{
Enhancement of Separation Methods in Nuclear Fuel Recycling
}

\author{
Terry Todd, Michael Simpson (INL); Thomas Hartmann, Josh Pak, Patricia Paviet- \\ Hartmann (ISU); Eric Brown, Dale Russell (BSU); Batric Pesic (U of I) \\ Developing a better understanding of lithium electrochemistry \\ CA114
}

This project was initiated in June 2007. The following four tasks were performed during Fiscal Year (FY) 2009:

1. Design and synthesis of several macrocompounds at Idaho State University (ISU) and Boise State University (BSU) by Dr. Pak and Dr. Brown, respectively

2. Extraction of technetium as anionic and cationic species by different macrocompounds (ISU)

3. Technetium waste forms (ISU)

4. Simultaneous reduction of uranium oxide and electrowinning of lithium oxide University of Idaho (U of I)

Results and Accomplishments

Task 1: Synthesis of new macrocylic ligands

New compounds have been synthesized in order to maximize the selective extraction and back-extraction of complexed technetium from acidic and basic solutions:

- Triethelene glycol monotosylate

- 2,2-Dimethylbenzo[1,3]dioxole (2)

- 5,6-Diiodo-2,2-dimethylbenzo[1,3]dixole (3)

- 4,5-Diiodocatechol (4)

- 4,5-diiodo-1,2-bis[2-[2-(2hydroxyethoxy)ethoxy]ethoxy]benzene (7)

- 4,5 diiodo-1,2-Bis[2-[2-[2-[[(4tosyl)sulfonyl]oxy] ethoxy] ethoxy] ethoxy]be nzene (8)

- $\quad 4,4^{\prime}, 5,5^{\prime}$-Tetraiododibenzo-24-crown-8 ether (9)

- $\quad$ Tetrakis $(N-2$-pyridonyl) methylresorcin[4] arene pentyl foot (12c)

- $\quad$ Tetrakis $(N$-2-benzothiazolonyl) methylresorcin[4] arene pentyl foot (12d)

- Different halogen substituted polyetherdiamides

Task 2: Extraction of technetium

Tetrakis( $N$-2-pyridonyl)

methylresorcin[4]arene pentyl foot (12c), and
Tetrakis $(N$-2-benzothiazolonyl) methylresorcin[4] arene pentyl foot (12d) were tested for the selective extraction of Tc-99 from nitric acid solution (0.1M). The low distribution coefficient of Tc-99 obtained with resorcinarenes implies that these types of macrocompounds may not be suitable for the selective extraction of Tc99. For this reason, we pursue the extraction of Tc-99 with other crown ethers and aza-crown ethers.

Seven crown ethers and aza-crown ethers with ring sizes of 12-crown-4 to 18-crown-6 (10 $\left.{ }^{3} \mathrm{M}\right)$ in methylisobutyl ketone medium were evaluated for efficiency of pertechnetate (620 Bq. $\mathrm{mL}^{-1}$ ) extraction from nitric acid solution (Table 1).

TABLE 1. Distribution Coefficient D for $\mathrm{TcO}_{4}^{-}$extraction with crown ethers from $0.1 \mathrm{~N} \mathrm{HNO}_{3}$.

\begin{tabular}{lccc}
\hline $\begin{array}{c}\text { Crown } \\
\text { Ether }\end{array}$ & $\begin{array}{c}\text { Cavity } \\
\text { Diameter } \\
(\AA)\end{array}$ & $\mathrm{D}_{\mathrm{Tc}-99}$ & $\begin{array}{c}\% \mathrm{D} /(\mathrm{D}+1) \\
\mathrm{D})\end{array}$ \\
\hline $\begin{array}{l}\text { Aza 12 } \\
\text { Crown 4 }\end{array}$ & $1.2-2.5$ & 0.82 & 45 \\
$\begin{array}{l}\text { Aza 15 } \\
\text { Crown 5 }\end{array}$ & $1.7-2.2$ & 0.785 & 44 \\
$\begin{array}{l}\text { Benzo 15 } \\
\text { Crown 5 }\end{array}$ & $1.7-2.2$ & $\mathbf{0 . 8 3}$ & 45 \\
$\begin{array}{l}\text { 1,2 Diaza } \\
\text { 15 Crown 5 }\end{array}$ & $1.7-2.2$ & 0.75 & 43 \\
$\begin{array}{l}\text { Aza 18 } \\
\text { Crown 6 }\end{array}$ & $2.6-3.2$ & 0.76 & 43 \\
$\begin{array}{l}\text { Diaza 18 } \\
\text { Crown 6 }\end{array}$ & $2.6-3.2$ & 0.78 & 44 \\
$\begin{array}{l}\text { DC18 } \\
\text { Crown 6 }\end{array}$ & $\mathbf{2 . 6 - 3 . 2}$ & $\mathbf{2 . 5}$ & $\mathbf{7 2}$ \\
\hline
\end{tabular}

DC18C6 was revealed to be the most efficient crown ether to extract Tc-99. It appears that the cyclohexano groups attached to the crown ether tend to increase the distribution coefficient of Tc-99. The presence of cyclohexano groups in DC18C6 tends to increase the basicity of the ether oxygens and also allows the ring to have more flexibility; thus, cyclohexano crowns tend to have higher binding constants for a given alkali metal ion than their benzo counterparts, which could explain the 
value of the distribution coefficient $\mathrm{D}_{\mathrm{Tc}-99}=2.5$ obtained with DC18C6 in methylisobutyl ketone.

\section{Task 3: Technetium Waste Forms}

Technetium waste forms were investigated, incorporating rare earth elements as potential fission products. Two advantages of these new waste- orms are their stability and their tolerance to radiation. In order to synthesize lanthanidetechnetium pyrochlore-type structures, pure $\mathrm{TcO}_{2}$ had to be synthesized. Therefore, anhydrous crystalline $\mathrm{TcO}_{2}$ was prepared by the thermal decomposition of freshly crystallized, white ammonium pertechnetate.

To synthesize lanthanum-technetium pyrochlore compounds, stoichiometric mixtures of $\mathrm{Ln}_{2} \mathrm{O}_{3}$ and $2 \mathrm{TcO}_{2}$ were ground in an agate mortar with methanol. After drying in air, the ground mixtures were folded in platinum foil which was sealed on both ends. The platinum coupons were inserted in Quartz tubes which were sealed under vacuum. Quartz ampoules with the platinum coupons were annealed at $1150^{\circ} \mathrm{C}$ for $48 \mathrm{hrs}$ under argon.

The sintered samples were analyzed by XRD/Rietveld (Fig. 1). The XRD pattern was taken at room temperature on a PANalytical X'Pert Pro Theta-Theta instrument using a Siwafer-type low background sample holder and a fast X'Celerator multi-Si strip detector. Highly accurate patterns were taken with step sizes of $0.008^{\circ} 2$ theta and $50 \mathrm{sec}$ per step in the 2theta range between $10^{\circ}$ and $120^{\circ} 2$ theta. The Rietveld analysis was performed using TOPAS 3 (BrukerAXS) which applied a full-parameter approach. This allowed us to calculate structure amplitudes and diffracted beam intensities and to quantify prospective impurities.

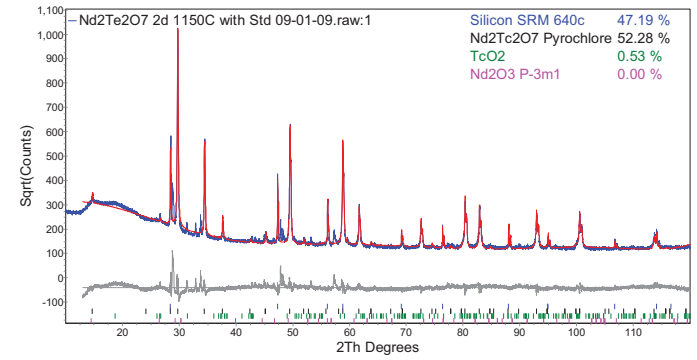

FIGURE 1. XRD/Rietveld Analysis of $\mathrm{Nd}_{2} \mathrm{Tc}_{2} \mathrm{O}_{7}$; blue pattern $=$ measurement, red pattern $=$ calculated intensities, grey pattern $=$ difference.
Task 4: Simultaneous reduction of uranium oxide and electrowinning of lithium oxide

As stated in the final report for Phase II, the understanding of reduction of $\mathrm{UO}_{2}$ with metallic lithium produced by electroreduction of $\mathrm{Li}_{2} \mathrm{O}$ depends on the understanding of electrochemistry of lithium oxide reduction. Reduction of $\mathrm{UO}_{2}$ with electrogenerated lithium happens as readily as lithium production. However, there are several mechanistic and practical problems relevant to lithium electrochemistry. The most important problem to resolve is the rate of lithium dissolution and solubility of lithium as a function of lithium oxide, uranium oxide, lithium chloride, metal, and chemical (water) impurities.

\section{Results and Accomplishments}

Significant achievements made this year include:

- The role of moisture is better understood, and a method to study lithium dissolution has been developed.

- The cathodic reaction is preoccupied with several reactions relevant to moisture, most predominantly to reduction of hydroxide from lithium hydroxide.

- Lithium hydroxide is produced by reaction of water with lithium oxide. The reaction of reduction of lithium hydroxide is characterized with extensive gaseous bubbling at the cathode.

- One of the final conclusions is that lithium dissolution cannot be studied in a single compartment cell. A two compartment cell, one for working electrode and one for counter electrode, has to be designed and used.

\section{Summary and Conclusions}

- $\quad$ Several ligands for metal ion and complex affinity studies were prepared and tested for the selective separation of Tc-99.

- Technetium waste forms were produced and characterized

- Lithium electrochemistry was studied and a better understanding developed. 


\title{
Investigation of Public Discourse Methods in Energy Policy Decision-making
}

\author{
S. Piet, J. Joe (INL); J. Freemuth, M. Louis, C. Nemnich, E. DeShazo, M. Bathrick, K. Vogt \\ (BSU); A. Hunter, S. Anderson, S. Sorensen (ISU); P. Wilson, T. Hall, P. Starkey (U of I) \\ Reducing project risk through public involvement \\ CA115
}

The ground is littered with projects that

failed because of strong public opposition. This LDRD project's objective is to add to the Energy

Policy Institute's tool box to reduce project risk through encouraging the public to engage in more critical thought and be more actively involved in public or social issues. Early in a project, project managers and decision-makers can talk with no one, pro and con stakeholder groups, or members of the public. Experience has shown that talking with no one incurs high risk because opposition stakeholders have many means to stop most (if not all) energy projects. Talking with organized stakeholder groups provides some risk reduction from mutual learning, but organized groups tend not to change positions except under conditions of a negotiated settlement. Achieving a negotiated settlement may be impossible. Furthermore, opposition often arises outside pre-existing groups. Standard public polling provides some information but does not reveal underlying motivations, intensity of attitudes, etc. Improved methods are needed that probe deeper into stakeholder (organized groups and members of the public) heuristics to increase the potential for change of opinions and/or out-of-box solutions. The term "heuristics" refers to the mental shortcuts, underlying beliefs, and paradigms that everyone uses to filter and interpret information, to interpret what is around us, and to guide our actions and decisions. Our team is two-thirds through the project to investigate stakeholder discourse methods in energy-policy decision making.

\section{Background and Motivation}

The traditional project approach is DecideAnnounce-Defend (DAD) in Figure 1. The traditional approaches to engaging stakeholder groups and individuals are neglect, education, or negotiate. "Neglect" is based on the premise that it is right and safe (from the project risk standpoint) to ignore those stakeholders potentially impacted by a decision and/or maintain that they have nothing to contribute to a decision. We believe it is neither right nor safe and that diverse stakeholders can meaningfully contribute. "Education" is based on the premise that "if only they understood, they would agree" and that many stakeholders are paying attention and are willing and able to change their positions. "Negotiation" is based on the premise that a straightforward in-between compromise between two opposite positions is worth pursuing and that those without prior positions nor organized groups representing their positions can be left out. "Negotiation" is often inadequate.

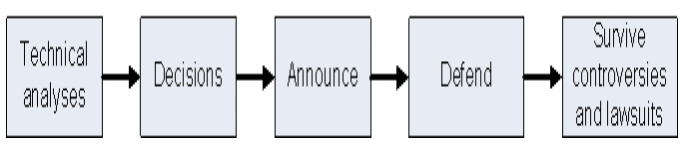

FIGURE 1. The traditional DAD project approach.

The literature and common experiences indicate that people are, and must be, "cognitive misers"; they only devote as much time to an action or decision as they perceive is required. The types of potential behavior have been described as follows with increasing cognitive effort required in going down the list: ${ }^{\text {b }}$

- Skill-based (auto pilot): Do it the way it has always been done; interpret new information on the basis of existing heuristics.

Appropriate for a familiar task or situation. Low attention required.

- Rule-based (if this, then do that): Recognize when to apply a different heuristic or weight heuristics differently.

- Knowledge-based (think it through):

Analyze the situation and develop a new approach or new heuristics if needed.

Appropriate for unfamiliar task or situation. High attention required.

A telephone poll tends to invoke skill-based behavior based on existing heuristics; there is no

\footnotetext{
a. For example, some of this team previously conducted focus groups for the potential next generation nuclear plant, which would produce hydrogen as one of its products. One participant considered hydrogen from his personal experience - the practice of making gas in his home safer by adding an odorant - and wanted to know if this would be used for hydrogen from the power plant. The "experts" had no answer.

b. Adapted from James Reason, Managing the Risks of Organizational Accidents, 1998.
} 
time for assimilation of new information or use of potential rule- or knowledge-based behavior. This tells us whether a project or energy option is supported, but does not tell us how those being sampled will respond to new information and discussion over the required years or decades of a project or $\mathrm{R} \& \mathrm{D}$ program. That would require understanding heuristics and how they may change.

So, we are left with a trap: we need to understand heuristics and how they may change, but this requires more cognitive effort than skillbased behavior and simple polling methods and thus heuristic change is both difficult to induce and difficult to measure. Only focus groups and other deliberative methods can trigger rule-based and knowledge-based behavior and probe into and possibly change heuristics. Even better would be longitudinal observations to measure time-dependent changes. This project conducted a detailed set of focus groups in FY 2009 and will probe longitudinal changes in FY 2010.

Why do we care about potential heuristics changes? Albert Einstein said, "Problems cannot be solved at the same level of awareness that created them." In other words, sometimes out-ofbox thinking is the only pathway to a sustainable solution. Second, if opinions are already polarized (as they tend to be in energy matters), only some type of change can lead to a sustainable solution that is not sabotaged by one side or the other. Third, energy projects require years if not decades to complete; knowledge, stakeholder values, and available resources often change significantly over such periods. Options that are initially in agreement or convergence with knowledge, values, and resources can drift out of convergence, leading to failure. ${ }^{c}$

Heuristics are difficult to change, as pointed out by Economics Nobel Prize winner D.

Kahneman. Heuristics change only with a shock to the system (punctuated equilibrium) or sustained pressure over time (incrementalism); change also depends on whether a person (or group) is sufficiently motivated and able to process new information. When new information

c. S. J. Piet et al, "Making Sustainable Decisions Using the KONVERGENCE Framework," Waste Management 2003, Tucson, Arizona, February 2003. is processed with existing heuristics, it can serve to merely anchor existing opinions. Heuristic changes are difficult to cause and therefore difficult to measure in semi-controlled situations. Also, the shocks or the time for changes to occur can be inadequate, as suggested in Figure 2.

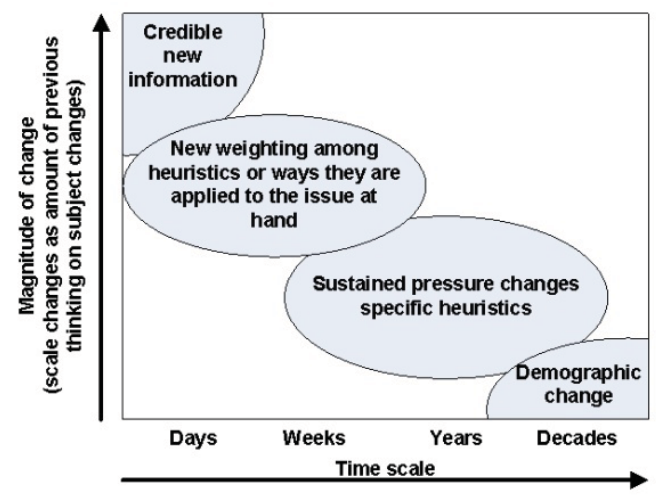

FIGURE 2. Changing heuristics, heuristic weighting, or how heuristics are applied to an issue is difficult.

\section{Technical Objectives and Approach}

The technical objectives of this research project were to answer the following questions:

- How do different types ("treatments") of public discourse affect the public's preference and resulting support for different options to meet electricity demand?

- How do different types of public discourse affect the public's support for technical research or policy alternatives that could eliminate or improve different options for meeting electricity demand?

- How do different types of public discourse and the public's intensity of opinion, psychological and demographic factors, social values, environmental factors, and assumptions affect their preference and support of different options for meeting electricity demand? As a corollary, how do these affect the likelihood and level of preference/support for improvements in energy options?

- How will the participants' evaluations of the different treatments, the speakers, mediators, and facilitators affect their support for 
different options for meeting electricity demand?

Our underlying model is shown in Figure 3. The survey instrument was designed to measure reflective (directly expressed) preference, importance among attributes, evaluation of energy options for each attribute (the weighted sum of attributes gives us the formative preference for each participant), and support measured by how much of a $\$ 100$ utility bill should be given to each energy option-fossil, nuclear, hydro, renewable, or energy conservation and efficiency. The survey instrument then asked participants to imagine a key improvement to each energy option and how that key improvement would change their preferences. This was posed to obtain "actionable" information in the sense of probing changeability of preferences and importance of potential $R \& D$ achievements. We also asked standard demographic questions, political viewpoint, and evaluation of energy expert panelists.

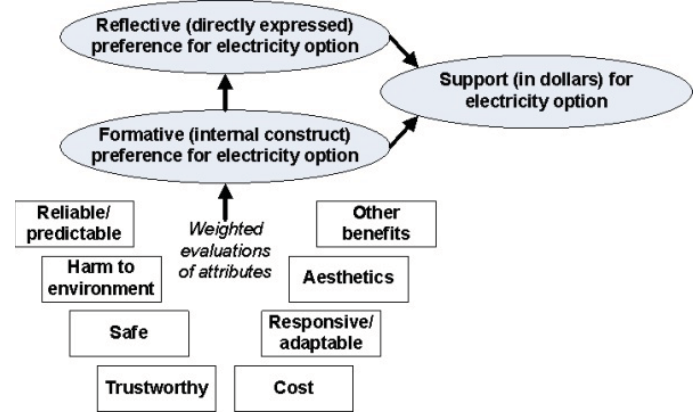

FIGURE 3. Our model motivating our experimental design.

We obtained mail-in responses and then asked a subset of responders to attend focus groups. The experimental matrix of the focus groups' varied discourse methods or "treatments" was as follows:

- Survey only — control group

- Session with seven experts (no deliberation)

- Session with seven experts and deliberation

- Briefing paper only

- Briefing paper, session with seven experts (no deliberation)
- Briefing paper, session with seven experts and deliberation.

\section{Results and Accomplishments}

This year's research effort culminated in March 2009 when 511 citizens returned a detailed energy policy survey and April 2009 when 76 Idaho citizens from the Treasure Valley (near Boise) participated in focus groups. Analysis of the huge data set is still underway, so it is premature to give a complete description of what we are still learning. Furthermore, in the last year of the project, we will interview some of the participants to obtain some longitudinal data, e.g., did their participation lead to more or less attention to energy issues and/or did they search out new information sources?

Preliminary observations include the following:

- Reflective preference, formative preference, and support were not totally consistent with each other, although there were positive correlations among them. The treatments and expression of importance of attributes were designed to test the relationships among these three preference/support measures.

- The percent of all respondents that expressed reflective preference for energy options was $92 \%$ for renewables, $92 \%$ conservation, $87 \%$ hydro, $69 \%$ nuclear, and $47 \%$ fossil. The distribution for nuclear and fossil showed polarization, with "strongly support" and "strongly oppose" the two most common answers $(-5$ and +5 on a 9 point scale).

- The percentages of all respondents that would put a non-zero amount of their $\$ 100$ toward each of the energy options were as follows: $89 \%$ renewables, $85 \%$ conservation, $81 \%$ hydro, $66 \%$ nuclear, and $52 \%$ fossil.

- Few among all respondents would put more than $50 \%$ of their $\$ 100$ to a single energy options. "Few" is $10 \%$ for renewables, $8 \%$ 
nuclear $^{\mathrm{d}}, 7 \%$ hydro, $2 \%$ conservation, and $0 \%$ fossil. That is, $10 \%$ of respondents would put $\$ 100$ of $\$ 100$ into renewables.

- $\quad$ The eight attributes in Figure 3 were independent of each other except for some clustering revealed by a preliminary factor analysis: safety, trustworthiness, environmental harm clustered for fossil and nuclear (indicating that these reflect a combined underlying view of each technology), and safety, environmental harm, aesthetics, and other benefits clustered for hydro.

- $\quad 90 \%$ of those who received the briefing paper viewed it positively, meaning our multi-discipline and multi-viewpoint approach to preparing the briefing paper achieved credibility among those with different energy option preferences.

- Participants' assessment of the credibility of the seven energy experts varied. We are investigating cause and effect-were experts with views matching pre-test opinions viewed more credibly or did the credibility of experts lead to different post-test opinions?

- There was no decisive change in opinion on any of the energy options among focus group participants. There were increases and decreases in reflective preference among treatments. Formative preference increased slightly for conservation and fossil. Support increased slightly for fossil and decreased slightly for renewables.

- We have not yet analyzed for consistency between attribute importance as directly expressed by participants versus attribute importance back-calculated from their reflective preferences and their assessments of each energy option for each attribute.

- We have not yet analyzed for the impact of postulated R\&D achievements.

d. Recall that participants were randomly selected from Ada county Idaho, which includes Boise and is 270 miles from the Idaho National Laboratory. So, we are unlikely to have any INL employees in the sample.
- In general, the changes were subtle and conclusions are limited by the statistical power of the experiment.

The team has made these public presentations and documents.

- $\quad$ M. C. Louis and C. De Sy, Letter to potentially interested stakeholders asking input, December 12, 2007.

- $\quad$ E. R. DeShazo, et al., "Investigation of Public Discourse Methods in Energy Policy Decision Making," Annual Meeting of the Idaho Academy of Sciences, March 27, 2008.

- $\quad$ Energy Policy Institute, "Options for Meeting Electricity In Idaho,” March 23, 2009.

- $\quad$ M. C. Louis, presenter, Energy Policy Institute, "Options for Meeting Electricity Demand in Idaho," April 18, 2009.

- $\quad$ M. C. Louis, presenter, "Comparing Methods to Inform Public Opinion: A MultiInstitution Study on Options to Meet Electricity Demand in Idaho," American Democracy Project meeting, Baltimore, Maryland, June 12, 2009.

We have also these internal working documents:

- J. C. Joe, E. R. DeShazo, "Stakeholder and Literature Question Database," February 5, 2008.

- J. C. Joe, E. R. DeShazo, "Literature Review Matrix," March 3, 2008.

- J. C. Joe, M. C. Louis, C. D. Nemnich, E. R. DeShazo, Research Plan, "Investigation of Public Discourse Methods in Energy Policy Decision-Making," July 25, 2008.

- $\quad$ Survey questionnaire, March 2009.

Summary and Conclusions

If existing decision support tools and approaches were adequate, energy policy would not be in the condition it is in. We aim to build on and integrate multi-disciplinary efforts to improve stakeholder and public engagement. The final year of the project will complete our 
analysis of focus group results, add longitudinal data, and produce various publications.

Improving the EPI toolbox strengthens its potential as a one-stop place to shop for energy policy and adds potential value and reduces risk to INL and CAES projects. The research goal of testing these deliberative processes is to understand how we might break the current gridlock on these issues and allow real policy deliberation and learning to occur to improve dialogue and understanding; guide technology research, development, and deployment; and most importantly, facilitate our ability to make the tough decisions that will ensure our energy future. 


\section{Development of Lignocellulosic Biofuels Production Potential in Idaho

\author{
Greg Bala (INL), Kevin Feris (BSU), Tim Magnuson (ISU), Jon Van Gerpen (UI) \\ Researching potential feedstocks in Idaho and improving current capabilities \\ CA116
}

There is a compelling need for new sources of clean, inexpensive, and renewable energy to replace petroleum-based energy. One promising route of exploration includes discovery and development of new bio-energy strategies to enhance the efficiency of biomass conversion and improve our ability to harness the biofuelproducing capabilities of microorganisms. Such bio-energy strategies have significant potential for expanding our current régime of energyproducing technologies.

Advancements in biofuels research and technology have the potential to provide economic benefits for the State of Idaho. Idaho's agricultural activities result in large amounts of potential feedstocks for biofuel production, feedstocks that are often viewed as "waste." Exploiting waste feedstocks via the coupling of new biotechnologies and biofuels production facilities can create a new industry base for Idaho.

This is a collaborative effort to:
1. Conduct a survey of the potential feedstocks for ethanol production within Idaho (i.e. starch, cellulose, and lignocellulosic-based materials)

2. Investigate means for improving established and developing novel biological systems that possess the highest potential for biofuel generation capabilities.

Our goal is to use expertise and collaboration among scientists at Idaho National Laboratory (INL), Boise State University (BSU), Idaho State University (ISU), and the University of Idaho (UI) to provide solutions for the problem of high feedstock cost for biofuels production. Novel feedstocks (e.g., lignocellulosic) available within the State of Idaho's agricultural wastes or products may allow biofuel producers within the state and region to increase production capacity and improve profitability.

\section{Technical Objectives}

\begin{tabular}{|c|c|c|c|c|}
\hline & \multicolumn{4}{|c|}{ Institution } \\
\hline Objective & BSU & INL & ISU & U of I \\
\hline $\begin{array}{l}\text { 1. Evaluate regional feedstocks for biofuel production. } \\
\text { - Characterize feedstock material with respect to chemical } \\
\text { composition and potential for microbiological conversion to } \\
\text { fermentable sugars. } \\
\text { - Determine promising feedstocks for biofuel production. } \\
\text { Feedstocks will be selected based on characterization outcome } \\
\text { for study, optimization, and integration into Objectives } 2 \text { and } 3 \text {. }\end{array}$ & & & & $\sqrt{ } *$ \\
\hline $\begin{array}{l}\text { 2. Evaluation of existing and development of novel enzymatic and/or } \\
\text { whole-cell saccharification strategies for biofuel production from } \\
\text { identified regional feedstocks. } \\
\text { - Evaluate conversion potential of feedstocks using } \\
\text { commercially available enzymes and organisms as well as } \\
\text { selected lignocellulytic isolates currently available in } \\
\text { institutional culture collections. }\end{array}$ & & & $\checkmark$ & \\
\hline $\begin{array}{l}\text { Investigate the application of encapsulated enzymes or whole } \\
\text { cells of known enzymes/organisms for accelerated and more } \\
\text { efficient processing of lignocellulosic content of regional } \\
\text { feedstocks. }\end{array}$ & $\checkmark$ & $\checkmark$ & & \\
\hline $\begin{array}{l}\text { - Incorporate known and novel lignocellulosic conversion } \\
\text { strategies into saccharification processes. }\end{array}$ & $\checkmark$ & $\checkmark$ & $\checkmark$ & $\checkmark$ \\
\hline $\begin{array}{l}\text { 3. Design and construct a bench-scale pretreatment unit for } \\
\text { lignocellulosic feedstocks. }\end{array}$ & & & & $\checkmark$ \\
\hline
\end{tabular}


This work supports the topical Department of Energy (DOE) Energy Mission statements to (1) advance the national, economic, and energy security of the US, and (2) promote scientific and technological innovation in support of economic and energy security. Additionally, the work directly supports the DOE Strategic Goal for Energy Efficiency and Renewable Energy "to protect our national and economic security by promoting a diverse supply of reliable, affordable and environmentally sound energy." Supported goals of the Biomass Program include the topical areas or feedstocks, sugar platforms, products, and integrated biorefineries.

Importantly, this research is aligned with the newly proposed Center for Advanced Energy Studies (CAES) Bioenergy Center. The principle focus areas of the proposed center are to (1) use Idaho materials, (2) apply life cycle assessment tools to identify and drive work, and (3) provide a connection interface between clients capable of problem resolution. This project forms the foundation for the use of Idaho materials as it strives to widen the applicable envelope of operations for biofuel production in Idaho. Additionally, it fully supports the strategic goals of the center in that it provides an existing, tangible connection between the state research institutions and CAES that is already focused on and engaged in bioenergy research. Further, we incorporate training opportunities for undergraduate, graduate, and postdoctoral students, thereby directly linking student training to enhancement of the biofuels industry in Idaho.

\section{Results and Accomplishments}

A primary objective has been to evaluate existing and develop novel enzymatic and whole-cell saccharification strategies for biofuel production. From identified regional feedstocks. Specifcally, we have been investigating the application of encapsulated enxymes/whole cells of known enzymes/organisms for accelerated and more efficient processing of lignocellulosic content of regional feedstocks. Our primary accomplishments to date have been in screening a number of encapsulation approaches for wholeorganism entrapment and subsequently evaluationg the hydrolytic capabilities of the encapsulated organisms or purified enxymes relative to free cell/enzyme treatments (i.e., nonencapsulated catalysts).

Prior to initiating encapsulation experiments, BSU established cultivation requirements, methods for assessing cellulytic capabilities, and lignocellulase enzyme stimulation conditions for a suite of known cellulose and lignin hydrolyzing microorganisms to investigate with encapsulation strategies (i.e., Trichoderma reesi, Anoxybacillus sp., Clostridium thermocellum, and Aspergillus niger). Hydrolytic capabilities of additional cultures of novel organisms are being evaluated as they become available from ISU.

Additionally, we have built the capacity for rapid quantitative assessment of cellulytic activity of known and novel microorganisms. Currently we are developing similar assays for quantitative assessments of both xylanase and lignin peroxidase activity. While these methods were developed elsewhere, this project has provided an opportunity for us to build expertise and thereby improve the technical capabilities of our research team, making us more competitive in this research area.

Initial encapsulation experiments compared growth rates and cellulytic activities of free and encapsulated $T$. reesei cells in the presence of purified cellulose (Avicell, CAS \# 9004-34-6). The $T$. reesei cells have been encapsulated in two different matrices: Biosep beads and calcium alginate.

Although we were able to encapsulate $T$. reesei in Biosep beads, little to no cellulase activity was detected. Additional experimentation suggested the lack of activity was due to sorption of extracellular enzymes to the powdered activated carbon present within the Biosep matrix.

Initial alginate encapsulation experiments were designed to test if $T$. reesei could be sustained and its cellulytic activity maintained in the calcium alginate beads. These experiments were unsuccessful in that alginate encapsulated cells didn't remain within the matrix during cultivation $\left(30^{\circ} \mathrm{C}, 100 \mathrm{rpm}\right)$ with cellulose as the carbon source even though good growth was observed. The fungi appeared to grow out of the alginate bead to colonize the bulk culture 
solution. Evaluation of relative levels of cellulase activity between encapsulated and nonencapsulated $T$. reesei cells as a ratio of cellulase activity:cell biomass showed no encapsulation treatment effect, likely due to the outgrowth from the alginate beads.

To prevent outgrowth from the beads, we performed an experiment in which the agitation rate was significantly increased. Entrapped and free-cell T. reesei cultures were grown in Avicell media on a 400 -rpm orbital shaker at $30^{\circ} \mathrm{C}$ for eight days. Treatments were periodically analyzed for biomass production and cellulase activity. The increased agitation rate had an appreciable effect on growth rates and colonization of the encapsulation matrix. Growth was much higher for the alginate bead treatments. Free-cell biomass increased by $147 \%$ whereas encapsulated organisms increased biomass more than 10 fold. Furthermore, $97 \%$ of the growth was contained in the bead. This was in contrast to the 100-rpm treatments which had only $4 \%$ of total biomass contained in the bead at the end of the experiment. Though the encapsulated treatments had higher amounts of biomass, the free-cell treatments had filter paper activity twice that of the encapsulation treatment. When cellulase activity was normalized for cellular dry weight, the free-cell treatments had 7 times the cellulytic activity as the encapsulated culture.

Our most recent experiments have involved modulation of the surface area:volume ratio of the alginate beads as a way to overcome the limitation in cellulase activity of the encapsulated organisms. Current literature suggests that at high agitation rates $T$. reesei will form tightly aggregated mycelia in liquid culture. Within these tightly aggregated mycelia, the cells in the center have low levels of metabolic activity, thought to be due to oxygen limitation within the aggregate. We hypothesized that similar conditions were occurring in our alginate beads. To limit the potential for metabolic inhibition within our encapsulation matrix, we modulated our encapsulation approach to increase the surface area:volume ratio of the alginate beads. Our initial experiments with this approach indicate that we can maintain high levels of biomass within the beads and high levels of cellulase activity by the encapsulated cells, effectively removing the inhibition we observed with the encapsulation matrices with lower surface area:volume ratios. On-going experiments are investigating a means for reducing the size of the alginate encapsulation matrix while maintaining high levels of metabolically active $T$. reesei.

In addition to these experiments, we have isolated several thermophiles that can use xylan as a sole carbon source by using solid materials such as filter paper, wood chips, and cellulose membranes suspended in thermal waters. These organisms are being evaluated with zymography and directed proteomics. Applying these tools to the xylanase enzymes of Anoxybacillus strain TWXYL3, we have discovered that the extracellular xylanolytic enzymes exist as a large molecular weight complex, an important finding when considering encapsulation strategies.

UI conducted a survey of the different types of feedstocks available for producing ethanol in Idaho. These included sugar, starch, cellulosic and lignocellulosic feedstocks. The survey evaluated the feedstocks using a rubric to determine those that would have advantages for Idaho ethanol plants. The general model was that existing starch-based ethanol plants could utilize other feedstocks by installing appropriate processes to convert the feedstock to fermentable sugars. The rubric includes transportation issues, the difficulty of converting the material to ethanol, the quantity of material available, and the current uses for the material. As part of the development of the survey, the rubric was compared to INL models to evaluate methodologies and results with similar studies. Although currently incomplete, we have determined that wood pulp-based cellulose from paper mills will be too expensive to use for ethanol production and recycled newspapers, although attractive, have the problem of limited supply. Research is ongoing to determine a suite of attractive feedstocks.

\section{Summary and Conclusions}

Initial findings suggest that by modulating the surface area:volume ratio of an alginate encapsulation matrix, we can maintain a similar level of cellulase activity as non-encapsulated 
cells with a lower level of cellular biomass, essentially doubling the volumetric productivity of cellulase activity in the encapsulated culture. Thus we may be able to increase the efficiency of cellulase production by $T$. reesei relative to standard cultivation conditions.

The biological culture collection that has arisen from this research is a valuable asset for other researchers in this area. Once published and described, these organisms can be provided to other groups for study, and genome sequences can be determined for comparison with other microorganisms being considered for larger- scale bio-fuel production. Additionally, directed proteomics methods will be an additional research tool for discovery of lignocelluloseconverting enzymes in many other systems.

Our experiments represent a novel approach to increasing rates of hydrolysis of lignocellulosic substrates for bio-fuels production. If successful, these studies may provide valuable input into the development of a biorefinery designed to convert regionally available lignocellulose feedstocks into fermentable sugars. 


\section{PAGE FOR CENTER FOR ADVANCED MODELING AND SIMULATION DIVIDER}

Center for Advanced Modeling and Simulation 


\title{
Structural and Electronic Properties of Adsorbed Metal Nanoparticles
}

\author{
Sergey N. Rashkeev
}

Improving the operational performance of materials and nuclear fuels under extreme conditions SH101

The goal of this research is to develop a basic understanding of scientific issues relating to catalysis, corrosion, and radiation effects. All of these phenomena are closely related to the structural transformations and electronic properties of materials. The properties of interest underlie important concerns related to both desirable and undesirable material modifications that occur in applications and the need to control them. These goals are achieved by applying advanced quantum-mechanical and atomic-scale computer simulation techniques to a broad class of materials in order to identify atomic-scale and nano-scale mechanisms that are responsible for the desirable properties of materials. The modeling is based on the state-of-art firstprinciples calculations and atomistic simulations for relevant surfaces and adsorbed metal particles. The emphasis of the project is to develop a general modeling approach to catalysis and corrosion based on a combination of firstprinciples simulations and statistical modeling. Our main goals are to increase: (1) the performance of catalysts used for hydrogen production and reduction of pollutants, and (2) the corrosion resistance of materials and fuels used in nuclear reactors.

\section{Technical Objectives}

The ultimate objectives of this work are to:

- Develop a strategy for designing long-lived catalytic materials which are resistant to the harsh reaction environments imposed by the process conditions

- Provide recommendations how to improve operational properties of materials and nuclear fuels in extreme conditions (high temperatures, chemically active environments, radiation damage) by controlled modification of corrosionresistant coatings.

\section{Results and Accomplishments}

1. Nuclear fuels modeling: Radiation defects, defects diffusion and phase segregation.

In Fiscal Year 2009 (FY-09), we performed modeling (Fig. 1). Formation energy for different interstitial configurations in UZr alloy system (nuclear fuel) including octo- and tetra- interstitials as well as dumbbells with different orientation (first-principles calculations) simulations for complex catalysts, polymer membranes, and nuclear fuels at different length scales. In particular, we calculated (from firstprinciples) formation energies and migration barriers for different defects in the UZr alloy that is used as nuclear fuel (Fig. 1). These results will be later used for kinetic Monte Carlo (KMC) calculations of diffusion and other kinetic coefficients as well as an input for phase modeling simulations. This work has been done in collaboration with researchers from the Materials and Fuels Complex (MFC) at Idaho National Laboratory (INL), Ohio State University, Georgia Tech (two graduate students

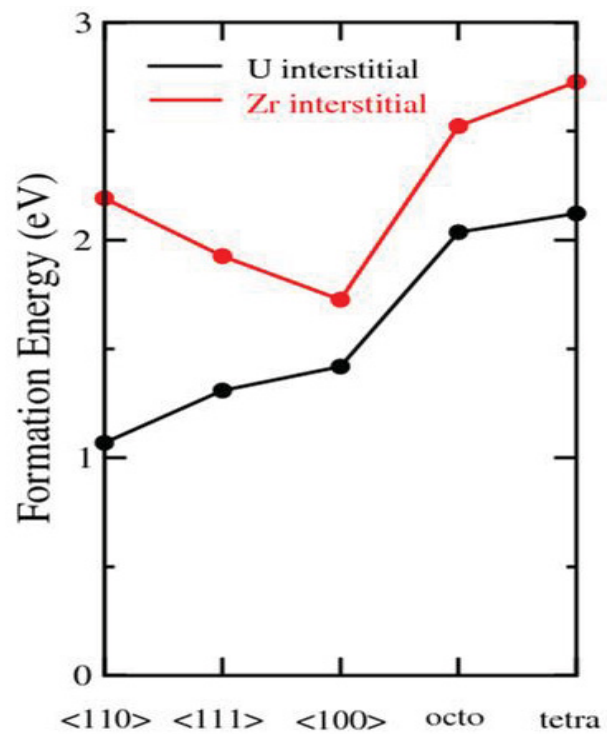

FIGURE 1. Formation energy for different interstitial configurations in UZr alloy system (nuclear fuel) including octo- and tetra-interstitials as well as dumbbells with different orientation (first-principles calculations).

from the Nuclear Engineering Division of this institution came to INL this summer and stayed with me as interns), University of Central Florida, and Los Alamos National Laboratory (LANL). In addition, first-principles calculations were performed for $(\mathrm{U}, \mathrm{Pu}) \mathrm{O}_{2}(\mathrm{MOX})$ nuclear fuels (in collaboration with Max-Planck-Institut, Stuttgart, Germany, and Institute for Transuranium Elements, Karlsruhe, Germany). The results of this research will be published in several papers that we plan to submit to high- 
ranked peer-reviewed journals. They were also presented at some conferences: TMS'2009 (San Francisco, CA), REI-15 (Padova, Italy), etc.

2. First-principles calculations and computer modeling \& simulation for complex catalytic materials: Towards the control of the catalyst activity and stability.

Theoretical/modeling investigation of the stability and catalytic activity for different catalytic materials and systems was performed (including porous oxides, metal nanoparticles on oxide substrates, complex oxide surfaces, thin oxide films positioned on the top of another oxide, etc.) working under extreme conditions (high temperatures and harsh environments, including steam, oxygen, acid vapor, high pressure, etc.). In particular, we (1) continued to perform first-principles calculations and computer simulations (started in FY-07) for high-temperature catalytic sulfuric acid decomposition in the sulfur-iodine thermochemical cycle for hydrogen production, (2) performed calculations for metal nanoparticle/oxide catalysts for ethanol oxidation, (3) investigated stability and catalytic activity of $\mathrm{Au} / \mathrm{TiO}_{2}$ catalysts modified by an incomplete monolayer of $\mathrm{SiO}_{2}$, and (4) started calculations for bimetallic catalysts for FischerTropsch reaction (synthetic fuel production).

Research efforts (1) and (2) significantly support experimental investigations on sulfuriodine cycles and catalysts for ethanol oxidation performed at the INL Chemical Sciences division and are consistent with the INL missions in nuclear energy and environment. Effort (3) has been performed in close collaboration with the Heterogeneous Catalysis Group (leader: Dr. Steven H. Overbury) at the Center for Nanophase Materials Sciences (CNMS), Oak Ridge National Laboratory (ORNL). We modeled the formation and performance of complex catalysts that consist of nanoparticles deposited at complex oxides (e.g., $\mathrm{TiO}_{2}$ substrate modified by an incomplete monolayer of $\mathrm{SiO}_{2}$, as shown in Fig. 2). We found that the stability of nanoparticles (against their sintering and agglomeration) may be much higher on such a modified complex substrate. This fact was experimentally discovered by ORNL researchers in the last few years, but the reason for such a behavior was unclear. We showed that an atomic-scale roughness introduced by a partial monolayer of $\mathrm{SiO}_{2}$ slows down the atomic surface diffusion and inhibit Au-nanoparticle growth/sintering. Effort (4) may be very useful for the INL HYTEST project.

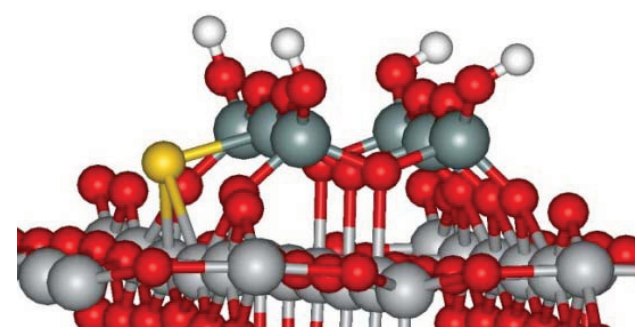

FIGURE 2. Schematics of an Au catalytic cluster formation at a comples $\mathrm{SiO} 2 / \mathrm{TiO} 2$ oxide surface - a gold atom binds to a double-defect (oxygen vacancy plus Si dangling bond) (first-principles calculations).

These results were presented at The 2009 March Meeting of the American Physical Society, $235^{\text {th }}$ and $236^{\text {th }}$ National Meetings of the American Chemical Society, North American Catalytic Society meeting, and at invited seminars in the CNMS, ORNL and Vanderbilt University. In FY-08 and FY-09, we won two CNMS-INL grant awards which provided access for me and my INL colleagues to the ORNL state-of-the-art characterization facilities and massive parallel computers (including the CRAY-XT4 "Jaguar" system).

\section{Gas interaction with polymers used for separation membranes.}

We performed atomic-scale, molecular dynamics simulations of gas interaction with several industrially important semi-crystalline and amorphous polymers used for gas separation membranes. In particular, we investigated interaction between the $\mathrm{H}_{2} \mathrm{O}, \mathrm{CO}_{2}$, and $\mathrm{N}_{2}$ molecules and the polybenzimidazole (PBI), bis(isobutylcarboxy)polybenzimidazole (PBIButyl), and Kapton polymers by using firstprinciple calculations (to find the possible binding energies between gas molecules and polymer matrices and to calculate their migration barriers and transition states) and by classical MD simulations (to study the evolution of large ensembles of gas molecules within crystalline polymers, as illustrated by Fig. 3). We investigated how the packing structure and the 
interlayer coupling of polymer matrices are related to the permeability of gas molecules. In particular, we showed that water molecules could penetrate into polymer and form water bubbles there which, in turn, may significantly weaken the polymer membrane and initiate formation of cracks and other extended defects in the material. The results were presented at the North American Membrane Society meeting (Charleston, SC, June 2009) where they attracted significant attention and stimulated several collaborations. Also, this research will be a part of the planned INL-ORNL Center on Membranes (proposal submitted to the Department of Energy [DOE]).

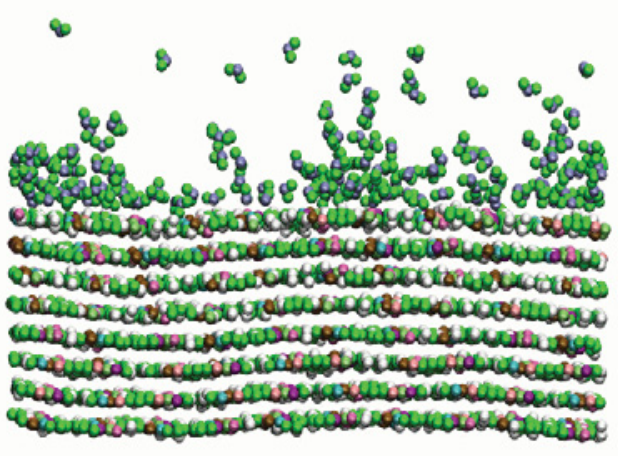

FIGURE 3. Gas interaction with polymer membranes: water drop formation in the bulk and at the surface of the polybenzimidazole (PBI) polymer (MD simulations).

\section{Advanced morphological analysis for surfaces with arbitrary texture and roughness.}

We developed an advanced morphologicalanalysis method for surfaces with arbitrary texture and roughness using a novel and original code for morphological decomposition (Fig. 4). This approach is based on recent developments in applied mathematics and allows performance of a complete multiscale analysis of morphology for rough surfaces (including surfaces with fractures and cracks). This method is much more efficient than any similar codes based on fast Fourier-transformation, wavelets, etc. Our preliminary results were recently published in the Applied Physics Letters magazine (which is probably the most prestigious U.S. journal on applied physics). This method will be used for the analysis of fractured and corroded surfaces that is widely performed at INL.

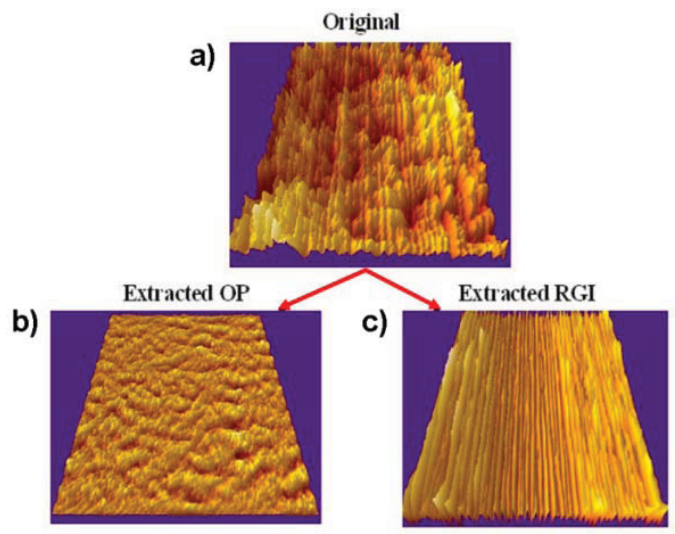

FIGURE 4. Three-dimensional (3D) visualization of the surface height function for rolled and formed aluminum alloy, AA6022. A total surface height function (a) and its morphological decomposition into orange peel $(O P)(b)$, and roll grind imprint (RGI) (c) components. The size of the imaged area is $1.2 \mathrm{~mm} \times 1.2 \mathrm{~mm}$.

\section{Summary and Conclusions}

INL has been given a leadership role in international revitalization of nuclear power. To develop nuclear reactors with a 60 -year life, INL must have an exceptional capability in research and development of the next generation of advanced materials and nuclear fuels that will deliver increased performance and reliability for increasingly demanding energy generation environments. This project contributes to design of active, long-living catalysts for hydrogen production and reduction and oxidation of pollutants and development of corrosion resistant materials and fuels. Also, it provides INL personnel an opportunity to compete for research and development funds from sources other than LDRD. The CNMS-INL grant awards provided INL personnel an access to the ORNL state-ofthe-art characterization facilities and massive parallel computers (including the CRAY-XT4 "Jaguar" system). We submitted and published several manuscripts to different highly ranked peer-review journals (including Catalysis Today, Journal of Physical Chemistry, Journal of Catalysis, Physical Review B-Condensed Matter, Applied Physics Letters, etc.) and we are preparing a large number of other publications. This should significantly increase the INL research and development potential. 


\section{Adaptive Modeling of Geometrically Complex Fuel Rods with a posteriori Error Control}

Michael Pernice

Extending goal-oriented adaptive modeling to nuclear fuel simulations SH103

Modeling and simulation is widely used to design and evaluate engineering systems. These simulations often form the basis for highconsequence decision making when experimentation is impractical or impossible, such as when assessing the safety of a nuclear reactor or designing fuel for the next generation of nuclear reactors. To increase the reliability of these simulations, it is essential to accurately estimate and reduce errors. Adjoint-based duality techniques account for both local and global contributions to simulation error and can also be used to identify regions where higher physical fidelity is needed to reduce the error in a desired output, a topic referred to as goal-oriented adaptive modeling. A principal objective of this project is to extend goal-oriented adaptive modeling from linear elliptic scalar problems that use Galerkin finite-element discretization to a broad class of nonlinear, time-dependent problems that use finite-volume discretization. This choice is motivated by nuclear fuel simulations, in which complex geometry can also be an important source of error. The technical approach is focused on creating new capabilities for applying adjoint-based techniques to finite volume discretizations on Cartesian grids that utilize locally irregular cells to represent complex geometry.

\section{Technical Objectives}

Our primary technical objectives in the final year of this project were:

- Disseminate results for new a posteriori error estimates for finite volume discretizations of semi-linear elliptic problems on regular Cartesian grids

- Extend these results to nonlinear problems of mixed type, specifically the incompressible Navier-Stokes equations

- Develop sharp error estimates for problems on locally irregular Cartesian grids.

\section{Results and Accomplishments}

In collaboration with colleagues Professors Don Estep (Department of Mathematics, Colorado State University) and Haiying Wang (initially a postdoctoral research assistant under the direction of Dr. Estep, currently at the
Department of Mathematical Sciences at Michigan Technological University), development of new a posteriori error estimates were completed, and the manuscript " $A$ posteriori error analysis of a cell-centered finite volume method for semi-linear elliptic problems" (INL/JOU-08-14300) was submitted for publication in the Journal of Computational and Applied Mathematics (JCAM). After incorporating modifications based on comments from the reviewers, a revised version of the manuscript was submitted; this revision was accepted for publication and will appear in hardcopy version of the journal in November 2009 (an electronic version has already posted to the JCAM web site).

Efforts were initiated to extend these results to the incompressible Navier-Stokes problem. A mixed formulation similar to that used for elliptic problems proved to be successful. Current efforts are directed at developing software to demonstrate the sharpness of the estimates. Results to date have been captured in a manuscript tentatively titled "MAC finite volume scheme and its a posteriori error estimate for incompressible Navier-Stokes equations." A final version of this manuscript that incorporates numerical demonstration of the sharpness of these estimates will be submitted for publication. These efforts have attracted interest from researchers at Lawrence Livermore National Laboratory, who have collaborated with Professor Wang to explore their use in flow problems with embedded discontinuities.

These techniques need to be modified in the presence of complex geometry. By themselves, Cartesian grids are incapable of representing complex geometry because of their physically rectangular nature. One common practice is to use body-fitted Cartesian grids that conform to the geometry but are, nevertheless, still logically rectangular. For simple circumstances (such as the exterior of an aircraft) this is tractable, but fine details and complex geometry cannot be captured by this technique. An alternative is to intersect a rectangular Cartesian grid against the geometry, which replaces the difficult problem of global grid generation with simpler local problems of calculating intersections between the grid and the geometry. This procedure creates a 
number of cut cells where the geometry intersects the grid. This is illustrated on the left of Figure 1, where the embedded boundary $\Gamma_{\mathrm{b}}$ separates two regions $\Omega_{1}$ and $\Omega_{2}$, creating a collection of cut cells in the region $\Omega_{\mathrm{b}}$. We have focused on problems where the geometry delineates a change in material properties, such as thermal conductivity. Developing accurate error estimates for these situations is difficult because solutions to this class of problems are generally not smooth, which places us outside the available mathematical frameworks for error estimation.

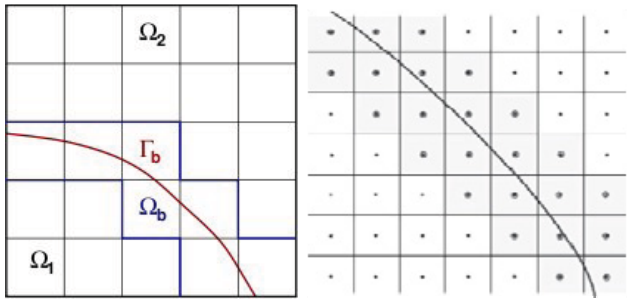

FIGURE 1. Left: An embedded boundary separating two regions with different material properties. Special treatment is needed for the cells that are cut by the interface. Right: The surrogate model is constructed by modifying the conductivity in the shaded region through appropriate interpolation.

Our approach to this is to solve a surrogate problem that is in some sense 'nearby' and is amenable to the error analysis techniques that we have already developed., The task is then to develop new techniques to accurately estimate the difference in the solutions of the two problems. We focused on steady-state heat conduction problems, and in particular on modeling the thermal conductivity. The key to the success of this approach was constructing a suitable surrogate for the actual thermal conductivity. This is illustrated on the right of Figure 1, where material properties in the unshaded regions are left unchanged, and material properties in the shaded region are defined by suitably weighted interpolations. These results have been summarized in a manuscript "A posteriori error analysis for a cut cell finite volume method" which has been submitted for publication in the journal Computer Methods in Applied Mechanics and Engineering.

\section{Summary and Conclusions}

New duality-based adjoint error estimators have been developed for partial differential equations that govern thermal conduction and incompressible fluid flow. These estimators rely on the smoothness of the solution, and we have successfully developed new estimators for problems with non-smooth solutions by trading off geometry errors and discretization errors through the introduction of a smooth surrogate problem. The robustness and low cost of Cartesian grid approximations to complex geometry make them an attractive option for multi-scale modeling strategies and CAD-based design optimization. 


\title{
Uncertainty Quantification for Nuclear Fuels Performance
}

\author{
Derek Gaston, Glen Hansen, Michael Pernice
}

Simulating the behavior of fuel rods

TM108

Assessment of the performance of fuel in nuclear reactors requires reliable prediction of fuel rod behavior. Fuel behavior is governed by the diffusion of chemical species, heat transport, and thermo-mechanical processes. One of the greatest challenges is in material properties, such as thermal conductivity and diffusivity of chemical species, which depend on temperature and fuel composition. Extrapolating these properties as fuel composition changes (for example, in high-burnup situations) also presents a significant challenge. Understanding how simulation-based predictions of fuel behavior are affected by uncertainty in these properties plays a key role in advanced modeling of nuclear fuel.

Further, all numerical simulations involve some amount of error. There is error in inputs, error in the model chosen, and error in finding a solution for that model with a numerical method. One large source of error in grid/mesh-based numerical methods, such as finite element or finite difference, is in discretization of the solution space. The resolution of the given mesh in areas with strong solution features (such as sharp gradients or curvature) will have a large impact on the overall numerical error incurred throughout the solution process. One approach to reducing this numerical error is to modify the mesh in areas of significant error to better capture these important solution features. This is typically referred to as adaptive mesh refinement (AMR), where the mesh adaptation is driven by some indicator of error.

Both of these key ideas, uncertainty quantification and adaptive mesh refinement, can be enhanced through employing an adjoint solution method. Adjoint methods involve finding a generalized Green's function for a set of partial differential equations (PDEs) and utilizing its properties for quantity of interest (QoI)-based sensitivity analysis and error estimation. This project's goals were to develop the foundational capability for these adjointbased methods, allowing them to be utilized by next-generation Idaho National Laboratory (INL) codes based on the Multiphysics Object Oriented Simulation Environment (MOOSE) computational science framework. This work will enable verification of predictive simulation capabilities for nuclear fuels and other INL missions.

To achieve these goals a strategic partnership with University of Texas (UT) at Austin was developed. INL's MOOSE simulation environment utilizes the LibMesh framework developed by the Institute for Computational Engineering and Sciences at UT. Dr. Graham Carey headed the development efforts at UT to build these advanced foundational capabilities and conduct the numerical experiments necessary to demonstrate them.

\section{Technical Objectives}

- Enable adjoint solution capability inside the LibMesh framework (MOOSE is based on LibMesh)

- Develop an interface for QoI calculations

- Use adjoint solution to form an errorestimator for use in AMR

- Verify error-estimator using model problems

- Implement adjoint-based sensitivity analysis

- Verify parameter sensitivity analysis using model problems

- $\quad$ Extend MOOSE and BISON (the nuclear fuels simulation code developed using MOOSE) to use these capabilities with model problems.

Results and Accomplishments

The main effort involved extending the formulation methodology and software implementation for adjoint-based implementations in LibMesh and enabling software for tests using BISON and MOOSE. Adjoint extensions have been developed and tested in LibMesh on representative model scalar field PDE problems. These first targeted improved error indicators and combined/extended prior jump and patch indicator capabilities in the adjoint setting. This was followed by a further extension to use LibMesh for adjoint based parameter sensitivity computations. Numerical studies revealed several important technical issues concerning both accuracy and convergence rates that were 
resolved during the course of the work. Tests on the model elliptic PDE and convection diffusion model were made. The infrastructure is being extended and applied with MOOSE/BISON on the same test problems.

Two model problems were used to test the accuracy and rate of convergence for the final combined adjoint approach. The case outlined below is the simpler one: a stationary diffusion problem with a source term governed by the Poisson equation,

$$
\begin{array}{cc}
-\nabla \cdot(\alpha \nabla u)=f & \text { on } \Omega \\
u=g & \text { on } \partial \Omega
\end{array}
$$

on the $\Omega-[0,1] \times[0,1]$ domain, where we chose $u$ to be the manufactured solution,

$u(x, y ; \alpha)=4\left(1-e^{\alpha x_{1}}-\left(1-e^{\alpha}\right) x_{1}\right)\left(x_{2}\right)\left(1-x_{2}\right)$

and calculated the corresponding f through differentiation. This manufactured solution exhibits multiscale spatial behavior due to a boundary layer near the left edge. It provides an exact analytic solution for comparison purposes and for verifying numerical rates of convergence. In the numerical simulations, the manufactured solution values on the boundary are specified as the Dirichlet data for the associated computational problem.

Figure 1 shows a contour plot of the manufactured solution. Note the strong gradients in the boundary layer near the left boundary. We chose the QoI to be the normal flux through the bottom horizontal edge of the domain,

$$
R\left(u\left(x_{1}, x_{2} ; \alpha\right)\right)--\left.\int_{0}^{1}(\alpha \nabla u \cdot \vec{n})\right|_{x_{2}=0} d x_{1} .
$$
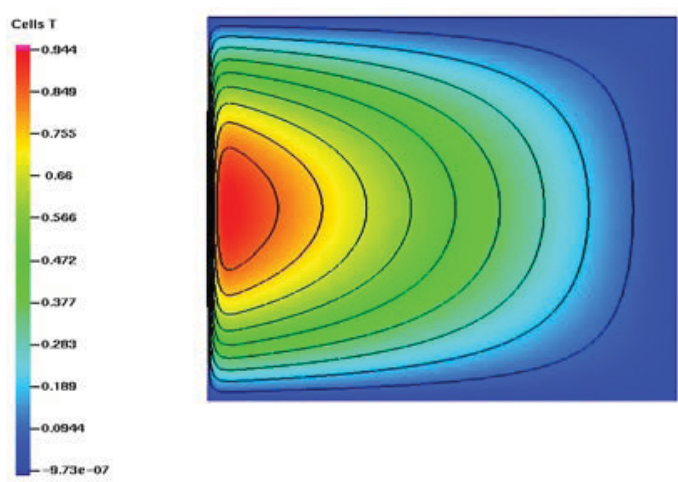

FIGURE 1. The manufactured solution $u$ with alpha $=100$ and corresponding source term.

The adaptively refined mesh is shown in Figure 2, superimposed on a color plot of the numerically computed solution of the adjoint problem. The mesh was obtained by using the Adjoint Residual method after 10 adaptive refinement steps, where the primal and dual estimates are obtained using a patch-recovery technique. Note the bias in the refinement pattern towards the boundary with specified QoI. This demonstrates the capability and the goal-oriented nature of the refinement, which implies more accuracy in the QoI at lower cost and, hence, a more efficient process due to the weighted mesh bias. This efficiency is shown in Figure 3 (next page) where all of the different adjoint-based adaptivity schemes do significantly better at reducing error than uniform refinement.
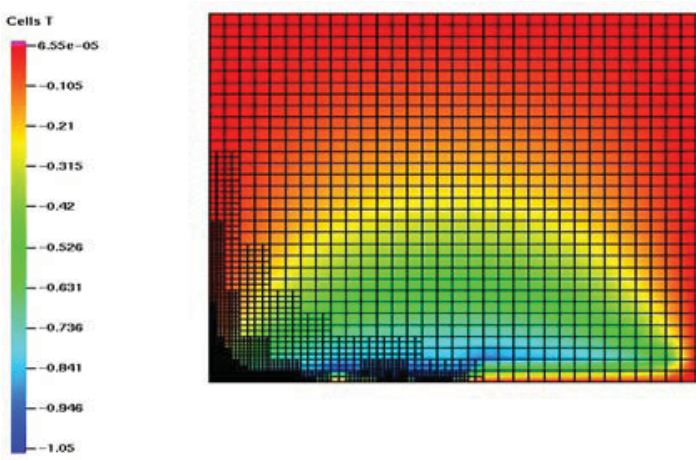

FIGURE 2. Adaptive mesh and numerically computed adjoint solution. 


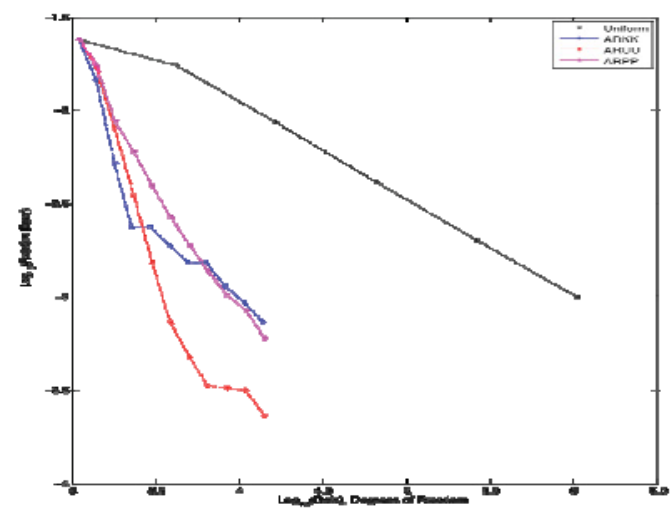

FIGURE 3. Convergence plot for the QoI for uniform refinement and several adjoint methods.

The next part of the work concerned the extension of this new adjoint formulation and implementation to compute parameter sensitivity more efficiently. An appropriate adjoint formulation was developed and implemented with LibMesh. Analytically, computing the sensitivity consists of taking the total derivative of the QoI with regard to alpha. Using this approach to numerically compute sensitivities and compare them to the analytic derivative, Figure 4 is obtained showing that adjoint-based mesh adaptivity can significantly reduce the error in sensitivity calculations.

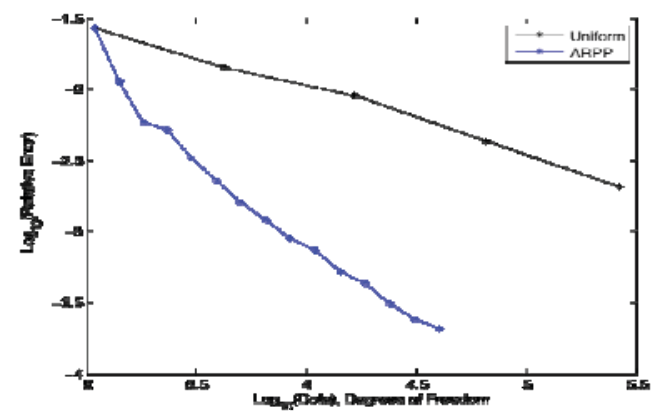

FIGURE 4. Convergence plot for the QoI sensitivity using uniform refinement and an adjoint-based method.

\section{Summary and Conclusions}

All of the above foundational capability was added to LibMesh. INL's MOOSE is built using LibMesh and, therefore, now has these advanced capabilities. The final objective is to utilize them with INL's BISON fuels-performance simulator that is built using MOOSE. The initial steps toward solving model problems with these new capabilities have been performed. Work is ongoing to provide a general capability that can be used by engineers to help design future fuels.

In addition, an ecosystem of simulation capabilities has been developed around MOOSE. These codes simulate everything from groundwater contamination to pebble-bed reactors. This foundational adjoint-based capability is now thereby poised to make a large impact in many areas of science at INL.

Finally, work at University of Texas at Austin is ongoing to enhance these capabilities and move into more advanced areas of adjointbased simulation, such as transient analysis. We will be leveraging contributions from NASA to allow rapid development of these advanced simulation techniques and enhance INL's ability to deliver predictive simulation. 


\section{DIVIDER FOR DISTINCTIVE SIGNATURES}

\section{Distinctive Signatures}




\section{INSTRUMENTATION, CONTROL AND INTELLIGENT SYSTEMS DISTINCTIVE SIGNATURE}




\title{
Exploration and Development of Automated Differential Equation-Based System Identification
}

\author{
Dr. Charles R. Tolle \\ A new class of nonlinear system identification \\ IC101
}

Our research objective is to explore and develop a new class of nonlinear system identification (SysID) methods that allow for the creation of differential-equation-based models via attractor space reconstruction techniques using sample data from actual systems. The exploration is focused on using Perona's reconstruction techniques (Perona et al., 2000) to perform Takens-based dynamics reconstruction of unknown differential equations. The developmental objective is based on our conjecture that Perona's approach can be extended to Sauer's spike train interval embedding methods. If this proves true, these methods will shed new insight into the relationship between time and dynamics. Both methods of reconstruction grew out of the past hundred years of nonlinear mathematics and, more recently, the study of chaotic system dynamics. These new reconstruction methods form a basis for a new class of SysID techniques that more closely obtains first-principle models of partially characterized or non-characterized dynamical systems. We plan to verify these techniques by comparing first-principle equations developed for prototypical dynamics problems, e.g., Rossler chaotic systems, inverted pendulums, magnetic-levitation stabilization experiments, modeling of hysteresis within motors, as well as more advanced gas metal arc welding (GMAW) systems, etc. Steps toward a new class of nonlinear observers will also be investigated. If the proposed work is successful, a new, more powerful set of nonlinear SysID methods will be opened up to the worldwide ICIS community. Furthermore, this research forms the basis for a new, automated, nonlinear modeling process, which has the potential to grow into a next-generation nonlinear adaptive control theory.

\section{Technical Objectives} include:

Objectives of the fiscal year's research

- Correcting Perona et al.'s optimization cost function

- Developing Perona et al.'s differentialequation reconstruction algorithm based on the corrected cost function
- Coding the new algorithm in MATLAB

- Testing the new algorithm against noisy data sets and writing a detailed paper on its implementation

- Coding the new algorithm in Distributed MATLAB

- Comparing and contrasting fractal dimension evaluation of embedding reconstructions with differential equation reconstruction methods

- Integrating time-based clustering (TBC) methods into our reconstruction algorithm to optimize dynamics sampling

- Developing an algorithm to discover the unique mapping function between two sets of ordinary differential equations (ODEs) that are guaranteed to have such a mapping to exist between them

- Using the new equation reconstruction algorithms and the new mapping discovery techniques, numerically testing Takens' and Sauer's embedding theorems

- Extending Perona et al.'s optimization cost function to include forced dynamical systems.

\section{Results and Accomplishments}

This project is part of the Instrumentation Control and Intelligent Systems (ICIS) Signature at INL. A continuing subcontract with our proposing collaborator, Dr. Doran Baker of the Rocky Mt. NASA Space Grant Consortium (RMNASASGC) and based out of Utah Sate University (USU), was extended to support our undergraduate researchers within the space grant. At the conclusion of last year's efforts, Mr. Warnick moved on to pursue his Ph.D. at Vanderbilt in physics (another traditional longstanding university partner of the INL's Energy Efficiency and Industrial Technologies department). In addition, Mr. Cory Larsen, an electrical engineering student at USU, continued his efforts on developing and porting clustering algorithms as well as upgrading the TBC software-analysis package from a TCL/TK-based graphical user interface (GUI) to a JAVA/OpenGL-based user interface. These 
efforts remain incomplete at this time. An additional student, Mr. Craig Bidstrup, an electrical engineering undergraduate at Brigham Young University (BYU), returned to the project in support of the fractal-analysis efforts this year. Significant work was accomplished towards the development of a new distributed-clustering algorithm. Detailed testing of this new algorithm is still ongoing by our university partners. This is of interest to this project because we are investigating the use of fractal dimension in the determination of a proper time embedment for discovery of system dynamics. Initial results that imply fractal dimension might achieve this goal were obtained during the prior year. The importance of this effort is further discussed below.

A new relationship with the South Dakota School of Mines and Technologies (SDSMT) has been established during this year's efforts. Dr. Tolle has, as part of these efforts, taken a part- time leave to join the faculty of the SDSMT's electrical and computer engineering department. Via this process, we have been able to extend the talent base applied to these efforts. As a reflection, Mr. Mark Pengitore, an undergrad student in mathematics (and considered one of the top-up-and-coming math students in the country due to his performance on the Putnam exam), joined the research team together Mr. Pengitore and Dr. Tolle. These researchers were able to extend the non-forced reconstruction cost function to include forcing terms. These efforts are based on Casdagli et al.'s ideas that extend Takens' and Sauer's embedding methods to include forcing terms. By formulating and extending the existing methods to include forcing terms, we are able to make a major step towards developing first-principle models that are useful for control applications.

Non-forced cost function is written in the following equation:

$Q_{2}=\frac{1}{2} \sum_{j=1}^{j_{\max }} \sum_{l=1}^{l_{\max }} \sum_{i=1}^{D}\left[\sum_{z=1}^{K}\left(a_{i, z} \int_{t_{j}}^{t_{j}+d t_{l}} A_{i, z}(\tau) d \tau\right)+\hat{x}_{i}\left(t_{j}\right)-x_{i}\left(t_{j}+d t_{l}\right)\right]^{2}$.

Our proposed forced-cost function adds to the equation in this way:

$Q_{3}=\frac{1}{2} \sum_{j=1}^{j_{\text {max }}} \sum_{l=1}^{l_{\text {max }}} \sum_{i=1}^{D}\left[\sum_{z=1}^{K}\left(a_{i, z} \int_{t_{j}}^{t_{j}+d t_{l}} A_{i, z}(\tau) d \tau\right)+\sum_{w=1}^{C} b_{i, w}\left(\int_{t_{j}}^{t_{j}+d t_{l}} B_{i, w}(\tau) d \tau\right)+\hat{x}_{i}-x_{i}\left(t_{j}+d t_{l}\right)\right]^{2}$.

In addition to these efforts, Dr. Randy Hoover, also new to SDSMT, has begun to provide in-kind support to the mapping efforts of the project. With Dr. Hoover's new insights, a generic linear method for relating the embedded phase space to the original phase space was developed. Initial investigations into the embedding process do, in fact, magnify our perceived issues of optimal embedding time. These initial insights appear to suggest that as one varies the embedding time, one actually increases a nonlinear warping of the reconstructed phase space. This may result in the ease of obtaining simplified first-principle-like models becoming significantly more difficult unless more optimal embeddings are sought, i.e., by optimizing the embedding time or extending these methods to include non-uniform time lags via a method like TBC. This may result in the relaxation of the non-linear warping effects due to time embedding.

In addition to our in-house research efforts, we continued to disseminate our findings via peer-review publications. Our first paper, entitled "Exploration of the Trajectory Method for Reconstructing Nonlinear Deferential Equations from Time Series," continues to be reviewed by Physica D (a top-ten physics/dynamics journal); no final action by Physica $D$ has been taken at this time. Two additional peer-reviewed papers were submitted during this year's efforts: the first, titled "Phase-Space Reconstruction: A Path Towards the Next Generation of Nonlinear Differential Equation Based Models and Its Implications Towards Non-Uniform Sampling Theory," was accepted and published in the 
IEEE Proceedings of the ISRCS 2009

Symposium. The second paper was entitled, "A Method for Finding Unknown Signals Using

Reinforcement FFT Differencing," and was accepted for publication in Oct./Nov. 2009 edition of the Journal of the Idaho Academy of Science. Finally, a new draft paper has been started which will address the proposed extensions of the reconstruction methods to forced dynamical systems. Nevertheless, this paper requires the further extension of the existing SysID application to include the solution of the newly proposed forced-dynamical system cost function. These efforts will become the focus of a new research project during FY 2010.

\section{Summary and Conclusions}

During the first year, we focused on implementing the basic reconstruction algorithm for ODEs. During the second year, this application has been refined via several enhancements, i.e., direct compilation of integration routines, a new user-friendly interface (Fig. 1), extension to distributed MATLAB, etc. The first and second years' efforts have resulted in obtaining a functional set of software tools for reconstructing nonlinear differential equations (Fig. 1). This provides a solid basis for performing new research into establish a new working relationship with a

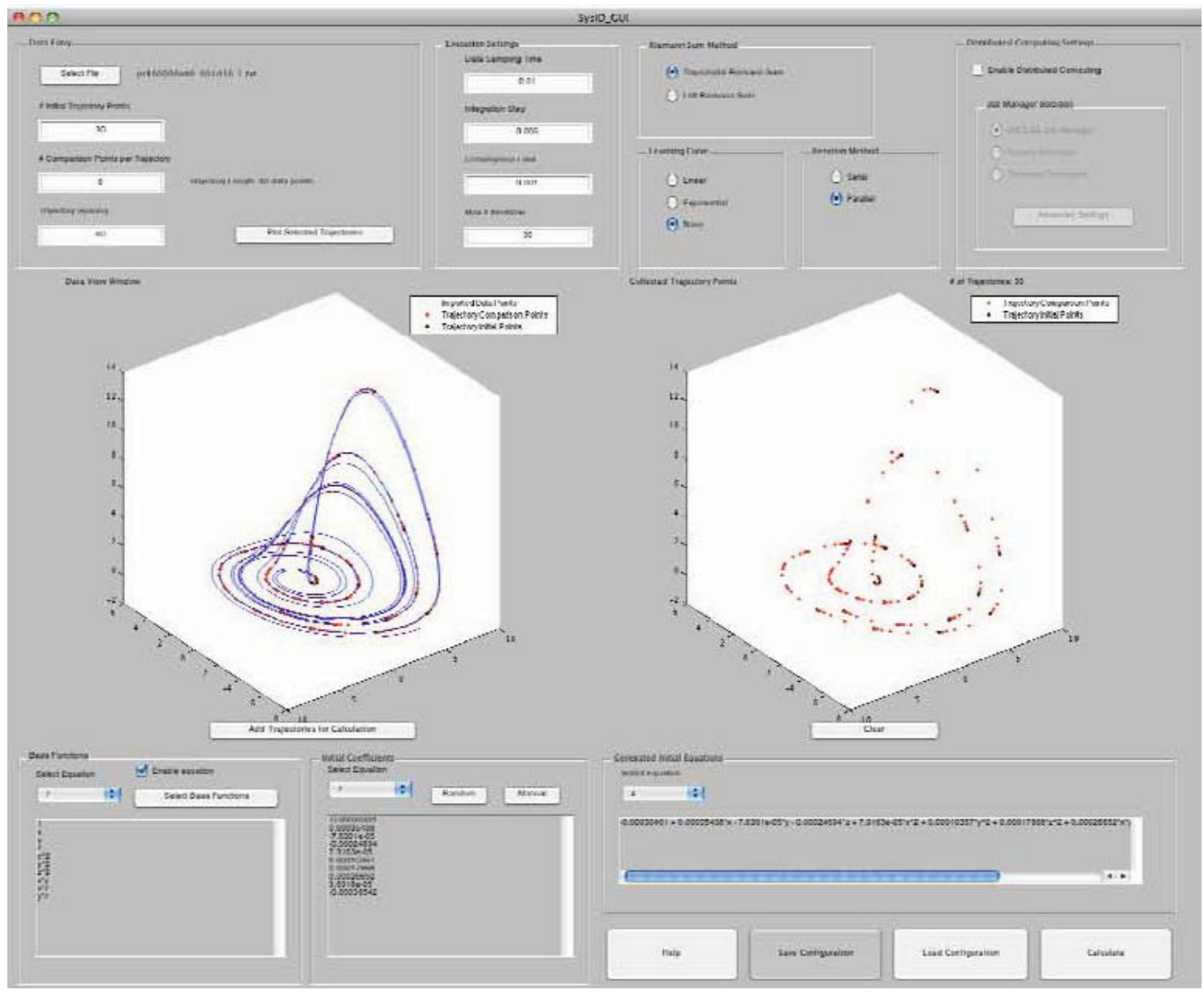

FIGURE 1. The new GUI interface for INL's nonlinear differential-equation reconstruction distributed MATLAB toolbox, showing an example reconstruction setup for the chaotic Rössler system. 
next-generation SysID of nonlinear dynamics. In the third year, we were able to investigate extending the existing methods to include forcing terms and to explore the fundamentals of the mapping and embedding processes themselves. In addition, we were able to well-established and respected educational institution and growing regional research institution: the South Dakota School of Mines and Technologies. By continuing to expand our regional relationships, we will significantly extend INL's external reputation within the mathematics, physics, and ICIS communities. Also, as discussed above, these new relationships provide us new insights into our research efforts as well as allow us to leverage unfunded research expertise within our external partnerships. This is in part seen through this project's engagement of numerous local university students and professors (from USU, BYU, ISU, and SDSMT) within these collaborative research efforts. These efforts will no doubt lead to joint follow-on funding proposals as the technology further develops. New discussion for joint projects between INL and SDSMT are beginning as this project closes.

One of the key accomplishments of the project is a MATLAB-based application that was developed (including a user manual and GUI, as shown in Figure 1.) This application stands as a formal useable product of this research effort. Moreover the concepts for using time embedding as a new nonlinear first-principle-style SysID method are better understood and have been disseminated via several peer-review articles. The current research lays the foundation for the further exploration of a new class of nonlinear observers based on time-based embedding and equation reconstruction. As the work continues, it is believed that these efforts have laid a solid foundation for a more powerful set of nonlinear SysID methods that will be developed for the next generation of adaptive control systems.

\section{References}

Perona, P., Porporato, A., and Ridolfi, L., “On the Trajectory Method for the Reconstruction of Differential Equations from Time Series," Nonlinear Dynamics, Vol. 23, pp. 13-33, 2000.

L. Noakes, "The Takens embedding theorem," International Journal of Bifurcation and Chaos, Vol. 1, No. 4, pp. 867-872, 1991.

T. Sauer, J. A. Yorke, and M. Casdagli, "Embedology," Journal of Statistical Physics, Vol. 65, No. 3/4, pp. 579-616, 1991.

M. Casdagli, "A dynamical systems approach to modeling input-output systems," in Nonlinear Modeling and Forecasting, ser. SFI Studies in the Sciences of Complexity, $\mathrm{M}$.

Casdagli and S. Eubank, Eds., Vol. XII, Santa Fe Institute. Redwood City, CA: AddisonWesley, 1992, pp. 265-281. 


\section{Tools for Proximal Human-Robot Interaction}

Curtis Nielsen, David Bruemmer, and Chetan Kapoor

Developing direct, unmediated human-robot interaction methods and tools that remove the need for a traditional operator interface

IC 102

The aim of the proposed research is to develop direct, unmediated human-robot interaction methods and tools that remove the need for a traditional operator interface. Historically, human/robot interaction has been dependent on intermediary representations, such as graphical user interfaces, keyboards, and joysticks. In actuality, in traditional interfaces, the human interacts with the interface, not with the robot. This "distal" interaction can contribute to human error and often requires significant training. Furthermore, in cases where the robot and human are proximal to each other and the operator is not sitting at a desk, traditional approaches are impractical. Eliminating the operator control unit enables humans and robots to work directly as peers, such that tasking and mutual understanding are grounded in the real environment and not an intermediary representation. The goal of this research is not to reproduce the mechanisms of natural humanhuman communication, but rather to develop synthetic, high-utility interaction methods and tools such that human-robot teaming is intrinsically linked to physical space. There are three major areas of emphasis to accomplish this research:

- Develop algorithms and perceptual capabilities to give the physical world a digital representation on board the robot that supports direct robot tasking and information sharing from human to robot.

- Develop algorithms and explore strategies to transmute the digital information collected by the robot into physical space for human understanding

- Assess the performance benefits of the developed methods and tools via pilot studies and human factors usability experiments with novices and domain area experts.

\section{Technical Objectives}

- Deliver the ability to recognize the human in robot space

- Instead of developing a new hand-held device, we used commercial off the shelf products including the Nintendo ${ }^{\circledR}$ Wii Remote and the Apple iPhone to task the robot.

- Built prototype of tasking beacon that will guide the robot with a wearable or thrown beacon

- Built prototype of tasking software to use an arm on a robot to automatically find doors and other objects.

- Represent the robot's digital world to the human

- Use a small hand-held device with a screen that illustrates information from the robot and supports simplified tasking.

- Used a personal dead reckoning system developed by the University of Michigan to put a trace of the human's path in the digital representation of the robot's environment.

Results and Accomplishments

During the first year of the LDRD, we learned that building a hand-held tasking device was not likely to yield a useable product. With that knowledge, efforts in this second year were focused on using commercial-off-the-shelf devices that could be modified to support proximal interaction. We found the Nintendo ${ }^{\circledR}$ Wii Remote to be of particular value to the work of proximal human-robot interaction because it simplified the operators' interactions with the robot and still allowed them to effectively accomplish some tasks (Fig. 1, next page). 

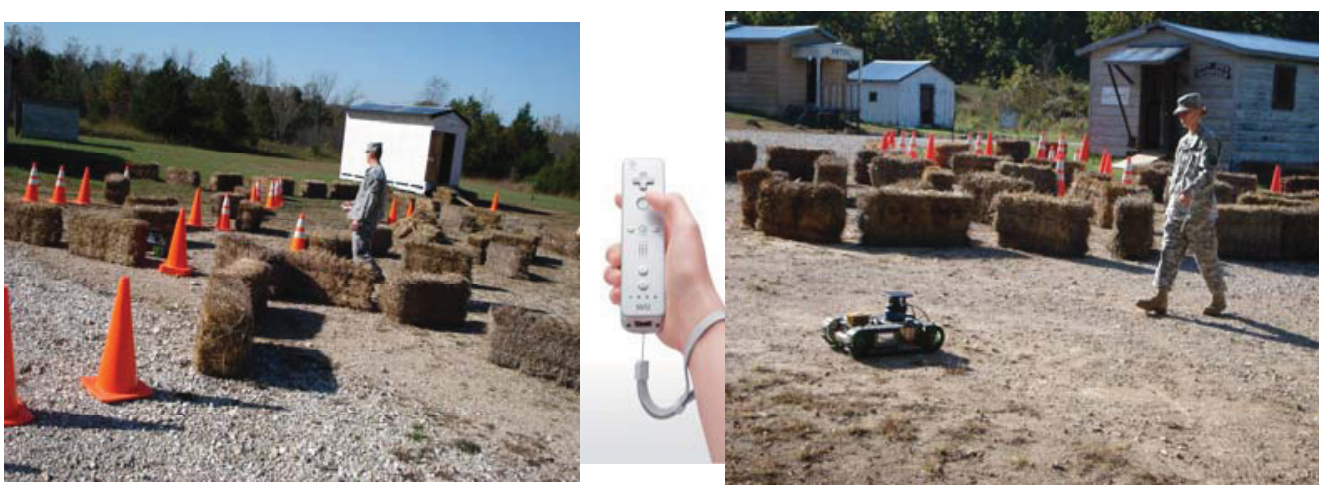

FIGURE 1. Soldiers using the Wii Remote to drive a robot at Ft. Leonard Wood, MO in October 2007.

Although a new hand-held tasking device was not a good direction for research, we determined that a beacon device was useful, especially since we could demonstrate a prototype array of antennae that could estimate the angle and distance to the beacon. During the first year, we constructed a proof-of-concept version, and during this year, we created a full prototype (Fig. 2).

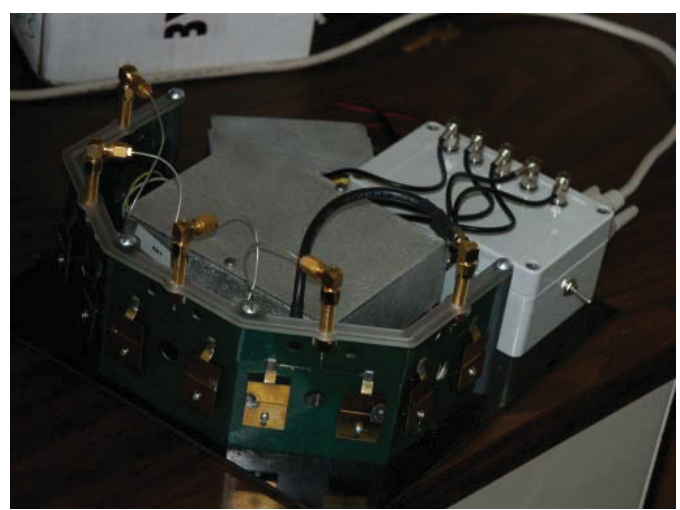

FIGURE 2. Antenna for robot tasking.

As we worked on the research problem of "proximal human-robot interaction" we realized that one measure of success is the ability to use the robot to accomplish real tasks while the operator is relatively close to the robot. This particularly hit home while attending an "unmanned ground capabilities" conference in Dallas, TX in November 2008. Operators were asked to drive robots through an obstacle course up stairs, down stairs, and around a pavilion, then to pick up an object and drop it into a container. The performance went very well through all the navigation tasks, but the manipulation task utterly failed (navigation took 30-60 seconds, manipulation took at least 2 minutes and most never finished). So, instead of focusing on the original theories of interaction where the human and robot need to share an understanding of the physical space around the robot, we changed our focus to find a solution to the problem of quickly controlling a mobile arm. To that end, we have been working closely with Chetan Kapoor, Amit Kulkarni, and Brian O'Neill at the University of Texas to develop solutions that will allow a robot with an arm (manipulator) to be tasked to open doors and pick up objects with minimal input from the operator. The approach used at the end of the second year was created for the manipulation of doors and used a three-dimensional (3-D) range sensor to detect flat surfaces (such as doors, walls, floors) and detect anomalies to the flat surface (door handles, items on the floor). Research efforts from the 2nd year led to the development of algorithms that will identify the doors and the handles on the door. Figure 3 shows some illustrations of the 3-D image of various doors. This year's effort on the project saw the development of algorithms for tasking the robot on both a macro and micro level. The macro tasking takes the robot to the general global direction and positions the arm in about the right location (Fig. 4). The micro tasking then allows the arm to be controlled specifically to accomplish a task with a simple point and click solution (Fig. 5). The University of Texas, under subcontract, has worked to augment the capabilities of the arm with obstacle avoidance algorithms so that if the robot or the user can identify places in the environment where the arm 
should not travel, then the logic around the arm movement would prohibit movement or pathplanning through that region. The demonstration of point-and-click capabilities was also shown at the unmanned ground capability conference and

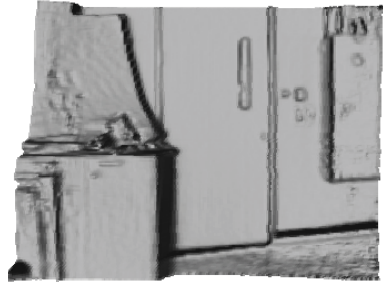

(a)

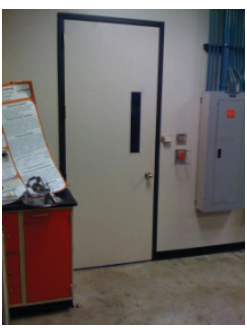

(b)

was referred by a robot manufacturer as one of the best technological demonstrations he had ever seen because of the observed failure of the prior approaches and the observed success of our approach.

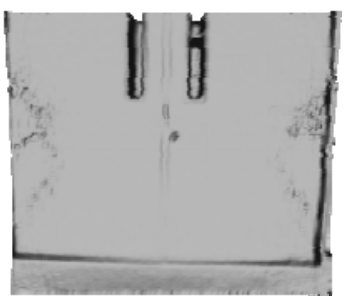

(c)

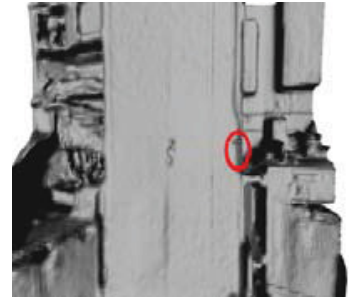

(d)

FIGURE 3. 3-D image of a regular door and its environment (a). Color photo of the environment (b). 3-D image of a double door (c), $3 D$ image of a cabinet door (d). Images courtesy of the University of Texas.

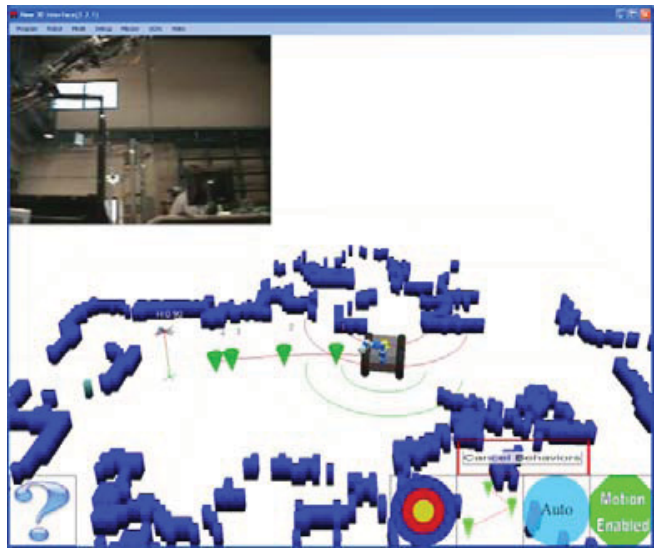

FIGURE 4. Interface for point and click arm positioning.

\section{Summary and Conclusions}

We have identified a need in the robotics R\&D community, namely quick and easy mobile-arm control. We have developed algorithms that can be used to support the
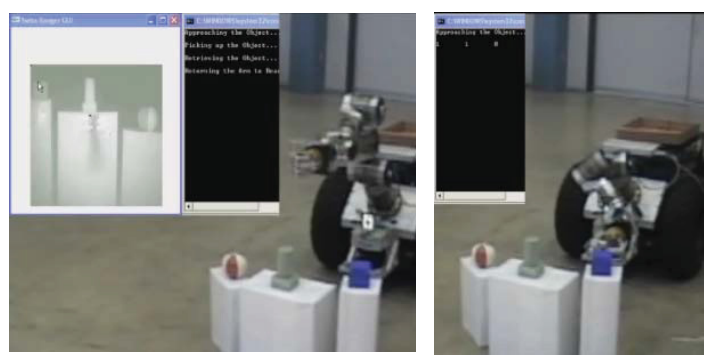

FIGURE 5. Robot responding to a click-and-retrieve task. building blocks of behavior development around arm control. This capability should support additional research efforts at the INL as we have a competitive advantage in the mobile manipulation research domains. Additionally, the prototype for the beacon device is complete and ready to be integrated and tested with a robot. There are many fundamental areas of radio frequency control that will need to be addressed once the prototype is integrated with a robot; specifically, how the information from the radio frequencies can and should be used to control a robot. The tools built and research performed will benefit the INL because outside parties interested in our robotics capabilities understand that we have not only solved difficult problems, but that we are continuing to design, test, and prove new theories and technologies with a focus towards producing solutions that improve the capabilities of end users in their assigned missions and tasks.
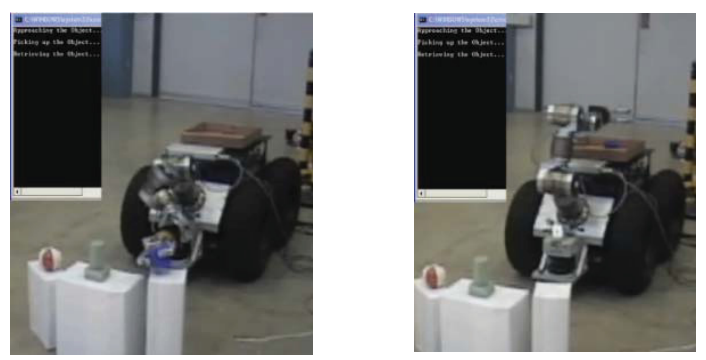


\title{
Haptic Interface for Remote Arc Welding
}

\author{
Corrie I. Nichol, Ph.D.
}

Developing and refining INL's haptics, controls, and industrial robotics program

IC104

The objective of this research is to further develop a research program for haptics, controls, and state-of-the-art industrial robotics at the Idaho National Laboratory (INL). The word "haptic" refers generally to a system that provides force and/or tactile feedback to the operator from a remote environment. The objectives for the second year of the project were as follows: first, to further refine and develop the system, including the addition of a second arm for bi-manual remote operations. The second objective was to add a more sophisticated camera system specifically for weld arc viewing. The third objective was the development of a highly dexterous remote manipulator, similar to a human hand. The fourth objective was to improve the haptic feel and response of the system, including reduction of haptic bounce.

Current State of the Art. The system developed in year one of this project embodies the current state of the art. This system incorporates a single remote manipulator for conducting haptically augmented remote robotic operations, specifically welding. This system is described in Figure 1.

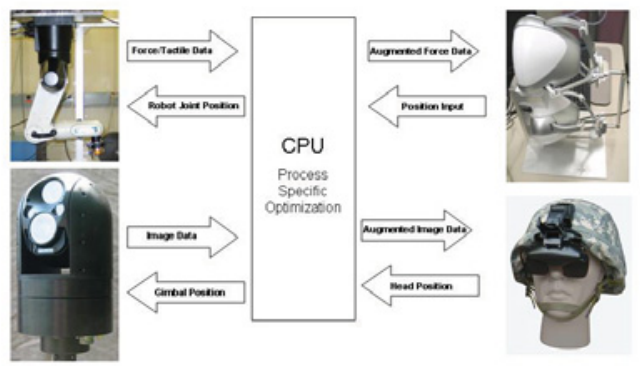

FIGURE 1. System developed in Year 1.

This system was developed to demonstrate the capabilities of such a system by enabling a user to perform welding remotely. The system has also been recognized as an important technology in many other areas, and for a variety of end applications currently limited by safety or other concerns. Some of these remote applications are in nuclear facilities and in bomb or improvised explosive device research and disposal.

Research Significance and Impact. The examples above represent applications that highlight the limitations of present technology for high-dexterity remote operations. This research seeks to overcome these limitations by giving the operator a more immersive experience with the remote environment by providing multimodal sensory feedback. This will provide a means whereby repair, maintenance, and research operations that must be conducted remotely can be conducted more quickly, more accurately and with less operator training than present methods. It will also expand the understanding of control theory and implementation. The system proposed will incorporate a user interface that will provide haptic (tactile and force feedback) information to the operator, enabling a trained welder to use the system in an intuitive manner, as well as allowing for a shared control strategy whereby the skilled motions of the user can be augmented by the system. This system will be designed to conduct welding and related activities remotely and will be used as a development platform for various control strategies.

This technology can be a great benefit to industry in performing remote operations as well as serving current and future research needs at INL.

\section{Technical Objectives}

The objective of this research project is to develop a system for providing tactile and force feedback ("haptic feedback") to a trained operator to allow the operator to perform welding and other industrial operations remotely. The major milestones for the project in Fiscal Year (FY) 2009 are listed below:

- $\quad$ Publication of results for FY 2008

- Expansion of system to include two robotic arms

- Separation of control to enable remote operations via a single network cable

- Incorporation of rudimentary gripper

- Incorporation of visual augmentation method

- Improved camera hardware for arc viewing

- Simulation and implementation of hapticbounce cancellation routine. 


\section{Results and Accomplishments}

The second robot was successfully integrated into the system, including design and assembly of all necessary hardware. The software was expanded to accommodate the second robotic manipulator, and the system was successfully tested with both arms active.

The software was also restructured such that it could be split to function on two separate computers. This effort was assisted by Brigham Young University-Idaho student Jesse Holdaway, who interned on the project during FY 2009. Separating the software to run on two computers accomplished the following two objectives. First, with both robotic arms active, the inverse kinematics routine became very resource intensive. This made it difficult to obtain the execution speed necessary for good haptic performance. Second, it was necessary to separate the software to make it possible to remove the operator from the presence of the robots, thus making the system truly remote.

The software was rewritten to require a minimum of communication between the two computers and to enable the best distribution of tasks between the two computers. The operator interface computer was programmed to interface with the input devices. Information from the input devices was processed, and an inverse kinematics solution was calculated. This information was communicated via Ethernet to the robot interface computer. This computer communicated these values to the robots, received and processed force information from the force sensors, and sent the final information via Ethernet to the operator interface computer, where this force information was displayed to the operator via the haptic controllers.

In parallel to this, the operator interface computer also interfaced with the head-aimed viewer. It was responsible for receiving inputs on operator head orientation and sending this on to the robot interface computer. A user interface merged an image from the computer with the camera image form the cell simultaneously and provided the user with a "heads-up" display, presenting pertinent information to the user in the periphery of the head worn display.
This computer also monitored the user inputs and sent commands to the robot interface computer to toggle the video feed from the standard camera to the arc viewing cameras located in the cell. By enabling the camera feed to be switched, the system requires only an Ethernet connection and a single camera feed for full multi-camera remote operations. The fully integrated system is shown in Figure 2.

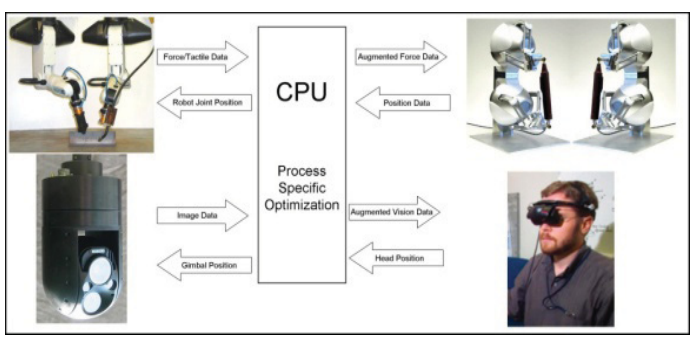

FIGURE 2. Bi-manual haptic system.

The system was then further augmented by incorporating a haptic bounce (contact instability) cancellation routine. The system was first modeled and simulated. It was characterized and compared empirically to the actual system. The software was then written to implement a passivity-based haptic-bounce correction algorithm. This algorithm was added to the system model and simulated. This enabled the tuning of the correction algorithm for optimal performance. The corrected algorithm was then incorporated into the physical system to compensate for contact instability.

Collaboration. Work this year has involved collaborative efforts with Dr. Milos Manic at the University of Idaho in further augmenting the feedback from the user and improving overall system performance. These efforts will continue for some time in the near future.

Research Benefits. This work, funded under the Instrumentation, Control, and Intelligent Systems (ICIS) signature at INL, has supported collaboration with Dr. Manic. Work is also being pursued with the other INL research groups in the field of explosives research.

The platform developed in this research project has also been demonstrated several times to many potential future customers and has been recognized as a necessary enabling technology for the future of the nuclear industry and for 
research in many hazardous applications and fields.

\section{Summary and Conclusions}

This project successfully developed and demonstrated the capabilities of a haptic telepresence system for the conduct of remote operations. The system is currently functional and has been used to develop better control and correction algorithms, and will serve in the future as a platform for conducting research in human-machine interaction, haptics, remote operations, and many other research uses.

Follow-on Work. Follow on funding has been applied for, and collaborative efforts continue with many different organizations. It is expected that this project will lead to another LDRD project and to a direct-funded research project with collaborators at INL.

Publications and Presentations. Publications and presentations resulting from this work are listed below.

Nichol, C.I., and Manic, M., 2009,'Video Game Device Haptic Interface for Robotic Arc Welding," HSI 2009, 2nd IEEE Conference on Human System Interaction, Catania, Italy, May 21-23, 2009. Available online: www.osti.gov/servlets/purl/957517sMWBDc/.
Invited Lectures/Talks. 2009 ICIS "Brew with the Crew" Invited talk: "Haptic Welding and You."

Invited Lecture: BYU Idaho ASME Student Section Forum "Robotics and Haptics," February 12, 2009.

Poster: 2009 INL Professional Exchange: "Haptic Interface for Remote Operations."

Feature Story: INL Web page "Engineer simulates a robot's sense of touch" by Roberta Kwok, published on INL's Nucleus home page and on Penn State's Mechanical Engineering Department Web page.

Accepted/Upcoming Presentation.

Nichol, C.I., 2009, “Telepresence System for Nuclear Industrial Processes," Presentation American Nuclear Society 2009 ANS Winter Meeting, November 2009, Abstract to be published in Transactions. 


\title{
Modeling Interface to Control System Designs
}

\author{
Kent McGillivary \\ Investigating the HLA framework for testing and modeling distributed control systems \\ IC105
}

This research continued to investigate

whether thehigh-level architecture (HLA)

framework is a tool that can be used to test and model distributed control systems. This project summary will discuss what was done during this fiscal year and then discuss several issues and needed improvements to make the HLA framework useful in modeling and testing distributed control systems.

Several steps were taken this year to determine whether the HLA framework could be used to model and test distributed control systems. The two main areas of research were modeling a process and control system in Matlab/Simulink and then breaking the modeling into components to be simulated on the HLA framework to see how this might work.

In modeling a simple process and control system, we chose to model the "Medium Chem" process used in Red and Blue training at INL. This was selected because it was a simple process and access to all the information that was needed to model the system was easily obtained. Matlab/Simulink was selected to model each portion of the system because it is a tool that is easy to use and also because its Real Time Workshop Toolbox allowed us to create generated code. This would be useful in hooking the model to the HLA bus.

The next step was designing and creating the HLA framework so that it could be used to model a simple process and control system. It was determined that there would be four different federates: simcontroller federate, process federate, control federate and HMI federate. The simcontroller federate controls the models loaded for each of the other federates and controls how fast the simulation ran. The process federate contained the model of how the physical process would behave. The control federate would contain the logic on monitoring signals for the process and controlling the process by opening and closing valves. Then the HMI federate would receive the signals shown on HMI. It contains the signals that could be changed by an operator.

Modification to some existing code that mapped inputs and outputs of Simulink model to HLA objects were required. The interface required modification to allow it to model three different federates. Configuration files that map the data from the Simulink Models were changed. A set of configuration files was created for each of the federates that mapped the inputs and outputs of the Simulink models to objects on the HLA bus. After this work, the code was ready for testing.

Tests were run to verify that models communicated and ran as expected. In this testing phase, several discoveries were made and issues identified in using HLA to experiment and model distributive control systems. These are discussed in the next section.

\section{Discoveries and Issues}

Issues discovered while doing this research involved HLA configuration problems in running a model. Some enhancements and tools were needed to make the HLA framework and the control-system model more realistic.

The first issue was to map the inputs and outputs of a model to published and subscribed objects on the HLA bus. Some work remains to improve the system to make it less time consuming and error prone. This may involve generating code automatically. Also, the configuration must be sufficiently generic that software tools other than Matlab may be used in modeling different parts of the process and control systems. Overcoming these issues would help improve the framework for testing of different control system configurations.

In order to model distributed control systems, a network modeling tool must be integrated on the HLA. This requires a network federate with additional research on modeling the different network configurations of control systems. Also, be research on how abnormal behavior might cause the control system to behave differently would be useful. A great deal of work could be done to model the control network system.

A need remains to incorporate different modeling tools in this HLA framework. Process modeling tools like HYSYS could be connected to HLA framework. Such tools may be more suited to modeling complex chemical processes than Matlab. A tool like this may allow for more 
complex processes, and more complex control system problems, to be modeled. More interesting control system models could then be created, a valuable addition to the HLA framework.

Overall, several issues and areas of development might make HLA a framework for modeling distributed-control systems. Most of these issues can be overcome in time.

\section{Conclusion}

The research this year has shown that the HLA framework can be used to test new control systems and to research activities in control systems. Several issues must be overcome, and development is needed to make the HLA bus a viable solution. Also several tools must be added to HLA framework - for example, network modeling software and chemical modeling tool like HYSYS. More complex systems must be modeled and tested to see how they would integrate with the HLA framework. 


\title{
Resilient Control System Network Agents
}

\author{
D. Todd Vollmer (INL), Milos Manic, Ph.D. (University of Idaho) \\ Developing a system with capabilities that exhibit adaptive traits to minimize consequences of \\ detrimental control-system network-based incidents \\ IC106
}

This is the second year of a proposed threeyear research project. The overall goal is to develop a system with capabilities that exhibit adaptive traits to minimize consequences of detrimental control-system network-based incidents. With a renewed national and international interest in new nuclear facilities with modern digital control designs, technologies that prove to enhance system security resiliency are necessary.

The project proposes to add intelligent detection schemes and response capability to an intrusion-detection system at the controller level instead of using a static rule-based system on the network segment. The focus will be on changes related to security incidents, but the anomaly detection algorithms developed by current research are flexible enough to identify other, more general issues as well. Normal system behaviors in control systems typically are simpler to characterize than those exhibited in traditional IT systems. Consequently, detecting abnormal behaviors is more likely with fewer false positives. Development of this technology can extend the normal reliability and functionality of control systems by localizing the detection and response to the individual component. Failures of the central authority or communication paths in a system will be mitigated.

Intrusion Detection Systems (IDS) generally fall into two main categories: network or host based. Network-based systems examine network traffic, looking for indicators of abnormal behavior. Most systems use predefined rules to perform this action. These rules are typically developed by a human expert after an incident. They are proficient in recognizing specific attacks, but variations can be missed. In contrast, anomaly-based systems examine network behavior to make determinations. Machineintelligence algorithms such as neural networks or support vector machines are trained and implemented. These systems can detect variants or even new attack vectors. The solutions examined in this effort are a member of the latter.

\section{Technical Objectives}

Specific milestones for this year and current status against each are presented in the list below:

1. Refined development of the software prototype implemented in year one, to include machine intelligence algorithms.

$\mathrm{A} \mathrm{C}++$ and $\mathrm{C \#}$ version of the previously developed Matlab ANN code was created. These implementations are portable across many different operating systems and hardware architectures. The code implements a dynamically defined ANN architecture (number of layers and neurons). A user interface that displays network device, selects one, and starts capturing the network traffic was added. The code is based on the WinPcap library and, as implemented, allows for stored pcap files to be used for inputs as well as live capture.

2. Identify and procure development hardware capable of running the prototype algorithm identified in 1.

A contract with the University of Idaho, under the direction of Dr. Milos Manic, has been executed. A development EMBEST PXA270DB Evaluation Board was obtained. Initial efforts of development met with several issues with development-tool version issues and driver incompatibility issues. Analysis of the board performance determined that the system was capable of processing the developed neuralnetwork analysis algorithm.

3. Targeted attack vectors more specific to control systems than used in year one development.

Refinement of the attack vectors designed in year one was accomplished. Nessus plug-ins specific to control system hardware were obtained and incorporated into the test system. ICMP test packets representative of five attack classes were created and implemented in a command line tool. In addition, a control-system specific network switch with IPv6 capabilities was acquired. This switch has port mirroring and other advanced monitoring capabilities for monitoring the systems reaction to the attack vectors. 
4. Determine response mechanisms and implement them in a prototype.

A prototype and paper have been created. The paper, titled "Human Interface for Cyber Security Intrusion Detection Systems," was presented at the HSI 2009 conference in Catania, Italy. This paper details a solution for presenting cyber-incident information to human operators from anomaly-based intrusion-detections systems. A similarity algorithm mapping anomaly results to signature-based intrusion system rules is presented. Categorizations of attacks found in rules created for the Snortintrusion system were used as a basis of information to present to the user. A proof-ofconcept system was developed using Perl and tested with generated Internet Control Message Protocol (ICMP) packets. A resulting identification on test data of $60 \%$ proved the efficacy of the proposed algorithm.

\section{Results and Accomplishments}

The majority of the work on this project occurred during the late spring and early summer months. This was a planned schedule, and it coincided with the availability of the University partners. However, three papers were submitted and presented at Institute of Electrical and Electronics Engineers (IEEE) conferences in this time frame. One paper was based primarily on work done the previous year. The other two papers were based on work performed this year. This has exposed the international community to the research capabilities and results funded by Idaho National Laboratory (INL). These papers have drawn the interest of several academics from the University of Kansas, and the possibility of collaboration is being explored. This research has advanced some new concepts in the area of network intrusion detection that are applicable for information technology systems everywhere.

Last year's findings showed that machineintelligence algorithms may be used in anomalybased intrusion detection systems on control systems. This year's work built on the previous system by adding functionality to the prototype and moving it closer to implementation on a hardware platform or any general-purpose computer. The creation of a test system was delayed, but finished by the end of the fiscal year. This system was to have been used to provide a broader set of test sets for the detection algorithm refinement. Not having this new test data led to one of the more innovative results of the research. Publicly available intrusiondetection rules detailing attack vectors were used as input for training a neural-network system. To our knowledge, this had not been done before.

A refined software prototype, based on the Intrusion Detection System using neuralnetwork-based modeling (IDS-NNM) implemented the previous year, was created. The algorithm was ported to $\mathrm{C}++$ and $\mathrm{CH}$. A neuralnetwork architecture was implemented, which is dynamically constructed based on a text file with a description of the network and its weight set. This text file can be extracted from Matlab's neural network toolbox. The WinPcap library was used to collect network data online. The packet headers are decoded and, based on the extracted information, the input vectors for the IDS-NNM algorithm are created. These are fed to the developed Neural Network. Overall, the algorithm is implemented in $\mathrm{C}++$ and it is capable of online network traffic filtering using the developed IDS-NNM algorithm. A paper titled "Neural Network Based Intrusion Detection System for Critical Infrastructures," based on the original prototype was presented in Atlanta, GA. at the International Joint Conference on Neural Networks (IJCNN). The majority of this effort was provided by the University partner.

A paper titled "Human Interface for Cyber Security Intrusion Detection Systems" was presented at the HSI 2009 conference in Catania, Italy. This paper details a solution for presenting cyber-incident information to human operators from anomaly based intrusion detections systems such as IDS-NNM. A similarity algorithm mapping anomaly results to signature-based intrusion system rules was detailed. Categorizations of attacks found in rules created for the Snort intrusion system were used as a basis of information to present to the user. A proof-of-concept system was developed using Perl and tested with generated ICMP packets. A resulting identification on test data of $60 \%$ proved the efficacy of the proposed algorithm. 
A refinement of the algorithm generated in the HSI 2009 paper was created. This work was presented in a paper titled "Computationally Efficient Neural Network Intrusion Security Awareness," at the International Symposium on Resilient Control Systems (ISRCS) 2009. The enhanced version of an algorithm to provide anomaly-based intrusion-detection alerts for cyber security state awareness is detailed. A unique aspect is the training of an error backpropagation neural network with intrusiondetection rule features to provide a recognition basis. Ethernet network packet details are subsequently provided to the trained network to produce a classification. This leverages rule knowledge sets to produce classifications for anomaly based systems. Several test cases executed on ICMP protocol revealed a $60 \%$ identification rate of true positives. This rate matched the previous work, but $70 \%$ less memory was used and the run time was reduced to less than 1 second from 37 seconds.

A new test system was completed at the end of the fiscal year. It consists of a Micrologix 1100 controller with a Moxa 505A IPv4/v6 managed-network switch. The system currently controls six LEDs, four push-button switches, two rotary potentiometers, two temperature sensors, a light source and a fan. All of this is packaged in a small, suitcase-sized portable container that has wheels and a pull handle. This portable system is roughly the size of a briefcase and will enable instrumentation and control research on real hardware. In addition, the researchers will no longer be confined to a fixed lab space. The physical lab can be moved at will and be exercised more efficiently in just about any location. This system has been made available to other LDRD researchers, and it is likely that it will be used.

\section{Summary and Conclusions}

INL has a strong presence in securing our nation's system of critical infrastructure networks, including control-systems cyber security. Our project has enhanced this position by discovering a mechanism to train anomalybased systems with knowledge gleaned from static-rule implementations. We have also implemented functionality that reports anomalydetection system results in understandable human terms. This type of information may also be provided to intelligent-system-monitoring tools utilizing data fusion. The knowledge of this system and the ability to integrate it with other systems resides in the principle investigator (PI) who is a laboratory employee. Furthermore, the three papers presented at international IEEE conferences resulting from this research have advanced the PI's efforts to obtain a Ph.D. in computer science.

The enhanced software prototype is now in a suitable form to be provided to other researchers. It does not restrict them to use of an expensive commercial application and can be the basis for future research. The plan for next year is to extend the prototype functionality to characterize the communication protocol data specific to command and control. This functionality will enable detection of more sophisticated attacks, such as subversion of the command systems. Two possible commercial applications for integration will be explored as well. 


\section{Integrated Control System Data Fusion}

Shane Cherry, David Gertman, Milos Manic, Miles McQueen, Jared Verba

Enhancing manager/operator response and increasing overall facility stability and efficiency

IC107

Modern critical infrastructure control and security systems have the capability to provide facility managers, operators, and security personnel with an abundance of monitoring data. This data comes from multiple sources, including process and control status as well as network and physical security information, which are deployed at different levels within the system to provide both situational awareness and defense in depth. However, due to the amount and complexity of the data, it is challenging for operators to quickly analyze situations and respond appropriately. This increasing volume of situational data and the potentially time-critical nature of related decisions makes data fusion a critical technology for transforming large amounts of information into timely, actionable intelligence. The hypothesis of this research is that a holistic assessment and prioritization of control system and security information will provide a basis for fusing data from various sources and in a specific manner so as to draw the relationships among them. As a result, this capability will enhance manager/operator response and increase overall facility stability and efficiency.

In addition, having this information is of no use unless there is an ability to be able to present it in a usable form and in such a way so that a human (or other machine) operator can understand it and take corrective action in a timely fashion. This requires a sophisticated interface that can present an accurate picture of the complete system as well as present viable resolution strategies should a problem occur.

This laboratory-directed research and development (LDRD) project attempts to address the above-mentioned issues using, as a basis, a simulated control-system process that involves related elements of cyber security, physical security, and process control measurements. The initial phase of this project was to develop the basic infrastructure and a framework demonstrating data fusion concepts along with presentation software and scenarios showing how such a system might operate. The envisioned data fusion system can be illustrated by the following 3-tier architecture shown in Figure 1.

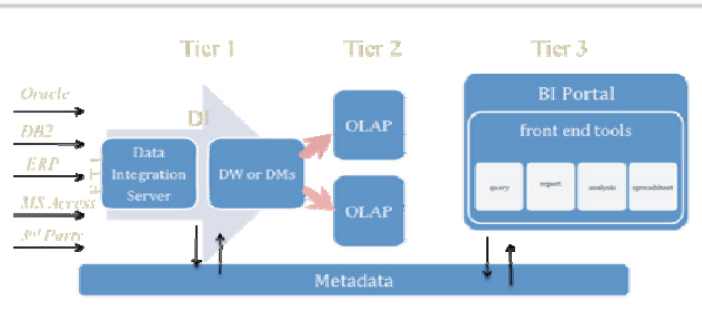

FIGURE 1. The 3-tier data fusion architecture system.

The main tiers of this system would execute: extraction, transformation, and loading (ETL) of disparate data sources along with creation of data warehouses (and data marts), followed by an online analytical processing (OLAP) tier, and resulting in a front-end suite of tools for query, analysis, and reporting.

\section{Technical Objectives}

The primary objective of this research is to develop an effective critical-infrastructure control and security data-fusion system that is scalable to be applied to full-scale facility control and security systems while maintaining operator and crew situational awareness and supporting decision making. Accomplishing this objective required meeting the following year one secondary objectives:

1. Develop methods and algorithms for effectively fusing cyber- and physicalsecurity situational data with control-system information and applying a suitable model for data abstraction and testing on a surrogate critical-infrastructure facility.

2. Apply probabilistic-risk and humanreliability methods for prioritization of control system and security data delivery to operator/manager and assess the efficacy of this approach by performing analytical human factors/HRA tests and human-in-theloop testing on different data fusion/abstraction approaches for a surrogate process system for which cyber- and physical-security data have been identified.

3. Provide efficient and effective presentation of data to process owner, reflecting integrated data fusion for both autonomous and human elements.

4. Provide capability to scale fusion system to address increased complexity and realism 
related to process control. This includes development of transient process model based on the Hybrid Energy Testing (HYTEST) laboratory being developed at Idaho National Laboratory (INL).

\section{Results and Accomplishments}

This data-fusion effort focuses on developing a way to fuse data from all possible information sources related to a critical infrastructure facility in such a way that it can be properly prioritized for presentation to and use by different levels of operators. Progress to date has been to develop a conceptual approach to the problem and to define a hypothetical physical facility with a baseline control system and scenarios of threats. Using this surrogate system, a series of scenarios was developed representative of normal, off-normal, and emergency operating conditions involving process, physical-security, and cyber-security factors. Consideration was given to blended attack, i.e., those having a physical security and cyber security components. Fusion techniques are being developed to present key information to operators, maintain high levels of situation awareness, and support the implementation of mitigating measures.

The accomplishments associated with these year one activities include:

- Using MatLab Simulink, developed notional control system and process model relative to chemical processing facility

- Developed notional physical security system and scenario-generation tool based on facility access technologies utilized at INL

- Developed cyber-security scenario generation capability aimed at adversely affecting control system and related processes

- Developed initial data fusion capability and graphical user interface that integrates cyber and physical security information with process control information

- Developed and validated HYTEST facility transient models for mass transport and chemical reaction in each of the following reactors: synthetic fuels (methanation) reactor, steam methane reforming reactor, high-temperature water-gas shift reactor

- Published two peer reviewed papers at international conferences:

○ Miles A. McQueen, Wayne F. Boyer, "Deception Used for Cyber Defense of Control Systems," IEEE International Conference on Human System Interaction, May 21-23, 2009.

- D.I. Gertman, "Human Factors and Data Fusion as Part of Control Systems Resilience," 2nd IEEE International Conference on Human System Interaction, Catania, Italy, May 2009.

Screen shots of the current system are shown below in Figure 2.

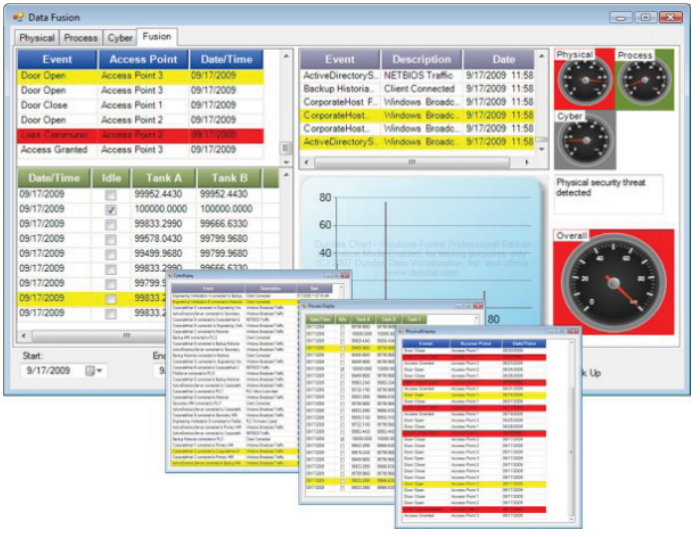

FIGURE 2. Current data fusion system interface.

\section{Summary and Conclusions}

The current system, though far from complete, allows for data input, rules-based evaluation and data retrieval. The data is "fused" by tying together relative time-based keys and using rules to evaluate the level of threat. Utilities exist for data input as well as some basic dashboard demonstrations of a prototypical implementation. Fact and dimension data are configured for future use in an OLAP system for data mining. This initial effort laid the groundwork for a more sophisticated implementation of a data-fusion system that is intended to combine existing rules-based evaluations with data-mining discoveries and predictive analysis. In addition, continued 
development of display tools for real-time analysis and interpretation of fused data will greatly enhance the usability and effectiveness of this system. The investigative and development process was useful in flushing out specific requirements as well as giving participants a much better view of the work involved and the potential utility of the approach.

The 3-year plan associated with this LDRD entails the development, as a proof of principle, of a set of algorithms based on data fusion, data mining and artificial intelligence aimed at enhancing the concept of holistic data fusion related to process control facilities. This includes continuing to refine data and information flow, increase complexity of process and security information, and design validation experiments. To accomplish these goals, integration of the HYTEST transient process model developed in year one into the fusion system will be a priority in year-two efforts. Follow-on efforts will include full hardware-in-the-loop data feeds off of the HYTEST system. Additional work will also entail enhancements of the existing user interface, i.e. front-end tools for querying, analysis, and reporting of fused and analyzed behavior. Once these goals are achieved, it is anticipated that the year-three activities will entail implementation of cutting-edge computational intelligence algorithms, such as neural networks, fuzzy-set theory, support vector machines, and others. 


\section{Wireless Sensor Testing}

John Buttles, Jason Wright

Developing approaches and solutions to resolve system degradation and instability

IC108

Wireless sensor network (WSN)

technologies have become a focus of development for instrumentation and control (I\&C) applications in many industries. The promise of lower installation cost combined with reliable monitoring of process parameters at virtually any location when needed has driven many existing instrumentation vendors, as well as new startup companies, to attempt to fill a need in wireless technology. These technologies are in their infancy and have not been fully tested for security, reliability, compatibility or cooperation. It is our hypothesis that radio frequency (RF) and/or cyber interference to a WSN can lead to destabilization of the control system by introducing latency or modifying data, which can undermine the control system design and amplify traditional concerns, where impact is limited to the target of the interference. The result is a domino effect that, while it may originate in loss of an individual sensor's data or bad data, will lead to facility-level changes that impact safety and/or production. Distributedcontrol networks and complex network theory, though a current topic of research, has not considered how multiple interference regimens can generate latencies or bad data that create destabilization and lead to a decrease in the resilience of control systems. To address this knowledge gap, this research proposal seeks to design and analyze full-scale WSN control systems that can be stressed by multiple interference types in order to develop approaches and solutions to resolve the expected system degradation and instability.

\section{Technical Objectives}

The work in this laboratory-directed research and development (LDRD) is divided into four tasks to be performed over three years:

- Define the state of the WSN market and prioritize WSN technology targets

- Set up multiple wireless sensor networks

- Perform experiments to measure cyber and $\mathrm{RF}$ interference effects on wireless sensor network communication

- Develop policies, best practices, and monitoring-device equipment specification to mitigate disruption of system data.

\section{Results and Accomplishments}

Fiscal Year (FY) 2009 was the first year of this LDRD; I received funding in March 2009. The first task was to define the state of the WSN market and identify the dominant WSN technologies that will be used in critical infrastructure industries. This task was completed using market-research studies, discussions with Idaho National Laboratory (INL) Supervisory Control and Data Acquisition (SCADA) partners, and surveys of equipment vendors.

Although many technologies were researched, it became apparent that three dominant technologies are widely used for WSNs. These are 802.11 (Wi-Fi), cellular, and IEEE 802.15.4. It is estimated in the reviewed market studies that over 5 million new wireless sensors will use these technologies deployed in many industrial applications by the year 2013 . These technologies are distributed as follows:

- 802.11 $2.5 \mathrm{M}$ sensors

- Cellular $1.5 \mathrm{M}$ sensors

- IEEE 802.15.4 $1 \mathrm{M}$ sensors.

\section{Summary and Conclusions}

The initial research to identify and support the original hypothesis is complete. The necessary test equipment, consisting of several WSN systems, signal simulators, RF analyzers, and PC control systems, has been purchased. A lab space in the Center for Advanced Energy Studies (CAES) building has been identified to begin testing, which will constitute much of next year's work.

The first year's work also informed WSN vendors, user groups, university researchers, and standards groups of my research. Many have offered support for my research over the next two years. This has resulted in a better understanding and awareness of WSN's and the problems they face when used in critical infrastructure industries. The work under this LDRD will expand our capabilities in the research of resilient control systems and become a valuable asset of the Department of Homeland Security Control Systems Security programs, and National SCADA Test Bed programs at INL. 


\section{Anomaly Detection, Diagnosis, and Resilient Control in Complex Engineered Systems}

V. Venkatasubramanian, Purdue University; H. Garcia, C. Rieger

Designing and implementing intelligent, resilient control systems that will be robust to accidental and intentional abnormal events IC109

One important challenge for control system engineers today is designing and implementing intelligent, resilient control systems that will be robust to accidental and intentional abnormal events. Such systems should also assist operators in making supervisory control decisions for such abnormal situations, in starting up and shutting down, performing controller assessment, and so on. Operators' failure to exercise the appropriate supervisory control decisions in abnormal conditions often have an adverse effect on product quality, process safety, occupational health, and environmental impact. The economic impact of such abnormal situations is enormous: about $\$ 20$ billion/year in losses in the petrochemical industry alone in the U.S. The cost is much more when one includes similar situations in other industries, such as pharmaceutical, specialty chemicals, power, and desalination. Thus, considerable incentive exists for developing intelligent, resilient control systems that can provide automated operator assistance for supervisory control in complex process plants. People in the process industries view this as the next major challenge in controlsystems research and application.

The goal is to develop a model-based hybrid framework for anomaly detection, diagnosis and resilient control in complex process systems. This hybrid framework will integrate signeddigraphs-based causal models of the process, dynamic-trend models, and statistical techniques for state awareness, and model-based supervisory control. Such an approach overcomes the shortcomings of the individual components and is a significant advancement over the state of the art. This framework will be tested and evaluated on both dynamic simulations and experimental testbeds. The lessons from this research can be generalized for other such process systems.

\section{Technical Objectives}

The technical objectives and project deliverables for this 3-year project would consist of developing and implementing:

- A theoretical framework for applying signed digraphs (SDG), principal-component analysis (PCA), and qualitative-trend analysis (QTA) for anomaly detection, diagnosis, and resilient control

- A hybrid Bayesian framework for integrating SDG, PCA and QTA

- Models of unit operations

- Two simulation testbeds: (i) Purdue testbed and (ii) MCM Small Loop testbed for various failure scenarios

- $\quad$ Papers, presentations, and reports to facilitate technology transfer to INL.

We anticipate that the lessons from this research can be generalized for other such process systems.

\section{Results and Accomplishments}

This report summarizes the progress we made in the first year - from April 1 through September 30, 2009. We focused on the development of the mathematical framework for PCA, SDG, and QTA as well as the Bayesian approach. We tested our ideas on a computer software technical review (CSTR) reactor system simulation for proof of concept study.

PCA Framework. Given a multivariate data set $\mathbf{Y}$ ( $n$ samples, $m$ variables), one seeks to find a number, $c$, of descriptors, $(\mathbf{T}(:, \mathrm{i})$ for $\mathrm{i}=1$ to $\mathrm{c})$ of the given data such that most of the information in $\mathbf{Y}$ is contained in these descriptors. One thus converts the matrix $\mathbf{Y}(\mathrm{n} \times$ $\mathrm{m})$ into a new matrix $\mathbf{T}(\mathrm{n} \times \mathrm{c})$ with lower dimensions. In PCA, each of the new descriptors is called a principal component (PC), which is a linear combination of the original data and is orthogonal to all other PCs. The coefficients defining these $\mathrm{PCs}$ are equal to the eigenvectors of the covariance matrix with the $\mathrm{c}$ largest eigenvalues. The SVD (singular value decomposition) algorithm is most commonly used to find the eigenvectors and eigenvalues of the covariance matrix.

Once the number $c$ is set, one writes:

$\mathbf{T}_{n \times c}=\mathbf{Y}_{n \times m} \times \mathbf{P}_{m \times c}$

with $\mathbf{P}$ defining the coefficients of the $c$ principal components as column vectors. Each column in T contains the values for each PC for the $n$ 
samples in $\mathbf{Y}$. The following properties of PCs and principal scores are proven:

$\mathbf{P}(., \mathrm{j})^{\mathrm{T}} \times \mathbf{P}(., \mathrm{k})=0$ if $\mathbf{j} \neq \mathrm{k}$ (Orthogonality)

$\mathbf{P}(., \mathrm{j})^{\mathrm{T}} \times \mathbf{P}(., \mathrm{j})=1$ (Eigenvectors are normalized)

$\operatorname{Var}(T(:, j))=\lambda_{j}($ Variance of principal score $=$ corresponding eigenvalue).

A crucial step in PCA modeling is the selection of the number of principal components. The underlying paradigm is that variance corresponds to information. One can thus discard those PCs with low variance.

Figure 1 shows an application of PCA to sensor and actuator failures.

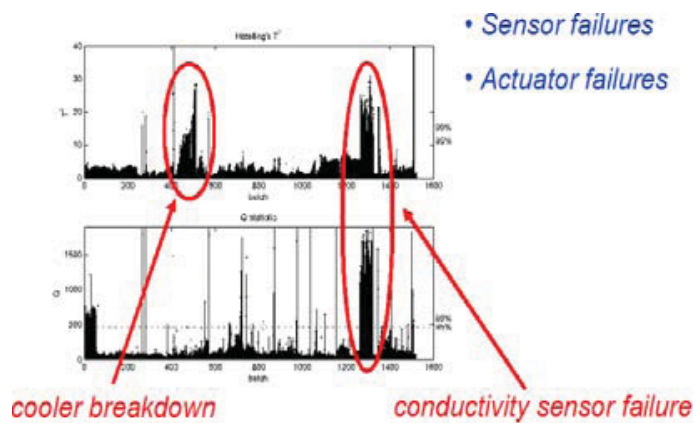

FIGURE 1. Hotelling's $T^{2}$ statistic and $Q$ statistic as a function of time. It is typical that sensor failures result in both statistics to be violating their limits, whereas other failures (process, actuator) may only affect the Hotelling's $T^{2}$ statistic.

Signed Directed Graphs. In the context of process supervision, SDGs are qualitative representations of a system based on qualitative but mechanistic knowledge. SDGs lend themselves to the presentation of such knowledge because graphs are easy to visualize. SDGs are special cases of general graphs as they have directed arcs (implies causality) and signs (implies direction of change). The sentence, "If A goes down, then B goes up," can thus be presented as follows:

\section{$\mathrm{A} \longrightarrow \mathrm{B}$.}

Such description does not imply that A will change if $\mathrm{B}$ changes, thus giving rise to a unidirectional arc. By inclusion of more variables $(\mathrm{C}, \mathrm{D}, \ldots)$ and more statements, one eventually obtains a more complex graph (Fig. 2).

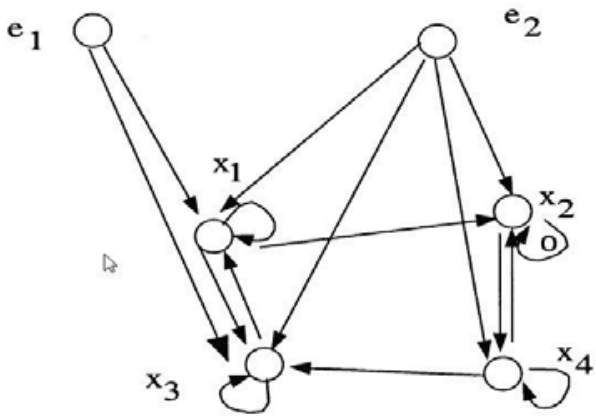

FIGURE 2. A moderately complex SDG model representation.

Once established, the SDG representation allows the emulation of the represented process qualitatively. This means that one can evaluate the effects of certain qualitative changes in one or a set of variables on the qualitative behavior in other variables. SGDs are suitable tools for rootcause analysis, i.e., the task of finding the variable or variables that are primarily responsible for a symptomatic behavior in other variables. Indeed, qualitative simulation of SDG allows questions of the form, "What if?" By emulating faulty conditions ("What if this sensor fails?" "Then this pump stops."), one obtains the expected behavior under these conditions. Following the simulation of several faults and the comparison with actual behavior, one can often exclude a whole range of potential faults. In extreme cases, one may be able to uniquely identify a single root cause, thereby finishing the diagnosis task. Figure 3 (next page) shows a typical table resulting from an SDG model simulation. For example, for the faulty condition " $\mathrm{w}_{1}$ high" one sees that the first three monitored variables $\left(\mathrm{c}_{1,1}, \mathrm{c}_{2,1}, \mathrm{q}_{\mathrm{f}}\right)$ have signs,+- , and + . For the fault " $\mathrm{w}_{2}$ high" one obtains,,-+ and + . Thus, if one observes a positive sign for $\mathrm{c}_{1,1}$, the fault " $\mathrm{w}_{2}$ high" can be excluded based on that information. To decide between " $\mathrm{w}_{1}$ high" and " $\mathrm{w}_{2}$ low" $(+,-,-)$ one will need additional information (e.g., on the sign of $\mathrm{q}_{\mathrm{f}}$ ). 


\begin{tabular}{|c|c|c|c|c|c|c|c|c|c|c|c|c|c|c|c|}
\hline \multirow[b]{2}{*}{ disturbance } & \multicolumn{15}{|c|}{ signs of system variables } \\
\hline & $\overline{c_{1,1}}$ & $c_{2,1}$ & $q_{\mathrm{r}}$ & $c_{1}$ & $c_{2}$ & $\mathrm{C}_{\mathrm{p}}$ & $T_{\mathrm{m}}$ & e & $T_{\mathrm{j}}$ & $T$ & $\mathrm{CS}_{1}$ & $k_{2}$ & $w_{\mathrm{p}}$ & CS & $\mathrm{VP}$ \\
\hline$w_{1}$ high & + & - & + & + & $?$ & - & - & + & + & - & + & - & + & + & + \\
\hline 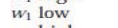 & - & + & - & - & ? & + & + & - & - & + & - & + & & & \\
\hline high & - & + & + & ? & + & - & - & + & + & - & + & - & + & + & + \\
\hline low & + & - & - & ? & - & + & + & - & - & + & - & + & - & - & - \\
\hline$T_{1}$ high & $\mathbf{0}$ & 0 & 0 & - & - & + & + & $\bar{t}$ & - & + & - & + & + & - & - \\
\hline & o & 0 & 0 & + & + & - & - & t & + & - & + & - & - & + & + \\
\hline igh & 0 & 0 & 0 & $\begin{array}{ll}+ \\
-\end{array}$ & & $\bar{t}$ & \pm & & - & $\bar{t}$ & - & - & - & - & - \\
\hline$T_{\text {m.bas low }}$ & $\mathrm{I}_{0}^{0}$ & $\begin{array}{l}0 \\
0\end{array}$ & $\begin{array}{l}0 \\
0\end{array}$ & - & - & $\begin{array}{lll}+ \\
+\end{array}$ & $\mp$ & $\begin{array}{llll}+ & \\
+\end{array}$ & $\begin{array}{llll}+ & & & \end{array}$ & $\begin{array}{llll}+ \\
+\end{array}$ & $\stackrel{+}{+}$ & $\stackrel{+}{+}$ & $\begin{array}{l}+ \\
+\end{array}$ & $\begin{array}{l}+ \\
+\end{array}$ & $\stackrel{+}{+}$ \\
\hline $\begin{array}{l}T_{\mathrm{m}} \mathrm{n} \text { tow } \\
T^{\text {set }} \text { low }\end{array}$ & 0 & 0 & 0 & + & + & - & - & - & - & - & - & - & - & - & - \\
\hline CS & 0 & 0 & 0 & - & - & + & + & - & + & + & - & + & + & + & + \\
\hline & & & 0 & + & + & - & - & + & - & - & + & & & - & - \\
\hline h & 0 & 0 & 0 & - & - & + & + & - & + & + & - & + & + & & + \\
\hline & 0 & 0 & 0 & + & + & - & - & + & 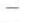 & - & + & - & 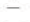 & . & - \\
\hline & & 0 & 0 & + & + & - & + & - & - & - & & - & & & - \\
\hline & & 0 & 0 & 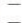 & - & + & + & + & + & + & + & + & + & + & + \\
\hline $\begin{array}{l}\mathrm{CS}_{\text {fanl hi }}^{\text {hio }} \\
\mathrm{CS}_{\text {sel }}\end{array}$ & $\begin{array}{l}0 \\
0\end{array}$ & $\begin{array}{l}0 \\
0\end{array}$ & $\begin{array}{l}0 \\
0\end{array}$ & + & + & $\begin{array}{l} \pm \\
\pm\end{array}$ & \pm & + & \pm & \pm & $\overline{+}$ & + & \pm & \pm & + \\
\hline & 0 & 0 & 0 & - & - & + & + & - & + & + & 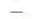 & + & + & & + \\
\hline 10 & 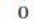 & 0 & 0 & + & + & - & - & + & & & + & & & + & - \\
\hline
\end{tabular}

FIGURE 3. A typical result obtained from SDG simulation. By comparing simulated trends with actual trends, one can exclude impossible fault scenarios and in some cases uniquely identify the root cause.

Qualitative Trend Analysis (QTA).A typical problem in the context of data analysis is that the user's perception and understanding of data is very different from proposed models that explain the same data. In particular, control engineers typically develop a qualitative model of how data should behave under normal and abnormal conditions. These descriptions are seldom exact; more often, they are qualitative in nature. "Up," "down," "faster," "slower" are terms regularly in use across many fields and applications. They allow humans to segment series into meaningful episodes where certain characteristic behaviors are dominant. Incorporating such abilities into automated, digitized systems is difficult, which explains why qualitative analysis is a relatively recent discipline.

One defines the qualitative description of a trend based on the first two derivatives. The first derivative determines whether the trend in a given process variable is steady, upwards or downwards. The second derivative determines whether the process variable is accelerating, decelerating or without change in the first derivative. For each possible combination for the signs of the two derivatives, one usually decides on a single alphabetic character, which then represents this behavior and referred to as a triangular primitive. If only the first derivative is used, we refer to the character as a monotonic primitive. Figure 4 shows how polynomial fitting results in a qualitative description of the trends present in the shown signal.

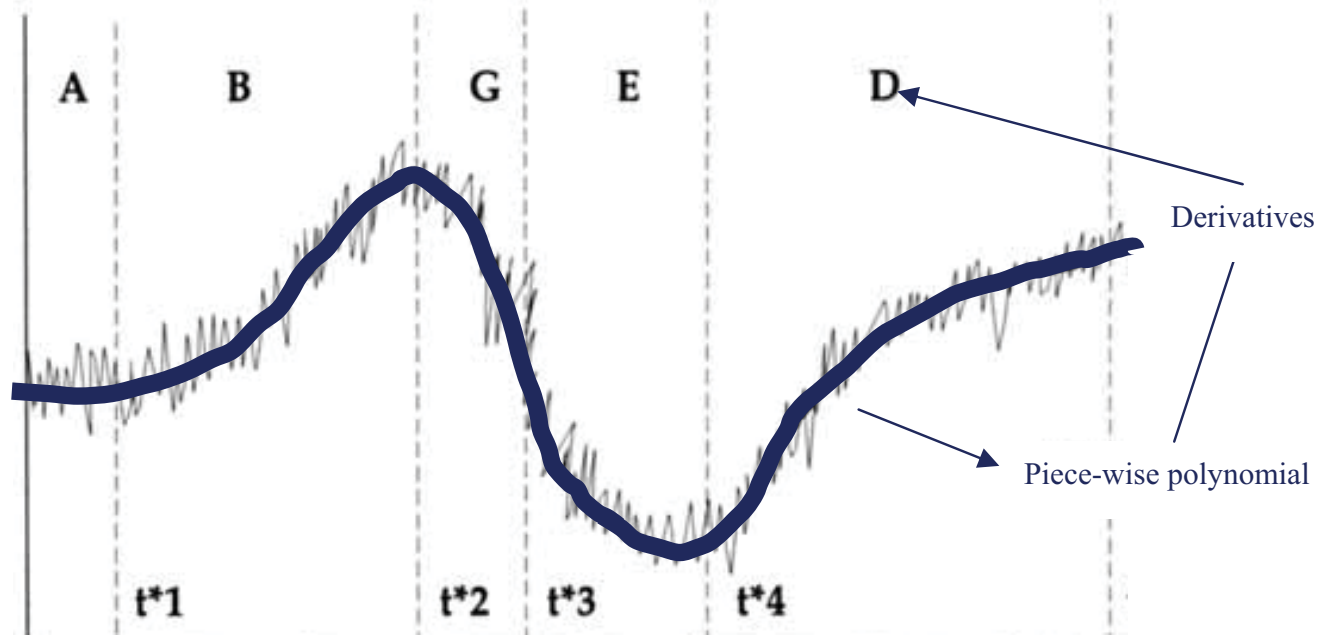

FIGURE 4. Converting a noisy time series into qualitative descriptors by means of polynomial fitting.

Benefits of QTA in-process monitoring follow when primitives or sequences of primitives can be linked to the experience of operators. Usually, a process operator recognizes a certain behavior, problematic or not, by matching the qualitative description of the recent process history with similar events in the past. To make QTA work for process diagnosis, one establishes a table that links series of primitives (series of characters, hence "words") with the appropriate diagnostic label. Better resolution may be obtained by including the time length associated with each primitive. 
Bayesian Fault-Tolerant Control. The essence of this technique lies within the application of a Kalman filter. Such a filter, using the mathematical model, predicts future measurements on the basis of current-state estimates. Each time one obtains new measurements, these measurements are used to update the current-state estimate. In the presence of a fault, the nominal or normal operation model will fail to predict future measurements correctly. High deviations between predictions and measurements are detected by means of a statistical test based on the $\chi^{2}$ statistic. Following such detection, one identifies the correct fault by fitting the fault parameters of each possible fault (magnitude, start time, duration) and selecting the fault corresponding to the best fit.

To account for model uncertainty in the diagnosis task, one uses not one model, but several realizations of the same model with different values for the uncertain parameters. For each of the models and for each possible parameter associated with a considered fault, one can compute the likelihood that the observed data, Y, stems from a system with given model parameters $(\mathrm{m})$, fault $(\mathrm{f})$ and associated parameters (b):

$\mathrm{L}(\mathrm{Y} \mid \mathrm{m}, \mathrm{b}, \mathrm{f})$.

One wishes to calculate the likelihood for a given fault, given the observed data $\mathrm{L}(\mathrm{f} \mid \mathrm{Y})$. Then, by evaluating this property for each possible fault, one can identify the most likely fault, i.e., the fault with maximal likelihood. One uses Bayes' rule for make this possible:

$\mathrm{L}(\mathrm{f} \mid \mathrm{Y})=\mathrm{L}(\mathrm{Y} \mid \mathrm{f})$. L (f) / L (Y)

In the latter, $\mathrm{L}(\mathrm{Y} \mid \mathrm{f})$ and $\mathrm{L}(\mathrm{Y})$ require that one integrates $\mathrm{L}(\mathrm{Y} \mid \mathrm{m}, \mathrm{b}, \mathrm{f})$ over the whole realm of models and parameters. For L(Y), one additionally needs to sum over all possible faults. Assuming that model parameters and fault parameters are not correlated, one writes:

$\mathrm{L}(\mathrm{Y} \mid \mathrm{f})=\int \mathrm{L}(\mathrm{Y} \mid \mathrm{m}, \mathrm{b}, \mathrm{f}) . \mathrm{L}(\mathrm{b}) . \mathrm{L}(\mathrm{m})$

$L(Y)=\sum L(Y \mid f) . L(f)$.

In these mathematical developments, $\mathrm{L}(\mathrm{m})$, $\mathrm{L}(\mathrm{b})$ and L(f) represent the so-called prior distributions. These distributions identify the prior belief that the practitioner has with respect to the probabilities associated with model parameters, fault parameters, and fault types respectively. While the theory is general, we base the $\mathrm{L}(\mathrm{M})$ prior on a model identification study while, for $\mathrm{L}(\mathrm{b})$ and $\mathrm{L}(\mathrm{f})$, a flat prior is used. This means that any value for $b$ or $f$ has equal probability.

Summing over all possible faults is relatively straightforward. However, the integration over the continuous set of parameters for faults and models cannot be done analytically in the general case. It is for this reason that one uses Monte Carlo sampling to obtain a representative subset of all possible values for the parameters. Then the integral for $\mathrm{L}(\mathrm{Y} \mid \mathrm{f})$ can be approximated by the following sum over all sampled model parameters and fault parameters:

$\mathrm{L}(\mathrm{Y} \mid \mathrm{f})=\sum \mathrm{L}(\mathrm{Y} \mid \mathrm{m}, \mathrm{b}, \mathrm{f}) . \mathrm{L}(\mathrm{b}) . \mathrm{L}(\mathrm{m})$.

CSTR Simulation Testbed. The developed framework is being currently tested for a linear Continuously Stirred Tank Reactor (CSTR). The reactor has a constant volume and constant equal in- and outflow. A reaction occurs in which component $\mathrm{A}$ is converted to component $\mathrm{B}$, and one wishes to control the component $\mathrm{B}$ concentration in the outgoing stream to a given set point. This is accomplished by a PID controller, which manipulates the concentration of $\mathrm{A}$ at the inlet. Figure 5 shows a typical simulation result.

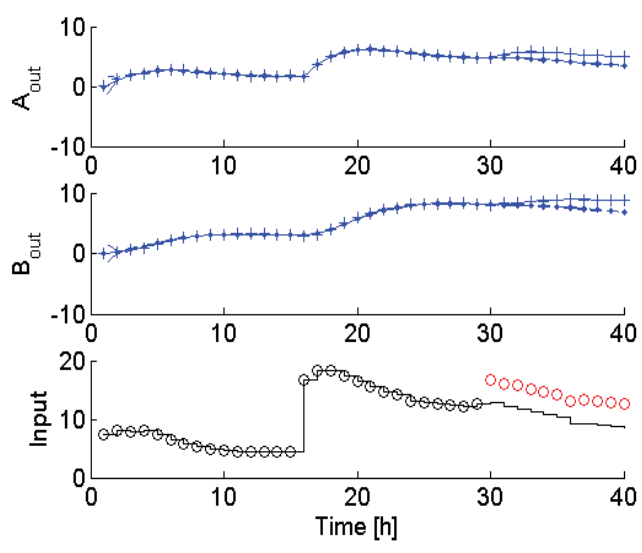

FIGURE 5. Typical simulation result for the CSTR testbed. 
In the two plots at the top, concentrations for $\mathrm{A}$ and $\mathrm{B}$ at the outlet are shown. Dots represent model predictions, and cross-hairs are the actual measurements. In the bottom plot, the input concentrations are shown. The line is the signal demanded by the controller, and the circles are the real values. At $16 \mathrm{~h}$ the controller setpoint for $\mathrm{B}_{\text {out }}$ is changed (from 3 to 8 ); consequently, the PID controller changes the inlet concentration. This is normal behavior, and one can see that the model is able to predict the measurements well. From $30 \mathrm{~h}$ onward, the actual real values of the inlet concentration are biased compared to the signal from the controller. Note that the real values are only shown to visualize the fault. They are not available for monitoring or diagnosis. After some time, the model fails to predict new measurements adequately. To test the diagnosis part of the system, it is assumed that a positive detection occurs at time $40 \mathrm{~h}$. At this point, one evaluates the likelihood, as explained above. Following this, one can plot the likelihood for a given fault type and start time.

This is shown in Figure 6. From this figure, one can see that single-fault type (bias $\mathrm{A}_{\text {in }}$ ) for a single start time $(30 \mathrm{~h})$ clearly dominates the other possibilities. This is exactly what was simulated, so the diagnosis task is done successfully.

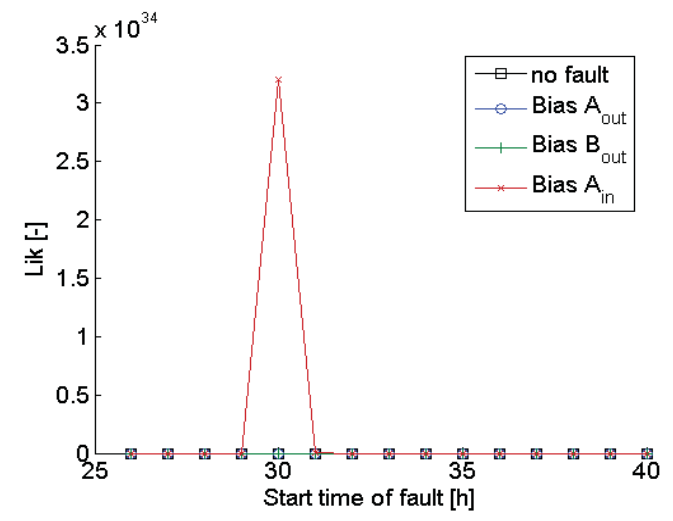

FIGURE 6. Likelihood as a function of time for different faults.

MCM Small Loop Simulation testbed. A second test bed is being developed for testing in the context of a cooling loop for a nuclear system. The scheme of the system, as modeled, is shown in Figure 7. The steady-state version of the model has been implemented, and the model allows initial testing of static techniques. Currently, the dynamic model is underway for a more realistic scenario simulation.

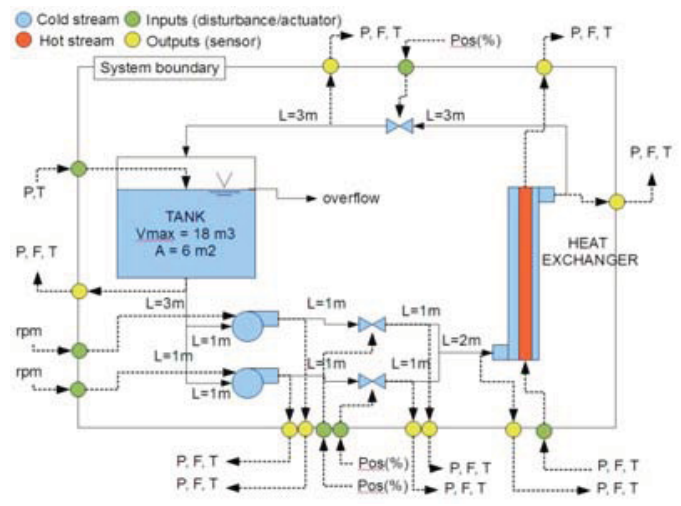

FIGURE 7. Safety critical system: nuclear plant cooling loop.

Such system is indeed safety critical, counts many components, and is characterized with severe non-linearity and hybrid behaviour; for example, one source is the simultaneous variation of temperature, flow, and pressure. The relationships between those variables are indeed non-linear, even under steady-state conditions. In addition, the heat-exchanger model consists of partial differential equations (PDE). The whole system can be approximated by differential algebraic equations (DAE) for which solvers in Matlab are available.

The model is sufficiently complex to emulate realistic challenges in fault detection and diagnosis. Still, the simulation test bed can be modified later according to specifications of the real-life system to enable the use of simulated data for training and validation of the real-life diagnostic system.

\section{Summary and Conclusions}

We accomplished the following so far:

- Developed a theoretical framework for using Principal Component Analysis (PCA)

- Developed a theoretical framework for using Qualitative Trend Analysis (QTA)

- Developed a theoretical framework for using Signed Digraphs (SDG) 
- Developed the Bayesian framework for fault tolerant control

- Developed a dynamic simulation model for a CSTR control system and a steady state model of the MCM 'small loop' test bed.

- Wrote a paper that has been accepted for presentation at the AIChE Annual Meeting in Nashville, TN, November 2009.

We believe that in spite of the 6-month time frame for this project in the first year, we have moved significantly towards accomplishing the 12-month first-year objectives originally listed in the proposal. We are confident that we are well positioned to meet the second-year objectives for this project. We are excited about the importance of this project and our collaboration with INL personnel. We look forward to developing and contributing new capabilities to INL in the form of mathematical models, computer algorithms and programs, and simulation case studies for anomaly detection, diagnosis, and resilient control in complex process systems. 


\section{MATERIALS AND NUCLEAR FUELS SIGNATURE}




\title{
Environmental Effects on Crack Growth in High-Temperature Alloys for Advanced Energy Systems
}

\author{
Richard Wright (INL), Ronald G. Ballinger (MIT), Project Team: Ji Hyun Kim, Peter Stahle, Julian Benz \\ (Graduate Student) Massachusetts Institute of Technology \\ Effects of oxyen partial pressure on crack growth rates in alloys \\ AF103
}

In this program, the effects of oxygen partial pressure on crack growth rates in Alloys $800 \mathrm{H}$, 617, Haynes 230 and Alloy 908 (with model alloy derivatives) are being studied using both static and fatigue loading. Tests are being performed over the temperature range of 500 $750^{\circ} \mathrm{C}$ (with a focus on $650^{\circ} \mathrm{C}$ ) and over an oxygen partial pressure range from $0.0-100 \mu$ bar oxygen partial pressure. Crack growth measurements are being performed using compact tension (CT) specimens in a specially designed, controlled atmosphere furnace system incorporated with a computer-controlled servohydraulic testing system. Tests are being conducted at either constant stress intensity factor, $K$, or constant stress intensity factor range, $\Delta K$. Crack length measurements are achieved using a reversing DC potential drop technique. The incoming gas mixture is controlled and measured. Oxygen potential is being measured using an in-situ probe located directly at the specimen surface.

\section{Work Plan}

The program consists of four major tasks: (1) materials procurement and precharacterization, (2) test system assembly and verification, (3) specimen fabrication and testing, (4) post-testing characterization and analysis of data and modeling.

\section{Program Progress: Crack Growth Rates}

Results for fatigue test performed on Inconel Alloy 617 at $650^{\circ} \mathrm{C}$ under constant $\Delta \mathrm{K}$ at different oxygen partial pressures, frequencies, and load ratios were reported last year. Figure 1 shows the results of the static crack growth behavior for material tested under constant $\mathrm{K}$ conditions ( $45 \mathrm{MPa} \sqrt{\mathrm{m}}$ ) as a function of oxygen potential. The data is plotted as crack length vs. time on the left vertical axis with the oxygen concentration plotted on the right vertical axis. Unlike the fatigue behavior at low $\mathrm{R}$ values, the crack growth rate increases as the oxygen concentration decreases over the range of oxygen concentration tested. The expected equivalent oxygen concentration for the Next Generation Nuclear Plant (NGNP) is in the range $1 \mathrm{ppm}$ and lower. As shown in Figure 2, the crack path also shifts from transgranular to intergranular when the loading mode is changed from cyclic to static. The transition is accompanied by extensive grain boundary cracking away from the immediate crack tip as well as extensive branching.

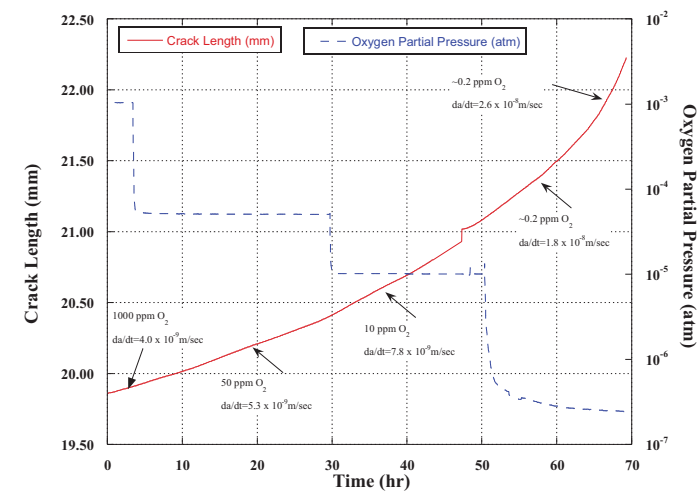

FIGURE 1. Static crack growth versus time as a function of oxygen partial pressure. $K=45 \mathrm{MPa} \mathrm{Vm}$. Analysis of chemistry ahead of crack tip.

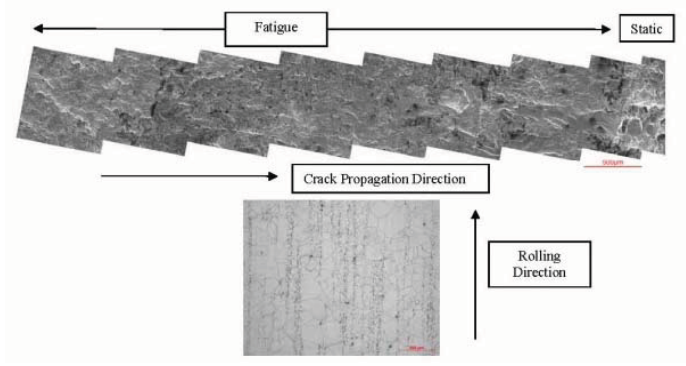

FIGURE 2. Scanning electron and optical micrographs of typical fracture surface and microstructure.

Auger line scans at a high resolution (512 points) were performed on the grain boundary ahead of the crack tip beyond the triple point. Figure 3 (next page) shows the region in which the first set of lines was placed with the first line directly ahead of the crack tip and the second line approximately $2 \mu \mathrm{m}$ from the crack tip perpendicular to the grain boundary. The surface near the crack tip again shows many of the small sub-micron particles that were analyzed in the previous survey analysis. The two lines were placed so that as few particles as possible were intersected on the line. The results for the line scan are shown superimposed on the corresponding secondary electron micrograph in Figure 4 (next page) for example. The boundary directly ahead of the crack tip reveals very little information about changes in composition given 
that the oxygen and carbon show no changes in intensity. There is an increase in the nickel intensity with a corresponding decrease in the chromium intensity directly at the grain boundary. Figure 5 (next page) shows very different results across the grain boundary than the previous line. A large spike in the chromium concentration is accompanied by a corresponding decrease in the nickel intensity directly at the grain boundary. Furthermore, a small increase in both the carbon and oxygen concentration coincides with the chromium peak. Both sides directly adjacent to the grain boundary on Line 2 appear to be populated by the $\sim 250$-nm particles, which are most likely chromium carbides. This would explain both the chromium and carbon peaks. However, the increase in oxygen has not been observed on these particles before. The difference may be that the particle intersected on Line 2 is directly ahead of the crack tip on the grain boundary.

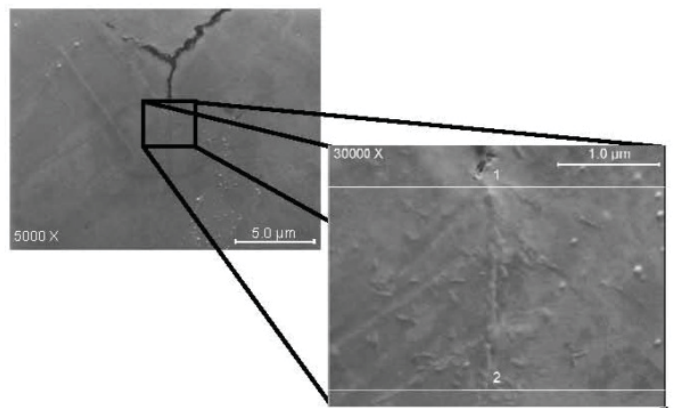

FIGURE 3. Image on the left showing the region in which the line scan analysis was performed ahead of an intergranular crack. The high magnification image on the right shows the placement of the lines with Line 1 directly ahead of the visibly oxidized surface and Line 2 on the grain boundary.

\section{Possible Crack Tip Damage Mechanisms}

In relating the above discussion of crack growth rates to the kinetics of oxygen penetration, the data compiled in Figure 6 (next page) needs to be considered. Most of the previous literature predicts that the grain boundary diffusivity of oxygen in nickel is approximately between $10^{-15}$ to $10^{-13} \mathrm{~m}^{2} / \mathrm{s}$. Sulfur, which is chemically similar to oxygen, has a measured intergranular diffusion coefficient of $4 \times 10^{-12} \mathrm{~m}^{2} / \mathrm{s}$ at $700^{\circ} \mathrm{C}$.

Furthermore, oxygen diffusion data on Ni270 from the work by Iococca and Woodford can be extrapolated to $650^{\circ} \mathrm{C}$ and predicts diffusivities of approximately $10^{-15} \mathrm{~m}^{2} / \mathrm{s}$. Lastly, work on IN718 at $650^{\circ} \mathrm{C}$ has found the intergranular diffusion coefficient to be approximately $10^{-15}$ $\mathrm{m}^{2} / \mathrm{s}$. For a simple comparison, we can assume that the oxygen diffusion depth follows the approximation for slab diffusion, $x \propto \sqrt{D t}$. This yields intergranular oxygen diffusion rates between $10^{-8}$ and $10^{-7} \mathrm{~m} / \mathrm{s}$ using the above data. From the crack growth data in this work, fatigue crack growth rates were approximately $10^{-8} \mathrm{~m} / \mathrm{s}$ for $0.1-\mathrm{Hz}$ loading and $2 \times 10^{-7} \mathrm{~m} / \mathrm{s}$ for $2-\mathrm{Hz}$ loading using the lower range of the data. In terms of the oxygen penetration depth ahead of the crack tip, the similar magnitudes of the crack growth and oxygen diffusion rates indicate that any long-range damage mechanisms are unlikely in the case of Alloy 617. Most of the literature discussing dynamic embrittlement argues that oxygen penetration can only occur a few nanometers (or atomic spacings) ahead of the crack tip, which appears to support these results.

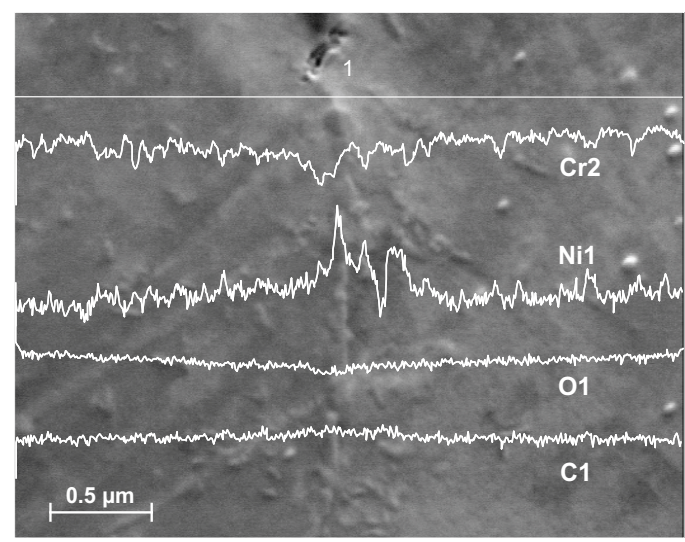

FIGURE 4. Line scan analysis results for Line 1 overlaid on the high magnification image in Figure 3 showing the intensities of chromium, nickel, oxygen, and carbon.

Continuing the discussion of dynamic embrittlement, the question arises of why the crack growth behavior appears to be insensitive to oxygen variations when approaching static loading conditions. If dynamic embrittlement is indeed the prominent damage mechanism, then oxygen penetration and subsequent decohesion can only occur on a very short scale. The crack growth rates for both trapezoidal and static loading at $\mathrm{K}=33.0 \mathrm{MPa} \sqrt{\mathrm{m}}$ approaches $10^{-10}$ 
$\mathrm{m} / \mathrm{s}$, which is two to three orders of magnitude less than the expected intergranular oxygen

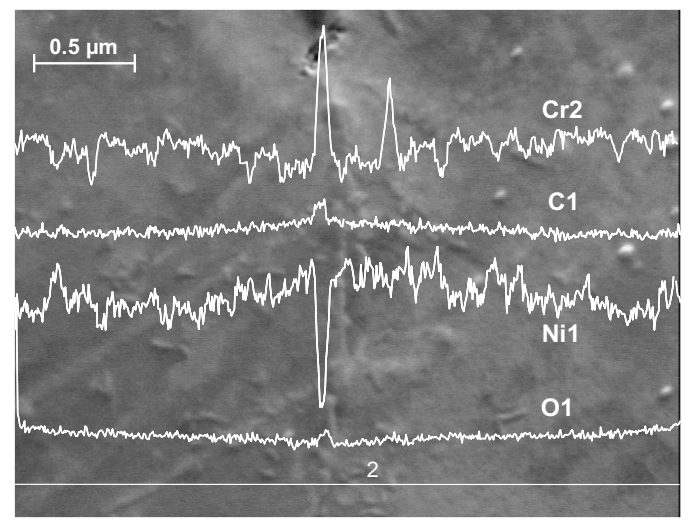

FIGURE 5. Line scan analysis results for Line 2 in Figure 3

diffusion rates. The difference between crack growth and oxygen diffusion rates possibly indicates that ample time was allowed for a protective oxide to form at the crack tip. The Auger spectroscopy results did not detect any intergranular oxide formation within 1 to $2 \mu \mathrm{m}$ ahead of the crack tip in the sensitivity of our analysis. Furthermore, the only detected oxide was in the crack wake, and the results indicated that this oxide was chromium rich, which according to the literature is protective against further oxygen ingress. If the penetration depth of the oxygen was only on the order of a few atomic spacings before a protective oxide formed, the moderate stresses at the crack tip may not have been sufficient to disrupt the oxide film and enhance the crack growth rate. The dynamic embrittlement crack growth process has been known to be intermittent and can vary with local grain boundary structure. As noted before, this is due to the constraint imposed on the crack tip by regions of uncracked material along the crack front which have yet to be fractured. The constraint imposed by these uncracked regions may keep the oxide film mostly intact when there are no oscillations in the load. This could also explain why all of the fatigue loading crack growth rates increased with oxygen potential. The disruption of the protective oxide film at the crack tip, even at the low $\Delta \mathrm{K}$ of $4.12 \mathrm{MPa} \sqrt{\mathrm{m}}$ used in the trapezoidal cycle, may have been sufficient to disrupt the brittle oxide and allow the embrittlement process to repeat itself.

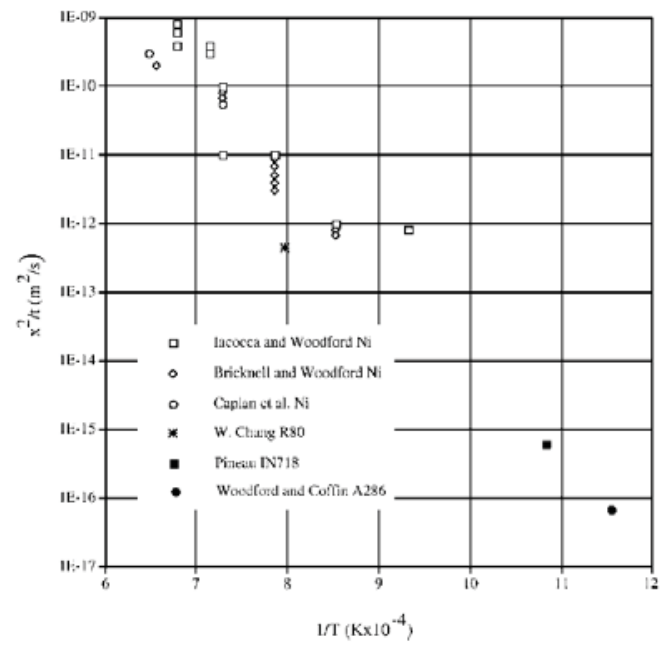

FIGURE 6. Intergranular oxygen penetration as a function of test temperature compiled from literature data on Ni270, Rene 80, IN718, and A286.

Lastly, the combination of the dynamic embrittlement process and protective chromium oxide formation can explain the diminishing change in magnitude of crack growth rates over the range of oxygen partial pressures when the trapezoidal loading cycle was used. The trapezoidal loading cycle was unique in that there was a 500-s hold time at maximum load while the rest of the fatigue data was under continuous cycle at $\mathrm{R}=0.5,0.625$, and 0.75 . The crack growth rates from the continuous fatigue all showed similar increases in crack growth rates over the oxygen concentration range from argon gas to $1000 \mathrm{vppm} \mathrm{O}_{2}$ mixtures in argon. However, the results from the trapezoidal cycle did not show as significant an increase in growth rates with increasing oxygen concentration. The hold time at maximum load may have been more similar to the static loading data where a protective oxide was allowed to form. In contrast, the segments of the trapezoidal waveform where load was being increased and decreased are more similar to the continuous sinusoidal fatigue and would have allowed the oxide to fracture slightly and oxygen to penetrate further along the boundary. Therefore, the enhancement in crack growth rates for a trapezoidal loading cycle was still detectable, unlike the static loading data, but ultimately exhibits only a minor change when compared to the continuous fatigue growth rates. 
Summary and Conclusions

The results of the work thus far, while preliminary, have both fundamental and engineering implications. At the fundamental level, if these initial results are confirmed, they show that if the oxygen potential becomes low enough it may be possible to destabilize the protective film such that crack propagation can occur. From an engineering perspective, the high chromium alloys such as Alloy 617 were designed for use in oxidizing environments. Use outside a highly oxidizing environment, such as will exist in the NGNP, may need to be approached with caution. 


\title{
Development of Advanced Burnup Measurement \& Nuclear Forensics ICP-MS Isotopics Analysis Techniques
}

\author{
B. Hilton, J. Giglio, I. Glagolenko, D. Cummings, J. Parry, M. Lillo, A. Robinson, J. Delmore, K. Carney \\ Leveraging the capability of inductively coupled plasma mass spectrometry (ICP-MS) to quantify \\ multiple fission products and actinides in a single scam \\ AF104
}

The goal of this research project is to develop a novel methodology of nuclear fuel burnup determination which is completely independent of model predictions and reactor types. This approach is a notable leap forward compared to the existing burnup determination practice that requires input of calculated fission fractions (identifying the specific fission source isotope and neutron energy that yielded fission, e.g., U-235 from thermal neutron, U-238 from fast neutron) from computational neutronics analysis in addition to the measured concentration of a single fission product isotope. The proposed method leverages the capability of inductively coupled plasma mass spectrometry (ICP-MS) to quantify multiple fission products and actinides in a single scan and uses these data to develop a system of linear burnup equations whose solution is the fission fractions. The fission fractions are substituted back in the equations to determine burnup and to benchmark physics models. The number of fission products required to solve the system of linear burnup equations is based on fuel composition so that the initial research stage will analyze simple oneto two-component fuels and subsequent years will increase the fuel complexity.

This research will result in a significant advancement of nuclear fuel burnup methodology with the following benefits:

- Establish a burnup measurement technique completely independent of computational reactor physics models and reactor type

- Establish a burnup measurement technique that can be used to benchmark computer physics models and investigate accuracy of basic physics data (i.e., cross sections and fission yields)

- Provide precise knowledge of the actinide inventory that is required for recycling, transport, storage, and safeguarding of spent nuclear fuel

- Identify the reactor sources of fission in forensic analysis of unknown/exotic nuclear materials

- Reduce the time and cost of the burnup analysis and required dissolutions/separations
- $\quad$ Reduce the dose rate to personnel.

Technical Objectives

The strategy selected for the development of the new method of burnup determination requires a multidisciplinary approach and consists of theoretical and experimental technical objectives, which are summarized below.

\section{Theoretical objectives}

- Research existing databases for energy dependence of fission yield data

- Improve an Idaho National Laboratory (INL)-developed Monte Carlo N-Particle Transport Code (MCNP) with Oak Ridge isotope generation (ORIGEN) (MCWO) neutronics code to model reducedenrichment research and test reactor (RERTR) irradiation experiments and calculate fission fractions, actinides, and fission product isotopes

- Perform neutronics calculations using REBUS modeling suite to predict isotopics mass inventory of fuel samples irradiated in Experimental Breeder Reactor-II (EBR-II) fast spectrum neutron reactor

- Enhance REBUS modeling suite by developing methodology to calculate fission fraction distributions of fuel samples irradiated in EBR-II fast reactor

- Develop system of linear burnup equations for solving the fission fraction using measured isotopics and nuclear data (i.e., fission yields)

- Perform test cases of linear burnup equation solver using the isotopics and nuclear data and compare with neutronics results

- Explore alternative approaches to determine fuel burnup, e.g., the ratio (or sum) of fission product isotopes.

\section{Experimental objectives}

- Analyze isotopics by ICP-MS of two to three (2-3) fuel types, e.g., U-Zr metallic alloy fast reactor fuel, U-Mo dispersion and monolithic research reactor plate fuel, and $\mathrm{UO}_{2}$ commercial light water reactor (LWR) 
fuel, demonstrating capability and efficiency of measuring multiple isotopes in a single scan

- Evaluate possible isobaric interferences and develop the required separation techniques

- Survey availability of analytical standards

- Characterize and quantify uncertainty and estimate sensitivity of the analysis

- Establish capability to perform standard ASTM E-321 "Nd-148 method" using thermal ionization mass spectrometry isotope dilution (TIMS ID) for analyzing nuclear fuel burnup

- Identify candidate fission product burnup monitors based on selection criteria.

\section{Results and Accomplishments}

The strategy implemented for developing a novel methodology of nuclear fuel burnup determination consisted of theoretical and experimental research objectives.

The theoretical portion continued developing the system of linear burnup equations and a solution method. The solution method research encompassed developing a numerical approach, identifying a mathematical modeling platform, and characterizing the energy dependence of the nuclear data and the reactor systems used for irradiating the experimental fuel samples.

The results of the neutronics models that calculate fission product and actinide isotopics and fission fraction distributions used to model the experimental samples were scrutinized for use in benchmarking comparisons. In collaboration with Argonne National Laboratory (ANL), the fission fractions of fifty-three EBR-II samples (chopper segments, blanket assemblies, etc.) were analyzed using REBUS modeling suite with an additional capability that calculates fission fractions. ANL also provided generic core modeling results describing the energy spectrum variation of individual fission rates radially and axially. The calculation results provided a comparison between state-of-the-art computer modeling and isotopic measurement capability and the solution of the system of linear burnup equations. Additional calculations were identified to reduce uncertainties and provide more directly comparable results to the "measured" burnup and multiple linear regression model (MLRM) measurement-derived fission fractions. The additional output data needed are fission fractions at multiple energy groups for ATR experiments and at the specific spatial location of the post-irradiation examination (PIE) sample and a larger number of fission products explicitly tracked. Options for completing the reanalysis have been identified and will be pursued in Year 3. A reanalysis that tallied fission fractions in multiple energy groups will also be used to assess the effect of the neutron energy spectrum on fission yield values.

The burnup equation system solution technique developed was a MLRM for solving overdetermined equation systems by linear least squares. MATLAB R2008b, a numerical computing environment and programming language, was used to solve the matrix system of linear burnup equations by least squares minimization. Other techniques that were investigated without success included Gaussian elimination, Truncated Singular Value Decomposition and Tikhonov, also known as Ridge Regression, method.

The burnup monitor MLRM using isotopic data from ICP-MS was demonstrated to determine fission fractions to within $1 \%$ of the neutronics model calculations for the RERTR UMo plate type fuel and to within $6 \%$ for the EBR-II fuel samples. The accuracy of the MLRM improves with the number of burnup monitor equations and the highest accuracy is achieved using 14 to 20 burnup monitor (fission product) equations. These fuel samples represent simple cases of two unknown fission sources (i.e., U-235 and Pu-239). For more complex fuel samples, additional fission product monitor burnup equations will be needed.

The ICP-MS experimental portion of the LDRD dealt with the evaluation of various fuel types for fission products by ICP-MS, expanding the number of isotopes that can be measured, and developing and performing separations on the sample solutions to eliminate isobaric interferences. 
The effort to compare ICP-MS technique with other analytical techniques continued. Four light water reactor fuels that have been extensively analyzed via ICP-MS will be analyzed by the American Society for Testing and Materials (ASTM) thermal ionization mass spectrometry (TIMS) method using 139La and Ce standards instead of $148 \mathrm{Nd}$. This will provide additional validation data for the ICP-MS and establish alternative fission product burnup monitors to increase confidence in burnup measurements. The twenty EBR-II samples (chopper segments, blanket assemblies, etc.) that were initially characterized last year were reanalyzed after performing $\mathrm{Nd} / \mathrm{Sm}$ separation. Four additional RERTR U-Mo samples were analyzed this year with and without the $\mathrm{Nd} / \mathrm{Sm}$ separation to characterize the effect of neutron capture. Eleven AFC-1 metallic fuel samples were analyzed using the new Perkin Elmer ICPMS-Dynamic Reaction Cell (ICP-MS-DRC), which has a much higher sensitivity. The initial analyses revealed significant differences in behavior between the new ICP-MS-DRC and the older instrument. The added sensitivity provides a capability to analyze lower yield fission products, but the sample solutions may require more separations due to the effect of interferences from neighbor isotopes, doubly charged heavy metal isotopes, and species oxidized in the flame. The data are still being evaluated due to the complexity of the higher actinides and the different performance of the new ICP-MS.

The measurement of nearly all stable fission products by ICP-MS has been successful and additional improvements were identified. Additional sample preparation steps and separations are needed to implement the advanced burnup determination MLRM method. While programmatic requirements have accommodated cladding and fuel co-dissolved, methods to separate the cladding components need to be developed for RERTR aluminum matrix and Al clad fuels and the Advanced Fuel Cycle Initiative (AFCI) stainless steel clad fuels. These advanced sample preparation steps will be developed in Year 3 and will also try to address Year 1 observations that some of the noble metal analytes (e.g., Mo, Tc, Ru and Rh) are particularly sensitive to the dissolution process.
The selection of a multiple linear regression model for the burnup equation system and solution method was motivated by Year 1's findings that a direct Gaussian elimination approach resulted in non-physical values. The accomplishment of developing a successful solution method for simple fuel cases was facilitated by the findings made in Year 1 .

The fission fractions of the fifty-three EBRII samples were calculated using the additional capability to the REBUS modeling suite that was developed in Year 1. These calculations are required to compare the "measured" burnup derived from the MLRM.

Year 1's development of neodymium $(\mathrm{Nd})$ /samarium $(\mathrm{Sm})$ chemical separation by the gas pressurized extraction chromatography (GPEC) instrument was applied to preparing fuel sample solutions of RERTR-9 U-Mo plate fuel and EBR-II metallic alloy fuel for ICP-MS analyses. The GPEC technique was also identified as a candidate method for developing separation techniques that are needed to analyze high cross section fission products (e.g., europium $[\mathrm{Eu}]$ /gadolinium $[\mathrm{Gd}]$ ) that are of interest to burnup credit and criticality hazard analysis of transportation and storage applications in the LWR industry. The expansion of analyzed fission products will further improve the capability of the burnup equation system solver when applied to more complex fuel systems such as actinide-bearing transmutation fuels.

The ASTM TIMS method using 148Nd, which was developed in Year 1, was modified to also use 139La and Ce standards. The application of the ASTM TIMS method with other than $148 \mathrm{Nd}$ expanded our ability to validate the ICP-MS methodology with the industry standard method.

The research of fission yield data in Year 1 motivated comparisons between the ENDF/BVII values and measured cumulative U-235 fission yield values derived from the RERTR UMo plates and EBR-II metallic alloy fuel. These comparisons showed that the measurements, which were not optimized for benchmarking, corresponded to within $10 \%$ of the tabulated data. This suggests the ICP-MS measurements 
can potentially result in a refinement and improvement of the old data, especially for those isotopes with a limited number of measured fission yields.

A system of linear burnup equations was developed and a solution method was successfully implemented using MLRM for solving an overdetermined system of equations.

For a simple case of irradiated fuel type with two unknown fission sources (U-235t and Pu-239t), an accuracy of $< \pm 1 \%$ was achieved when 14-20 fission product monitor equations were implemented. For a fast reactor fuel system, the solution accuracy was typically $< \pm 5 \%$, but some sample results were less accurate. Improvements to the MLRM solver are needed and future plans for Year 3 include (1) investigating alternative solution algorithms such as Least Absolute Deviations, which is less sensitive to outliers, (2) improving the accuracy of fission yield values that have uncertainties greater than $\pm 5 \%$, and (3) increasing the number of measurable fission products using the INL state-of-the art quadrupole dynamic reaction cell ICP-MS which has higher sensitivity $(1000 \times)$ than the instrument used in Years 1 and 2.

The hypothesis that the ICP-MS can provide multiple fission products at a fraction of the cost as current methods has continued to be proven based on Year 1 and Year 2 experience. The measured fission products have been evaluated regarding their importance as input in the burnup equation system solver. The more than forty measured fission products were ranked and established sets of eight, fourteen, and twenty that can be used in deriving the fission product monitor burnup equations from the ICP-MS measurements.

The preliminary results of the experimental portion of the laboratory-directed research and development (LDRD) have reinforced INL's importance in the nuclear science and engineering community for leading the characterization of nuclear fuels. Some of the methods and experience gained in the LDRD will benefit PIE isotopic and burnup analyses of current programs such as Global Nuclear Energy Partnership (GNEP)/AFCI, RERTR, Next Generation Nuclear Plant (NGNP), and future programs of LWR Sustainability and burnup credit funded by the Department of Energy (DOE), the Nuclear Regulatory Commission (NRC) and nuclear electric industry.

The development of the GPEC rare earth separation and the expansion of the ASTM method for TIMS using La and Ce and ICP-MS Isotope Dilution (ID) will be extremely beneficial in the characterization of PIE samples in the future. This is an extremely marketable capability to support future fuel characterization work as well as support programs such as the Yucca Mountain Burnup Credit program. The Electric Power Research Institute (EPRI) has contacted us regarding a work-for-others (WFO) contract using the capabilities we are developing. Additionally, the evaluation of the large amount of data will allow future PIE work to illustrate the applicability of easy-to-measure fission products rather than relying on the standard $148 \mathrm{Nd}$.

\section{Summary and Conclusions}

The following are the major results and accomplishments of this research:

1. Developed system of linear burnup equations and successfully demonstrated a solution method using MLRM for solving an overdetermined system of linear burnup equations. For a simple case of irradiated fuel type with two unknown fission sources (U-235t and Pu-239t), an accuracy of $< \pm 1 \%$ was achieved when 14-20 fission product monitor equations were implemented.

2. Further demonstrated the hypothesis that the ICP-MS can provide multiple fission products at a fraction of the cost as current methods.

3. Demonstrated feasibility of using data collected from ICP-MS to improve the accuracy of the fission yield data of candidate fission product burnup monitors.

4. Demonstrated feasibility of ICP-MS to analyze low concentration, high crosssection isotopes for burnup credit in LWR fuels.

5. Expanded the capability of TIMS ID technique consistent with standard ASTM E-321 to analyze lanthanum and cerium 
isotopes. Characterized and purified $\mathrm{La}$ and $\mathrm{Ce}$ isotopic standards for isotope dilution technique.

6. Identified additional enhancements needed for neutronics code models (MCNP w/ORIGEN for RERTR and AFCI) that will provide more directly comparable results to the "measured" burnup and MLRM measurement derived fission fractions.
7. Presented four conference papers, submitted two journal articles, and have two journal articles in preparation derived from this research. 


\section{Development of a Small Sample Volume Mechanical Properties Testing Technique for Irradiated Fuels and Materials}

James I. Cole, Dennis D. Keiser (INL), Dave Bahr (Washington State University)

A mechanical testing system and methodology to rapidly assess the strength, stiffness, and, in some cases, toughness of materials after irradiation AF105

This project is targeted at developing a mechanical testing system and methodology that can rapidly assess the strength, stiffness, and, in some cases, toughness of materials after irradiation. We are developing a micromechanical tester using instrumented indentation. The goal will be to deliver an instrument that can be placed within a limited volume glove box environment so that the mechanical response of small irradiated samples can assessed at a cost which is significantly lower than that using macroscopic tensile testing methodologies. Rapid screening of fuels and materials in consideration for Generation IV reactors will require an assessment of a wide range of metals and ceramics. A simple testing instrument and method that could provide initial guidance in down-selecting materials to a few for further, more expensive testing would greatly improve the options available for researchers. In addition, with many new materials, the volume available is small; a micromechanical testing system allows limited experimental alloys to be assessed while providing statistically significant results.

To meet this goal, we are developing an instrument that will have the capability to probe flow stress at various effective strains using multiple indentation tips. Currently there is a gap in available instrumentation for this purpose. Commercially available systems all have replaceable tips and a single loading mechanism; while excellent for high quality materials assessment, it is very challenging in a glove box environment to exchange small, mm-scale tips regularly. Upon completion of the project, a calibrated, working prototype will be installed into an environment that can be used to significantly hasten and broaden the number of materials that can be tested after exposure to irradiation. This will have two fundamental benefits: (1) it will enable further understanding of the response of materials to radiation, and (2) it will enhance the ability to measure basic mechanical properties using rapid testing and indentation techniques.

\section{Technical Objectives}

The objectives for the three-year project are:
- Develop software for controlling and collecting data during instrumented indentation system

- Carry out multiple indentations with tips of various angles to ensure strain hardening behavior

- Design indentation system suitable for insertion into a glove box environment (i.e., meet space requirements of approximately 1 cubic foot)

- Construct prototype multi-tip indentation system

- Conduct baseline testing between existing commercial systems and smaller footprint, more flexible prototype

- Deliver prototype to INL.

Results and Accomplishments

The initial software has been developed in LabView to control the motion and data collection of instrumented indentation. The key development was to standardize the partial unload segment so that material properties can be evaluated at multiple depths (similar to published methods by Weppleman and Swain). A typical load-depth curve is shown in Figure 1. This development used a stepper motor driving system and a linear variable differential transducer (LVDT) for measuring displacement. Indentations can be scaled directly to

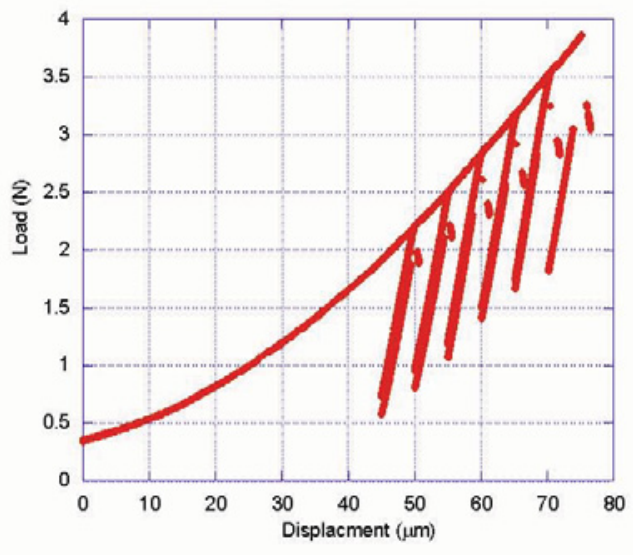

FIGURE 1. Typical load-depth data showing successful control of partial unloading sequences. 
microhardness testing scales, such as the indentation in Figure 1 in a $6061 \mathrm{Al}$ alloy. While this general software development was successful, several issues arose. In particular, the challenges in switching directions and ensuring uniform unloading conditions suggested that a different loading mechanism was appropriate.

For the prototype system to insert into the glove box, the system has been designed and simulated. The basic structure is a single loading mechanism using a voice coil for loading rather than a stepper motor with a displacement transducer in the loading train. Under the loading mechanism, we have two indenter tips suspended by orthoplanar springs, which provide the appropriate spring constant while maintaining the small profile needed for the device. Figure 2 shows the basic structure. The orthoplanar spring

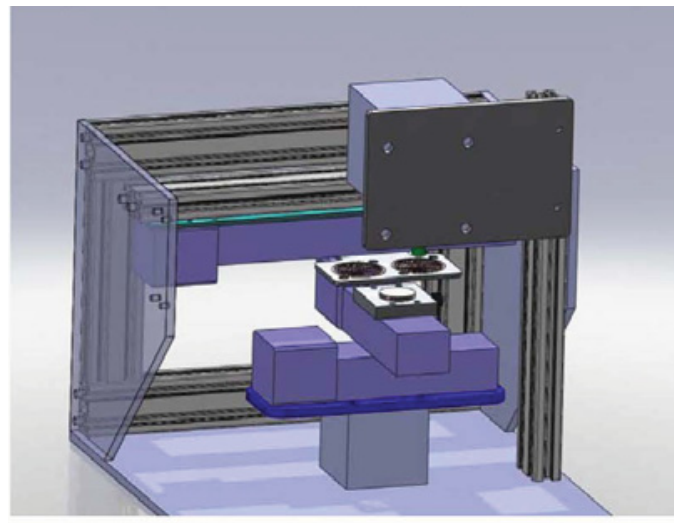

FIGURE 2. Assembly view of prototype. Front view of the assembly showing the XYZ positioner configuration (bottom center), cage, voice coil assembly (top right), specimen holder and spring carriage (top center), and the upper horizontal positioner (top left).

design was adopted as a way of securing the indenter tips while minimizing the possibility of lateral deflections. Beryllium-copper UNS 17200 was chosen for the spring material for its low spring constant, which enables the spring to deflect a significant amount under low loads. Two of these springs are incorporated into the spring carriage, which holds the indenter tips as they are moved underneath the voice coil punch. With these springs, the majority of the load from the voice coil actuator should be transferred from the voice coil to the indenter tips, with little carried by the spring. Figure 3 shows a typical finite element analysis of the stresses and resulting displacement for the orthoplanar springs specially constructed for this project.

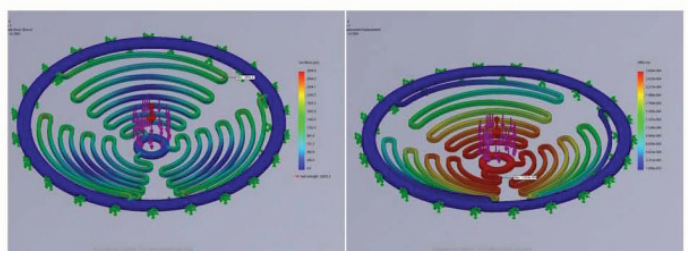

FIGURE 3. Static loading test including the weight of the indenter tip, gravity, and a 0.05-N force on the orthoplanar spring. Left: Stress results of static. Max stress $=2884.9 \mathrm{psi}$, and $Y S=32053.3$ psi. Right (displacement results of static test) Total deflection $=2.654 \mathrm{e}-04 \mathrm{~m}$.

All parts have now been ordered and are being delivered for the construction of the prototype multi-tip instrumented indenter, including optics and vibration isolation padding. All development and design work has been carried out by graduate students at Washington State University.

\section{Summary and Conclusions}

At the end of the first year, we have successfully developed the software to control an instrumented indenter, designed a new multi-tip indenter system, and performed simulations on an unconventional spring design to meet the stringent space requirements for the system. In the next year we will construct and troubleshoot the entire system and provide baseline data in metallic systems with two different tips, one with a standard Vickers geometry and one with a sharper conical tip. 


\section{Microstructure and Deformation Physics of Nuclear Materials by Atomistically Informed Mesoscale Simulation}

Dieter Wolf

Developing a fundamental understanding of the basic point-defect, dislocation, and grain-boundary mechanisms controlling radiation damage and its effects on the thermo-mechanical behavior of the material SH100

The main goal of this work is to develop atomistically informed mesoscale simulations of the effects of radiation damage on the thermomechanical behavior of polycrystalline materials in order to elucidate the physical mechanisms that control the degradation behavior of model fission-reactor materials under irradiation. At the atomic level, this requires the combination of the expertise in the simulation of radiation damage developed for single crystals with state-of-the-art molecular-dynamics (MD) simulations of microstructural evolution in nanocrystalline materials. These simulations are being developed for both nanocrystalline $\mathrm{UO}_{2}$ (as model fuel material) and for metals, such as body-centeredcubic molybdenum (as a model material representing the cladding). Although inherently limited to a grain size of nanometer dimensions, the MD simulations provide a detailed materialsphysics-based understanding of the fundamental mechanisms that control the behavior of fuel and cladding model materials and how this behavior is affected by radiation damage. In order to extend both the MD length and time scales to those governing the behavior of usually coarsegrained engineering materials, the insights gained from the MD simulations have to be captured quantitatively. This requires development of theoretical models describing, for example, the grain-size and strain-rate dependence of the point-defect and microstructural processes observed in the MD simulations. These models provide the fundamental basis for the simultaneous development of the mesoscale simulations that enable prediction of microstructural evolution on the length and time scales of the actual engineering material.

\section{Technical Objectives}

The focus of the atomistic simulations is on developing a fundamental understanding of the basic point-defect, dislocation, and grainboundary mechanisms controlling radiation damage and its effects on the thermo-mechanical behavior of the material. As model materials we consider nanocrystalline $\mathrm{UO}_{2}$ (as a model nuclear-fuel material) and body-centered-cubic Mo (as a model cladding material).

Due to well-known computational limitations, such atomic-level simulations are necessarily limited to nanocrystalline materials, i.e., polycrystals with a grain size of sub-micron dimensions. Therefore, in order to reach engineering length-scale and time-scale regimes controlling the coarse-grained actual materials, the insights gained from these simulations have to be captured quantitatively in the form of theoretical models that describe explicitly, for example, the grain-size and strain-rate dependence of the MD results. These models then provide the basis for the development of mesoscale simulations of course-grained fuel and cladding materials.

\section{Results and Accomplishments}

We have made significant progress in the following three areas:

1. Molecular dynamics simulation of irradiation-induced point-defect clustering in uranium dioxide.

We have recently developed a novel approach for performing molecular-dynamics (MD) simulations of irradiation damage that circumvents the cascade simulation and, instead, insert Frenkel pairs directly into the crystal. We have now applied this approach to elucidate kinetically evolving irradiation-induced point-defect processes during cluster formation in $\mathrm{UO}_{2}$. Our simulations revealed that in the absence of defects on the cation sublattice in $\mathrm{UO}_{2}$, the defects initially present on the anion sublattice recombine and annihilate completely during equilibration. However, in the simultaneous presence of the defects on both sublattices, separate vacancy and interstitial clusters on the anion sublattice are formed. We also found that a high concentration of cation defects results in the formation of new Frenkel pairs on the anion sublattice. We concluded that the cation sublattice is primarily responsible for the radiation tolerance/intolerance of the material.

2. Molecular dynamics simulation of void migration in $\mathrm{UO}_{2}$ in a temperature gradient.

We have used MD simulations to study the mechanism of migration of voids in $\mathrm{UO}_{2}$ in a temperature gradient. It is well known that within a few hours after startup of a nuclear reactor, the temperature gradient within a fuel 
element causes migration of voids/bubbles radially inwards to form a central hole. To understand the atomic processes that control this migration of voids, we performed molecular MD simulations on single-crystal $\mathrm{UO}_{2}$ with voids of diameter $2.2 \mathrm{~nm}$. An external temperature gradient was applied across the simulation cell. At the end of the simulation run, it was observed that the voids had moved towards the hot end of the simulation cell. The void-migration velocity obtained from the simulations was compared with the available phenomenological equations for void migration due to different transport mechanisms. Surface diffusion of the slowest moving specie, i.e., uranium, was found to be the dominant mechanism for void migration. The contribution from lattice diffusion and the thermal stress gradient to the void migration was analyzed and found to be negligible. By extrapolation, a crossover from the surfacediffusion controlled mechanism to the latticediffusion controlled mechanism was predicted to occur for voids with sizes in the $\mu \mathrm{m}$ range.

3. Phase-field simulation of void nucleation in a polycrystalline metal.

We have extended our previously developed mesoscopic phase-field modeling approach for single crystals to study void nucleation in polycrystalline metals. Again based on a coupled set of Cahn-Hilliard and Allen-Cahn equations, the model captures several relevant processes, including vacancy annihilation and nucleation at grain boundaries, vacancy diffusion towards sinks (including grain boundaries and void surfaces), as well as void nucleation and growth due to vacancy super-saturations occurring in the grain interiors. In order to capture all these processes, the model receives input from MD simulations on the nucleation of vacancies from and annihilation at the grain boundaries as well as on the vacancy-diffusion behavior in the grain interiors. Illustrative results are presented in Figure 1, demonstrating that annihilation of the vacancy population at the grain-boundary sinks results in the formation of void-denuded zones adjacent to the grain boundaries. The width of these zones was found to increase with the vacancy diffusivity and decrease with the vacancy production rate (i.e., with the number of cascades per unit volume and unit time). A quantified model capturing these effects was developed.

Atomistically-informed phase-field approach for void nucleation and growth in a polycrystal
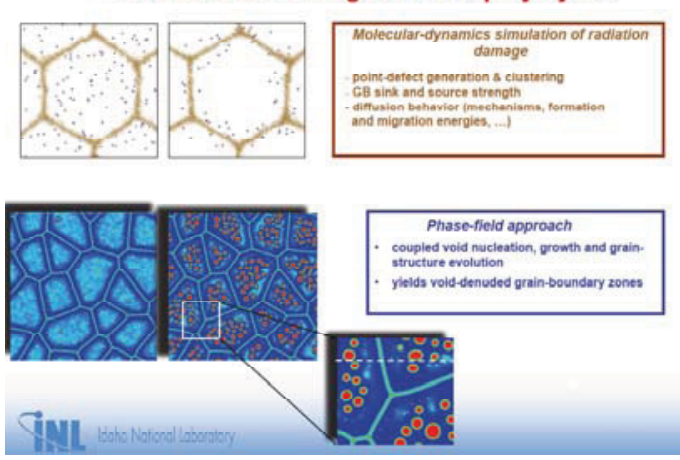

FIGURE 1. Concurrent void nucleation and growth in a polycrystalline metal super-saturated by continuous addition of irradiation-induced vacancies. The grain boundaries exhibit void-denuded zones that are formed because the grain boundaries act as vacancy sinks.

\section{Summary and Conclusions}

Our high-temperature molecular-dynamics simulations have provided novel insight into the mechanism by which irradiation-induced point defects in $\mathrm{UO}_{2}$ evolve kinetically to form defect clusters that involve both sublattices. Our MD simulations also revealed the mechanism by which voids in $\mathrm{UO}_{2}$ move in a temperature gradient. These simulations provide the necessary input for mesoscale simulations of microstructure evolution under the effects of irradiation. The application of these simulations to capture void nucleation and migration in a polycrystalline material represents an important step forward towards the development of microstructurally designed fission-reactor fuel and cladding materials. 
SEPARATIONS AND ACTINIDE SCIENDE DISTINCTIVE SIGNATURE 


\title{
Utility of Unusual Oxidation States of Americium for Separations
}

\author{
Bruce J. Mincher, Leigh Martin, and Nick Schmitt \\ Oxidizing americium to higher oxidation states as a basis for separations \\ AS102
}

The presence of americium (Am)

complicates attribution determinations in nuclear forensics and the development of an advanced fuel cycle. The mass spectrometric determination of curium $(\mathrm{Cm})$ ratios will be necessary to determine origins of the next generation of nuclear fuels, thus isobaric Am will need to be separated prior to $\mathrm{Cm}$ analysis. For the fuel cycle, initial separation of Am along with uranium $(\mathrm{U})$, neptunium $(\mathrm{Np})$, and plutonium $(\mathrm{Pu})$ extracted in the UREX process would greatly simplify later processing by obviating the need for additional actinide extraction and actinide/lanthanide separation stages.

Unfortunately, under normal conditions, Am, $\mathrm{Cm}$, and the lanthanides are trivalent and their aqueous chemistry is very similar, making separation a difficult challenge. Oxidation of Am to higher oxidation states could provide a basis for the needed separations in both cases. The objectives of this proposal were to demonstrate the existence of both AmV and AmVI at the high nitric acid concentrations encountered in realistic analytical and separations environments and to demonstrate a solvent extraction separation with utility for separations on the process and analytical scales. This provides the opportunity to improve attribution analytical capabilities and design a greatly simplified and more costeffective fuel cycle.

\section{Technical Objectives}

- Demonstrate Am oxidation at high nitric acid concentrations encountered in real separations

- Demonstrate the solvent extraction of oxidized Am using a process compatible with current Fuel Cycle Research and Development solvent extraction schemes

- Prepare manuscripts describing the results above for publication in peer-reviewed journals.

\section{Results and Accomplishments}

Few reagents suitable for the oxidation of Am to the hexavalent state $\left(\mathrm{AmO}_{2}{ }^{+2}\right)$, and none for the oxidation of Am to the pentavalent state $\left(\mathrm{AmO}_{2}{ }^{+}\right)$in acidic solution were known prior to this work. We reported in Fiscal Year (FY) 2008 the successful oxidation of americium to quantitative AmVI or AmV in highly acidic solution under specific conditions using sodium bismuthate $\left(\mathrm{NaBiO}_{3}\right)$ powder. The TBP solvent extraction behavior of hexavalent americium was found to follow the known trends established by $\mathrm{U}, \mathrm{Np}, \mathrm{Pu}$. These data are shown in Figure 1. Figure 2 shows the solvent extraction behavior of all three oxidation states. These results are new and significant for three reasons: (1) this is the first demonstration of AmVI solvent extraction using the UREX solvent from the nitric acid concentrations actually used under process conditions, (2) spectroscopic measurements were made using a liquid waveguide capillary cell (LWCC - World Precision Instruments) with an effective pathlength of $1 \mathrm{~m}$ allowing for spectroscopic investigation of low americium chemical concentrations (gloveboxes are unnecessary), and (3) techniques have been developed for the production of quantitative AmVI or AmV. None of this has been previously reported by others.

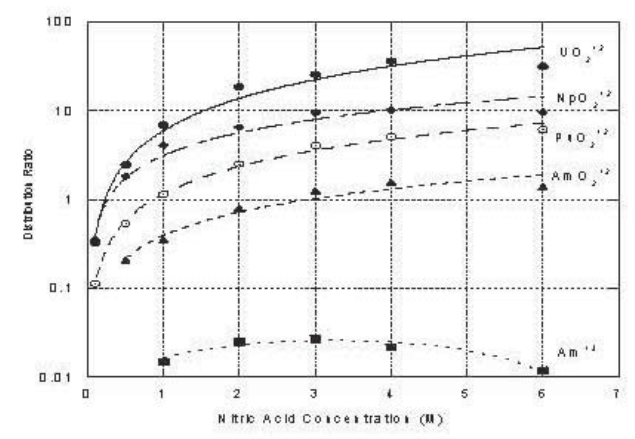

FIGURE 1. The solvent extraction of uranyl, neptunyl, plutonyl, americyl and $\mathrm{Am}^{+3}$ from nitric acid solution using $30 \%$ TBP in dodecane.

In FY 2009 we extended the investigation of the extraction of AmVI using more basic analogs of tributylphosphATE (TBP) in an attempt to elevate the distribution ratios. As seen in Figure 2 (next page), a maximum $D_{\mathrm{Am}} \sim 2$ was achievable with TBP, which is adequate for analytical chemistry but marginal for process design. The results using $0.1-\mathrm{M}$ concentrations of two basic organophosphorous extractants are compared to the same concentration of TBP in Figure 3 (next page). Dibutylbutylphosphonate provided elevated distribution ratios for AmVI, while the increase in basicity did not result in a 


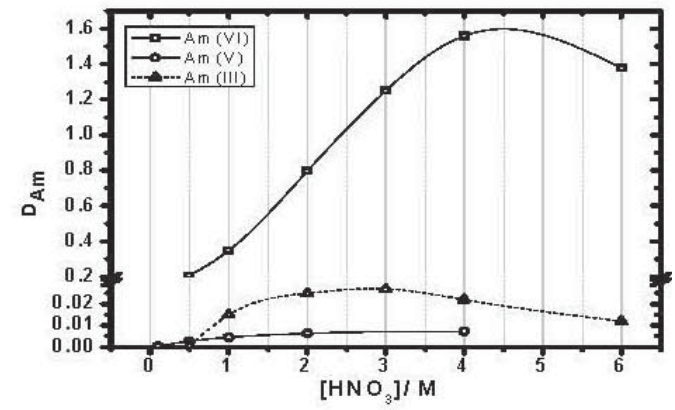

FIGURE 2. A plot of distribution ratio versus absorbed dose for the three americium oxidation states prepared in acidic solution, extracted with $30 \%$ TBP in dodecane.

significant increase in extraction of trivalent americium. This suggests that a separation from the trivalent $\mathrm{Cm}$ and lanthanides is possible. When dibutylbutylphosphonate was used at a concentration of $30 \%$ in dodecane, in analogy with the concentration of TBP in the UREX process, a satisfactory result of $D_{\mathrm{Am}} \sim 6$ was obtained. This is shown in Figure 4. A solvation number plot of the $\log D_{\mathrm{Am}}$ versus $\log$ [DBP] concentration resulted in a slope of 2 , in analogy with the stoichiometry of the TBP complex.

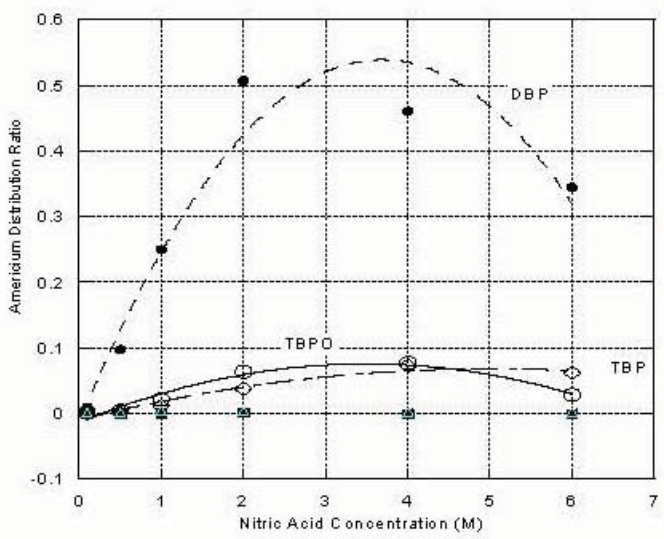

FIGURE 3. The solvent extraction $\mathrm{AmO}_{2}{ }^{2+}$ and $\mathrm{Am}^{+3}$ from nitric acid solution using $0.1 \mathrm{MTBP}$,

dibutylbutylphosphonate, or tributylphosphine oxide in dodecane.

The success of an AmVI solvent extraction process depends on avoiding the introduction of trace reducing agents, including radiolysis products, into solution. We observed this in the necessity to use short contact times during our extractions. Since this might be problematic under process conditions, we initiated investigations of the extraction behavior of more

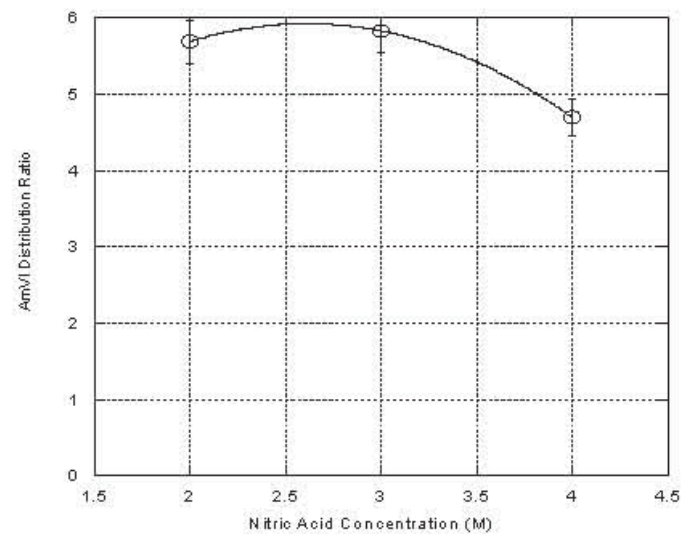

FIGURE 4. The solvent extraction $\mathrm{AmO}_{2}{ }^{2+}$ from nitric acid solution using $30 \%$ dibutylbutylphosphonate in dodecane.

stable AmV under TRUEX process conditions. The success of this approach would allow the separation of Am from the lanthanides with only minor modification to the currently proposed Fuel Cycle Research and Development partitioning scheme. The success of this laboratory-directed research and development (LDRD) has now allowed us to receive direct funding from the Fuel Cycle Research and Development program to continue this effort and also to investigate the kinetics of americium oxidation/reduction kinetics. Thus, this program has been successful in that it has generated new information and techniques for use in actinide science and has led to direct follow-on funding.

The experimental results from this work were published in the following peer-reviewed venues:

1. Martin, L.R., B. J. Mincher, and N. C. Schmitt, 2009, "Extraction of Am(VI) with a neutral organophosphorous ligand." $J$. Radioanal. Nucl. Chem. DOI 10.1007/s 10967-009-0153-o.

2. Mincher, B.J., L. R. Martin, and N. C. Schmitt, 2008, "Tributylphosphate extraction behavior of bismuthate-oxidized americium." Inorg. Chem, Vol. 47, pp. 6984-6989. 
3. Martin, L.R., B. J. Mincher, and N. C. Schmitt, 2008, "Investigating the use of bismuth (V) for the oxidation and subsequent solvent extraction of americium (VI)," Proceedings of the International Solvent Extraction Conference 2008, Tucson, AZ, Vol. 1, pp. 605-610, Moyer, B.A., ed. Canadian Institute of Mining, Metallurgy and Petroleum, Montréal.

4. Mincher, B.J., L. R. Martin, and N. C. Schmitt, 2007, "Co-extraction of Am(VI) and the major actinides with tributyl phosphate," Proceedings of Global 2007, Advanced Nuclear Fuel Cycles and Systems, American Nuclear Society, Boise, ID, September 9-13, 2007, pp. 1500-1502.

5. Martin L.R., B. J. Mincher, and N. C. Schmitt, 2007, "Understanding the chemistry of uncommon americium oxidation states for application to actinide/lanthanide separations," Proceedings of Global 2007, Advanced Nuclear Fuel Cycles and Systems, American Nuclear Society, Boise, ID, September 913, 2007. pp. 719-722.
Summary and Conclusions

Although previous attempts by others to oxidize americium in acidic solution have failed, we have successfully demonstrated quantitative production of both $\mathrm{Am}(\mathrm{V})$ and $\mathrm{Am}(\mathrm{VI})$. We have also developed spectroscopic techniques to view these oxidation states directly using safe americium concentrations in both the organic and aqueous phases. We have published the results in high-impact factor venues. Our results have lead to the real possibility of designing an Am/lanthanide separation that can be immediately adapted to analytical procedures and has promise for process scale separations. Consequently we have received follow-on funding from the Fuel Cycle Research and Development program. This program has also contributed to the fundamental understanding of the chemistry of the higher oxidation states of americium. 


\title{
Rapid Detection of Plutonium, Neptunium, and Technetium in Water Samples
}

\author{
Sarah Pepper, Dean Peterman, Troy Tranter \\ Using CPE coupled to radiometric/spectrometric detection methods to identify radionuclides in aqueous \\ samples
}

AS105

The identification of nuclear weapons production activities, nuclear fuel reprocessing operations, disposal of nuclear waste, and accidents involving nuclear materials can be achieved through the detection of plutonium $\left({ }^{239} \mathrm{Pu}\right.$ and $\left.{ }^{240} \mathrm{Pu}\right)$, neptunium $\left({ }^{237} \mathrm{~Np}\right)$ or fission products such as technetium $\left({ }^{99} \mathrm{Tc}\right)$. Their concentrations can be extremely low in aqueous samples, and consequently large volumes of solution may need to be collected and preconcentrated prior to analysis. Extractionseparation techniques, such as solvent extraction, are commonly employed to isolate and preconcentrate the analyte of interest. However, there is a move towards replacing this technique due to the generation of mixed wastes and the hazards associated with organic solvents. Cloud point extraction (CPE) based on the clouding phenomenon of surfactants above a certain temperature offers an attractive alternative. The technique employs water as the predominant solvent along with small amounts of inexpensive, environmentally benign surfactants. In the case of metal extraction, a chelating ligand is considered essential to facilitate quantitative separation (Fig. 1). To date, CPE has not been applied to the isolation of $\mathrm{Pu}, \mathrm{Np}$, and $\mathrm{Tc}$, but since it utilizes the formation of metal-ligand complexes, it should be ideally suited to separating these radionuclides. The objective of this project is to design a pre-concentration scheme, using CPE coupled to radiometric or spectrometric detection methods to facilitate the rapid identification of radionuclides in aqueous samples.

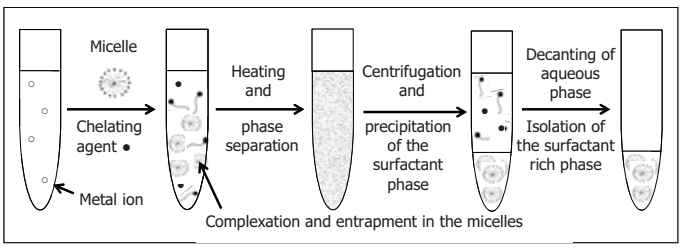

FIGURE 1. Cloud point extraction procedure.

The results obtained in this project will enable the development of a detection method for $\mathrm{Pu}, \mathrm{Np}$, and $\mathrm{Tc}$ in aqueous samples. We will also advance the study of $\mathrm{CPE}$ as a viable alternative to traditional separation methods as applied to radionuclide separations. With the move towards techniques that minimize sample manipulation, analyte losses and use of toxic solvents, the development of CPE applicable to actinide/fission product detection would place INL at the forefront of this emerging technology.

\section{Technical Objectives}

- Investigated various ligands for compatibility with cloud point extraction, including bis-(2-ethylhexyl) phosphoric acid (HDEHP), octylphenyl-N,Ndisobutylcarbamoylmethyl phosphine oxide (CMPO), tri-n-butyl phosphate (TBP), ammonium diethyl dithiophosphate (DDTP), ammonium diethyldithiocarbamate (DDTC), 1-(2-pyridylazo)-2-naphthol (PAN), 2-(5bromo-2-pyridylazo)-5-(diethylamino)phenol (Br-PADAP), 8-hydroxyquinoline (8-HQ) and ammonium pyrrolodinedithiocarbamate (APDC)

- Investigated various experimental parameters, including equilibration time, incubation time, water bath temperature, $\mathrm{pH}$, ionic strength, and metal-to-ligand ratio, to optimize separation of trivalent lanthanides using the ligand APDC with the surfactant Triton X-114

- Attempted cloud point extraction of plutonium using the ligand 8-

hydroxyquinoline with the surfactant Triton $\mathrm{X}-114$

- Presented experimental results at the following conferences:

- $237^{\text {th }}$ American Chemical Society National Meeting in Salt Lake City, UT

- Methods and Applications of Radioanalytical Chemistry conference (MARC VIII) in Kailua-Kona, HI

- $238^{\text {th }}$ American Chemical Society National Meeting in Washington D.C.

- Authored peer-reviewed article published in Journal of Radioanalytical and Nuclear Chemistry (DOI 10.1007/s10967-0090303-4)

Results and Accomplishments

Ligand selection: In the CPE literature, the chelating ligands used for metal extraction are 
similar or the same as those used in traditional solvent extraction schemes. Therefore, one of the hypotheses of this laboratory-directed research and development (LDRD) was that ligands used for the extraction of actinides and fission products in solvent extraction would be suitable for use in CPE and could be exploited in the design of a CPE scheme to allow maximum complexation, and therefore maximum separation, while minimizing competition from other metals. Consequently, the ligands HDEHP, CMPO, and TBP were initially investigated. Unfortunately, these ligands significantly interfered with the clouding phenomenon of the surfactant, i.e., they prevented the sample from becoming turbid which resulted in a lack of separation between the bulk aqueous phase and the surfactant rich phase. Subsequently, the ligands DDTP, DDTC, PAN, Br-PADAP, 8-HQ and APDC were investigated and all appeared compatible with the surfactant. However, APDC was chosen as the ligand for initial experiments since it is a known complexant with trivalent lanthanides and uranium and has been used in CPE for the separation of transition metals. In addition, it dissolves in water, thus minimizing the presence of organic solvents, such as ethanol or methanol, and does not complex Group I and II metals, which can interfere in detection of analytes in environmental samples.

Optimization of experimental parameters for the detection of trivalent lanthanides in solution: In a typical CPE scheme, a number of experimental parameters require optimization. Typical experimental parameters may include equilibration time, incubation time, water bath temperature, metal:ligand (M:L) ratio, $\mathrm{pH}$, and ionic strength. This section describes experiments that were performed to optimize several of these experimental parameters for the detection of trivalent lanthanides in aqueous solution using the ligand APDC.

Equilibration time is defined as the period of time prior to heating during which the metal and/or metal-ligand complex forms and partitions into the micelle. The effect of this time was investigated for $1 \times 10^{-5} \mathrm{M}$ of $\mathrm{La}(\mathrm{III})$ in the absence and presence of APDC at a metal:ligand ratio of 1:100. The samples were left for various times on a rotary shaker at room temperature prior to being placed in a water bath at $50^{\circ} \mathrm{C}$ for 1 hour for the cloud point separation. In the absence of APDC, less than $30 \%$ of the metal extracted into the surfactant-rich phase at all equilibration times investigated. However, in the presence of APDC over 24 hours, the amount of $\mathrm{La}(\mathrm{III})$ extracted increases from around $60 \%$ to about $90 \%$. This increase in the measured extraction efficiency in the presence of the ligand suggests that complex formation does occur and is essential to efficient separation. However, the formation of the complex and its subsequent partitioning into the micelle is slow.

Consequently, a 24-hour equilibration time was chosen for all subsequent experiments.

Incubation time is the period of time that the sample is held at a temperature above the cloud point temperature during which time the phases separate. In addition, overheating by more than $10^{\circ} \mathrm{C}$ is considered essential to obtain an efficient separation. Hence, the effect of these parameters on the separation of $\mathrm{La}(\mathrm{III})$ in the presence of a 100-fold excess of APDC was investigated (Fig. 2). Increasing both the incubation time and temperature generally increased the amount of $\mathrm{La}(\mathrm{III})$ extracted into the surfactant rich phase, with an almost quantitative separation occurring after 2 hours at $60^{\circ} \mathrm{C}$.

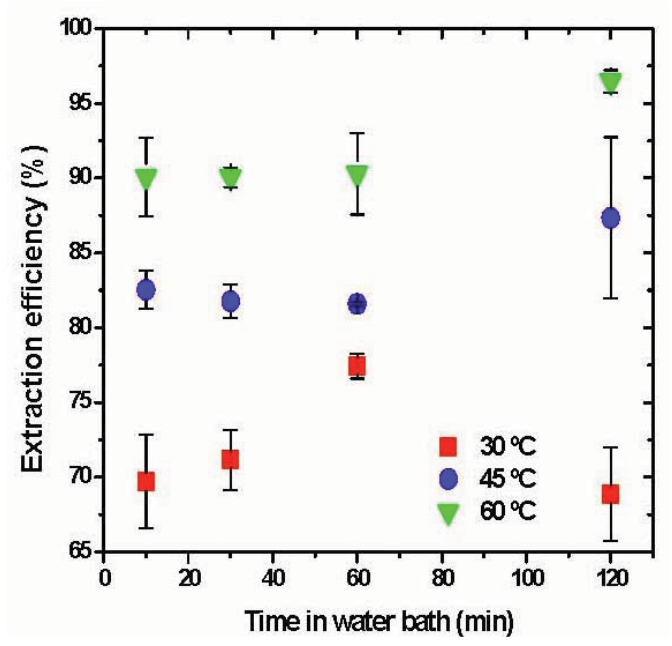

FIGURE 2. Effect of incubation time and water bath temperature on the extraction of La(III) in the presence of 100-fold excess of APDC.

Ligand concentration is considered one of the most important parameters to optimize, and 
its effect on the extraction of the $\operatorname{Ln}(\mathrm{III})$

individually is shown in Figure 3, while Figure 4 shows the effect when all are present in solution together. Samples were equilibrated for 24 hours prior to being placed in the water bath at $50^{\circ} \mathrm{C}$ for one hour. As the amount of ligand increases from a M:L ratio of 1:0 to 1:1000, the amount of

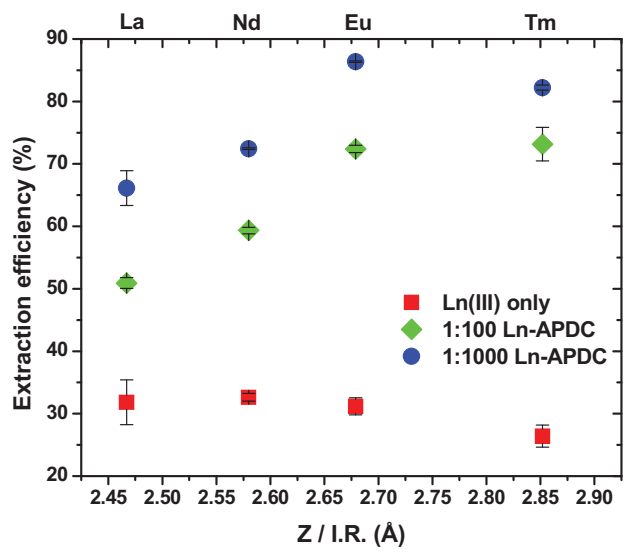

FIGURE 3. Extraction efficiency for individual Ln(III) as a function of M:L ratio, where $[$ Ln(III) initial $]=1 \times 10^{-5} \mathrm{M}$.

Ln(III) extracted increases. This further supports the assumption that a metal-ligand complex is being extracted, possibly as a neutral complex containing 1 metal center and 3 ligand units similar to that proposed for the SE of metals by dithiocarbamates. For the Ln(III) investigated, the data appear to reach a maximum extraction efficiency at $\mathrm{Eu}(\mathrm{III})$ before decreasing at Tm(III), possibly as a result of the competition between complexation of the metal center by the ligand and dehydration of metal-coordinated water. When the $\operatorname{Ln}(\mathrm{III})$ are present in solution separately, the larger M:L ratio-i.e., 1:1000gives the greater separation. However, when $\mathrm{Ln}(\mathrm{III})$ are present in solution together, there appears to be little difference in the extraction efficiency at M:L ratios above 1:0. At present, the reason for this difference is unknown. In addition, when present together, the CPE system shows greater selectivity for $\mathrm{Eu}(\mathrm{III})$.

The experimental results to date indicate that $\mathrm{CPE}$ is a promising alternative to solvent extraction for the pre-concentration of aqueous samples, but there are a number of parameters that still need to be optimized.

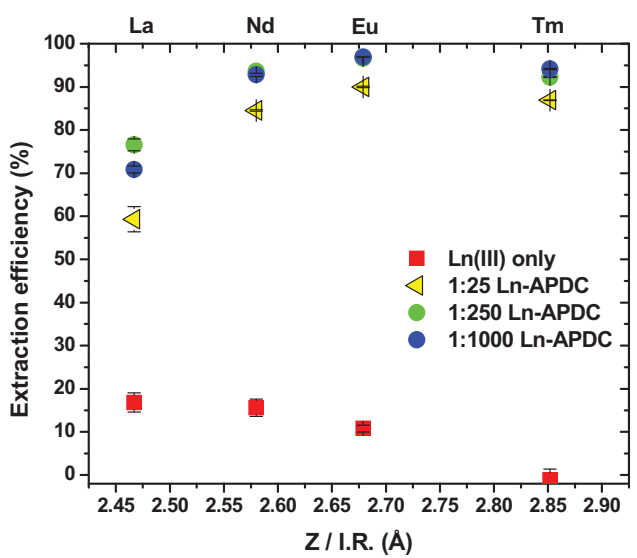

FIGURE 4. Extraction efficiency for Ln(III) in solution as a function of M:L ratio, where total [Ln(III)initial] $=4 \times 10^{-5}$ M.

Cloud point extraction of plutonium: This experiment attempted to assess the ability of CPE to extract plutonium. A solution containing ${ }^{239} \mathrm{Pu}$ in $1 \%$ nitric acid was added to an excess of the ligand 8-hydroxyquinoline dissolved in Triton X-114. Extraction was approximately $20 \%$, but the oxidation state of $\mathrm{Pu}$ was unknown. However, this experiment was performed to demonstrate whether CPE can be applied to the separation of $\mathrm{Pu}$.

\section{Summary and Conclusions}

Much of this year was spent attempting to find a system that worked before applying it to the target analytes, i.e. plutonium, neptunium, and technetium. This proved to be more problematic than first assumed, despite reports that suggested that adding surfactant to an aqueous solution of the metal and heating for a period of time in the presence of a chelating ligand results in a quantitative separation. While a system to separate trivalent lanthanides has been partially optimized, there are parameters that still must be investigated, e.g., $\mathrm{pH}$ and ionic strength. A preliminary experiment was performed to assess the applicability of CPE to $\mathrm{Pu}$ separation, and next year the system will be further explored starting with well-characterized $\mathrm{Pu}$ solutions (principally of known oxidation state). 


\title{
Evaluation of Covalent Interactions in Actinide Coordination Compounds
}

\author{
Dean R. Peterman, Dale D. Russell (BSU) \\ Electrochemical and spectroscopic techniques to study the coordination and separations chemistry of the \\ INL DPAH compounds with several An(III) and Ln(III) species
} AS106

Complexing agents containing soft-base (e.g., $\mathrm{N}$ or $\mathrm{S}$ ) donors such as dithiophosphinic acids have significant potential for the efficient separation of actinides, An(III), from lanthanides, Ln(III). A family of dithiophosphinic acids (DPAH) developed at INL has shown unprecedented selectivity for americium (Am) and curium $(\mathrm{Cm})$ over lanthanides in acidic solution. We have used electrochemical and spectroscopic techniques to study the coordination and separations chemistry of the INL DPAH compounds with several An(III) and Ln(III) species. As the actinide series is crossed from uranium $(\mathrm{U})$ to curium, the extent of covalency in the metal ligand bond is expected to decrease due the spatial contraction of the $5 \mathrm{f}$ orbitals. UV-vis, infrared, and Raman spectroscopies will be used to quantify the extent of covalency in the An(III)-DPAH coordination compounds. By systematically studying the variations in An(III)-DPAH coordination complexes, we plan to develop a detailed understanding of the interactions of $\mathrm{Ln}(\mathrm{III})$ and An(III) ions with soft donor ligand systems. This detailed understanding holds the promise of providing great insight into the nature of actinide interactions with soft-donor bases.

\section{Technical Objectives}

- Optimized methodology for the electrochemical detection of americium in aqueous and organic solvents

- Designed and procured a flow-through electrochemical cell for the bulk electrolysis of actinide materials

- Designed and procured an inert atmosphere glovebox for application of UV-Vis-nIR, IR, and Raman spectroscopy to samples containing macroscopic concentrations of uranium, neptunium, plutonium, americium, and curium

- Presented experimental results at the Methods and Applications of Radioanalytical Chemistry Conference (MARC VIII) in Kailua-Kona, HI

- $\quad$ Authored peer-reviewed paper titled "Selective Separation of Minor Actinides and Lanthanides Using Aromatic Dithiophosphinic and Phosphinic Acid
Derivatives," J. Radioanalytical Nuc.

Chem., DOI 10.1007/s10967-009-0288-z.

\section{Results and Accomplishments}

For a systematic comparison of lanthanide coordination chemistry with that of the actinides, it is necessary to use the same oxidation states of the elements being studied. The lanthanide elements are readily available in their +3 oxidation states. The heavier actinides form stable trivalent compounds, but lighter actinides, notably uranium, are not thermodynamically stable in this oxidation state in aqueous solution. Uranium(III), for example, has been classified as a substantial reductant because it is so readily oxidized to higher oxidation states; oxidation of $\mathrm{U}^{3+}$ occurs in air, and in water in the presence of oxidizing acids or dissolved oxygen. Actinides used in this work include $\mathrm{U}$, plutonium $(\mathrm{Pu})$, neptunium (Np), Am and Cm. Several protocols have been reported in the literature for the preparation of $\mathrm{U}^{3+}, \mathrm{Np}^{3+}$ and $\mathrm{Pu}^{3+}$ from the higher oxidation states. These methods include use of chemical redox reagents, enzyme catalyzed processes, and electrolytic methods. Of the methods reported, electrochemical methods have the least interferences and are capable of generating large quantities of a high-purity product in the desired oxidation state.

Prof. Dale Russell, of the Chemistry Department at Boise State University, spent her sabbatical at INL performing research on the electrochemistry of actinide species in aqueous and organic media. The aim of her experimental work was the development of an understanding of the electrochemical conditions necessary for the production of trivalent actinide species in aqueous and organic media. The production method chosen is controlled potential electrolysis (CPE). This is an ideal method for generating the product of a redox reaction. Electrodes are exceptionally clean "reagents" as they provide only electrons (or, alternatively, a "sink" for electrons) while side reactions and contaminating side products are minimized. It is customary to isolate the oxidation and reduction reaction products in separate compartments of the electrochemical cell, simplifying the task of product purification. In principle, the +3 oxidation state for each actinide can be generated in this way. 
One anticipated problem with the use of $\mathrm{CPE}$ for the production of trivalent actinides is that the +3 oxidation state of some actinides is not particularly stable, and these may be challenging to isolate. In that case, a ligand may be introduced into the CPE solution to stabilize the desired oxidation state. The stabilizing ligand is unlikely to be any of the dithiophosphinic acid ligands of this study as they are essentially insoluble in aqueous media. Several different classes of ligand are known which may serve, and in this case, a ligand exchange would be performed after generation of the An(III) state. One problem to anticipate with ligand stabilization during CPE is the possibility that the ligand itself might be electroactive at the redox potential of one or more of the metals. If this occurs, an alternate ligand would need to be selected for that metal to stabilize the incipient An(III) state. Alternatively, the CPE experiment could conceivably be conducted in a specially designed apparatus that permits continuous extraction into the solvent, trifluoromethylphenyl sulfone (FS-13), during the CPE generation of An(III) state.

The electrochemistry of americium and europium were studied using the DPAH ligand, bis(o-trifluoromethylphenyl dithiophosphinic acid, and the solvent FS-13 (Fig. 1). For each metal, the electrochemical response in both aqueous and nonaqueous solutions was determined. The metal concentrations before and
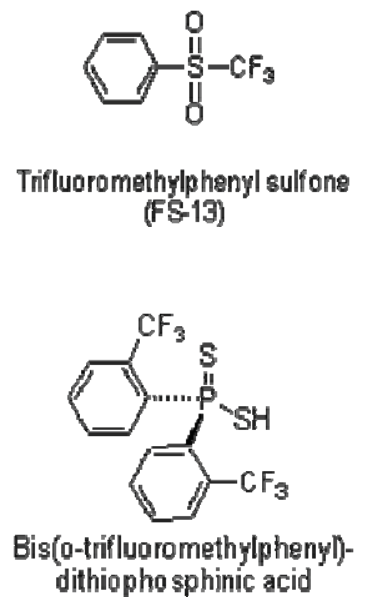

FIGURE 1. Structure of DPAH ligand and solvent used in this study. after extraction were determined in both phases via both radiochemical methods and electrochemical detection. The experimental conditions necessary for electrochemical detection at low concentration $\left(\sim 10^{-7} \mathrm{M}\right)$ were determined for both metals. These experimental conditions and the electrode preconditioning are now optimized and will be used for experiments involving the less-stable actinides in the future. For most of the electrochemical work, the reference electrode was $\mathrm{Ag} / \mathrm{AgCl}(4 \mathrm{M} \mathrm{NaCl})$ from BioAnalytical Systems (BAS). A silver wire pseudo reference electrode was also used on occasion and gave a similar reference potential. The potential of this electrode in FS-13 was very stable and did not have a liquid junction component to it. Also, it did not contaminate the FS-13 as the $\mathrm{Ag} / \mathrm{AgCl}$ electrode would. The counter electrode in all cases was a platinum wire spiral coil.

The working electrode in much of the work was a platinum rotating disk electrode with a Teflon $^{\mathrm{TM}}$ sheath, supplied by BAS; however, in later work it became apparent that the FS-13 solutions caused serious degradation of the platinum working electrode in potential regimes where some metals of interest had their redox potentials. This necessitated the identification of alternate electrode materials for the $\mathrm{CPE}$ experiments. Two acceptable alternatives were identified, graphite and indium-tin oxide (NESA glass). Cyclic voltammetry experiments were performed at scan rates of either $50 \mathrm{mV} / \mathrm{s}$, or 100 $\mathrm{mV} / \mathrm{s}$. Solutions were purged with nitrogen gas for 30-40 minutes prior to scans, and nitrogen was streamed over the solution during scans. Tetrabutylammonium hexafluorophosphate (TBAHFP) (0.1 M) was added to all FS-13 solutions immediately preceding the voltammetric procedure to serve as an electrolyte. Typical cyclic voltammetry traces for three different concentrations of Am(III) dissolved in FS-13 are presented in Figure 2.

An inert atmosphere glovebox was procured and installed in the laboratories in the CFA-625 facility. This glovebox is equipped with optical and electrical feedthroughs which will enable spectroscopic and electrochemical experiments with samples containing large amounts of 
actinides and other radionuclides with minimal risk of personnel exposure of contamination.

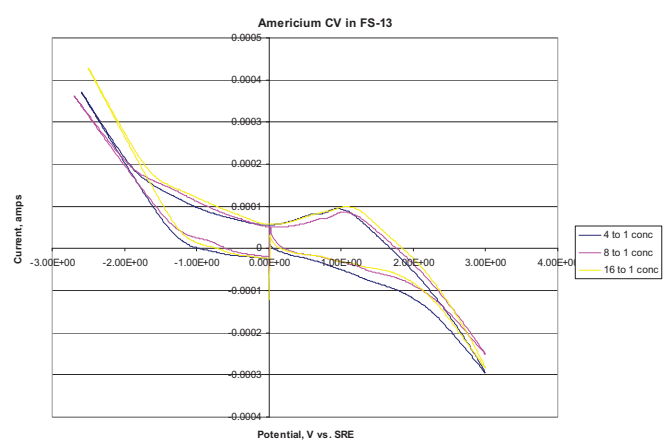

FIGURE 2. Cyclic voltammetry traces of three different concentrations of americium dissolved in FS-13.

\section{Summary and Conclusions}

The DPAH ligand, bis-(otrifluoromethylphenyl) dithiophosphinic acid was electrochemically characterized in FS-13. The extraction of several metals, both actinides and lanthanides, was performed using this ligand. Electrochemical methods were demonstrated for detection and quantitation at the submicromolar concentration levels. Several electrode materials were investigated for use in the electrochemical detection and quantitation of the actinides in aqueous and nonaqueous solutions, with the outcome that graphite and NESA glass gave the best results. In addition, the experimental conditions necessary for the electrochemical production of macroscopic quantities of trivalent actinide species in aqueous and organic solution have been optimized. Using these conditions, experiments will be performed in the inert atmosphere glovebox in order to produce large amounts of actinide species in the desired $3+$ oxidation state. This will permit the spectroscopic investigation of the extent of covalent versus ionic interactions present in the An(III) soft-donor ligand systems. 


\title{
Measuring Actinide Speciation in High-pH Solutions
}

\author{
Gary S. Groenewold, Anthony D. Appelhans, Garold L. Gresham \\ Using electrospray ionization mass spectrometry to determine metal speciation in solutions \\ AS107
}

In a given environment, metal speciation is the envelope of chemical forms that an element can exist in. It can encompass various coordination complexes, oxidation states, adsorbates, precipitates, or particulate states, and the ultimate disposition of that element will depend on its speciation chemistry. Hence, methods for identifying and quantifying metal speciation are needed, particularly for actinide metals where speciation envelopes can be extremely diverse. Actinide solutions with high ionic strength (I) or extreme $\mathrm{pH}$ are a speciation challenge.

All current methods for measuring actinide speciation in solution have significant drawbacks that are related to inadequate specificity and sensitivity or constraining experimental or apparatus requirements. Two salient examples of approaches currently used for actinide separation are UV-visible spectroscopy (UV-vis) and x-ray absorption spectroscopy (XAS). Changes in solution coordination can be measured using UV-vis, but peak shapes are broad and the presence of multiple or unexpected chemicals in the samples can make measurement difficult. UV-vis does benefit from the fact that the experimental apparatus is relatively inexpensive and can occupy a relatively small laboratory footprint, and thus is compatible with many radiological laboratory settings. XAS techniques can exactly determine atomic positions within coordination complexes. However, it also has difficulty differentiating multiple species present in the same sample, which makes data interpretation difficult. And XAS requires synchrotron radiation, making analysis of many types of samples difficult or impossible; examples include those having a high degree of chemical complexity and those having significant radiological control constraints.

Electrospray ionization mass spectrometry (ESI-MS) is an alternative approach for determining metal speciation in solutions, and has significant potential for measuring speciation in complex mixtures. ESI-MS works by spraying an analyte solution through a high potential (e.g., $4 \mathrm{kV}$ ) capillary where charged droplets will desolvate at atmospheric pressure, leaving behind gaseous ionic solutes that are transferred via a skimmer cone to the high vacuum of a mass spectrometer. Many solution species are transferred intact from solution to the gas phase and are unequivocally identified on the basis of their mass-to-charge ratio. On account of the dramatic changes in the physical environment, some controversy in the literature has focused on whether the gas phase ions accurately reflect those in solution. Indeed ESI of metal complexes has been shown to cause changes in numbers and types of ligands, and in some cases oxidation state, as the molecules go from solution to the gas phase during the ESI process. However, multiple studies have shown that gas phase ion populations very accurately reflect solution phase speciation. On the basis of these arguments, ESI might be ideally suited for measuring actinide solution speciation. However, two factors relevant to this project are potential obstacles to using ESI for speciation measurement in actinide solutions having high $\mathrm{pH}$ or ionic strength:

- $\quad$ Spray of a high $\mathrm{I} / \mathrm{pH}$ solution resulting in plugging and/or corrosion of the capillary

- Spray of a solution containing significant radiological activity within the spray housing.

One approach for overcoming these obstacles is to utilize desorption electrospray ionization, or DESI. Here, molecules on surfaces are picked up by ESI droplets as they impact and then recoil from the surface. This approach can eliminate the spray of highly concentrated salt solutions, which could be instead deposited on the target surfaces. Furthermore, it could function with significantly reduced sample sizes, making characterization of the species much more tractable due to lower activity.

\section{Technical Objectives}

This laboratory-directed research and development (LDRD) project pursues an ambitious course of research that is designed to:

- Modify an existing INL ESI-MS to enable it to function in the DESI mode

- Evaluate the performance of the instrument for characterizing metal-solvent coordination complexes, strongly 
complexing ligands, and hydroxide-

carbonate media

- Demonstrate instrument function for characterizing TRU species.

\section{Results and Accomplishments}

\section{DESI Modification}

A recent development showed that utilization of a screen for holding the analyte solution offered a much-simplified approach for conducting DESI experiments because of easier geometric constraints. Instead of requiring a droplet to impact and recoil from a target surface at specified angles, the droplet is instead passed through a screen, picking up analyte from the screen mesh surface in the process (Fig. 1). This is referred to as transmission mode DESI, or TM-DESI. A commercial ESI instrument was modified, changing the

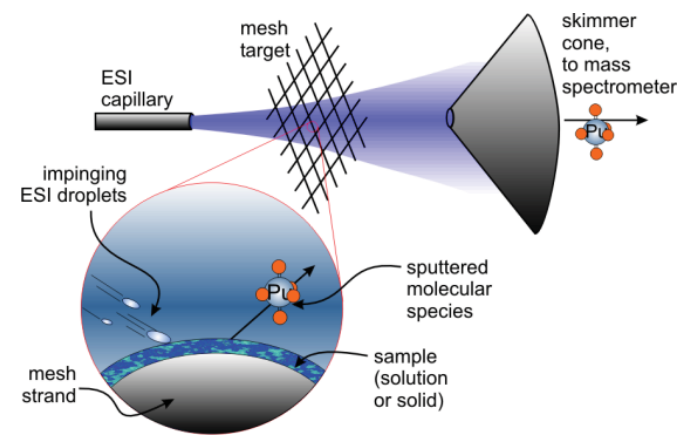

FIGURE 1. Schematic diagram of the TM-DESI process: droplets impacting and recoiling from a screen target.

orientation of the spray capillary relative to the skimmer cone by inserting a screen mount and an adjustable cradle that would hold the screen in place between the capillary and the skimmer cone. An aluminum platform was fabricated that enabled the ESI capillary to be mounted coaxially with the aperture of the skimmer cone, and the screen cradle was then mounted in between, as shown in Figure 2.

\section{Analytical Scoping Studies using TM-DESI}

Initial testing of the TM-DESI instrument showed facile production of sample ions. Application of a quaternary ammonium cation to the target screen followed by bombardment with $\mathrm{MeOH} / \mathrm{H}_{2} \mathrm{O}$ droplets produced abundant tetrahexylammonium cations at $\mathrm{m} / \mathrm{z} 354$, with an iodide-bound dimer at $\mathrm{m} / \mathrm{z} 835$ (Fig. 3). These ions accurately reflect the composition of the organic salt on the target surface. In addition to demonstrating the functionality of the instrument, the experiment showed that the technique was amenable to compounds typical of those encountered in ionic liquids.

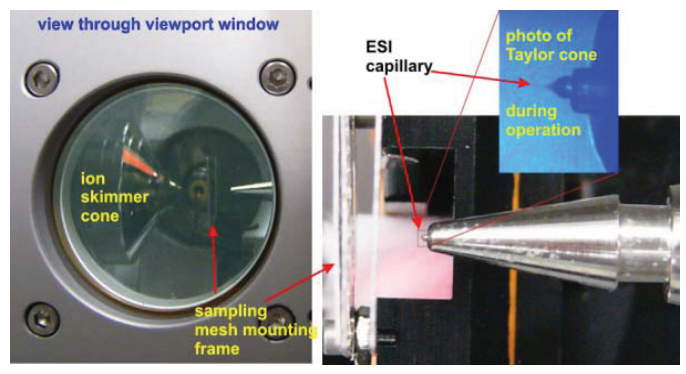

FIGURE 2. Photographs of the modified source region of the ESI instrument. Droplets are formed from a Taylor cone at the capillary tip during ESI.

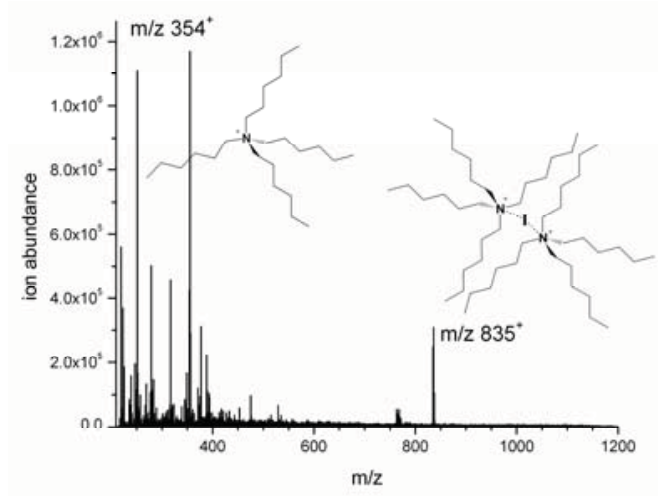

FIGURE 3. Positive ion TM-DESI mass spectrum of tetrahexylammonium iodide.

TM-DESI analysis of 1-M solutions of lanthanide nitrate salts produced characteristic metal coordination complexes in both the positive and negative ion mass spectra. The spectrum of lanthanum nitrate contained both singly and doubly charged ion pair complexes of $\mathrm{La}^{3+}$ and $\mathrm{NO}_{3}{ }^{-}$, together with small quantities of acetate- and methoxide-containing complexes (Fig. 4, next page). The remainder of the inner coordination sphere was occupied with solvent, in this case methanol from the spray solution; up to four methanol molecules were observed (accounting for $m / z$ 390.3), making the $\mathrm{La}^{3+}$ cation octacoordinate, a result consistent with 
coordination observed in solution. In the negative ion mode, analysis of a $\mathrm{La}\left(\mathrm{NO}_{3}\right)_{3} / \mathrm{NaNO}_{3}$ mixture produced predominantly $\left[\mathrm{La}\left(\mathrm{NO}_{3}\right)_{4}\right]^{-}$, but also clusters of lanthanum and sodium nitrate. The conclusion of these experiments was that the TM-DESI method was producing complexes in the gas phase that were in accord with those expected to be present in the condensed phase target.
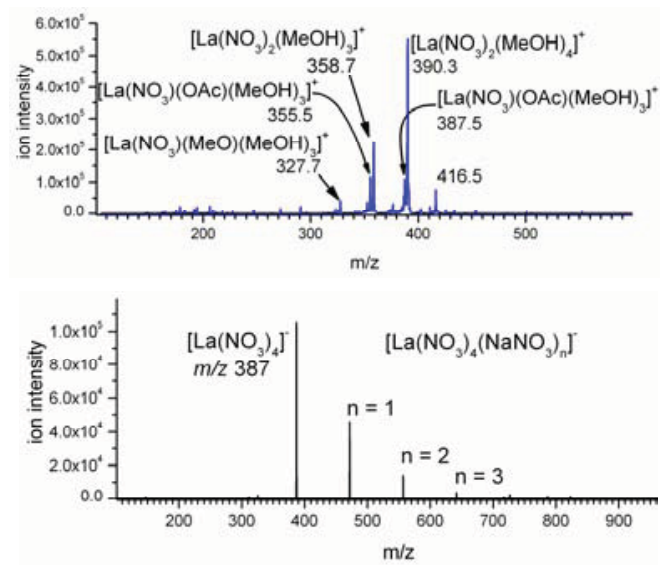

FIGURE 4. TM-DESI analysis of $\mathrm{La}\left(\mathrm{NO}_{3}\right)$. Top, positive ion. Bottom, negative ion, with $\mathrm{NaNO}_{3}$.

Solutions at high $\mathrm{pH}$, or solids derived from these, are challenging characterization problems in that high spectroscopic background and chemically aggressive behavior are associated with these materials. High $\mathrm{pH}$ surfaces fix $\mathrm{CO}_{2}$, forming bicarbonate and carbonate species; consequently, these surfaces tend to have similar chemical speciation. Prior studies have shown that generating gas phase carbonate ions that are indicative of the condensed phase speciation is difficult, probably owing to the relatively facile elimination of $\mathrm{CO}_{2}$ that occurs from discrete carbonate-bearing species in the mass spectrometer.

March had previously demonstrated that carbonate-containing ions could be generated using ESI, but failed to observe bicarbonate clusters. Analysis of sodium bicarbonate solutions produced abundant cation series corresponding to $\left[\left(\mathrm{NaHCO}_{3}\right)_{\mathrm{n}} \mathrm{M}\right]^{+}$, where $\mathrm{M}=$ $\mathrm{Na}^{+}$and $\mathrm{Na}_{3} \mathrm{CO}_{3}^{+}$(Fig. 5). This result demonstrates the applicability of TM-DESI for species that are too fragile to be detected using other mass spectrometric approaches and suggests that TM-DESI may be less prone to perturb speciation compared to standard ESI.

The final group of scoping studies emphasized measurement of samples containing strongly complexing ligands. Measurement of ethylenediamine tetraacetic acid (abbreviated $\mathrm{H}_{4} \mathrm{Y}$ ) species has traditionally been challenging on account of four Bronsted acid sites with the ability to strongly bind in a multi-dentate fashion. TM-DESI of $\mathrm{Na}_{2} \mathrm{H}_{2} \mathrm{Y}$ produced $\mathrm{Na}^{+}-$ cationized salts, as well as clusters that were typified by multiple $\mathrm{Na}_{2} \mathrm{H}_{2} \mathrm{Y}$ units. As cluster size increased, so did substitution of $\mathrm{Na}$ for $\mathrm{H}$, producing molecules enriched in $\mathrm{Na}$. This suggests that larger clusters prefer additional $\mathrm{Na}$ cations, and they may be required to hold the cluster together. The conclusion of the $\mathrm{H}_{4} \mathrm{Y}$ experiments is that TM-DESI can readily produce gas phase ions that are highly indicative of the condensed phase speciation.

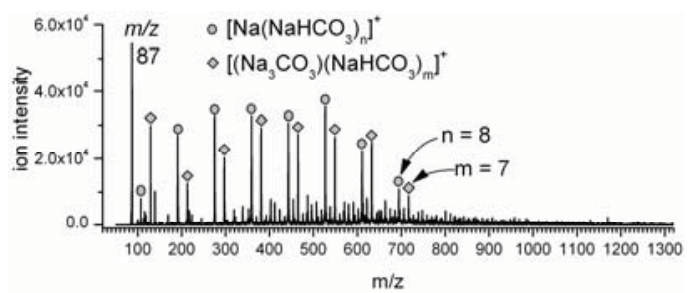

FIGURE 5. Positive ion TM-DESI spectrum of sodium bicarbonate solution, showing bicarbonate and mixed bicarbonate / carbonate cluster species out to eight $\mathrm{CO}_{3}{ }^{2}$ units.

\section{Summary and Conclusions}

This research project has produced a new capability for INL, enabling mass spectrometric analyses of metal speciation from solutions and solids that are at high I and or extreme $\mathrm{pH}$. The research will enable INL to compete more effectively for programs in the areas of nuclear fuel reprocessing, metal contamination, and renewable fuels (since the TM-DESI approach is also very likely applicable to rapid characterization of biological molecules). In the remaining years of this project, the path forward will be to more quantitatively define speciation measurements and to develop a method for conducting TM-DESI analyses that will accommodate measurement of transuraniccontaining samples. 


\section{Isotope Ratio Measurement Methods for Direct Analysis of Samples}

Jill R. Scott, Timothy R. McJunkin, Andrew Merriam

Innovative alternatives to improve isotope mass spectrometry

AS108

Current methods to characterize nuclear materials require time consuming, expensive sample preparation. Sample preparation is necessary to remove interferences that limit the performance (e.g., limits of detection) of thermal ionization mass spectrometers (TIMS). In addition, the number of different types of ions that can be analyzed by TIMS is limited to $<10$ because each ion type requires its own detector. Thus, the goal of our project is to develop innovative alternatives for isotope ratio mass spectrometry that not only will provide dramatic improvement in sensitivity, but will also provide enhanced resolution and simultaneous detection of all ion types over a wide mass-to-charge $(\mathrm{m} / \mathrm{z})$ range $(>500)$ for analysis of environmental samples with little or no sample preparation. Environmental samples are challenging because they are typically heterogeneous, and the concentration of isotopes of interest tends to be low. To address the heterogeneity aspect, our approach is to take advantage of our current imaging capabilities associated with the laserbased optical and chemical imager (LOCI), which includes a laser desorption Fourier transform ion cyclotron resonance mass spectrometer (FTICR-MS) as well as optical detection capability for laser-induced fluorescence and Raman. FTICR-MS is a good choice for low detection limits because it only requires $\sim 400$ ions for a signal-to-noise $(\mathrm{S} / \mathrm{N})$ of 3 and it provides unparalleled mass resolution $(70,000+)$ and mass accuracy $(\sim 1 \mathrm{ppm})$ over a wide mass range $(>500 \mathrm{u})$; thus, there will be little or no isobaric interferences for the isotope measurements. However, taking advantage of LOCI for accurate isotope ratios requires addressing isotope fractionation that occurs during the ionization process by changing from a multiphoton ionization (MPI) to a single photon ionization (SPI) process, which is the primary focus of this year's activities.

This project also includes incorporation of laser-induced breakdown spectroscopy (LIBS), because LIBS has been identified as an emerging technology for determining isotope ratios and it can be acquired using the optical detection capability of LOCI. While the use of LIBS for isotope ratios is immature, it was chosen because it has the potential for future field-portable instrumentation even though its isotope ratios may not be highly accurate. The proposed project would provide an instrument for determining isotope ratios from heterogeneous samples without any sample preparation by both laser desorption FTICR-MS and LIBS. The isotope ratio accuracy of the LIBS will be compared to the more accurate FTICR-MS ratios. Because the LIBS analysis can be performed under various environmental conditions (e.g., pressure) and then compared with the FTICR-MS data for the same sample, it will be possible to assess what conditions are most appropriate for developing field-portable LIBS systems to assist in even more rapid isotope ratio measurements in the future.

\section{Technical Objectives}

- Integration of laser source for SPI

$\circ$ Acquisition and development of SPI (118-nm) light source components

- Adapting vacuum housing of FTICRMS to accommodate new 118-nm light source

- Initial testing of SPI source with our proprietary virtual infinite parallel plate (ViPP) FTICR cell

- Setup and initial LIBS testing

- Modifications to optical detection software and electronics

- Fabrication of independent test chamber

- Presented results at conferences.

Results and Accomplishments

Two key issues have prevented laser desorption/ionization FTICR-MS from being used for isotope ratio measurements. The first issue is related to the FTICR-MS method because of difficulties related to ratio accuracy that stem from the fact that ions are not all located at the same radius upon detection in traditional FTICR cells. Previously, we determined how to overcome the limitation related to the FTICR cell so that all ions of all types will be detected at the same radius; thus, the observed signals will accurately reflect the number of ions. Our novel virtual infinite parallel plate (ViPP) ${ }^{1}$ FTICR-cell will also result 
in very low detection limits (attogram), high accuracy isotope ratio measurements, simultaneous multiple-ion capability with high mass resolution, and mass accuracy for analysis of heterogeneous environmental samples. The second issue is related to fractionation or bias that occurs during the ionization event that are associated with optical isotope shifts and hyperfine structures, especially when using MPI. Overcoming this bias requires shifting from a MPI laser desorption/ionization approach to one that will provide single photon ionization. ${ }^{2-4} \mathrm{SPI}$ requires producing photons with high energy, such as $118-\mathrm{nm}(\sim 10.5 \mathrm{eV})$ photons, so that interaction of desorbed species with a single photon will result in ionization. Another advantage of SPI at $118 \mathrm{~nm}$ is that it increases detection limits because most species generated by single-laser desorption/ionization alone are neutrals. ${ }^{5}$ SPI would be a vast improvement over traditional multiphoton techniques that produce isotope fractionation and have poor ionization efficiencies $(<10 \%)$.

A single photon ionization (SPI) source was added to the current laser desorption FTICR-MS to allow virtually all elemental isotopes and molecular species with ionization energies $<10.49 \mathrm{eV}$ to ionize with a single photon, eliminating the fractionation inherent with multiphoton ionization and enhancing the ionization efficiency to $\sim 100 \%$. Figure 1 is a photograph of the vacuum ultravioltet (VUV) source of 118.2-nm photons supplied by Lumeras (Santa Cruz, California) and is similar in design to previously described 118-nm laser light sources. ${ }^{2-5}$ Briefly, the high-energy $118-\mathrm{nm}$ photons are generated via gas-phase nonlinear frequency conversion of a high-power $\mathrm{Nd}$ :YAG pump laser $(354.7 \mathrm{~nm})$ in a xenon (Xe) gas chamber. The 118-nm photons will exit the Xe gas chamber through a $\mathrm{MgF}_{2}$ window directly into the vacuum chamber of the FTICR-MS.

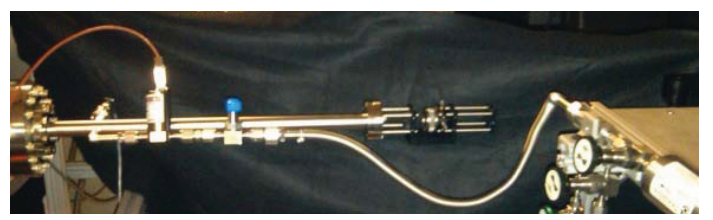

FIGURE 1. Photograph of VUV 118-nm single photon ionization source.
Because of the geometry of the FTICR-MS vacuum chamber, it is not practical to locate the SPI unit in close proximity to the sample as is the case for most other mass spectrometers. Therefore, the 118-nm photons must travel a much longer distance $(\sim 190 \mathrm{~cm})$ before encountering the sample, which leads to the potential for the photons to interact with any neutrals in the vacuum chamber. Indeed, initial tests of the SPI with the ViPP FTICR cell revealed that some of the electronic components of the detection circuit located within the vacuum chamber were outgassing, producing an array of ions even when no sample was present. Thus, it will be necessary to eliminate the outgassing issue (e.g., either by replacing the components inside the vacuum chamber or redesigning the chamber to move the circuit outside of the vacuum). The extended vacuum length before the 118-nm photons can react with analytes of interests also mandates a greater photon flux than the $<10^{10}$ photons $/ \mathrm{cm}^{2}$ produced by typical 118-nm VUV sources.

Experiments using a traditional cubic FTICR cell and associated electronics were performed with gaseous analytes to estimate the $118-n m$ photon flux. Given $1 \mu \mathrm{J}$ of $118-n m$ pulse energy and $10.49 \mathrm{eV} /$ photon, there are $6 \times 10^{11}$ photons/pulse. Assuming diffraction-limited propagation from the SPI unit to the FTICR cell, then the 118-nm beam diameter in the cell is $2.2 \mathrm{~mm}$ for a propogation length (L) of $190 \mathrm{~cm}$. If the vacuum in the chamber were perfect (i.e., no absorbers), the photon flux at the cell is between $5 \times 10^{12}$ and $1 \times 10^{13}$ photons $/ \mathrm{cm}^{2}$. Addition of a density $\mathrm{n}$ of absorbing gas reduces this value by $n^{*} L / 3$. Thus, density $n=3.53 \times$ $10^{16}$ atoms $/ \mathrm{cm}^{3} /$ Torr. Therefore, the photon flux is $\sim 5 \times 10^{12}$ photons $/ \mathrm{cm}^{2}$ at the cell, for gas pressures between $1 \times 10^{-8}$ and $1 \times 10^{-7}$ Torr. As illustrated in Figure 2, acetyl acetate at $5 \times 10^{-8}$ Torr produced the expected radical cations as $\mathrm{m} / \mathrm{z} 100$ as well as dimers at m/z 200; thus, there are collisional associations occurring in the gas phase between neutrals and ions. 


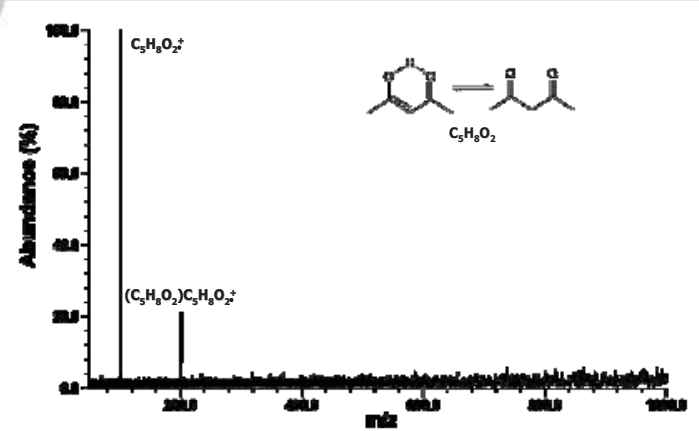

FIGURE 2. FTICR-MS spectrum of acetyl acetate ionized with $118 \mathrm{~nm}$ photons showing the expected radical cation at $\mathrm{m} / \mathrm{z} 100$ and the dimer radical cation at $\mathrm{m} / \mathrm{z} 200$ with no other species present, which means that the vacuum has few, if any, extraneous gas-phase absorbers present.

The second focus of this project has been on understanding LIBS as an emerging technology for nuclear forensics, which is still an immature isotope ratio method because of the high resolution that is required, which includes controlling the atmospheric conditions to reduce Doppler and Stark line broadening. LIBS isotope determination has been reported for ${ }^{238} \mathrm{U} /{ }^{235} \mathrm{U}$ isotopes that have a shift of $0.025 \mathrm{~nm}$ and was acquired at a reduced pressure of $3 \mathrm{~Pa}^{6}{ }^{6}$ For the ${ }^{239} \mathrm{Pu} /{ }^{240} \mathrm{Pu}$ isotopes, the shift is only $\sim 0.005 \mathrm{~nm}$ and could not be resolved under the same conditions as the uranium experiment. However, a team at Los Alamos National Laboratory was able to resolve these isotopes by using a carefully controlled helium bath gas at reduced pressure. ${ }^{7}$ Therefore, while LIBS is a promising technique for more portable nuclear forensics, research into appropriate conditions remains to be done. Toward this end, a separate vacuum chamber was fabricated for LIBS experiments. While our FTICR-MS vacuum housing can be used as a controllable reduced-pressure chamber that can also be backfilled with various collision gases (e.g., He, $\mathrm{N}_{2}$, Ar, etc.) and it is already equipped with high resolution optical detection capability, the separate vacuum chamber is necessary to independently evaluated effects of pressure on LIBS from magnetic field effects that have been shown to increase the $\mathrm{S} / \mathrm{N}$ of LIBS spectra. ${ }^{8}$

\section{Summary and Conclusions}

During the this first year of this project, a 118-nm SPI laser light source was integrated into our imaging instrument and tested to ensure that sufficient photon flux was produced. Initial tests were performed using the SPI source with our proprietary ViPP FTICR cell to determine compatibility issues. The key compatibility issue is the outgassing of electronic components of the current detection circuit, which will be addressed in the second year of the project. The apparatus (e.g., hardware, electronics, and software) and experiments for understanding LIBS-related isotope ratio analyses have also been initiated to allow the optimal conditions for LIBS analysis of nuclear forensic samples to be explored. The ultimate goal is to develop the capability at INL to analyze heterogeneous samples for the presence of nuclear-material-related isotopes with a method that requires no sample preparation to map the chemical signatures on the sample, provides high resolution $\left(>10^{5}\right)$ to avoid isobaric interferences, and simultaneously detects all isotopes of all elements with detection limits of $10^{3}$ atoms or less with an isotope accuracy of $<1 \%$ - and does all of this with automated data collection and interpretation. When the project is completed, LOCI will be a transformational and integrated "one-stop-shop" for characterizing nuclear material samples.

\section{References}

1. Dahl, D. A., J. R. Scott, and T. R. McJunkin, U.S. Patent Pending (11833079, 08/02/2007).

2. Trevor, J. L., K. R. Lykke, M. J. Pellin, and L. Hanley, 1998, "Two Laser Mass Spectrometry of Thiolate, Disulfide, and Sulfide Self-Assembled Monolayers," Langmuir, Vol. 14, p. 1664-1673.

3. Ayre, C. R., L. Moro, and C. H. Becker, 1994, "Effects of Desorption Method and Photoionizing Laser Characteristics on Molecular Fragmentation," Anal. Chem. Vol. 66, pp. 1610-1619.

4. Kornienko, O., E. T. Ada, J. Tinka, M. B. J. Wijesundara, and L. Hanley, 1998, “Organic surface analysis by two-laser ion trap mass spectrometry. 2. Improved desorption/photoionization configuration," Anal. Chem. Vol. 70, p. 1208.

5. Pallix, J. B., U. Schuhle, C. H. Becker and D. L. Huestis, 1989, “Advantages of Single- 
Photon Ionization over Multiphoton Ionization for Mass-Spectrometric SurfaceAnalysis of Bulk Organic Polymers," Anal. Chem. Vol. 61, p. 805.

6. Smith, C. A., 2000, The Actinide Research Quarterly, 4th quarter, 1.

7. Smith, C. A., M. A. Martinez, D. K. Veirs, and D. A. Cremers, 2002, "Pu-239/Pu-240 isotope ratios determined using high resolution emission spectroscopy in a laserinduced plasma," Spectrochim. Acta B, Vol. 57, p. 929.

8. Rai, V. N., H. Zhang, F. Y. Yueh, J. P. Singh, and A. Kumar, 2003, "Effect of steady magnetic-field on laser-induced breakdown spectroscopy," Appl. Optics, Vol. 42, p. 3662. 
Page for Appendices Divider

Appendices 
Appendix A -

Authors 


\section{Appendix A - Authors}

Project ID Principal Investigator Page \#

A

ST135 Anderson, M. O.

FF108 Apel, W. A.

GB103 Apel, W. A.

B

ST132

AE124

ST138

IC 108

C

CA104 Carroll, M. C. 259

IC107

NN113

$\mathrm{NN} 116$

NS163

AE119

AF 105

EI109

Bala, G. A.

274

Benson, M. T.

135

Berry, R. A.

Breckenridge, R. P.

Buttles, J. W.

C

Cherry, S. J.

308

Chichester, D. L.

85

243

235

159

330

216

D

PH101

Deaton, J. D.

96

NE156

Dinh, N. T.

F

IC102 Few, D.

297

G

EI102 Garcia, H. E. 198

TM108 Gaston, D. R. 286

NU100

AS107

Gertman, D. I.

53

229

NN110

Groenewold, G. S.

EI104

EI104
346

Groenewold, G. S.

Guillen, D. P.

14

206

\section{Project ID}

Principal Investigator

Page \#

$\mathrm{H}$

PH104 Harris, B. K. 62

NE157 Herrmann, S. D. 46

AF104 Hilton, B. A. 325

TM106 Huang, H. 154

ST136 Hurley, D. H. 12

ST136 Hurley, D. H. 145

K

PH102 Kelly, D. L. 97

EI105 Kenney, K. L. 209

NN117 Kinlaw, M. T. 245

ST131 Klaehn, J. R. 132

SH104 Knoll, D. 190

NE158 Knudson, D. L. 49

L

BS105 Lacey, J. A. 101

BS103 Lacy, J. M. 71

NS156 Lacy, J. M. 75

NS161 Landon, M. D. 7

NS161 Landon, M. D. 78

AE117 Law, J. D. 23

GB104 Lee, B. D. 117

GB105 Lee, B. D. 120

ST133 Lee, R. D. 137

NU101 Lessing, P. A. 57

NN118 Li, S. X. 249

M

AE118 Marsden, K. C. 26

PH103 Marshall, D. W. 60

AE121 Martineau, R. C. 162

IC105 McGillivary, K. E. 303

NN114 McGrath, C. 238

PH105 McJunkin, T. R. 125

CA105 McLing, T. L. 262

FF105 Meakin, P. 107 


\section{Appendix A - Authors}

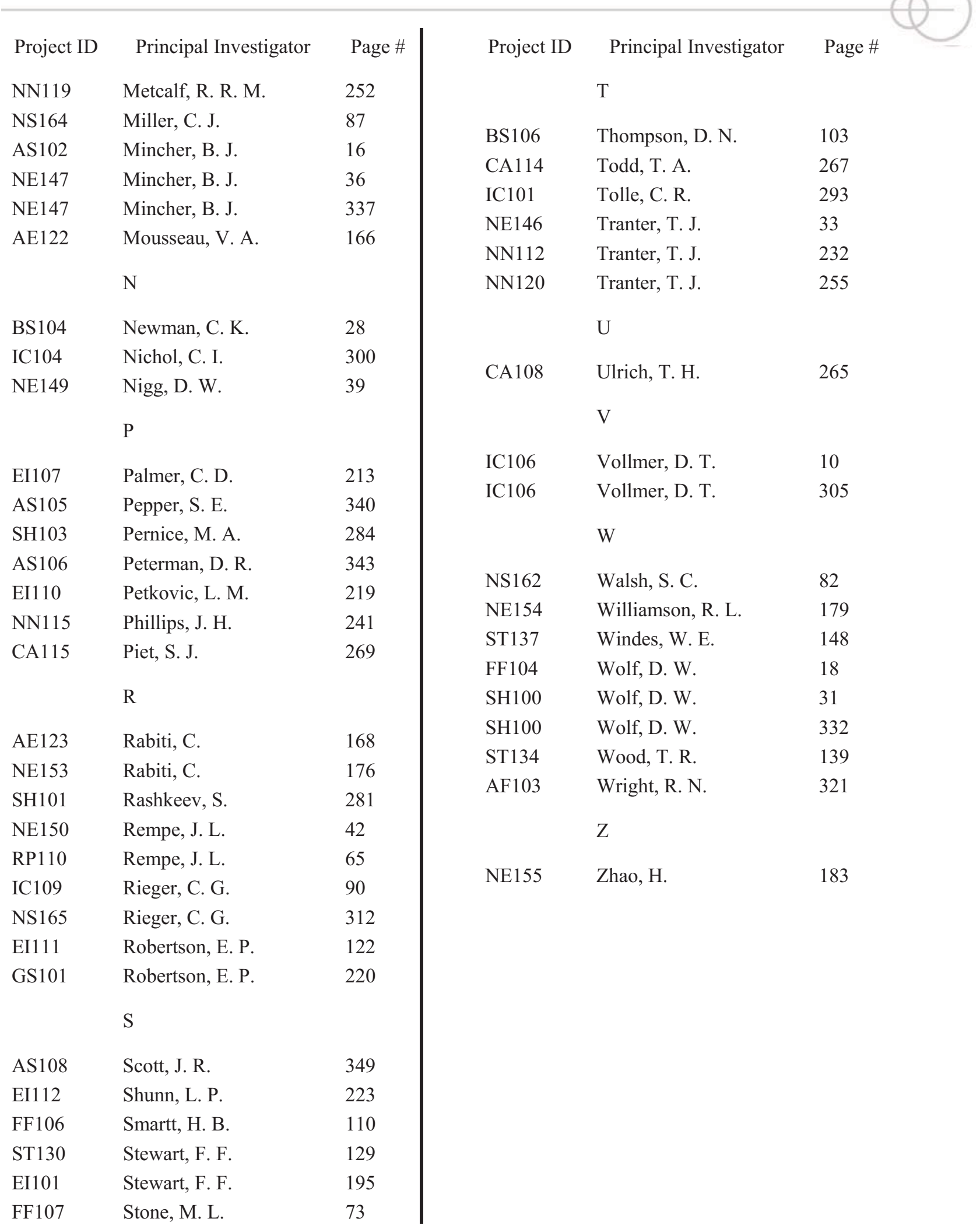


Appendix B -

Refereed Publications 


\section{Appendix B - Refereed Publications}

Berry, AE124, Unified Two-phase CFD Modeling of Boiling, Cavitation and Bubble Collapse. Richard Saurel, Fabien Petitpas, Ray A. Berry, "Simple and efficient relaxation methods for interfaces separating compressible fluids, cavitating flows and shocks in multiphase mixtures," Journal of Computational Physics 228:5, 1678-1712, March, 2009.

Berry, AE124, Unified Two-phase CFD Modeling of Boiling, Cavitation and Bubble Collapse. Kurt D. Hamman and Ray A. Berry, "A CFD simulation process for fast reactor fuel assemblies," Nuclear Engineering and Design, Accepted, August, 2009.

Berry, AE124, Unified Two-phase CFD Modeling of Boiling, Cavitation and Bubble Collapse. Richard C. Martineau, Ray A. Berry, Aurelia Esteve, Kurt D. Hamman, Dana A. Knoll, Ryosuke Park and William Taitano, "Comparative analysis of natural convection flows simulated by both the conservation and incompressible forms of the Navier-Stokes equations in a differentially-heated cavity, Nuclear Engineering and Design, Accepted, September, 2009.

Chichester, NN113, Active Interrogation DieAway Assay Development Program. D. L. Chichester and E. H. Seabury, "Using Electronic Neutron Generators in Active...," IEEE Transactions on Nuclear Science 52:2 pp. 441447, April, 2009.

Cogliati, AE119, Multi-reactor design and analysis platform. Samuel E. Bays, Hongbin Zhang and Haihua Zhao, "The Industrial Sodium Cooled Fast Reactor," Advances in Nuclear Fuel Management IV (ANFM 2009), April, 2009

Deaton, PH101, Cognitive Network Engine and Simulation Framework. S. Amaar Ahmad, Juan Deaton, Umesh Shukla, Ryan Irwin, Luiz A.

DaSilva and Allen B. MacKenzie, " A Comparison of Channel Assignment Techniques with Power Control in Ad Hoc Networks," IC3N Cognets Conference, June, 2009.

Dinh, NE156, D. R. Nourgaliev, H. Park and V. Mousseau, "Recovery Discontinuous Galerkin Jacobian-free Newton Krylov Method for Multiphysics Problems," CFD Review 2008 (Chapter in book), In Press.
Dinh, NE156, Next-Generation Code for Nuclear Systems. R. Nourgaliev, S. Kadioglu and V. Mousseau, "Marker Re-distancing Level Set Method for High-Fidelity Implicit Interface Tracking," SIAM Journal of Scientific Computing, In Press.

Dinh, NE156, Next-Generation Code for Nuclear Systems. H. Park, R. Nourgaliev, R. Martineau and D. Knoll, "On Physics-based Preconditioning of the Navier-Stokes Equations," Journal of Computational Physics, In Press.

Dinh, NE156, Next-Generation Code for Nuclear Systems. H. Zhao and P. F. Peterson, "OneDimensional Analysis of Thermal Stratification in the AHR Coolant Pool," Nuclear Engineering and Technology 41:7, pp. 953-968, September, 2009.

Dinh, NE156, Next-Generation Code for Nuclear Systems. H. Zhao, H. Zhang, V. A. Mousseau and P. F. Peterson, "Improving SFR Economics through Innovations from Thermal Design and Analysis Aspects," Nuclear Engineering and Design Volume 239, pp. 1042-1055, June, 2009.

Garcia, EI102, Advanced Predictive Condition Monitoring and Control for Modern Energy Systems: Gasification-based Processes. B. Liu, H. E. Garcia, L. L. Baxter, "A Simplified Phase Equilibrium Algorithm Used to Predict Ash/Slag Behaviors in Slagging Gasifiers/Combustors," 25th Annual International Pittsburgh Coal Conference, October, 2008.

Guillen, EI104, CFD-Based Simulation Capability of Fischer-Tropsch Reactors and Process Equipment. Donna Guillen, "Development And Validation of a Multifield Model of Churn-Turbulent Gas/Liquid Flows, Proceedings of ICONE17, Volume 75210, September, 2009.

Guillen, EI104, CFD-Based Simulation Capability of Fischer-Tropsch Reactors and Process Equipment. Guillen, D. P. et al., "Optimization of a Two-Fluid Hydrodynamic Model of a Churn-Turbulent Flow," Proceedings of ICONE17, 17th International Conference on Nuclear Engineering, September, 2009. 


\section{Appendix B - Refereed Publications}

Hilton, AF104, "Development of Advanced Burnup Measurement and Nuclear Forensics using Inductively Coupled Mass Spectroscopy Isotopics Analysis Techniques. I. Glagolenko, B.Hilton, J.Giglio, and D.Cummings, "Fission Yield Measurements by Inductively Coupled Plasma Mass-Spectrometry" Journal of Radioanalytical and Nuclear Chemistry, July, 2009.

Hilton, AF104, "Development of Advanced Burnup Measurement and Nuclear Forensics using Inductively Coupled Mass Spectroscopy Isotopics Analysis Techniques. B.A. Hilton, I.Y. Glagolenko, J.J. Giglio and D.G. Cummings, "Advanced Burnup Determination Method using Inductively Coupled Plasma Mass

Spectrometry," Global 2009, p. 9354, September, 2009.

Knoll, SH104, Multi-Physics Simulation Methods for Advanced Reactor Analysis. Kadioglu, Knoll and deOliveria, "Multiphysics Analysis of Spherical Fast Burst Reactors," Nuclear Science and Engineering 163, pp. 132-143, 2009.

Knoll, SH104, Multi-Physics Simulation Methods for Advanced Reactor Analysis. Newman, Hansen and Gaston, "Three dimensional coupled simulation of thermomechanics, heat, and oxygen diffusion in UO2 nuclear fuel rods, Journal of Nuclear Materials, Volume 392, January, 2009.

Knudson, NE158. Instrumentation for in-pile detection of thermal conductivity. J. Rempe and M. Meyer, "ATR NSUF Instrumentation Enhancement Efforts," NPIC HMIT 2009

Topical Meeting on Nuclear Plant Instrumentation, 2009.

Knudson, NE158. Instrumentation for in-pile detection of thermal conductivity. Joy L. Rempe, Darrell L. Knudson, Keith G. Condie, Joshua E. Daw, Heng Ban, Brandon Fox, and Gordon E. Kohse, "New Sensors for the Advanced Test Reactor National Scientific User Facility," Controls, June, 2009.
Knudson, NE158. Instrumentation for in-pile detection of thermal conductivity. JB. Fox, H. Ban, J. Rempe, J. Daw, K. Condie, and D. Knudson, "In-Pile Thermal Conductivity Measurement Method for Nuclear Fuels," 30th International Thermal Conductivity Conference and 18th International Thermal Expansion Symposium, September, 2009.

Law, AE117, Group Actinide Separation from Spent Nuclear Fuel Using a Modified Universal Solvent Extraction Process. A. Paulenova, M.Yu. Alyapyshev, V.A. Babain, R.S. Herbst, J.D. Law, "Extraction of Lanthanides with Diamides of Dipicolinic Acid from Nitric Acid Solutions," Separation Science and Technology Volume 3, pp. 206-2618, July, 2008.

Law, AE117, Group Actinide Separation from Spent Nuclear Fuel Using a Modified Universal Solvent Extraction Process. J.L. Lapka, A. Paulenova1, M.Yu. Alyapyshev, V.A. Babain, R.S. Herbst and J.D. Law, "Extraction of Uranium(VI) with Diamides of Dipicolinic Acid from Nitric Acid Solutions," Radiochimica acta 97:6, pp. 291-296, June, 2009.

Law, AE117, Group Actinide Separation from Spent Nuclear Fuel Using a Modified Universal Solvent Extraction Process. J.L. Lapka, A. Paulenova, M.Yu. Alyapyshev, V.A. Babain, R.S. Herbst and J.D. Law, "Extraction of Mo and Tc with Diamides of Dipicolinic Acid from Nitric Acid Solutions, J. Radioanal. Nucl. Chem 280:2, 307-313, February, 2009.

Martineau, AE121, A General Framework for Simulating Fully-Coupled Mass and Energy Transport Phenomena in Nuclear Energy Systems. HyeongKae Park, Robert Nourgaliev, Richard Martineau and Dana Knoll, "On Physics-Based Preconditioning for the NavierStokes Equations," Journal of Computational Physics, Accepted, September, 2009.

Martineau, AE121, A General Framework for Simulating Fully-Coupled Mass and Energy Transport Phenomena in Nuclear Energy Systems. Derek Gaston, Chris Newman, Glen Hansen and Damien Lebrun-Grand, "A Parallel Computational Framework for Coupled Systems of Nonlinear Equations," Nuclear Engineering and Design 239:1768, September, 2009. 


\section{Appendix B - Refereed Publications}

Martineau, AE121, A General Framework for Simulating Fully-Coupled Mass and Energy Transport Phenomena in Nuclear Energy Systems. R. Martineau, R. Berry, A. Esteve, K. Hammon, D. Knoll, H. Park, and W. Taitano, "Comparative Analysis of Natural Convection Flows Simulated by both the Conservation and Incompressible Forms of the Navier-Stokes Equations in a Differentially-Heated Square Cavity," Nuclear Engineering and Design, Accepted, September, 2009.

Mincher, NE147, Reactivity of radiolytically produced nitrogen oxide radicals toward aromatic compounds. B. J. Micher, G. Elias, L. R. Martin and S, P. Mezyk, "Radiation chemistry and the nuclear fuel cycle," J. Radioanal. Nucl. Chem. DOI 10.1007/s 10967-009-0156-x, August, 2009.

Mincher, AS102, Utility of unsusal oxidation states of americium for separations. L. R. Martin, B. J. Mincher and N. C. Schmitt, "Extraction of Am(VI) with a neutral organophosphorous ligand," J. Radioanal. Nucl. Chem., DOI 10.1007/s 10967-009-0153, August, 2009.

Mincher, AS102, Utility of unsusal oxidation states of americium for separations. B. Mincher, L. R. Martin and N. C. Schmitt,

"Tributylphosphate extraction behavior of bismuthate-oxidized americium," Inorg. Chem. 47:6984-6989, June, 2008.

Mousseau, AE122, System Analysis for Reactor Applications with High Fidelity. Michael A. Pope and Vincent A. Mousseau, "Accuracy and Efficiency of a Coupled Neutronics and Thermal Hydraulics Model," Nuclear Engineering and Technology 41:7, September, 2009

Mousseau, AE122, System Analysis for Reactor Applications with High Fidelity. Jean C. Ragusa and Vijay S. Mahadevan, "Consistent and accurate schemes for coupled neutronics thermal hydraulics reactor analysis," Nuclear Engineering and Design 239:3, pp. 566-579, March, 2009.
Pepper, AS105, Rapid detection of plutonium, neptunium and technetium in water samples. S. E. Pepper, D. R. Peterman, T. J. Tranter and B. M. White, "Detection of radionuclides in aqueous samples using cloud point extraction," Journal of Radioanalytical and Nuclear Chemistry, 7, 10967-009-0303-4, July 2009.

Pernice, SH103, Adaptive Modeling of Geometrically Complex Fuel Rods with a posterior Error Control. D. Estep, M. Pernice, D. Pham, S. Taverner and H. Wang, "Error analysis of a cell-centered finite volume method for semilinear elliptic problems," Journal of Computational and Applied Mathematics 233:2, 459-472, November, 2009.

Peterman, AS106, Evaluation of covalent interactions in actinide coordination compounds. Dean Peterman, Leigh Martin, John Klaehn, Mason Harrup, Mitchell Greenhalgh and Thomas Luther, "Selective separation of minor actinides and lanthanides using aromatic dithiophosphinic and phosphinic acid derivatives", Journal of Radioanalytical and Nuclear Chemistry 282(2): 527-531.

Rashkeev, SH101, Structural and electronic properties of surfaces and adsorbed metal particles: application to catalysis, corrosion and radiation effects. Michael V. Glazoff, Sergey N. Rashkeev, Yuri P. Pytey, Jeong-Whan Yoon and Simon Sheu, "Interplay between plastic deformations and optical properties of surfaces," Appl. Phys. Lett. 95:8:8146, October, 2009.

Rashkeev, SH101, Structural and electronic properties of surfaces and adsorbed metal particles: application to catalysis, corrosion and radiation effects. S. J. Pennycook et al., "Aberration-corrected scanning transmission electron microscopy: from atomic imaging and analysis to solving energy problems," Philosophical Transactions of the Royal Society A:3673709-3733, January, 2009. 


\section{Appendix B - Refereed Publications}

Rashkeev, SH101, Structural and electronic properties of surfaces and adsorbed metal particles: application to catalysis, corrosion and radiation effects. E. A. Kotomin, Yu. A. Mastrikov, S. N. Rashkeev and P. Van Uffelen, "Implementing first principles calculations of defect migration in a fuel performance code for UN simulations," Journal of Nuclear Materials 393:2, 292-299, June, 2009.

Rashkeev, SH101, Structural and electronic properties of surfaces and adsorbed metal particles: application to catalysis, corrosion and radiation effects. Yanting Wang and Sergey N. Rashkeev, "Melting phase transitions and catalytic activity of bilayer gold nanoclusters," Journal of Physical Chemistry C113:24, 1051710520, September, 2009.

Rashkeev, SH101, Structural and electronic properties of surfaces and adsorbed metal particles: application to catalysis, corrosion and radiation effects. L. M. Petkovic, S. N. Rashkeev and D. M. Ginosar, "Ethanol oxidation on metal oxide supported platinum catalysts," Catalysis Today 147:2, 107-114, September, 2009.

Rashkeev, SH101, Structural and electronic properties of surfaces and adsorbed metal particles: application to catalysis, corrosion and radiation effects. G. M. Veith, A. R. Lupini, S. Rashkeev, S. Pennycook, D. R. Mullins, V. Schwartz, C. A. Bridges and N. J. Dudney, "Thermal stability and catalytic activity of gold nanoparticles supported on silica," Journal of Catalysis 262:1, 92-101, September, 2009

Rashkeev, SH101, Structural and electronic properties of surfaces and adsorbed metal particles: application to catalysis, corrosion and radiation effects. M. M. Kuklja and S. N. Rashkeev, "Interplay of decomposition mechanisms at shear-strain interface," The Journal of Physical Chemistry C113:1, 17-20, September, 2009

Rashkeev, SH101, Structural and electronic properties of surfaces and adsorbed metal particles: application to catalysis, corrosion and radiation effects. S. N. Rashkeev, D. M. Ginosar, L. M. Petkovic and H. H. Farrell, "Catalytic activity of supported metal particles for sulfuric acid decomposition reaction," Catalysis Today 139:4, 291-298, September, 2009.
Rashkeev, SH101, Structural and electronic properties of surfaces and adsorbed metal particles: application to catalysis, corrosion and radiation effects. I.G. Batyrev, D. Hughart, R. Durand, M. Bounaser, B. R. Tuttle, D. M. Fleetwood, R. D. Schrimpf, S. N. Rashkeev, G. W. Dunham, M. Law and S. T. Pantelides, "Effects of hydrogen on the radiation response of bipolar transistors: experiment and modeling," IEEE Trans. Nucl. Sci 55:6, 309-3045, Oct, 2008.

Rashkeev, SH101, Structural and electronic properties of surfaces and adsorbed metal particles: application to catalysis, corrosion and radiation effects. R. D. Shrimpf, K. M. Warren, D. R. Ball, R. A. Weller, R. A. Reed, D. M. Fleetwood, L. W. Massengill, M. H. Mendenhall, S. N. Rashkeev, S. T. Pantelides and M. A. Alles, "Multi-scale simulation of radiation effects in electronic devices," IEEE Trans Nucl. Sci. 55:4, 1891-1902, October, 2008.

Rashkeev, SH101, Structural and electronic properties of surfaces and adsorbed metal particles: application to catalysis, corrosion and radiation effects. K. Griffin Roberts, M. Varela, S. Rashkeev, S. T. Pantelides, S. J Pennycook and Kannan M. Krishnan, "Defect-mediated ferromagnetism in insulating co-doped anatase TiO2 thin films," Phys. Rev. B78, pp. 14409, October, 2008.

Rempe, NE150, Viability evaluations of linear variable differential transformers (LVDTs) and capacitive micro-machined ultrasonic transducers (CMUTs) for in-pile instrumentation. J. Rempe and M. Meyer, "ATR NSUF Instrumentation Enhancement Efforts," NPIC HMIT 2009 Topical Meeting on Nuclear Plant Instrumentation Special Edition, August, 2009.

Rempe, NE150, Viability evaluations of linear variable differential transformers (LVDTs) and capacitive micro-machined ultrasonic transducers (CMUTs) for in-pile instrumentation. J. Rempe, D. Knudson and K. Condie, "New Sensors for In-Pile Testing at the ATR NSUF," Proceedings of the First International Conference on Advancements in Nuclear Instrumentation, June, 2009. 


\section{Appendix B - Refereed Publications}

Rempe, RP110, High Temperature In-Pile Instrumentation Enhancements. J. Daw, J. Crepeau, J. L. Rempe, S. C. Wilkins, D. Knudson and K. G. Condie, "Initial Results from Investigations to Enhance the Performance of High Temperature Irradiation-Resistant Thermocouples," Journal of the Japanese Society of Mechanical Engineers (JSME) 2:2, pp. 854863, 2008.

Rempe, RP110, High Temperature In-Pile Instrumentation Enhancements J. L. Rempe, D. L. Knudson and J. E. Daw, "Type C Thermocouple Performance at $1500{ }^{\circ} \mathrm{C}$," Measurement Science and Technology 119:115201, September, 2008.

Rempe, RP110, High Temperature In-Pile Instrumentation Enhancements. J. L. Rempe, D. L. Knudson and K. G. Condie, "Options Extending the Applicability of High Temperature Irradiation Resistant Thermocouples," NURETH12 Special Edition 167:169-177, July, 2009.

Rempe, RP110, High Temperature In-Pile Instrumentation Enhancements J. E. Daw, J. L. Rempe, D. L. Knudson, S. C. Wilkins, and J. C. Crepeau, "High Temperature IrradiationResistant Thermocouples Improvements" NPIC HMIT 2009 Topical Meeting on Nuclear Plant Instrumentation, April, 2009.

Rempe, RP110, High Temperature In-Pile Instrumentation Enhancements J. Rempe, D. Knudson, K. Condie, J. Daw,and S. C. Wilkins, "New Sensors for In-Pile Temperature Detection at the ATR NSUF," Proceedings of the 13th International Topical Meeting on Nuclear Reactor Thermal-Hydraulics (NURETH13), October, 2009.
Tolle, IC101, Exploration and Development of Automated Differential Equation-Based System Identification. Charles R. Tolle, and Mark Pengitore, "Phase-Space Reconstruction: a Path Towards the Next Generation of Nonlinear Differential Equation Based Models and Its Implications Towards Non-Uniform Sampling Theory Phase-Space Reconstruction: a Path Towards the Next Generation of Nonlinear Differential Equation Based Models and Its Implications Towards Non-Uniform Sampling Theory," ISRCS 2009 2nd International Symposium on Resilience Control Systems , August 2009.

Tolle, IC101, Exploration and Development of Automated Differential Equation-Based System Identification. Charles R. Tolle, and John W. James, "A Method for Finding Unknown Signals Using Reinforcement FFT Differencing," Journal of the Idaho Academy of Science, Accepted, September, 2009.

Vollmer, IC106. Resilient Control System Network Agents. O. Linda, T. Vollmer, M. Manic, "Neural Network Based Intrusion Detection System for Critical Infrastructures" Accepted, February, 2009.

Vollmer, IC106. Resilient Control System Network Agents. T. Vollmer, and M. Manic, "Human Interface for Cyber Security Intrusion Detection Systems," HSI2009, March, 2009.

Vollmer, IC106. Resilient Control System Network Agents. T. Vollmer and M. Manic, "Computationally Efficient Neural Network Intrusion Security Awareness," ISRCS2009, July, 2009.

Wolf, SH100, Microstructure and Deformation Physics of Fission-Reactor Model Materials by Atomistically Informed Mesoscale Simulation. Dilpuneet S. Aidhy (UFL), Paul C. Millett, Dieter Wolf, Simon R. Phillpot (UFL) and Hanchen Huang (RPI), "Kinetically driven pointdefect clustering in irradiated $\mathrm{MgO}$ by molecular-dynamics simulation," Scripta Materialia 60(2009): 691-694, January, 2009. 


\section{Appendix B - Refereed Publications}

Wolf, SH100, Microstructure and Deformation Physics of Fission-Reactor Model Materials by Atomistically Informed Mesoscale Simulation. Millett, Srujan Rokkam (FSU), Anter El-Azab (FSU), Michael Tonks and Dieter Wolf, "Void Nucleation and Growth in Irradiated Polycrystalline Metals: A Phase-Field Model," Modelling and Simulation in Materials Science and Eng. 17(2009): 64003, August, 2009.

Wolf, SH100, Microstructure and Deformation Physics of Fission-Reactor Model Materials by Atomistically Informed Mesoscale Simulation. Tapan G. Desai, Paul Millett, Michael Tonks and Dieter Wolf, "Atomistic Simulations of Void Migration Under a Thermal Gradient in UO2," ACTA MATERIALIA, December, 2009.
Wolf, SH100, Microstructure and Deformation Physics of Fission-Reactor Model Materials by Atomistically Informed Mesoscale Simulation. Tapan G Desai, Pankaj Nerikar (LANL) and Blas P Uberuaga (LANL), "The role of grain boundary structure in stress-induced phase transformation in UO2," Modelling and Simulation in Materials Science and Engineering, November, 2009.

Zhao, NE155, Use of Ice Thermal Storage Systems to Improve LWR Plant Efficiency. H. Zhao, H. Zhang, R. Szilard and W. Yan, "Use of Ice Thermal Storage Systems to Address LWR Cooling Issues," Transactions of the American Nuclear Society 199:103-104, June, 2009. 
Appendix C -

Key Terms 
2-D lattice physics code, 168

3-tier data fusion architecture system, 308

A Strategy to Tightly Couple Neutronics and Thermal Hydraulics Models, 28

a transient hot-wire thermal method (THWM), 49

adaptive mesh refinement (AMR), 286

adaptive traits to minimize consequences of detrimental control-system network-based incidents, 305

adjoint-based simulation, 288

advanced armor concepts, 77

advanced material research and

development, 72

advanced modeling of nuclear fuel, 286

Advanced morphological analysis for

surfaces with arbitrary texture and

roughness, 283

advanced nuclear reactors, 162

advanced simulation techniques, 288

Advanced Test Reactor (ATR), 39

algorithms for thermal hydraulics, 186

Am oxidation at high nitric acid

concentrations, 337

Amberlyst-15, 197

AmVI solvent extraction process, 338

an adjoint solution method, 286

analytical methods for metabolome profiling

of bacteria, 119

anomaly-based intrusion detection systems, 306

apomixis, 265

armor development, 71

ATTILA 3D transport code, 39

attribution determinations in nuclear

forensics, 337

bacterial enzyme stability, 113

basic infrastructure and a framework

demonstrating data fusion concepts, 308

Bayesian Fault-Tolerant Control, 315

Bi-manual haptic system, 301

bi-manual remote operations, 300

biofuel production in Idaho, 275

bioinformatic and data-analysis tools, 120

biomass conversion, 274 bioprocess for the conversion of

lignocellulosic biomass to value-added

chemicals, 117

Biorefinery Systems, 195

blast-resistant technologies, 75

carbohydrate-induced differential gene

expression patterns, 120

Carbon sequestration in mafic (basalt) lava

flows, 262

catalysis, corrosion, and radiation effects, 281

catalytically active membranes, 195

channel-assignment algorithms, 96

chaotic system dynamics, 293

characterizing nuclear material samples, 351

Chemical Signatures of Nuclear

Proliferation, 229

chemistry of the higher oxidation states of americium, 339

Cloud point extraction (CPE), 340

coalbed methane (CBM) production operations, 122

cognitive networking research, 96

Complex Resilient Networks Testbeds, 91

consolidated bioprocessing (CBP)

microorganism, 117

control room design for nuclear facilities, 53

Controlling and monitoring groundwater

quality during oil shale development, 139

controlling projectile speed for precise material evaluation studies, 72

coupled neutronics and thermal hydraulics, 167

creation of differential-equation-based models via attractor space reconstruction techniques, 293

critical infrastructure control and security systems, 308

Critical Infrastructure Protection, 96

crop genetic engineering and seed

production systems, 265

decision support tools, 272

decision-analysis tool, 151

desorption electrospray ionization, 346

detecting organic reprocessing signatures, 231 


\section{Appendix C - Key Terms}

detection method for $\mathrm{Pu}, \mathrm{Np}$, and $\mathrm{Tc}$ in aqueous samples, 340

detection of high-energy prompt fission neutrons, 245

diffuse interface methods, 173

Digital Control Systems and Interfaces, 53

discrete element method (DEM), 214

discrete equations method (DEM), 173

distribution model of basalt hydraulic properties, 263

Dynamic Bayesian Networks, 97

efficient extraction processes, 213

electrochemical processing, 46

electrospray ionization mass spectrometry, 346

Electrospray ionization mass spectrometry

(ESI-MS), 346

energy development in the western U.S., 141

engineering analysis of the coupled neutronic and thermal-hydraulic behavior of the ATR core, 39

enhance the efficiency of ion production and simplify sample preparation, 73

entrained-flow slagging gasifiers, 198 environmental and socioeconomic impacts of a hybrid energy system, 151

environmental protection of water resources, 139

ERANOS code system, 177

explosive fingerprint characteristics, 87

explosives detection, 89

facilitate code connectivity, 161

field-scale computer model of a full-scale

CBM reservoir, 124

fine spatial-resolution hyperspectral imagery from small, Class-II UAVs, 144

flexible, fully coupled, accurate code for transient reactor multiphysics analysis, 29

fluid dynamic interface problems, 172

fluid flow through CBM fields, 124

flux of a gram-positive thermoacidophile, 117

focus groups, 271

Fourier transform ion cyclotron resonance mass spectrometer (FTICR-MS), 349 framework to model and analyze complex resilient distributed control networks, 90

fuel reprocessing for the purpose of recovering fissionable radionuclides, 229

fuel-depletion capability, 41

functionalized PMOs that have a high capacity for binding copper ions, 106 gas injection system, 71

Gas interaction with polymers used for separation membranes, 282

gene expression in sorghum, 265

genomic, transcriptomic, proteomic, and metabolomic techniques, 115

GeoFracFH Model, 140

geologic carbon sequestration, 262

geologic sequestration of $\mathrm{CO}_{2}, 262$

geomechanics model, 215

gram-positive thermoacidophile, 121

haptic bounce (contact instability) cancellation routine, 301

Haptic Interface for Remote Arc Welding, 300

haptic telepresence system for the conduct of remote operations, 302

HELIOS code suite, 168

HELIOS transport codes, 39

High Temperature In-Pile Instrumentation, 65

High Temperature Irradiation Resistant ThermoCouple (HTIR-TC), 65

high-energy impact research, 71

high-frequency, small-length scale eddycurrent system, 145

High-performance (HP) polymers, 132

high-sensitivity surface analysis, 229

high-temperature HP-membrane transport properties, 133

High-Temperature Irradiation-Resistant ThermoCouples (HTIR-TCs), 50

high-utility interaction methods and tools, 297

HP polymeric-membrane systems for gasseparation applications, 132

human performance, 53

human system simulation (HSS), 53

human-in-the-loop performance testing, 53 
human-machine interaction, 302

human-reliability analysis (HRA), 97

hybrid nondestructive examination (NDE)

approach, 145

hydrolysis of lignocellulosic feedstocks, 115

hydrolysis of lignocellulosic substrates for

bio-fuels production, 277

Ice Thermal Storage Systems, 183

immersive visualization environment capability, 56

Impacts of In-Situ Oil-Shale Development on Water Quality, 139

improved ion production efficiencies in praseodymium metal ion samples, 74

increased membrane system performance, 129

inductively coupled plasma mass

spectrometry (ICP-MS), 325

innovative alternatives for isotope ratio mass

spectrometry, 349

in-pile real-time sensors, 42

integrated gasifier model, 198

intelligent detection schemes and response capability, 305

intelligent, resilient control systems, 312

interconnections between membrane structure, polymer glass transition temperature ( $\mathrm{Tg})$, and system operating temperature, 129

Intrusion Detection Systems, 305

Jacobian-free Newton-Krylov (JFNK) method, 162

Kapton membranes, 133

large-scale computational evaluation of carbon sequestration capacity in plainsstyle volcanic terrains, 263

laser-based optical and chemical imager (LOCI), 349

laser-induced breakdown spectroscopy

(LIBS), 349

life cycle assessment tools, 275

light water reactors (LWRs), 162

lignocellulose-degrading enzymes, 101

Lignocellulose-derived Carbon Metabolism, 115

lignocellulosic residues, 103 lignocellulosic-carbon sources, 115

linear burnup equations for solving the fission fraction, 325

Linear Variable Differential Transformers, 42

Low-Temperature Performance in Membrane Materials, 129

LWR Plant Efficiency, 183

mass spectrometric analyses of metal speciation from solutions and solids that are at high I and or extreme $\mathrm{pH}, 348$

material strength and failure characteristics for hard materials, 76

mechanism of nitration of aromatic groups in irradiated, aqueous nitric acid, 36

metabolic-engineering tools, 117

metal speciation, 346

methods for identifying and quantifying metal speciation, 346

microstructural evolution of the powders following varying sintering cycles, 260

mining simulator data in support of HRA, 98

mobile channel assignment, 96

model sensitivity analysis for single-phase

flow, 166

model-based hybrid framework for anomaly detection, diagnosis and resilient control in complex process systems, 312

modeling and simulation, 176

Modeling and simulation, 284

modeling and simulation tools to evaluate impacts on groundwater, 139

Modeling the synergies of hybrid energy systems, 151

Modern Lattice Physics Capability, 168

Monte Carlo N-Particle Transport Code (MCNP), 325

multi-modal sensory feedback, 300

multiphysics HPC framework, 162

Multiphysics Object Oriented Simulation

Environment (MOOSE), 162

Multiphysics Object Oriented Simulation Environment (MOOSE) computational science framework, 286

Multi-Reactor Design and Analysis Platform, 159 


\section{Appendix C - Key Terms}

Navier-Stokes equation, 162

$\mathrm{N}$-centered radicals in irradiated, aqueous nitric acid, 36

network interaction model, 120

Neural Network Based Intrusion Detection

System for Critical Infrastructures, 306

neutronics calculations, 325

new a posteriori error estimates for finite volume discretizations of semi-linear elliptic problems on regular Cartesian grids, 284

new architecture for reactor multiphysics codes, 29

New duality-based adjoint error estimators, 285

next generation of adaptive control systems, 296

next-generation, high-fidelity simulation tools, 162

nitrated products in irradiated solution, 38

nitric acid radiolysis, 36

nondestructive examination, 128

non-proliferation, 231

notional physical security system and scenario-generation tool, 309

novel methodology of nuclear fuel burnup determination, 325

nuclear energy production, 231

Nuclear fuels modeling, 281

oil-shale development, 139

particle-based discrete-element model (DEM), 141

Perona's reconstruction techniques, 293

Phased-array ultrasonic examination, 125

Phosphazenes, 131

PIE isotopic and burnup analyses, 328

pilot-in-the-loop UAV sensor platforms, 142

plant risk-informed safety decision making, 186

plug-in-based code to link together multiple nuclear codes, 161

polyoxometalate (POM) catalysts, 195

Polyoxometalate catalysts, 197

posttranslational modifications, 113

potential for geologic sequestration of $\mathrm{CO}_{2}$

for a given mafic rock site, 262 predictive condition monitoring and control, 198

predictive fuel performance simulation, 179

Principal Component Analysis (PCA), 316

prismatic VHTR, 168

process to remove enzymatic poisons from

biomass hydrolysate, 195

process toxins, 195

production of value-added chemicals, 117

Purdue networks testbed prototype,

PurNETS ver 1.0, 93

pyrolysis of oil shale, 213

quadrupole dynamic reaction cell ICP-MS, 328

Qualitative Trend Analysis (QTA), 316

quantitative production of both $\mathrm{Am}(\mathrm{V})$ and

Am(VI), 339

quantum-mechanical and atomic-scale

computer simulation techniques, 281

quick and easy mobile-arm control, 299

radiolytically enhanced nitrous acid-

catalyzed nitration, 38

reactor physics design, 176

Reactor Physics Sensitivity Analysis, Uncertainty Quantification, and Data

Assimilation Capability, 176

reactor-system safety-analysis tools, 186

reduction of haptic bounce, 300

Remote sensing technologies, 142

renewable energy, 274

reusable, oligosaccharide-templated

materials, 103

selective metal binding, 106

Sensitivity analysis and uncertainty evaluation, 176

shale-oil resources, 213

Signed Digraphs (SDG), 316

simplified flow regime topological maps, 171

simulate the coupling between fluid generation, geomechanics, and fluid flow, 107

simulation-based predictions of fuel

behavior, 286 
single remote manipulator for conducting haptically augmented remote robotic operations, 300

sintering materials at microbar vacuum levels, increasing the potential for compaction of ceramic or composite materials that simply cannot be produced elsewhere, 260

smeared-cracking models, 179

software tools for sensitivity analysis, uncertainty quantification, and data assimilation, 177

Spark plasma sintering (SPS), 259

state-of-the-art capabilities to model nuclear fuel performance, 179

state-of-the-art fracture-mechanics computational methods, 179

state-of-the-art industrial robotics, 300

steady-state 3-D neutron-transport discreteordinates code, 39

streamlining data transfer between codes, 159

subsurface energy and environmental applications, 107

sustainable closed nuclear-fuel cycle, 46

SysID techniques, 293

System Analysis for Reactor Applications with High Fidelity (SARAH), 166

systematic methodology to estimate the potential for geologic sequestration of greenhouse gasses, 262

Takens-based dynamics reconstruction, 293

Taylor cylinder apparatus, 75

The Multi-Reactor Design and Analysis Platform (MRDAP), 159

Thermal and Acid Activated In-plant Lignocellulose-degrading System, 101 thermal ionization mass spectrometry isotope dilution (TIMS ID), 326

Thermus thermophilus, 114

tightly couple several physical phenomena in nuclear reactors, 28

time-based clustering (TBC) methods, 293 trace explosive signature, 87

trace-explosive fingerprint morphology, 87

two bubble-dynamic phenomena, 171

two-step oxide reduction and electrorefining process, 46

two-thermocouple steady-state, 49

UAV potential for environmental and scientific applications, 142

UAV-based hyperspectral remote sensing system, 142

ultrahigh-frequency laser ultrasonic system, 145

uncertainty quantification and reduction, 167

uncertainty quantification and reduction methods, 166

unconventional fuels, 213

unconventional strategic fuels, 141

unified physical/mathematical and numerical model development of compressible, all-speed multiphase flows, 171

Unmanned Aerial Vehicles, 142

unmanned aerial vehicles (UAVs), 142

unmediated human-robot interaction

methods and tools, 297

value-added chemicals, 115

verification and validation (V\&V), 178

very high temperature reactor (VHTR), 168

VHTR graphite-specific HELIOS library, 168

visualization and modeling methodologies, 55

WAG injection scheme in coalbed methane production operations, 122

water-alternating-gas (WAG) injection scheme, 122

Wireless sensor network (WSN) technologies, 311

with plastic scintillator-based and liquid scintillator-based acquisition systems, 247

WSN technologies, 311 


\section{Appendix C - Key Terms}


Appendix D -
Project Performance 


\section{Appendix D - Project Performance}

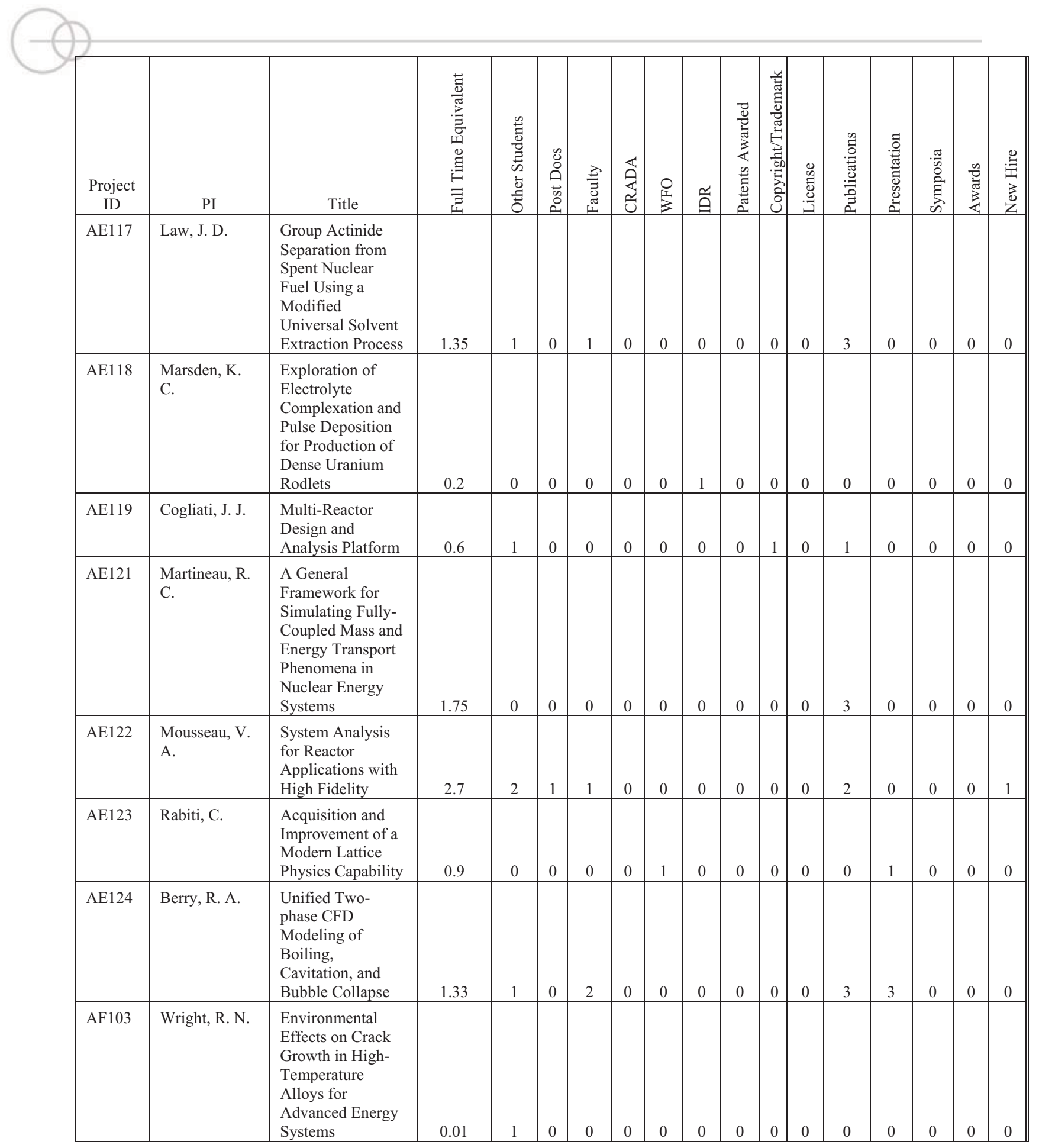




\section{Appendix D - Project Performance}

\begin{tabular}{|c|c|c|c|c|c|c|c|c|c|c|c|c|c|c|c|c|c|}
\hline $\begin{array}{l}\text { Project } \\
\text { ID }\end{array}$ & PI & Title & 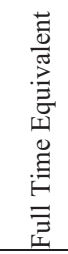 & 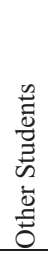 & $\begin{array}{l}\tilde{0} \\
0 \\
0 \\
\tilde{n} \\
0 \\
0\end{array}$ & 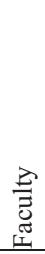 & 岕 & $\sum_{3}^{0}$ & 光 & 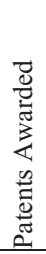 & 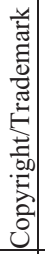 & 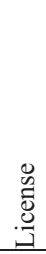 & 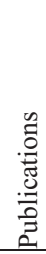 & 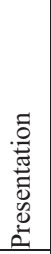 & 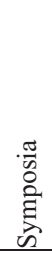 & 总 & $\begin{array}{l}\stackrel{0}{\Xi} \\
3 \\
3 \\
0 \\
\end{array}$ \\
\hline AF104 & Hilton, B. A. & $\begin{array}{l}\text { Development of } \\
\text { Advanced Burnup } \\
\text { Measurement \& } \\
\text { Nuclear Forensics } \\
\text { ICP-MS Isotopics } \\
\text { Analysis } \\
\text { Techniques }\end{array}$ & 1.03 & 0 & 0 & 0 & 0 & 0 & 0 & 0 & 0 & 0 & 2 & 0 & 0 & 0 & 0 \\
\hline AF105 & Cole, J. I. & $\begin{array}{l}\text { Development of a } \\
\text { Small Sample } \\
\text { Volume } \\
\text { Mechanical } \\
\text { Properties Testing } \\
\text { Technique for } \\
\text { Irradiated Fuels } \\
\text { and Materials }\end{array}$ & 0.3 & 1 & 0 & 1 & 0 & 0 & 0 & 0 & 0 & 0 & 0 & 0 & 0 & 0 & 0 \\
\hline AS102 & Mincher, B. J. & $\begin{array}{l}\text { Utility of Unusual } \\
\text { Oxidation States } \\
\text { of Americium for } \\
\text { Separations }\end{array}$ & 0.85 & 0 & 0 & 1 & 0 & 0 & 0 & 0 & 0 & 0 & 2 & 0 & 0 & 0 & 0 \\
\hline AS105 & Pepper, S. E. & $\begin{array}{l}\text { Rapid Detection of } \\
\text { Plutonium, } \\
\text { Neptunium and } \\
\text { Technetium in } \\
\text { Water Samples }\end{array}$ & 0.97 & 0 & 0 & 0 & 0 & 0 & 0 & 0 & 0 & 0 & 1 & 3 & 0 & 0 & 0 \\
\hline AS106 & $\begin{array}{l}\text { Peterman, D. } \\
\text { R. }\end{array}$ & $\begin{array}{l}\text { Evaluation of } \\
\text { Covalent } \\
\text { Interactions in } \\
\text { Actinide } \\
\text { Coordination } \\
\text { Compounds } \\
\end{array}$ & 0.2 & 0 & 0 & 1 & 0 & 0 & 0 & 0 & 2 & 1 & 1 & 1 & 1 & 0 & 0 \\
\hline AS107 & $\begin{array}{l}\text { Groenewold, } \\
\text { G. S. }\end{array}$ & $\begin{array}{l}\text { Measuring } \\
\text { Actinide } \\
\text { Speciation in High } \\
\text { pH Solutions }\end{array}$ & 0.55 & 0 & 0 & 0 & 0 & 0 & 0 & 0 & 0 & 0 & 0 & 2 & 0 & 0 & 0 \\
\hline AS108 & Scott, J. R. & $\begin{array}{l}\text { Isotope Ratio } \\
\text { Measurements } \\
\text { Methods for } \\
\text { Direct Analysis of } \\
\text { Samples } \\
\end{array}$ & 0.6 & 0 & 0 & 0 & 0 & 0 & 0 & 0 & 0 & 0 & 0 & 0 & 0 & 0 & 0 \\
\hline BS103 & Lacy, J. M. & $\begin{array}{l}\text { Multi-Stage } \\
\text { Sequential } \\
\text { Injection Gas Gun }\end{array}$ & 0.2 & 0 & 0 & 0 & 0 & 0 & 0 & 0 & 0 & 0 & 0 & 0 & 0 & 0 & 0 \\
\hline BS104 & $\begin{array}{l}\text { Newman, C. } \\
\text { K. }\end{array}$ & $\begin{array}{l}\text { A Strategy to } \\
\text { Tightly Couple } \\
\text { Neutronics and } \\
\text { Thermal- } \\
\text { Hydraulics Models } \\
\text { for Next } \\
\text { Generation } \\
\text { Reactor Analysis }\end{array}$ & 0.1 & 2 & 0 & 1 & 0 & 0 & 0 & 0 & 0 & 0 & 0 & 0 & 0 & 0 & 0 \\
\hline
\end{tabular}




\section{Appendix D - Project Performance}

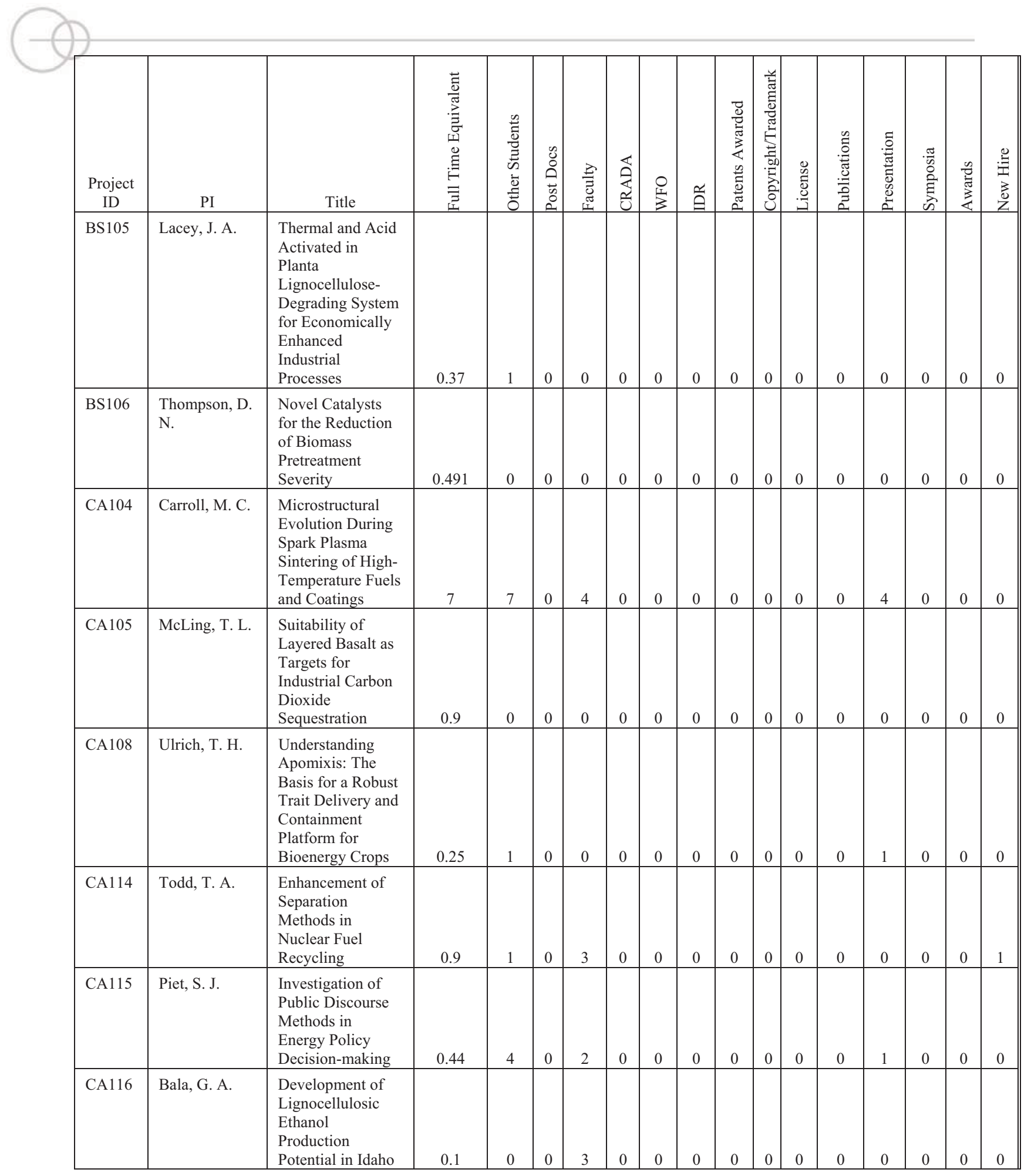




\section{Appendix D - Project Performance}

\begin{tabular}{|c|c|c|c|c|c|c|c|c|c|c|c|c|c|c|c|c|c|}
\hline $\begin{array}{l}\text { Project } \\
\text { ID }\end{array}$ & PI & Title & 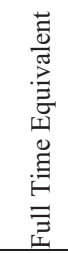 & $\frac{5}{0}$ & d & 름 & 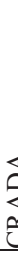 & 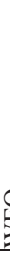 & 号 & 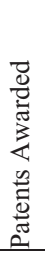 & 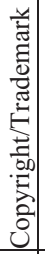 & 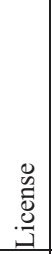 & 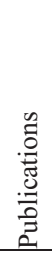 & 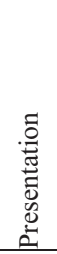 & 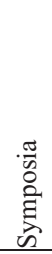 & 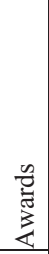 & $\begin{array}{l}\stackrel{0}{\Xi} \\
3 \\
3 \\
0 \\
\end{array}$ \\
\hline EI101 & Stewart, F. K. & $\begin{array}{l}\text { Chemical } \\
\text { Separations and } \\
\text { Process Research } \\
\text { to Enable } \\
\text { Biorefinery } \\
\text { Systems }\end{array}$ & 0.26 & 1 & 0 & 1 & $\underline{0}$ & c & 0 & 0 & 0 & 0 & 0 & 1 & 0 & 0 & 0 \\
\hline EI102 & Garcia, H. E. & $\begin{array}{l}\text { Advanced } \\
\text { Predictive } \\
\text { Condition } \\
\text { Monitoring and } \\
\text { Control for } \\
\text { Modern Energy } \\
\text { Systems: } \\
\text { Gasification-based } \\
\text { Processes }\end{array}$ & 1.3 & 0 & 0 & 1 & 0 & c & 0 & 0 & 0 & 0 & 1 & 1 & 0 & 0 & 0 \\
\hline EI104 & Guillen, D. P. & $\begin{array}{l}\text { CFD-Based } \\
\text { Simulation } \\
\text { Capability of } \\
\text { Fischer-Tropsch } \\
\text { Reactors and } \\
\text { Process } \\
\text { Equipment }\end{array}$ & 0.7 & 1 & 0 & 1 & $\underline{0}$ & c & 0 & 0 & 0 & 0 & 2 & 0 & 3 & 0 & 0 \\
\hline EI105 & Kenney, K. L. & $\begin{array}{l}\text { Biomass } \\
\text { Feedstock } \\
\text { Assembly to } \\
\text { Gasification } \\
\text { Process } \\
\text { Computational } \\
\text { Interface } \\
\text { Development }\end{array}$ & 1 & 1 & 0 & 1 & 0 & c & 0 & 0 & 0 & 0 & 0 & 0 & 0 & 0 & 0 \\
\hline EI107 & Palmer, C. D. & $\begin{array}{l}\text { Generation and } \\
\text { Expulsion of } \\
\text { Hydrocarbons } \\
\text { from Oil Shale }\end{array}$ & 0.78 & 2 & 0 & 0 & 0 & c & 0 & 0 & 0 & 0 & 0 & 11 & 2 & 0 & 0 \\
\hline EI109 & Cooper, D. C. & $\begin{array}{l}\text { Pathways to } \\
\text { Energy } \\
\text { Independence }\end{array}$ & 0.5 & 0 & 0 & $\underline{0}$ & 0 & c & 0 & 0 & 0 & 0 & 0 & 4 & 0 & 0 & 0 \\
\hline EI110 & $\begin{array}{l}\text { Petkovic, L. } \\
\text { M. }\end{array}$ & $\begin{array}{l}\text { Investigation of } \\
\text { Fischer Tropsch } \\
\text { Catalyst } \\
\text { Deactivation }\end{array}$ & 0.51 & 1 & 0 & 0 & 0 & c & 0 & 0 & 0 & 0 & 0 & 0 & 0 & 0 & 0 \\
\hline EI111 & $\begin{array}{l}\text { Robertson, E. } \\
\text { P. }\end{array}$ & $\begin{array}{l}\text { Altering } \\
\text { Wettability by } \\
\text { Chemical } \\
\text { Amendments to } \\
\text { Improve Gas } \\
\text { Production from } \\
\text { Tight Sands }\end{array}$ & 0.75 & 0 & 0 & 1 & 0 & c & 0 & 0 & 0 & 0 & 0 & 0 & 0 & 0 & 0 \\
\hline
\end{tabular}




\section{Appendix D - Project Performance}

\begin{tabular}{|c|c|c|c|c|c|c|c|c|c|c|c|c|c|c|c|c|c|c|}
\hline $\begin{array}{c}\text { Project } \\
\text { ID }\end{array}$ & PI & Title & 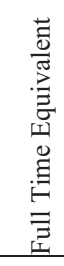 & 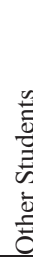 & & & & 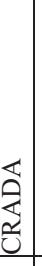 & 早 & 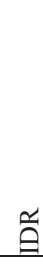 & 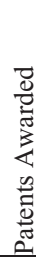 & 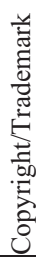 & 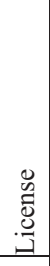 & 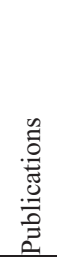 & 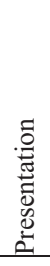 & 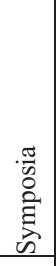 & 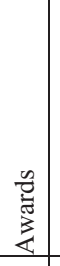 & $\begin{array}{l}0 \\
.0 \\
z \\
z \\
z\end{array}$ \\
\hline EI112 & Shunn, L. P. & $\begin{array}{l}\text { Hybrid Energy } \\
\text { System Neural } \\
\text { Reactance } \\
\text { Dynamic Control } \\
\text { System }\end{array}$ & 0.58 & 0 & 0 & & & 0 & 0 & 0 & 0 & 0 & 0 & 0 & 0 & 0 & 0 & \\
\hline FF104 & Wolf, D. W. & $\begin{array}{l}\text { Integrated } \\
\text { Mesoscale } \\
\text { Approach for the } \\
\text { Simulation of } \\
\text { Nuclear-Fuel } \\
\text { Behavior }\end{array}$ & 0.1 & 0 & 0 & & & 0 & 0 & 0 & 0 & 0 & 0 & 0 & 0 & 0 & 0 & 0 \\
\hline FF105 & Meakin, P. & $\begin{array}{l}\text { Particle-Discrete } \\
\text { Element Model } \\
\text { Simulation of the } \\
\text { Coupling between } \\
\text { Material Failure/ } \\
\text { Deformation and } \\
\text { Fluid Generation/ } \\
\text { Flow }\end{array}$ & 0.25 & 0 & 0 & & & 0 & 0 & 0 & 0 & 0 & 0 & 0 & 2 & 0 & 0 & 0 \\
\hline FF106 & Smartt, H. B. & $\begin{array}{l}\text { Scaling of } \\
\text { Welding Processes }\end{array}$ & 0.3 & 0 & 0 & & & 0 & 0 & 0 & 0 & 0 & 0 & 0 & 0 & 0 & 0 & 0 \\
\hline FF107 & Stone, M. L. & $\begin{array}{l}\text { Enhanced Metal } \\
\text { Ion Analysis }\end{array}$ & 0.3 & 0 & 0 & & & 0 & 0 & 0 & 0 & 0 & 0 & 0 & 0 & 0 & 0 & 0 \\
\hline FF108 & Apel, W. A. & $\begin{array}{l}\text { Effect of } \\
\text { Glycosylation on } \\
\text { the Cctivity and } \\
\text { Stability of } \\
\text { Bacterial Enzymes }\end{array}$ & 0.55 & 1 & 0 & & & 0 & 0 & 0 & 0 & 0 & 0 & 0 & 0 & 0 & 0 & 0 \\
\hline GB103 & Apel, W. A. & $\begin{array}{l}\text { A Systems } \\
\text { Biology Approach } \\
\text { to Understanding } \\
\text { Lignocellulose } \\
\text { Derived Carbon } \\
\text { Metabolism by } \\
\text { Alicyclobacillus } \\
\text { acidocaldarius }\end{array}$ & 0.6 & 1 & 0 & & & 0 & 0 & 0 & 0 & 0 & 1 & 0 & 0 & 0 & 0 & 0 \\
\hline GB104 & Lee, B. D. & $\begin{array}{l}\text { Metabolic } \\
\text { Engineering of } \\
\text { Alicyclobacillus } \\
\text { acidocaldarius for } \\
\text { Lactic Acid } \\
\text { Production from } \\
\text { Biomass Derived } \\
\text { Monosaccharides }\end{array}$ & 1.6 & 0 & ( & & & 0 & 0 & 0 & 0 & 0 & 0 & 0 & 0 & 0 & 0 & 0 \\
\hline GB105 & Lee, B. D. & $\begin{array}{l}\text { Network } \\
\text { Interaction In the } \\
\text { Thermoacidophile } \\
\text { Alicyclobacillus } \\
\text { acidocaldarius In } \\
\text { Response to } \\
\text { Different Complex } \\
\text { Carbon Sources }\end{array}$ & 0.6 & 0 & c & & & 0 & 0 & 0 & 0 & 0 & 0 & 0 & 0 & 0 & 0 & 0 \\
\hline
\end{tabular}




\section{Appendix D - Project Performance}

\begin{tabular}{|c|c|c|c|c|c|c|c|c|c|c|c|c|c|c|c|c|c|}
\hline $\begin{array}{l}\text { Project } \\
\text { ID }\end{array}$ & PI & Title & 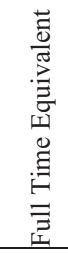 & 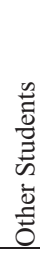 & $\begin{array}{l}\tilde{0} \\
0 \\
0 \\
\tilde{0} \\
0 \\
0\end{array}$ & 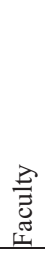 & 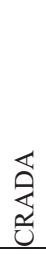 & $\sum_{3}^{0}$ & 光 & 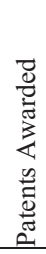 & 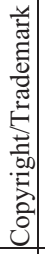 & $\begin{array}{l} \\
0 \\
.0 \\
0 \\
.0 \\
\end{array}$ & 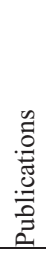 & 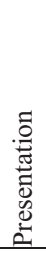 & 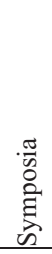 & 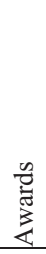 & 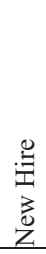 \\
\hline GS101 & $\begin{array}{l}\text { Robertson, E. } \\
\text { P. }\end{array}$ & $\begin{array}{l}\text { Reducing CBM } \\
\text { Water Discharge } \\
\text { Volume }\end{array}$ & 0.5 & 0 & 0 & 0 & 0 & 0 & 0 & 0 & 0 & 0 & 0 & 1 & 0 & 0 & 0 \\
\hline IC101 & Tolle, C. R. & $\begin{array}{l}\text { Exploration and } \\
\text { Development of } \\
\text { Automated } \\
\text { Differential } \\
\text { Equation-Based } \\
\text { System } \\
\text { Identification }\end{array}$ & 0.5 & 5 & 0 & 4 & 0 & 0 & 0 & 0 & 0 & 0 & 2 & 2 & 2 & 0 & 0 \\
\hline IC102 & Few, D. & $\begin{array}{l}\text { A Toolset for } \\
\text { Proximal Human- } \\
\text { Robot Interaction }\end{array}$ & 0.2 & 2 & 0 & 1 & 0 & 0 & 0 & 0 & 0 & 0 & 0 & 0 & 0 & 0 & 0 \\
\hline IC104 & Nichol, C. I. & $\begin{array}{l}\text { Haptic Interface } \\
\text { for Robotic Arc } \\
\text { Welding }\end{array}$ & 0.5 & 0 & 0 & 1 & 0 & 0 & 0 & 0 & 0 & 0 & 0 & 3 & 0 & 0 & 0 \\
\hline IC105 & $\begin{array}{l}\text { McGillivary, } \\
\text { K. E. }\end{array}$ & $\begin{array}{l}\text { Modeling } \\
\text { Interface to } \\
\text { Control System } \\
\text { Designs }\end{array}$ & 0.3 & 0 & 0 & 0 & 0 & 0 & 0 & 0 & 0 & 0 & 0 & 0 & 0 & 0 & 0 \\
\hline IC106 & Vollmer, D. T. & $\begin{array}{l}\text { Resilient Control } \\
\text { System Network } \\
\text { Agents }\end{array}$ & 0.25 & 0 & 0 & 1 & 0 & 0 & 0 & 0 & 0 & 0 & 3 & 0 & 0 & 0 & 0 \\
\hline IC107 & Cherry, S. J. & $\begin{array}{l}\text { Integrated Control } \\
\text { System Data } \\
\text { Fusion }\end{array}$ & 0.83 & 1 & 0 & 1 & 0 & 0 & 0 & 0 & 0 & 0 & 0 & 0 & 0 & 0 & 0 \\
\hline IC 108 & Buttles, J. W. & $\begin{array}{l}\text { Wireless Sensor } \\
\text { Testing }\end{array}$ & 0.4 & 1 & 0 & 0 & 0 & 0 & 0 & 0 & 0 & 0 & 0 & 0 & 0 & 0 & 0 \\
\hline IC109 & Rieger, C. G. & $\begin{array}{l}\text { Anomaly } \\
\text { Detection, } \\
\text { Diagnosis, and } \\
\text { Resilient Control } \\
\text { in Complex } \\
\text { Engineered } \\
\text { Systems } \\
\end{array}$ & 0.06 & 0 & 1 & 1 & 0 & 0 & 0 & 0 & 0 & 0 & 0 & 0 & 0 & 0 & 0 \\
\hline NE146 & Tranter, T. J. & $\begin{array}{l}\text { Process Modeling } \\
\text { of Solvent } \\
\text { Extraction } \\
\text { Separations for } \\
\text { Advanced Nuclear } \\
\text { Fuel Cycles }\end{array}$ & 0.5 & 0 & 0 & 0 & 0 & 0 & 0 & 0 & 0 & 0 & 0 & 0 & 0 & 0 & 0 \\
\hline NE147 & Mincher, B. J. & $\begin{array}{l}\text { Reactivity of } \\
\text { Radiolytically } \\
\text { Produced Nitrogen } \\
\text { Oxide Radicals } \\
\text { toward Aromatic } \\
\text { Compounds }\end{array}$ & 0.75 & 0 & 0 & 2 & 1 & 1 & 0 & 0 & 0 & 0 & 1 & 1 & 0 & 0 & 0 \\
\hline
\end{tabular}




\section{Appendix D - Project Performance}

\begin{tabular}{|c|c|c|c|c|c|c|c|c|c|c|c|c|c|c|c|c|c|}
\hline $\begin{array}{l}\text { Project } \\
\text { ID }\end{array}$ & PI & Title & 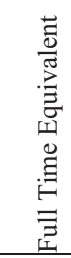 & 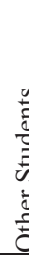 & & & 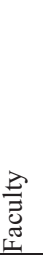 & 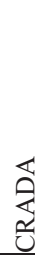 & 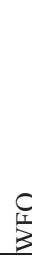 & & | & 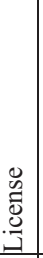 & 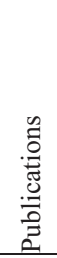 & 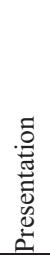 & 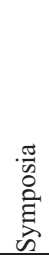 & 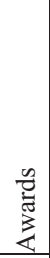 & $\begin{array}{l}\text { 号 } \\
z \\
z \\
z \\
z\end{array}$ \\
\hline NE149 & Nigg, D. W. & $\begin{array}{l}\text { Modernization/Op } \\
\text { timization of the } \\
\text { Advanced Test } \\
\text { Reactor's Core- } \\
\text { Analysis } \\
\text { Capability to } \\
\text { Facilitate Its } \\
\text { Operation as a } \\
\text { National Scientific } \\
\text { User Facility }\end{array}$ & 0.85 & 2 & ( & & 0 & 0 & 0 & 0 & 0 & 0 & 0 & 0 & 2 & 0 & 0 \\
\hline NE150 & Rempe, J. L. & $\begin{array}{l}\text { Viability } \\
\text { Evaluations of } \\
\text { Linear Variable } \\
\text { Differential } \\
\text { Transformers } \\
\text { (LVDTs) and } \\
\text { Capacitive Micro- } \\
\text { Machined } \\
\text { Ultrasonic } \\
\text { Transducers } \\
\text { (CMUTs) for In- } \\
\text { Pile } \\
\text { Instrumentation }\end{array}$ & 0.8 & 1 & c & & 1 & 0 & 0 & 0 & 0 & 0 & 2 & 8 & 1 & 0 & 0 \\
\hline NE153 & Rabiti, C. & $\begin{array}{l}\text { Reactor Physics } \\
\text { Sensitivity } \\
\text { Analysis, } \\
\text { Uncertainty } \\
\text { Quantification, } \\
\text { and Data } \\
\text { Assimilation } \\
\text { Capability }\end{array}$ & 1.2 & 1 & ( & & 1 & 0 & 0 & 0 & 0 & 0 & 0 & 3 & 0 & 0 & 1 \\
\hline NE154 & $\begin{array}{l}\text { Williamson, } \\
\text { R. L. }\end{array}$ & $\begin{array}{l}\text { Fracture Methods } \\
\text { for Reactor Fuel } \\
\text { Performance } \\
\text { Analysis }\end{array}$ & 0.5 & 0 & ( & & 0 & 0 & 0 & 0 & b & 0 & 0 & 0 & 0 & 0 & 0 \\
\hline NE155 & Zhao, H. & $\begin{array}{l}\text { Use of Ice } \\
\text { Thermal Storage } \\
\text { Systems to } \\
\text { Improve LWR } \\
\text { Plant Efficiency }\end{array}$ & 0.787 & 2 & (1) & & 1 & 0 & 0 & ( & 0 & 0 & 1 & 0 & 0 & 0 & 0 \\
\hline NE156 & Dinh, N. T. & $\begin{array}{l}\text { Development of a } \\
\text { Next-Generation } \\
\text { Production Code } \\
\text { for Nuclear } \\
\text { Reactor System } \\
\text { Analysis and } \\
\text { Safety Margin } \\
\text { Quantification }\end{array}$ & 3.68 & 1 & ( & & 4 & 0 & 0 & ( & 0 & 0 & 5 & 5 & 2 & 0 & 0 \\
\hline
\end{tabular}




\section{Appendix D - Project Performance}

\begin{tabular}{|c|c|c|c|c|c|c|c|c|c|c|c|c|c|c|c|c|c|}
\hline $\begin{array}{l}\text { Project } \\
\text { ID }\end{array}$ & PI & Title & 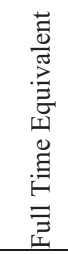 & 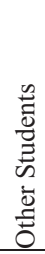 & $\begin{array}{l}\tilde{0} \\
0 \\
0 \\
\tilde{n} \\
0 \\
0\end{array}$ & 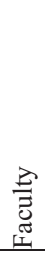 & 芯 & $\sum_{3}^{0}$ & $\stackrel{\widetilde{a}}{\mathrm{G}}$ & 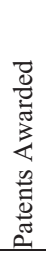 & 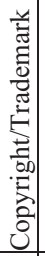 & 泀 & 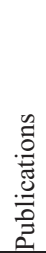 & 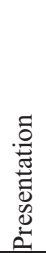 & 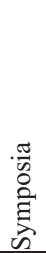 & 党 & $\begin{array}{l}\stackrel{0}{0} \\
3 \\
3 \\
0 \\
z\end{array}$ \\
\hline NE157 & $\begin{array}{l}\text { Herrmann, S. } \\
\text { D. }\end{array}$ & $\begin{array}{l}\text { Characterization } \\
\text { of a Consolidated } \\
\text { Electrochemical } \\
\text { Technique for } \\
\text { Separation and } \\
\text { Recovery of } \\
\text { Actinides from } \\
\text { Fission Products }\end{array}$ & 0.6 & 0 & 0 & 0 & 0 & 0 & 0 & 0 & 0 & 0 & 0 & 0 & 0 & 0 & 0 \\
\hline NE158 & $\begin{array}{l}\text { Knudson, D. } \\
\text { L. }\end{array}$ & $\begin{array}{l}\text { Advanced } \\
\text { Instrumentation } \\
\text { for In-pile } \\
\text { Detection of } \\
\text { Thermal } \\
\text { Conductivity }\end{array}$ & 0.6 & 1 & 0 & 1 & 0 & 0 & 0 & 0 & 0 & 0 & 3 & 4 & 1 & 0 & 1 \\
\hline NN110 & $\begin{array}{l}\text { Groenewold, } \\
\text { G. S. }\end{array}$ & $\begin{array}{l}\text { Chemical } \\
\text { Signatures of } \\
\text { Nuclear } \\
\text { Proliferation on } \\
\text { Particles } \\
\end{array}$ & 0.5 & 0 & 0 & 1 & 0 & 0 & 0 & 0 & 0 & 0 & 0 & 0 & 0 & 0 & 0 \\
\hline NN112 & Tranter, T. J. & $\begin{array}{l}\text { Technetium } \\
\text { Signature Analysis }\end{array}$ & 0.38 & 0 & 0 & 0 & 0 & 0 & 0 & 0 & 0 & 0 & 0 & 0 & 0 & 0 & 0 \\
\hline NN113 & $\begin{array}{l}\text { Chichester, D. } \\
\text { L. }\end{array}$ & $\begin{array}{l}\text { Active } \\
\text { Interrogation Die- } \\
\text { Away Assay } \\
\text { Development } \\
\text { Program }\end{array}$ & 0.5 & 0 & 0 & 0 & 0 & 0 & 0 & 0 & 0 & 0 & 1 & 1 & 0 & 0 & 0 \\
\hline NN114 & McGrath, C. & $\begin{array}{l}135 \mathrm{Xe} \text { Recovery } \\
\text { from the } \\
\text { Spontaneous } \\
\text { Fission of } 252 \mathrm{Cf}\end{array}$ & 0 & 0 & 0 & 0 & 0 & 0 & 0 & 0 & 0 & 0 & 0 & 0 & 0 & 0 & 0 \\
\hline NN115 & Phillips, J. H. & $\begin{array}{l}\text { Developing a Next } \\
\text { Generation, Risk- } \\
\text { Informed } \\
\text { Approach for } \\
\text { Robust and } \\
\text { Resilient Design } \\
\text { Development } \\
\text { (R2D2) } \\
\end{array}$ & 1.01 & 0 & 0 & 2 & 0 & 0 & 0 & 0 & 0 & 0 & 0 & 0 & 0 & 0 & 0 \\
\hline NN116 & $\begin{array}{l}\text { Chichester, D. } \\
\text { L. }\end{array}$ & $\begin{array}{l}\text { Neutron } \\
\text { Spectrometer } \\
\text { Development }\end{array}$ & 0.55 & 0 & 0 & 1 & 0 & 0 & 0 & 0 & 0 & 0 & 0 & 0 & 0 & 0 & 0 \\
\hline NN117 & Kinlaw, M. T. & $\begin{array}{l}\text { Nuclear Material } \\
\text { Detection Using } \\
\text { Neutron Time-of- } \\
\text { Flight }\end{array}$ & 0.4 & 1 & 0 & 1 & 0 & 0 & 0 & 0 & 0 & 0 & 0 & 1 & 0 & 0 & 0 \\
\hline
\end{tabular}




\section{Appendix D - Project Performance}

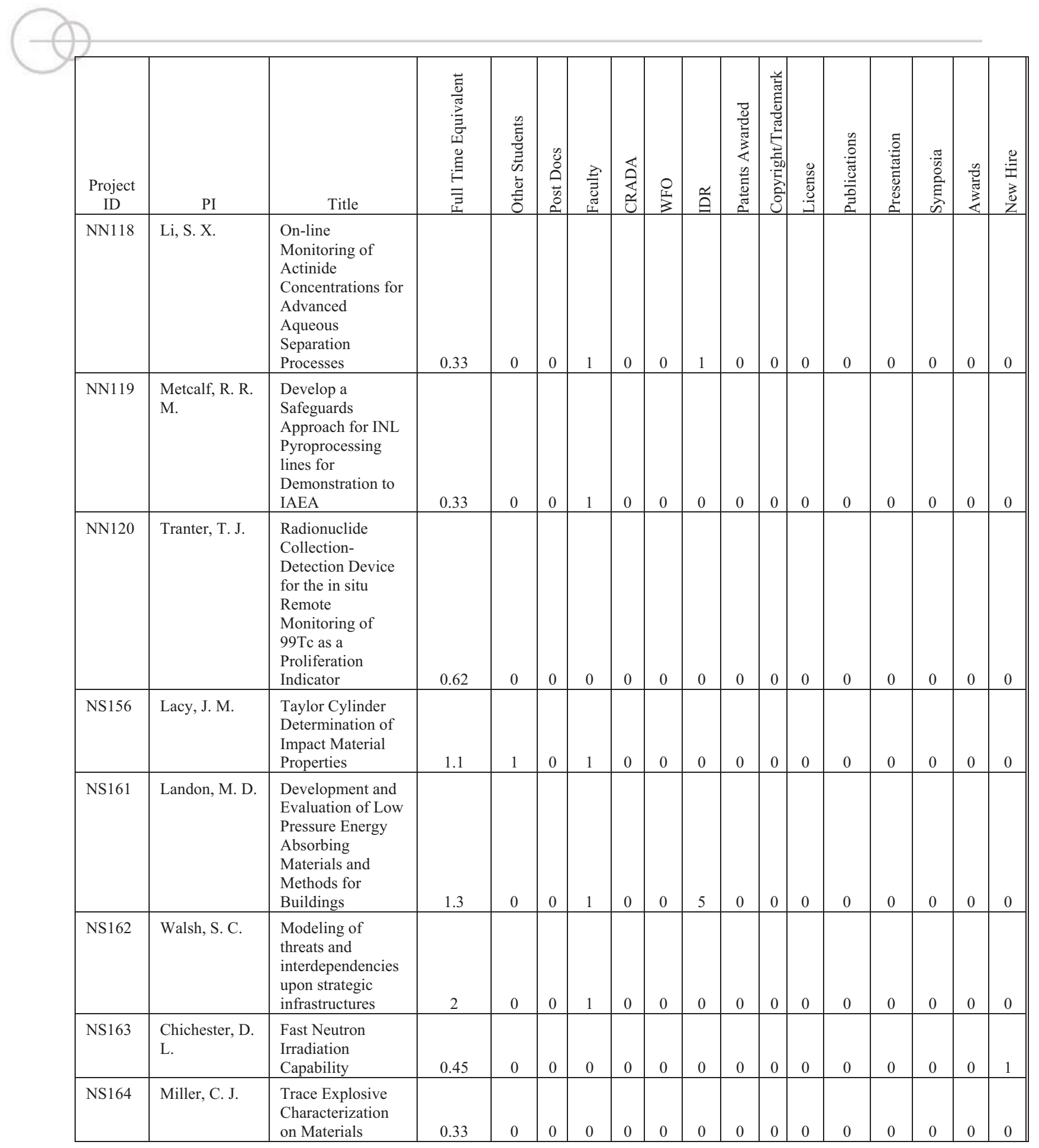




\section{Appendix D - Project Performance}

\begin{tabular}{|c|c|c|c|c|c|c|c|c|c|c|c|c|c|c|c|c|c|}
\hline $\begin{array}{c}\text { Project } \\
\text { ID }\end{array}$ & PI & Title & 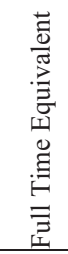 & 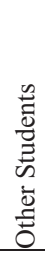 & $\begin{array}{l}n \\
0 \\
0 \\
0 \\
\\
\tilde{w} \\
0 \\
0\end{array}$ & 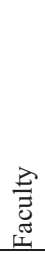 & 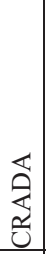 & $\sum_{3}^{0}$ & 号 & 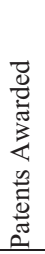 & 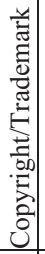 & 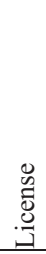 & 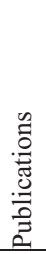 & 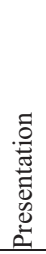 & 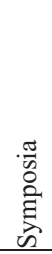 & 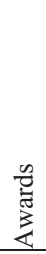 & $\begin{array}{l}\stackrel{0}{\Xi} \\
3 \\
3 \\
Z\end{array}$ \\
\hline NS165 & Rieger, C. G. & $\begin{array}{l}\text { Methodologies for } \\
\text { the Design, } \\
\text { Analysis, and } \\
\text { Validation for } \\
\text { Operation of } \\
\text { Complex Resilient } \\
\text { Networks }\end{array}$ & 0.13 & 1 & 0 & 1 & 0 & 0 & 0 & 0 & 0 & 0 & 0 & 0 & 0 & 0 & 0 \\
\hline NU100 & Gertman, D. I. & $\begin{array}{l}\text { Human } \\
\text { Performance } \\
\text { Assessment for } \\
\text { Technology } \\
\text { Neutral } \\
\text { Evaluation: } \\
\text { Combining Virtual } \\
\text { and Physical } \\
\text { Testing for } \\
\text { Design, } \\
\text { Development and } \\
\text { Review of Digital } \\
\text { Control Systems } \\
\text { and Interfaces } \\
\end{array}$ & 1.2 & 0 & 0 & 0 & 0 & 0 & 0 & 0 & 0 & 0 & 0 & 0 & 1 & 0 & 0 \\
\hline NU101 & Lessing, P. A. & $\begin{array}{l}\text { Advanced } \\
\text { Ceramic Nuclear } \\
\text { Fuels }\end{array}$ & 2.4 & 0 & 0 & 2 & 0 & 0 & 0 & 0 & 0 & 0 & 0 & 0 & 0 & 0 & 1 \\
\hline PH101 & Deaton, J. D. & $\begin{array}{l}\text { Cognitive } \\
\text { Network Engine } \\
\text { and Simulation } \\
\text { Framework,Ph.D. } \\
\text { Candidate } \\
\text { Proposal for Juan } \\
\text { Deaton }\end{array}$ & 0.1 & 2 & 0 & 2 & 0 & 0 & 0 & 0 & 0 & 0 & 1 & 0 & 0 & 0 & 0 \\
\hline PH102 & Kelly, D. L. & $\begin{array}{l}\text { Application of } \\
\text { Dynamic Bayesian } \\
\text { Networks to } \\
\text { Systems with } \\
\text { Ambient } \\
\text { Intelligence }\end{array}$ & 0.5 & 0 & 1 & 1 & 0 & 0 & 0 & 0 & 0 & 0 & 0 & 0 & 0 & 0 & 0 \\
\hline PH103 & $\begin{array}{l}\text { Marshall, D. } \\
\text { W. }\end{array}$ & $\begin{array}{l}\text { Characterization } \\
\text { of Fluidized Beds } \\
\text { via Pressure- } \\
\text { Fluctuation } \\
\text { Analysis }\end{array}$ & 0.18 & 1 & 0 & 2 & 0 & 0 & 0 & 0 & 0 & 0 & 0 & 0 & 0 & 0 & 0 \\
\hline
\end{tabular}




\section{Appendix D - Project Performance}

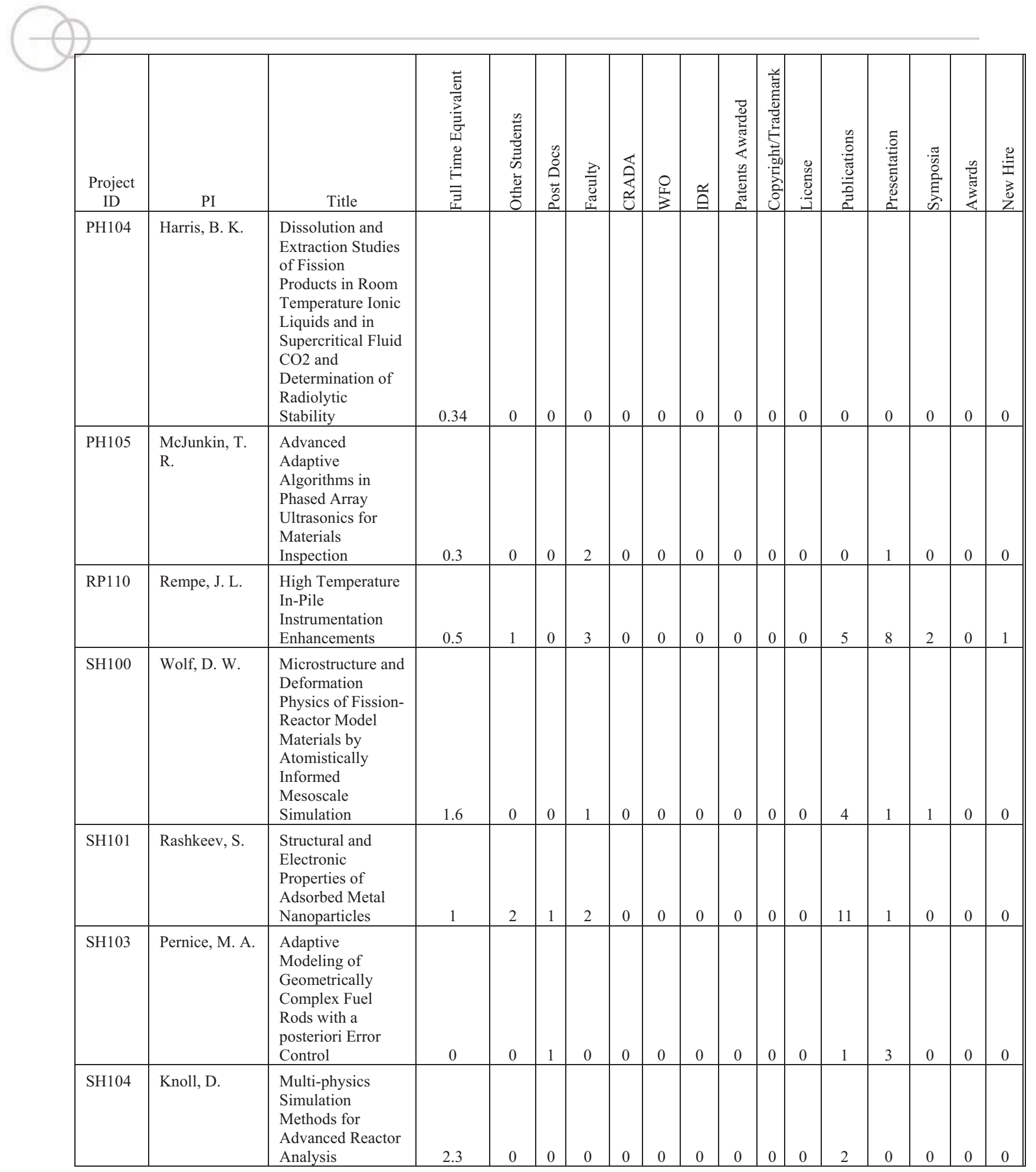




\section{Appendix D - Project Performance}

\begin{tabular}{|c|c|c|c|c|c|c|c|c|c|c|c|c|c|c|c|c|c|}
\hline $\begin{array}{c}\text { Project } \\
\text { ID }\end{array}$ & PI & Title & 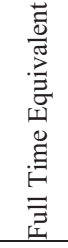 & 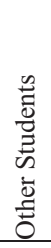 & $\begin{array}{l}n \\
0 \\
0 \\
0 \\
\\
\tilde{y} \\
0 \\
\end{array}$ & 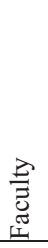 & 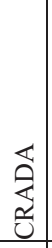 & $\sum_{3}^{0}$ & 亘 & 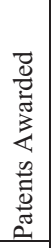 & 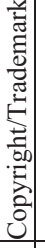 & 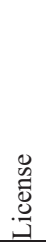 & 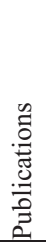 & 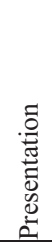 & 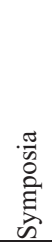 & 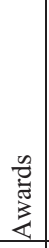 & 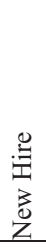 \\
\hline ST130 & Stewart, F. F. & $\begin{array}{l}\text { Investigation of } \\
\text { Low Temperature } \\
\text { Performance in } \\
\text { Membrane } \\
\text { Materials and } \\
\text { Processes for Gas } \\
\text { Separations }\end{array}$ & 0.35 & 0 & 1 & 1 & 0 & 0 & 0 & 0 & 0 & 0 & 0 & 1 & 0 & 0 & 0 \\
\hline ST131 & Klaehn, J. R. & $\begin{array}{l}\text { High-Performance } \\
\text { Polymer } \\
\text { Membranes for } \\
\text { High Temperature } \\
\text { Gas Separations }\end{array}$ & 0.39 & 0 & 0 & 2 & 2 & 0 & 0 & 0 & 0 & 0 & 0 & 1 & 0 & 0 & 0 \\
\hline ST132 & Benson, M. T. & $\begin{array}{l}\text { Triazine-Based } \\
\mathrm{CO} 2 \text { Capture } \\
\text { Agents }\end{array}$ & 0.45 & 0 & 0 & 0 & 0 & 0 & 0 & 0 & 0 & 0 & 0 & 0 & 0 & 0 & 0 \\
\hline ST133 & Lee, R. D. & $\begin{array}{l}\text { Dynamic Impact } \\
\text { Model and } \\
\text { Information } \\
\text { System to support } \\
\text { Unconventional } \\
\text { Fuels } \\
\text { Development }\end{array}$ & 0.58 & 1 & 0 & 1 & 0 & 0 & 0 & 0 & 0 & 0 & 0 & 0 & 0 & 0 & 0 \\
\hline ST134 & Wood, T. R. & $\begin{array}{l}\text { Near Field } \\
\text { Impacts of In-Situ } \\
\text { Oil Shale } \\
\text { Development on } \\
\text { Water Quality- } \\
\end{array}$ & 0.8 & 0 & 0 & 1 & 0 & 0 & 0 & 0 & 0 & 0 & 0 & 12 & 1 & 0 & 0 \\
\hline ST135 & $\begin{array}{l}\text { Anderson, M. } \\
\text { O. }\end{array}$ & $\begin{array}{l}\text { Advanced Remote } \\
\text { Sensing for } \\
\text { Energy and } \\
\text { Environmental } \\
\text { Applications using } \\
\text { Unmanned Aerial } \\
\text { Vehicles }\end{array}$ & 1 & 2 & 0 & 1 & 0 & 0 & 0 & 0 & 0 & 0 & 0 & 0 & 0 & 0 & 0 \\
\hline ST136 & Hurley, D. H. & $\begin{array}{l}\text { Addressing the } \\
\text { Spectrum of } \\
\text { Nuclear Related } \\
\text { NDE Needs: A } \\
\text { Hybrid laser } \\
\text { Ultrasonic and } \\
\text { Eddy Current } \\
\text { Approach }\end{array}$ & 0.95 & 0 & 0 & 0 & 0 & 0 & 0 & 0 & 0 & 0 & 0 & 0 & 0 & 0 & 1 \\
\hline ST137 & Windes, W. E. & $\begin{array}{l}\text { Research of } \\
\text { Advanced VHTR } \\
\text { Core Components }\end{array}$ & 0.5 & 2 & 0 & 5 & 0 & 0 & 0 & 0 & 0 & 0 & 0 & 0 & 0 & 0 & 0 \\
\hline
\end{tabular}




\section{Appendix D - Project Performance}

\begin{tabular}{|c|c|c|c|c|c|c|c|c|c|c|c|c|c|c|c|c|c|}
\hline $\begin{array}{l}\text { Project } \\
\text { ID }\end{array}$ & PI & Title & 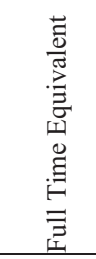 & 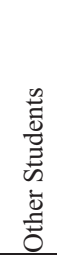 & $\begin{array}{l}0 \\
0 \\
0 \\
0 \\
\tilde{n} \\
0 \\
0\end{array}$ & 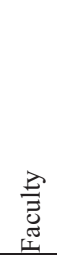 & 志 & $\sum_{3}^{0}$ & 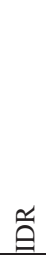 & 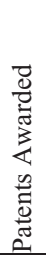 & 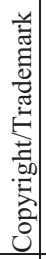 & 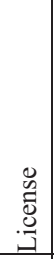 & 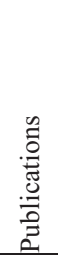 & 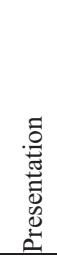 & 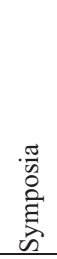 & 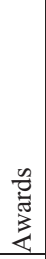 & $\begin{array}{l}0 \\
. \\
3 \\
0 \\
z\end{array}$ \\
\hline ST138 & $\begin{array}{l}\text { Breckenridge, } \\
\text { R. P. }\end{array}$ & $\begin{array}{l}\text { Development of a } \\
\text { Sustainable } \\
\text { Ecological } \\
\text { Engineering } \\
\text { approach to } \\
\text { support Hybrid } \\
\text { energy Systems } \\
\text { Development in } \\
\text { the Western Inland } \\
\text { Energy Corridor }\end{array}$ & 0.2 & 0 & 0 & 2 & 0 & 0 & 0 & 0 & 0 & 0 & 0 & 1 & 0 & 0 & 0 \\
\hline TM106 & Huang, $\mathrm{H}$. & $\begin{array}{l}\text { Development of } \\
\text { 3D Multiphase } \\
\text { Flow and Reactive } \\
\text { Transport Codes } \\
\text { and their } \\
\text { Applications to } \\
\text { Reactive Flow in } \\
\text { Porous Media and } \\
\text { Fracture Apertures }\end{array}$ & 0.69 & 0 & 0 & 0 & 0 & 0 & 0 & 0 & 0 & 0 & 0 & 0 & 0 & 0 & 0 \\
\hline TM108 & Gaston, D. R. & $\begin{array}{l}\text { Uncertainty } \\
\text { Quantification for } \\
\text { Nuclear Fuels } \\
\text { Performance }\end{array}$ & 0.05 & 2 & 1 & 2 & 0 & 0 & 0 & 0 & 0 & 0 & 0 & 0 & 0 & 0 & 0 \\
\hline & & & 74.888 & 64 & 7 & 91 & 3 & 2 & 7 & 0 & 3 & 2 & 63 & 93 & 19 & 0 & 9 \\
\hline
\end{tabular}




\section{Appendix D - Project Performance}


Appendix $\mathbf{E}$

Relevance to Major DOE Missions 


\section{Appendix E - Relevance to Major DOE Missions}

\begin{tabular}{|c|c|c|c|c|c|c|}
\hline $\begin{array}{l}\text { Project } \\
\text { ID }\end{array}$ & $\begin{array}{l}\text { Principal } \\
\text { Investigator }\end{array}$ & Title & 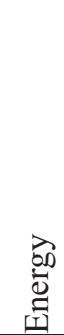 & 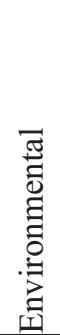 & 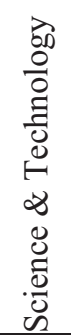 & 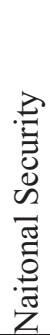 \\
\hline AE117 & Law, J. D. & $\begin{array}{l}\text { Group Actinide Separation from Spent } \\
\text { Nuclear Fuel Using a Modified Universal } \\
\text { Solvent Extraction Process }\end{array}$ & $\mathrm{X}$ & $\mathrm{X}$ & $\mathrm{X}$ & $X$ \\
\hline AE118 & Marsden, K. C. & $\begin{array}{l}\text { Exploration of Electrolyte Complexation and } \\
\text { Pulse Deposition for Production of Dense } \\
\text { Uranium Rodlets }\end{array}$ & $X$ & & & \\
\hline AE119 & Cogliati, J. J. & Multi-Reactor Design and Analysis Platform & & $\mathrm{X}$ & $\mathrm{X}$ & \\
\hline AE121 & Martineau, R. C. & $\begin{array}{l}\text { A General Framework for Simulating Fully- } \\
\text { Coupled Mass and Energy Transport } \\
\text { Phenomena in Nuclear Energy Systems }\end{array}$ & $\mathrm{X}$ & & & \\
\hline AE122 & Mousseau, V. A. & $\begin{array}{l}\text { System Analysis for Reactor Applications } \\
\text { with High Fidelity }\end{array}$ & $\mathrm{X}$ & $\mathrm{X}$ & & $X$ \\
\hline AE123 & Rabiti, C. & $\begin{array}{l}\text { Acquisition and Improvement of a Modern } \\
\text { Lattice Physics Capability }\end{array}$ & $\mathrm{X}$ & $\mathrm{X}$ & $\mathrm{X}$ & $\mathrm{X}$ \\
\hline AE124 & Berry, R. A. & $\begin{array}{l}\text { Unified Two-phase CFD Modeling of } \\
\text { Boiling, Cavitation, and Bubble Collapse }\end{array}$ & $\mathrm{X}$ & & $\mathrm{X}$ & $\mathrm{X}$ \\
\hline AF103 & Wright, R. N. & $\begin{array}{l}\text { Environmental Effects on Crack Growth in } \\
\text { High-Temperature Alloys for Advanced } \\
\text { Energy Systems }\end{array}$ & $\mathrm{X}$ & & & \\
\hline AF104 & Hilton, B. A. & $\begin{array}{l}\text { Development of Advanced Burnup } \\
\text { Measurement \& Nuclear Forensics ICP-MS } \\
\text { Isotopics Analysis Techniques }\end{array}$ & $\mathrm{X}$ & & & $X$ \\
\hline AF105 & Cole, J. I. & $\begin{array}{l}\text { Development of a Small Sample Volume } \\
\text { Mechanical Properties Testing Technique for } \\
\text { Irradiated Fuels and Materials }\end{array}$ & $\mathrm{X}$ & & $\mathrm{X}$ & \\
\hline AS102 & Mincher, B. J. & $\begin{array}{l}\text { Utility of Unusual Oxidation States of } \\
\text { Americium for Separations }\end{array}$ & $\mathrm{X}$ & & & $\mathrm{X}$ \\
\hline AS105 & Pepper, S. E. & $\begin{array}{l}\text { Rapid Detection of Plutonium, Neptunium } \\
\text { and Technetium in Water Samples }\end{array}$ & & $\mathrm{X}$ & $\mathrm{X}$ & $\mathrm{X}$ \\
\hline AS106 & Peterman, D. R. & $\begin{array}{l}\text { Evaluation of Covalent Interactions in } \\
\text { Actinide Coordination Compounds }\end{array}$ & $\mathrm{X}$ & $\mathrm{X}$ & $\mathrm{X}$ & $\mathrm{X}$ \\
\hline AS107 & Groenewold, G. S. & $\begin{array}{l}\text { Measuring Actinide Speciation in High } \mathrm{pH} \\
\text { Solutions }\end{array}$ & $\mathrm{X}$ & $X$ & $\mathrm{X}$ & \\
\hline AS108 & Scott, J. R. & $\begin{array}{l}\text { Isotope Ratio Measurements Methods for } \\
\text { Direct Analysis of Samples }\end{array}$ & $X$ & $X$ & $\mathrm{X}$ & $X$ \\
\hline BS103 & Lacy, J. M. & Multi-Stage Sequential Injection Gas Gun & & & $\mathrm{X}$ & X \\
\hline
\end{tabular}




\section{Appendix E - Relevance to Major DOE Missions}

\begin{tabular}{|c|c|c|c|c|c|c|}
\hline $\begin{array}{l}\text { Project } \\
\text { ID }\end{array}$ & $\begin{array}{l}\text { Principal } \\
\text { Investigator }\end{array}$ & Title & $\begin{array}{l}\text { 感 } \\
\text { 島 }\end{array}$ & 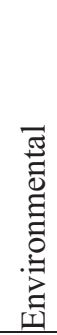 & 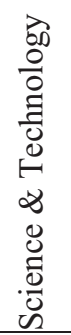 & 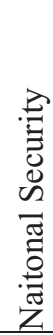 \\
\hline BS104 & Newman, C. K. & $\begin{array}{l}\text { A Strategy to Tightly Couple Neutronics and } \\
\text { Thermal-Hydraulics Models for Next } \\
\text { Generation Reactor Analysis }\end{array}$ & $X$ & $X$ & $X$ & \\
\hline BS105 & Lacey, J. A. & $\begin{array}{l}\text { Thermal and Acid Activated in Planta } \\
\text { Lignocellulose-Degrading System for } \\
\text { Economically Enhanced Industrial Processes }\end{array}$ & $X$ & $X$ & $\mathrm{X}$ & $X$ \\
\hline BS106 & Thompson, D. N. & $\begin{array}{l}\text { Novel Catalysts for the Reduction of } \\
\text { Biomass Pretreatment Severity }\end{array}$ & $\mathrm{X}$ & & $\mathrm{X}$ & \\
\hline CA104 & Carroll, M. C. & $\begin{array}{l}\text { Microstructural Evolution During Spark } \\
\text { Plasma Sintering of High-Temperature Fuels } \\
\text { and Coatings }\end{array}$ & $X$ & $X$ & $X$ & \\
\hline CA105 & McLing, T. L. & $\begin{array}{l}\text { Suitability of Layered Basalt as Targets for } \\
\text { Industrial Carbon Dioxide Sequestration }\end{array}$ & $X$ & $X$ & $X$ & \\
\hline CA108 & Ulrich, T. H. & $\begin{array}{l}\text { Understanding Apomixis: The Basis for a } \\
\text { Robust Trait Delivery and Containment } \\
\text { Platform for Bioenergy Crops }\end{array}$ & $X$ & & $X$ & $X$ \\
\hline CA114 & Todd, T. A. & $\begin{array}{l}\text { Enhancement of Separation Methods in } \\
\text { Nuclear Fuel Recycling }\end{array}$ & $X$ & $X$ & $X$ & $X$ \\
\hline CA115 & Piet, S. J. & $\begin{array}{l}\text { Investigation of Public Discourse Methods in } \\
\text { Energy Policy Decision-making }\end{array}$ & $\mathrm{X}$ & $\mathrm{X}$ & $X$ & $\mathrm{X}$ \\
\hline CA116 & Bala, G. A. & $\begin{array}{l}\text { Development of Lignocellulosic Ethanol } \\
\text { Production Potential in Idaho }\end{array}$ & $\mathrm{X}$ & & & \\
\hline EI101 & Stewart, F. K. & $\begin{array}{l}\text { Chemical Separations and Process Research } \\
\text { to Enable Biorefinery Systems }\end{array}$ & $\mathrm{X}$ & $\mathrm{X}$ & & \\
\hline EI102 & Garcia, H. E. & $\begin{array}{l}\text { Advanced Predictive Condition Monitoring } \\
\text { and Control for Modern Energy Systems: } \\
\text { Gasification-based Processes }\end{array}$ & $X$ & $X$ & $X$ & $X$ \\
\hline EI104 & Guillen, D. P. & $\begin{array}{l}\text { CFD-Based Simulation Capability of } \\
\text { Fischer-Tropsch Reactors and Process } \\
\text { Equipment }\end{array}$ & $\mathrm{X}$ & $X$ & $\mathrm{X}$ & $\mathrm{X}$ \\
\hline EI105 & Kenney, K. L. & $\begin{array}{l}\text { Biomass Feedstock Assembly to Gasification } \\
\text { Process Computational Interface } \\
\text { Development }\end{array}$ & $X$ & & $X$ & \\
\hline EI107 & Palmer, C. D. & $\begin{array}{l}\text { Generation and Expulsion of Hydrocarbons } \\
\text { from Oil Shale }\end{array}$ & $X$ & $X$ & $\mathrm{X}$ & $X$ \\
\hline EI109 & Cooper, D. C. & Pathways to Energy Independence & $\mathrm{X}$ & $\mathrm{X}$ & $\mathrm{X}$ & $\mathrm{X}$ \\
\hline EI110 & Petkovic, L. M. & $\begin{array}{l}\text { Investigation of Fischer Tropsch Catalyst } \\
\text { Deactivation }\end{array}$ & $X$ & $X$ & $X$ & $X$ \\
\hline
\end{tabular}




\section{Appendix E - Relevance to Major DOE Missions}

\begin{tabular}{|c|c|c|c|c|c|c|}
\hline $\begin{array}{l}\text { Project } \\
\text { ID }\end{array}$ & $\begin{array}{l}\text { Principal } \\
\text { Investigator }\end{array}$ & Title & 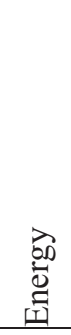 & 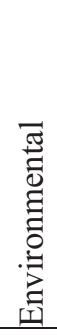 & 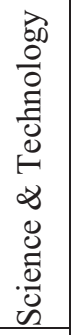 & 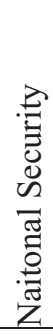 \\
\hline EI111 & Robertson, E. P. & $\begin{array}{l}\text { Altering Wettability by Chemical } \\
\text { Amendments to Improve Gas Production } \\
\text { from Tight Sands }\end{array}$ & $\mathrm{X}$ & & & \\
\hline EI112 & Shunn, L. P. & $\begin{array}{l}\text { Hybrid Energy System Neural Reactance } \\
\text { Dynamic Control System }\end{array}$ & $\mathrm{X}$ & $\mathrm{X}$ & $\mathrm{X}$ & $\mathrm{X}$ \\
\hline FF104 & Wolf, D. W. & $\begin{array}{l}\text { Integrated Mesoscale Approach for the } \\
\text { Simulation of Nuclear-Fuel Behavior }\end{array}$ & & & $\mathrm{X}$ & \\
\hline FF105 & Meakin, $\mathrm{P}$. & $\begin{array}{l}\text { Particle-Discrete Element Model Simulation } \\
\text { of the Coupling Between Material } \\
\text { Failure/Deformation and Fluid } \\
\text { Generation/Flow }\end{array}$ & $\mathrm{X}$ & $X$ & $\mathrm{X}$ & $\mathrm{X}$ \\
\hline FF106 & Smartt, H. B. & Scaling of Welding Processes & $\mathrm{X}$ & & $\mathrm{X}$ & \\
\hline FF107 & Stone, M. L. & Enhanced Metal Ion Analysis & & $\mathrm{X}$ & $\mathrm{X}$ & $\mathrm{X}$ \\
\hline FF108 & Apel, W. A. & $\begin{array}{l}\text { Effect of Glycosylation on the Activity and } \\
\text { Stability of Bacterial Enzymes }\end{array}$ & $\mathrm{X}$ & & $\mathrm{X}$ & \\
\hline GB103 & Apel, W. A. & $\begin{array}{l}\text { A Systems Biology Approach to } \\
\text { Understanding Lignocellulose Derived } \\
\text { Carbon Metabolism by Alicyclobacillus } \\
\text { acidocaldarius }\end{array}$ & $\mathrm{X}$ & $\mathrm{X}$ & $\mathrm{X}$ & $\mathrm{X}$ \\
\hline GB104 & Lee, B. D. & $\begin{array}{l}\text { Metabolic Engineering of Alicyclobacillus } \\
\text { acidocaldarius for Lactic Acid Production } \\
\text { from Biomass Derived Monosaccharides }\end{array}$ & $X$ & $X$ & $X$ & $X$ \\
\hline GB105 & Lee, B. D. & $\begin{array}{l}\text { Network Interaction In the Thermoacidophile } \\
\text { Alicyclobacillus acidocaldarius In Response } \\
\text { to Different Complex Carbon Sources }\end{array}$ & $\mathrm{X}$ & $\mathrm{X}$ & $\mathrm{X}$ & $\mathrm{X}$ \\
\hline GS101 & Robertson, E. P. & Reducing CBM Water Discharge Volume & $\mathrm{X}$ & & & \\
\hline IC101 & Tolle, C. R. & $\begin{array}{l}\text { Exploration and Development of Automated } \\
\text { Differential Equation-Based System } \\
\text { Identification }\end{array}$ & $\mathrm{X}$ & $\mathrm{X}$ & $\mathrm{X}$ & $\mathrm{X}$ \\
\hline IC102 & Few, D. & $\begin{array}{l}\text { A Toolset for Proximal Human-Robot } \\
\text { Interaction }\end{array}$ & & & $\mathrm{X}$ & $\mathrm{X}$ \\
\hline IC104 & Nichol, C. I. & Haptic Interface for Robotic Arc Welding & $\mathrm{X}$ & $\mathrm{X}$ & $\mathrm{X}$ & $\mathrm{X}$ \\
\hline IC105 & McGillivary, K. E. & $\begin{array}{l}\text { Modeling Interface to Control System } \\
\text { Designs }\end{array}$ & $X$ & & & $X$ \\
\hline IC106 & Vollmer, D. T. & Resilient Control System Network Agents & $\mathrm{X}$ & & $\mathrm{X}$ & $\mathrm{X}$ \\
\hline IC107 & Cherry, S. J. & Integrated Control System Data Fusion & $\mathrm{X}$ & & & $\mathrm{X}$ \\
\hline IC108 & Buttles, J. W. & Wireless Sensor Testing & $\mathrm{X}$ & $\mathrm{X}$ & $\mathrm{X}$ & $\mathrm{X}$ \\
\hline
\end{tabular}




\section{Appendix E - Relevance to Major DOE Missions}

\begin{tabular}{|c|c|c|c|c|c|c|}
\hline $\begin{array}{l}\text { Project } \\
\text { ID }\end{array}$ & $\begin{array}{l}\text { Principal } \\
\text { Investigator }\end{array}$ & Title & 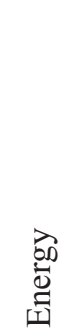 & 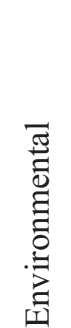 & 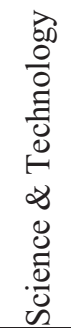 & 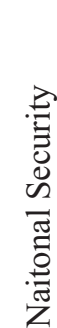 \\
\hline IC109 & Rieger, C. G. & $\begin{array}{l}\text { Anomaly Detection, Diagnosis, and Resilient } \\
\text { Control in Complex Engineered Systems }\end{array}$ & $\mathrm{X}$ & & $\mathrm{X}$ & $\mathrm{X}$ \\
\hline NE146 & Tranter, T. J. & $\begin{array}{l}\text { Process Modeling of Solvent Extraction } \\
\text { Separations for Advanced Nuclear Fuel } \\
\text { Cycles }\end{array}$ & $\mathrm{X}$ & $\mathrm{X}$ & $\mathrm{X}$ & $\mathrm{X}$ \\
\hline NE147 & Mincher, B. J. & $\begin{array}{l}\text { Reactivity of Radiolytically Produced } \\
\text { Nitrogen Oxide Radicals toward Aromatic } \\
\text { Compounds }\end{array}$ & $\mathrm{X}$ & $\mathrm{X}$ & $\mathrm{X}$ & $\mathrm{X}$ \\
\hline NE149 & Nigg, D. W. & $\begin{array}{l}\text { Modernization/Optimization of the } \\
\text { Advanced Test Reactor's Core-Analysis } \\
\text { Capability to Facilitate Its Operation as a } \\
\text { National Scientific User Facility }\end{array}$ & $\mathrm{X}$ & & $\mathrm{X}$ & $\mathrm{X}$ \\
\hline NE150 & Rempe, J. L. & $\begin{array}{l}\text { Viability Evaluations of Linear Variable } \\
\text { Differential Transformers (LVDTs) and } \\
\text { Capacitive Micro-Machined Ultrasonic } \\
\text { Transducers (CMUTs) for In-Pile } \\
\text { Instrumentation }\end{array}$ & $\mathrm{X}$ & & & $\mathrm{X}$ \\
\hline NE153 & Rabiti, C. & $\begin{array}{l}\text { Reactor Physics Sensitivity Analysis, } \\
\text { Uncertainty Quantification, and Data } \\
\text { Assimilation Capability }\end{array}$ & $\mathrm{X}$ & & $\mathrm{X}$ & \\
\hline NE154 & Williamson, R. L. & $\begin{array}{l}\text { Fracture Methods for Reactor Fuel } \\
\text { Performance Analysis }\end{array}$ & $\mathrm{X}$ & & & \\
\hline NE155 & Zhao, H. & $\begin{array}{l}\text { Use of Ice Thermal Storage Systems to } \\
\text { Improve LWR Plant Efifciency }\end{array}$ & $\mathrm{X}$ & $\mathrm{X}$ & & \\
\hline NE156 & Dinh, N. T. & $\begin{array}{l}\text { Development of a Next-Generation } \\
\text { Production Code for Nuclear Reactor System } \\
\text { Analysis and Safety Margin Quantification }\end{array}$ & $\mathrm{X}$ & & $\mathrm{X}$ & \\
\hline NE157 & Herrmann, S. D. & $\begin{array}{l}\text { Characterization of a Consolidated } \\
\text { Electrochemical Technique for Separation } \\
\text { and Recovery of Actinides from Fission } \\
\text { Products }\end{array}$ & $\mathrm{X}$ & & & \\
\hline NE158 & Knudson, D. L. & $\begin{array}{l}\text { Advanced Instrumentation for In-pile } \\
\text { Detection of Thermal Conductivity }\end{array}$ & $\mathrm{X}$ & & & $\mathrm{X}$ \\
\hline NN110 & Groenewold, G. S. & $\begin{array}{l}\text { Chemical Signatures of Nuclear Proliferation } \\
\text { on Particles }\end{array}$ & & $\mathrm{X}$ & & $\mathrm{X}$ \\
\hline NN112 & Tranter, T. J. & Technetium Signature Analysis & & & & $\mathrm{X}$ \\
\hline NN113 & Chichester, D. L. & $\begin{array}{l}\text { Active Interrogation Die-away Assay } \\
\text { Development Program }\end{array}$ & $\mathrm{X}$ & & & $\mathrm{X}$ \\
\hline
\end{tabular}




\section{Appendix E - Relevance to Major DOE Missions}

\begin{tabular}{|c|c|c|c|c|c|c|}
\hline $\begin{array}{l}\text { Project } \\
\text { ID }\end{array}$ & $\begin{array}{l}\text { Principal } \\
\text { Investigator }\end{array}$ & Title & 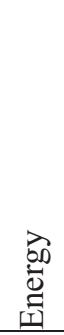 & 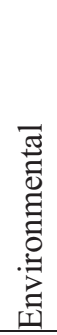 & 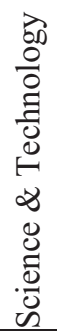 & 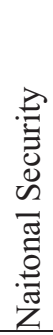 \\
\hline NN114 & McGrath, C. & $\begin{array}{l}135 \mathrm{Xe} \text { Recovery from the Spontaneous } \\
\text { Fission of } 252 \mathrm{Cf}\end{array}$ & & & & $X$ \\
\hline NN115 & Phillips, J. H. & $\begin{array}{l}\text { Developing a Next Generation, Risk- } \\
\text { Informed Approach for Robust and Resilient } \\
\text { Design Development (R2D2) }\end{array}$ & $\mathrm{X}$ & & & $\mathrm{X}$ \\
\hline NN116 & Chichester, D. L. & Neutron Spectrometer Development & $\mathrm{X}$ & $\mathrm{X}$ & $\mathrm{X}$ & $\mathrm{X}$ \\
\hline NN117 & Kinlaw, M. T. & $\begin{array}{l}\text { Nuclear Material Detection Using Neutron } \\
\text { Time-of-Flight }\end{array}$ & & & & $X$ \\
\hline NN118 & Li, S. X. & $\begin{array}{l}\text { On-line Monitoring of Actinide } \\
\text { Concentrations for Advanced Aqueous } \\
\text { Separation Processes }\end{array}$ & $X$ & & & \\
\hline NN119 & Metcalf, R. R. M. & $\begin{array}{l}\text { Develop a Safeguards Approach for INL } \\
\text { Pyroprocessing lines for Demonstration to } \\
\text { IAEA }\end{array}$ & $\mathrm{X}$ & & & $\mathrm{X}$ \\
\hline NN120 & Tranter, T. J. & $\begin{array}{l}\text { Radionuclide Collection-Detection Device } \\
\text { for the in situ Remote Monitoring of 99Tc as } \\
\text { a Proliferation Indicator }\end{array}$ & & $X$ & X & X \\
\hline NS156 & Lacy, J. M. & $\begin{array}{l}\text { Taylor Cylinder Determination of Impact } \\
\text { Material Properties }\end{array}$ & $\mathrm{X}$ & & $\mathrm{X}$ & $\mathrm{X}$ \\
\hline NS161 & Landon, M. D. & $\begin{array}{l}\text { Development and Evaluation of Low } \\
\text { Pressure Energy Absorbing Materials and } \\
\text { Methods for Buildings }\end{array}$ & $X$ & $X$ & & \\
\hline NS162 & Walsh, S. C. & $\begin{array}{l}\text { Modeling of Threats and Interdependencies } \\
\text { upon Strategic Infrastructures }\end{array}$ & & & & X \\
\hline NS163 & Chichester, D. L. & Fast Neutron Irradiation Capability & $\mathrm{X}$ & & $\mathrm{X}$ & $\mathrm{X}$ \\
\hline NS164 & Miller, C. J. & $\begin{array}{l}\text { Trace Explosive Characterization on } \\
\text { Materials }\end{array}$ & $\mathrm{X}$ & & & $X$ \\
\hline NS165 & Rieger, C. G. & $\begin{array}{l}\text { Methodologies for the Design, Analysis, and } \\
\text { Validation for Operation of Complex } \\
\text { Resilient Networks }\end{array}$ & $\mathrm{X}$ & $\mathrm{X}$ & $\mathrm{X}$ & $X$ \\
\hline NU100 & Gertman, D. I. & $\begin{array}{l}\text { Human Performance Assessment for } \\
\text { Technology Neutral Evaluation: Combining } \\
\text { Virtual and Physical Testing for Design, } \\
\text { Development and Review of Digital Control } \\
\text { Systems and Interfaces }\end{array}$ & $\mathrm{X}$ & & & \\
\hline NU101 & Lessing, P. A. & Advanced Ceramic Nuclear Fuels & $\mathrm{X}$ & & & \\
\hline
\end{tabular}




\section{Appendix E - Relevance to Major DOE Missions}

\begin{tabular}{|c|c|c|c|c|c|c|}
\hline $\begin{array}{l}\text { Project } \\
\text { ID }\end{array}$ & $\begin{array}{l}\text { Principal } \\
\text { Investigator }\end{array}$ & Title & 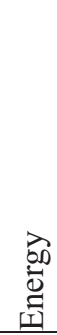 & 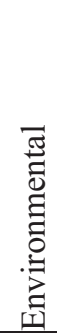 & 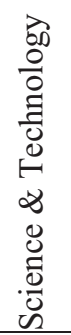 & 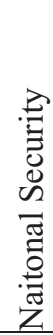 \\
\hline PH101 & Deaton, J. D. & $\begin{array}{l}\text { Cognitive Network Engine and Simulation } \\
\text { Framework,Ph.D. Candidate Proposal for } \\
\text { Juan Deaton }\end{array}$ & $\mathrm{X}$ & $\mathrm{X}$ & & $\mathrm{X}$ \\
\hline PH102 & Kelly, D. L. & $\begin{array}{l}\text { Application of Dynamic Bayesian Networks } \\
\text { to Systems with Ambient Intelligence }\end{array}$ & $X$ & & & $X$ \\
\hline PH103 & Marshall, D. W. & $\begin{array}{l}\text { Characterization of Fluidized Beds via } \\
\text { Pressure-Fluctuation Analysis }\end{array}$ & $X$ & & & \\
\hline PH104 & Harris, B. K. & $\begin{array}{l}\text { Dissolution and Extraction Studies of Fission } \\
\text { Products in Room Temperature Ionic Liquids } \\
\text { and in Supercritical Fluid CO2 and } \\
\text { Determination of Radiolytic Stability }\end{array}$ & $\mathrm{X}$ & $\mathrm{X}$ & $\mathrm{X}$ & $\mathrm{X}$ \\
\hline PH105 & McJunkin, T. R. & $\begin{array}{l}\text { Advanced Adaptive Algorithms in Phased } \\
\text { Array Ultrasonics for Materials Inspection }\end{array}$ & $\mathrm{X}$ & $\mathrm{X}$ & $X$ & $X$ \\
\hline RP110 & Rempe, J. L. & $\begin{array}{l}\text { High Temperature In-Pile Instrumentation } \\
\text { Enhancements }\end{array}$ & $\mathrm{X}$ & & & $\mathrm{X}$ \\
\hline SH100 & Wolf, D. W. & $\begin{array}{l}\text { Microstructure and Deformation Physics of } \\
\text { Fission-Reactor Model Materials by } \\
\text { Atomistically Informed Mesoscale } \\
\text { Simulation }\end{array}$ & & & $\mathrm{X}$ & \\
\hline SH101 & Rashkeev, S. & $\begin{array}{l}\text { Structural and Electronic Properties of } \\
\text { Adsorbed Metal Nanoparticles }\end{array}$ & $\mathrm{X}$ & $\mathrm{X}$ & $\mathrm{X}$ & $\mathrm{X}$ \\
\hline SH103 & Pernice, M. A. & $\begin{array}{l}\text { Adaptive Modeling of Geometrically } \\
\text { Complex Fuel Rods with a posteriori Error } \\
\text { Control }\end{array}$ & $X$ & $X$ & $X$ & \\
\hline SH104 & Knoll, D. & $\begin{array}{l}\text { Multi-physics Simulation Methods for } \\
\text { Advanced Reactor Analysis }\end{array}$ & $\mathrm{X}$ & $\mathrm{X}$ & $\mathrm{X}$ & $\mathrm{X}$ \\
\hline ST130 & Stewart, F. F. & $\begin{array}{l}\text { Investigation of Low Temperature } \\
\text { Performance in Membrane Materials and } \\
\text { Processes for Gas Separations }\end{array}$ & $\mathrm{x}$ & & $X$ & \\
\hline ST131 & Klaehn, J. R. & $\begin{array}{l}\text { High-Performance Polymer Membranes for } \\
\text { High Temperature Gas Separations }\end{array}$ & $\mathrm{X}$ & $\mathrm{X}$ & $\mathrm{X}$ & $\mathrm{X}$ \\
\hline ST132 & Benson, M. T. & Triazine-Based CO2 Capture Agents & $\mathrm{x}$ & & $\mathrm{X}$ & \\
\hline ST133 & Lee, R. D. & $\begin{array}{l}\text { Dynamic Impact Model and Information } \\
\text { System to support Unconventional Fuels } \\
\text { Development }\end{array}$ & $X$ & $\mathrm{X}$ & $\mathrm{X}$ & $\mathrm{X}$ \\
\hline ST134 & Wood, T. R. & $\begin{array}{l}\text { Near Field Impacts of In-Situ Oil Shale } \\
\text { Development on Water Quality- }\end{array}$ & $X$ & $X$ & $X$ & $X$ \\
\hline
\end{tabular}




\section{Appendix E - Relevance to Major DOE Missions}

\begin{tabular}{|c|c|c|c|c|c|c|}
\hline $\begin{array}{l}\text { Project } \\
\text { ID }\end{array}$ & $\begin{array}{l}\text { Principal } \\
\text { Investigator }\end{array}$ & Title & 悹 & 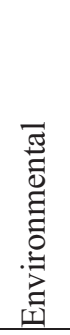 & 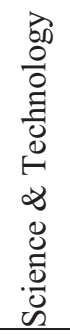 & 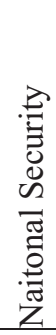 \\
\hline ST135 & Anderson, M. O. & $\begin{array}{l}\text { Advanced Remote Sensing for Energy and } \\
\text { Environmental Applications using } \\
\text { Unmanned Aerial Vehicles }\end{array}$ & $X$ & $X$ & $X$ & $X$ \\
\hline ST136 & Hurley, D. H. & $\begin{array}{l}\text { Addressing the Spectrum of Nuclear Related } \\
\text { NDE Needs: A hybrid laser ultrasonic and } \\
\text { eddy current approach }\end{array}$ & $X$ & & & \\
\hline ST137 & Windes, W. E. & $\begin{array}{l}\text { Research of Advanced VHTR Core } \\
\text { Components }\end{array}$ & $X$ & & $X$ & \\
\hline ST138 & Breckenridge, R. P. & $\begin{array}{l}\text { Development of a Sustainable Ecological } \\
\text { Engineering approach to support Hybrid } \\
\text { energy Systems Development in the Western } \\
\text { Inland Energy Corridor }\end{array}$ & $\mathrm{X}$ & $\mathrm{X}$ & $\mathrm{X}$ & $X$ \\
\hline TM106 & Huang, $\mathrm{H}$. & $\begin{array}{l}\text { Development of 3D Multiphase Flow and } \\
\text { Reactive Transport Codes and their } \\
\text { Applications to Reactive Flow in Porous } \\
\text { Media and Fracture Apertures }\end{array}$ & $\mathrm{X}$ & $X$ & $X$ & \\
\hline TM108 & Gaston, D. R. & $\begin{array}{l}\text { Uncertainty Quantification for Nuclear Fuels } \\
\text { Performance }\end{array}$ & $X$ & $X$ & $X$ & \\
\hline
\end{tabular}




\section{Appendix E - Relevance to Major DOE Missions}


Appendix F -

Relevance to Major National Programs 


\section{Appendix F - Relevance to Major National Programs}

\begin{tabular}{|c|c|c|c|c|c|c|c|c|c|c|c|c|c|}
\hline Project ID & $\begin{array}{l}\text { Principle } \\
\text { Investigator }\end{array}$ & Title & 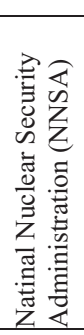 & 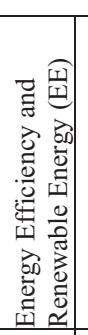 & 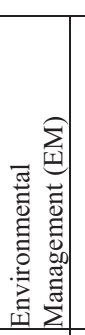 & 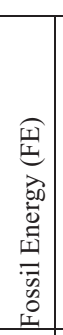 & 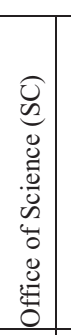 & 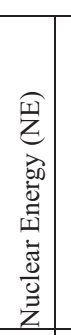 & 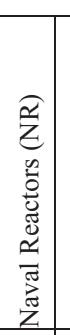 & 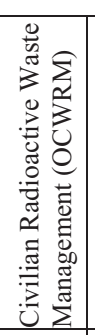 & 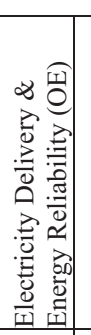 & 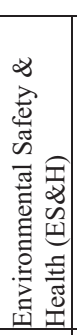 & 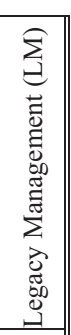 \\
\hline AE117 & Law, J. D. & $\begin{array}{l}\text { Group Actinide } \\
\text { Separation from } \\
\text { Spent Nuclear Fuel } \\
\text { Using a Modified } \\
\text { Universal Solvent } \\
\text { Extraction Process }\end{array}$ & & & $\mathrm{S}$ & & $\mathrm{S}$ & $\mathrm{P}$ & & $\mathrm{S}$ & & & \\
\hline AE118 & Marsden, K. C. & $\begin{array}{l}\text { Exploration of } \\
\text { Electrolyte } \\
\text { Complexation and } \\
\text { Pulse Deposition for } \\
\text { Production of Dense } \\
\text { Uranium Rodlets }\end{array}$ & & & & & & $\mathrm{P}$ & & & & & \\
\hline AE119 & Cogliati, J. J. & $\begin{array}{l}\text { Multi-reactor design } \\
\text { and analysis platform }\end{array}$ & & & & & $\mathrm{S}$ & $\mathrm{P}$ & & & & & \\
\hline AE121 & $\begin{array}{l}\text { Martineau, R. } \\
\text { C. }\end{array}$ & $\begin{array}{l}\text { A General } \\
\text { Framework for } \\
\text { Simulating Fully- } \\
\text { Coupled Mass and } \\
\text { Energy Transport } \\
\text { Phenomena in } \\
\text { Nuclear Energy } \\
\text { Systems }\end{array}$ & & $\mathrm{S}$ & & & $\mathrm{S}$ & $\mathrm{P}$ & & & & & \\
\hline AE122 & $\begin{array}{l}\text { Mousseau, V. } \\
\text { A. }\end{array}$ & $\begin{array}{l}\text { System Analysis for } \\
\text { Reactor Applications } \\
\text { with High Fidelity }\end{array}$ & & & & & $\mathrm{S}$ & $\mathrm{P}$ & & & & & \\
\hline AE123 & Rabiti, C. & $\begin{array}{l}\text { Acquisition and } \\
\text { Improvement of a } \\
\text { Modern Lattice } \\
\text { Physics Capability }\end{array}$ & S & & & & & $\mathrm{P}$ & & $\mathrm{S}$ & & & \\
\hline AE124 & Berry, R. A. & $\begin{array}{l}\text { Unified Two-phase } \\
\text { CFD Modeling of } \\
\text { Boiling, Cavitation, } \\
\text { and Bubble Collapse }\end{array}$ & $\underline{S}$ & & & & $\mathrm{~S}$ & $\mathrm{P}$ & $\mathrm{S}$ & & & & \\
\hline AF 103 & Wright, R. N. & $\begin{array}{l}\text { Environmental } \\
\text { Effects on Crack } \\
\text { Growth in High- } \\
\text { Temperature Alloys } \\
\text { for Advanced Energy } \\
\text { Systems }\end{array}$ & & & & $\mathrm{S}$ & $\mathrm{S}$ & $\mathrm{P}$ & & & & & \\
\hline AF 104 & Hilton, B. A. & $\begin{array}{l}\text { Development of } \\
\text { Advanced Burnup } \\
\text { Measurement \& } \\
\text { Nuclear Forensics } \\
\text { ICP-MS Isotopics } \\
\text { Analysis Techniques }\end{array}$ & S & & & & & $\mathrm{P}$ & & & & & \\
\hline
\end{tabular}




\section{Appendix F - Relevance to Major National Programs}

\begin{tabular}{|c|c|c|c|c|c|c|c|c|c|c|c|c|c|}
\hline Project ID & $\begin{array}{l}\text { Principle } \\
\text { Investigator }\end{array}$ & Title & 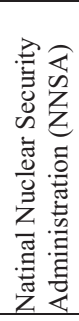 & 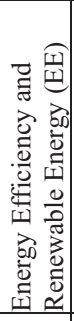 & 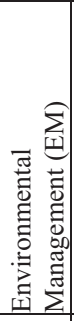 & 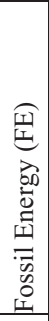 & 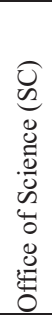 & 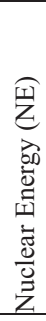 & 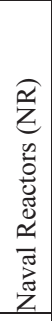 & 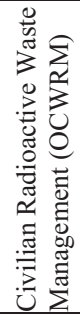 & 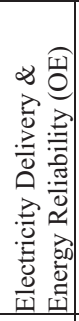 & 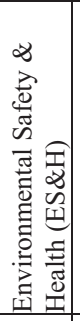 & 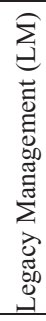 \\
\hline AF105 & Cole, J. I. & $\begin{array}{l}\text { Development of a } \\
\text { Small Sample } \\
\text { Volume Mechanical } \\
\text { Properties Testing } \\
\text { Technique for } \\
\text { Irradiated Fuels and } \\
\text { Materials }\end{array}$ & & & & & $\mathrm{S}$ & $\mathrm{P}$ & $\mathrm{S}$ & & & & \\
\hline AS102 & Mincher, B. J. & $\begin{array}{l}\text { Utility of Unusual } \\
\text { Oxidation States of } \\
\text { Americium for } \\
\text { Separations }\end{array}$ & $\mathrm{P}$ & & $\mathrm{S}$ & & & $\mathrm{S}$ & & & & & \\
\hline AS105 & Pepper, S. E. & $\begin{array}{l}\text { Rapid Detection of } \\
\text { Plutonium, } \\
\text { Neptunium and } \\
\text { Technetium in Water } \\
\text { Samples }\end{array}$ & $\mathrm{P}$ & & & & $\mathrm{S}$ & $\mathrm{S}$ & & & & & \\
\hline AS106 & Peterman, D. R. & $\begin{array}{l}\text { Evaluation of } \\
\text { Covalent Interactions } \\
\text { in Actinide } \\
\text { Coordination } \\
\text { Compounds }\end{array}$ & $\mathrm{S}$ & & $\mathrm{S}$ & & $\mathrm{S}$ & $\mathrm{P}$ & & $\mathrm{S}$ & & & \\
\hline AS107 & $\begin{array}{l}\text { Groenewold, G. } \\
\text { S. }\end{array}$ & $\begin{array}{l}\text { Measuring Actinide } \\
\text { Speciation in High } \\
\text { pH Solutions }\end{array}$ & & & $\mathrm{S}$ & & $\mathrm{P}$ & $\mathrm{S}$ & & & & & \\
\hline AS108 & Scott, J. R. & $\begin{array}{l}\text { Isotope Ratio } \\
\text { Measurements } \\
\text { Methods for Direct } \\
\text { Analysis of Samples }\end{array}$ & $\mathrm{P}$ & $\mathrm{S}$ & $\mathrm{S}$ & & $\mathrm{S}$ & $\mathrm{S}$ & $\mathrm{S}$ & & & & $\mathrm{S}$ \\
\hline BS103 & Lacy, J. M. & $\begin{array}{l}\text { Multi-Stage } \\
\text { Sequential Injection } \\
\text { Gas Gun }\end{array}$ & $P$ & & & & $\mathrm{~S}$ & & & & & & \\
\hline BS104 & Newman, C. K. & $\begin{array}{l}\text { A Strategy to Tightly } \\
\text { Couple Neutronics } \\
\text { and Thermal- } \\
\text { Hydraulics Models } \\
\text { for Next Generation } \\
\text { Reactor Analysis }\end{array}$ & & & & & $\mathrm{S}$ & $\mathrm{P}$ & & & & & \\
\hline BS105 & Lacey, J. A. & $\begin{array}{l}\text { Thermal and Acid } \\
\text { Activated in Planta } \\
\text { Lignocellulose- } \\
\text { Degrading System } \\
\text { for Economically } \\
\text { Enhanced Industrial } \\
\text { Processes }\end{array}$ & & $\mathrm{P}$ & & & $\mathrm{S}$ & & & & & & \\
\hline BS106 & $\begin{array}{l}\text { Thompson, D. } \\
\text { N. }\end{array}$ & $\begin{array}{l}\text { Novel Ccatalysts for } \\
\text { the Reduction of } \\
\text { Biomass } \\
\text { Pretreatment Severity }\end{array}$ & & $\mathrm{P}$ & & & $\mathrm{S}$ & & & & & & \\
\hline
\end{tabular}




\section{Appendix F - Relevance to Major National Programs}

\begin{tabular}{|c|c|c|c|c|c|c|c|c|c|c|c|c|c|}
\hline Project ID & $\begin{array}{c}\text { Principle } \\
\text { Investigator }\end{array}$ & Title & 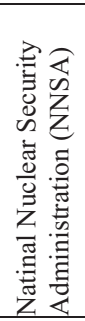 & 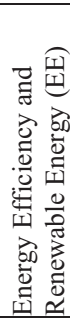 & 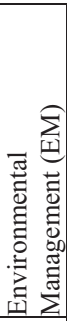 & 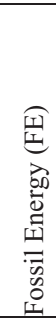 & 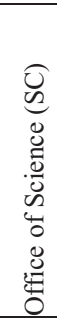 & 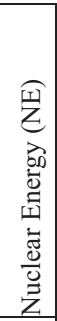 & 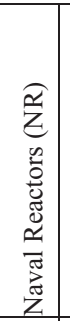 & 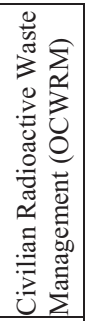 & 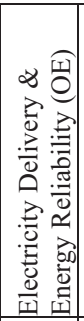 & 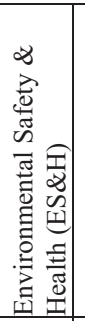 & 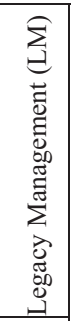 \\
\hline CA104 & Carroll, M. C. & $\begin{array}{l}\text { Microstructural } \\
\text { Evolution During } \\
\text { Spark Plasma } \\
\text { Sintering of High- } \\
\text { Temperature Fuels } \\
\text { and Coatings }\end{array}$ & & & $\mathrm{S}$ & $\mathrm{S}$ & $\mathrm{S}$ & $\mathrm{P}$ & & $\mathrm{S}$ & & & S \\
\hline CA105 & McLing, T. L. & $\begin{array}{l}\text { Suitability of } \\
\text { Layered Basalt as } \\
\text { Targets for Industrial } \\
\text { Carbon Dioxide } \\
\text { Sequestration }\end{array}$ & & & & $\mathrm{P}$ & & & & & $\mathrm{S}$ & $\mathrm{S}$ & \\
\hline CA108 & Ulrich, T. H. & $\begin{array}{l}\text { Understanding } \\
\text { Apomixis: The Basis } \\
\text { for a Robust Trait } \\
\text { Delivery and } \\
\text { Containment } \\
\text { Platform for } \\
\text { Bioenergy Crops } \\
\end{array}$ & & $\mathrm{P}$ & $\mathrm{S}$ & & $\mathrm{S}$ & & & & & & \\
\hline CA114 & Todd, T. A. & $\begin{array}{l}\text { Enhancement of } \\
\text { Separation Methods } \\
\text { in Nuclear Fuel } \\
\text { Recycling }\end{array}$ & & & & & & $\mathrm{P}$ & & & & & \\
\hline CA115 & Piet, S. J. & $\begin{array}{l}\text { Investigation of } \\
\text { Public Discourse } \\
\text { Methods in Energy } \\
\text { Policy Decision- } \\
\text { making }\end{array}$ & & $\mathrm{S}$ & $\mathrm{S}$ & & & $\mathrm{P}$ & & $\mathrm{S}$ & & & \\
\hline CA116 & Bala, G. A. & $\begin{array}{l}\text { Development of } \\
\text { Lignocellulosic } \\
\text { Ethanol Production } \\
\text { Potential in Idaho }\end{array}$ & & $P$ & & & & & & & & & \\
\hline EI101 & Stewart, F. F. & $\begin{array}{l}\text { Chemical Separations } \\
\text { and Process Research } \\
\text { to Enable Biorefinery } \\
\text { Systems }\end{array}$ & & $\mathrm{P}$ & & & $\mathrm{S}$ & & & & & & \\
\hline EI102 & Garcia, H. E. & $\begin{array}{l}\text { Advanced Predictive } \\
\text { Condition } \\
\text { Monitoring and } \\
\text { Control for Modern } \\
\text { Energy Systems: } \\
\text { Gasification-based } \\
\text { Processes }\end{array}$ & & $\mathrm{P}$ & $\mathrm{S}$ & $\mathrm{S}$ & & $\mathrm{S}$ & & & $\mathrm{S}$ & $\mathrm{S}$ & \\
\hline EI104 & Guillen, D. P. & $\begin{array}{l}\text { CFD-Based } \\
\text { Simulation } \\
\text { Capability of } \\
\text { Fischer-Tropsch } \\
\text { Reactors and Process } \\
\text { Equipment }\end{array}$ & & $\mathrm{P}$ & & $\mathrm{S}$ & & & & & & & \\
\hline
\end{tabular}




\section{Appendix F - Relevance to Major National Programs}

\begin{tabular}{|c|c|c|c|c|c|c|c|c|c|c|c|c|c|}
\hline Project ID & $\begin{array}{l}\text { Principle } \\
\text { Investigator }\end{array}$ & Title & 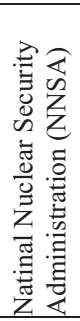 & 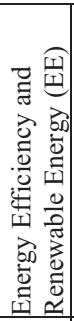 & 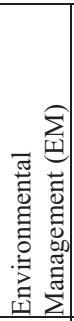 & 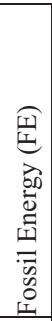 & 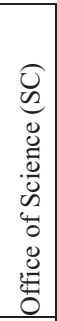 & 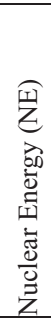 & 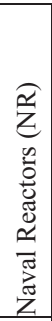 & 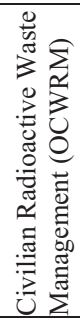 & 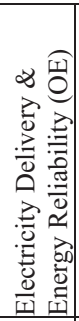 & 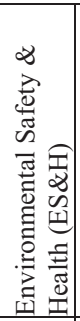 & 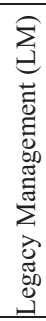 \\
\hline EI105 & Kenney, K. L. & $\begin{array}{l}\text { Biomass Feedstock } \\
\text { Assembly to } \\
\text { Gasification Process } \\
\text { Computational } \\
\text { Interface } \\
\text { Development }\end{array}$ & & $\mathrm{P}$ & & & & & & & & & \\
\hline EI107 & Palmer, C. D. & $\begin{array}{l}\text { Generation and } \\
\text { Expulsion of } \\
\text { Hydrocarbons from } \\
\text { Oil Shale }\end{array}$ & & & & $\mathrm{P}$ & $\mathrm{S}$ & & & & & & \\
\hline EI109 & Cooper, D. C. & $\begin{array}{l}\text { Pathways to Energy } \\
\text { Independence }\end{array}$ & & $P$ & & $\mathrm{~S}$ & $\mathrm{~S}$ & $\mathrm{~S}$ & & & $\mathrm{~S}$ & & \\
\hline EI110 & Petkovic, L. M. & $\begin{array}{l}\text { Investigation of } \\
\text { Fischer Tropsch } \\
\text { Catalyst Deactivation }\end{array}$ & & $\mathrm{P}$ & & $\mathrm{S}$ & $\mathrm{S}$ & & & & & & \\
\hline EI111 & $\begin{array}{l}\text { Robertson, E. } \\
\text { P. }\end{array}$ & $\begin{array}{l}\text { Altering Wettability } \\
\text { by Chemical } \\
\text { Amendments to } \\
\text { Improve Gas } \\
\text { Production from } \\
\text { Tight Sands }\end{array}$ & & & $\mathrm{S}$ & $\mathrm{P}$ & & & & & & & \\
\hline EI112 & Shunn, L. P. & $\begin{array}{l}\text { Hybrid Energy } \\
\text { System Neural } \\
\text { Reactance Dynamic } \\
\text { Control System }\end{array}$ & & & & $\mathrm{S}$ & & $\mathrm{P}$ & & & & & \\
\hline FF104 & Wolf, D. W. & $\begin{array}{l}\text { Integrated Mesoscale } \\
\text { Approach for the } \\
\text { Simulation of } \\
\text { Nuclear-Fuel } \\
\text { Behavior }\end{array}$ & & & & & $\mathrm{P}$ & $\mathrm{S}$ & & & & & \\
\hline FF105 & Meakin, P. & $\begin{array}{l}\text { Particle-Discrete } \\
\text { Element Model } \\
\text { Simulation of the } \\
\text { Coupling between } \\
\text { Material } \\
\text { Failure/Deformation } \\
\text { and Fluid } \\
\text { Generation/Flow } \\
\end{array}$ & & & $\mathrm{S}$ & $\mathrm{P}$ & $\mathrm{S}$ & & & & & & \\
\hline FF106 & Smartt, H. B. & $\begin{array}{l}\text { Scaling of Welding } \\
\text { Processes }\end{array}$ & & $\mathrm{P}$ & & $\mathrm{S}$ & $\mathrm{S}$ & $\mathrm{S}$ & & & & & \\
\hline FF107 & Stone, M. L. & $\begin{array}{l}\text { Enhanced Metal Ion } \\
\text { Analysis }\end{array}$ & $\mathrm{P}$ & & $\mathrm{S}$ & & $\mathrm{S}$ & $\mathrm{S}$ & & & & $S$ & \\
\hline FF108 & Apel, W. A. & $\begin{array}{l}\text { Effect of } \\
\text { Glycosylation on the } \\
\text { Activity and Stability } \\
\text { of Bacterial Enzymes }\end{array}$ & & $\mathrm{S}$ & & & $P$ & & & & & & \\
\hline
\end{tabular}




\section{Appendix F - Relevance to Major National Programs}

\begin{tabular}{|c|c|c|c|c|c|c|c|c|c|c|c|c|c|}
\hline Project ID & $\begin{array}{l}\text { Principle } \\
\text { Investigator }\end{array}$ & Title & 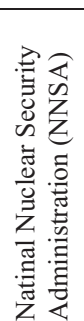 & 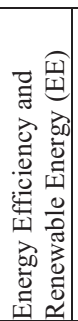 & 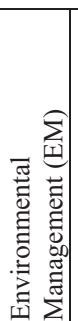 & 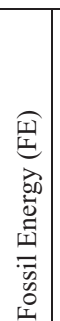 & 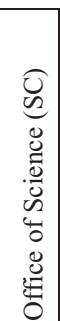 & 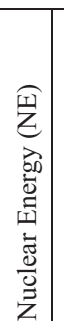 & 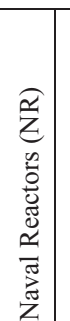 & 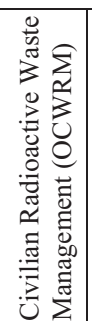 & 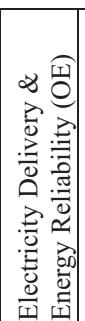 & 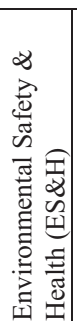 & 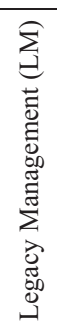 \\
\hline GB103 & Apel, W. A. & $\begin{array}{l}\text { A Systems Biology } \\
\text { Approach to } \\
\text { Understanding } \\
\text { Lignocellulose } \\
\text { Derived Carbon } \\
\text { Metabolism by } \\
\text { Alicyclobacillus } \\
\text { acidocaldarius }\end{array}$ & & $\mathrm{P}$ & & $\mathrm{S}$ & $\mathrm{S}$ & & & & & & \\
\hline GB104 & Lee, B. D. & $\begin{array}{l}\text { Metabolic } \\
\text { Engineering of } \\
\text { Alicyclobacillus } \\
\text { acidocaldarius for } \\
\text { Lactic Acid } \\
\text { Production from } \\
\text { Biomass Derived } \\
\text { Monosaccharides }\end{array}$ & & $\mathrm{P}$ & & & $\mathrm{S}$ & & & & & & \\
\hline GB105 & Lee, B. D. & $\begin{array}{l}\text { Network Interaction } \\
\text { In the } \\
\text { Thermoacidophile } \\
\text { Alicyclobacillus } \\
\text { acidocaldarius In } \\
\text { Response to } \\
\text { Different Complex } \\
\text { Carbon Sources }\end{array}$ & & $\mathrm{P}$ & & & $\mathrm{S}$ & & & & & & \\
\hline GS101 & $\begin{array}{l}\text { Robertson, E. } \\
\text { P. }\end{array}$ & $\begin{array}{l}\text { Reducing CBM } \\
\text { Water Discharge } \\
\text { Volume }\end{array}$ & & & $\mathrm{S}$ & $\mathrm{P}$ & & & & & & & \\
\hline IC101 & Tolle, C. R. & $\begin{array}{l}\text { Exploration and } \\
\text { Development of } \\
\text { Automated } \\
\text { Differential } \\
\text { Equation-Based } \\
\text { System Identification }\end{array}$ & $\mathrm{S}$ & $\mathrm{S}$ & $\mathrm{S}$ & $\mathrm{S}$ & $P$ & $\mathrm{~s}$ & & & $\mathrm{~S}$ & & \\
\hline IC102 & Few, D. & $\begin{array}{l}\text { A Toolset for } \\
\text { Proximal Human- } \\
\text { Robot Interaction }\end{array}$ & $\mathrm{S}$ & & $\mathrm{S}$ & & $P$ & $\mathrm{~S}$ & & & & & \\
\hline IC104 & Nichol, C. I. & $\begin{array}{l}\text { Haptic Interface for } \\
\text { Robotic Arc Welding }\end{array}$ & & & S & & $\mathrm{S}$ & $\mathrm{P}$ & $\mathrm{S}$ & $\mathrm{S}$ & & & $\mathrm{S}$ \\
\hline IC105 & $\begin{array}{l}\text { McGillivary, K. } \\
\text { E. }\end{array}$ & $\begin{array}{l}\text { Modeling Interface to } \\
\text { Control System } \\
\text { Designs }\end{array}$ & $\mathrm{S}$ & & & & $\mathrm{S}$ & $\mathrm{S}$ & & & $\mathrm{P}$ & & \\
\hline IC106 & Vollmer, D. T. & $\begin{array}{l}\text { Resilient Control } \\
\text { System Network } \\
\text { Agents }\end{array}$ & $\mathrm{S}$ & $\mathrm{S}$ & & & & $\mathrm{S}$ & & & $\mathrm{p}$ & & \\
\hline IC107 & Cherry, S. J. & $\begin{array}{l}\text { Integrated Control } \\
\text { System Data Fusion }\end{array}$ & & & & & $\mathrm{S}$ & $\mathrm{S}$ & & & $\mathrm{P}$ & & \\
\hline IC108 & Buttles, J. W. & $\begin{array}{l}\text { Wireless Sensor } \\
\text { Testing }\end{array}$ & $\mathrm{S}$ & & $\mathrm{S}$ & & & $\mathrm{P}$ & & & $\mathrm{S}$ & $\underline{S}$ & \\
\hline
\end{tabular}




\section{Appendix F - Relevance to Major National Programs}

\begin{tabular}{|c|c|c|c|c|c|c|c|c|c|c|c|c|c|}
\hline Project ID & $\begin{array}{c}\text { Principle } \\
\text { Investigator }\end{array}$ & Title & 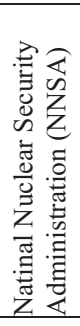 & 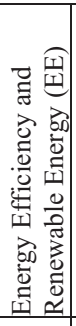 & 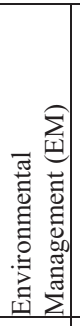 & 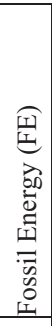 & 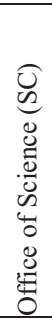 & 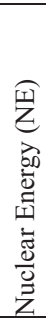 & 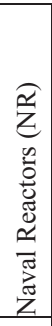 & 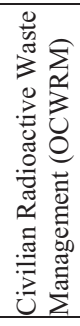 & 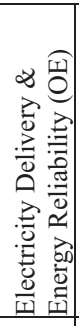 & 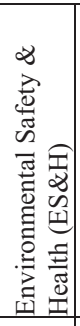 & 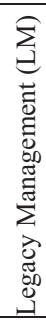 \\
\hline IC109 & Rieger, C. G. & $\begin{array}{l}\text { Anomaly Detection, } \\
\text { Diagnosis, and } \\
\text { Resilient Control in } \\
\text { Complex Engineered } \\
\text { Systems }\end{array}$ & & $\mathrm{S}$ & & $\mathrm{S}$ & $\mathrm{S}$ & $\mathrm{P}$ & & & $\mathrm{S}$ & $\mathrm{S}$ & \\
\hline NE146 & Tranter, T. J. & $\begin{array}{l}\text { Process Modeling of } \\
\text { Solvent Extraction } \\
\text { Separations for } \\
\text { Advanced Nuclear } \\
\text { Fuel Cycles }\end{array}$ & $\mathrm{S}$ & & S & & & $\mathrm{P}$ & & S & & & \\
\hline NE147 & Mincher, B. J. & $\begin{array}{l}\text { Reactivity of } \\
\text { Radiolytically } \\
\text { Produced Nitrogen } \\
\text { Oxide Radicals } \\
\text { toward Aromatic } \\
\text { Compounds }\end{array}$ & $\mathrm{S}$ & & & & $\mathrm{S}$ & $\mathrm{P}$ & & & & & \\
\hline NE149 & Nigg, D. W. & $\begin{array}{l}\text { Modernization/Optim } \\
\text { ization of the } \\
\text { Advanced Test } \\
\text { Reactor's Core- } \\
\text { Analysis Capability } \\
\text { to Facilitate Its } \\
\text { Operation as a } \\
\text { National Scientific } \\
\text { User Facility }\end{array}$ & $\mathrm{S}$ & $\mathrm{S}$ & & & $\mathrm{S}$ & $\mathrm{P}$ & $\mathrm{S}$ & & & & \\
\hline NE150 & Rempe, J. L. & $\begin{array}{l}\text { Viability Evaluations } \\
\text { of Linear Variable } \\
\text { Differential } \\
\text { Transformers } \\
\text { (LVDTs) and } \\
\text { Capacitive Micro- } \\
\text { Machined Ultrasonic } \\
\text { Transducers } \\
\text { (CMUTs) for In-Pile } \\
\text { Instrumentation }\end{array}$ & & & & & & $\mathrm{S}$ & $\mathrm{P}$ & & & & \\
\hline NE153 & Rabiti, C. & $\begin{array}{l}\text { Reactor Physics } \\
\text { Sensitivity Analysis, } \\
\text { Uncertainty } \\
\text { Quantification, and } \\
\text { Data Assimilation } \\
\text { Capability }\end{array}$ & & & & & & $\mathrm{P}$ & & & & & \\
\hline NE154 & $\begin{array}{l}\text { Williamson, R. } \\
\text { L. }\end{array}$ & $\begin{array}{l}\text { Fracture Methods for } \\
\text { Reactor Fuel } \\
\text { Performance } \\
\text { Analysis } \\
\end{array}$ & & & & & & $\mathrm{P}$ & & & & & \\
\hline NE155 & Zhao, H. & $\begin{array}{l}\text { Use of Ice Thermal } \\
\text { Storage Systems to } \\
\text { Improve LWR Plant } \\
\text { Efficiency }\end{array}$ & & $\mathrm{S}$ & $\mathrm{S}$ & $\mathrm{S}$ & & $\mathrm{P}$ & & & & & \\
\hline
\end{tabular}




\section{Appendix F - Relevance to Major National Programs}

\begin{tabular}{|c|c|c|c|c|c|c|c|c|c|c|c|c|c|}
\hline Project ID & $\begin{array}{l}\text { Principle } \\
\text { Investigator }\end{array}$ & Title & 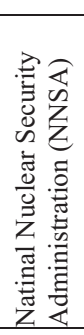 & 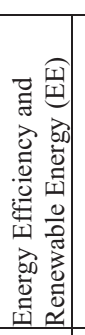 & 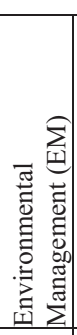 & 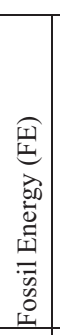 & 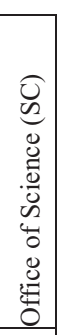 & 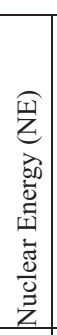 & 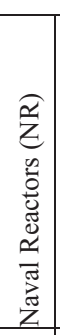 & 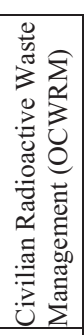 & 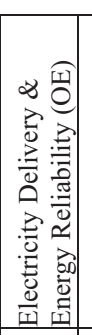 & 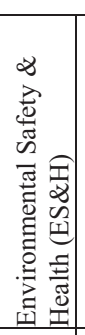 & 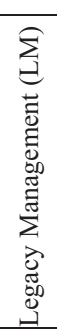 \\
\hline NE156 & Dinh, N. T. & $\begin{array}{l}\text { Development of a } \\
\text { Next-Generation } \\
\text { Production Code for } \\
\text { Nuclear Reactor } \\
\text { System Analysis and } \\
\text { Safety Margin } \\
\text { Quantification }\end{array}$ & & & & & & $\mathrm{P}$ & $\mathrm{S}$ & & & & \\
\hline NE157 & $\begin{array}{l}\text { Herrmann, S. } \\
\text { D. }\end{array}$ & $\begin{array}{l}\text { Characterization of a } \\
\text { Consolidated } \\
\text { Electrochemical } \\
\text { Technique for } \\
\text { Separation and } \\
\text { Recovery of } \\
\text { Actinides from } \\
\text { Fission Products }\end{array}$ & & & & & & $\mathrm{P}$ & & & & & \\
\hline NE158 & Knudson, D. L. & $\begin{array}{l}\text { Advanced } \\
\text { Instrumentation for } \\
\text { In-pile Detection of } \\
\text { Thermal } \\
\text { Conductivity }\end{array}$ & & & & & & $\mathrm{P}$ & $\mathrm{S}$ & & & & \\
\hline NN110 & $\begin{array}{l}\text { Groenewold, G. } \\
\text { S. }\end{array}$ & $\begin{array}{l}\text { Chemical Signatures } \\
\text { of Nuclear } \\
\text { Proliferation on } \\
\text { Particles }\end{array}$ & $\mathrm{P}$ & & & & & & & & & & \\
\hline NN112 & Tranter, T. J. & $\begin{array}{l}\text { Technetium } \\
\text { Signature Analysis }\end{array}$ & $\mathrm{P}$ & & & & & & & & & & \\
\hline NN113 & $\begin{array}{l}\text { Chichester, D. } \\
\text { L. }\end{array}$ & $\begin{array}{l}\text { Active Interrogation } \\
\text { Die-away Assay } \\
\text { Development } \\
\text { Program }\end{array}$ & P & & & & & $\mathrm{S}$ & & $\mathrm{S}$ & & & \\
\hline NN114 & McGrath, C. & $\begin{array}{l}\text { 135Xe recovery from } \\
\text { the spontaneous } \\
\text { fission of } 252 \mathrm{Cf}\end{array}$ & $\mathrm{P}$ & & & & & & & & & & \\
\hline NN115 & Phillips, J. H. & $\begin{array}{l}\text { Developing a Next } \\
\text { Generation, Risk- } \\
\text { Informed Approach } \\
\text { for Robust and } \\
\text { Resilient Design } \\
\text { Development (R2D2) }\end{array}$ & $\mathrm{S}$ & & & & & $\mathrm{P}$ & & & & & \\
\hline NN116 & $\begin{array}{l}\text { Chichester, D. } \\
\text { L. }\end{array}$ & $\begin{array}{l}\text { Neutron } \\
\text { Spectrometer } \\
\text { Development }\end{array}$ & $\mathrm{P}$ & & $\mathrm{S}$ & & $\mathrm{S}$ & $\mathrm{S}$ & $\mathrm{S}$ & $\mathrm{S}$ & & $\mathrm{S}$ & \\
\hline NN117 & Kinlaw, M. T. & $\begin{array}{l}\text { Nuclear Material } \\
\text { Detection Using } \\
\text { Neutron Time-of- } \\
\text { Flight }\end{array}$ & $\mathrm{P}$ & & & & & & & & & & \\
\hline
\end{tabular}




\section{Appendix F - Relevance to Major National Programs}

\begin{tabular}{|c|c|c|c|c|c|c|c|c|c|c|c|c|c|}
\hline Project ID & $\begin{array}{l}\text { Principle } \\
\text { Investigator }\end{array}$ & Title & 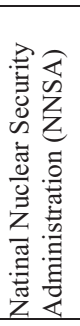 & 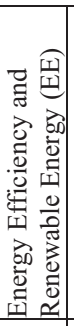 & 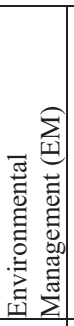 & 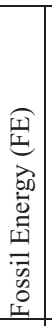 & 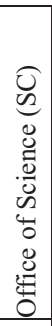 & 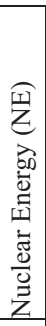 & 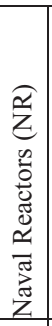 & 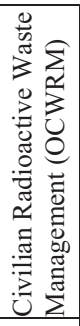 & 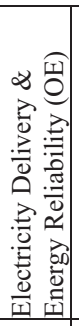 & 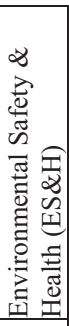 & 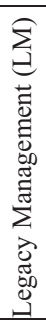 \\
\hline NN118 & Li, S. X. & $\begin{array}{l}\text { On-line Monitoring } \\
\text { of Actinide } \\
\text { Concentrations for } \\
\text { Advanced Aqueous } \\
\text { Separation Processes }\end{array}$ & $P$ & & & & & $\mathrm{~S}$ & & & & & \\
\hline NN118 & $\begin{array}{l}\text { Metcalf, R. R. } \\
\text { M. }\end{array}$ & $\begin{array}{l}\text { Develop a Safeguards } \\
\text { Approach for INL } \\
\text { Pyroprocessing lines } \\
\text { for Demonstration to } \\
\text { IAEA }\end{array}$ & $P$ & & & & & $\mathrm{~S}$ & & & & & \\
\hline NN120 & Tranter, T. J. & $\begin{array}{l}\text { Radionuclide } \\
\text { Collection-Detection } \\
\text { Device for the in situ } \\
\text { Remote Monitoring } \\
\text { of } 99 \mathrm{Tc} \text { as a } \\
\text { Proliferation } \\
\text { Indicator }\end{array}$ & $\mathrm{P}$ & & $\mathrm{S}$ & & $\mathrm{S}$ & $\mathrm{S}$ & & $\mathrm{S}$ & & $S$ & \\
\hline NS156 & Lacy, J. M. & $\begin{array}{l}\text { Taylor Cylinder } \\
\text { Determination of } \\
\text { Impact Material } \\
\text { Properties }\end{array}$ & $\mathrm{S}$ & & & & $\mathrm{S}$ & $\mathrm{P}$ & & & & & \\
\hline NS161 & Landon, M. D. & $\begin{array}{l}\text { Development and } \\
\text { Evaluation of Low } \\
\text { Pressure Energy } \\
\text { Absorbing Materials } \\
\text { and Methods for } \\
\text { Buildings }\end{array}$ & $\mathrm{S}$ & & & & $\mathrm{P}$ & & & & & S & \\
\hline NS162 & Walsh, S. C. & $\begin{array}{l}\text { Modeling of threats } \\
\text { and } \\
\text { interdependencies } \\
\text { upon strategic } \\
\text { infrastructures }\end{array}$ & & & & & $\mathrm{S}$ & & & & $\mathrm{P}$ & & \\
\hline NS163 & $\begin{array}{l}\text { Chichester, D. } \\
\text { L. }\end{array}$ & $\begin{array}{l}\text { Fast Neutron } \\
\text { Irradiation Capability }\end{array}$ & $\mathrm{P}$ & & $\mathrm{S}$ & & $\mathrm{S}$ & $\mathrm{S}$ & & $\mathrm{S}$ & & $\mathrm{S}$ & \\
\hline NS164 & Miller, C. J. & $\begin{array}{l}\text { Trace Explosive } \\
\text { Characterization on } \\
\text { Materials }\end{array}$ & $P$ & & & & & & & & & & \\
\hline NS165 & Rieger, C. G. & $\begin{array}{l}\text { Methodologies for } \\
\text { the Design, Analysis, } \\
\text { and Validation for } \\
\text { Operation of } \\
\text { Complex Resilient } \\
\text { Networks }\end{array}$ & & $S$ & & $\mathrm{~S}$ & $\mathrm{~S}$ & $\mathrm{~S}$ & & & $P$ & S & \\
\hline
\end{tabular}




\section{Appendix F - Relevance to Major National Programs}

\begin{tabular}{|c|c|c|c|c|c|c|c|c|c|c|c|c|c|}
\hline Project ID & $\begin{array}{l}\text { Principle } \\
\text { Investigator }\end{array}$ & Title & 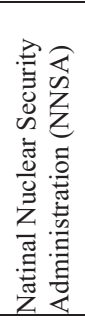 & 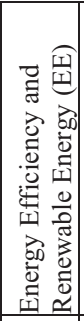 & 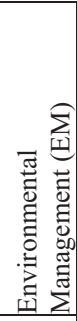 & 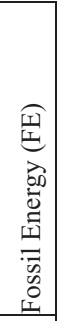 & 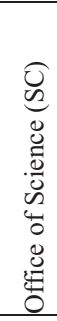 & 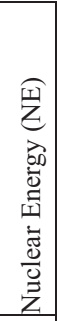 & 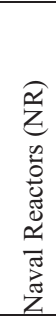 & 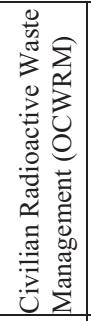 & 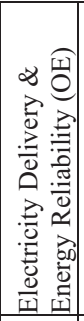 & 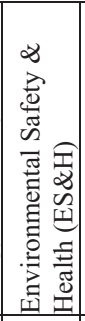 & 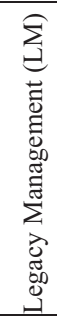 \\
\hline NU100 & Gertman, D. I. & $\begin{array}{l}\text { Human Performance } \\
\text { Assessment for } \\
\text { Technology Neutral } \\
\text { Evaluation: } \\
\text { Combining Virtual } \\
\text { and Physical Testing } \\
\text { for Design, } \\
\text { Development and } \\
\text { Review of Digital } \\
\text { Control Systems and } \\
\text { Interfaces }\end{array}$ & $\mathrm{S}$ & & & & $\mathrm{S}$ & $\mathrm{P}$ & & & & & \\
\hline NU101 & Lessing, P. A. & $\begin{array}{l}\text { Advanced Ceramic } \\
\text { Nuclear Fuels }\end{array}$ & & & & & & $\mathrm{P}$ & & & & & \\
\hline PH101 & Deaton, J. D. & $\begin{array}{l}\text { Cognitive Network } \\
\text { Engine and } \\
\text { Simulation } \\
\text { Framework,Ph.D. } \\
\text { Candidate Proposal } \\
\text { for Juan Deaton }\end{array}$ & $\mathrm{P}$ & $\mathrm{S}$ & & & & & & & $\mathrm{S}$ & & \\
\hline PH102 & Kelly, D. L. & $\begin{array}{l}\text { Application of } \\
\text { Dynamic Bayesian } \\
\text { Networks to Systems } \\
\text { with Ambient } \\
\text { Intelligence }\end{array}$ & $\mathrm{S}$ & & & & & $\mathrm{P}$ & & & $\mathrm{S}$ & & \\
\hline PH103 & Marshall, D. W. & $\begin{array}{l}\text { Characterization of } \\
\text { Fluidized Beds via } \\
\text { Pressure-Fluctuation } \\
\text { Analysis }\end{array}$ & & $\mathrm{S}$ & & $\mathrm{S}$ & & $\mathrm{P}$ & & & & & \\
\hline PH104 & Harris, B. K. & $\begin{array}{l}\text { Dissolution and } \\
\text { Extraction Studies of } \\
\text { Fission Products in } \\
\text { Room Temperature } \\
\text { Ionic Liquids and in } \\
\text { Supercritical Fluid } \\
\mathrm{CO} 2 \text { and } \\
\text { Determination of } \\
\text { Radiolytic Stability } \\
\end{array}$ & $\mathrm{S}$ & & $\mathrm{P}$ & & & $\mathrm{S}$ & & & & & \\
\hline PH105 & $\begin{array}{l}\text { McJunkin, T. } \\
\text { R. }\end{array}$ & $\begin{array}{l}\text { Advanced Adaptive } \\
\text { Algorithms in Phased } \\
\text { Array Ultrasonics for } \\
\text { Materials Inspection }\end{array}$ & & & $\mathrm{S}$ & $\mathrm{S}$ & $\mathrm{S}$ & $\mathrm{P}$ & & $\mathrm{S}$ & & & \\
\hline RP110 & Rempe, J. L. & $\begin{array}{l}\text { High Temperature In- } \\
\text { Pile Instrumentation } \\
\text { Enhancements }\end{array}$ & & & & & & $\mathrm{P}$ & $\mathrm{s}$ & & & & \\
\hline SH100 & Wolf, D. W. & $\begin{array}{l}\text { Microstructure and } \\
\text { Deformation Physics } \\
\text { of Fission-Reactor } \\
\text { Model Materials by } \\
\text { Atomistically } \\
\text { Informed Mesoscale } \\
\text { Simulation }\end{array}$ & & & & & $\mathrm{S}$ & $P$ & & & & & \\
\hline
\end{tabular}




\section{Appendix F - Relevance to Major National Programs}

\begin{tabular}{|c|c|c|c|c|c|c|c|c|c|c|c|c|c|}
\hline Project ID & $\begin{array}{l}\text { Principle } \\
\text { Investigator }\end{array}$ & Title & 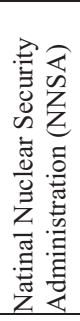 & 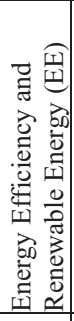 & 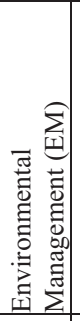 & 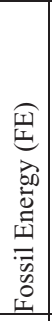 & 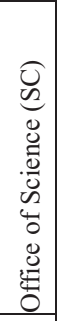 & 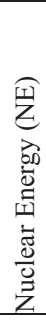 & 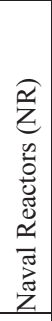 & 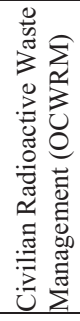 & 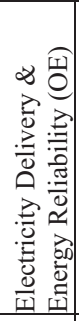 & 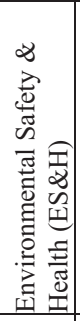 & 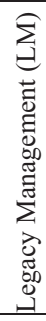 \\
\hline SH101 & Rashkeev, S. & $\begin{array}{l}\text { Structural and } \\
\text { Electronic Properties } \\
\text { of Adsorbed Metal } \\
\text { Nanoparticles }\end{array}$ & & $\mathrm{S}$ & $\mathrm{S}$ & $\mathrm{S}$ & $\mathrm{P}$ & $\mathrm{S}$ & & & & & \\
\hline SH103 & Pernice, M. A. & $\begin{array}{l}\text { Adaptive Modeling } \\
\text { of Geometrically } \\
\text { Complex Fuel Rods } \\
\text { with a posteriori } \\
\text { Error Control }\end{array}$ & $\mathrm{S}$ & & S & $\mathrm{S}$ & $\mathrm{P}$ & $\mathrm{S}$ & & & & & \\
\hline SH104 & NULL & $\begin{array}{l}\text { Multi-physics } \\
\text { Simulation Methods } \\
\text { for Advanced } \\
\text { Reactor Analysis }\end{array}$ & & & & & $\mathrm{S}$ & $\mathrm{P}$ & & & & & \\
\hline ST130 & Stewart, F. F. & $\begin{array}{l}\text { Investigation of Low } \\
\text { Temperature } \\
\text { Performance in } \\
\text { Membrane Materials } \\
\text { and Processes for } \\
\text { Gas Separations }\end{array}$ & & $P$ & $\mathrm{~S}$ & $\mathrm{~S}$ & & $\mathrm{~S}$ & & & & $\mathrm{~S}$ & \\
\hline ST131 & Klaehn, J. R. & $\begin{array}{l}\text { High-Performance } \\
\text { Polymer Membranes } \\
\text { for High Temperature } \\
\text { Gas Separations }\end{array}$ & & & $\mathrm{S}$ & $\mathrm{P}$ & & $\mathrm{S}$ & & & $\mathrm{S}$ & & \\
\hline ST132 & Benson, M. T. & $\begin{array}{l}\text { Triazine-Based CO2 } \\
\text { Capture Agents }\end{array}$ & & $\mathrm{S}$ & & $\mathrm{P}$ & & & & & & & \\
\hline ST133 & Lee, R. D. & $\begin{array}{l}\text { Dynamic Impact } \\
\text { Model and } \\
\text { Information System } \\
\text { to support } \\
\text { Unconventional } \\
\text { Fuels Development }\end{array}$ & & & $\mathrm{S}$ & $\mathrm{P}$ & $\mathrm{S}$ & & & & & & $\mathrm{S}$ \\
\hline ST134 & Wood, T. R. & $\begin{array}{l}\text { Near Field Impacts of } \\
\text { In-Situ Oil Shale } \\
\text { Development on } \\
\text { Water Quality- }\end{array}$ & & $\mathrm{S}$ & $\mathrm{S}$ & $\mathrm{P}$ & $\mathrm{S}$ & & & & & $\mathrm{S}$ & \\
\hline ST135 & $\begin{array}{l}\text { Anderson, M. } \\
\text { O. }\end{array}$ & $\begin{array}{l}\text { Advanced Remote } \\
\text { Sensing for Energy } \\
\text { and Environmental } \\
\text { Applications using } \\
\text { Unmanned Aerial } \\
\text { Vehicles }\end{array}$ & $\mathrm{S}$ & & $\mathrm{P}$ & $\mathrm{S}$ & $\mathrm{S}$ & $\mathrm{S}$ & & & & & $\mathrm{S}$ \\
\hline ST136 & Hurley, D. H. & $\begin{array}{l}\text { Addressing the } \\
\text { Spectrum of Nuclear } \\
\text { Related NDE Needs: } \\
\text { A hybrid laser } \\
\text { ultrasonic and eddy } \\
\text { current approach }\end{array}$ & & & & $\mathrm{S}$ & & $\mathrm{P}$ & $\mathrm{S}$ & & & & \\
\hline ST137 & Windes, W. E. & $\begin{array}{l}\text { Research of } \\
\text { Advanced VHTR } \\
\text { Core Components }\end{array}$ & & & & $\mathrm{S}$ & $\mathrm{S}$ & $\mathrm{P}$ & $\mathrm{S}$ & & & & \\
\hline
\end{tabular}




\section{Appendix F - Relevance to Major National Programs}

\begin{tabular}{|c|c|c|c|c|c|c|c|c|c|c|c|c|c|}
\hline Project ID & $\begin{array}{c}\text { Principle } \\
\text { Investigator }\end{array}$ & Title & 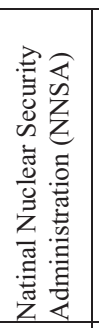 & 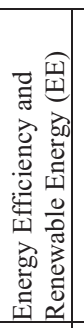 & 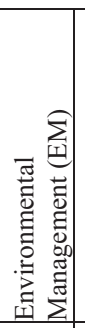 & 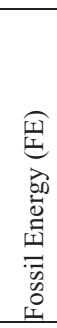 & 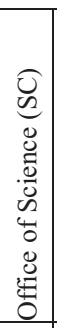 & 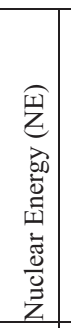 & 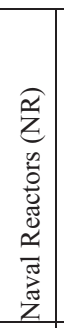 & 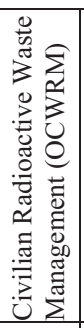 & 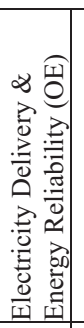 & 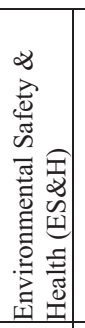 & 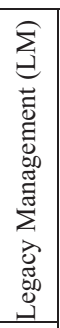 \\
\hline ST138 & $\begin{array}{l}\text { Breckenridge, } \\
\text { R. P. }\end{array}$ & $\begin{array}{l}\text { Development of a } \\
\text { Sustainable } \\
\text { Ecological } \\
\text { Engineering } \\
\text { approach to support } \\
\text { Hybrid energy } \\
\text { Systems } \\
\text { Development in the } \\
\text { Western Inland } \\
\text { Energy Corridor }\end{array}$ & & S & & $\mathrm{P}$ & & & & & & & \\
\hline TM106 & Huang, H. & $\begin{array}{l}\text { Development of 3D } \\
\text { Multiphase Flow and } \\
\text { Reactive Transport } \\
\text { Codes and their } \\
\text { Applications to } \\
\text { Reactive Flow in } \\
\text { Porous Media and } \\
\text { Fracture Apertures }\end{array}$ & & & $\mathrm{P}$ & $\mathrm{S}$ & $\mathrm{S}$ & $\mathrm{S}$ & & $\mathrm{S}$ & $\mathrm{S}$ & & $\mathrm{S}$ \\
\hline TM108 & Gaston, D. R. & $\begin{array}{l}\text { Uncertainty } \\
\text { quantification for } \\
\text { nuclear fuels } \\
\text { performance } \\
\end{array}$ & & & & & $\mathrm{S}$ & $\mathrm{P}$ & $\mathrm{S}$ & & & & \\
\hline
\end{tabular}

